\title{
Leveraging the Domino Skeletal Expansion of Thia- /Selenazolidinones via Nitrogen-Atom Transfer in Hexafluoroisopropanol: Room Temperature Access to Six- Membered S/Se,N-Heterocycles
}

\author{
Vandana Jaiswal, ${ }^{1}$ Mangilal Godara, ${ }^{1}$ Dinabandhu Das, ${ }^{2}$ Vincent Gandon ${ }^{* 3}$ Jaideep Saha ${ }^{* 1}$ \\ ${ }^{1}$ Division of Molecular Synthesis and Drug Discovery, Centre of Biomedical Research \\ (CBMR), SGPGIMS Campus, Lucknow 226014, Uttar Pradesh. India. \\ ${ }^{2}$ School of Physical Sciences, Jawaharlal Nehru University, New Delhi, India. \\ ${ }^{3}$ †'nstitut de Chimie Moléculaire et des Matériaux d'Orsay (ICMMO), CNRS UMR 8182, \\ Université Paris-Saclay, Bâtiment 420, 91405 Orsay cedex, France \& ${ }^{\ddagger}$ Laboratoire de Chimie \\ Moléculaire (LCM), CNRS UMR 9168, Ecole Polytechnique, Institut Polytechnique de Paris, \\ route de Saclay, 91128 Palaiseau cedex, France.
}

\section{Table of Contents}

\begin{tabular}{|c|c|c|}
\hline Entry & Description & Page \\
\hline 1 & Optimization Studies. & $\mathrm{S} 2$ \\
\hline 2 & Preparation of starting materials...... & S3-S26 \\
\hline & Gram scale preparation of compound $\mathbf{2 a} \ldots \ldots \ldots \ldots$ & S27 \\
\hline 4 & Control Experiments... & $\mathrm{S} 28-\mathrm{S} 30$ \\
\hline 5 & NMR Spectra of New Compounds.. & S31-S131 \\
\hline 6 & Computational studies. & S132-S155 \\
\hline & Crystallographic Data.. & S156-S167 \\
\hline 8 & References.. & S168 \\
\hline
\end{tabular}




\section{Optimization Table ${ }^{a}$}

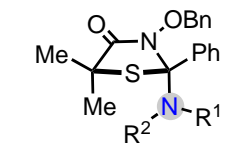

$\mathrm{R}^{1}=\mathrm{R}^{2}=-\left(\mathrm{CH}_{2}\right)_{4^{-}}(\mathbf{1} \mathbf{a})$

$\mathrm{R}^{1}=\mathrm{R}^{2}=\mathrm{Et} \quad(\mathbf{1 b})$

or HFIP adduct (1c)

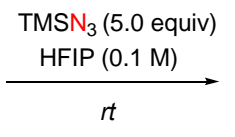

$2 a$
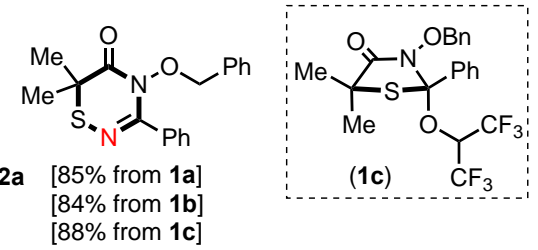

\begin{tabular}{|c|c|c|c|}
\hline Entry & Substrate & Deviation from standard conditions & Yield[\%] $]^{b}$ \\
\hline 1 & $1 \mathrm{a}$ & 2.0 equiv. of $\mathrm{TMSN}_{3}$ & 75 \\
\hline 2 & $1 a$ & None & 85 \\
\hline 3 & $1 a$ & 10 equiv. of $\mathrm{TMSN}_{3}$ & 88 \\
\hline 4 & $1 \mathrm{~b}$ & None & 84 \\
\hline 5 & $1 a$ & Conc. [0.5 M] & 69 \\
\hline 6 & $1 a$ & Conc. [0.1 M] & 33 \\
\hline 7 & $1 a$ & heating at $50^{\circ} \mathrm{C}$ & 85 \\
\hline $8^{c}$ & $1 a$ & TFE as solvent & 31 \\
\hline 9 & $1 a$ & 'PrOH as solvent & 0 \\
\hline 10 & $1 a$ & ACN or DMF as solvent & 0 \\
\hline 11 & $1 a$ & $\mathrm{HFIP}-\mathrm{H}_{2} \mathrm{O}$ mixture $(1: 1)$ & 0 \\
\hline 12 & $1 a$ & $\mathrm{NaN}_{3}$ instead of $\mathrm{TMSN}_{3}$ & 0 \\
\hline $13^{d}$ & $1 a$ & ${ }^{n} \mathrm{Bu}_{4} \mathrm{NN}_{3}$ instead of $\mathrm{TMSN}_{3}$ & 40 \\
\hline $14^{e}$ & $1 a$ & $\mathrm{BnN}_{3}$ instead of $\mathrm{TMSN}_{3}$ & 0 \\
\hline $15^{e}$ & $1 a$ & $\mathrm{PhN}_{3}$ instead of $\mathrm{TMSN}_{3}$ & 0 \\
\hline 16 & 1c & None & 88 \\
\hline 17 & 1c & $\mathrm{DCM}$ or $\mathrm{ACN}$ or DMF as solvent & 0 \\
\hline
\end{tabular}

TFE: trifluoroethanol; ACN: acetonitrile; DMF: dimethylformamide

Table S1: aptimized conditions: Compound 1 a (1.0 equiv), $\operatorname{TMSN}_{3}$ (5.0 equiv), $\mathrm{HFIP}(0.1 \mathrm{M})$, rt; ' considerably sluggish. ${ }^{e}$ Formation of compound $1 \mathbf{c}$ was observed. [Note: Due to insignificant difference in yield for the use of 5 and 10 equivalents of $\mathrm{TMSN}_{3}$ in the reaction, reaction conditions with 5 equivalents was chosen to be optimal for subsequent development] 


\section{Preparation of Starting Materials}

2a. List of 2-aminothiazolidin-4-one derivatives or 1,1,1,3,3,3-hexafluoropropan-2-yl)oxy)thiazolidin-4-ones used in the study:

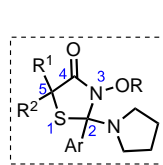

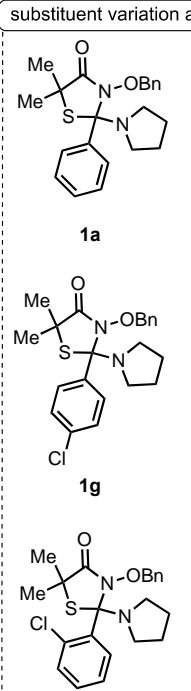

$1 \mathrm{~m}$

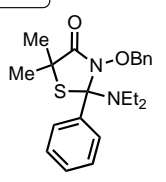

1b
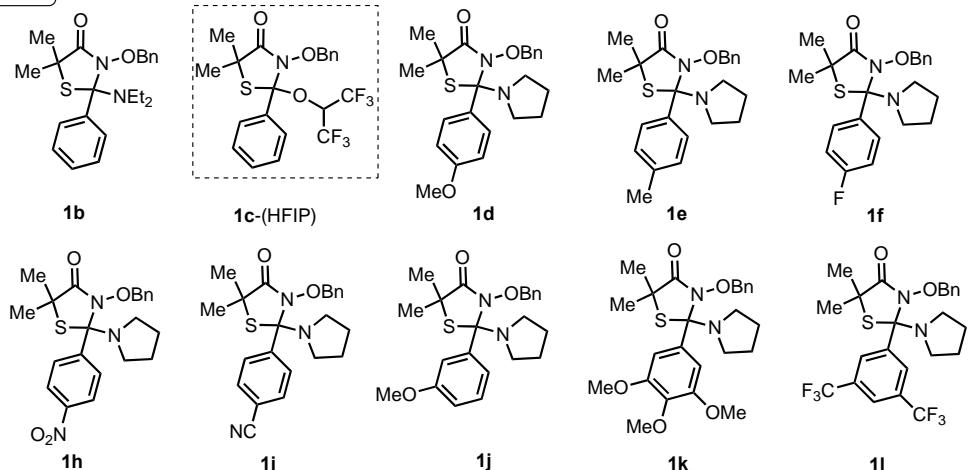

$1 \mathrm{k}$

11

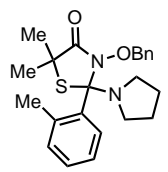

1n
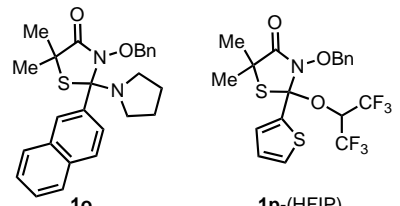

1p-(HFIP)

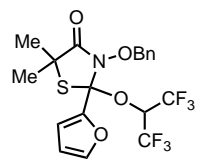

1q-(HFIP)
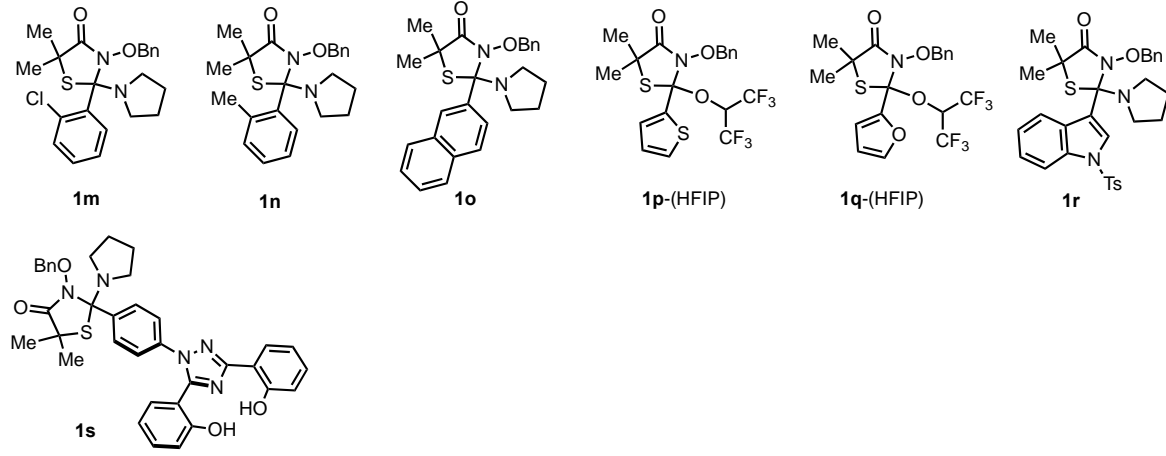

substituent variation at C5
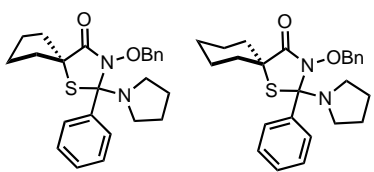

(a)
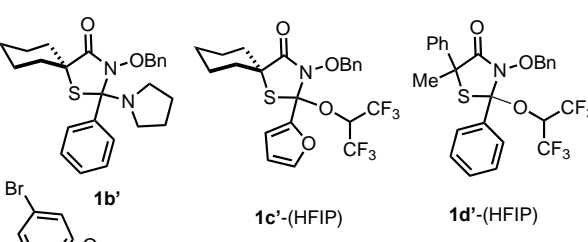

1d'-(HFIP)
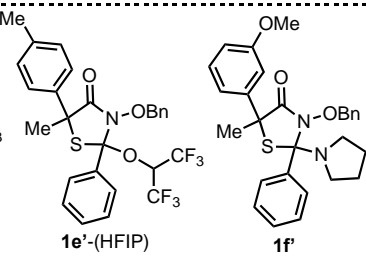

19'-(HFIP)

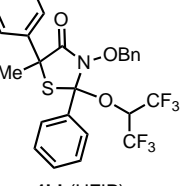

substituent variation at $\mathrm{N}-\mathrm{OR}$

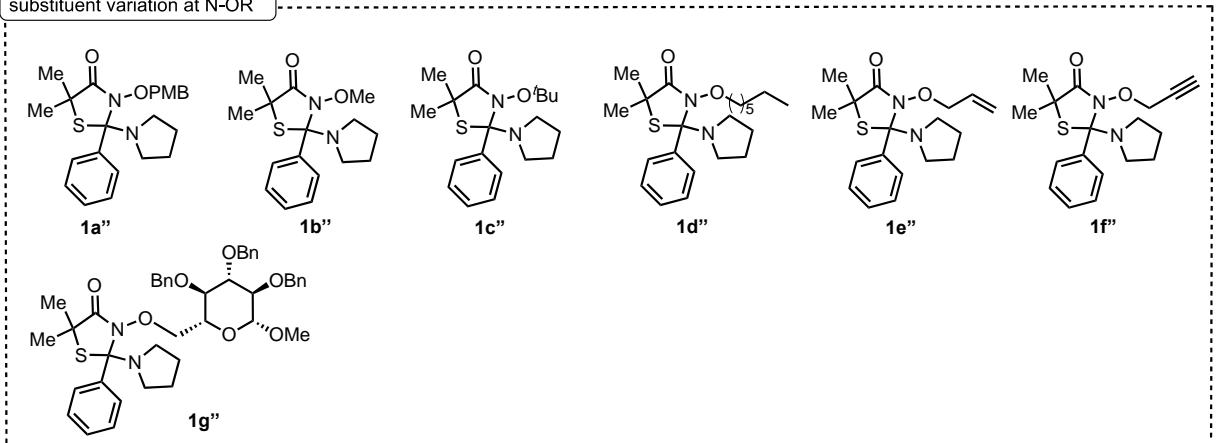

Figure S1-A. Structures of new and reported thiazolidinones used in the current study. 
<smiles>CC(Br)(Br)C(=O)NOCc1ccccc1</smiles>

$4 a$

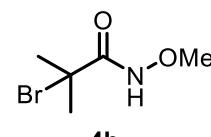

4b

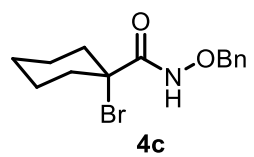

$4 \mathrm{c}$<smiles>CC(C)(Br)C(=O)NO[14CH3]</smiles>

4d<smiles>CCOONC(=O)C(C)(C)Br</smiles>

$4 e$<smiles>COc1ccc(C(=S)N2CCCC2)cc1</smiles>

$5 a$<smiles>[Se]=C(c1ccccc1)N1CCC1</smiles>

$5 b$

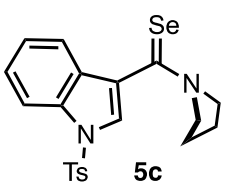

TS 5

Figure S1-B. Structures of new and reported $\alpha$-halohydroxamates and selenamides used in the current study.

2b. General procedure for the synthesis of 2-aminothiazolidin-4-one derivatives or 1,1,1,3,3,3hexafluoropropan-2-yl)oxy)-thiazolidin-4-one (1):

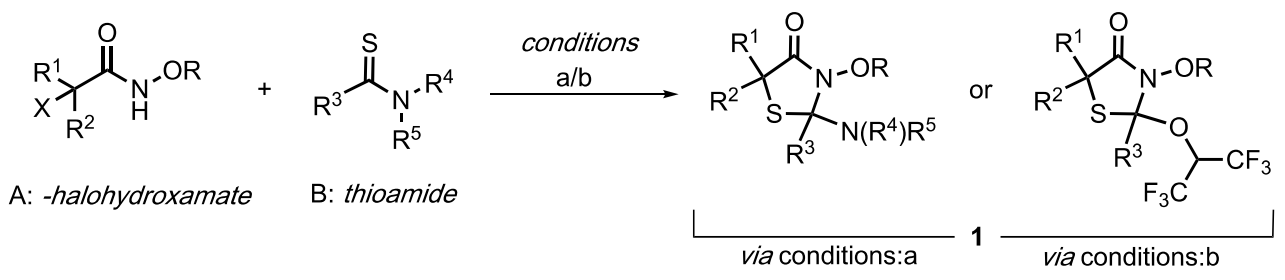

Scheme S1. General procedure for 2-aminothiazolidin-4-one derivatives or HFIP-adducts.

(i) 2-Aminothiazolidin-4-ones: These compounds were prepared following our previous report. ${ }^{1}$ To a solution of thioamide (1.0 equiv) and $\alpha$-halohydroxamate (2-3 equiv) in acetonitrile $(0.5 \mathrm{M})$ was added $\mathrm{Na}_{2} \mathrm{CO}_{3}$ (4.0 equiv) and the reaction mixture was heated at 80 ${ }^{\circ} \mathrm{C}$ for $1 \mathrm{~h}$. Upon completion of the reaction (TLC controlled), reaction mixture was passed through celite and thoroughly washed with ethyl acetate. The filtrate was concentrated under vacuo and the crude product was purified by silica gel column chromatography to afford the 2-aminothiazolidin-4-one derivatives (1).

(ii) 1,1,1,3,3,3-Hexafluoropropan-2-yl)oxy)-thiazolidin-4-ones: To a solution of $\alpha$ halohydroxamate (1.0 equiv) and thioamide (1.0 equiv) in $\left(\mathrm{CF}_{3}\right)_{2} \mathrm{CHOH}(0.4 \mathrm{M})$, was added DIPEA (2.0 equiv) dropwise. The reaction mixture was stirred at room temperature and the reaction progress was monitored by thin layer chromatography (TLC). Upon completion of the reaction, HFIP was removed under reduced pressure and the crude was purified by silica gel column chromatography to afford the corresponding 1,1,1,3,3,3-hexafluoropropan-2-yl)oxy)thiazolidin-4-ones (1) . 
2c. Characterization of Newly Synthesized Thiazolidin-4-ones.

3-(Benzyloxy)-2-(4-methoxyphenyl)-5,5-dimethyl-2-(pyrrolidin-1-yl)thiazolidin-4-one (1d)

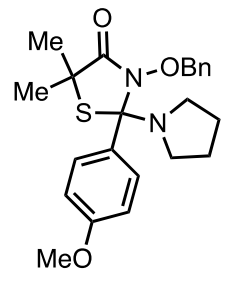

Following the general procedure $(3 \mathbf{b}-\mathbf{i})$, reaction between thioamide, [(4methoxyphenyl)(pyrrolidin-1-yl)methanethione (0.075 g, $0.33 \mathrm{mmol}, 1.0$ equiv)] and corresponding a-halohydroxamate $(0.184 \mathrm{~g}, 0.67 \mathrm{mmol}, 2.0$ equiv) afforded desired 4thiazolidinone 1d, which was purified by silica gel column chromatography (3:97

EtOAc:Hexane as eluent) to give the title compound as white solid in $64 \%(0.090 \mathrm{~g})$ yield. $R_{f}$ 0.3 (1:9 EtOAc:Hexane); ${ }^{1} \mathbf{H}$ NMR (400 MHz, $\left.\mathrm{CDCl}_{3}\right) \delta 8.01(\mathrm{~d}, J=8.8 \mathrm{~Hz}, 2 \mathrm{H}), 7.28-7.23$ (m, $5 \mathrm{H}), 6.91(\mathrm{~d}, J=8.8 \mathrm{~Hz}, 2 \mathrm{H}), 4.82(\mathrm{~d}, J=8.8 \mathrm{~Hz}, 1 \mathrm{H}), 3.83-3.80(\mathrm{~m}, 4 \mathrm{H}), 2.65-2.57(\mathrm{~m}, 2 \mathrm{H})$, 2.52-2.45 (m, 2H), 1.80-1.75 (m, 4H), $1.71(\mathrm{~s}, 3 \mathrm{H}), 1.66(\mathrm{~s}, 3 \mathrm{H}) ;{ }^{13} \mathrm{C}\{\mathbf{1 H}\}$ NMR $(100 \mathrm{MHz}$, $\left.\mathrm{CDCl}_{3}\right) \delta 170.6,160.3,134.5,131.9,131.3,129.8,128.7,128.3,113.5,92.2,76.9,55.4,48.0$, 44.9, 31.8, 27.6, 24.0;HRMS (ESI-TOF) m/z: [M+H] ${ }^{+}$calculated for $\mathrm{C}_{23} \mathrm{H}_{29} \mathrm{~N}_{2} \mathrm{O}_{3} \mathrm{~S}$ 413.1899, mass found 413.1893 .

3-(Benzyloxy)-5,5-dimethyl-2-(pyrrolidin-1-yl)-2-(p-tolyl)thiazolidin-4-one (1e)

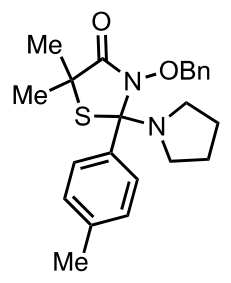

Following the general procedure $(3 b-i)$, reaction between thioamide, [pyrrolidin-1-yl(ptolyl)methanethione $(0.200 \mathrm{~g}, 0.97 \mathrm{mmol}, 1.0$ equiv)] and $\alpha$-halohydroxamate $(0.528 \mathrm{~g}, 1.94$ mmol, 2.0 equiv) afforded desired 4-thiazolidinone 1e, which was purified by silica gel column chromatography (2:98 EtOAc:Hexane as eluent) to give the title compound as semi solid in $84 \%(0.334 \mathrm{~g})$ yield. $R_{f} 0.2$ (5:95 EtOAc:Hexane); ${ }^{1} \mathbf{H}$ NMR $\left(400 \mathrm{MHz}, \mathrm{CDCl}_{3}\right) \delta 8.00$ (d, $\mathrm{J}=$ $8.0 \mathrm{~Hz}, 2 \mathrm{H}), 7.28-7.23(\mathrm{~m}, 5 \mathrm{H}), 7.22(\mathrm{~J}=8.0 \mathrm{~Hz}, 2 \mathrm{H}), 4.83(\mathrm{~d}, J=8.4 \mathrm{~Hz}, 1 \mathrm{H}), 3.83(\mathrm{~d}, J=$ $8.8 \mathrm{~Hz}, 1 \mathrm{H}), 2.69-2.61(\mathrm{~m}, 2 \mathrm{H}), 2.55-2.48(\mathrm{~m}, 2 \mathrm{H}), 2.37(\mathrm{~s}, 3 \mathrm{H}), 1.80-1.74(\mathrm{~m}, 4 \mathrm{H}), 1.73(\mathrm{~s}$, $3 \mathrm{H}), 1.68(\mathrm{~s}, 3 \mathrm{H}) ;{ }^{13} \mathrm{C}\{1 \mathrm{H}\}$ NMR $\left(100 \mathrm{MHz}, \mathrm{CDCl}_{3}\right) \delta 170.4,138.9,136.7,134.1,129.6,128.8$, 128.5, 128.1, 92.0, 76.6, 47.7, 44.6, 31.5, 27.3, 23.7, 21.0; HRMS (ESI-TOF) m/z: [M+H] ${ }^{+}$ calculated for, $\mathrm{C}_{23} \mathrm{H}_{29} \mathrm{~N}_{2} \mathrm{O}_{2} \mathrm{~S}$ 397.1950, mass found 397.1942 .

3-(Benzyloxy)-2-(4-fluorophenyl)-5,5-dimethyl-2-(pyrrolidin-1-yl)thiazolidin-4-one (1f) 


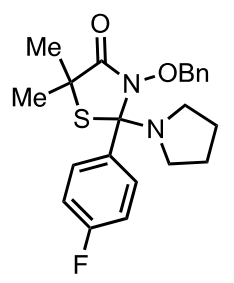

Following the general procedure $(\mathbf{3 b}-\mathbf{i})$, reaction between thioamide [(4fluorophenyl)(pyrrolidin-1-yl)methanethione $(0.285 \mathrm{~g}, 1.36 \mathrm{mmol}, 1.0$ equiv)] and $\alpha-$ halohydroxamate $(0.742 \mathrm{~g}, 2.72 \mathrm{mmol}, 2.0$ equiv) afforded desired 4-thiazolidinone 1f, which was purified by silica gel column chromatography (3:97 EtOAc:Hexane as eluent) to give the title compound as white solid in $79 \%(0.430 \mathrm{~g})$ yield. $R_{f} 0.4(1: 9 \mathrm{EtOAc:Hexane}) ;{ }^{1} \mathbf{H}$ NMR $\left(400 \mathrm{MHz}, \mathrm{CDCl}_{3}\right) \delta 8.09(\mathrm{dd}, J=8.4,5.6 \mathrm{~Hz}, 2 \mathrm{H}), 7.31-7.27(\mathrm{~m}, 3 \mathrm{H}), 7.27-7.24(\mathrm{~m}, 2 \mathrm{H})$, $7.09(\mathrm{~d}, J=8.8 \mathrm{~Hz}, 2 \mathrm{H}), 4.88(\mathrm{~d}, J=8.8 \mathrm{~Hz}, 1 \mathrm{H}), 3.89(\mathrm{~d}, J=8.8 \mathrm{~Hz}, 1 \mathrm{H}), 2.65-2.58(\mathrm{~m}, 2 \mathrm{H})$, 2.54-2.48 (m, 2H), 1.84-1.76 (m, 4H), $1.72(\mathrm{~s}, 3 \mathrm{H}), 1.69(\mathrm{~s}, 3 \mathrm{H}) ;{ }^{13} \mathbf{C}\{1 \mathrm{H}\} \quad \mathrm{NMR}(100 \mathrm{MHz}$, $\left.\mathrm{CDCl}_{3}\right) \delta 170.8,163.3(\mathrm{~d}, J=247.5 \mathrm{~Hz}), 135.7(\mathrm{~d}, J=3.1 \mathrm{~Hz}), 134.3,131.9(\mathrm{~d}, J=8.2 \mathrm{~Hz})$, 129.8, 128.8, 128.4, 115.2 (d, $J=21.2 \mathrm{~Hz}$ ), 91.7, 77.0, 48.1, 45.0, 31.8, 27.6, 24.0; HRMS (ESI-TOF) m/z: [M+H] $]^{+}$calculated for $\mathrm{C}_{22} \mathrm{H}_{26} \mathrm{FN}_{2} \mathrm{O}_{2} \mathrm{~S}$ 401.1699, mass found 401.1691.

3-(Benzyloxy)-2-(4-chlorophenyl)-5,5-dimethyl-2-(pyrrolidin-1-yl)thiazolidin-4-one (1g)<smiles></smiles>

Following the general procedure $(3 \mathbf{b}-\mathbf{i})$, reaction between thioamide [(4chlorophenyl)(pyrrolidin-1-yl)methanethione $(0.200 \mathrm{~g}, 0.88 \mathrm{mmol}, 1.0$ equiv)] and $\alpha-$ halohydroxamate $(0.480 \mathrm{~g}, 1.76 \mathrm{mmol}, 2.0$ equiv) afforded desired 4-thiazolidinone $1 \mathrm{~g}$, which was purified by silica gel column chromatography (3:97 EtOAc:Hexane as eluent) to give the title compound as white solid in $89 \%$ ( $0.330 \mathrm{~g})$ yield. $R_{f} 0.4$ (1:9 EtOAc:Hexane); ${ }^{1} \mathrm{H}$ NMR (400 $\left.\mathrm{MHz}, \mathrm{CDCl}_{3}\right) \delta 8.03(\mathrm{~d}, J=8.8 \mathrm{~Hz}, 2 \mathrm{H}), 7.37(\mathrm{~d}, J=8.8 \mathrm{~Hz}, 2 \mathrm{H}), 7.32-7.28(\mathrm{~m}, 3 \mathrm{H}), 7.27-7.23$ $(\mathrm{m}, 2 \mathrm{H}), 4.89(\mathrm{~d}, J=8.8 \mathrm{~Hz}, 1 \mathrm{H}), 3.98(\mathrm{~d}, J=8.8 \mathrm{~Hz}, 1 \mathrm{H}), 2.66-2.57(\mathrm{~m}, 2 \mathrm{H}), 2.55-2.48(\mathrm{~m}$, $2 \mathrm{H}), 1.85-1.76(\mathrm{~m}, 4 \mathrm{H}), 1.72(\mathrm{~s}, 3 \mathrm{H}), 1.69(\mathrm{~s}, 3 \mathrm{H}) ;{ }^{13} \mathrm{C}\{\mathbf{1 H}\} \mathbf{N M R}\left(100 \mathrm{MHz}, \mathrm{CDCl}_{3}\right) \delta 171.0$, 138.6, 135.2, 134.3, 131.4, 129.8, 128.9, 128.5, 128.5(2), 91.7, 77.0, 48.1, 45.1, 31.8, 27.6, 24.0; HRMS (ESI-TOF) m/z: [M+H] $]^{+}$calculated for $\mathrm{C}_{22} \mathrm{H}_{26} \mathrm{CIN}_{2} \mathrm{O}_{2} \mathrm{~S}$ 417.1404, mass found 417.1398. 
4-(3-(Benzyloxy)-5,5-dimethyl-4-oxo-2-(pyrrolidin-1-yl)thiazolidin-2-yl)benzonitrile (1i)<smiles>CCCCON1C(=O)C(C)(C)SC1(c1ccc(C#N)cc1)N1CCCC1</smiles>

This compound was prepared in HFIP following the general procedure (3b-ii). Accordingly, reaction between thioamide [4-(pyrrolidine-1-carbonothioyl)benzonitrile $(0.050 \mathrm{~g}, 0.23 \mathrm{mmol}$, 1.0 equiv)] and $\alpha$-halohydroxamate $(0.063 \mathrm{~g}, 0.23 \mathrm{mmol}, 1.0$ equiv) afforded desired 4thiazolidinone 1i, which was purified by silica gel column chromatography (3:97 EtOAc:Hexane as eluent) to give the title compound as colorless oil in $51 \%(0.048 \mathrm{~g})$ yield. $R_{f}$ 0.3 (1:9 EtOAc:Hexane); ${ }^{1} \mathrm{H}$ NMR (400 MHz, CDCl $) \delta 8.17$ (d, $\left.J=8.3 \mathrm{~Hz}, 2 \mathrm{H}\right), 7.67$ (d, $J=$ 8.3 Hz, 2H), 7.33-7.27 (m, 3H), 7.24-7.19 (m, 2H), $4.92(\mathrm{~d}, J=9.0 \mathrm{~Hz}, 1 \mathrm{H}), 4.09(\mathrm{~d}, J=9.0$ $\mathrm{Hz}, 1 \mathrm{H}), 2.66-2.59(\mathrm{~m}, 2 \mathrm{H}), 2.58-2.50(\mathrm{~m}, 2 \mathrm{H}), 1.87-1.78(\mathrm{~m}, 4 \mathrm{H}), 1.71(\mathrm{~s}, 3 \mathrm{H}), 1.70(\mathrm{~s}, 3 \mathrm{H})$; ${ }^{13} \mathrm{C}\{1 \mathrm{H}\}$ NMR $\left(100 \mathrm{MHz}, \mathrm{CDCl}_{3}\right) \delta 171.3,145.2,134.1,132.1,130.5,129.6,128.9,128.5$, 118.5, 113.0, 91.3, 77.1 , 48.0, 45.3, 31.6, 27.7, 24.1;HRMS (ESI-TOF) m/z: [M+H] calculated for $\mathrm{C}_{23} \mathrm{H}_{26} \mathrm{~N}_{3} \mathrm{O}_{2} \mathrm{~S}$ 408.1746, mass found 408.1740 .

3-(Benzyloxy)-5,5-dimethyl-2-(pyrrolidin-1-yl)-2-(3,4,5-trimethoxyphenyl)thiazolidin-4-one $(1 k)$

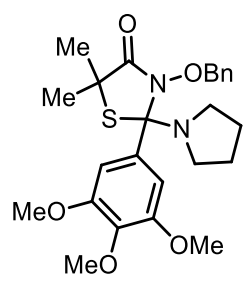

Following the general procedure $(3 b-i)$, reaction between thioamide [pyrrolidin-1-yl(3,4,5trimethoxyphenyl)methanethione $(0.238 \mathrm{~g}, 0.84 \mathrm{mmol}, 1.0$ equiv)] and $\alpha$-halohydroxamate ( $0.458 \mathrm{~g}, 1.68 \mathrm{mmol}, 2.0$ equiv) afforded desired 4-thiazolidinone $\mathbf{1} \mathbf{k}$, which was purified by silica gel column chromatography (1:9 EtOAc:Hexane as eluent) to give the title compound as white solid in 60\% (0.242 g) yield. $R_{f} 0.2$ (1:9 EtOAc:Hexane); ${ }^{1} \mathbf{H}$ NMR (400 MHz, $\left.\mathrm{CDCl}_{3}\right) \delta$ $7.33(\mathrm{~s}, 2 \mathrm{H}), 7.24-7.20(\mathrm{~m}, 3 \mathrm{H}), 7.18-7.14(\mathrm{~m}, 2 \mathrm{H}), 4.92(\mathrm{~d}, J=8.9 \mathrm{~Hz}, 1 \mathrm{H}), 3.82(\mathrm{~s}, 3 \mathrm{H}), 3.80-$ $3.74(\mathrm{~m}, 7 \mathrm{H}), 2.64-2.57(\mathrm{~m}, 2 \mathrm{H}), 2.51-2.43(\mathrm{~m}, 2 \mathrm{H}), 1.77-1.72(\mathrm{~m}, 4 \mathrm{H}), 1.68(\mathrm{~s}, 3 \mathrm{H}), 1.63(\mathrm{~s}$, $3 \mathrm{H}) ;{ }^{13} \mathrm{C}\{1 \mathrm{H}\}$ NMR $\left(100 \mathrm{MHz}, \mathrm{CDCl}_{3}\right) \delta 170.4,152.8,138.5,135.1,134.5,129.5,128.7,128.3$, 107.1, 92.6, 76.8, 60.9, 56.1, 48.1, 44.8, 31.7, 27.4, 24.0; HRMS (ESI-TOF) m/z: [M+H] ${ }^{+}$ calculated for $\mathrm{C}_{25} \mathrm{H}_{33} \mathrm{~N}_{2} \mathrm{O}_{5} \mathrm{~S} 473.2110$, mass found 473.2108 . 
3-(Benzyloxy)-2-(3,5-bis(trifluoromethyl)phenyl)-5,5-dimethyl-2-(pyrrolidin-1-yl)thiazolidin-4one (11)<smiles>CC(C)(C)C(=O)N1CCCC1</smiles>

Following the general procedure $(3 \mathbf{b}-\mathbf{i})$, reaction between thioamide $[(3,5-$ bis(trifluoromethyl)phenyl)(pyrrolidin-1-yl)methanethione $(0.345 \mathrm{~g}, 1.05 \mathrm{mmol}, 1.0$ equiv)] and a-halohydroxamate $(0.574 \mathrm{~g}, 2.10 \mathrm{mmol}, 2.0$ equiv) afforded desired 4-thiazolidinone 1I, which was purified by silica gel column chromatography (2:98 EtOAc:Hexane as eluent) to give the title compound as colorless oil in $69 \%(0.356 \mathrm{~g})$ yield. $R_{f} 0.35$ (1:9 EtOAc:Hexane); ${ }^{1} \mathrm{H}$ NMR $\left(400 \mathrm{MHz}, \mathrm{CDCl}_{3}\right) \delta 8.62(\mathrm{~s}, 2 \mathrm{H}), 7.96(\mathrm{~s}, 1 \mathrm{H}), 7.36-7.30(\mathrm{~m}, 3 \mathrm{H}), 7.29-7.24(\mathrm{~m}, 2 \mathrm{H})$, $5.02(\mathrm{~d}, J=8.8 \mathrm{~Hz}, 1 \mathrm{H}), 4.36(\mathrm{~d}, J=9.2 \mathrm{~Hz}, 1 \mathrm{H}), 2.72-2.60(\mathrm{~m}, 4 \mathrm{H}), 1.97-1.90(\mathrm{~m}, 4 \mathrm{H}), 1.82$ $(\mathrm{s}, 3 \mathrm{H}), 1.81(\mathrm{~s}, 3 \mathrm{H}) ;{ }^{13} \mathrm{C}\{1 \mathrm{H}\}$ NMR $\left(100 \mathrm{MHz}, \mathrm{CDCl}_{3}\right) \delta 171.5,142.6,133.7,131.7$ (q, $J=33.0$ $\mathrm{Hz}$ ), 130.4-130.2(m), 129.8, 129.1, 128.5, 123.4 (q, $J=271.0 \mathrm{~Hz}$ ), 123.2 (quint., $J=4.0 \mathrm{~Hz}$ ), 91.3, 77.2, 48.3, 45.4, 31.7, 27.6, 24.1; HRMS (ESI-TOF) $\mathrm{m} / \mathrm{z}:[\mathrm{M}+\mathrm{H}]^{+}$calculated for, $\mathrm{C}_{24} \mathrm{H}_{25} \mathrm{~F}_{6} \mathrm{~N}_{2} \mathrm{O}_{2} \mathrm{~S}$ 519.1541, mass found 519.1534 .

3-(Benzyloxy)-2-(2-chlorophenyl)-5,5-dimethyl-2-(pyrrolidin-1-yl)thiazolidin-4-one (1m)<smiles>CC(C)(C(N)=O)C(c1ccccc1Cl)(N1CCCC1)N1CCCC1</smiles>

Following the general procedure $(3 \mathbf{b}-\mathbf{i})$, reaction between thioamide [(2chlorophenyl)(pyrrolidin-1-yl)methanethione $\quad(0.2 \mathrm{~g}, 0.89 \mathrm{mmol}, 1.0$ equiv)] and $\alpha-$ halohydroxamate $(0.48 \mathrm{~g}, 1.78 \mathrm{mmol}, 2.0$ equiv) afforded desired 4-thiazolidinone $1 \mathrm{~m}$, which was purified by silica gel column chromatography (2:98 EtOAc:Hexane as eluent) to give the title compound as white solid in 57\% (0.210 g) yield. $R_{f}$ 0.3 (5:95 EtOAc:Hexane); ${ }^{1} \mathbf{H}$ NMR (400 MHz, $\left.\mathrm{CDCl}_{3}\right)$ ठ 7.54-7.48 (m, 3H), 7.41-7.35 (m, 4H), 7.22-7.13 (m, 2H), 5.40 (d, J=8.8 $\mathrm{Hz}, 1 \mathrm{H}), 4.98(\mathrm{~d}, J=8.8 \mathrm{~Hz}, 1 \mathrm{H}), 3.36-3.16(\mathrm{~m}, 2 \mathrm{H}), 3.01-2.86(\mathrm{~m}, 2 \mathrm{H}), 1.93-1.85(\mathrm{~m}, 4 \mathrm{H}), 1.62$ $(\mathrm{s}, 3 \mathrm{H}), 1.28(\mathrm{~s}, 3 \mathrm{H}) ;{ }^{13} \mathrm{C}\{\mathbf{1 H}\}$ NMR $\left(100 \mathrm{MHz}, \mathrm{CDCl}_{3}\right) \delta 172.3,140.7,134.7,133.1,131.6$, $129.4,129.0,128.9,128.6,126.9,126.8,88.2,77.2,47.4,46.2,29.6,29.2,26.1$; HRMS (ESITOF) $\mathrm{m} / \mathrm{z}:[\mathrm{M}+\mathrm{H}]^{+}$calculated for $\mathrm{C}_{22} \mathrm{H}_{26} \mathrm{CIN}_{2} \mathrm{O}_{2} \mathrm{~S} 417.1404$ mass found 417.1398. 
<smiles>Cc1ccccc1C1(N2CCCC2)SC(C)(C)C(=O)N1OCc1ccccc1</smiles>

Following the general procedure, reaction between thioamide pyrrolidin-1-yl(otolyl)methanethione $(0.200 \mathrm{~g}, 0.98 \mathrm{mmol}, 1.0$ equiv) and halohydroxamate $(0.536 \mathrm{~g}, 1.96$ $\mathrm{mmol}, 2.0$ equiv) afforded the corresponding thiazolidin-4-one $\mathbf{1 n}$, which was purified by silica gel column chromatography (2:98 EtOAc:Hexane as eluent) to give the title compound as white solid in $61 \%(0.237 \mathrm{~g})$ yield. $R, 0.25$ (5:95 EtOAc:Hexane); ${ }^{1} \mathbf{H}$ NMR $\left(400 \mathrm{MHz}, \mathrm{CDCl}_{3}\right) \delta$ 7.52-7.46 (m, 3H) , 7.40-7.33 (m, 3H), 7.19-7.16 (m, 2H), 7.14-7.07 (m, 1H), $5.31(\mathrm{~d}, J=8.8$ $\mathrm{Hz}, 1 \mathrm{H}), 5.01(\mathrm{~d}, J=8.8 \mathrm{~Hz}, 1 \mathrm{H}), 3.47-2.66(\mathrm{~m}, 4 \mathrm{H}), 2.48(\mathrm{~s}, 3 \mathrm{H}), 1.94-1.79(\mathrm{~s}, 4 \mathrm{H}), 1.63$ (s, $3 \mathrm{H}), 1.32(\mathrm{~s}, 3 \mathrm{H}) .{ }^{13} \mathrm{C}\{\mathbf{1 H}\}$ NMR $\left(100 \mathrm{MHz}, \mathrm{CDCl}_{3}\right) \delta 173.1,141.2,136.7,134.8,132.4,129.4$, 128.8, 128.6, 128.0, 125.8, 125.6, 89.6, 76.7, 47.6, 46.3, 29.4, 29.3, 25.7, 21.0; HRMS (ESITOF) $\mathrm{m} / \mathrm{z}:[\mathrm{M}+\mathrm{H}]^{+}$calculated for $\mathrm{C}_{23} \mathrm{H}_{29} \mathrm{~N}_{2} \mathrm{O}_{2} \mathrm{~S} 397.1950$, mass found 397.1945.

3-(Benzyloxy)-5,5-dimethyl-2-(naphthalen-2-yl)-2-(pyrrolidin-1-yl)thiazolidin-4-one (10)

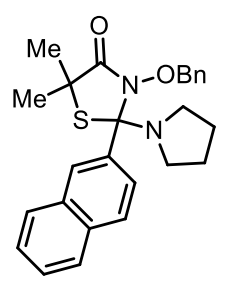

Following the general procedure $(3 \mathbf{b}-\mathbf{i})$, reaction between thioamide, [naphthalen-2$\mathrm{yl}($ pyrrolidin-1-yl)methanethione $(0.200 \mathrm{~g}, 0.83 \mathrm{mmol}, 1.0$ equiv)] and $\alpha$-halohydroxamate ( $0.449 \mathrm{~g}, 1.66 \mathrm{mmol}, 2.0$ equiv) afforded desired 4-thiazolidinone 10, which was purified by silica gel column chromatography (2:98 EtOAc:Hexane as eluent) to give the title compound as colorless oil in $87 \%(0.311 \mathrm{~g})$ yield. $R_{f} 0.2$ (5:95 EtOAc:Hexane); ${ }^{1} \mathbf{H} \mathbf{N M R}\left(400 \mathrm{MHz}, \mathrm{CDCl}_{3}\right)$ $\delta 8.55(\mathrm{~s}, 1 \mathrm{H}), 8.17(\mathrm{~d}, J=8.7 \mathrm{~Hz}, 1 \mathrm{H}), 7.87-7.76(\mathrm{~m}, 3 \mathrm{H}), 7.47-7.41(\mathrm{~m}, 2 \mathrm{H}), 7.17-7.11(\mathrm{~m}$, $5 \mathrm{H}), 4.82(\mathrm{~d}, J=8.7 \mathrm{~Hz}, 1 \mathrm{H}), 3.83(\mathrm{~d}, J=8.7 \mathrm{~Hz}, 1 \mathrm{H}), 2.69-2.61(\mathrm{~m}, 2 \mathrm{H}), 2.58-2.50(\mathrm{~m}, 2 \mathrm{H})$, 1.79-1.71(m,7H), $1.68(\mathrm{~s}, 3 \mathrm{H}) ;{ }^{13} \mathrm{C}\{\mathbf{1 H}\}$ NMR $\left(100 \mathrm{MHz}, \mathrm{CDCl}_{3}\right) \delta 170.8,137.3,134.2,133.5$, 132.9, 129.7, 129.3, 128.7, 128.6 , 128.1, 127.8, 127.4, 127.2, 126.8, 126.2, 92.2, 76.8, 47.9, 44.9, 31.7, 27.5, 23.9; HRMS (ESI-TOF) m/z: $[\mathrm{M}+\mathrm{H}]^{+}$calculated for $\mathrm{C}_{26} \mathrm{H}_{29} \mathrm{~N}_{2} \mathrm{O}_{2} \mathrm{~S} 433.1950$, mass found 433.1945 . 
3-(Benzyloxy)-2-((1,1,1,3,3,3-hexafluoropropan-2-yl)oxy)-5,5-dimethyl-2-(thiophen-2-

yl)thiazolidin-4-one (1p-HFIP)

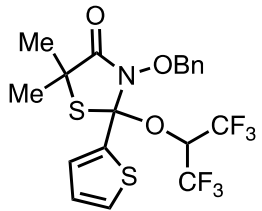

Following the general procedure (3b-ii), reaction between pyrrolidin-1-yl(thiophen-2$\mathrm{yl})$ methanethione $(0.200 \mathrm{~g}, 1.01 \mathrm{mmol}, 1.0$ equiv)] and $\alpha$-halohydroxamate $(0.274 \mathrm{~g}, 1.01$ mmol, 1.0 equiv) afforded desired 4-thiazolidinone 1p-HFIP, which was purified by silica gel column chromatography (2:98 EtOAc:Hexane as eluent) to give the title compound as colorless oil in $52 \%(0.254 \mathrm{~g})$ yield. $R_{f} 0.6$ (5:95 EtOAc:Hexane); ${ }^{1} \mathbf{H}$ NMR (400 MHz, $\mathrm{CDCl}_{3}$ ) $\delta 7.53-7.50(\mathrm{~m}, 1 \mathrm{H}), 7.42(\mathrm{~d}, J=5.2 \mathrm{~Hz}, 1 \mathrm{H}), 7.31(\mathrm{~d}, J=7.2 \mathrm{~Hz}, 2 \mathrm{H}), 7.26(\mathrm{t}, J=7.2 \mathrm{~Hz}, 2 \mathrm{H})$, 7.21 (t, J=7.2 Hz, 1H), 6.93 (t, J=4.8 Hz, 1H), 5.54 (sept, J=6.0 Hz, 1H), 5.09 (s, 2H), 1.54 $(\mathrm{s}, 6 \mathrm{H}) ;{ }^{13} \mathbf{C}\{\mathbf{1 H}\} \mathbf{N M R}\left(100 \mathrm{MHz}, \mathrm{CDCl}_{3}\right) \delta 171.1,145.0,137.4,131.5,131.2,130.6,128.5$, 128.4, 128.1, 126.0, 124.8-116.4 ( $\left.\mathrm{m}, \mathrm{CF}_{3}\right), 77.0,67.5$ (hept, $\left.J_{\mathrm{FC}}=34.4 \mathrm{~Hz}\right), 50.8,25.8$. HRMS (ESI-TOF) m/z: [M+H] ${ }^{+}$calculated for, $\mathrm{C}_{19} \mathrm{H}_{18} \mathrm{~F}_{6} \mathrm{NO}_{3} \mathrm{~S}_{2} 486.0632$ mass found 486.0627 .

3-(Benzyloxy)-2-(furan-2-yl)-2-((1,1,1,3,3,3-hexafluoropropan-2-yl)oxy)-5,5-

dimethylthiazolidin-4-one (1q-HFIP)

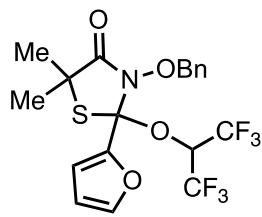

Following the general procedure $(\mathbf{3 b}$-ii), reaction between thioamide [phenyl(pyrrolidin-1$\mathrm{yl})$ methanethione $(0.100 \mathrm{~g}, 0.55 \mathrm{mmol}, 1.0$ equiv)] and $\alpha$-halohydroxamate $(0.150 \mathrm{~g}, 0.55$ mmol, 1.0 equiv) afforded desired 4-thiazolidinone 1q-HFIP, which was purified by silica gel column chromatography (2:98 EtOAc:Hexane as eluent) to give the title compound as colorless oil in 58\% (0.152 g) yield. $R_{f} 0.4$ (5:95 EtOAc:Hexane); ${ }^{1} \mathbf{H}$ NMR (400 MHz, $\mathrm{CDCl}_{3}$ ) $\delta 7.43(\mathrm{~d}, J=1.2 \mathrm{~Hz}, 1 \mathrm{H}), 7.39-7.31(\mathrm{~m}, 5 \mathrm{H}), 7.28(\mathrm{dd}, J=3.6,0.4 \mathrm{~Hz}, 1 \mathrm{H}), 6.50(\mathrm{dd}, J=3.6$, $0.2 \mathrm{~Hz}, 1 \mathrm{H}), 5.65$ (sept, $J=6.0 \mathrm{~Hz}, 1 \mathrm{H}), 5.13(\mathrm{~s}, 2 \mathrm{H}), 1.66(\mathrm{~s}, 6 \mathrm{H}) ;{ }^{13} \mathrm{C}\{\mathbf{1 H}\} \mathbf{N M R}(100 \mathrm{MHz}$, $\left.\mathrm{CDCl}_{3}\right) \delta 171.2,143.7,143.1,141.6,137.5,128.5,128.5(2), 128.2,124.8-116.4\left(\mathrm{~m}, \mathrm{CF}_{3}\right)$, 118.1, 112.4, 77.2, 67.54 (hept, $J_{F C}=35.0 \mathrm{~Hz}$ ), 49.8, 25.9; HRMS (ESI-TOF) m/z: [M+H] ${ }^{+}$ calculated for $\mathrm{C}_{19} \mathrm{H}_{18} \mathrm{~F}_{6} \mathrm{NO}_{4} \mathrm{~S} 470.0861$, mass found 470.0855 . 
<smiles>CCCCCN1C(=O)C(C)(C)SC1(c1cn(C)c2ccccc12)N1CCCC1</smiles>

Following the general procedure $(\mathbf{3 b}-\mathbf{i})$, reaction between thioamide [pyrrolidin-1-yl(1-tosyl$1 \mathrm{H}$-indol-3-yl)methanethione $(0.200 \mathrm{~g}, 0.52 \mathrm{mmol}, 1.0$ equiv)] and $\alpha$-halohydroxamate $(0.423$ $\mathrm{g}, 1.04 \mathrm{mmol}, 3.0$ equiv) afforded desired 4-thiazolidinone 1r, which was purified by silica gel column chromatography (1:9 EtOAc:Hexane as eluent) to give the title compound as white solid in $71 \%(0.212 \mathrm{~g})$ yield. $R_{f} 0.25$ (1:9 EtOAc:Hexane); ${ }^{1} \mathbf{H}$ NMR $\left(400 \mathrm{MHz}, \mathrm{CDCl}_{3}\right) \delta 8.22$ (d, $J=8.0 \mathrm{~Hz}, 1 \mathrm{H}), 8.06(\mathrm{~s}, 1 \mathrm{H}), 8.02(\mathrm{~d}, J=8.4 \mathrm{~Hz}, 1 \mathrm{H}), 7.74(\mathrm{~d}, J=8.0 \mathrm{~Hz}, 2 \mathrm{H}), 7.43-7.31$ $(\mathrm{m}, 2 \mathrm{H}), 7.22(\mathrm{~d}, J=6.4 \mathrm{~Hz}, 1 \mathrm{H}), 7.18(\mathrm{~d}, J=8.0 \mathrm{~Hz} 1 \mathrm{H}), 7.15(\mathrm{~d}, J=8.8 \mathrm{~Hz} 3 \mathrm{H}), 6.82(\mathrm{~d}, J=$ $7.6 \mathrm{~Hz} 2 \mathrm{H}$ ), $4.66(\mathrm{~d}, J=6.4 \mathrm{~Hz}, 1 \mathrm{H}), 3.67(\mathrm{~d}, J=8.4 \mathrm{~Hz}, 1 \mathrm{H}), 2.59-2.49(\mathrm{~m}, 4 \mathrm{H}), 2.20(\mathrm{~s}, 3 \mathrm{H})$, 1.78-1.72 (m, 7H), $1.69(\mathrm{~s}, 3 \mathrm{H}) ;{ }^{13} \mathbf{C}\{\mathbf{1 H}\}$ NMR $\left(100 \mathrm{MHz}, \mathrm{CDCl}_{3}\right) \delta 170.7,145.4,136.1,135.0$, 133.9, 130.1, 130.0, 129.6, 128.8, 128.3, 128.1, 126.8, 125.7, 125.4, 124.0, 123.7, 113.8, 86.6, 76.9, 47.3, 45.4, 31.9, 27.4, 24.0, 21.5; HRMS (ESI-TOF) m/z: [M+H] ${ }^{+}$calculated for $\mathrm{C}_{31} \mathrm{H}_{34} \mathrm{~N}_{3} \mathrm{O}_{4} \mathrm{~S}_{2}$ 576.1991, mass found 576.1988.

Synthesis of 1s: vide infra

3-(Benzyloxy)-2-phenyl-2-(pyrrolidin-1-yl)-1-thia-3-azaspiro[4.4]nonan-4-one (1a')

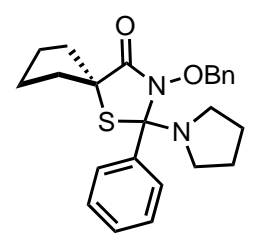

Following the general procedure (3b-i), reaction between thioamide [phenyl(pyrrolidin-1yl)methanethione $(0.130 \mathrm{~g}, 0.68 \mathrm{mmol}, 1.0$ equiv)] and $\alpha$-halohydroxamate $(0.405 \mathrm{~g}, 1.36$ mmol, 2.0 equiv) afforded desired 4-thiazolidinone 1a', which was purified by silica gel column chromatography (2:98 EtOAc:Hexane as eluent) to give the title compound as white solid in $51 \%(0.141 \mathrm{~g})$ yield. $R_{f} 0.25$ (5:95 EtOAc:Hexane); ${ }^{1} \mathbf{H}$ NMR $\left(400 \mathrm{MHz}, \mathrm{CDCl}_{3}\right) \delta 8.09$ (d, $\mathrm{J}=$ 7.6 Hz, 2H), 7.44-7.36 (m, 3H), 7.29-7.25 (m, 3H), 7.24-7.21 (m, 2H), $4.83(\mathrm{~d}, J=8.4 \mathrm{~Hz}, 1 \mathrm{H})$, $3.74(\mathrm{~d}, J=8.4 \mathrm{~Hz}, 1 \mathrm{H}), 2.69-2.60(\mathrm{~m}, 2 \mathrm{H}), 2.56-2.46(\mathrm{~m}, 3 \mathrm{H}), 2.45-2.36(\mathrm{~m}, 1 \mathrm{H}), 2.19-2.05$ $(\mathrm{m}, 2 \mathrm{H}), 1.96-1.75(\mathrm{~m}, 8 \mathrm{H}) ;{ }^{13} \mathbf{C}\{\mathbf{1 H}\}$ NMR $\left(100 \mathrm{MHz}, \mathrm{CDCl}_{3}\right) \delta 171.1,139.7,134.4,130.0$, 129.9, 129.3, 128.7, 128.3, 93.0, 77.1, 54.7, 48.1, 44.3, 40.7, 25.6, 25.5, 24.1; HRMS (ESITOF) m/z: $[M+H]^{+}$calculated for $\mathrm{C}_{24} \mathrm{H}_{29} \mathrm{~N}_{2} \mathrm{O}_{2} \mathrm{~S} 409.1950$, mass found 409.1941. 
3-(Benzyloxy)-2-phenyl-2-(pyrrolidin-1-yl)-1-thia-3-azaspiro[4.5]decan-4-one (1 b')<smiles>CCCC=C1SC(c2ccccc2)(N2CCCC2)N(CCCC)C1=O</smiles>

Following the general procedure (3b-i), reaction between thioamide [phenyl(pyrrolidin-1$\mathrm{yl}$ )methanethione $(0.075 \mathrm{~g}, 0.39 \mathrm{mmol}, 1.0$ equiv)] and $\alpha$-halohydroxamate $(0.366 \mathrm{~g}, 1.17$ mmol, 3.0 equiv) afforded desired 4-thiazolidinone 1b', which was purified by silica gel column chromatography (2:98 EtOAc:Hexane as eluent) to give the title compound as white solid in $72 \%(0.119 \mathrm{~g})$ yield. $R_{f} 0.25$ (5:95 EtOAc:Hexane); ${ }^{1} \mathbf{H}$ NMR $\left(400 \mathrm{MHz}, \mathrm{CDCl}_{3}\right) \delta 8.09$ (d, $\mathrm{J}=$ $6.8 \mathrm{~Hz}, 2 \mathrm{H}), 7.43-7.37(\mathrm{~m}, 3 \mathrm{H}), 7.28-7.25(\mathrm{~m}, 3 \mathrm{H}), 7.25-7.21(\mathrm{~m}, 2 \mathrm{H}), 4.82(\mathrm{~d}, J=8.8 \mathrm{~Hz}, 1 \mathrm{H})$, $3.75(\mathrm{~d}, J=8.8 \mathrm{~Hz}, 1 \mathrm{H}), 2.68-2.61(\mathrm{~m}, 2 \mathrm{H}), 2.56-2.50(\mathrm{~m}, 2 \mathrm{H}), 2.15-2.05(\mathrm{~m}, 2 \mathrm{H}), 2.03-1.97$ $(\mathrm{m}, 1 \mathrm{H}), 1.90-1.85(\mathrm{~m}, 1 \mathrm{H}), 1.84-1.76(\mathrm{~m}, 6 \mathrm{H}), 1.76-1.70(\mathrm{~m}, 2 \mathrm{H}), 1.56-1.43(\mathrm{~m}, 1 \mathrm{H}), 1.38-$ $1.29(\mathrm{~m}, 1 \mathrm{H}) .{ }^{13} \mathrm{C}\{1 \mathrm{H}\}$ NMR $\left(100 \mathrm{MHz}, \mathrm{CDCl}_{3}\right) \delta 170.5,140.1,134.5,130.0,129.9,129.3$, 128.7, 128.4, 92.8, 77.0, 52.5, 48.0, 38.8, 35.2, 25.2, 24.0, 23.1, 22.9; HRMS (ESI-TOF) m/z: $[\mathrm{M}+\mathrm{H}]^{+}$calculated for $\mathrm{C}_{25} \mathrm{H}_{31} \mathrm{~N}_{2} \mathrm{O}_{2} \mathrm{~S} 423.2106$, mass found 423.2105 .

3-(Benzyloxy)-2-(furan-2-yl)-2-((1,1,1,3,3,3-hexafluoropropan-2-yl)oxy)-1-thia-3azaspiro[4.5]decan-4-one (10'-HFIP)<smiles>CCCCN1C(=O)[C@H](CCC)SC1(OC(F)(F)F)c1ccco1</smiles>

Following the general procedure (3b-ii), reaction between furan-2-yl(pyrrolidin-1$\mathrm{yl})$ methanethione $(0.125 \mathrm{~g}, 0.69 \mathrm{mmol}, 1.0$ equiv)] and $\alpha$-halohydroxamate $(0.215 \mathrm{~g}, 0.69$ mmol, 1.0 equiv) afforded desired 4-thiazolidinone 1c'-HFIP, which was purified by silica gel column chromatography (2:98 EtOAc:Hexane as eluent) to give the title compound as white solid in 46\% (0.161 g) yield. $R_{f} 0.55$ (5:95 EtOAc:Hexane); ${ }^{1} \mathbf{H}$ NMR $\left(400 \mathrm{MHz}, \mathrm{CDCl}_{3}\right) \delta 7.39$ (s, 1H), 7.37-7.27 (m, 5H), $7.24(\mathrm{~d}, J=3.2 \mathrm{~Hz}, 1 \mathrm{H}), 6.48-6.43(\mathrm{~m}, 1 \mathrm{H}), 5.61$ (sept, $J=6.0 \mathrm{~Hz}$, $1 \mathrm{H}), 5.12(\mathrm{~s}, 2 \mathrm{H}), 2.10-2.02(\mathrm{~m}, 2 \mathrm{H}), 2.00-1.92(\mathrm{~m}, 2 \mathrm{H}), 1.74-1.64(\mathrm{~m}, 2 \mathrm{H}), 1.52-1.43(\mathrm{~m}, 3 \mathrm{H})$, 1.42-1.34 (m, 1H); ${ }^{13} \mathrm{C}\{1 \mathrm{H}\}$ NMR $\left(100 \mathrm{MHz}, \mathrm{CDCl}_{3}\right) \delta 170.3,144.2,143.1,141.1,137.4$, 128.5, 128.5(2), 128.1, 124.8-116.4 ( $\mathrm{m}, \mathrm{CF}_{3}$ ), 118.0, 112.4, 77.3, 67.2 (hept, JFC = 35.0 Hz), 55.2, 33.7, 25.3, 22.5; HRMS (ESI-TOF) $\mathrm{m} / \mathrm{z}$ : $[\mathrm{M}+\mathrm{H}]^{+}$calculated for $\mathrm{C}_{22} \mathrm{H}_{22} \mathrm{~F}_{6} \mathrm{NO}_{4} \mathrm{~S} 510.1174$, mass found 510.1172 . 
3-(Benzyloxy)-2-((1,1,1,3,3,3-hexafluoropropan-2-yl)oxy)-5-methyl-2,5-diphenylthiazolidin-4one (1d'-HFIP)

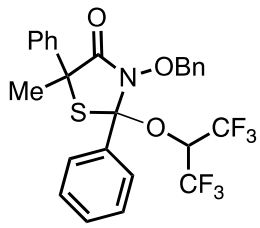

Following the general procedure $(\mathbf{3 b}$-ii), reaction between thioamide [phenyl(pyrrolidin-1$\mathrm{yl})$ methanethione $(0.100 \mathrm{~g}, 0.52 \mathrm{mmol}, 1.0$ equiv)] and $\alpha$-halohydroxamate $(0.151 \mathrm{~g}, 0.52$ mmol, 1.0 equiv) afforded desired 4-thiazolidinone 1d'-HFIP, which was purified by silica gel column chromatography (2:98 EtOAc:Hexane as eluent) to give the title compound as white solid in 60\% (0.169 g) yield. $R_{f} 0.5$ (5:95 EtOAc:Hexane); ${ }^{1} \mathbf{H}$ NMR (400 MHz, $\left.\mathrm{CDCl}_{3}\right) \delta 7.65$ (d, $J=7.6 \mathrm{~Hz}, 2 \mathrm{H}), 7.61-7.56(\mathrm{~m}, 2 \mathrm{H}), 7.46-7.33(\mathrm{~m}, 11 \mathrm{H}), 5.65$ (sept, $J=6.0 \mathrm{~Hz}, 1 \mathrm{H}$ ), 5.08 (s, 2H), 2.25 (s, 3H); ${ }^{13} \mathrm{C}\{1 \mathrm{H}\}$ NMR $\left(100 \mathrm{MHz}, \mathrm{CDCl}_{3}\right) \delta 169.0,153.1,138.2,137.4,131.6$, 130.1, 128.9, 128.9(2), 128.6, 128.6(2), 128.4, 127.9, 126.8, 124.8-116.3 (m, $\left.\mathrm{CF}_{3}\right), 76.3,67.9$ (hept, $J=35.0 \mathrm{~Hz}$ ), 58.2, 24.5;HRMS (ESI-TOF) $\mathrm{m} / \mathrm{z}$ : $[\mathrm{M}+\mathrm{H}]^{+}$calculated for $\mathrm{C}_{26} \mathrm{H}_{21} \mathrm{~F}_{6} \mathrm{NO}_{3} \mathrm{~S}$ 542.1225, mass found 542.1221.

3-(Benzyloxy)-2-((1,1,1,3,3,3-hexafluoropropan-2-yl)oxy)-5-methyl-2-phenyl-5-(ptolyl)thiazolidin-4-one (1e'-HFIP)

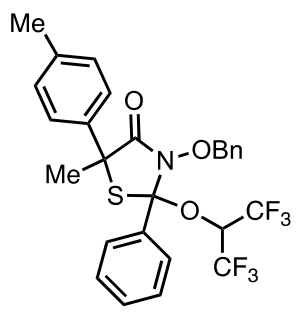

Following the general procedure $(\mathbf{3 b}$-ii), reaction between thioamide [phenyl(pyrrolidin-1$\mathrm{yl})$ methanethione $(0.031 \mathrm{~g}, 0.16 \mathrm{mmol}, 1.0$ equiv)] and $\alpha$-halohydroxamate $(0.050 \mathrm{~g}, 016$. mmol, 1.0 equiv) afforded desired 4-thiazolidinone 1e'-HFIP, which was purified by silica gel column chromatography (2:98 EtOAc:Hexane as eluent) to give the title compound as colorless oil in 55\% (0.050 g) yield. $R_{f} 0.35$ (5:95 EtOAc:Hexane); ${ }^{1} \mathbf{H}$ NMR (400 MHz, $\mathrm{CDCl}_{3}$ ) ठ 7.56-7.50 (m, 2H), $7.48(\mathrm{~d}, J=8.0 \mathrm{~Hz}, 2 \mathrm{H}), 7.42-7.38(\mathrm{~m}, 3 \mathrm{H}), 7.38-7.29(\mathrm{~m}, 5 \mathrm{H}), 7.17(\mathrm{~d}, J$ $=8.0 \mathrm{~Hz}, 2 \mathrm{H}), 5.59$ (sept, $J=6.0 \mathrm{~Hz}, 1 \mathrm{H}), 5.02(\mathrm{~s}, 2 \mathrm{H}), 2.35(\mathrm{~s}, 3 \mathrm{H}), 2.18(\mathrm{~s}, 3 \mathrm{H}) ;{ }^{13} \mathrm{C}\{\mathbf{1 H}\}$ NMR $\left(100 \mathrm{MHz}, \mathrm{CDCl}_{3}\right) \delta 169.1,153.2,138.9,138.2,134.3,131.6,130.1,129.6,128.6,128.5$, 128.4, 127.9, 126.7, 124.8-115.5 (m, $\mathrm{CF}_{3}$ ), 76.2, 67.9 (hept, $J_{\mathrm{FC}}=35.0 \mathrm{~Hz}$ ), 57.9, 24.4, 21.1; HRMS (ESI-TOF) m/z: [M+H] ${ }^{+}$calculated for $\mathrm{C}_{27} \mathrm{H}_{24} \mathrm{~F}_{6} \mathrm{NO}_{3} \mathrm{~S} 556.1381$, mass found 556.1374 . 
3-(Benzyloxy)-5-(4-fluorophenyl)-2-((1,1,1,3,3,3-hexafluoropropan-2-yl)oxy)-5-methyl-2-

phenylthiazolidin-4-one (19'-HFIP)

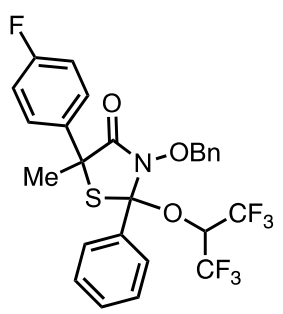

Following the general procedure (3b-ii), reaction between thioamide [phenyl(pyrrolidin-1$\mathrm{yl})$ methanethione $(0.030 \mathrm{~g}, 0.16 \mathrm{mmol}, 1.0$ equiv)] and $\alpha$-halohydroxamate $(0.048 \mathrm{~g}, 0.16$ mmol, 1.0 equiv) afforded desired 4-thiazolidinone 19'-HFIP, which was purified by silica gel column chromatography (3:97 EtOAc:Hexane as eluent) to give the title compound as colorless oil in $56 \%$ (0.049 g) yield. $R_{f} 0.5$ (1:9 EtOAc:Hexane); ${ }^{1} \mathbf{H}$ NMR (400 MHz, $\left.\mathrm{CDCl}_{3}\right) \delta$ 7.61-7.56 (m, 2H), 7.53-7.49 (m, 2H), 7.42-7.38 (m, 3H), 7.37-7.28 (m, 5H), $7.04(\mathrm{t}, J=8.8$ $\mathrm{Hz}, 2 \mathrm{H}), 5.56$ (sept, J=6.0 Hz, 1H), $5.00(\mathrm{~s}, 2 \mathrm{H}), 2.16(\mathrm{~s}, 3 \mathrm{H}) ;{ }^{13} \mathbf{C}\{1 \mathrm{H}\} \mathbf{N M R}\left(100 \mathrm{MHz}, \mathrm{CDCl}_{3}\right)$ $\delta 168.8,162.8$ (d, $J=247.6 \mathrm{~Hz}$ ), 152.8, 138.1, 133.1 (d, $J=3.3 \mathrm{~Hz}), 131.4,130.2,129.0$ (d, $J$ $=8.4 \mathrm{~Hz}), 128.6,128.5,128.4,128.4(2), 127.9,124.7-115.4\left(\mathrm{~m}, \mathrm{CF}_{3}\right), 115.8$ (d, J=21.5 Hz), 76.3, 67.9 (hept, $J_{\mathrm{FC}}=34.4 \mathrm{~Hz}$ ), 57.4, 24.4;HRMS (ESI-TOF) $\mathrm{m} / \mathrm{z}:[\mathrm{M}+\mathrm{H}]^{+}$calculated for $\mathrm{C}_{26} \mathrm{H}_{21} \mathrm{~F}_{7} \mathrm{NO}_{3} \mathrm{~S} 560.1130$, mass found 560.1122

3-(Benzyloxy)-5-(4-bromophenyl)-2-((1,1,1,3,3,3-hexafluoropropan-2-yl)oxy)-5-methyl-2phenylthiazolidin-4-one (1 $\boldsymbol{h}^{\prime}$-HFIP)

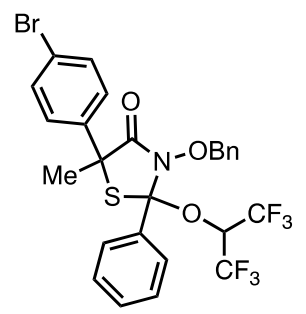

Following the general procedure $(3 \mathbf{b}-\mathrm{ii})$, reaction between thioamide $\mathrm{N}, \mathrm{N}$ diethylbenzothioamide $(0.055 \mathrm{~g}, 0.28 \mathrm{mmol}, 1.0$ equiv)] and $\alpha$-halohydroxamate $(0.106 \mathrm{~g}, 0.28$ mmol, 1.0 equiv) afforded desired 4-thiazolidinone 1h'-HFIP, which was purified by silica gel column chromatography (2:98 EtOAc:Hexane as eluent) to give the title compound as white solid in 53\% (0.094 g) yield. $R_{f} 0.3$ (5:95 EtOAc:Hexane); ${ }^{1} \mathrm{H}$ NMR (400 MHz, $\left.\mathrm{CDCl}_{3}\right) \delta$ 7.54$7.50(\mathrm{~m}, 2 \mathrm{H}), 7.50-7.45(\mathrm{~m}, 4 \mathrm{H}), 7.43-7.39(\mathrm{~m}, 3 \mathrm{H}), 7.39-7.29(\mathrm{~m}, 5 \mathrm{H}), 5.58$ (sept, $J=6.0 \mathrm{~Hz}$ 1H), 5.01 (s, 2H), $2.15(\mathrm{~s}, 3 \mathrm{H}) ;{ }^{13} \mathbf{C}\{\mathbf{1 H}\}$ NMR $\left(100 \mathrm{MHz}, \mathrm{CDCl}_{3}\right) \delta$ 168.6, 152.7, 138.1, 136.5, 132.0, 131.3, 130.2, 128.7, 128.6, 128.5, 128.4, 128.4(2), 128.0, 124.6-116.3 (m, CF $)$, 123.2, 76.3, 67.9 (hept, $J_{F C}=35.0 \mathrm{~Hz}$ ), 57.6, 24.4;HRMS (ESI-TOF) m/z: [M+H] ${ }^{+}$calculated for $\mathrm{C}_{26} \mathrm{H}_{21} \mathrm{BrF}_{6} \mathrm{NO}_{3} \mathrm{~S}$ 620.0330, mass found 620.0330. 
3-(Benzyloxy)-5-(3-methoxyphenyl)-5-methyl-2-phenyl-2-(pyrrolidin-1-yl)thiazolidin-4-one

$\left(\mathbf{1 f}^{\prime}\right)$



This compound was prepared in HFIP, following the general procedure $(3 \mathbf{b}-\mathbf{i})$, reaction between thioamide [phenyl(pyrrolidin-1-yl)methanethione $(0.120 \mathrm{~g}, 0.63 \mathrm{mmol}, 1.0$ equiv)] and a-halohydroxamate $(0.201 \mathrm{~g}, 0.63 \mathrm{mmol}, 1.0$ equiv) at $1 \mathrm{M}$ concentration afforded desired 4thiazolidinone 1f', which was purified by silica gel column chromatography (3:97 EtOAc:Hexane as eluent) to give the title compound as mixture of diastereomers (d.r.=1:0.8) as colorless oil in $40 \%(0.119 \mathrm{~g})$ yield. $R_{f} 0.4$ (1:9 EtOAc:Hexane); ${ }^{1} \mathbf{H}$ NMR (400 MHz, $\left.\mathrm{CDCl}_{3}\right)$ $\delta 8.15(\mathrm{~d}, J=6.8 \mathrm{~Hz}, 1.6 \mathrm{H}), 8.03-7.98(\mathrm{~m}, 2 \mathrm{H}), 7.48-7.41(\mathrm{~m}, 2.4 \mathrm{H}), 7.40-7.37(\mathrm{~m}, 3.2 \mathrm{H}), 7.33-$ $7.27(\mathrm{~m}, 12.5 \mathrm{H}), 7.13(\mathrm{~d}, J=8.0 \mathrm{~Hz}, 2 \mathrm{H}), 6.85-6.82(\mathrm{~m}, 1.6 \mathrm{H}), 4.99(\mathrm{~d}, J=8.4 \mathrm{~Hz}, 0.8 \mathrm{H}), 4.96$ (d, $J=8.4 \mathrm{~Hz}, 1 \mathrm{H}), 4.00$ (d, $J=8.4 \mathrm{~Hz}, 0.8 \mathrm{H}$ ), $3.84(\mathrm{~s}, 2 \mathrm{H}), 3.81$ (d, $J=8.4 \mathrm{~Hz}, 1 \mathrm{H}), 3.76$ (s, $3 \mathrm{H})$, 2.76-2.69 (m, 2H), 2.63-2.56 (m, 3.4H), 2.56-2.49 (m, 2H), $2.10(\mathrm{~s}, 5.4 \mathrm{H}), 1.86-1.81(\mathrm{~m}$, 4H), 1.74-1.70 (m, 3H); ${ }^{13} \mathrm{C}\{1 \mathrm{H}\}$ NMR $\left(100 \mathrm{MHz}, \mathrm{CDCl}_{3}\right) \delta$ 168.8, 168.8(2), 159.8, 159.6, $146.1,144.2,139.7,134.4,134.3,130.1,130.0,129.9,129.8,129.7,129.4,129.4(2)$, 129.4(3),128.9, 128.8, 128.4, 128.4(2), 119.0, 118.2, 113.1, 112.8, 112.7, 111.9, 92.9, 92.6, 77.0, 55.4, 55.3, 52.03, 51.3, 48.5, 48.0, 32.8, 27.6, 24.1, 24.0; HRMS (ESI-TOF) m/z: $[\mathrm{M}+\mathrm{H}]^{+}$calculated for $\mathrm{C}_{28} \mathrm{H}_{31} \mathrm{~N}_{2} \mathrm{O}_{3} \mathrm{~S} 475.2055$, mass found 475.2054.

\section{3-((4-Methoxybenzyl)oxy)-5,5-dimethyl-2-phenyl-2-(pyrrolidin-1-yl)thiazolidin-4-one (1a”)}

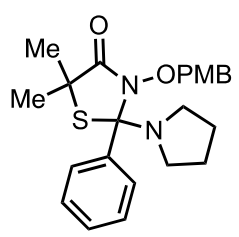

Following the general procedure (3b-i), reaction between thioamide [phenyl(pyrrolidin-1$\mathrm{yl})$ methanethione $(0.100 \mathrm{~g}, 0.52 \mathrm{mmol}, 1.0$ equiv)] and $\alpha$-halohydroxamate $(0.315 \mathrm{~g}, 1.04$ mmol, 2.0 equiv) afforded desired 4-thiazolidinone 1a", which was purified by silica gel column chromatography (3:97 EtOAc:Hexane as eluent) to give the title compound as semisolid in $91 \%$ (0.196 g) yield. $R_{f} 0.2$ (1:9 EtOAc:Hexane); ${ }^{1} \mathbf{H}$ NMR (400 MHz, $\left.\mathrm{CDCl}_{3}\right) \delta$ $8.12(\mathrm{~d}, J=7.2 \mathrm{~Hz}, 2 \mathrm{H}), 7.47-7.39(\mathrm{~m}, 3 \mathrm{H}), 7.17$ (d, $J=8.4 \mathrm{~Hz}, 2 \mathrm{H}), 6.81$ (d, J = 8.4 Hz, 2H), $4.77(\mathrm{~d}, J=8.8 \mathrm{~Hz}, 1 \mathrm{H}), 3.78(\mathrm{~s}, 3 \mathrm{H}), 3.69(\mathrm{~d}, J=8.8 \mathrm{~Hz}, 1 \mathrm{H}), 2.69-2.61(\mathrm{~m}, 2 \mathrm{H}), 2.56-2.49$ $(\mathrm{m}, 2 \mathrm{H}), 1.84-1.78(\mathrm{~m}, 4 \mathrm{H}), 1.74(\mathrm{~s}, 3 \mathrm{H}), 1.69(\mathrm{~s}, 3 \mathrm{H}) ;{ }^{13} \mathrm{C}\{1 \mathrm{H}\} \mathrm{NMR}\left(100 \mathrm{MHz}, \mathrm{CDCl}_{3}\right) \delta 170.8$, 160.1 , 139.9, 131.6, 130.1, 129.3, 128.4, 126.6, 113.8, 92.4, 76.5, 55.3, 48.0, 45.0, 31.8, 27.6, 
24.0; HRMS (ESI-TOF) m/z: $[\mathrm{M}+\mathrm{H}]^{+}$calculated for $\mathrm{C}_{23} \mathrm{H}_{29} \mathrm{~N}_{2} \mathrm{O}_{3} \mathrm{~S}$ 413.1899, mass found 413.1891.

3-Methoxy-5,5-dimethyl-2-phenyl-2-(pyrrolidin-1-yl)thiazolidin-4-one (16")

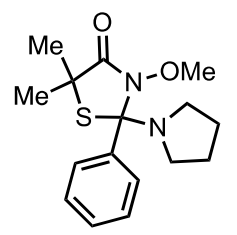

Following the general procedure (3b-i), reaction between thioamide [phenyl(pyrrolidin-1$\mathrm{yl})$ methanethione $\quad(0.100 \mathrm{~g}, 0.52 \mathrm{mmol}, 1.0$ equiv)] and a-halohydroxamate $(0.306 \mathrm{~g}, 1.56$ mmol, 3.0 equiv) afforded desired 4-thiazolidinone 1b", which was purified by silica gel column chromatography (3:97 EtOAc:Hexane as eluent) to give the title compound as white solid in 90\% (0.144 g) yield. $R_{f} 0.3$ (1:9 EtOAc:Hexane); ${ }^{1} \mathrm{H}$ NMR (400 MHz, $\left.\mathrm{CDCl}_{3}\right) \delta 8.02$ (d, $J=6.8 \mathrm{~Hz}, 2 \mathrm{H}), 7.39-7.31(\mathrm{~m}, 3 \mathrm{H}), 3.26(\mathrm{~s}, 3 \mathrm{H}), 2.69-2.60(\mathrm{~m}, 2 \mathrm{H}), 2.53-2.46(\mathrm{~m}, 2 \mathrm{H}), 1.85-1.78$ $(\mathrm{m}, 4 \mathrm{H}), 1.67(\mathrm{~s}, 3 \mathrm{H}), 1.63(\mathrm{~s}, 3 \mathrm{H}) ;{ }^{13} \mathbf{C}\{\mathbf{1 H}\} \mathbf{N M R}\left(100 \mathrm{MHz}, \mathrm{CDCl}_{3}\right) \delta 170.5,139.8,129.5$, 129.1, 128.3, 92.0, 62.9, 47.9, 44.8, 31.5, 27.6, 23.9; HRMS (ESI-TOF) m/z: [M+H]+calculated for $\mathrm{C}_{16} \mathrm{H}_{23} \mathrm{~N}_{2} \mathrm{O}_{2} \mathrm{~S} 307.1480$, mass found 307.1497.

3-(tert-Butoxy)-5,5-dimethyl-2-phenyl-2-(pyrrolidin-1-yl)thiazolidin-4-one (1c")

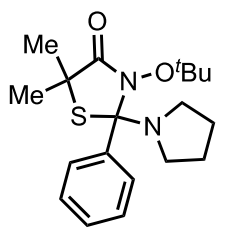

Following the general procedure (3b-i), reaction between thioamide [phenyl(pyrrolidin-1$\mathrm{yl})$ methanethione $(0.100 \mathrm{~g}, 0.52 \mathrm{mmol}, 1.0$ equiv)] and $\alpha$-halohydroxamate $(0.248 \mathrm{~g}, 1.04$ mmol, 2.0 equiv) afforded desired 4-thiazolidinone 1c", which was purified by silica gel column chromatography (2:98 EtOAc:Hexane as eluent) to give the title compound as colorless oil in $66 \%(0.182 \mathrm{~g})$ yield. $R_{f} 0.3$ (5:95 EtOAc:Hexane); ${ }^{1} \mathbf{H}$ NMR (400 MHz, $\left.\mathrm{CDCl}_{3}\right)$ $\delta 8.06(\mathrm{~d}, J=7.2 \mathrm{~Hz}, 2 \mathrm{H}), 7.40-7.31(\mathrm{~m}, 3 \mathrm{H}), 2.60-2.50(\mathrm{~m}, 4 \mathrm{H}), 1.86-1.78(\mathrm{~m}, 4 \mathrm{H}), 1.77(\mathrm{~s}$, $3 \mathrm{H}), 1.65(\mathrm{~s}, 3 \mathrm{H}), 0.89(\mathrm{~s}, 9 \mathrm{H}) ;{ }^{13} \mathrm{C}\{\mathbf{1 H}\} \mathbf{N M R}\left(100 \mathrm{MHz}, \mathrm{CDCl}_{3}\right) \delta 176.1,139.7,131.4,129.2$, 127.9, 93.0, 85.2, 48.1, 44.3, 33.2, 28.1, 26.9, 23.8; HRMS (ESI-TOF) m/z: $[\mathrm{M}+\mathrm{H}]^{+}$calculated for $\mathrm{C}_{19} \mathrm{H}_{29} \mathrm{~N}_{2} \mathrm{O}_{2} \mathrm{~S} 349.1950$, mass found 349.1948. 
3-(Heptyloxy)-5,5-dimethyl-2-phenyl-2-(pyrrolidin-1-yl)thiazolidin-4-one (1d'”)

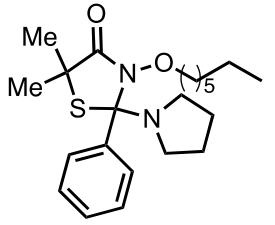

Following the general procedure (3b-i), reaction between thioamide [phenyl(pyrrolidin-1$\mathrm{yl})$ methanethione $(0.150 \mathrm{~g}, 0.78 \mathrm{mmol}, 1.0$ equiv)] and $\alpha$-halohydroxamate $(0.440 \mathrm{~g}, 1.56$ mmol, 2.0 equiv) afforded desired 4-thiazolidinone 1d", which was purified by silica gel column chromatography (2:98 EtOAc:Hexane as eluent) to give the title compound as colorless oil in $80 \%(0.240 \mathrm{~g})$ yield. $R_{f} 0.35$ (5:95 EtOAc:Hexane); ${ }^{1} \mathbf{H}$ NMR (400 MHz, $\left.\mathrm{CDCl}_{3}\right)$ $\delta 8.03(\mathrm{~d}, J=8.0 \mathrm{~Hz}, 2 \mathrm{H}), 7.40-7.32(\mathrm{~m}, 3 \mathrm{H}), 3.86(\mathrm{q}, J=7.2 \mathrm{~Hz}, 1 \mathrm{H}), 2.86(\mathrm{q}, J=7.2 \mathrm{~Hz}, 1 \mathrm{H})$, 2.70-2.62 (m, 2H), 2.56-2.48 (m, 2H), 1.85-1.80 (m, 4H), $1.68(\mathrm{~s}, 3 \mathrm{H}), 1.65(\mathrm{~s}, 3 \mathrm{H}), 1.49-1.35$ $(\mathrm{m}, 2 \mathrm{H}), 1.27-1.14(\mathrm{~m}, 8 \mathrm{H}), 0.84(\mathrm{t}, J=7.2 \mathrm{~Hz}, 3 \mathrm{H}) ;{ }^{13} \mathbf{C}\{\mathbf{1 H}\} \mathbf{N M R}\left(100 \mathrm{MHz}, \mathrm{CDCl}_{3}\right) \delta 170.8$, 134.0, 129.7, 129.1, 128.2, 92.3, 75.3, 48.0, 44.9, 31.7, 31.6, 29.1, 28.0, 27.7, 25.7, 24.1, 22.7, 14.2; HRMS (ESI-TOF) m/z: [M+H] ${ }^{+}$calculated for $\mathrm{C}_{22} \mathrm{H}_{35} \mathrm{~N}_{2} \mathrm{O}_{2} \mathrm{~S} 391.2419$, mass found 391.2417.

3-(Allyloxy)-5,5-dimethyl-2-phenyl-2-(pyrrolidin-1-yl)thiazolidin-4-one (1e”)<smiles>C=CCON(C(=O)C(C)(C)C)C(c1ccccc1)(c1ccccc1)N1CCCC1</smiles>

Following the general procedure (3b-i), reaction between thioamide [phenyl(pyrrolidin-1$\mathrm{yl})$ methanethione $(0.100 \mathrm{~g}, 0.52 \mathrm{mmol}, 1.0$ equiv)] and $\alpha$-halohydroxamate $(0.231 \mathrm{~g}, 1.04$ mmol, 2.0 equiv) afforded desired 4-thiazolidinone 1e", which was purified by silica gel column chromatography (3:97 EtOAc:Hexane as eluent) to give the title compound as colorless oil in $92 \%(0.160 \mathrm{~g})$ yield. $R_{f} 0.25$ (5:95 EtOAc:Hexane); ${ }^{1} \mathrm{H}$ NMR $\left(400 \mathrm{MHz}, \mathrm{CDCl}_{3}\right) \delta 8.06$ (d, $\mathrm{J}=$ $6.4 \mathrm{~Hz}, 2 \mathrm{H}), 7.42-7.34(\mathrm{~m}, 3 \mathrm{H}), 5.64$ (ddt, $J=17.2,10.6,6.8 \mathrm{~Hz}, 1 \mathrm{H}), 5.12-5.00(\mathrm{~m}, 2 \mathrm{H}), 4.18$ (dd, $J=10.4,6.4 \mathrm{~Hz}, 1 \mathrm{H}$ ), 3.51 (dd, $J=10.4,6.4 \mathrm{~Hz}, 1 \mathrm{H}$ ), 2.69-2.62 (m, 2H), 2.56-2.49 (m, $2 \mathrm{H}), 1.86-1.80(\mathrm{~m}, 4 \mathrm{H}), 1.70(\mathrm{~s}, 3 \mathrm{H}), 1.66(\mathrm{~s}, 3 \mathrm{H}) ;{ }^{13} \mathbf{C}\{\mathbf{1 H}\} \mathbf{N M R}\left(100 \mathrm{MHz}, \mathrm{CDCl}_{3}\right) \delta 171.2$, 139.8, 131.5, 129.9, 129.3, 128.3, 120.7, 92.3, 76.5, 48.0, 44.9, 31.9, 27.5, 24.0;HRMS (ESITOF) $\mathrm{m} / \mathrm{z}$ : $[\mathrm{M}+\mathrm{H}]^{+}$calculated for $\mathrm{C}_{18} \mathrm{H}_{25} \mathrm{~N}_{2} \mathrm{O}_{2} \mathrm{~S} 333.1637$, mass found 333.1631 . 


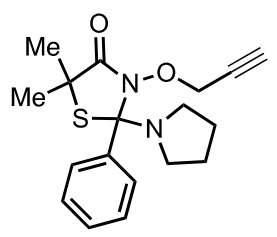

Following the general procedure $(\mathbf{3 b}-\mathbf{i})$, reaction between thioamide [phenyl(pyrrolidin-1$\mathrm{yl})$ methanethione $(0.050 \mathrm{~g}, 0.26 \mathrm{mmol}, 1.0$ equiv)] and $\alpha$-halohydroxamate $(0.173 \mathrm{~g}, 0.78$ mmol, 3.0 equiv) afforded desired 4-thiazolidinone 1f'", which was purified by silica gel column chromatography (3:97 EtOAc:Hexane as eluent) to give the title compound as colourless oil in $93 \%(0.080 \mathrm{~g})$ yield. $R_{f} 0.4$ (1:9 EtOAc:Hexane); ${ }^{1} \mathbf{H}$ NMR $\left(400 \mathrm{MHz}, \mathrm{CDCl}_{3}\right) \delta 8.05$ (d, $J=$ $7.2 \mathrm{~Hz}, 2 \mathrm{H}), 7.41-7.34(\mathrm{~m}, 3 \mathrm{H}), 4.35$ (dd, $J=14.0,2.0 \mathrm{~Hz}, 1 \mathrm{H}), 3.73(\mathrm{dd}, J=14.0,2.0 \mathrm{~Hz}, 1 \mathrm{H})$, 2.68-2.61 (m, 2H), 2.54-2.48 (m, 2H), 2.37 (brs, 1H), 1.85-1.79 (m, 4H), 1.69 (s, 3H), 1.65 (s, $3 \mathrm{H}) ;{ }^{13} \mathrm{C}\{1 \mathrm{H}\}$ NMR $\left(100 \mathrm{MHz}, \mathrm{CDCl}_{3}\right) \delta 171.3,139.5,129.6,129.3,128.4,92.2,76.8,76.6$, 62.8, 48.0, 44.7, 31.7, 27.5, 24.0;HRMS (ESI-TOF) m/z: [M+H] $]^{+}$calculated for $\mathrm{C}_{18} \mathrm{H}_{22} \mathrm{~N}_{2} \mathrm{O}_{2} \mathrm{~S}$ 331.1480 , mass found 331.1484 .

Synthesis of 19": (vide infra)

2d. Synthesis of Deferasirox Derived 4-thiazolidinone (1s):

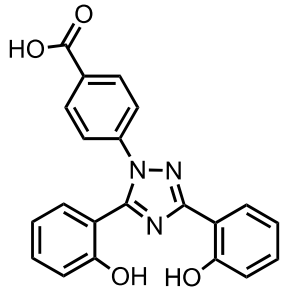

Deferasirox (Exjade)

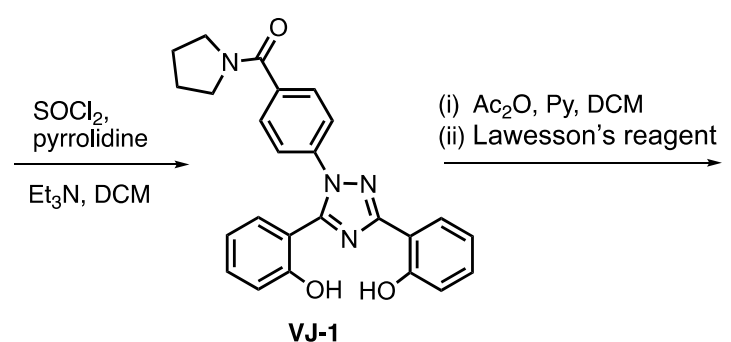

VJ-1

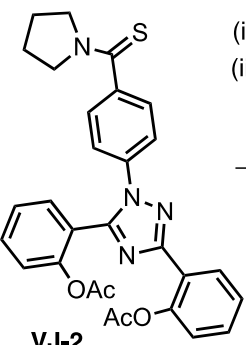

(i) $\mathrm{K}_{2} \mathrm{CO}_{3}, \mathrm{MeOH}$

(ii)<smiles>CC(C)(Br)C(=O)NOCc1ccccc1</smiles>

$\mathrm{Na}_{2} \mathrm{CO}_{3}, \mathrm{ACN}$

VJ-2

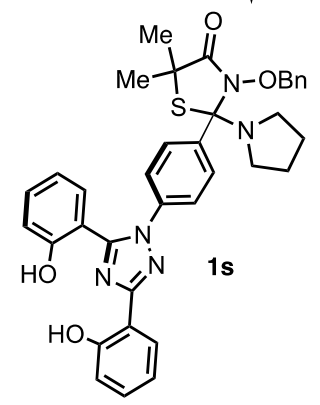

Scheme S2. Synthetic steps to key 2-amino-4-thiazolidinone precursor compound 1 s. 
Preparation of compound VJ-1 [(4-(3,5-bis(2-hydroxyphenyl)-1H-1,2,4-triazol-1yl)phenyl)(pyrrolidin-1-yl)methanone]<smiles>O=C(c1ccc(-n2nc(-c3ccccc3O)nc2-c2ccccc2O)cc1)N1CCCC1</smiles>

This compound was prepared following a reported procedure. ${ }^{2}$ Thionyl chloride $(2.0 \mathrm{~mL}, 26.87$ $\mathrm{mmol})$ was added to Deferasirox $(1.0 \mathrm{~g}, 2.68 \mathrm{mmol})$ in $\mathrm{CH}_{2} \mathrm{Cl}_{2}(100.0 \mathrm{~mL})$, and the reaction mixture was refluxed for $22 \mathrm{~h}$ at $50^{\circ} \mathrm{C}$. After completion, the reaction mixture was concentrated in vacuo to render the corresponding acid chloride as dark brown solid. The crude material was dissolved in $\mathrm{CH}_{2} \mathrm{Cl}_{2}(50.0 \mathrm{~mL})$ and to this solution were added pyrrolidine $(0.21 \mathrm{~g}, 2.98$ $\mathrm{mmol}$ ) followed by triethylamine $(0.30 \mathrm{~mL}, 2.30 \mathrm{mmol})$. The reaction mixture was stirred at room temperature for $18 \mathrm{~h}$ and then was quenched by the addition of saturated $\mathrm{NaHCO}_{3}$ solution. The aqueous layer was extracted with $\mathrm{CH}_{2} \mathrm{Cl}_{2}(50 \mathrm{~mL} \times 2)$ and combined organic portion was dried (with anhydrous $\mathrm{Na}_{2} \mathrm{SO}_{4}$ ) and concentrated in vacuo. The crude was purified by silica gel column chromatography (40:60 EtOAc:Hexane as eluent) to afford the amide VJ1 as white solid in $61 \%(0.598 \mathrm{~g})$ yield. $R_{f} 0.2$ (2:3 EtOAc:Hexane); ${ }^{1} \mathbf{H}$ NMR (400 MHz, $\left.\mathrm{CDCl}_{3}\right)$ $\delta 11.40$ (s, 1H), 9.67 (s, 1H), 8.13 (dd, $J=7.6,1.2 \mathrm{~Hz}, 1 \mathrm{H}), 7.71$ (d, J = 8.2 Hz, 2H), 7.56 (d, $J=8.2 \mathrm{~Hz}, 2 \mathrm{H}), 7.40-7.31(\mathrm{~m}, 2 \mathrm{H}), 7.12(\mathrm{~d}, J=8.0 \mathrm{~Hz}, 1 \mathrm{H}) 7.08-7.01(\mathrm{~m}, 2 \mathrm{H}), 6.99(\mathrm{dd}, J=$ 8.0, 1.6 Hz, 1H), 6.71-6.65 (m, 1H), $3.69(\mathrm{t}, J=6.8 \mathrm{~Hz}, 2 \mathrm{H}), 3.49(\mathrm{t}, J=6.4 \mathrm{~Hz}, 2 \mathrm{H}), 2.04-1.92$ $(\mathrm{m}, 4 \mathrm{H}) .{ }^{13} \mathbf{C}\{\mathbf{1 H}\}$ NMR $\left(100 \mathrm{MHz}, \mathrm{CDCl}_{3}\right) \delta 168.3,159.5,158.1,156.6,152.2,139.2,138.8$, 133.1, 131.9, 128.9, 127.8, 127.7, 126.2, 120.0, 119.3, 118.4, 117.2, 113.3, 110.1, 49.8, 46.6, 26.6, 24.5; HRMS (ESI-TOF) m/z: [M+H] $]^{+}$calculated for $\mathrm{C}_{25} \mathrm{H}_{23} \mathrm{~N}_{4} \mathrm{O}_{3} 427.1770$, mass found 427.1764 .

Preparation of compound VJ-2 [(1-(4-(pyrrolidine-1-carbonothioyl)phenyl)-1H-1,2,4triazole-3,5-diyl)bis(2,1-phenylene) diacetate]

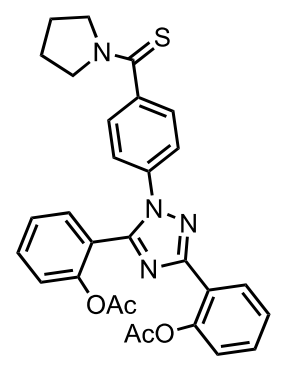

To a solution of compound $\mathbf{V J}-1(0.500 \mathrm{~g}, 1.17 \mathrm{mmol})$ in $\mathrm{CH}_{2} \mathrm{Cl}_{2}(5.0 \mathrm{~mL})$ at $0{ }^{\circ} \mathrm{C}$ were successively added pyridine (1.13 mL, $14.08 \mathrm{mmol}, 12.0$ equiv) and acetic anhydride $(1.0 \mathrm{~mL}$, 
$10.56 \mathrm{mmol}, 9.0$ equiv) and reaction mixture was allowed to warm to room temperature. The stirring was continued for $48 \mathrm{~h}$ and then it was quenched with the addition of $0.5 \mathrm{~N} \mathrm{HCl}$ solution. Organic layer was washed with brine $(2 \times 20 \mathrm{~mL})$, collected and dried over anhydrous $\mathrm{Na}_{2} \mathrm{SO}_{4}$. After the solvent was removed in vacuo, the crude was purified by silica gel column chromatography (40:60 EtOAc:Hexane as eluent) to afford the corresponding diacetate (VJ1-diacetate) as white solid in $89 \%(0.530 \mathrm{~g})$ yield. $R_{f} 0.18$ (2:3 EtOAc:Hexane); ${ }^{1} \mathrm{H}$ NMR (400 $\left.\mathrm{MHz}, \mathrm{CDCl}_{3}\right) \delta 8.40(\mathrm{~d}, J=7.6 \mathrm{~Hz}, 1 \mathrm{H}), 7.58(\mathrm{~d}, J=8.2 \mathrm{~Hz}, 2 \mathrm{H}), 7.54-7.48(\mathrm{~m}, 4 \mathrm{H}), 7.41$ (t, J $=7.6 \mathrm{~Hz}, 1 \mathrm{H}), 7.33-7.27(\mathrm{~m}, 3 \mathrm{H}), 7.22(\mathrm{t}, J=7.6 \mathrm{~Hz}, 1 \mathrm{H}), 3.68(\mathrm{t}, J=6.8 \mathrm{~Hz}, 2 \mathrm{H}), 3.44(\mathrm{t}, J=$ $6.4 \mathrm{~Hz}, 2 \mathrm{H}), 2.38(\mathrm{~s}, 3 \mathrm{H}), 2.12(\mathrm{~s}, 3 \mathrm{H}), 2.03-1.91(\mathrm{~m}, 4 \mathrm{H}) .{ }^{13} \mathrm{C}\{1 \mathrm{H}\} \mathbf{N M R}\left(100 \mathrm{MHz}, \mathrm{CDCl}_{3}\right) \delta$ $170.6,169.1,168.5,159.4,150.7,148.9,148.5,138.9,137.2,131.7,131.3,130.7,129.8$, 128.4, 126.5, 126.2, 124.0, 123.9, 123.5, 123.4, 121.6, 49.7, 46.5, 26.6, 24.5, 21.6, 21.0; HRMS (ESI-TOF) m/z: [M+H] ${ }^{+}$calculated for $\mathrm{C}_{29} \mathrm{H}_{27} \mathrm{~N}_{4} \mathrm{O}_{5} 511.1981$, mass found 511.1975 .

Next, the diacetate $(0.450 \mathrm{~g}, 0.88 \mathrm{mmol})$ was dissolved in dry toluene $(36.0 \mathrm{~mL})$ and was added Lawesson's reagent $(0.196 \mathrm{~g}, 0.48 \mathrm{mmol}, 0.55$ equiv $)$ in one portion. The reaction mixture was refluxed for 2-3 $\mathrm{h}$. The solvent was removed in vacuo and crude was purified by silica gel column chromatography (20:80 EtOAc:Hexane as eluent) to give corresponding thioamide, VJ-2 as a pale yellow crystal in $78 \%(0.361 \mathrm{~g}, 0.68)$ yield. $R_{f} 0.15$ (1:4 EtOAc:Hexane); ${ }^{1} \mathbf{H}$ NMR (400 MHz, $\left.\mathrm{CDCl}_{3}\right) \delta 8.35$ (d, $\left.J=7.6 \mathrm{~Hz}, 1 \mathrm{H}\right), 7.48(\mathrm{t}, J=7.2 \mathrm{~Hz}$, $1 \mathrm{H}), 7.45-7.33(\mathrm{~m}, 6 \mathrm{H}), 7.31(\mathrm{~d}, J=7.6 \mathrm{~Hz}, 1 \mathrm{H}), 7.25-7.20(\mathrm{~m}, 2 \mathrm{H}), 7.16(\mathrm{~d}, J=8.0 \mathrm{~Hz}, 1 \mathrm{H})$, $3.93(\mathrm{t}, J=6.8 \mathrm{~Hz}, 2 \mathrm{H}), 3.43(\mathrm{t}, J=6.8 \mathrm{~Hz}, 2 \mathrm{H}), 2.34(\mathrm{~s}, 3 \mathrm{H}), 2.08-2.01(\mathrm{~m}, 5 \mathrm{H}), 2.01-1.92(\mathrm{~m}$, $2 \mathrm{H}) .{ }^{13} \mathrm{C}\{1 \mathrm{H}\}$ NMR $\left(100 \mathrm{MHz}, \mathrm{CDCl}_{3}\right) \delta 195.5,170.5,168.9,159.3,150.6,148.8,148.4,143.7$, 137.9, 131.6, 131.3, 130.6, 129.7, 126.9, 126.4, 126.1, 123.8, 123.7, 123.3, 123.3(2), 121.6, 53.8, 53.6, 26.6, 24.6, 21.5, 20.9; HRMS (ESI-TOF) m/z: [M+H] ${ }^{+}$calculated for $\mathrm{C}_{29} \mathrm{H}_{27} \mathrm{~N}_{4} \mathrm{O}_{4} \mathrm{~S}$ 527.1753 , mass found 527.1750 .

3-(Benzyloxy)-2-(4-(3,5-bis(2-hydroxyphenyl)-1H-1,2,4-triazol-1-yl)phenyl)-5,5-dimethyl-2(pyrrolidin-1-yl)thiazolidin-4-one (1s)

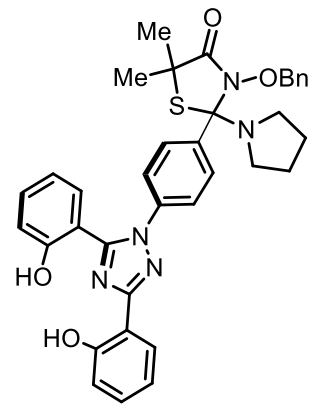

Compound VJ-2 (0.340 g, $0.65 \mathrm{mmol})$ was taken in $5.0 \mathrm{~mL}$ of $\mathrm{MeOH}$ and was added solid $\mathrm{K}_{2} \mathrm{CO}_{3}(0.214 \mathrm{~g}, 1.55 \mathrm{mmol}, 2.4$ equiv). The reaction mixture was stirred at room temperature for overnight. After removal of volatiles, the crude residue was treated with saturated aqueous 
$\mathrm{NH}_{4} \mathrm{Cl}$ solution $(15.0 \mathrm{~mL})$ and extracted in ether $(10 \mathrm{~mL} \times 3)$. The combined organic layer was washed with brine and dried over anhydrous $\mathrm{Na}_{2} \mathrm{SO}_{4}$. The filtrate was concentrated in vacuo and purified by silica gel column chromatography (30:70 EtOAc:Hexane as eluent) to give corresponding thioamide diol (VJ-2-diol) as pale yellow solid in $91 \%\left(0.260 \mathrm{~g}\right.$,) yield. $R_{f} 0.2$ (30:70 EtOAc:Hexane); ${ }^{1} \mathrm{H}$ NMR $\left(400 \mathrm{MHz}, \mathrm{CDCl}_{3}\right) \delta 11.44(\mathrm{~s}, 1 \mathrm{H}), 9.63(\mathrm{~s}, 1 \mathrm{H}), 8.14$ (d, $\mathrm{J}=$ $7.6 \mathrm{~Hz}, 1 \mathrm{H}), 7.57(\mathrm{~d}, J=8.4 \mathrm{~Hz}, 2 \mathrm{H}), 7.51(\mathrm{~d}, J=8.8 \mathrm{~Hz}, 2 \mathrm{H}), 7.41-7.32(\mathrm{~m}, 2 \mathrm{H}), 7.14(\mathrm{~d}, J=$ $8.4 \mathrm{~Hz}, 1 \mathrm{H}), 7.11-7.02(\mathrm{~m}, 3 \mathrm{H}), 6.72(\mathrm{t}, J=8.0 \mathrm{~Hz}, 1 \mathrm{H}), 4.02(\mathrm{t}, J=7.2 \mathrm{~Hz}, 2 \mathrm{H}), 3.55$ (t, $J=$ $6.8 \mathrm{~Hz}, 2 \mathrm{H}), 2.18-2.02(\mathrm{~m}, 4 \mathrm{H}) ;{ }^{13} \mathbf{C}\{\mathbf{1 H}\} \mathbf{N M R}\left(100 \mathrm{MHz}, \mathrm{CDCl}_{3}\right) \delta 195.2,159.5,158.2,156.7$, 152.2, 145.5, 138.3, 133.2, 131.9, 127.8, 127.8(2), 127.5, 126.2, 120.1, 119.3, 118.5, 117.3, 113.4, 110.1, 54.1, 53.8, 26.7, 24.8; HRMS (ESI-TOF) m/z: [M+H] calculated for $\mathrm{C}_{25} \mathrm{H}_{23} \mathrm{~N}_{4} \mathrm{O}_{2} \mathrm{~S}$ 443.1542, mass found 443.1535.

Following the general procedure $(3 \mathrm{~b}-\mathrm{i})$, reaction of the thioamide diol $(0.260 \mathrm{~g}, 0.59 \mathrm{mmol}, 1.0$ equiv) with $\alpha$-halohydroxamate $(0.478 \mathrm{~g}, 1.77 \mathrm{mmol}, 3.0$ equiv) afforded desired 4thiazolidinone 1s, which was purified by silica gel column chromatography (1:4 EtOAc:Hexane as eluent) to give the title compound as white solid in $94 \%(0.349 \mathrm{~g})$ yield. $R_{f} \quad 0.18(1: 4$ EtOAc:Hexane); $\mathbf{m p} 96-98 \stackrel{\circ}{\circ}$; FT-IR ( $\left.\mathrm{v} \mathrm{cm}^{-1}\right)$ : 3286, 2966, 2924, 2858, 1697, 1587, 1492, 1461, 1249, 752; ${ }^{1} \mathrm{H}$ NMR (400 MHz, $\left.\mathrm{CDCl}_{3}\right) \delta 11.36$ (s, 1H), 9.62 (s, 1H), 8.27 (d, J=8.4 Hz, 2H), 8.10 (dd, $J=7.6,0.8 \mathrm{~Hz}, 2 \mathrm{H}$ ), 7.51 (d, $J=8.8 \mathrm{~Hz}, 2 \mathrm{H}$ ), $7.33(\mathrm{td}, J=8.4,1.2 \mathrm{~Hz}, 1 \mathrm{H}$ ), 7.28$7.24(\mathrm{~m}, 4 \mathrm{H}), 7.21(\mathrm{td}, J=8.0,0.8 \mathrm{~Hz} 1 \mathrm{H}), 7.07(\mathrm{~d}, J=8.0 \mathrm{~Hz} 1 \mathrm{H}), 7.03(\mathrm{~d}, J=8.4 \mathrm{~Hz}, 1 \mathrm{H})$, $7.00(\mathrm{t}, J=7.6 \mathrm{~Hz} 1 \mathrm{H}), 6.89(\mathrm{~d}, J=8.0 \mathrm{~Hz}, 1 \mathrm{H}), 6.36(\mathrm{t}, J=7.6 \mathrm{~Hz}, 1 \mathrm{H}), 4.97(\mathrm{~d}, J=8.8 \mathrm{~Hz}$, $1 \mathrm{H}), 4.12(\mathrm{~d}, J=8.8 \mathrm{~Hz}, 1 \mathrm{H}), 2.69-2.61(\mathrm{~m}, 2 \mathrm{H}), 2.60-2.53(\mathrm{~m}, 2 \mathrm{H}), 1.84-1.79(\mathrm{~m}, 4 \mathrm{H}), 1.72$ $(\mathrm{s}, 3 \mathrm{H}), 1.69(\mathrm{~s}, 3 \mathrm{H}) ;{ }^{13} \mathrm{C}\{1 \mathrm{H}\}$ NMR $\left(100 \mathrm{MHz}, \mathrm{CDCl}_{3}\right) \delta 171.3,159.5,158.2,156.6,152.2$, 142.3, 138.7, 134.1, 133.0, 131.9, 131.6, 129.7, 129.1, 128.6, 127.8, 127.5, 126.1, 120.0, 119.0, 118.5, 117.3, 113.4, 110.2, 91.6, 77.2, 48.2, 45.3, 31.8, 27.7, 24.1; HRMS (ESI-TOF) $\mathrm{m} / \mathrm{z}:[\mathrm{M}+\mathrm{H}]^{+}$calculated for $\mathrm{C}_{36} \mathrm{H}_{36} \mathrm{~N}_{5} \mathrm{O}_{4} \mathrm{~S}$ 634.2488, mass found 634.2476. 
2e. Synthesis of D-glucose linked 4-thiazolidinone (1 g"):

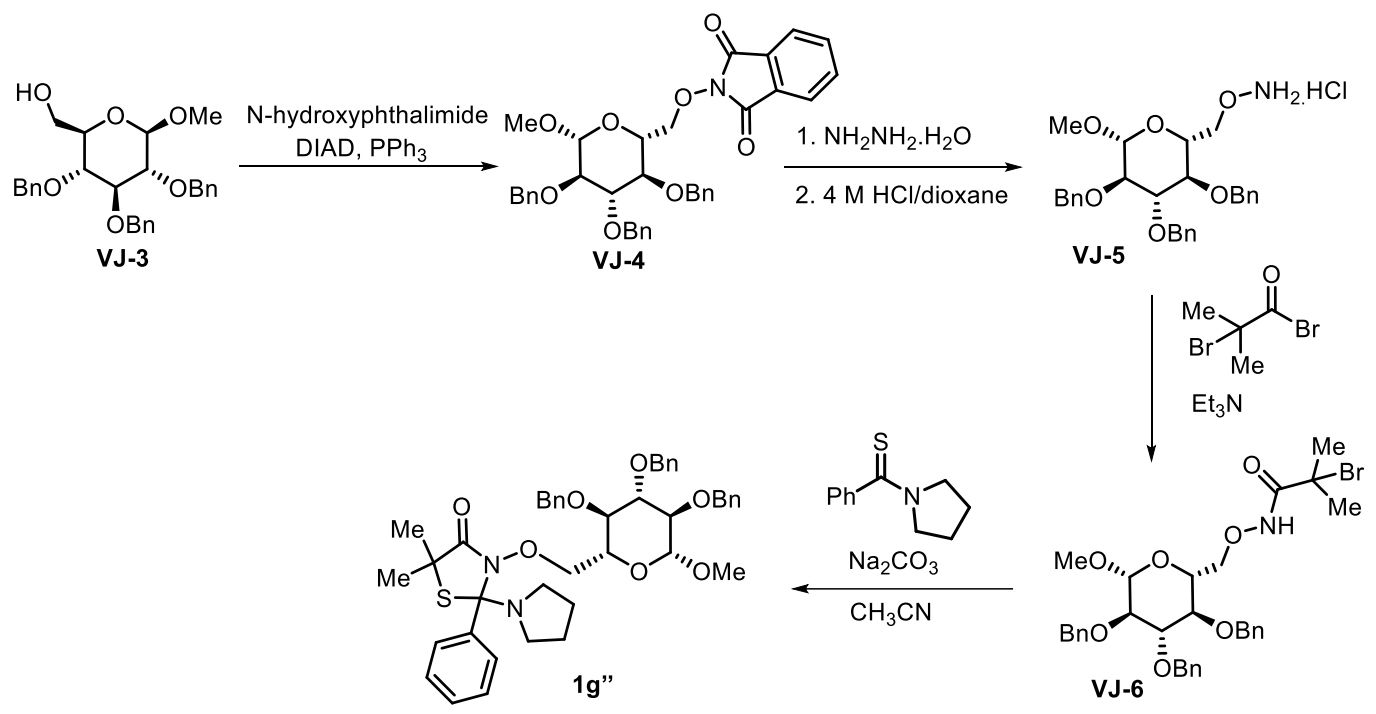

Scheme S3. Synthetic scheme for the preparation of methyl-glucoside linked 4-thiazolidinone.

Preparation of compound VJ-4 [2-((2S,3S,4R,5S,6S)-3,4,5-tris(benzyloxy)-6methoxytetrahydro-2H-pyran-2-yl)methoxy)isoindoline-1,3-dione]<smiles>CO[C@H]1O[C@H](ON2C(=O)c3ccccc3C2=O)[C@H]([OH2+])[C@@H](O)[C@H]1O</smiles>

To a stirred solution of commercially available methyl 2,3,4-tri-O-benzyl- $\beta$-D-glucopyranoside ${ }^{3}$ VJ-3 (0.662 g, $1.43 \mathrm{mmol}, 1.0$ equiv) in anhydrous THF (8.0 mL) were added $\mathrm{N}$ hydroxyphthalimide $(0.372 \mathrm{~g}, 2.28 \mathrm{mmol}, 1.6$ equiv) and triphenylphosphine $(0.635 \mathrm{~g}, 2.42$ mmol, 1.7 equiv) and the mixture was stirred for 10 minutes until the solution is homogeneous. Diisopropyl azodicarboxylate $\left(0.6 \mathrm{~mL}, 2.99 \mathrm{mmol}, 2.1\right.$ equiv) was then added dropwise at $0^{\circ} \mathrm{C}$ and the mixture was stirred for $18 \mathrm{~h}$ at room temperature. The solvents were removed in vacuo and the yellow residue was purified by silica gel column chromatography (Ether: $\mathrm{CH}_{2} \mathrm{Cl}_{2}$ :Hexane 10: 20: 70 as eluent) to afford C6-functionalized glucopyranoside VJ-4 as a white solid in $69 \%(0.598 \mathrm{~g})$ yield. $R_{f} 0.2$ (Ether: $\left.\mathrm{CH}_{2} \mathrm{Cl}_{2}: \mathrm{Hexane} 10: 20: 70\right) ;{ }^{1} \mathrm{H}$ NMR (400 $\left.\mathrm{MHz}, \mathrm{CDCl}_{3}\right) \delta$ 7.85-2.80 (m, 2H), 7.77-7.72 (m, 2H), 7.37-7.28 (m, 15H), 4.99-4.89 (m, 3H), $4.82(\mathrm{~d}, J=11.2 \mathrm{~Hz}, 1 \mathrm{H}), 4.71(\mathrm{~d}, J=11.2 \mathrm{~Hz}, 2 \mathrm{H}), 4.51$ (d, $J=11.6 \mathrm{~Hz}, 1 \mathrm{H}), 4.39-4.28(\mathrm{~m}$, 2H), 3.80-3.74 (m, $1 \mathrm{H}), 3.70$ (t, $J=8.8 \mathrm{~Hz}, 1 \mathrm{H}), 3.58(\mathrm{t}, J=9.2 \mathrm{~Hz}, 1 \mathrm{H}), 3.48-3.41(\mathrm{~m}, 4 \mathrm{H})$; ${ }^{13} \mathbf{C}\{\mathbf{1 H}\}$ NMR $\left(100 \mathrm{MHz}, \mathrm{CDCl}_{3}\right) \delta 163.4,138.6,138.5,137.8,134.5,129.1,128.6,128.5$, 
128.4, 128.2, 128.1, 128.0, 128.0(2), 127.7, 123.5, 104.7, 84.7, 82.2, 77.2, 76.6, 75.8, 75.2, 74.8, 74.1, 57.2; HRMS (ESI-TOF) m/z: [M+Na] ${ }^{+}$calculated for $\mathrm{C}_{36} \mathrm{H}_{35} \mathrm{NNaO}_{8} 632.2260$, mass found 632.2257 .

Preparation of compound VJ-5 [O-((2S,3S,4R,5S,6S)-3,4,5-tris(benzyloxy)-6methoxytetrahydro-2H-pyran-2-yl)methyl)hydroxylamine hydrochloride]

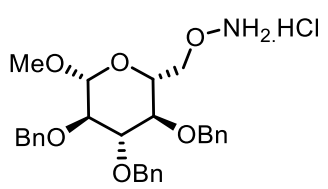

To a suspension of VJ-4 (0.400 g, $0.66 \mathrm{mmol}, 1.0$ equiv) in a mixture of $\mathrm{MeOH}(4.0 \mathrm{~mL})$ and $\mathrm{CH}_{2} \mathrm{Cl}_{2}(1.5 \mathrm{~mL})$ was added hydrazine monohydrate $(0.085 \mathrm{~mL}, 1.98 \mathrm{mmol}, 3.0$ equiv) and the mixture was stirred at room temperature for $4 \mathrm{~h}$. A saturated aqueous solution of $\mathrm{NaHCO}_{3}(10$ $\mathrm{mL})$ was then added and extracted with EtOAc $(3 \times 10 \mathrm{~mL})$. The organic layer was washed with brine $(15.0 \mathrm{~mL})$, dried over anhydrous $\mathrm{Na}_{2} \mathrm{SO}_{4}$ and concentrated in vacuo. The residue was passed through short silica gel column (using 1:9 EtOAc/Hexane as eluent) to afford compound VJ-5 in $84 \%(0.265 \mathrm{~g})$ yield. The material was again dissolved in a mixture of $\mathrm{CH}_{2} \mathrm{Cl}_{2}$ and MTBE $(9.0 \mathrm{~mL}, 3: 1)$ and then treated with $4 \mathrm{M} \mathrm{HCl}$ solution in dioxane $(0.5 \mathrm{~mL})$. After stirring for 10 minutes, solvent was removed in vacuo to obtain a solid residue which was directly used in the next step.

Preparation of compound VJ-6 [2-bromo-2-methyl-N-((2S,3S,4R,5S,6S)-3,4,5tris(benzyloxy)-6-methoxytetrahydro-2H-pyran-2-yl)methoxy)propenamide]

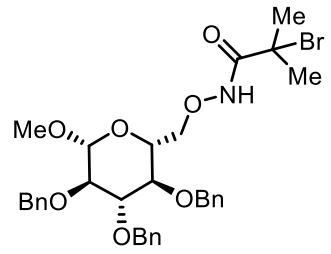

To a cooled $\left(0^{\circ} \mathrm{C}\right)$ suspension of compound VJ-5 (0.285 g, $0.55 \mathrm{mmol} 1.0$ equiv) and triethylamine $\left(0.092 \mathrm{~mL}, 0.66 \mathrm{mmol}, 1.2\right.$ equiv) in dry $\mathrm{CH}_{2} \mathrm{Cl}_{2}(0.25 \mathrm{M})$ was added $\alpha-$ bromoisobutyryl bromide ( $0.08 \mathrm{~mL}, 0.66 \mathrm{mmol}, 1.2$ equiv) and the reaction was continued at same temperature until consumption of starting material was observed (TLC controlled). After completion, reaction mixture was quenched with water, and extracted with DCM. The combined organic layer was washed with brine, dried over $\mathrm{Na}_{2} \mathrm{SO}_{4}$ and concentrated in vacuo. The crude residue was purified by column chromatography (1:4 EtOAc/Hexane as eluent) to afford the corresponding $\alpha$-halohydroxamate, VJ-6 in $51 \%(0.177 \mathrm{~g})$ yield. $R_{f} 0.25(1: 4$ EtOAc:Hexane); ${ }^{1} \mathrm{H}$ NMR (400 MHz, $\left.\mathrm{CDCl}_{3}\right) \delta 9.76(\mathrm{~s}, 1 \mathrm{H}), 7.34-7.27(\mathrm{~m}, 15 \mathrm{H}), 4.94$ (t, $\mathrm{J}=$ $11.2 \mathrm{~Hz}, 2 \mathrm{H}), 4.88(\mathrm{~d}, J=10.8 \mathrm{~Hz}, 1 \mathrm{H}), 4.82(\mathrm{~d}, J=11.2 \mathrm{~Hz}, 1 \mathrm{H}), 4.70(\mathrm{t}, J=11.6 \mathrm{~Hz}, 2 \mathrm{H})$, 
4.35 (d, J=8.0 Hz, 1H), 4.23 (dd, $J=12.4,4.0 \mathrm{~Hz}, 1 \mathrm{H}$ ), 4.14 (dd, $J=12.1,2.8 \mathrm{~Hz}, 1 \mathrm{H}$ ), 3.69$3.64(\mathrm{~m}, 2 \mathrm{H}), 3.61(\mathrm{~s}, 3 \mathrm{H}), 3.58-3.53(\mathrm{~m}, 1 \mathrm{H}), 3.46(\mathrm{t}, J=8.4 \mathrm{~Hz}, 1 \mathrm{H}), 1.96(\mathrm{~s}, 3 \mathrm{H}), 1.95$ (s, $3 \mathrm{H}) .{ }^{13} \mathbf{C}\{\mathbf{1 H}\}$ NMR $\left(100 \mathrm{MHz}, \mathrm{CDCl}_{3}\right) \delta 168.9,138.5,138.4,137.9,128.6,128.5,128.3,128.2$, 128.1, 127.9, 127.9(2), 127.8, 105.1, 84.4, 82.3, 77.3, 75.8, 75.3, 75.0, 74.7, 74.6, 59.3, 57.6, 32.5, 32.4; HRMS (ESI-TOF) m/z: [M+Na] ${ }^{+}$calculated for $\mathrm{C}_{32} \mathrm{H}_{38} \mathrm{BrNNaO}_{7} 650.1729$, mass found 650.1725 .

5,5-Dimethyl-2-phenyl-2-(pyrrolidin-1-yl)-3-(((2S,3S,4R,5R,6R)-4,5,6-tris(benzyloxy)-3methoxytetrahydro-2H-pyran-2-yl)methoxy)thiazolidin-4-one (19")

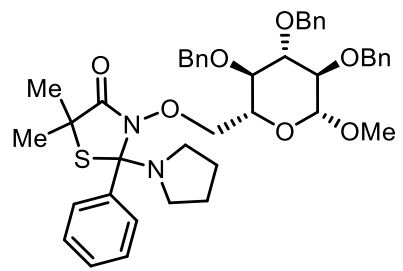

Thiazolidin-4-one derivative 19" was prepared using compound VJ-6 and thioamide by following the general procedure as described earlier (see subsection 3b). Accordingly, reaction between phenyl(pyrrolidin-1-yl) methanethione $(0.020 \mathrm{~g}, 0.10 \mathrm{mmol}, 1.0$ equiv) and VJ-6 (0.132 g, 0.20 mmol, 2.0 equiv) afforded the desired thiazolidin-4-one 19", which was purified by silica gel column chromatography (1:9 EtOAc:Hexane as eluent) to give the title compound as mixture of diastereomers (d.r. $=1: 0.8)$ as white solid in $68 \%(0.053 \mathrm{~g})$ yield. $R_{f}$ 0.2 (1:9 EtOAc:Hexane); ${ }^{1} \mathrm{H}$ NMR $\left(400 \mathrm{MHz}, \mathrm{CDCl}_{3}\right) \delta 8.03$ (d, J=8.8 Hz, 2H), 7.98-7.93 (m, 1.6H), 7.25-7.22 (m, 33H), $4.924 .82(\mathrm{~m}, 4 \mathrm{H}), 4.80-4.77(\mathrm{~m}, 2 \mathrm{H}), 4.76-4.74(\mathrm{~m}, 1 \mathrm{H}), 4.72(\mathrm{~d}, J$ $=3.6 \mathrm{~Hz} 0.8 \mathrm{H}$ ), $4.67(\mathrm{dd}, J=10.8,8.4 \mathrm{~Hz}, 1.8 \mathrm{H}), 4.56(\mathrm{~d}, J=10.8 \mathrm{~Hz}, 0.8 \mathrm{H}), 4.27(\mathrm{~d}, J=$ $8.0 \mathrm{~Hz}, 0.8 \mathrm{H}), 4.19(\mathrm{~d}, J=7.6 \mathrm{~Hz}, 1 \mathrm{H}), 4.13(\mathrm{dd}, J=9.2,6.0 \mathrm{~Hz}, 1 \mathrm{H}), 3.87(\mathrm{~d}, J=8.8 \mathrm{~Hz}$, $0.8 \mathrm{H}), 3.64-3.54(\mathrm{~m}, 9 \mathrm{H}), 3.47(\mathrm{t}, J=8.8 \mathrm{~Hz}, 2 \mathrm{H}), 3.39(\mathrm{t}, J=8.0 \mathrm{~Hz}, 1 \mathrm{H}), 3.33(\mathrm{~d}, J=10.8$ $\mathrm{Hz}, 1.6 \mathrm{H}), 3.26(\mathrm{t}, J=9.2 \mathrm{~Hz}, 0.8 \mathrm{H}), 2.75-2.63(\mathrm{~m}, 3.6 \mathrm{H}), 2.58-2.51(\mathrm{~m}, 1.6 \mathrm{H}), 2.49-2.42(\mathrm{~m}$, $2 \mathrm{H}), 1.80-1.76(\mathrm{~m}, 7.5 \mathrm{H}), 1.68-1.62,(\mathrm{~m}, 11 \mathrm{H}) ;{ }^{13} \mathrm{C}\{1 \mathrm{H}\} \mathbf{N M R}\left(100 \mathrm{MHz}, \mathrm{CDCl}_{3}\right) \delta 171.8,171.3$, 140.2, 139.7, 138.6, 138.6 (2), 138.6(3), 138.6(4), 138.3, 138.2, 129.9(2), 129.5, 129.0, 129.0(2), 128.6, 128.5, 128.5(2), 128.5(3), 128.5(4), 128.4, 128.3, 128.2, 128.2(2), 128.2(3), 128.1, 128.0, 128.0(2), 127.8, 127.8(2), 127.8(3), 127.7, 127.7(2), 104.4, 104.3, 92.6, 92.2, 84.9, 84.6, 82.3, 82.3(2), 77.9, 77.1, 75.9, 75.8, 75.3, 74.9, 74.8, 74.7, 73.7, 73.5, 73.3(2), 57.2, 57.0, 48.4, 48.3, 44.9, 44.8, 31.3. 31.2, 28.2, 28.1, 24.3, 24.2; HRMS (ESI-TOF) m/z: $[\mathrm{M}+\mathrm{H}]^{+}$calculated for $\mathrm{C}_{43} \mathrm{H}_{51} \mathrm{~N}_{2} \mathrm{O}_{7} \mathrm{~S}$ 739.3417, mass found 739.3407 . 
Synthesis of a-hydroxamates and selenamides:

Compound $\mathbf{4 a - 4 e}$ were prepared by a reported procedure $\mathrm{4}^{4}$. Compound $\mathbf{4 d}$ and $4 \mathrm{e}$ are new compounds. Selenamide (5a-5c) were prepared following reported procedure ${ }^{5}$, data for new compounds are provided (list is provided in Scheme1-B; vide supra)

\section{2-Bromo-N-((4-methoxybenzyl)oxy)-2-methylpropanamide (4d)}<smiles>CC(C)(Br)C(=O)N[13CH3]</smiles>

O-(4-methoxybenzyl)hydroxylamine hydrochloride $(1.5 \mathrm{~g}, 7.91 \mathrm{mmol}, 1.0$ equiv) and triethylamine $\left(0.800 \mathrm{~g}, 7.91 \mathrm{mmol}, 1.0\right.$ equiv) were taken in in dry $\mathrm{CH}_{2} \mathrm{Cl}_{2}(0.25 \mathrm{M})$ and cooled to $0{ }^{\circ} \mathrm{C}$. To this solution was added $\alpha$-bromoisobutyryl bromide $(1.8 \mathrm{~g}, 7.91 \mathrm{mmol}, 1.0$ equiv) and reaction was continued until consumption of starting material was observed while maintaining the same temperature (TLC controlled). After completion, it was quenched with water, and extracted with DCM. The combined organic layer was washed with brine, dried over $\mathrm{Na}_{2} \mathrm{SO}_{4}$ and concentrated in vacuo. The crude residue was purified by column chromatography (1:9 EtOAc/Hexane as eluent) to afford the title compound as white foam in $70 \%\left(1.794\right.$ g) yield. ${ }^{1} \mathrm{H}$ NMR $\left(400 \mathrm{MHz}, \mathrm{CDCl}_{3}\right) \delta 9.09(\mathrm{~s}, 1 \mathrm{H}), 7.34(\mathrm{~d}, J=8.4 \mathrm{~Hz}, 2 \mathrm{H}), 6.90$ $(\mathrm{d}, J=8.4 \mathrm{~Hz}, 2 \mathrm{H}), 4.86(\mathrm{~s}, 2 \mathrm{H}), 3.81(\mathrm{~s}, 3 \mathrm{H}), 1.93(\mathrm{~s}, 6 \mathrm{H}) .{ }^{13} \mathrm{C}\{1 \mathrm{H}\} \mathrm{NMR}\left(100 \mathrm{MHz}, \mathrm{CDCl}_{3}\right) \delta$ 160.3, 131.3, 126.9, 114.1, 77.9, 55.4, 32.5, 30.8; HRMS (ESI-TOF) m/z: [M+Na] ${ }^{+}$calculated for $\mathrm{C}_{12} \mathrm{H}_{16} \mathrm{BrNNaO}_{3}, 324.0206$, mass found 324.0204 .

2-Bromo-2-methyl-N-propoxypropanamide (4e)<smiles>CCCNC(=O)NCC</smiles>

Similar to the procedure for compound $4 \mathrm{~d}$, O-heptylhydroxylamine hydrochloride $(0.600 \mathrm{~g}, 3.58$ mmol with a-bromoisobutyryl bromide $(0.811 \mathrm{~g}, 3.58 \mathrm{mmol}, 1.0$ equiv) delivered compound $4 \mathrm{e}$ as pink oil in $76 \%(0.761 \mathrm{~g})$ yield. Column chromatographic conditions: (5:95 EtOAc/Hexane as eluent). ${ }^{1} \mathrm{H}$ NMR $\left(400 \mathrm{MHz}, \mathrm{CDCl}_{3}\right) \delta 9.22(\mathrm{~s}, 1 \mathrm{H}), 3.92(\mathrm{t}, J=6.8 \mathrm{~Hz}, 2 \mathrm{H}), 1.95(\mathrm{~s}, 6 \mathrm{H})$, 1.70-1.61 (m, 2H), 1.42-1.34 (m, 2H), 1.31-1.25 (m, 6H), $0.87(\mathrm{t}, J=6.8 \mathrm{~Hz}, 3 \mathrm{H}) .{ }^{13} \mathrm{C}\{1 \mathrm{H}\} \mathrm{NMR}$ $\left(100 \mathrm{MHz}, \mathrm{CDCl}_{3}\right) \delta 169.8,77.03,32.6,31.8,29.2,28.1,25.9,22.7,14.2 ;$ HRMS (ESI-TOF) $\mathrm{m} / \mathrm{z}:[\mathrm{M}+\mathrm{H}]^{+}$calculated for $\mathrm{C}_{11} \mathrm{H}_{23} \mathrm{BrNO}_{2} 280.0907$, mass found 280.0908 . 
<smiles>Cc1ccc(C(=O)N2CCCC2)cc1</smiles>

To a solution of pyrrolidin-1-yl(p-tolyl)methanone $(0.200 \mathrm{~g}, 1.06 \mathrm{mmol}, 1.0$ equiv $)$ in toluene $(16 \mathrm{~mL})$ was added Woollins reagent $(0.338 \mathrm{~g}, 0.64 \mathrm{mmol}, 0.6$ equiv $)$, and the mixture was stirred for $4 \mathrm{~h}$ at $130{ }^{\circ} \mathrm{C}$. After cooling to room temperature, the resulting mixture was concentrated in vacuo. Purification by silica gel column chromatography (1:9 EtOAc/hexane as eluent) to give the title compound as yellow foam in $92 \%(0.245 \mathrm{~g})$ yield. ${ }^{1} \mathbf{H}$ NMR (400 $\left.\mathrm{MHz}, \mathrm{CDCl}_{3}\right) \delta 7.27(\mathrm{~d}, J=8.0 \mathrm{~Hz}, 2 \mathrm{H}), 7.14(\mathrm{~d}, J=8.0 \mathrm{~Hz}, 2 \mathrm{H}), 3.97(\mathrm{t}, J=7.2 \mathrm{~Hz}, 2 \mathrm{H}), 3.37$ $(\mathrm{t}, J=6.8 \mathrm{~Hz}, 2 \mathrm{H}), 2.34(\mathrm{~s}, 3 \mathrm{H}), 2.16-2.08(\mathrm{~m}, 2 \mathrm{H}), 2.05-1.98(\mathrm{~m}, 2 \mathrm{H}) \cdot{ }^{13} \mathrm{C}\{\mathbf{1 H}\} \mathbf{N M R}(100 \mathrm{MHz}$, $\left.\mathrm{CDCl}_{3}\right) \delta 200.5,144.0,138.8,128.8,124.9,57.4,54.7,26.6,24.8,21.4$; HRMS (ESI-TOF) $\mathrm{m} / \mathrm{z}$ : $[\mathrm{M}+\mathrm{H}]^{+}$calculated for $\mathrm{C}_{12} \mathrm{H}_{16} \mathrm{NSe} 254.0448$, mass found 254.0439 .

Pyrrolidin-1-yl(1-tosyl-1H-indol-3-yl)methaneselenone (5c)<smiles></smiles>

To a solution of pyrrolidin-1-yl(1-tosyl-1H-indol-3-yl)methanone $(0.150 \mathrm{~g}, 0.41 \mathrm{mmol}, 1.0$ equiv ) in toluene $(7 \mathrm{~mL})$ was added $\mathrm{WR}(0.130 \mathrm{~g}, 0.24 \mathrm{mmol}, 0.6$ equiv $)$, and the mixture was stirred for $4 \mathrm{~h}$ at $130{ }^{\circ} \mathrm{C}$. After cooling to room temperature, the resulting mixture was concentrated in vacuo. Purification by silica gel column chromatography (1:4 EtOAc/hexane as eluent) to give the title compound yellow foam in $88 \%(0.154 \mathrm{~g})$ yield. ${ }^{1} \mathbf{H}$ NMR $(400 \mathrm{MHz}$, $\left.\mathrm{CDCl}_{3}\right) \delta 7.95(\mathrm{~d}, J=8.4 \mathrm{~Hz}, 1 \mathrm{H}), 7.80(\mathrm{~d}, J=8.4 \mathrm{~Hz}, 2 \mathrm{H}), 7.73(\mathrm{~s}, 1 \mathrm{H}), 7.34(\mathrm{~d}, J=8.0 \mathrm{~Hz}$, $1 \mathrm{H}), 7.32-7.28(\mathrm{~m}, 1 \mathrm{H}), 7.27-7.22(\mathrm{~m}, 3 \mathrm{H}), 3.98(\mathrm{t}, J=6.8 \mathrm{~Hz}, 2 \mathrm{H}), 3.41(\mathrm{t}, J=6.8 \mathrm{~Hz}, 2 \mathrm{H})$, $2.35(\mathrm{~s}, 3 \mathrm{H}), 2.17-2.09(\mathrm{~m}, 2 \mathrm{H}), 2.04-1.97(\mathrm{~m}, 2 \mathrm{H}) .{ }^{13} \mathrm{C}\{1 \mathrm{H}\} \mathbf{N M R}\left(100 \mathrm{MHz}, \mathrm{CDCl}_{3}\right) \delta 189.9$, 145.7, 134.8, 134.5, 130.2, 128.3, 127.3, 127.2, 125.4, 123.9, 122.3, 121.6, 113.5, 57.3, 54.8, 26.6, 24.7, 21.7; HRMS (ESI-TOF) m/z: [M+H] calculated for $\mathrm{C}_{20} \mathrm{H}_{21} \mathrm{~N}_{2} \mathrm{O}_{2} \mathrm{SSe}$ 433.0489, mass found 433.0485 . 


\section{Gram-scale synthesis of compound 2a}

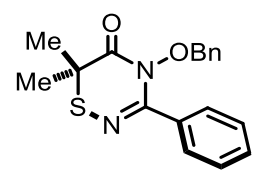

This reaction was performed following the general procedure as illustrated in section-4 of the SI. To a solution of compound $2 a(2.0 \mathrm{~g}, 5.22 \mathrm{mmol}, 1.0$ equiv) in HFIP ( $52.0 \mathrm{~mL}, 0.1 \mathrm{M})$ was added $\mathrm{TMSN}_{3}(3.01 \mathrm{~g}, 26.1 \mathrm{mmol}, 5.0$ equiv) and stirred at room temperature until complete consumption of starting material was observed (TLC monitored). Upon completion of the reaction, solvent was recovered through vacuum distillation and the crude residue was purified by silica gel column chromatography (using 2:98 ethyl acetate/hexane mixture as eluent) to afford compound $\mathbf{2 a}$ as white solid in $87 \%(1.49 \mathrm{~g})$ yield. [Note: recovered HFIP was used for same transformation without any affect in reaction efficiency] 


\section{Control Experiments}

(a)

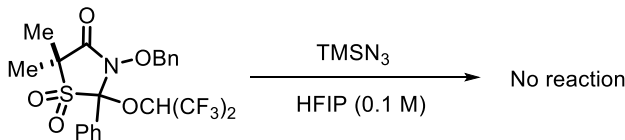
14

(b)<smiles>CC1(C)S[C@@](Nc2ccccc2)(N2CC[C@H](c3ccccc3)C2)C(C)(C)C1=O</smiles>

(c)<smiles>CC1(C)S[C@@](c2ccccc2)(N2CCCC2)N(OCc2ccccc2)C1=O</smiles>

(d)

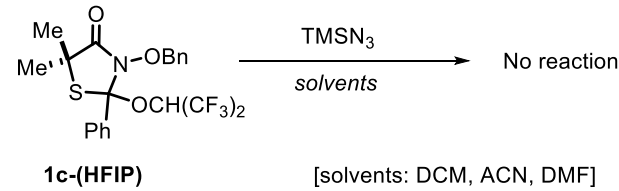

(e) Azidation-ring expansion with Lewis/Bronsted acids<smiles>CC1(C)S[C@@](c2ccccc2)(N2CCCC2)N(OCc2ccccc2)C1=O</smiles>

$1 \mathrm{a}$

$$
\begin{aligned}
& \mathrm{TMSN}_{3} \\
& \stackrel{\mathrm{BF}_{3} . \mathrm{Et}_{2} \mathrm{O} \text { or } \mathrm{TfOH}}{\longrightarrow} \text { No desired reaction } \\
& \text { for } \mathrm{BF}_{3} . \mathrm{Et}_{2} \mathrm{O} ; 1 \text { a was mostly unreacted } \\
& \text { for } \mathrm{TfOH}, 1 \mathrm{a} \text { was decomposed }
\end{aligned}
$$

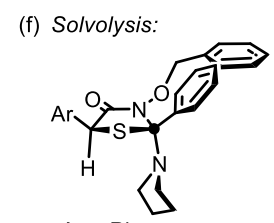

$\mathrm{Ar}=\mathrm{Ph}$

$\mathrm{Ar}=4-\mathrm{ClC}_{6} \mathrm{H}_{4}$

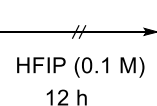

$12 \mathrm{~h}$

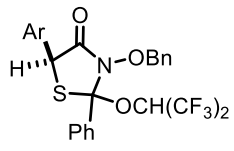

HFIP adduct not observed

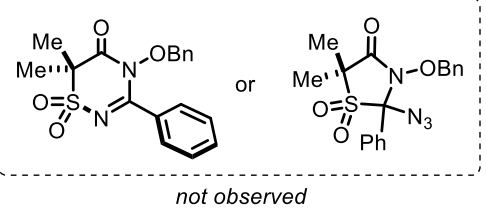

Comparative solvolysis of thia-/oxazolidinone in HFIP

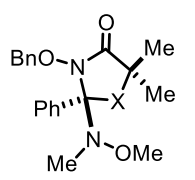

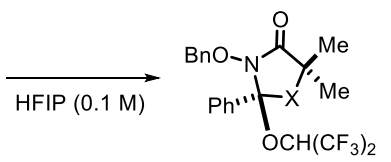

$\mathrm{X}=\mathrm{S}$ (quantitative yield) $\mathrm{X}=\mathrm{O}(0 \%)$

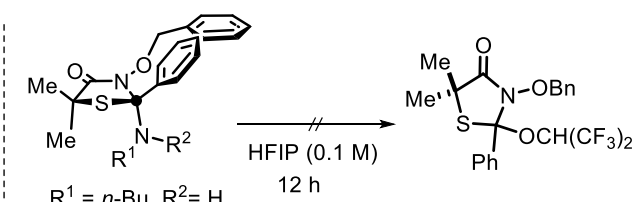

$\mathrm{R}^{1}=n-\mathrm{Bu}, \mathrm{R}^{2}=\mathrm{H} \quad 12 \mathrm{~h}$

$\mathrm{R}^{1}=\mathrm{Ph}, \mathrm{R}^{2}=\mathrm{Et}$

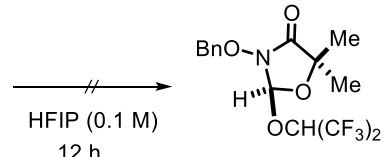

Scheme S4. Control experiments.

Experiment (a):

Compound $14(0.05 \mathrm{~g})$ was subjected to standard reaction conditions (5.0 equiv $\mathrm{TMSN}_{3}, 0.1$ $\mathrm{M} \mathrm{HFIP)} \mathrm{at} \mathrm{room} \mathrm{temperature.} \mathrm{Most} \mathrm{of} \mathrm{the} \mathrm{starting} \mathrm{material} \mathrm{was} \mathrm{found} \mathrm{unreacted} \mathrm{and} \mathrm{no} \mathrm{trace}$ of the ring-expanded product or azide addition was observed (as shown in the inset box) by MS analysis. 
Synthesis of compound 14:

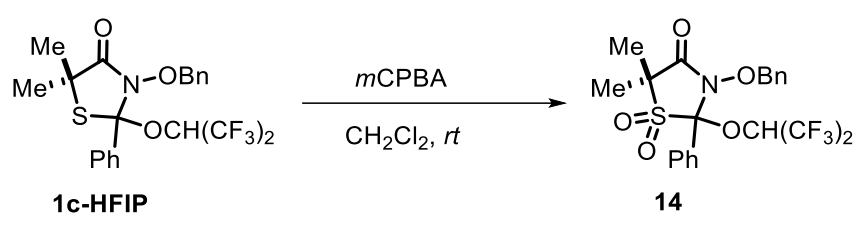

To a solution of compound $1 \mathrm{c}-\mathrm{HFIP}(0.033 \mathrm{~g}, 0.07 \mathrm{mmol}, 1.0$ equiv) in dry DCM $(0.7 \mathrm{~mL}, 0.1$ $\mathrm{M})$, was added $\mathrm{mCPBA}\left(0.068 \mathrm{~g}, 0.28 \mathrm{mmol}, 4.0\right.$ equiv) at $0^{\circ} \mathrm{C}$ and the reaction was stirred at room temperature until complete consumption of starting material (TLC monitored). The reaction mixture was quenched with saturated aqueous solution of $\mathrm{NaHCO}_{3}$ and extracted with DCM. Combined organic layers was dried with anhydrous $\mathrm{Na}_{2} \mathrm{SO}_{4}$ and concentrated in vacuo. Crude residue was purified by silica gel column chromatography (3:97 EtOAc/hexane) to afford compound 14 as colourless oil in $86 \%(0.030 \mathrm{~g})$ yield. $R_{f} 0.3$ (5:95 EtOAc:Hexane); ${ }^{1} \mathbf{H}$ NMR $\left(400 \mathrm{MHz}, \mathrm{CDCl}_{3}\right)$ ठ 7.64-7.59 (m, 2H), 7.52-7.43 (m, 3H), 7.40-7.34 (m, 3H), 7.33-7.29 $(\mathrm{m}, 2 \mathrm{H}), 5.64$ (hept, $J=6.0 \mathrm{~Hz}, 1 \mathrm{H}), 5.15(\mathrm{~s}, 2 \mathrm{H}), 1.72(\mathrm{~s}, 6 \mathrm{H}) \cdot{ }^{13} \mathbf{C}\{\mathbf{1 H}\} \mathbf{N M R}\left(101 \mathrm{MHz}, \mathrm{CDCl}_{3}\right)$ $\delta 167.2,155.6,136.1,131.4,129.7,128.7(2), 128.6(3), 128.6,124.9,124.5-116.1$ (m), 78.5, 68.6, 67.6 (hept, $J=35.0 \mathrm{~Hz}$ ), 20.9; HRMS (ESI-TOF) $\mathrm{m} / \mathrm{z}:[\mathrm{M}+\mathrm{H}]^{+}$calculated for $\mathrm{C}_{21} \mathrm{H}_{20} \mathrm{~F}_{6} \mathrm{NO}_{5} \mathrm{~S} 512.0966$ mass found 512.0961.

Experiment (b):

This reaction was performed with compound $2 \mathrm{a}\left(0.05 \mathrm{~g}, 1.0\right.$ equiv) and 5.0 equiv. of $\mathrm{PhN}_{3}$ in HFIP (0.1M). Unreacted starting material and HFIP adduct were found in the reaction mixture after $8 \mathrm{~h}$.

Experiment (c):

This was set up akin to experiment- $b$ except 5.0 equiv. of $\mathrm{BnN}_{3}$ was used. Unreacted starting material (along with HFIP adduct) were found at both room temperature or under heating at $50{ }^{\circ} \mathrm{C}$, with no trace of new products.

\section{Experiment (d):}

Reaction of the HFIP-adduct (1c) with $\mathrm{TMSN}_{3}$ (5.0 equiv) in various solvents such as $\mathrm{DCM}$, ACN, DMF etc. did not afford desired ring-expansion product, and unreacted starting material was left in the reaction mixture. In DMF, formation of some intractable product mixture was observed.

Experiment (e):

Reactions of aminothiazolidinone (1a) with $\mathrm{TMSN}_{3}$ (5.0 equiv) were performed in presence of $\mathrm{BF}_{3}$. $\mathrm{Et}_{2} \mathrm{O}$ (0.5 and 1.0 equiv) or $\mathrm{TfOH}\left(0.2 / 0.5 / 1.0\right.$ equiv) in $\mathrm{DCM}$. Reaction with $\mathrm{BF}_{3}$. $\mathrm{Et}_{2} \mathrm{O}$ did 
not show any progress of the reaction. Whereas, for $\mathrm{TfOH}$, with 0.2 equiv. no significant progress of reaction was noted and with 0.5-1.0 equiv. compound 1a was decomposed. No azide addition product or ring-expanded product was observed by NMR and LC-MS analysis.

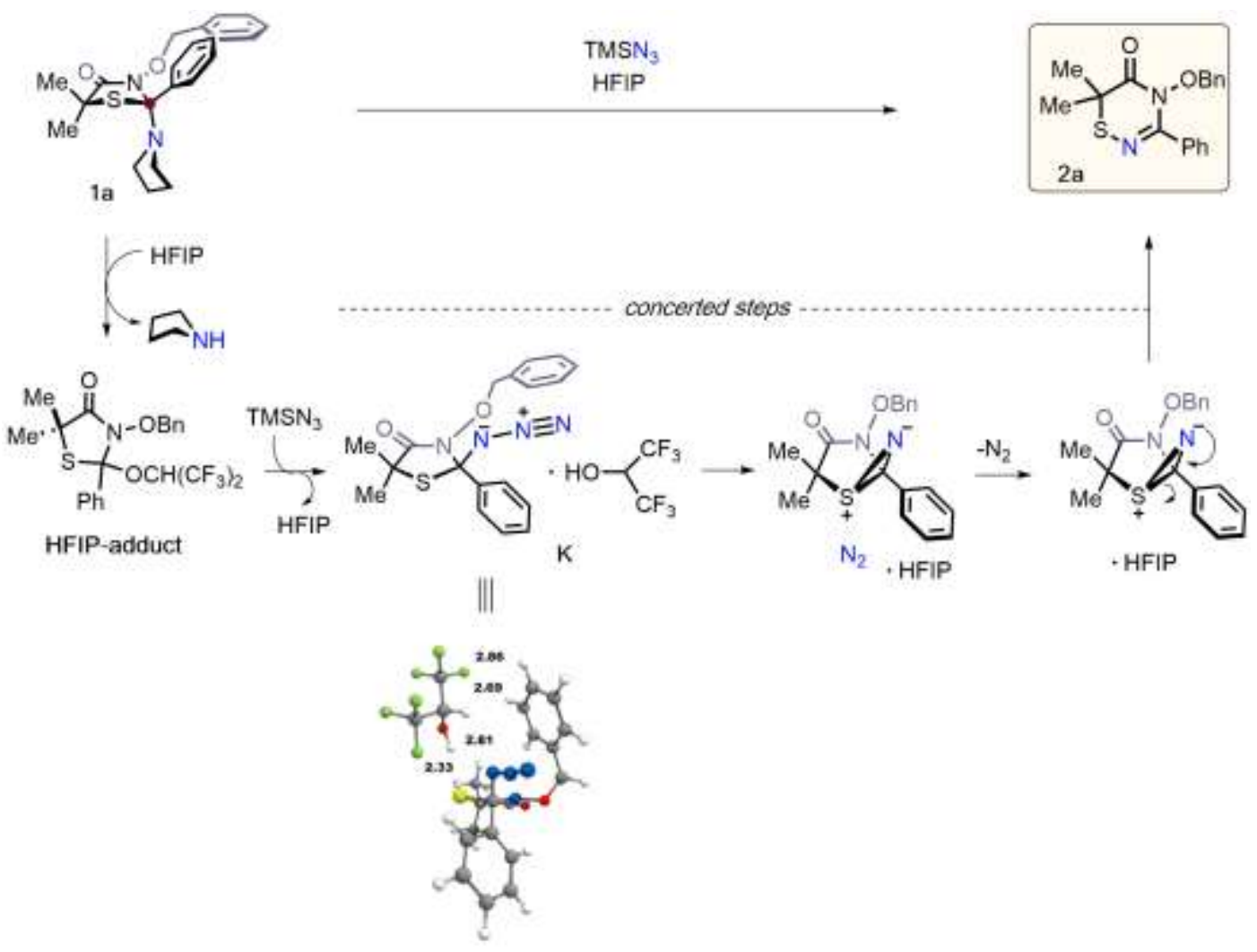

Scheme S5: Proposed Mechanism 


\section{NMR spectra of new compounds}
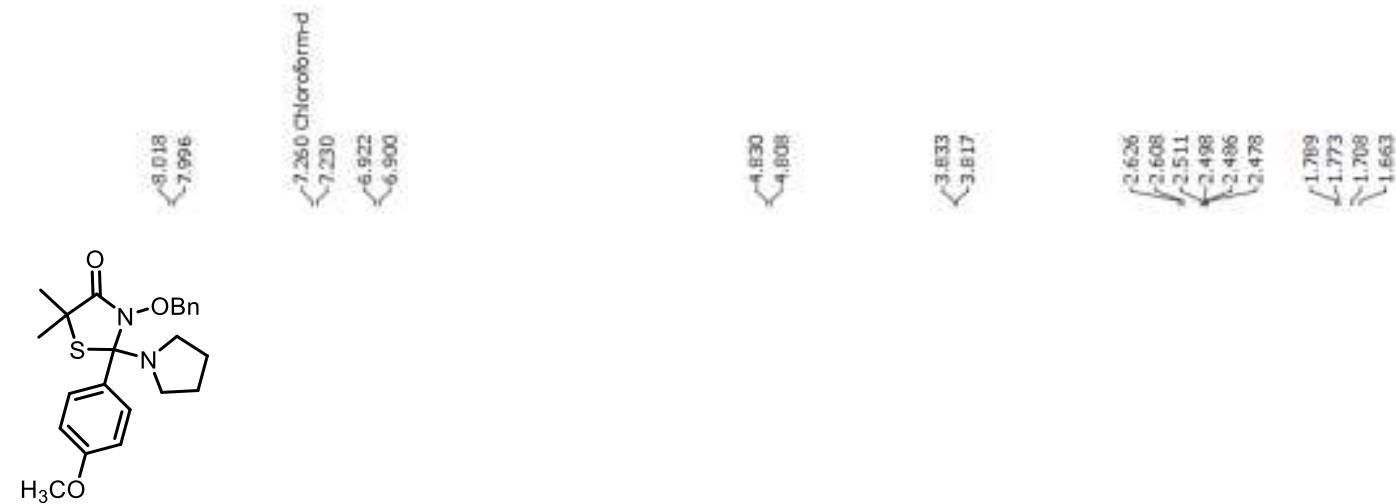

1d $\left(400 \mathrm{MHz}_{\mathrm{CDCl}}\right)$
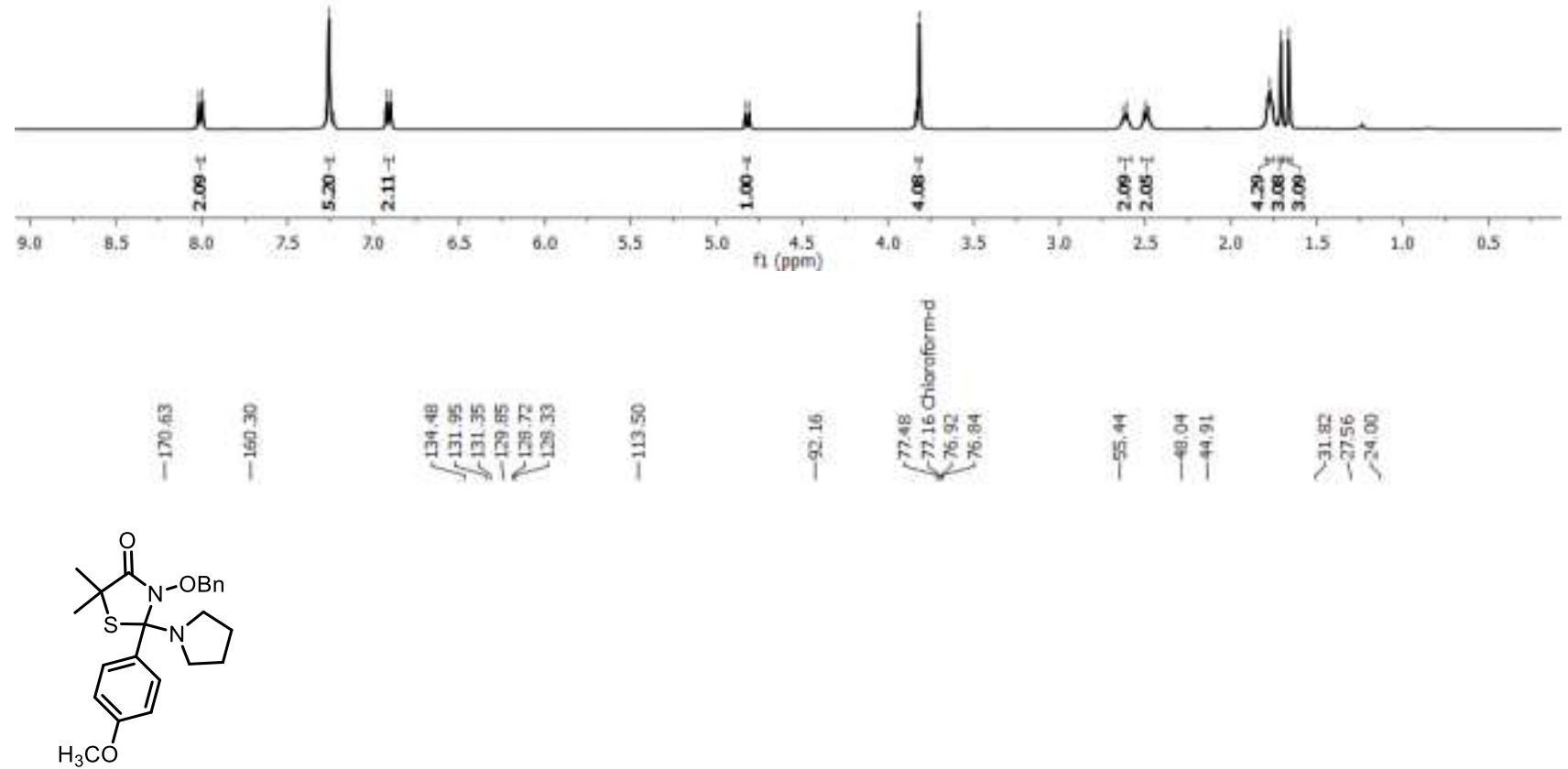

1d $\left(100 \mathrm{MHz}^{\mathrm{CDCl}}\right)_{3}$

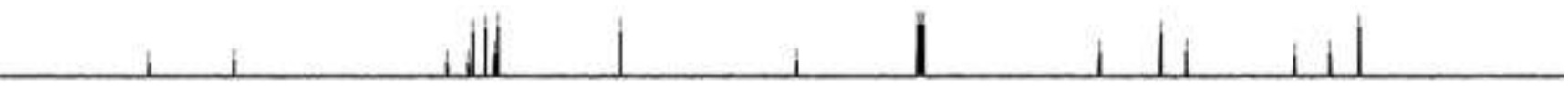

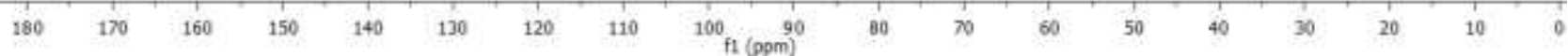



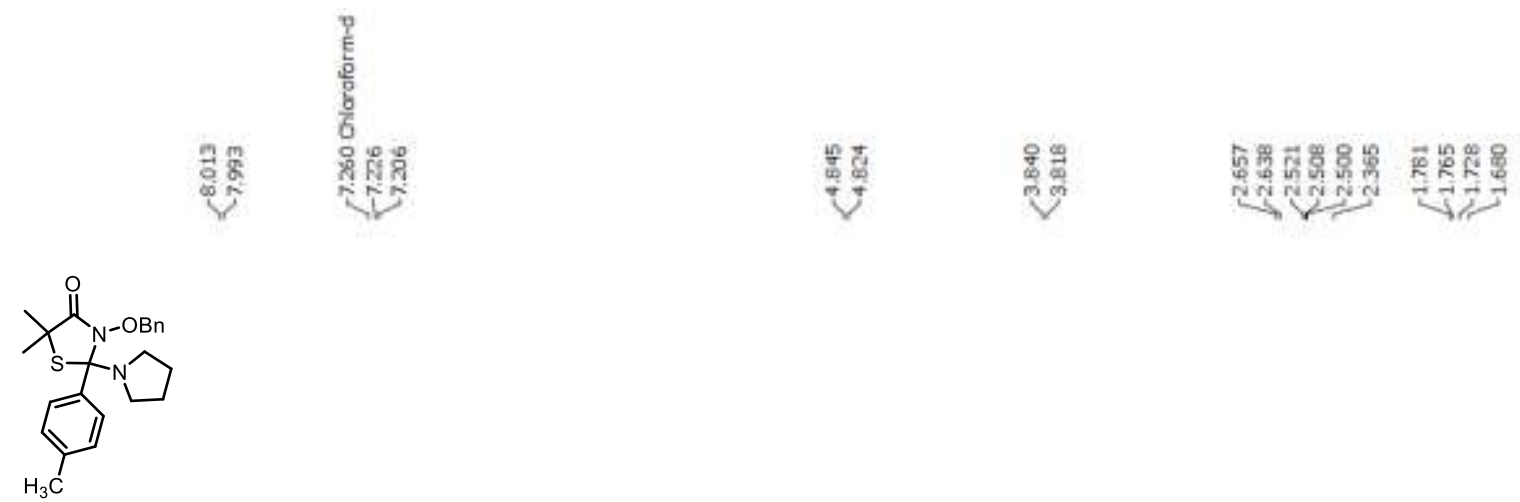

1e $\left(400 \mathrm{MHz}, \mathrm{CDCl}_{3}\right)$
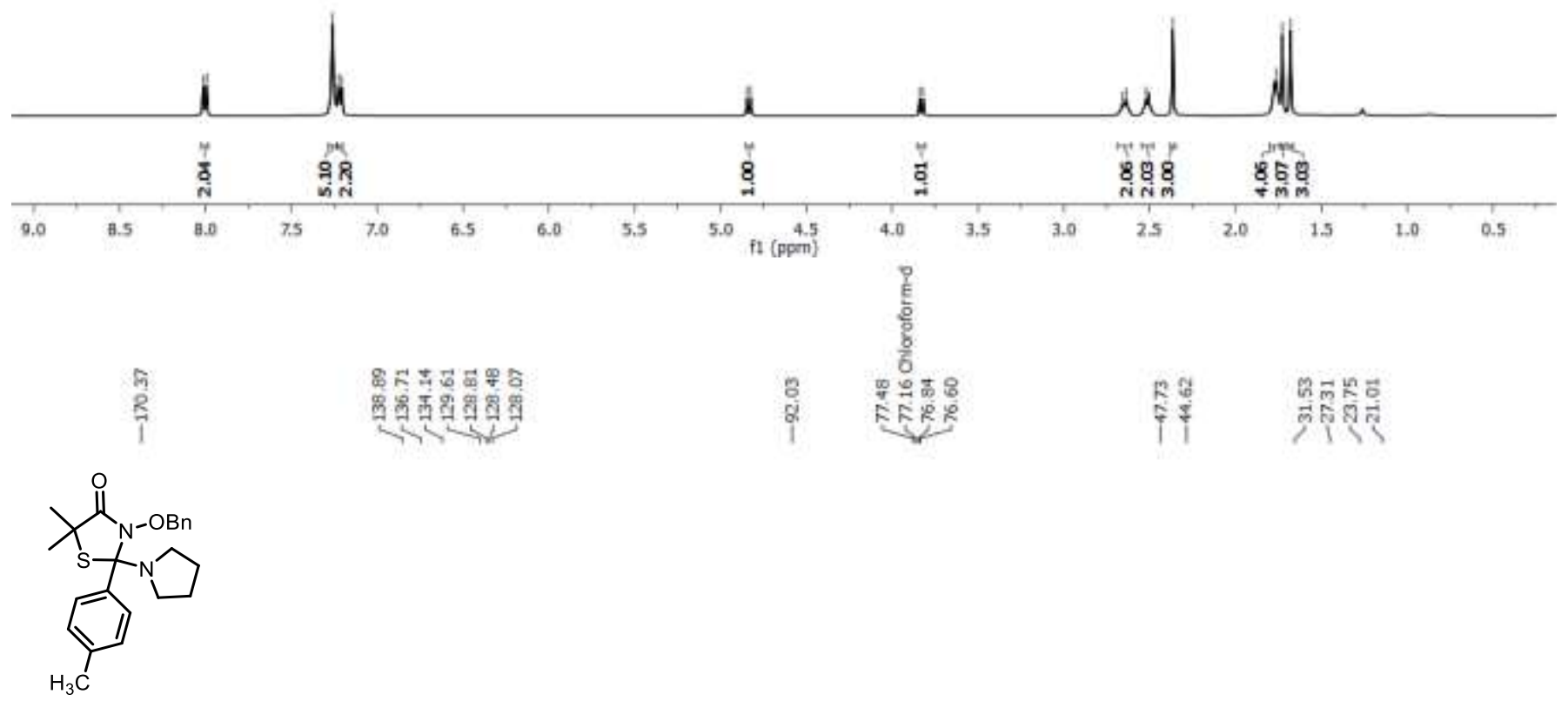

1e $\left(100 \mathrm{MHz}, \mathrm{CDCl}_{3}\right)$
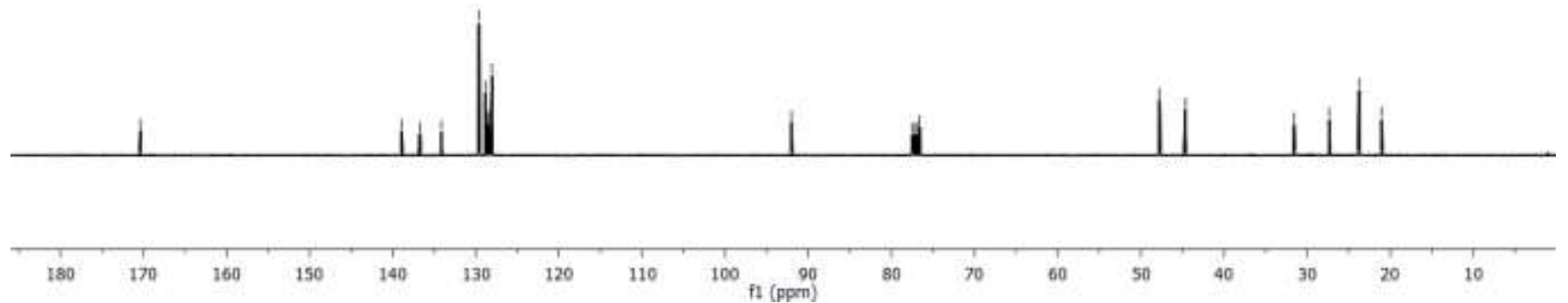


\section{$\frac{1}{\frac{1}{6}}$}

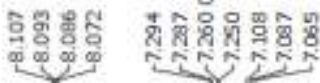

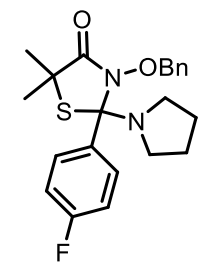

If $\left(400 \mathrm{MHz}, \mathrm{CDCl}_{3}\right)$

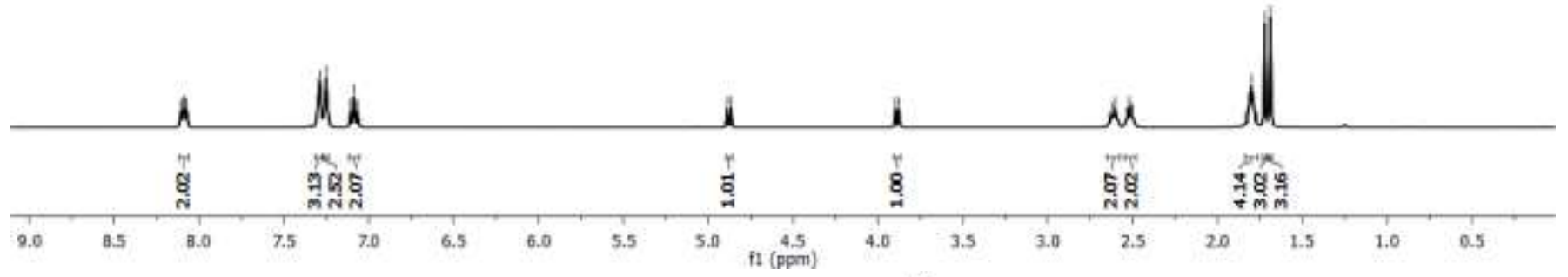



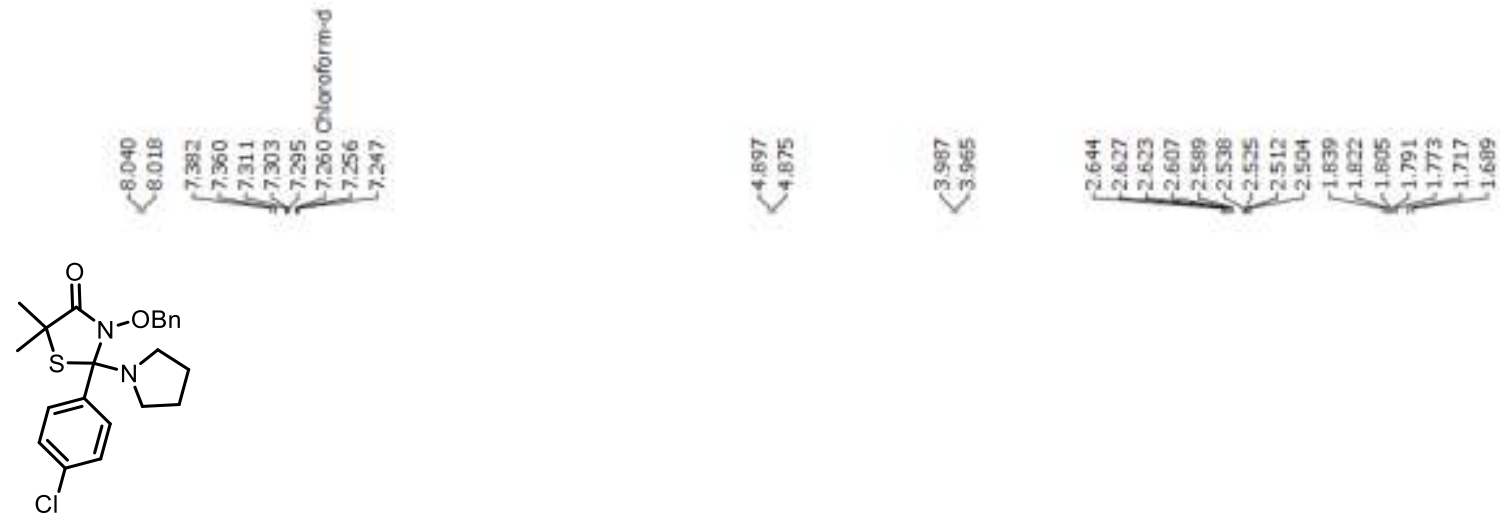

$1 \mathrm{~g}\left(400 \mathrm{MHz}, \mathrm{CDCl}_{3}\right)$
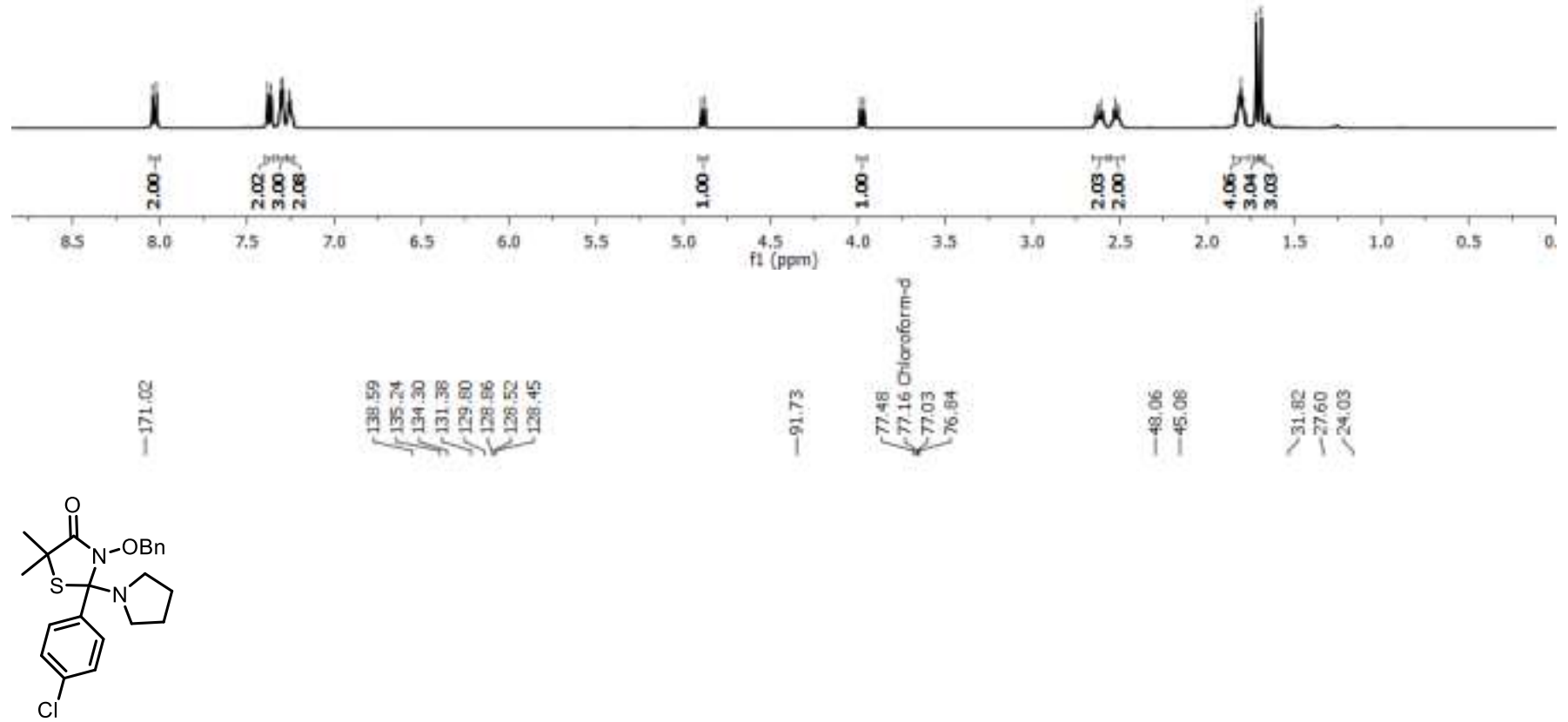

$1 \mathrm{~g}\left(100 \mathrm{MHz}, \mathrm{CDCl}_{3}\right)$
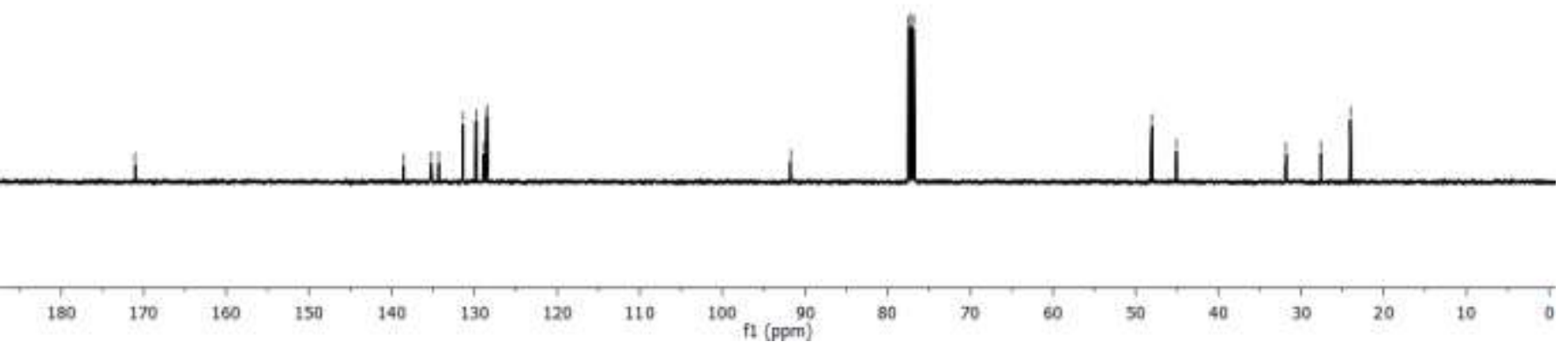


\section{要哣}

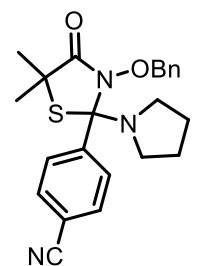

1 i (400 $\mathrm{MHz}, \mathrm{CDCl}_{3}$ )
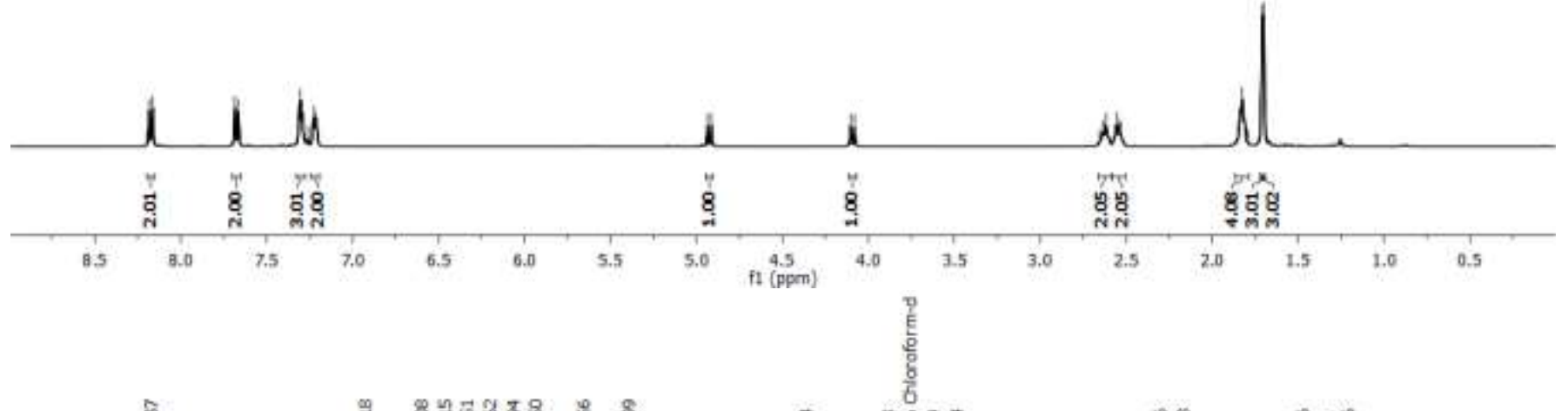

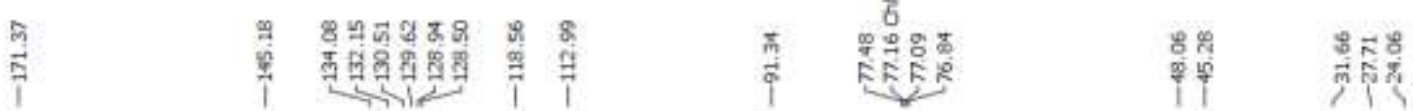

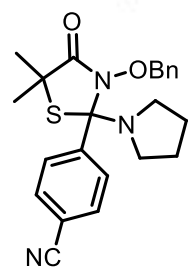

1i $\left(100 \mathrm{MHz}, \mathrm{CDCl}_{3}\right)$
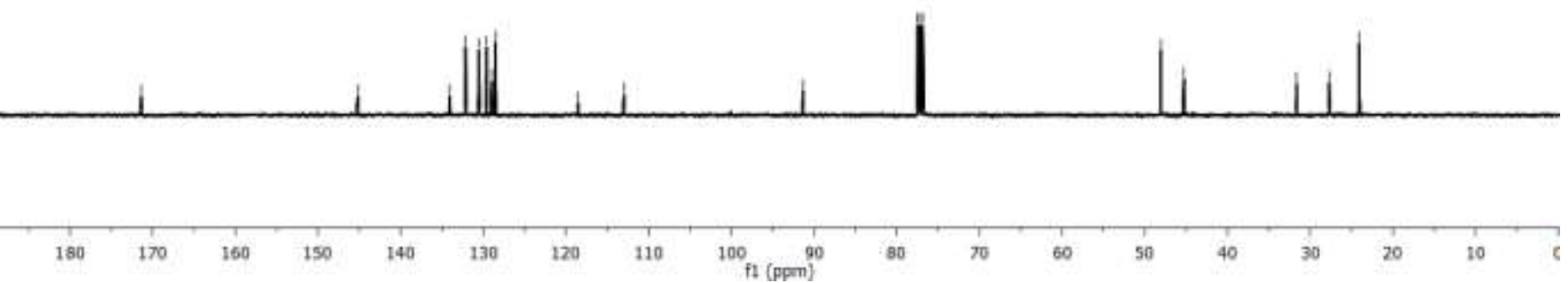

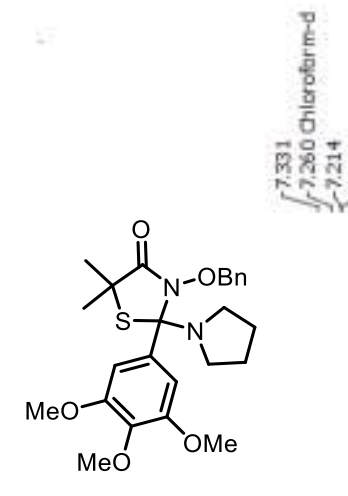

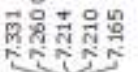

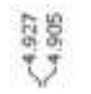

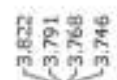

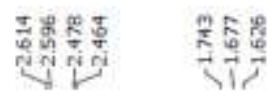

$1 \mathrm{k}\left(400 \mathrm{MHz}, \mathrm{CDCl}_{3}\right)$
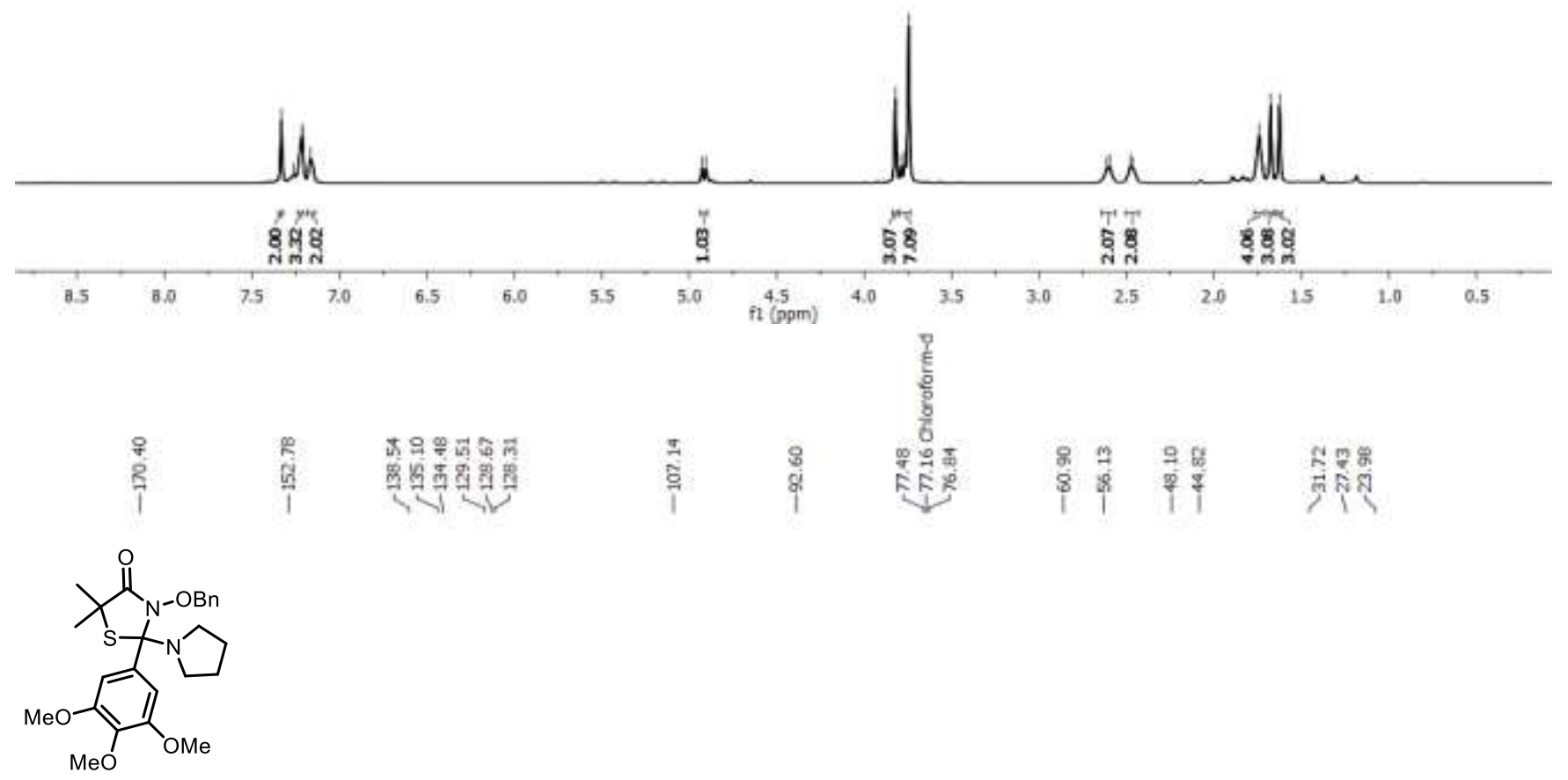

1k (100 $\left.\mathrm{MHz}, \mathrm{CDCl}_{3}\right)$

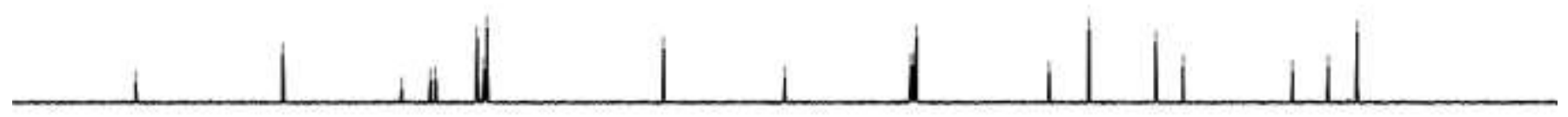

$\begin{array}{lllllllll}180 & 170 & 160 & 150 & 140 & 130 & 120 & 110 & 100 \quad \begin{array}{l}90 \\ \mathrm{f} 1\end{array}(\mathrm{ppm})\end{array}$ 


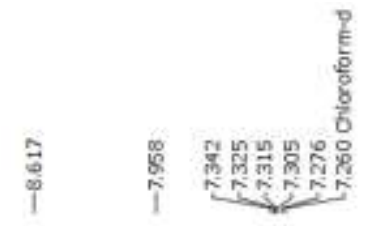

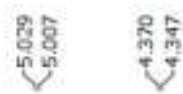

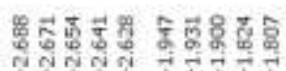

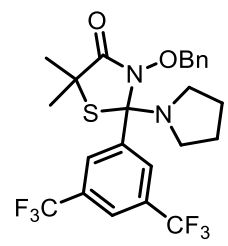

1 ( $\left(400 \mathrm{MHz}, \mathrm{CDCl}_{3}\right)$
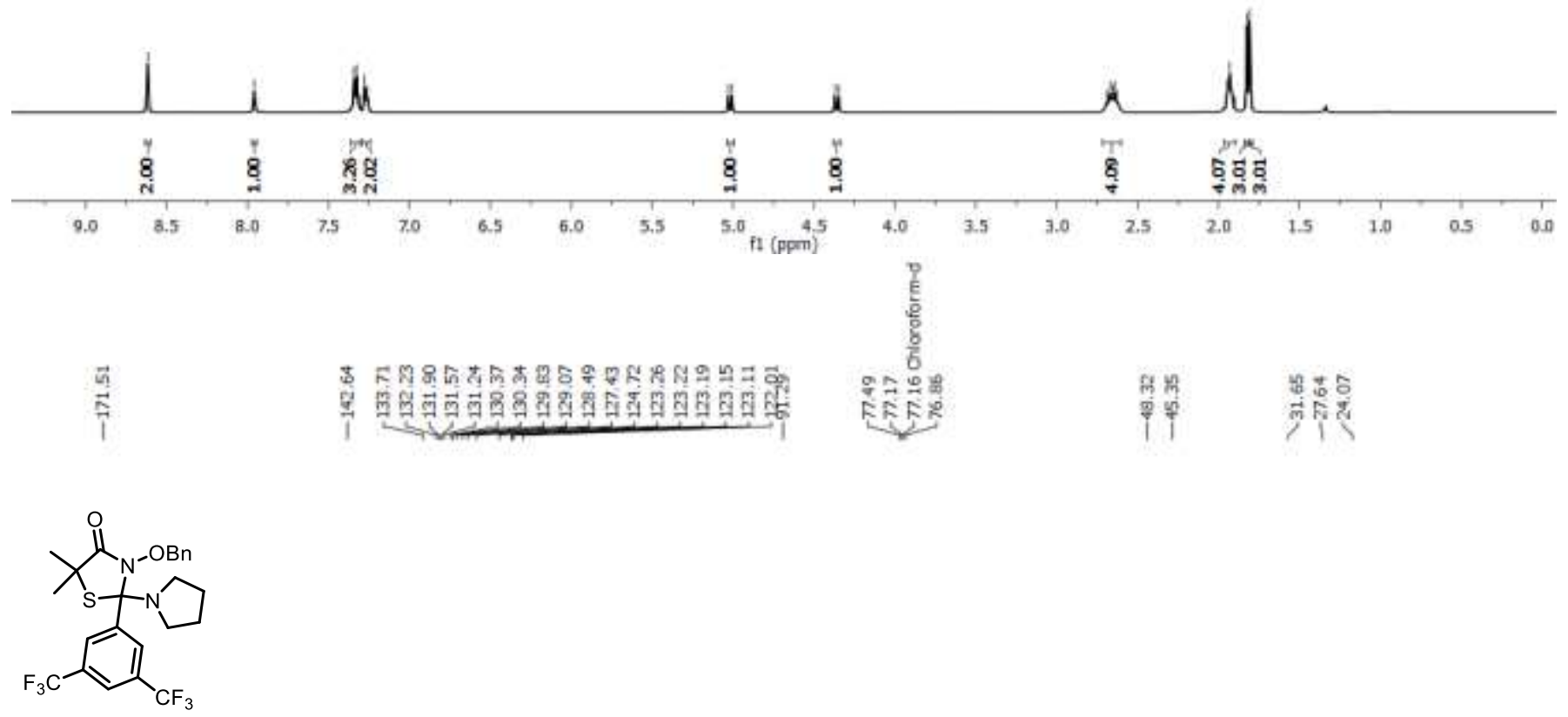

1) $\left(100 \mathrm{MHz}, \mathrm{CDCl}_{3}\right)$
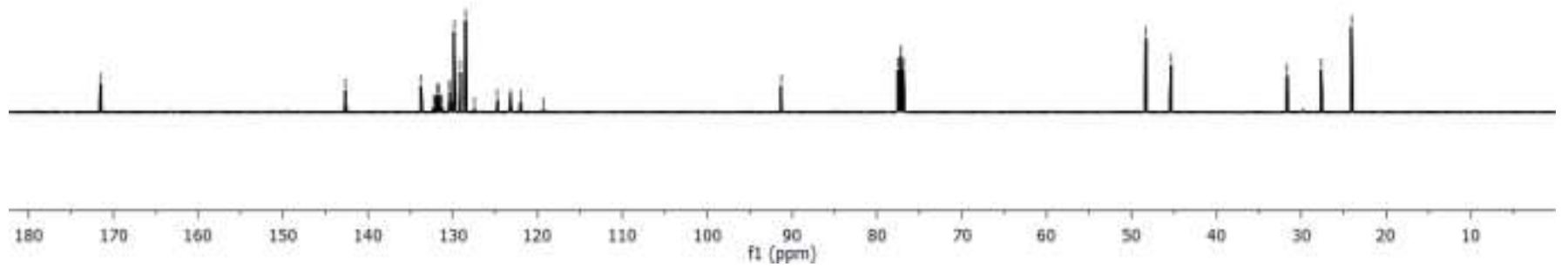


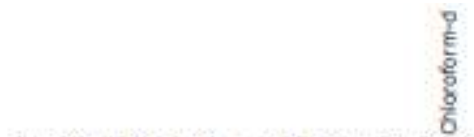<smiles>CC1(C)C(=O)N([18OH])C(c2ccccc2Cl)(N2CCCC2)C1(C)C</smiles>

$1 \mathrm{~m}\left(400 \mathrm{MHz}, \mathrm{CDCl}_{3}\right)$

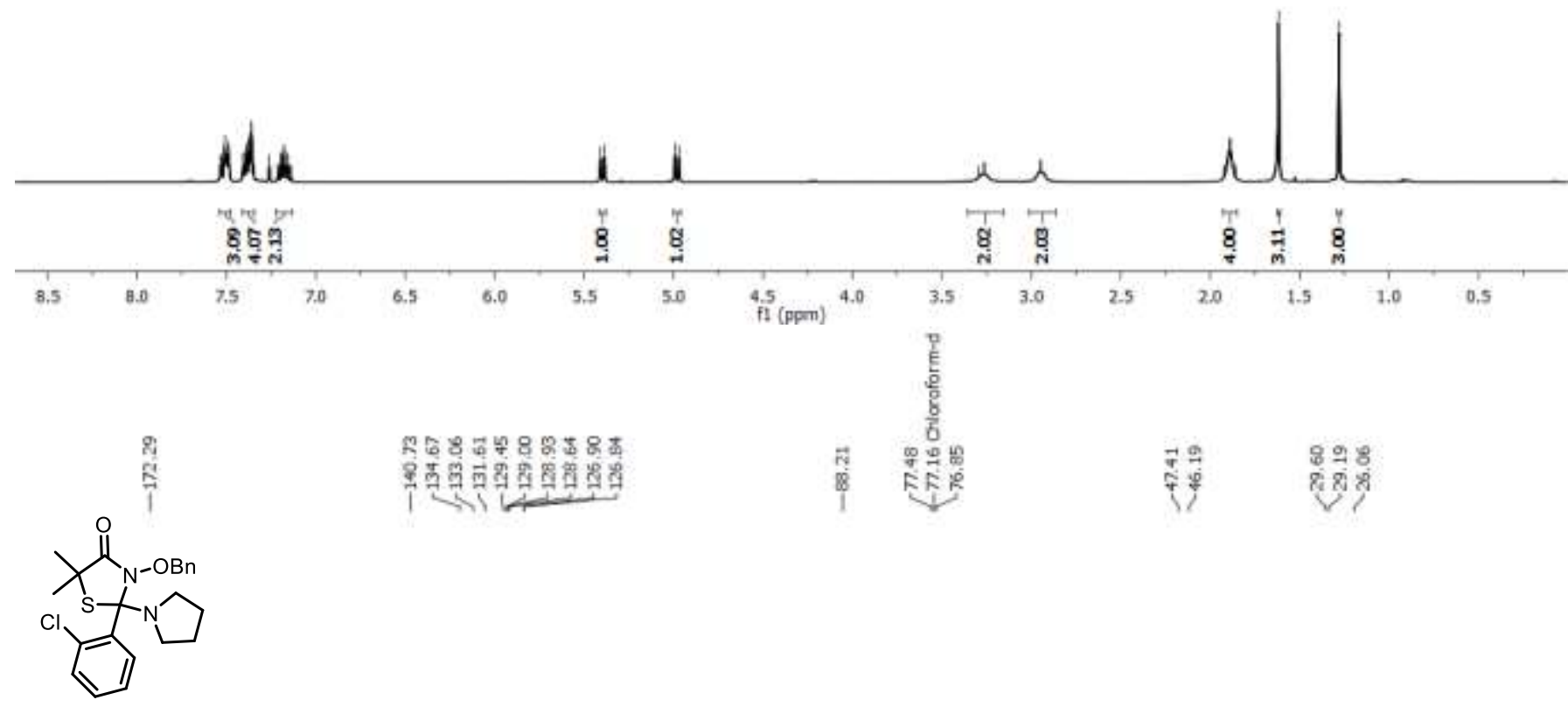

$1 \mathrm{~m}\left(100 \mathrm{MHz}, \mathrm{CDCl}_{3}\right)$

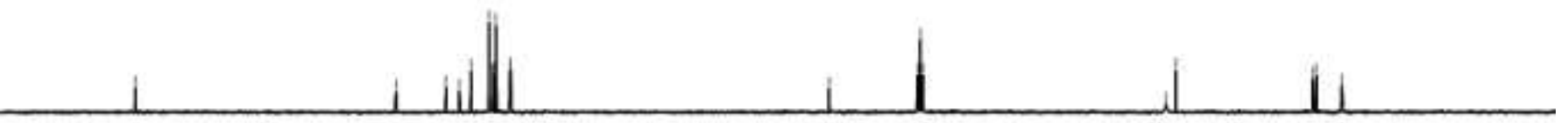




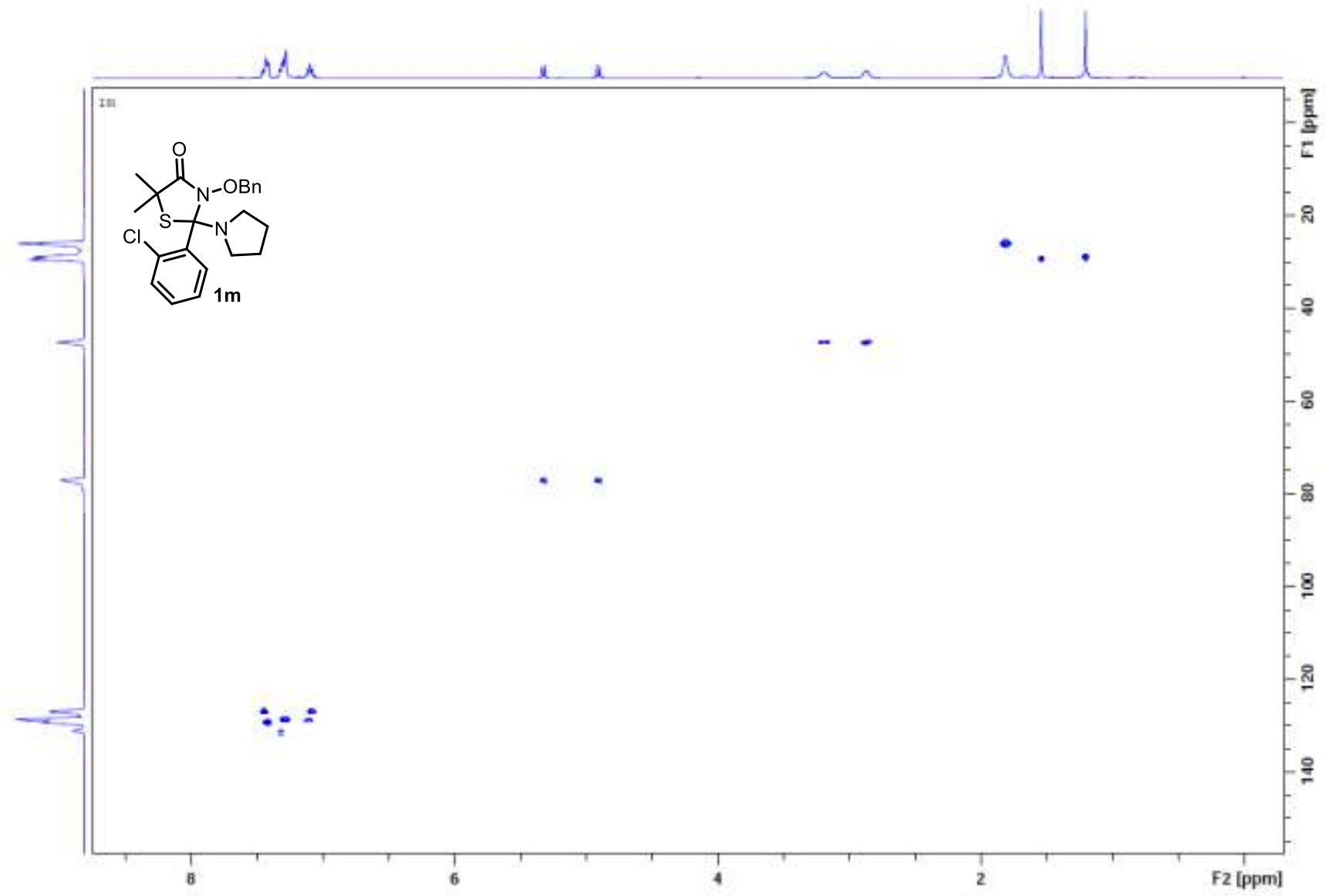




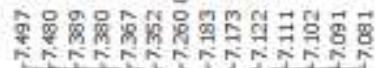

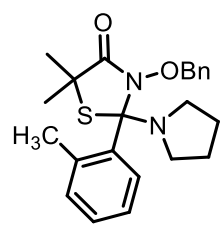

1n $\left(400 \mathrm{MHz}, \mathrm{CDCl}_{3}\right)$
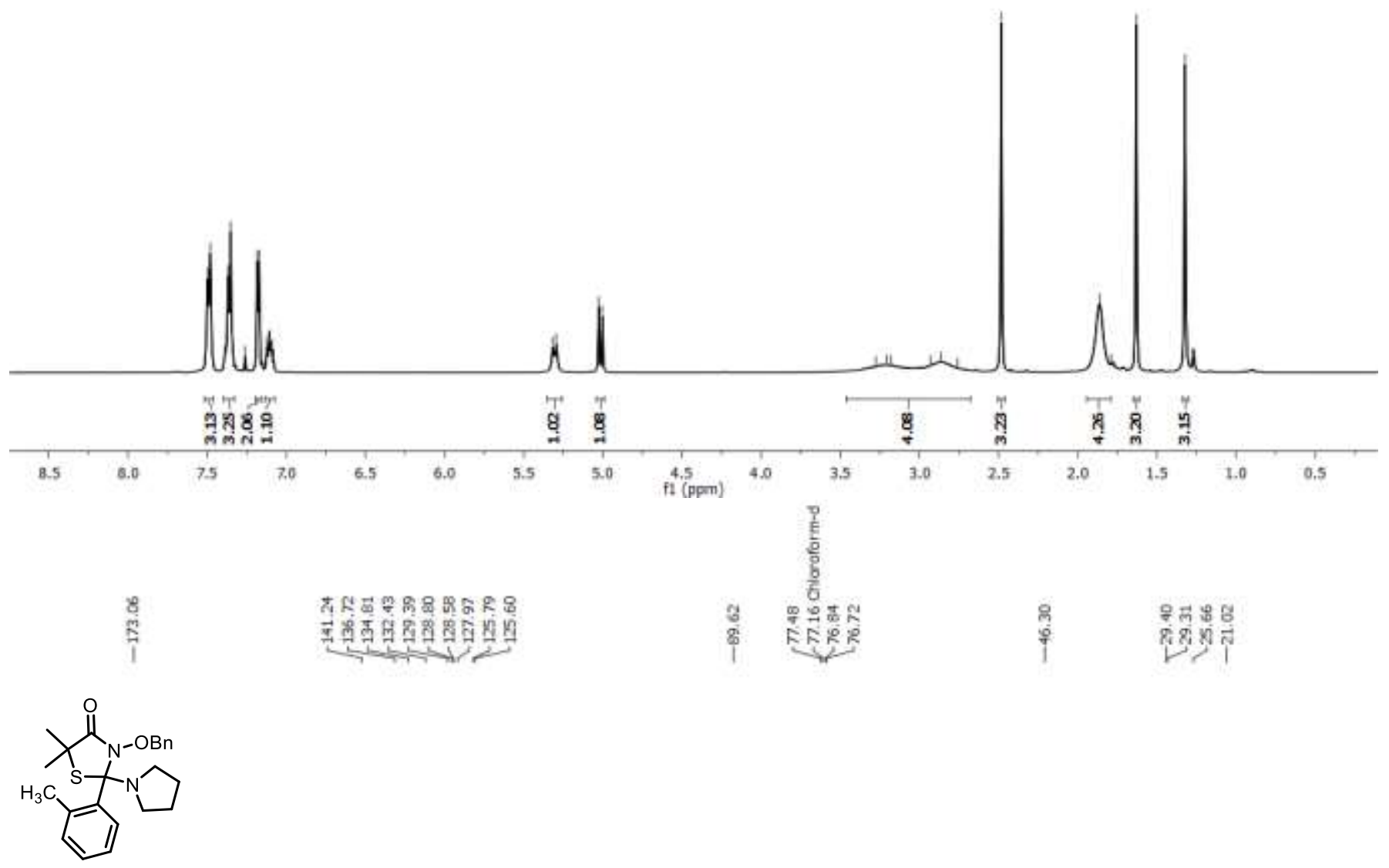

1n $\left(100 \mathrm{MHz}, \mathrm{CDCl}_{3}\right)$
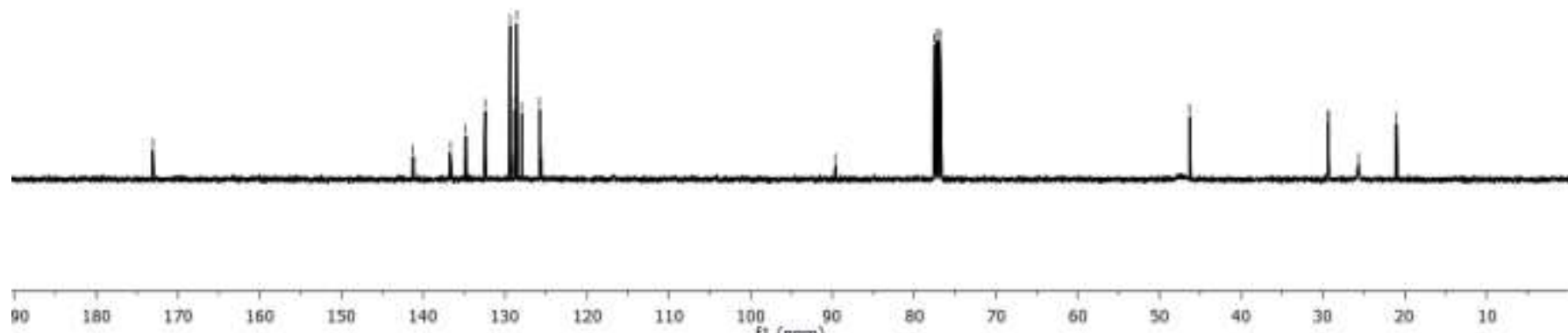

$150 \quad 1$

130

$120 \quad 110 \quad 100090$

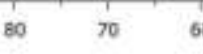

60

$\begin{array}{lll}10 & 1 \\ 40 & 20\end{array}$ 


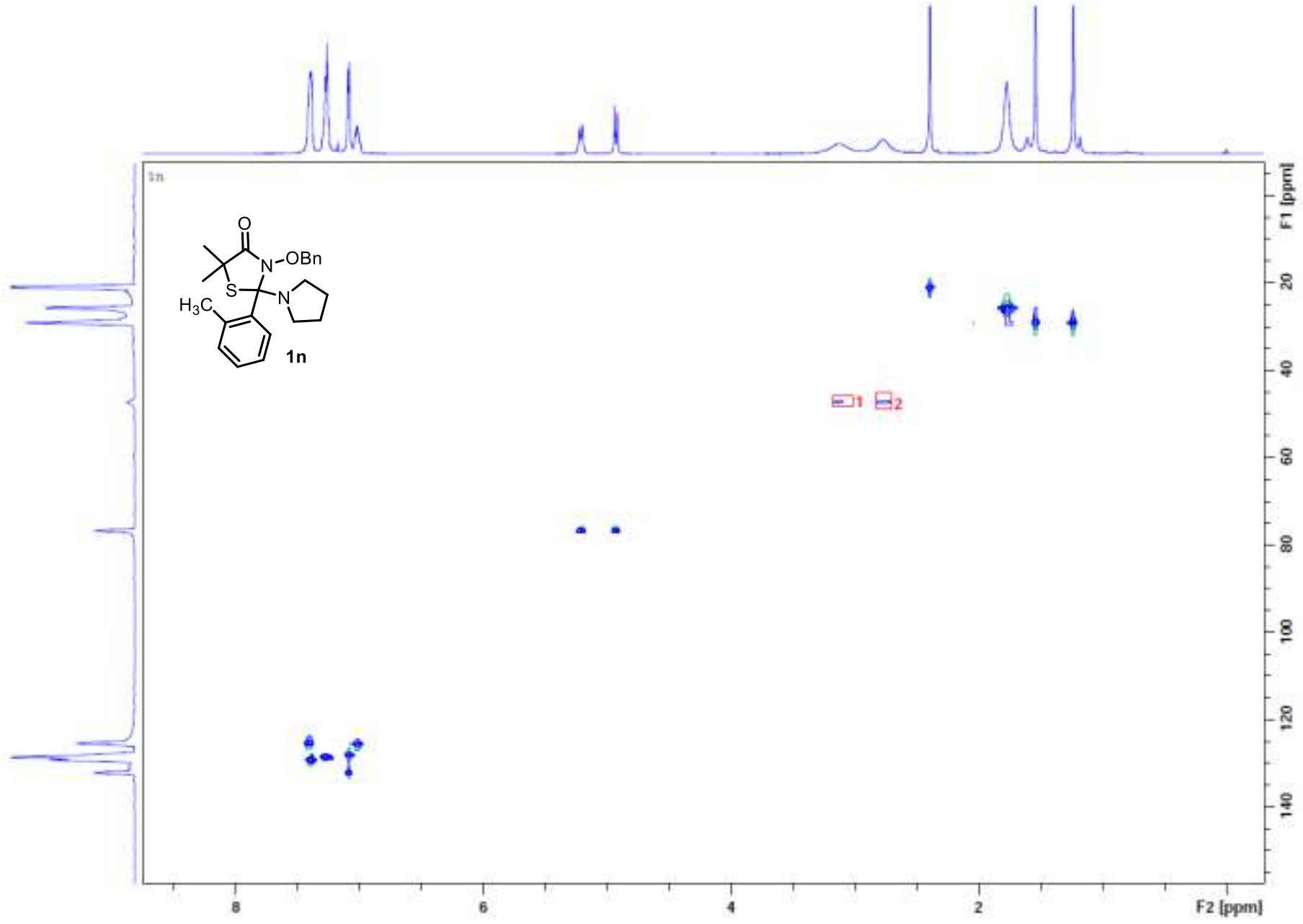




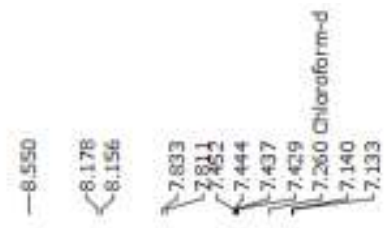

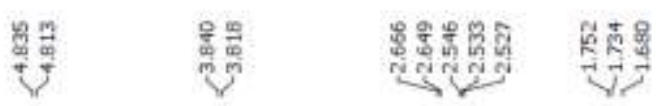

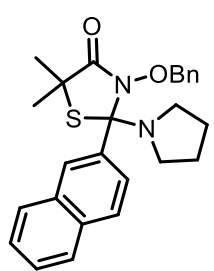

10 $\left(400 \mathrm{MHz}, \mathrm{CDCl}_{3}\right)$
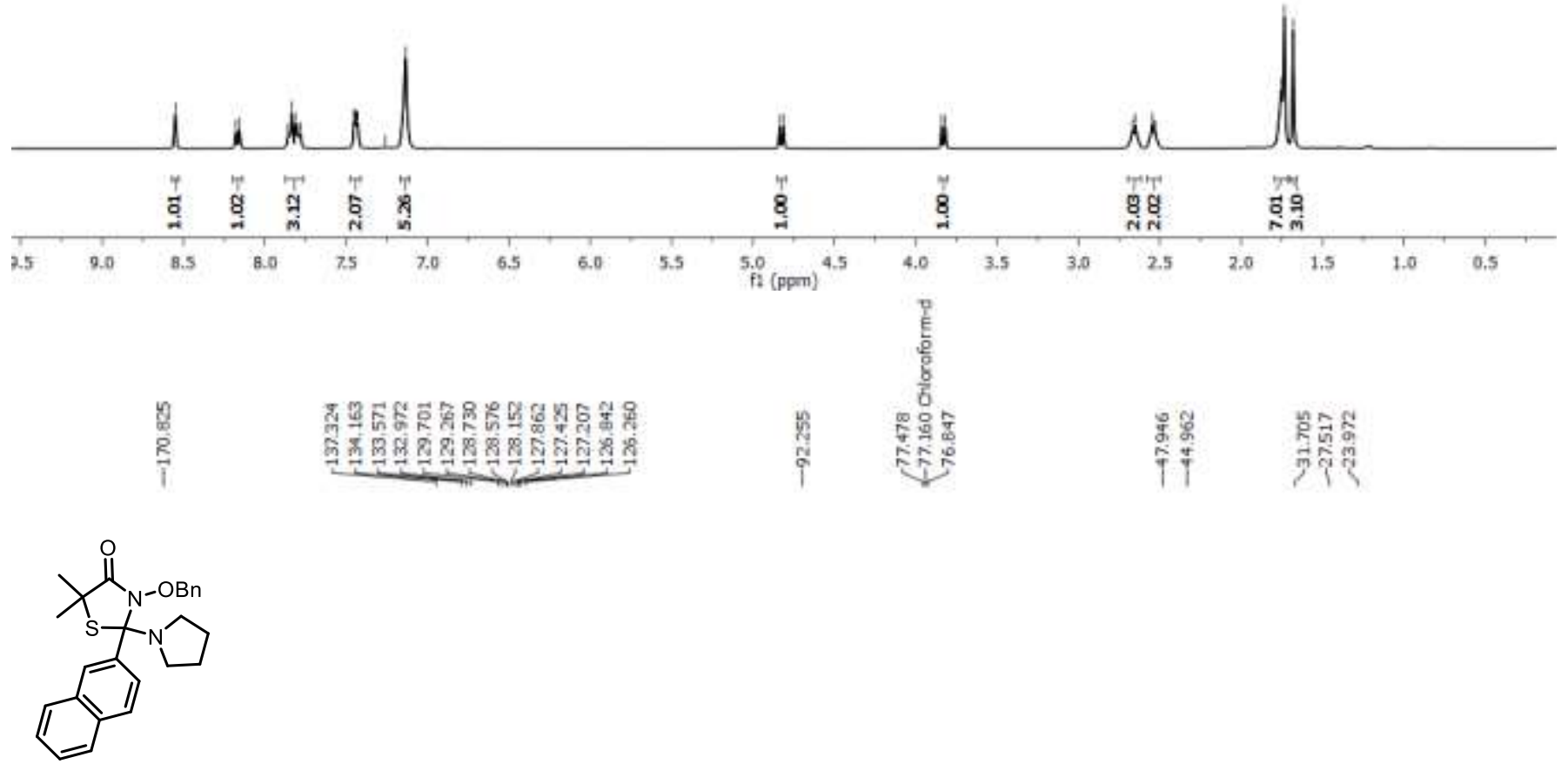

10 $\left(100 \mathrm{MHz}, \mathrm{CDCl}_{3}\right)$
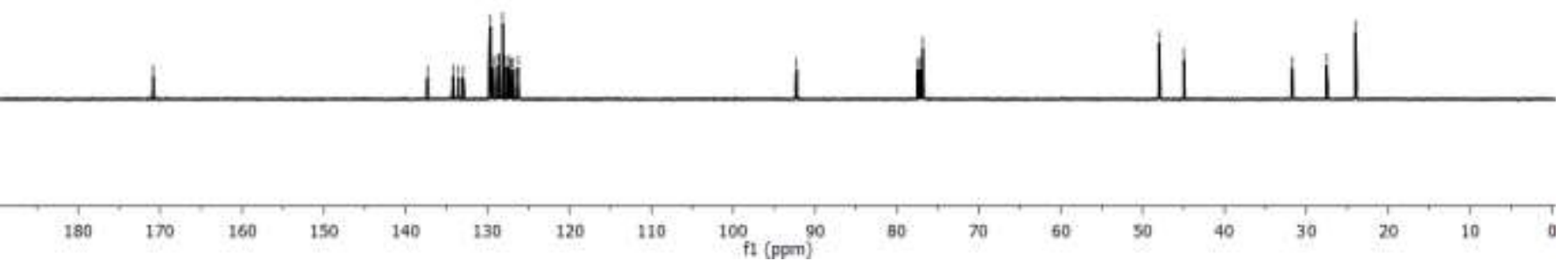
है

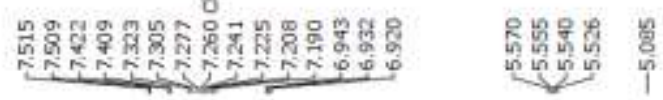

$\mathrm{S}_{\mathrm{N}}^{\mathrm{O}-\mathrm{OBn}}$

1p-HFIP $\left(400 \mathrm{MHz}, \mathrm{CDCl}_{3}\right)$

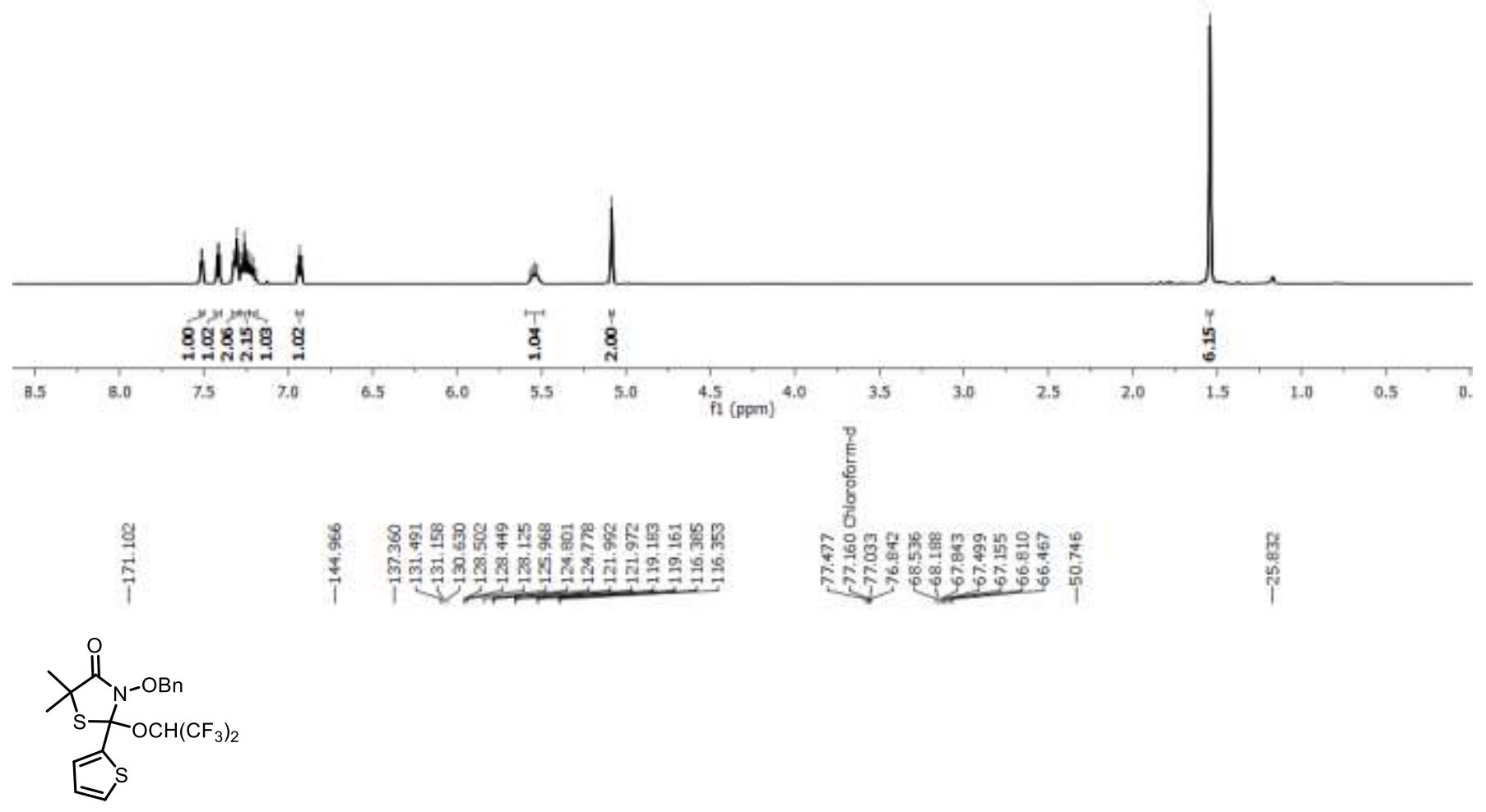

1p-HFIP (100 MHz, $\left.\mathrm{CDCl}_{3}\right)$
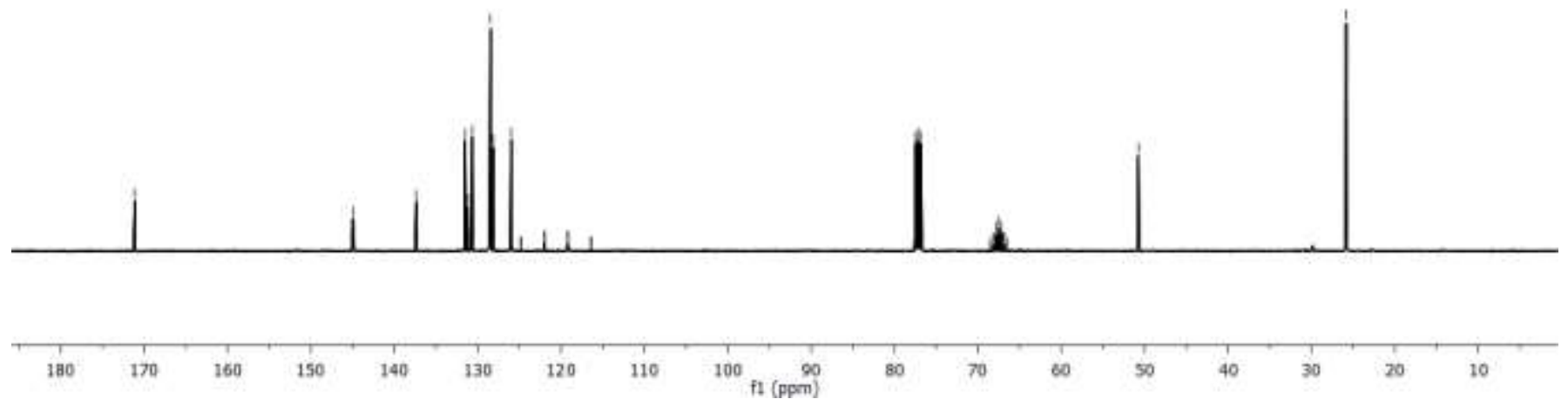
$\overbrace{\mathrm{N}}^{\mathrm{N}-\mathrm{OBn}}$

1q-HFIP (400 MHz, $\mathrm{CDCl}_{3}$ )

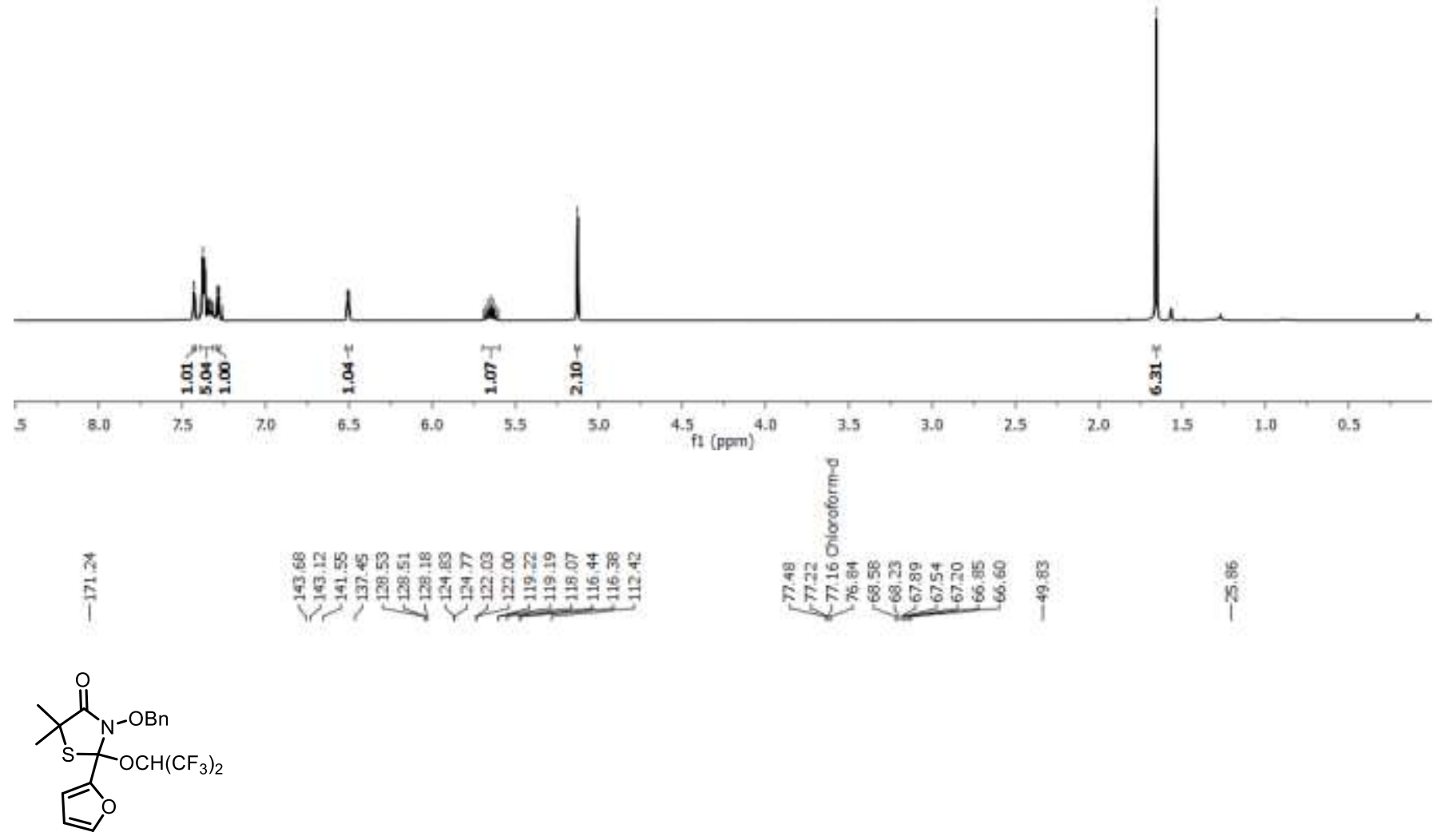

1q-HFIP (100 MHz, $\mathrm{CDCl}_{3}$ )
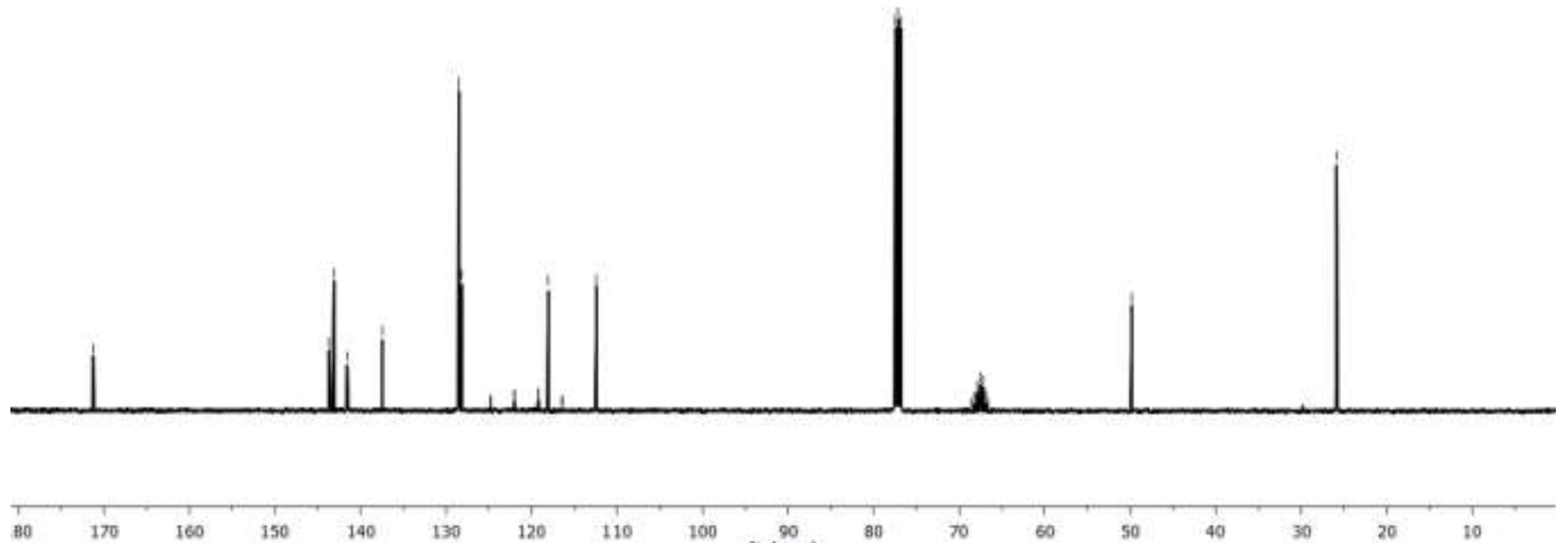

$110 \quad 100 \quad$ f1 90

80 


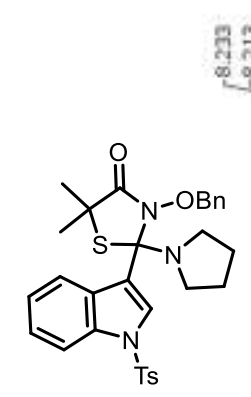

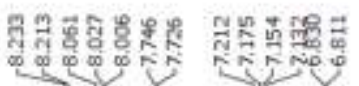

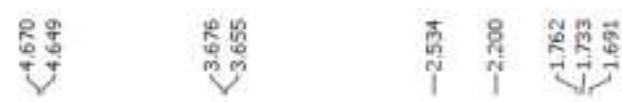

1r $\left(400 \mathrm{MHz}, \mathrm{CDCl}_{3}\right)$
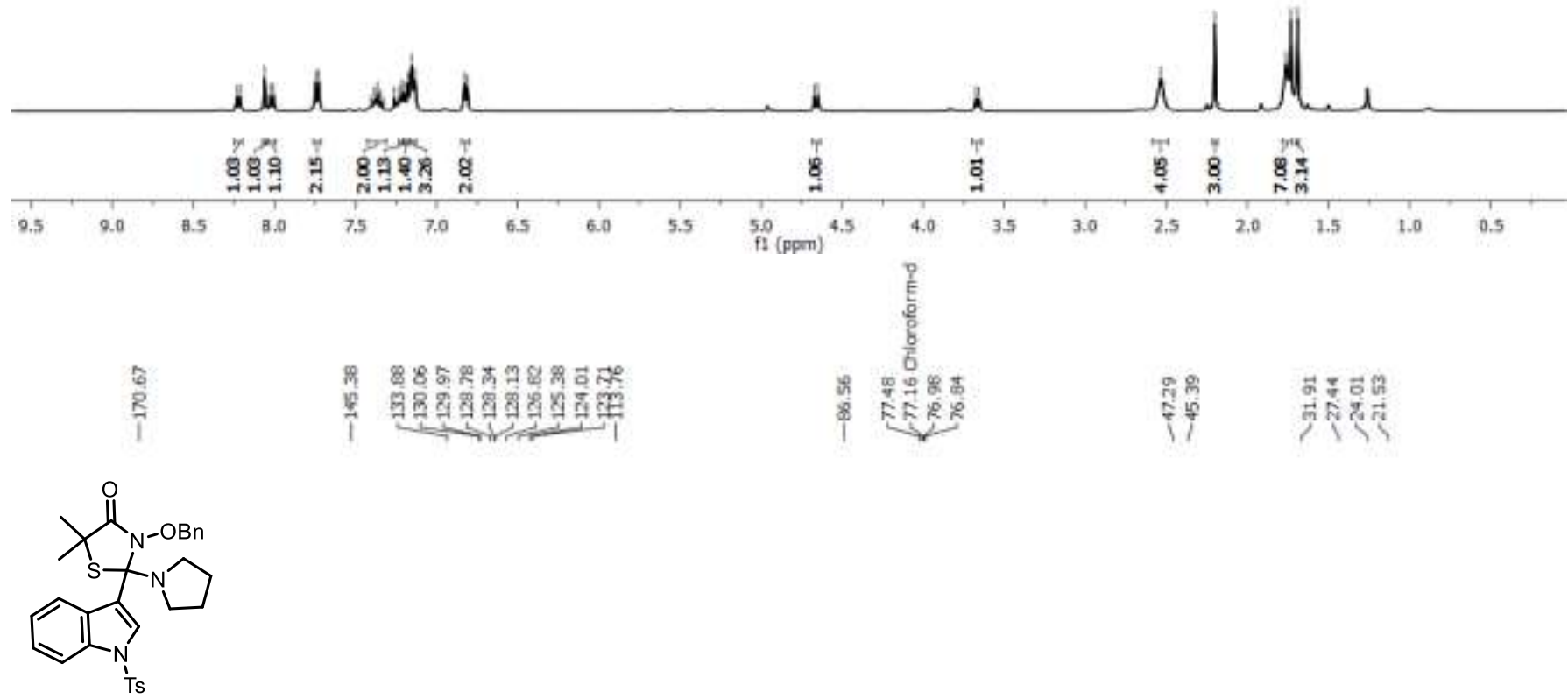

1r $\left(100 \mathrm{MHz}, \mathrm{CDCl}_{3}\right)$
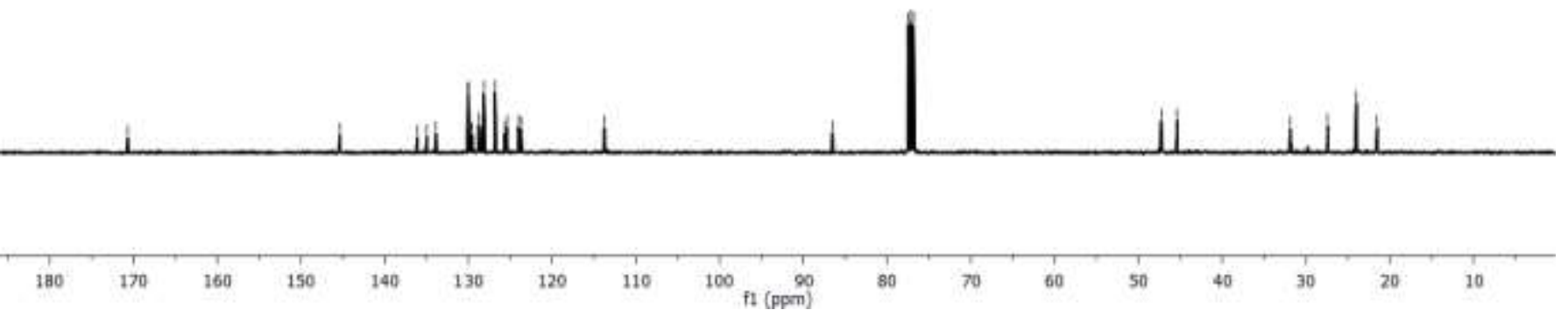


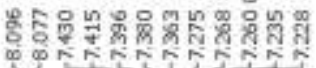
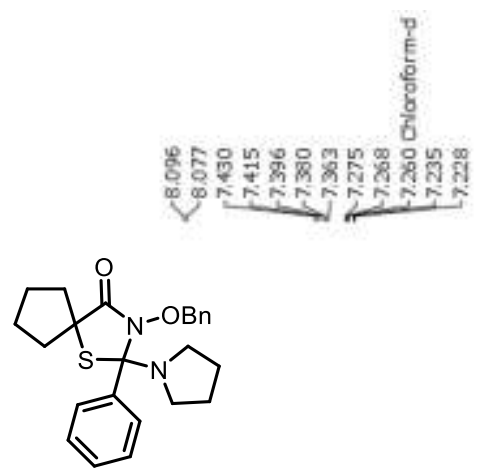

1a' (400 MHz, $\left.\mathrm{CDCl}_{3}\right)$
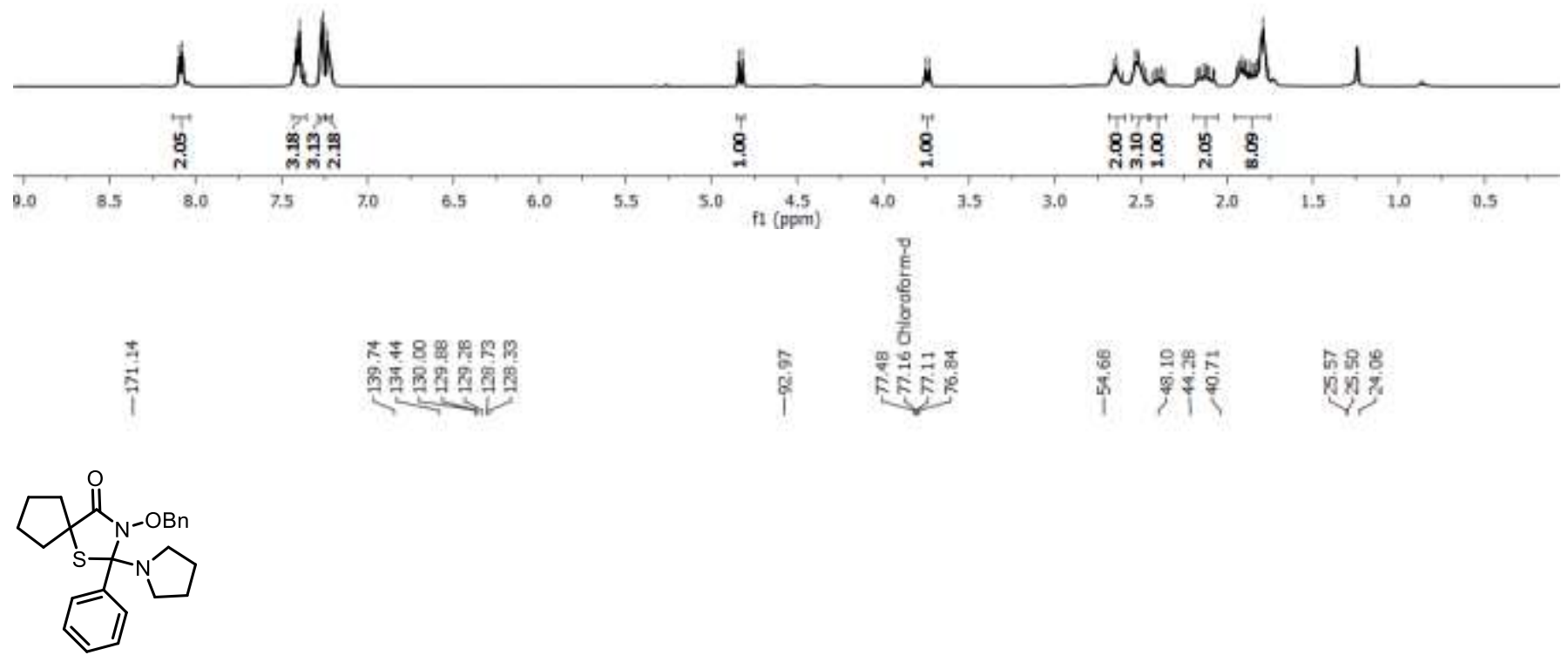

1a' (100 MHz, $\mathrm{CDCl}_{3}$ )
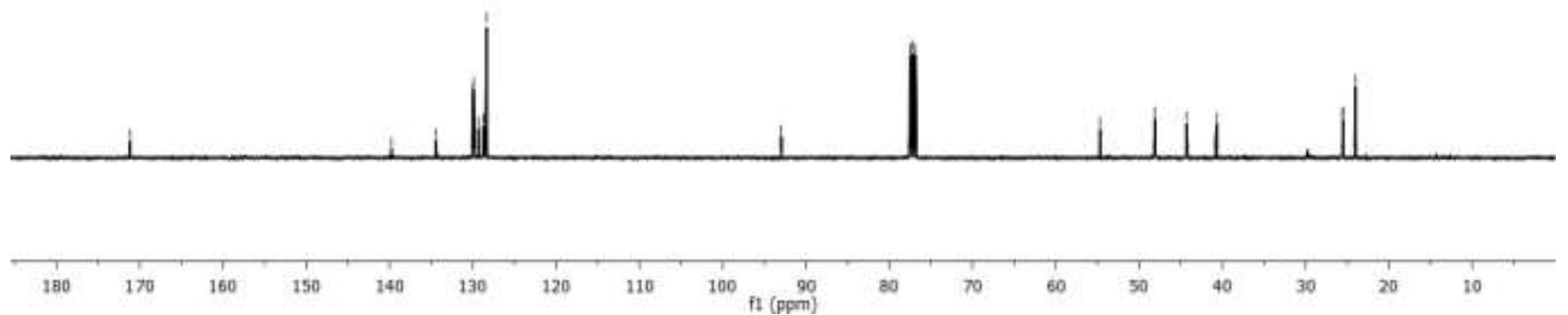

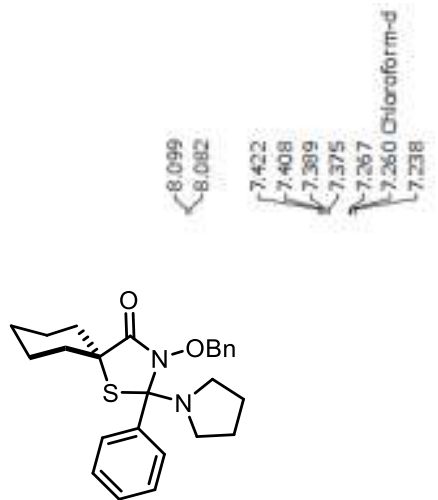

1b' $\left(400 \mathrm{MHz}, \mathrm{CDCl}_{3}\right)$
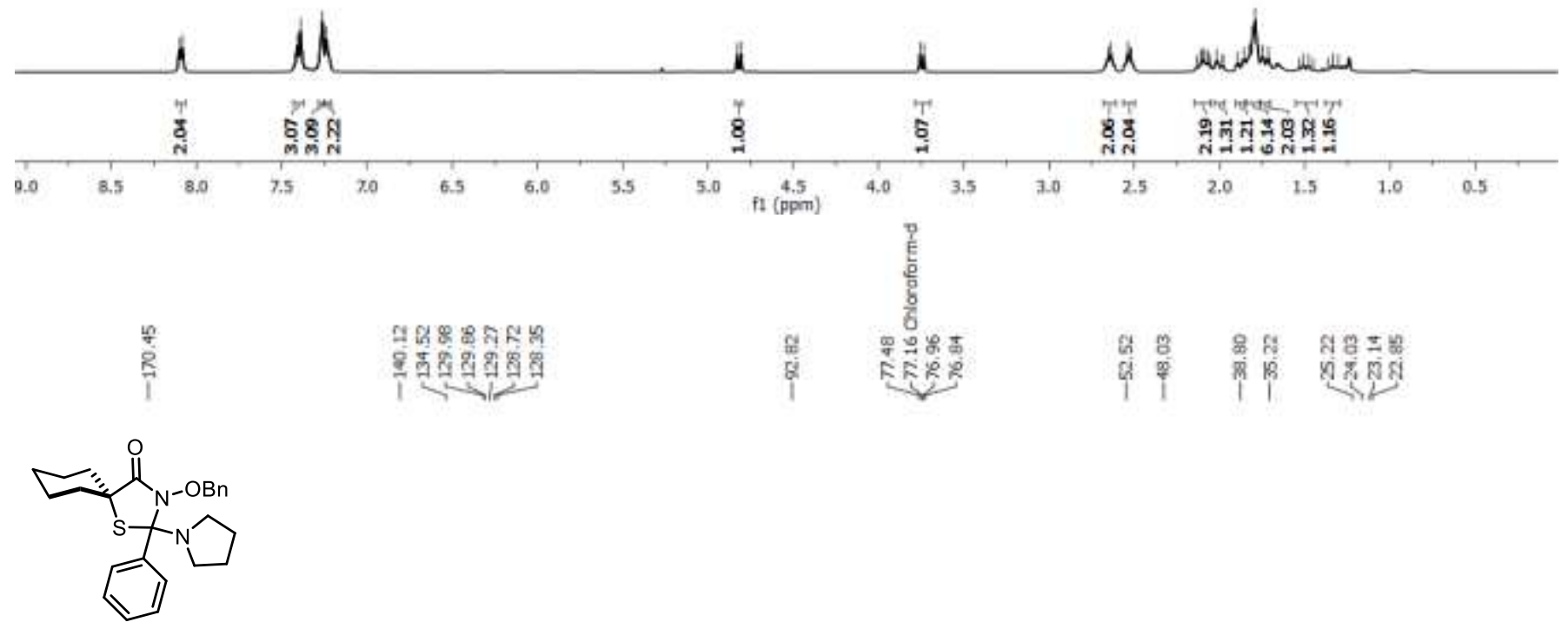

1b' (100 $\mathrm{MHz}, \mathrm{CDCl}_{3}$ )

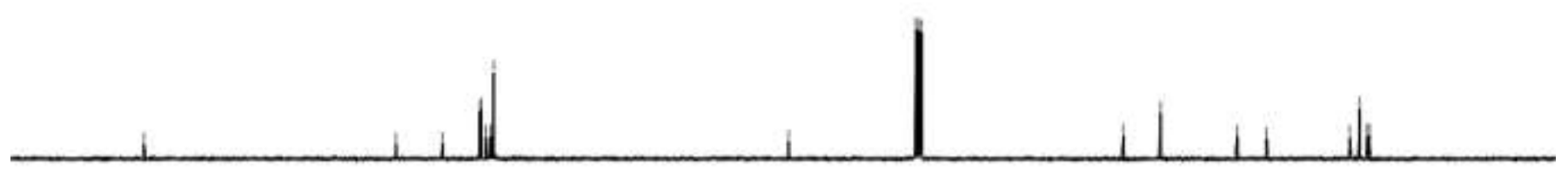

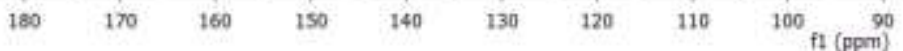



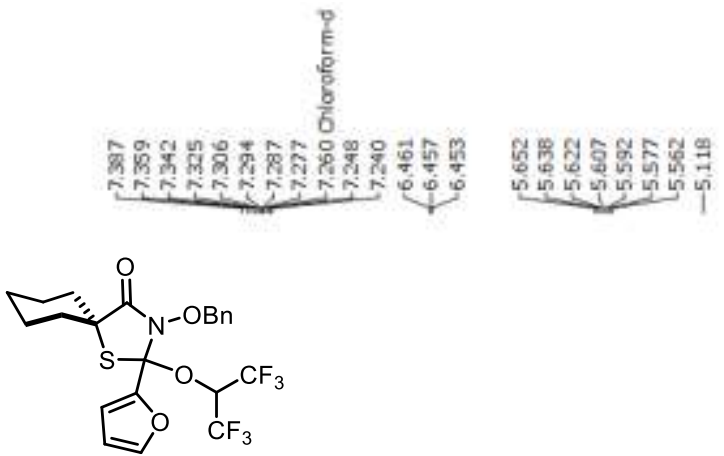

1c'-(HFIP) (400 MHz, $\left.\mathrm{CDCl}_{3}\right)$
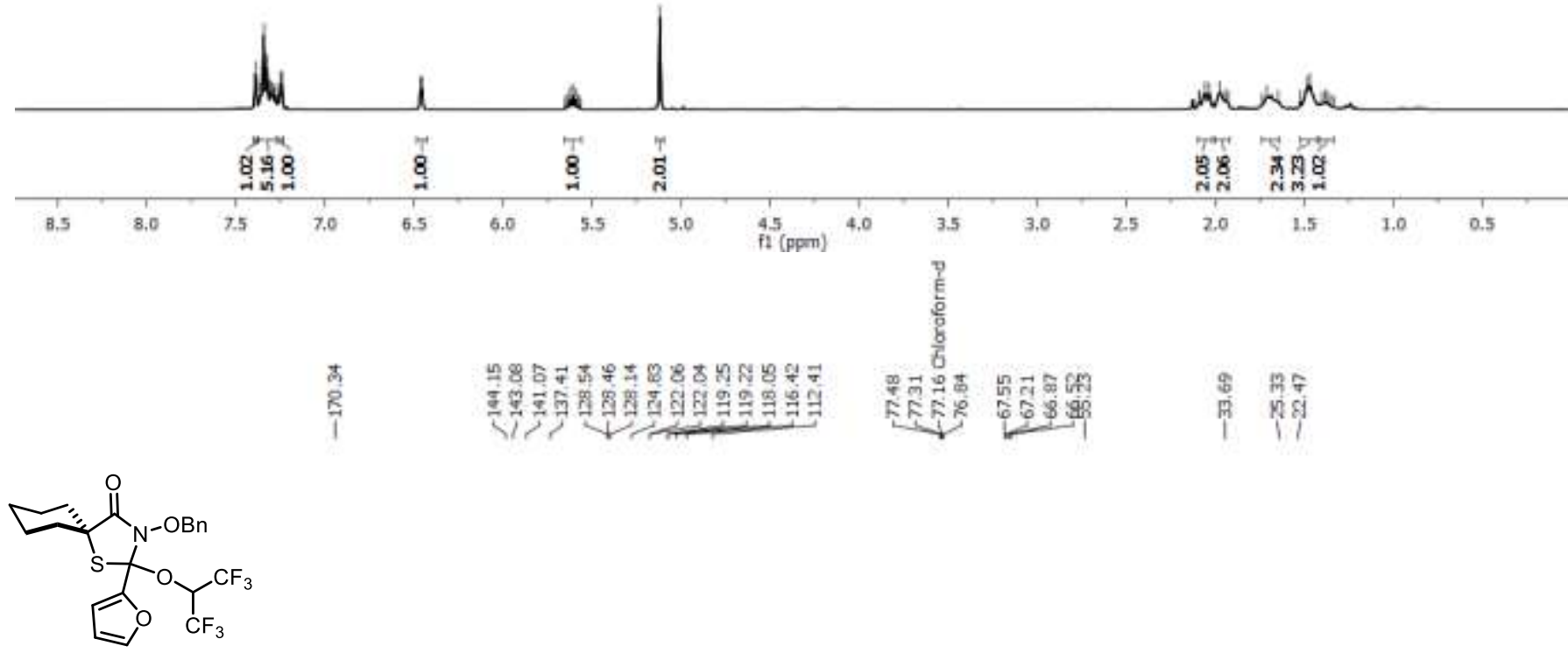

1c'-(HFIP) (100 MHz, $\left.\mathrm{CDCl}_{3}\right)$

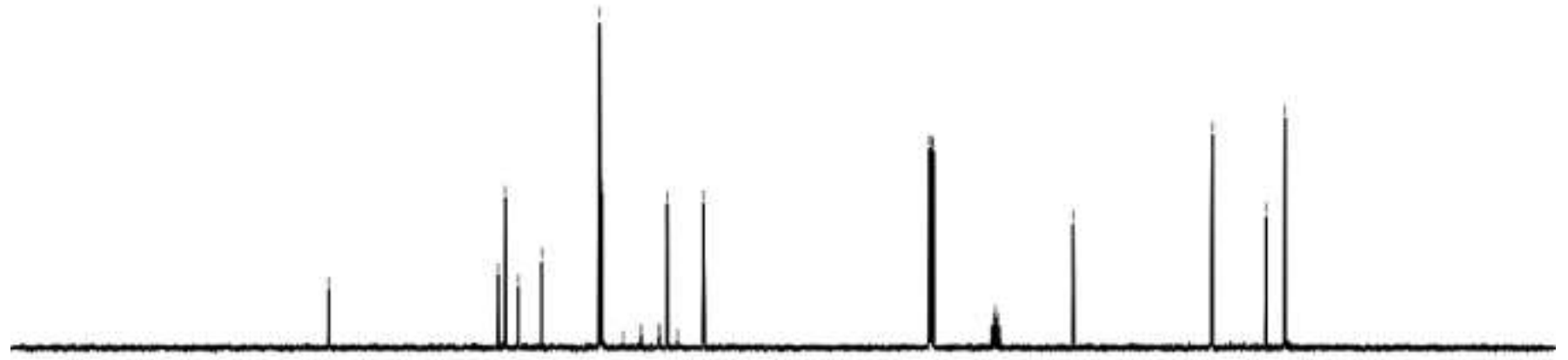

$\begin{array}{llllllllllllllllllllllllll}210 & 200 & 190 & 180 & 170 & 160 & 150 & 140 & 130 & 120 & 110 & 100 & 90 & 80 & 70 & 60 & 50 & 40 & 30 & 20 & 10 & 0 & -10 & 100\end{array}$ 


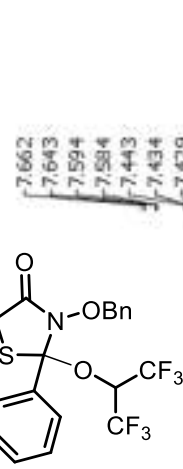

1d'-(HFIP) (400 MHz, $\mathrm{CDCl}_{3}$ )

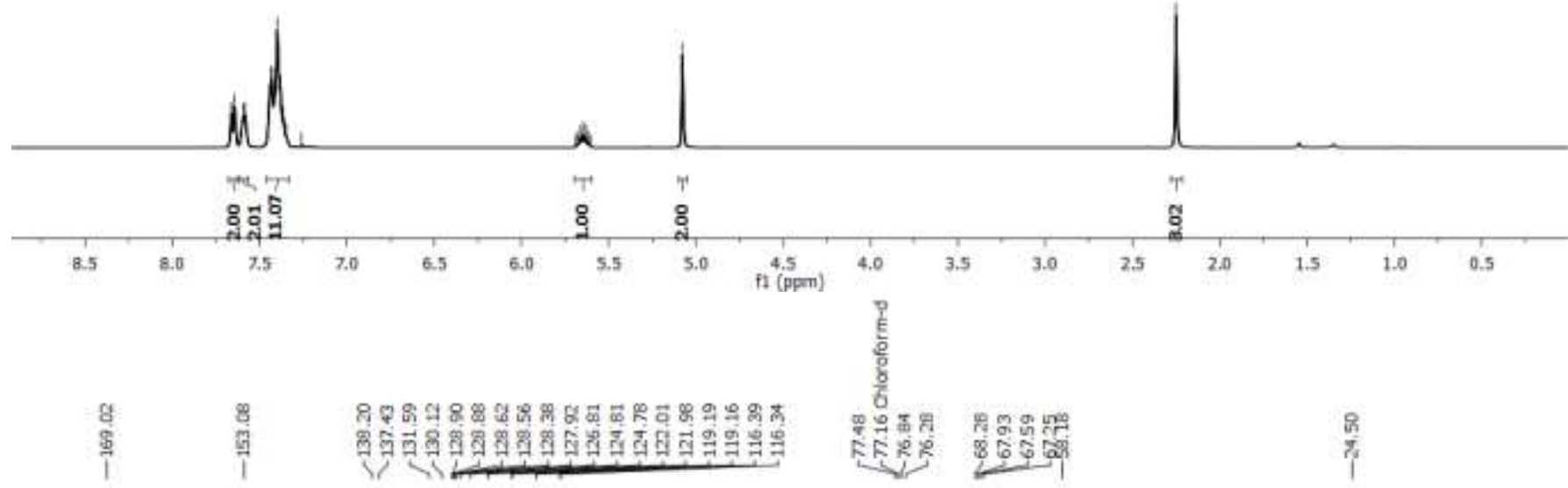<smiles>CC1(c2ccccc2)SC(OC(C(F)(F)F)C(F)(F)F)(c2ccccc2)N(Cc2ccccc2)C1=O</smiles>

1d'-(HFIP) (100 MHz, $\left.\mathrm{CDCl}_{3}\right)$
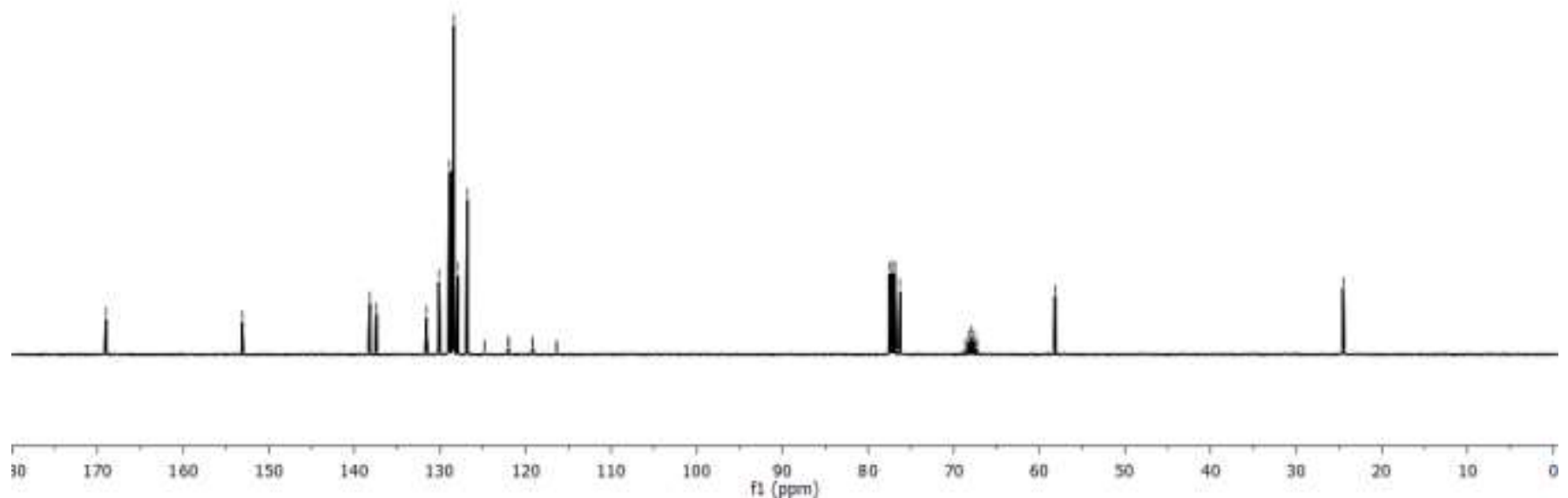


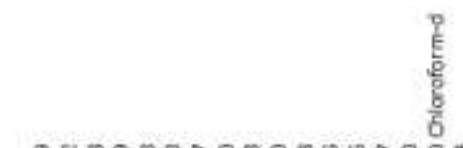

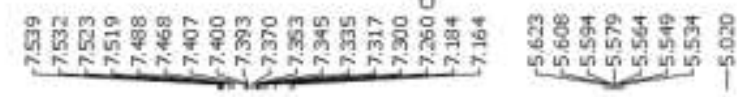

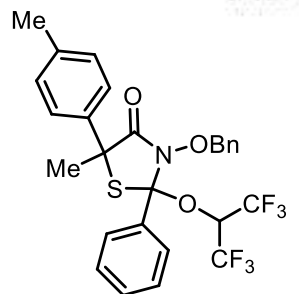

1e'-(HFIP) (400 MHz, $\left.\mathrm{CDCl}_{3}\right)$
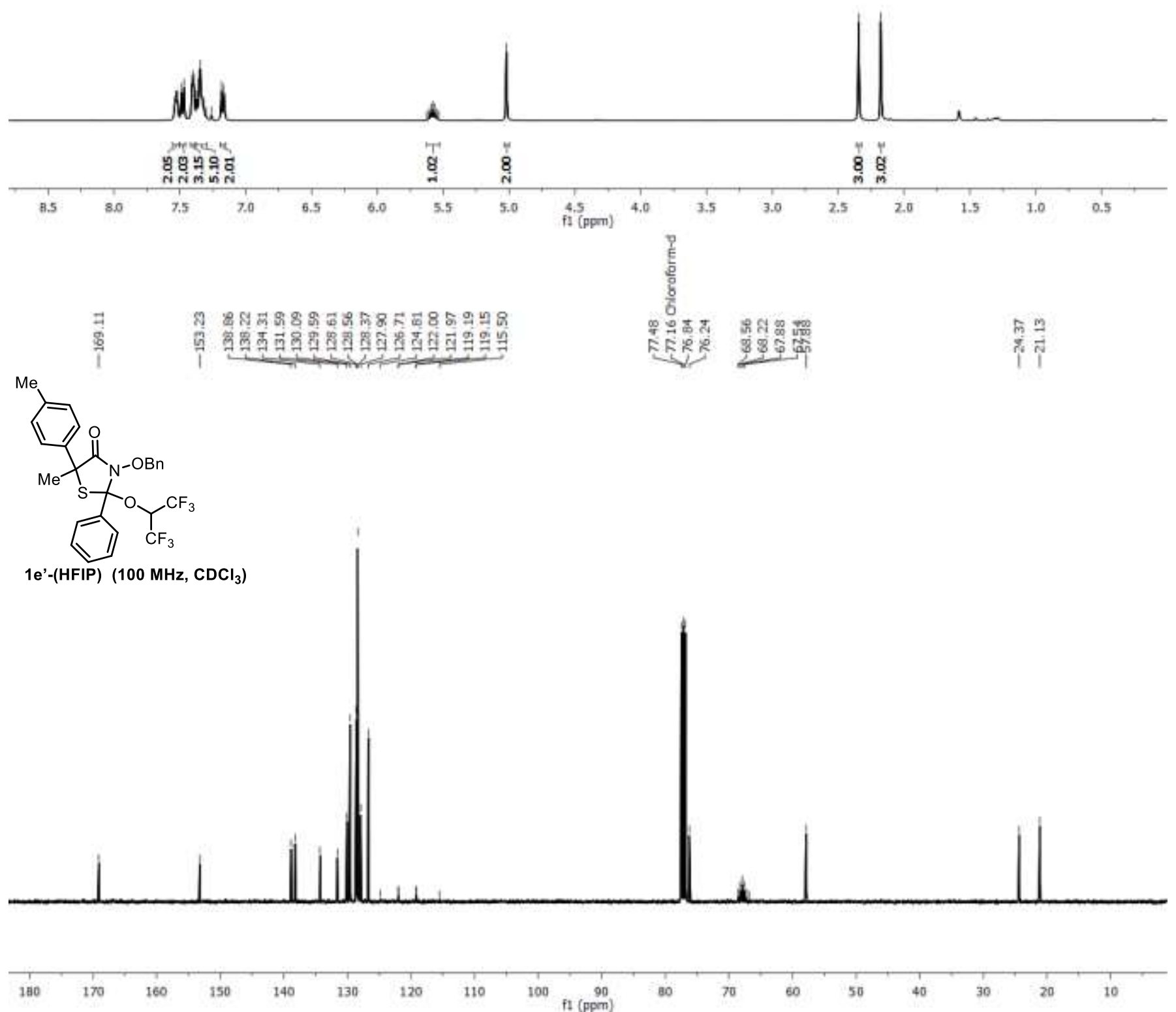


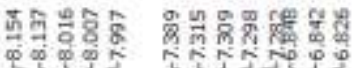

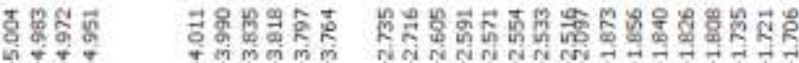

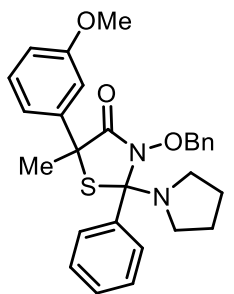

1f' (400 $\left.\mathrm{MHz}, \mathrm{CDCl}_{3}\right)$

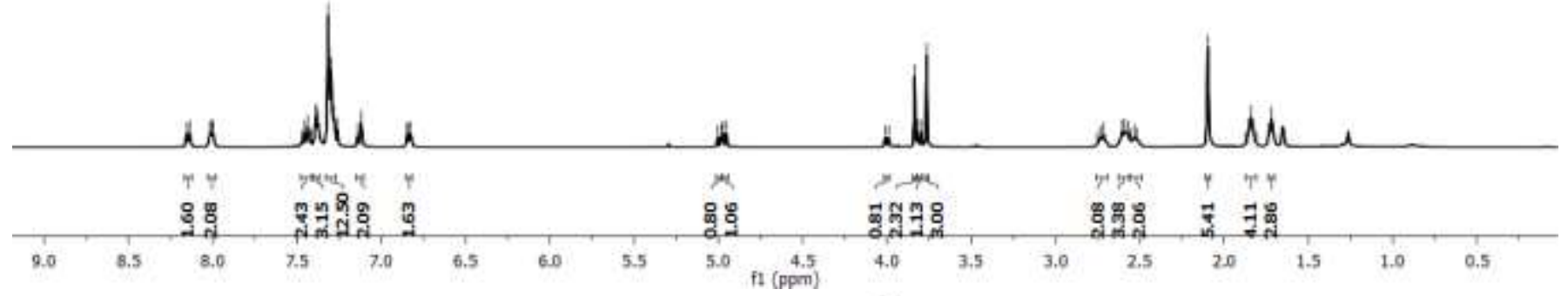

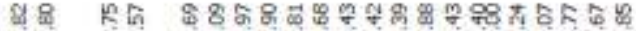

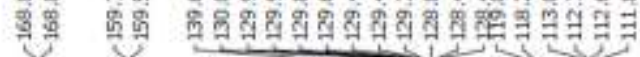

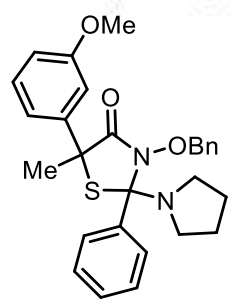

1f' $\left(100 \mathrm{MHz}, \mathrm{CDCl}_{3}\right)$

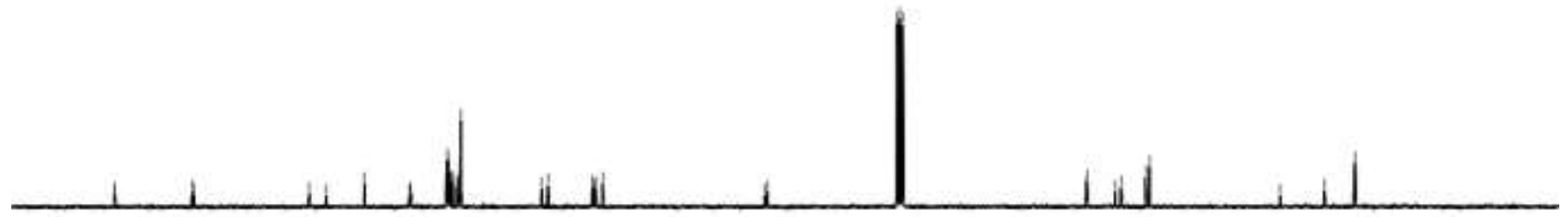

Bo
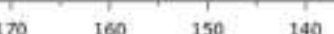

\section{S马}

gig हिए

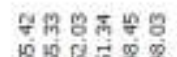

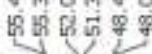

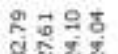

भिर्ये 


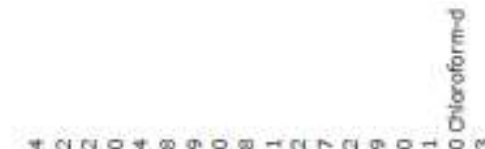

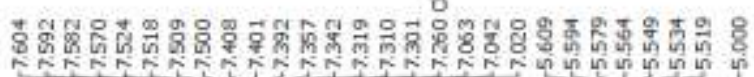

욜

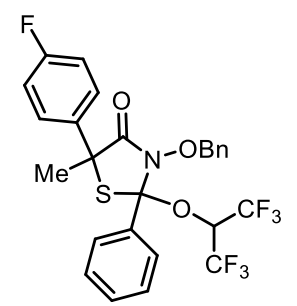

1g'-(HFIP) $\left(400 \mathrm{MHz}, \mathrm{CDCl}_{3}\right)$
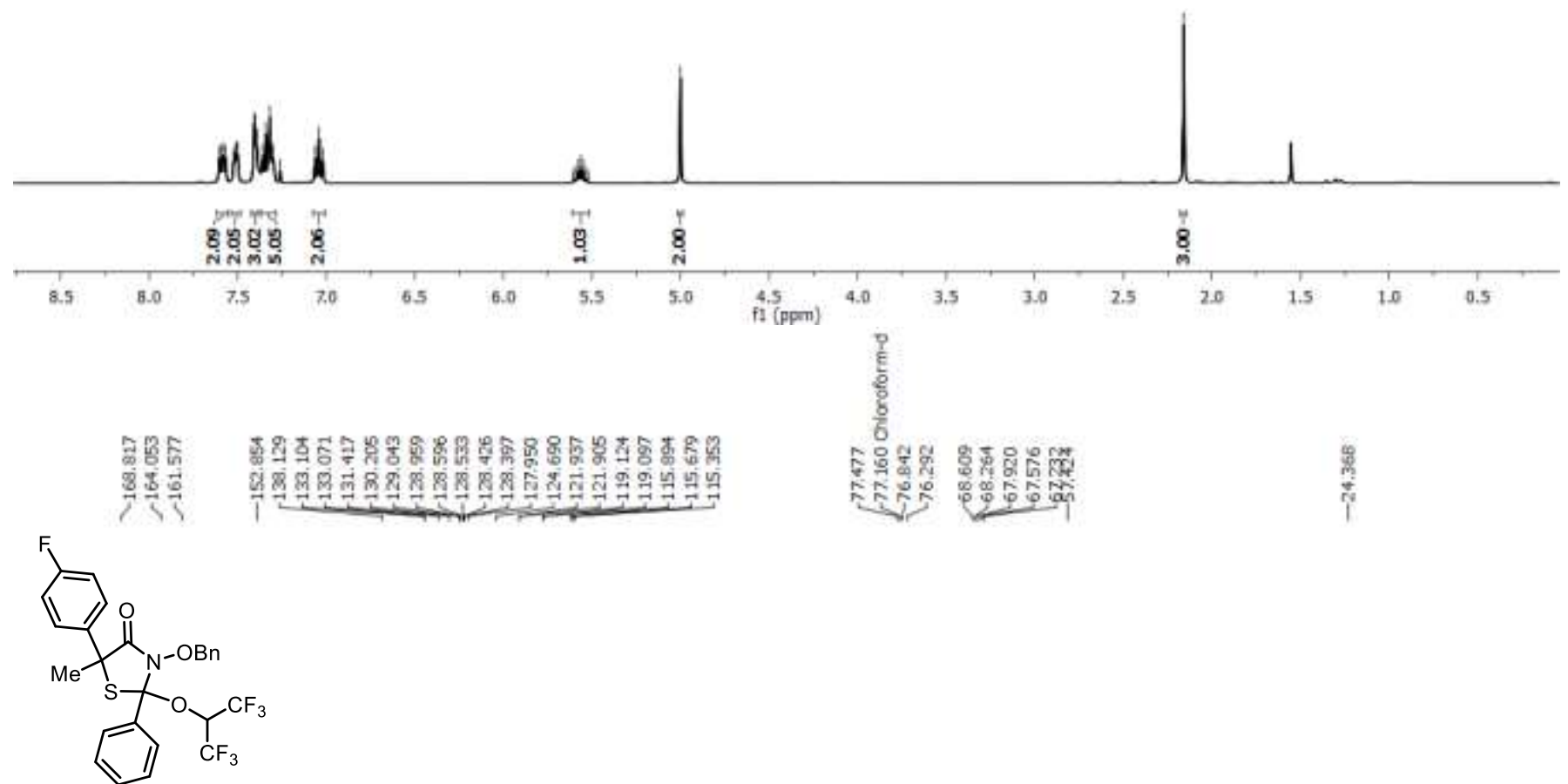

19'-(HFIP) (100 MHz, $\left.\mathrm{CDCl}_{3}\right)$
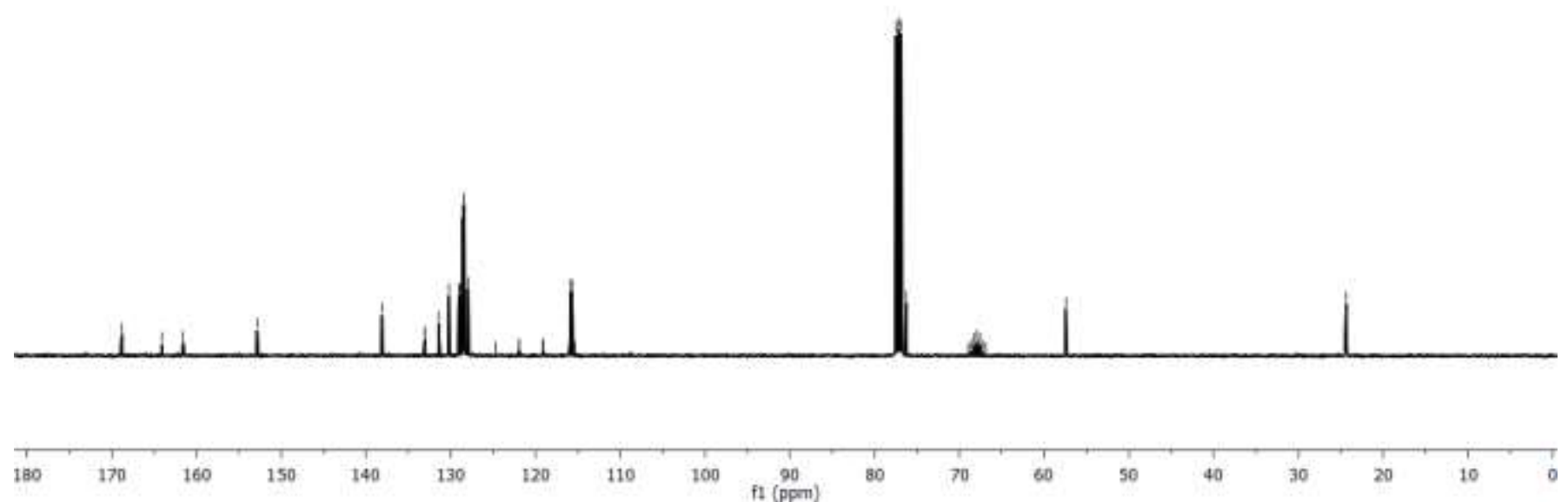


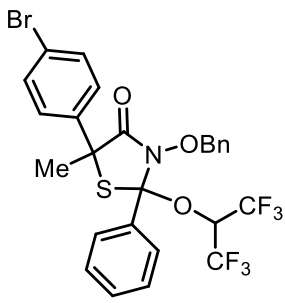

1h'-(HFIP) (400 MHz, $\left.\mathrm{CDCl}_{3}\right)$
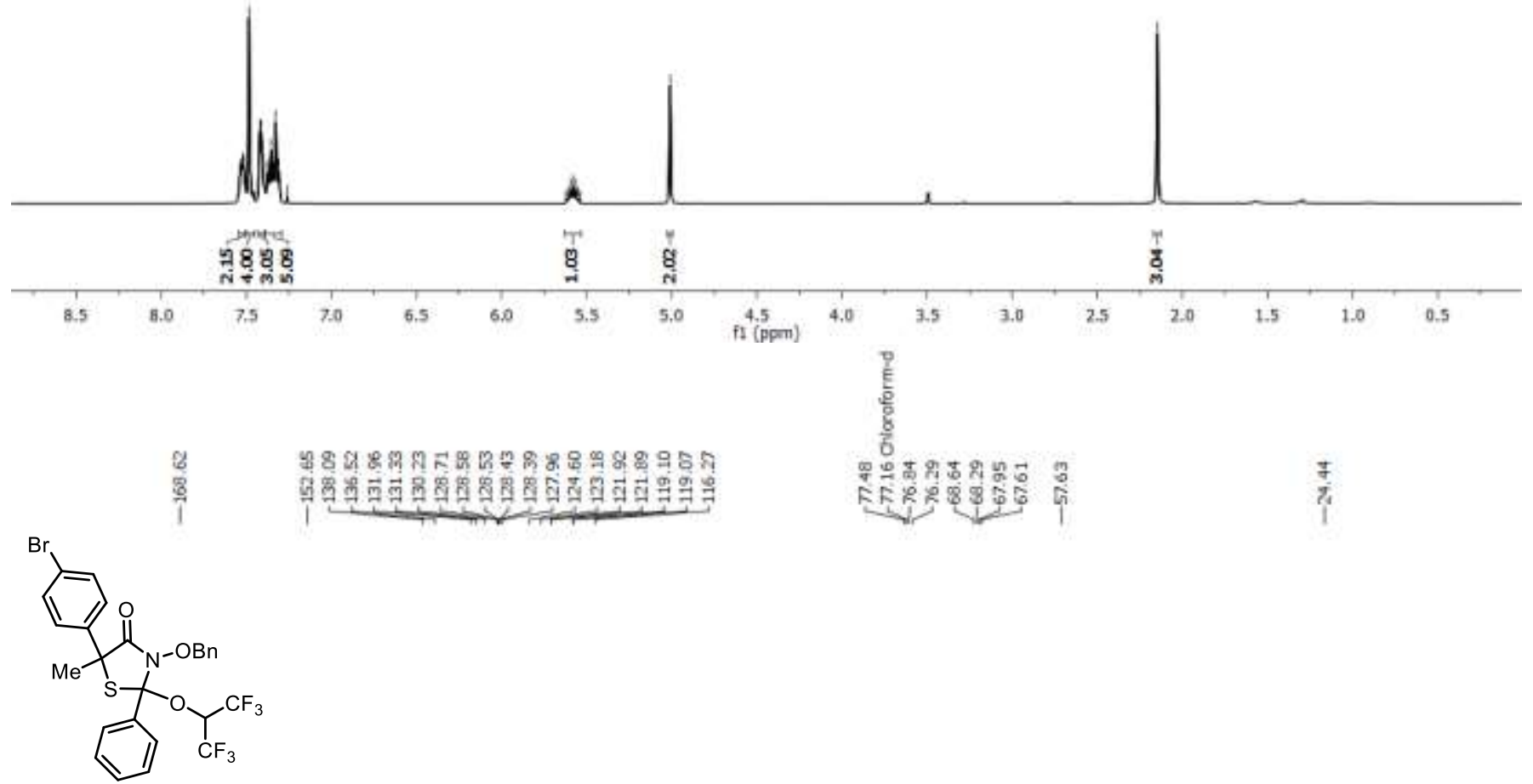

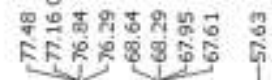

$\frac{7}{7}$

1h'-(HFIP) (100 MHz, $\left.\mathrm{CDCl}_{3}\right)$

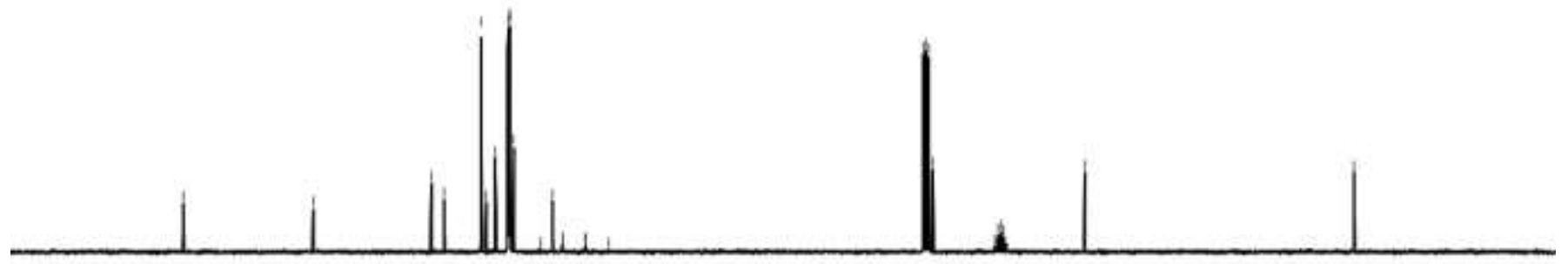

$\begin{array}{lllllllll}180 & 170 & 160 & 150 & 140 & 130 & 120 & 110 & 100 \\ \mathrm{f1} & (\mathrm{pps} n)^{90}\end{array}$ 


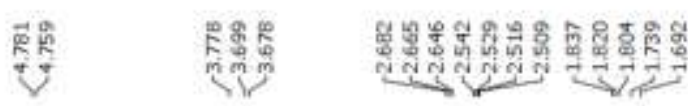

1a" $\left(400 \mathrm{MHz}, \mathrm{CDCl}_{3}\right)$
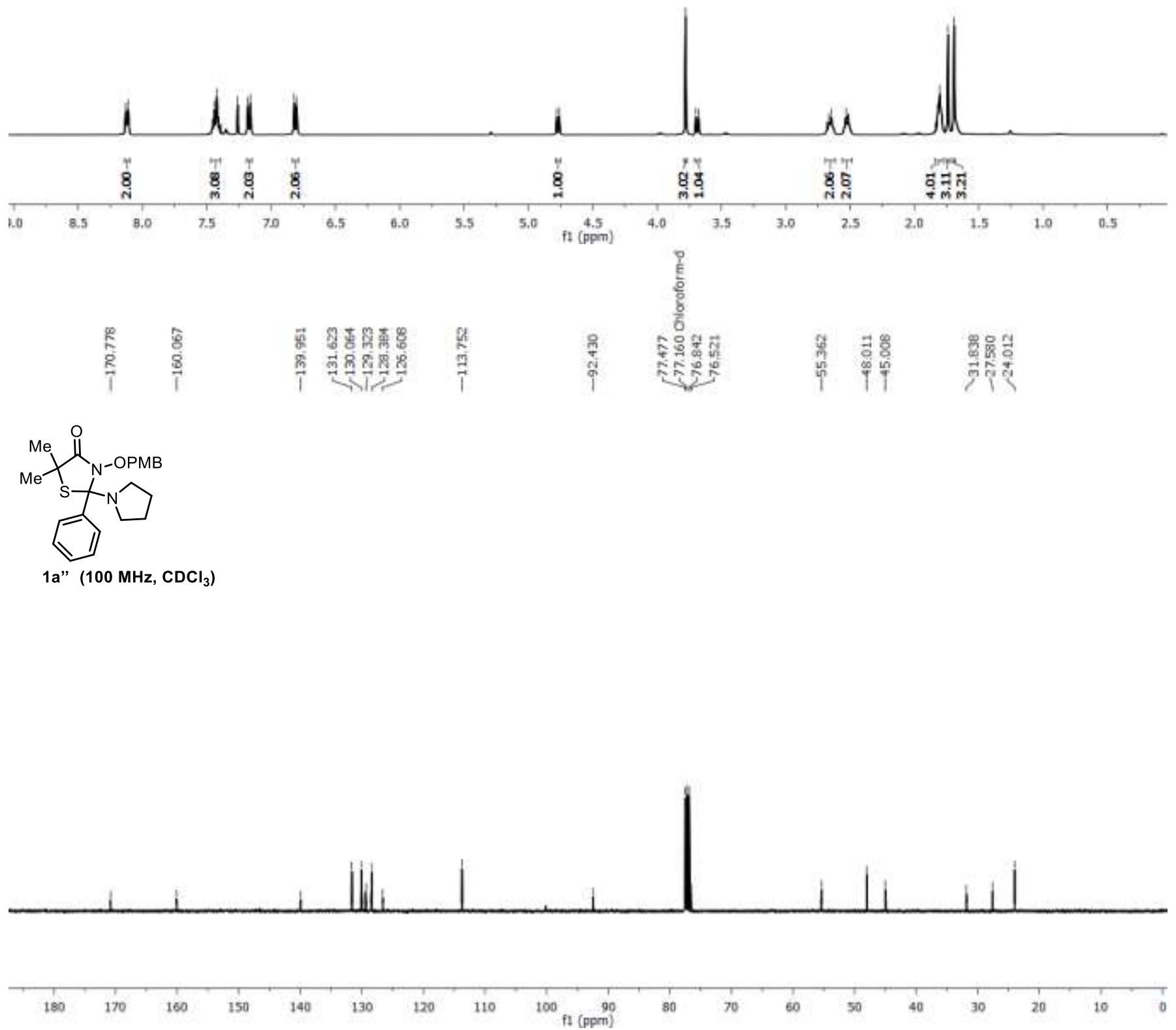
해용

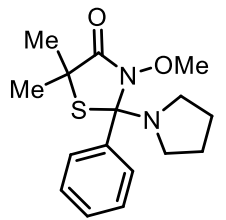

1b" (400 MHz, $\mathrm{CDCl}_{3}$ )
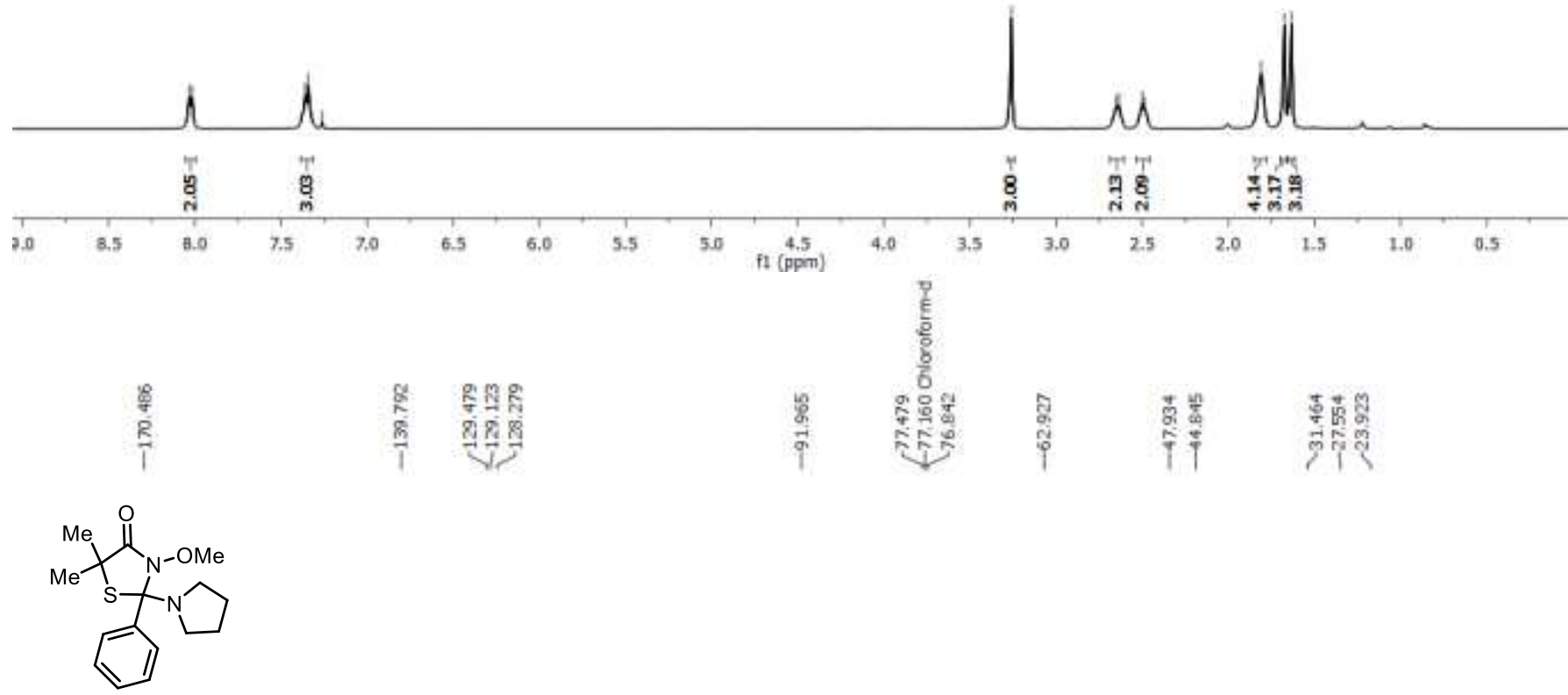

1b" (100 MHz, $\mathrm{CDCl}_{3}$ )
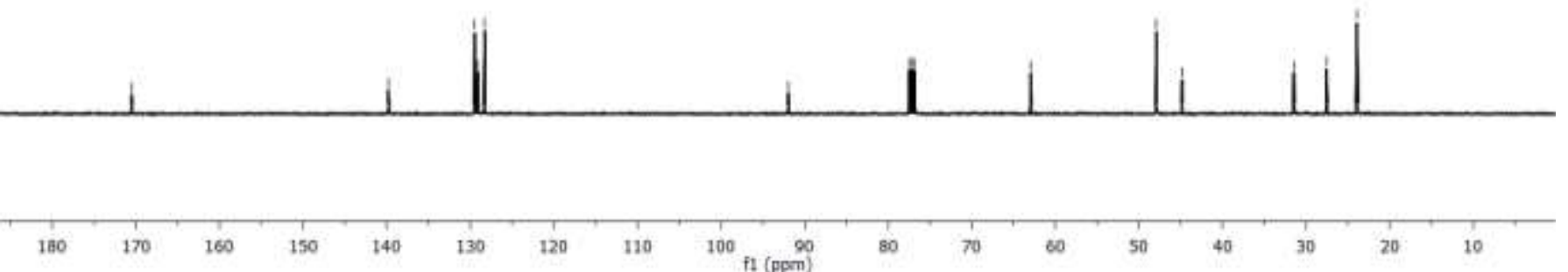

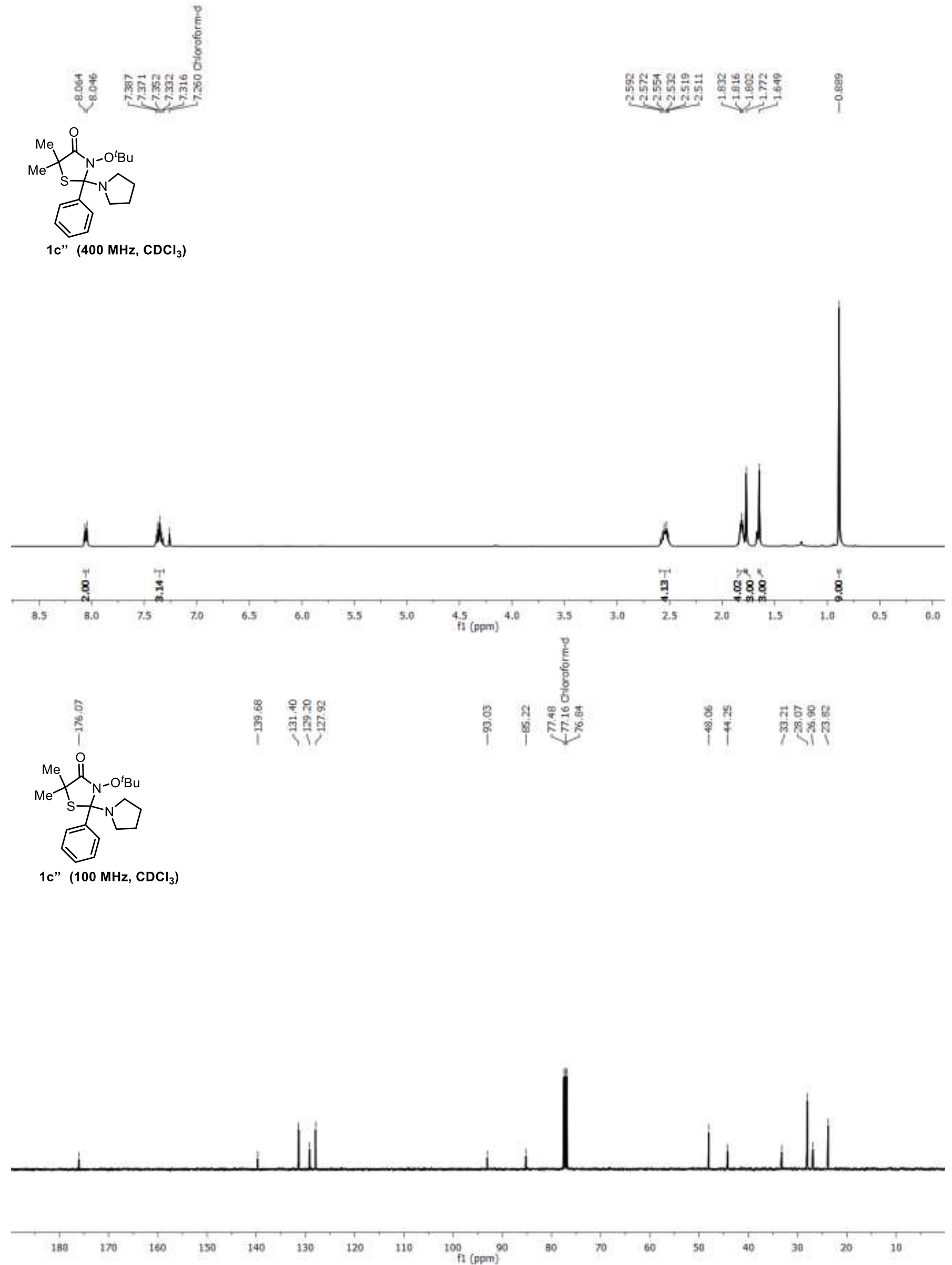


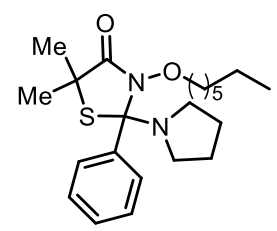

1d" (400 MHz, $\mathrm{CDCl}_{3}$ )
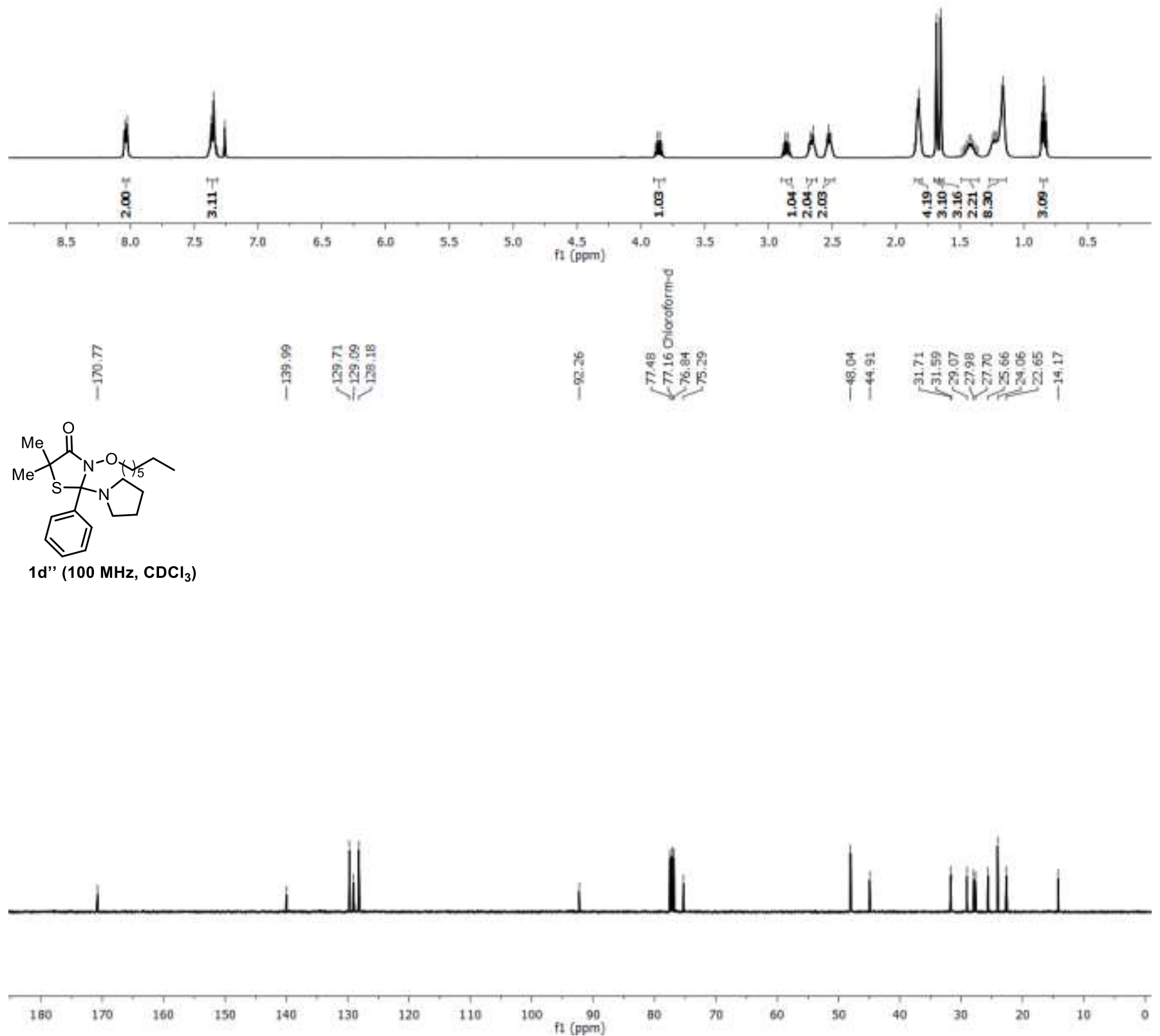

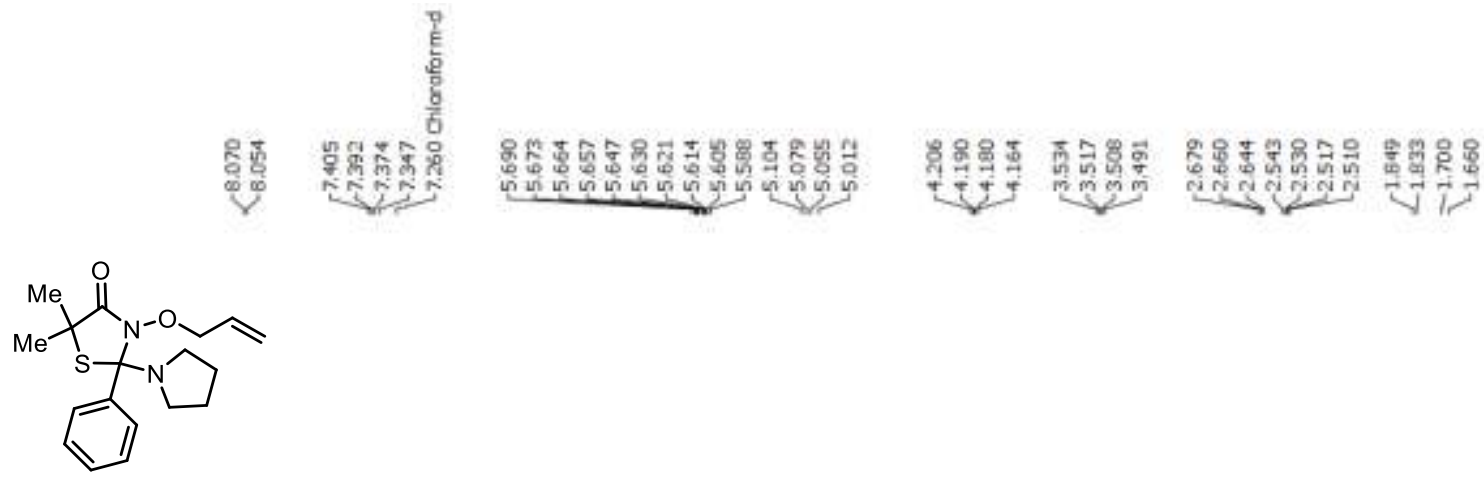

1e" (400 MHz, $\left.\mathrm{CDCl}_{3}\right)$
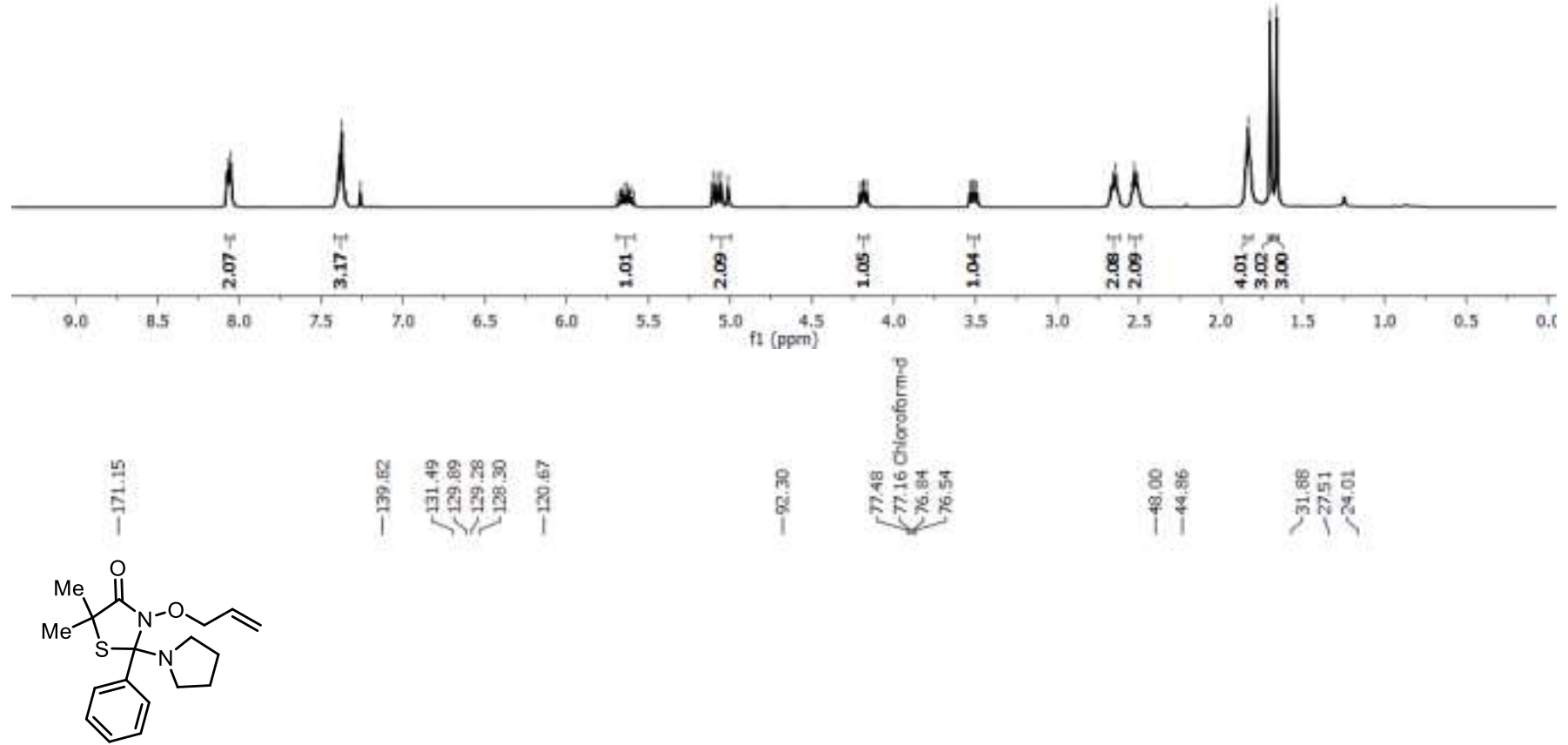

1e" (100 MHz, $\left.\mathrm{CDCl}_{3}\right)$
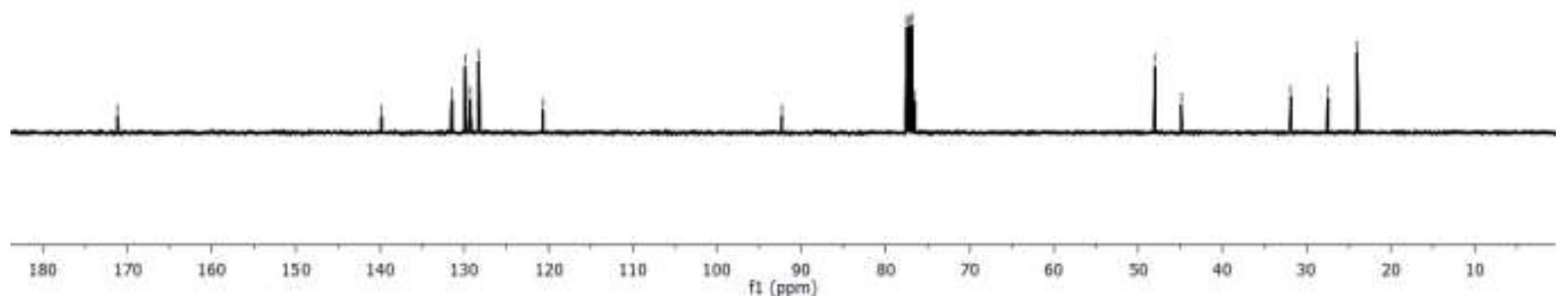


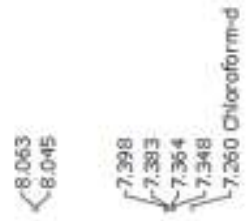

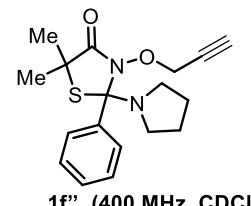

1f" $\left(400 \mathrm{MHz}, \mathrm{CDCl}_{3}\right)$
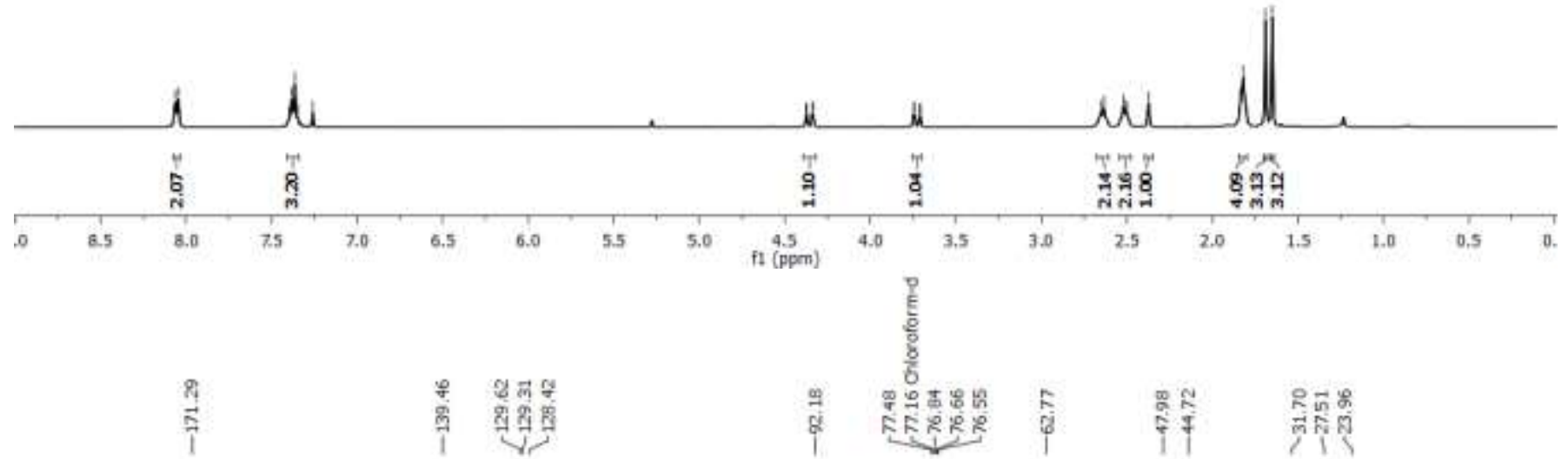

$\frac{1}{8}$

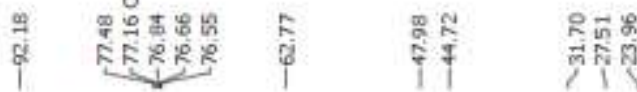

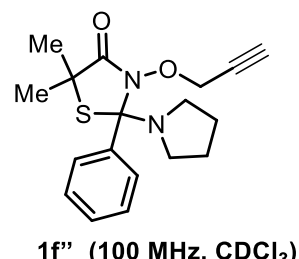

1f" $\left(100 \mathrm{MHz}, \mathrm{CDCl}_{3}\right)$
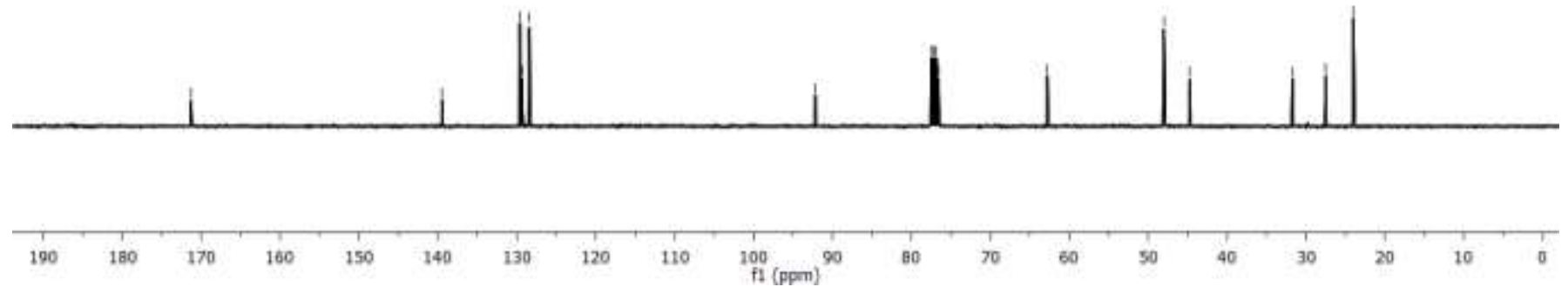


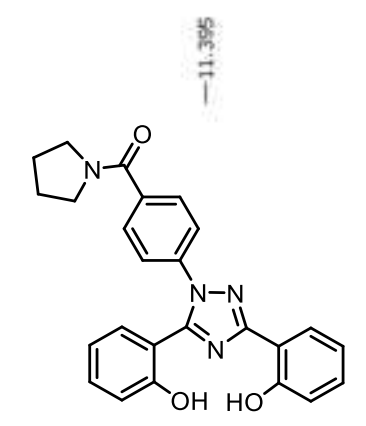

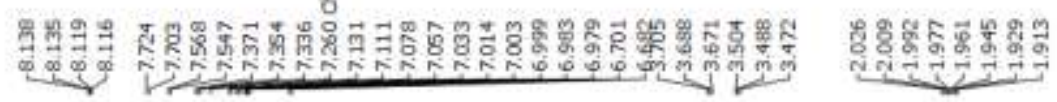

VJ-1 (400 MHz, $\left.\mathrm{CDCl}_{3}\right)$
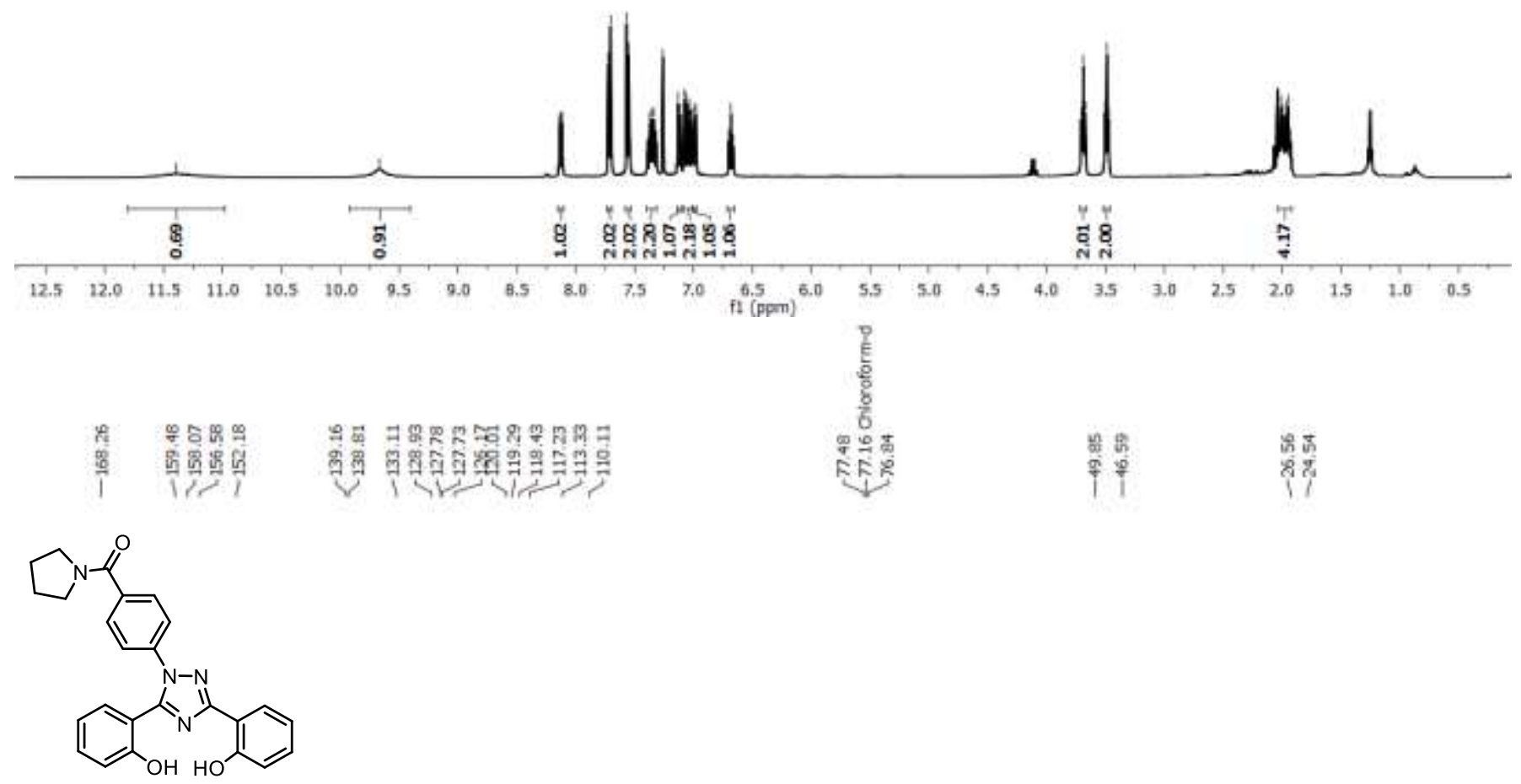

VJ-1 $\left(100 \mathrm{MHz}, \mathrm{CDCl}_{3}\right)$

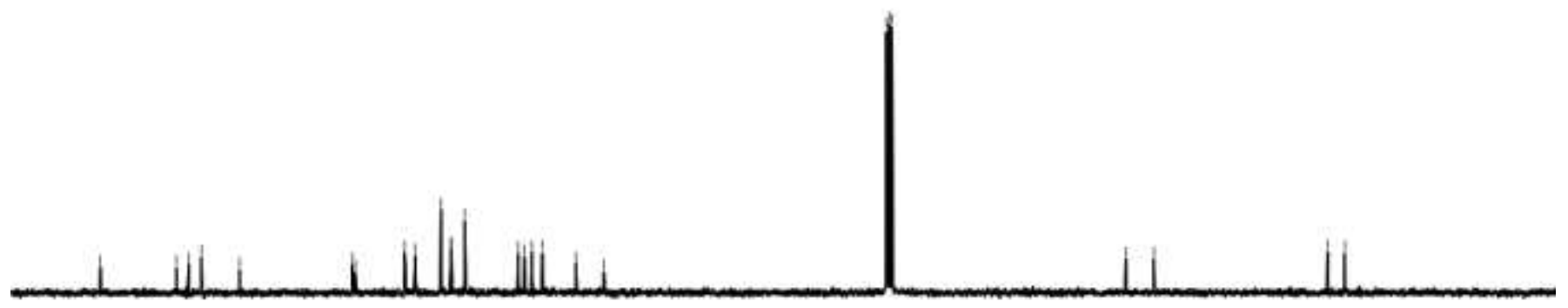

$\begin{array}{llllllllllllllll}170 & 160 & 150 & 140 & 130 & 120 & 110 & 100 & 90 & 80 & 70 & 60 & 50 & 40 & 30 & 20\end{array}$ 

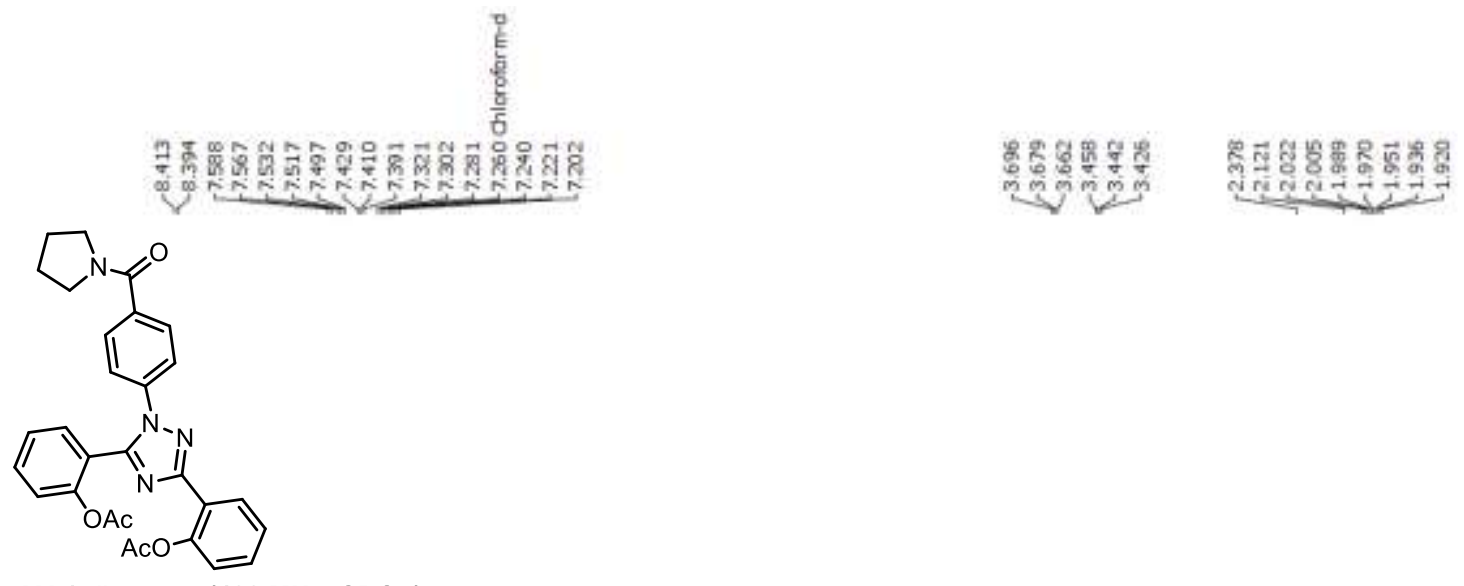

VJ-1-diacetate $\left(400 \mathrm{MHz}, \mathrm{CDCl}_{3}\right)$
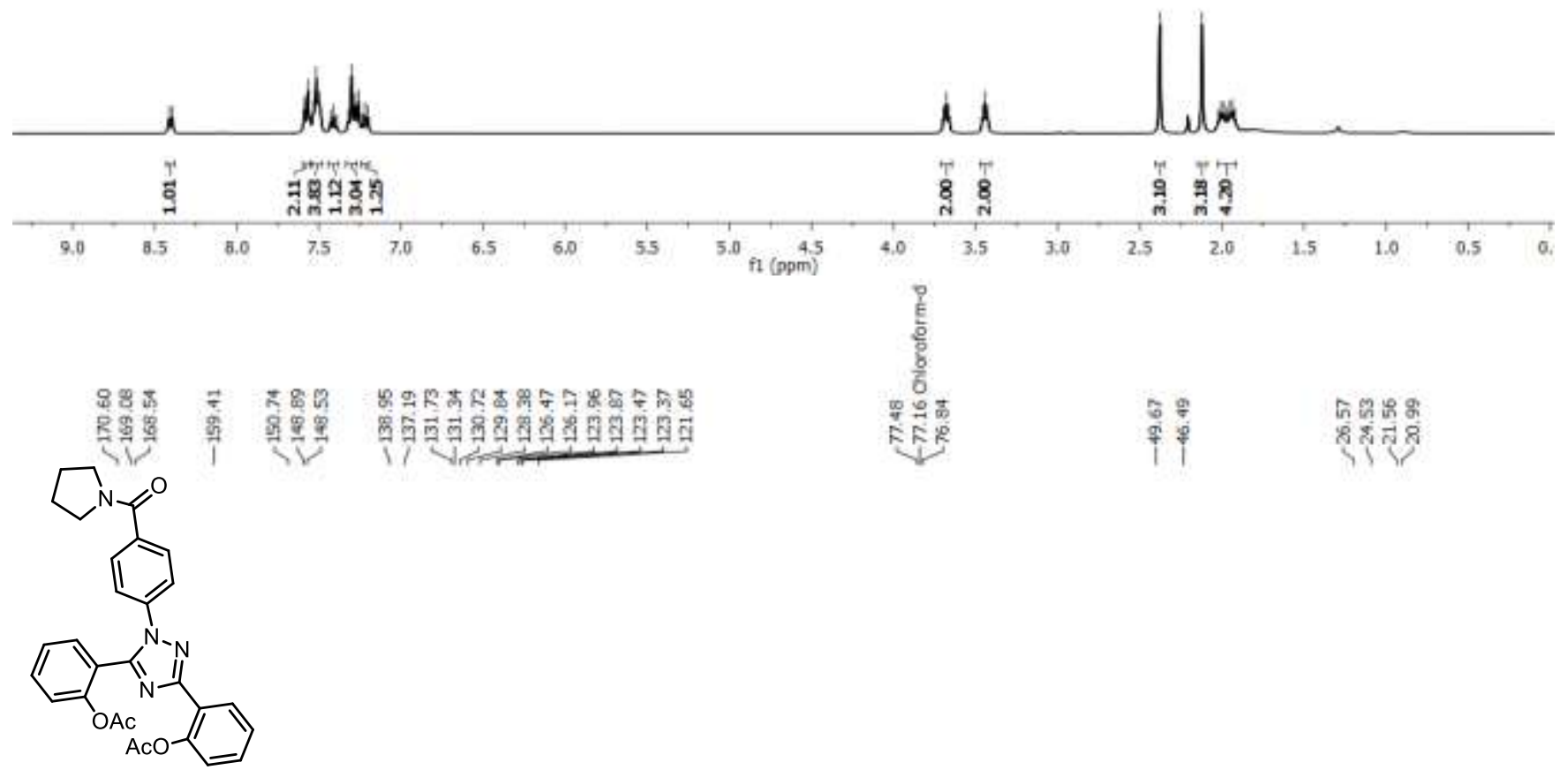

VJ-1-diacetate $\left(100 \mathrm{MHz} \mathrm{CDCl}_{3}\right)$
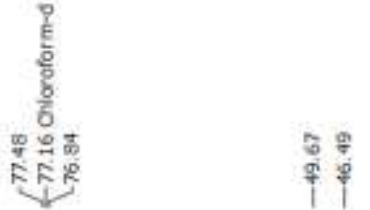

กิ

존죠

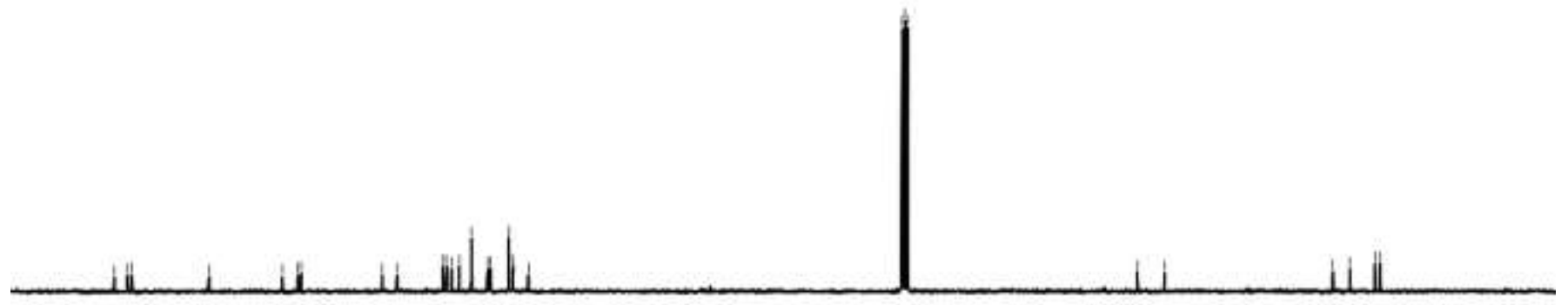




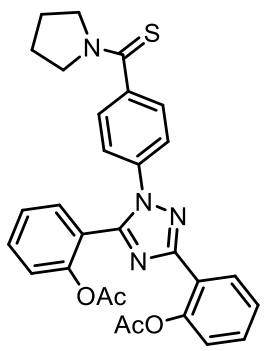

VJ-2 (400 $\mathrm{MHz}, \mathrm{CDCl}_{3}$ )
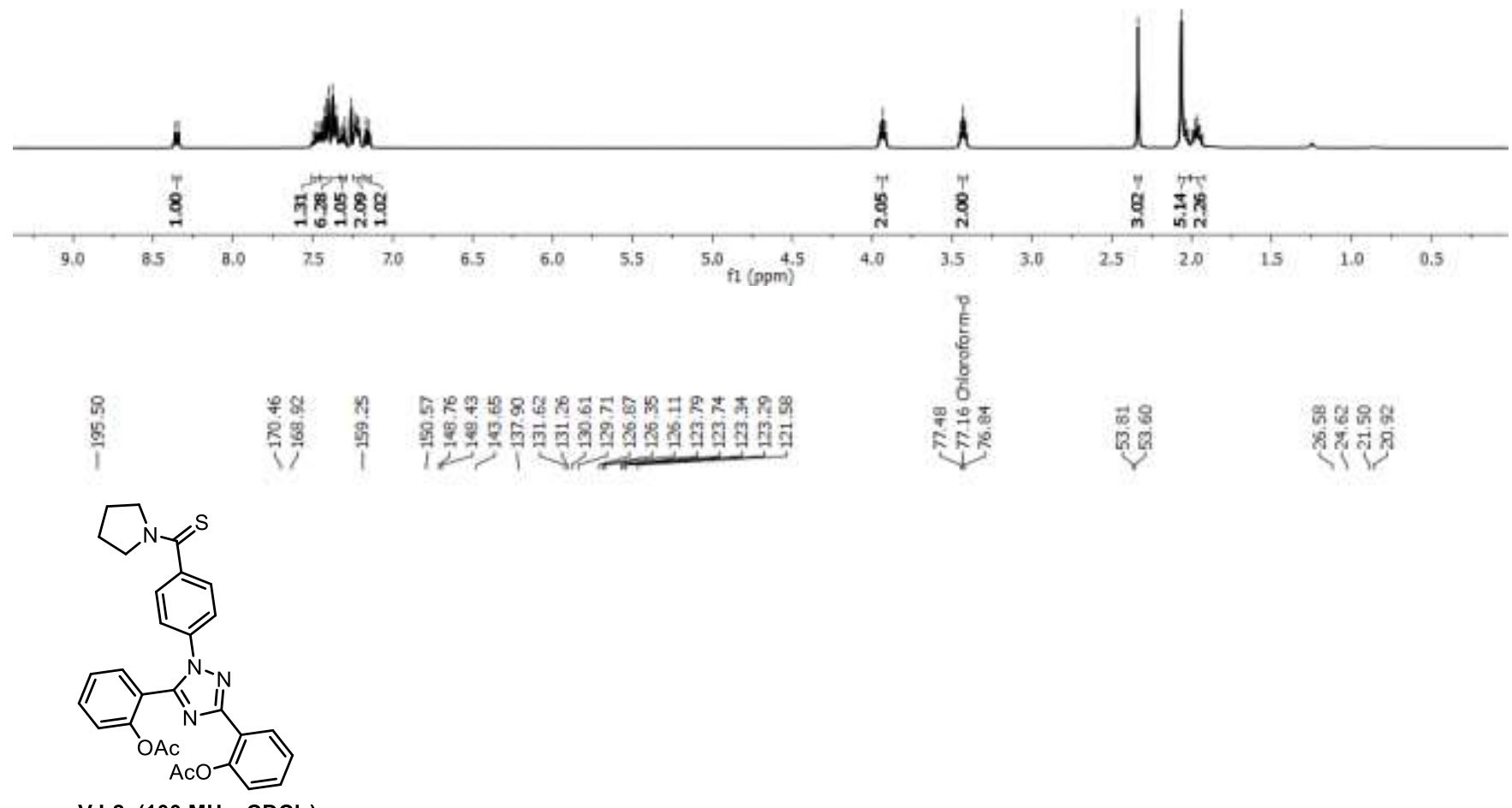

VJ-2 $\left(100 \mathrm{MHz}, \mathrm{CDCl}_{3}\right)$

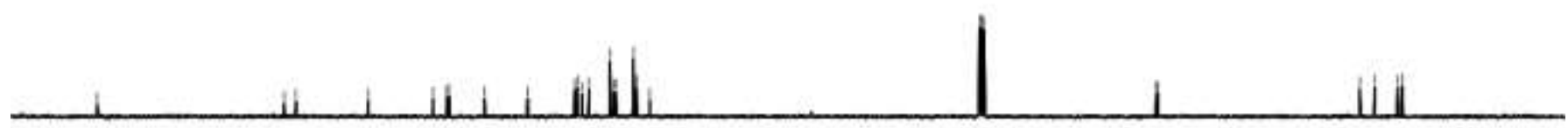

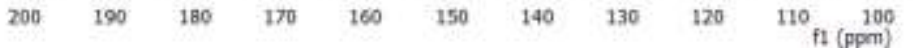




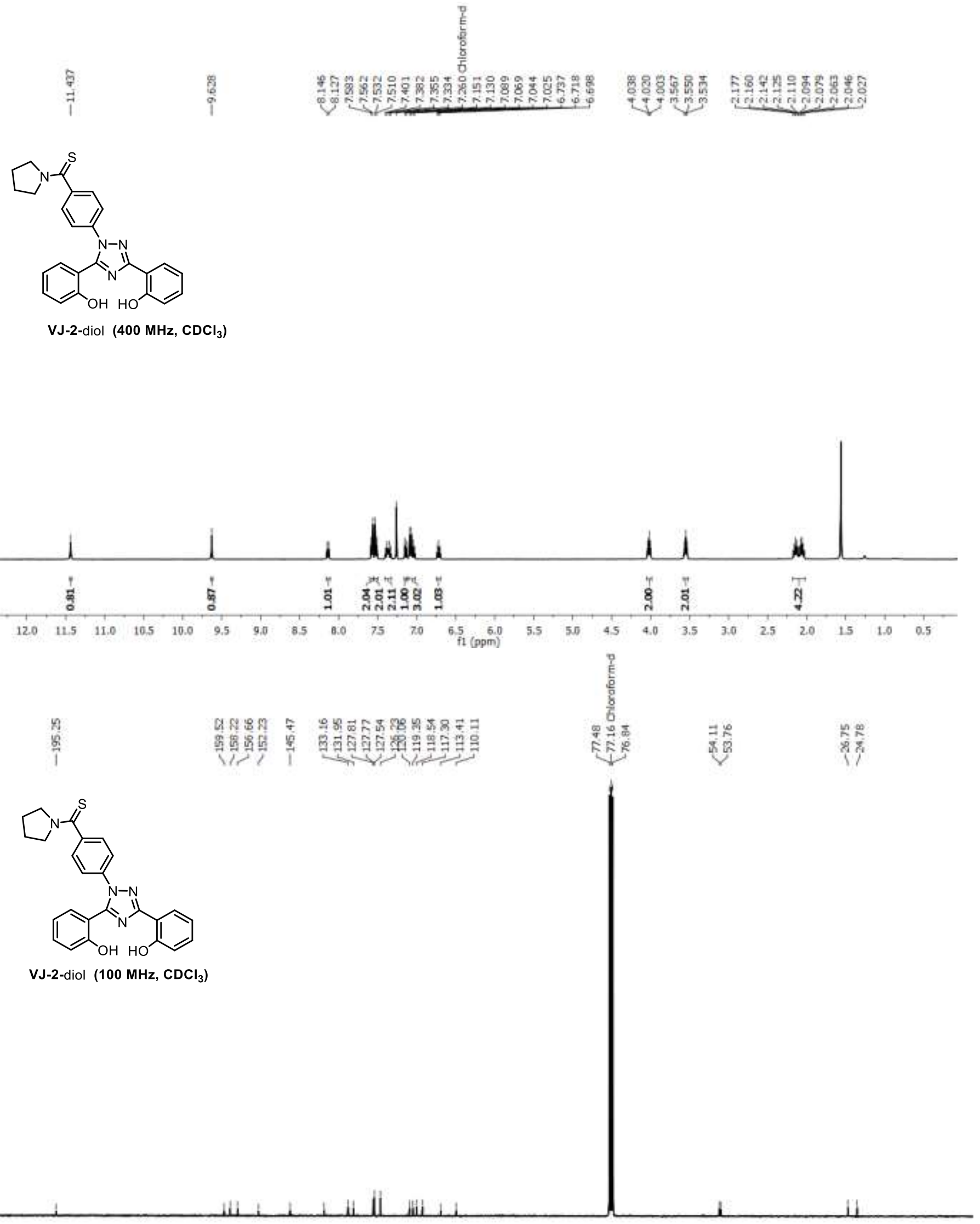

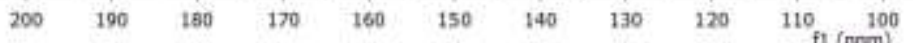



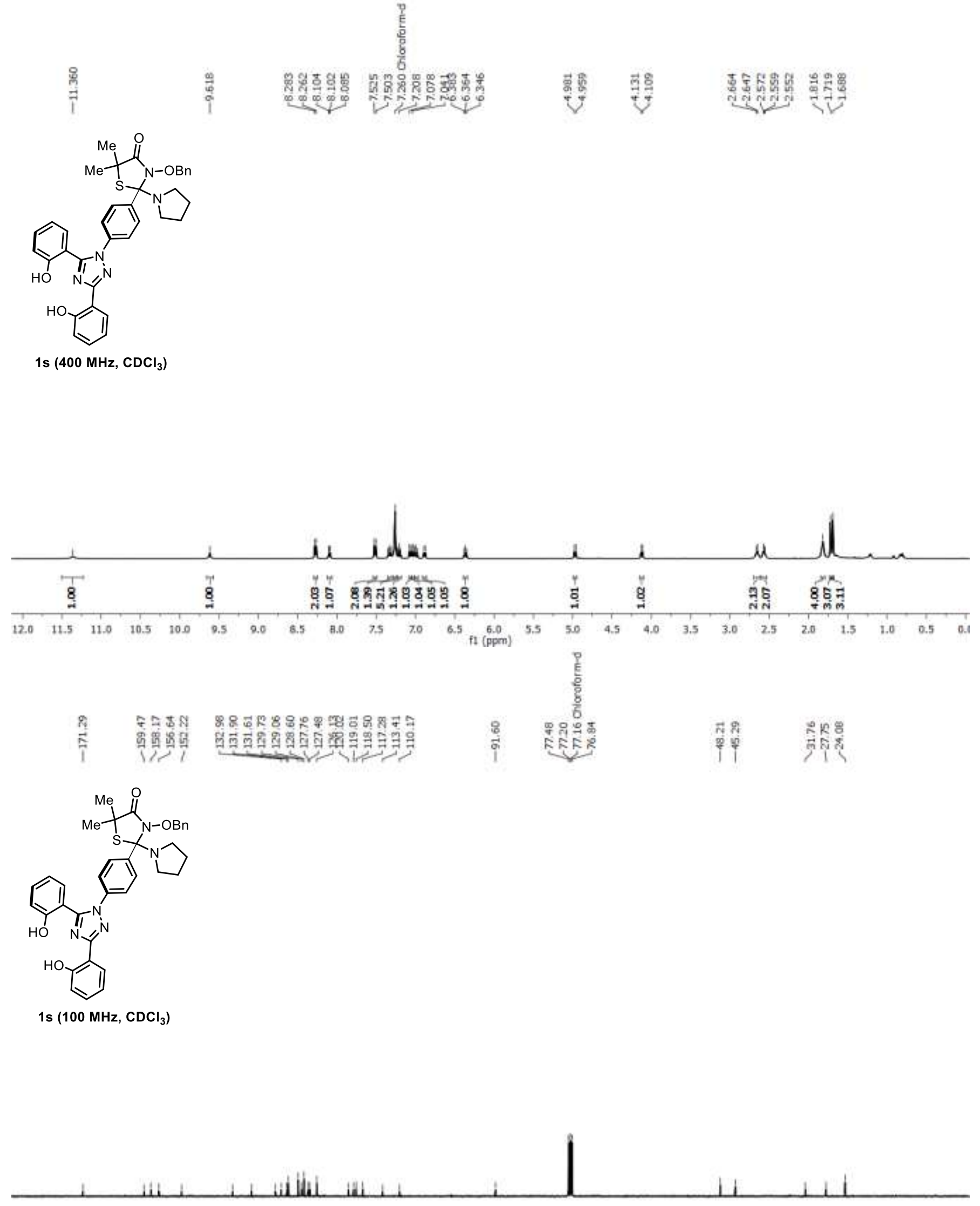

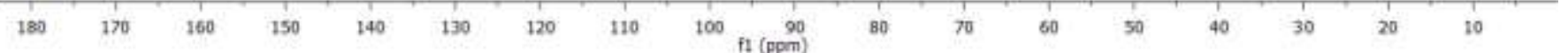



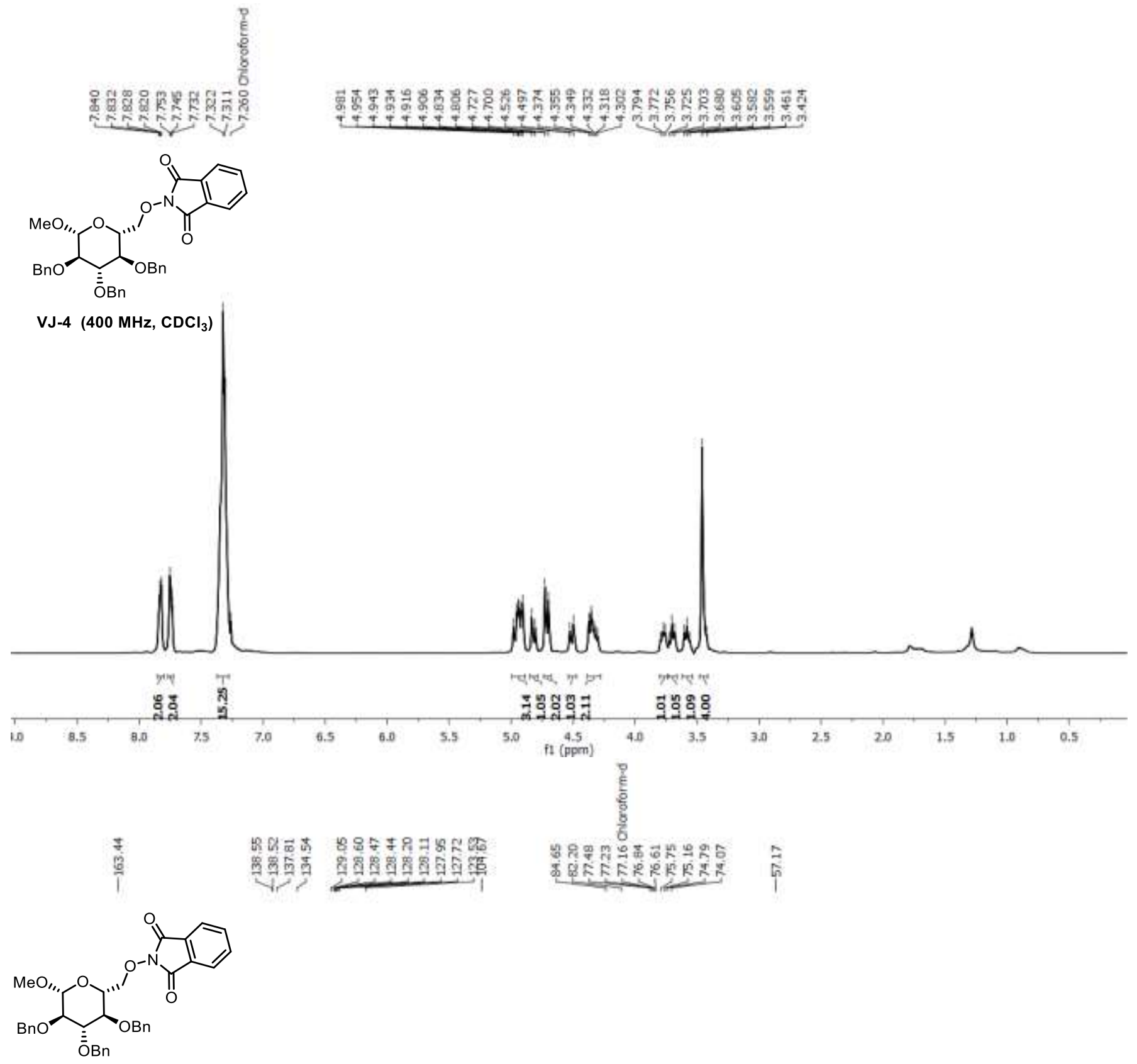

VJ-4 (100 $\left.\mathrm{MHz}, \mathrm{CDCl}_{3}\right)$

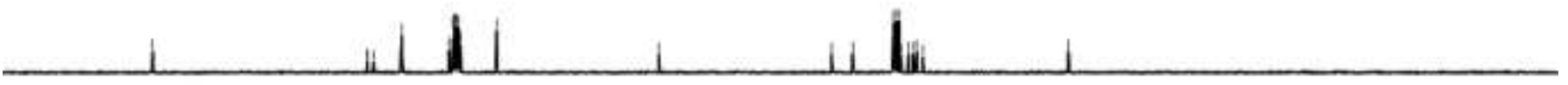

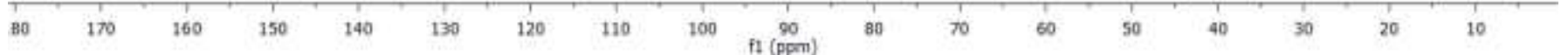




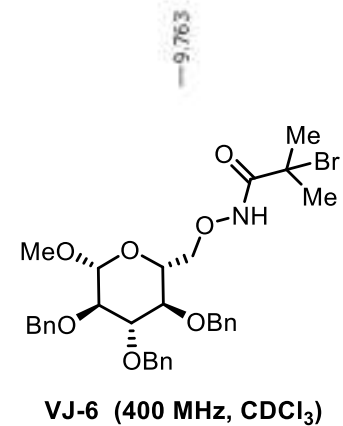

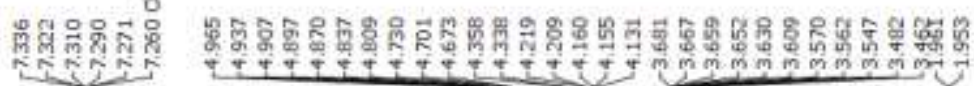

J-6 (400 MHz, $\left.\mathrm{CDCl}_{3}\right)$
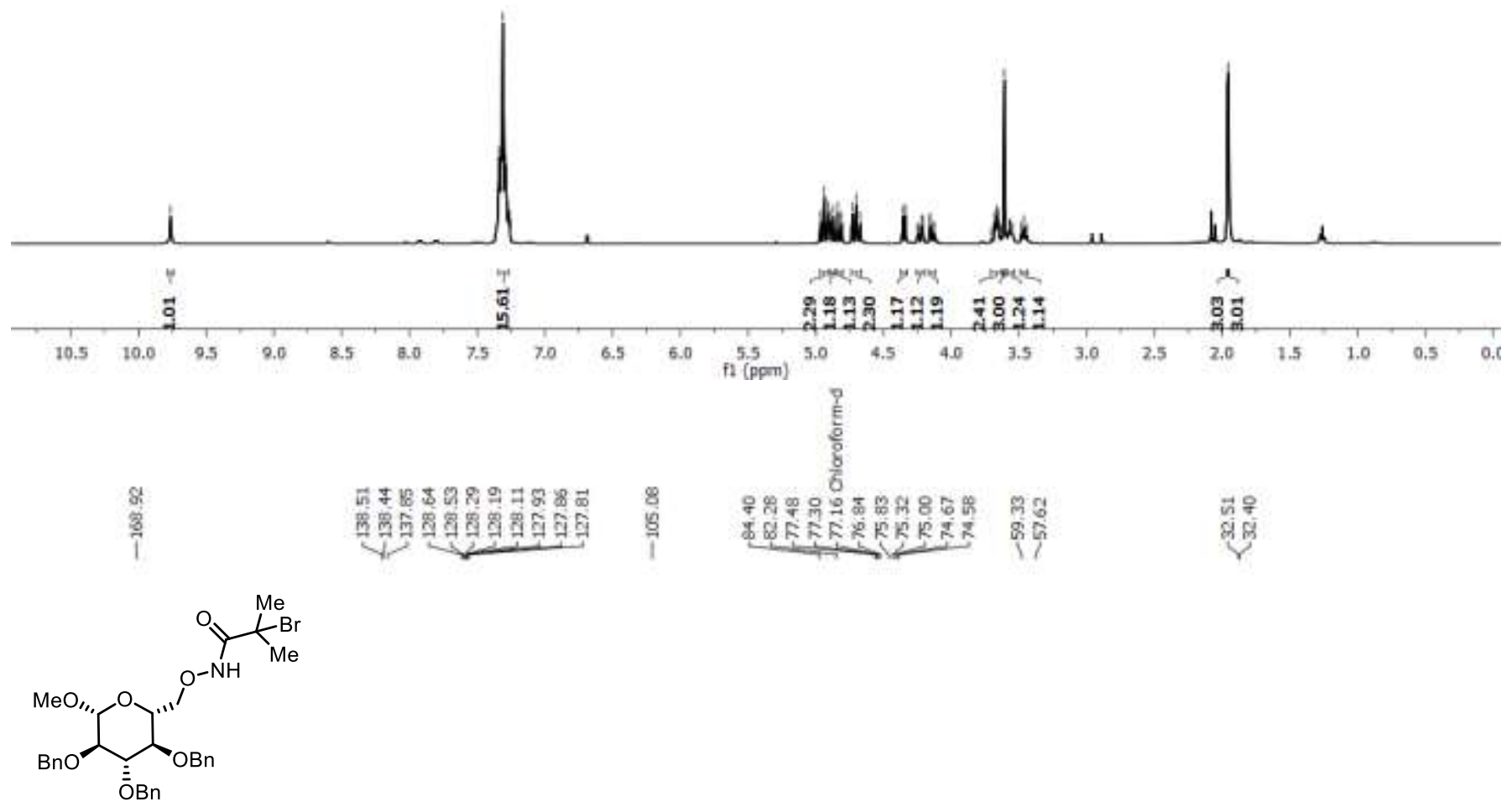

VJ-6 (100 MHz, $\left.\mathrm{CDCl}_{3}\right)$
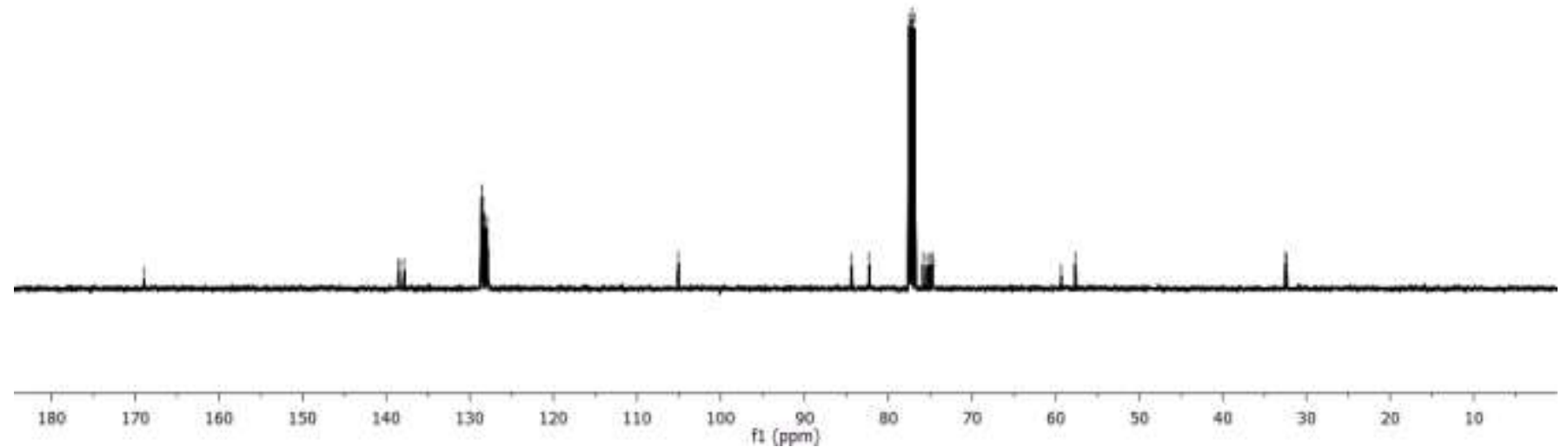

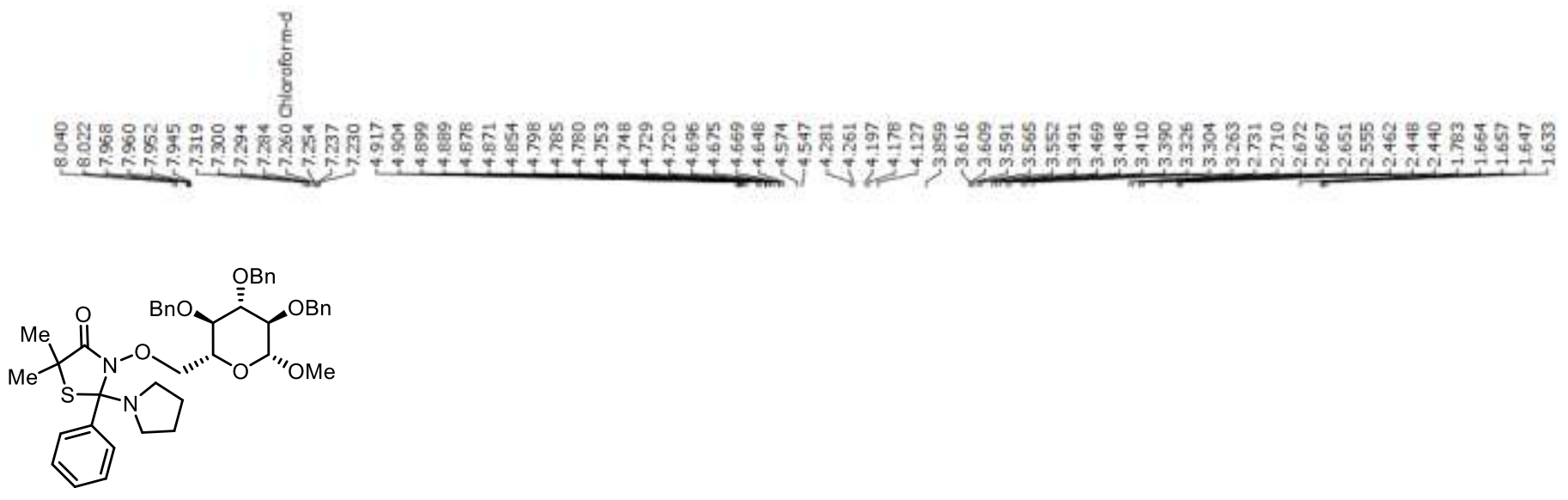

$1 \mathrm{~g} "\left(400 \mathrm{MHz}, \mathrm{CDCl}_{3}\right)$
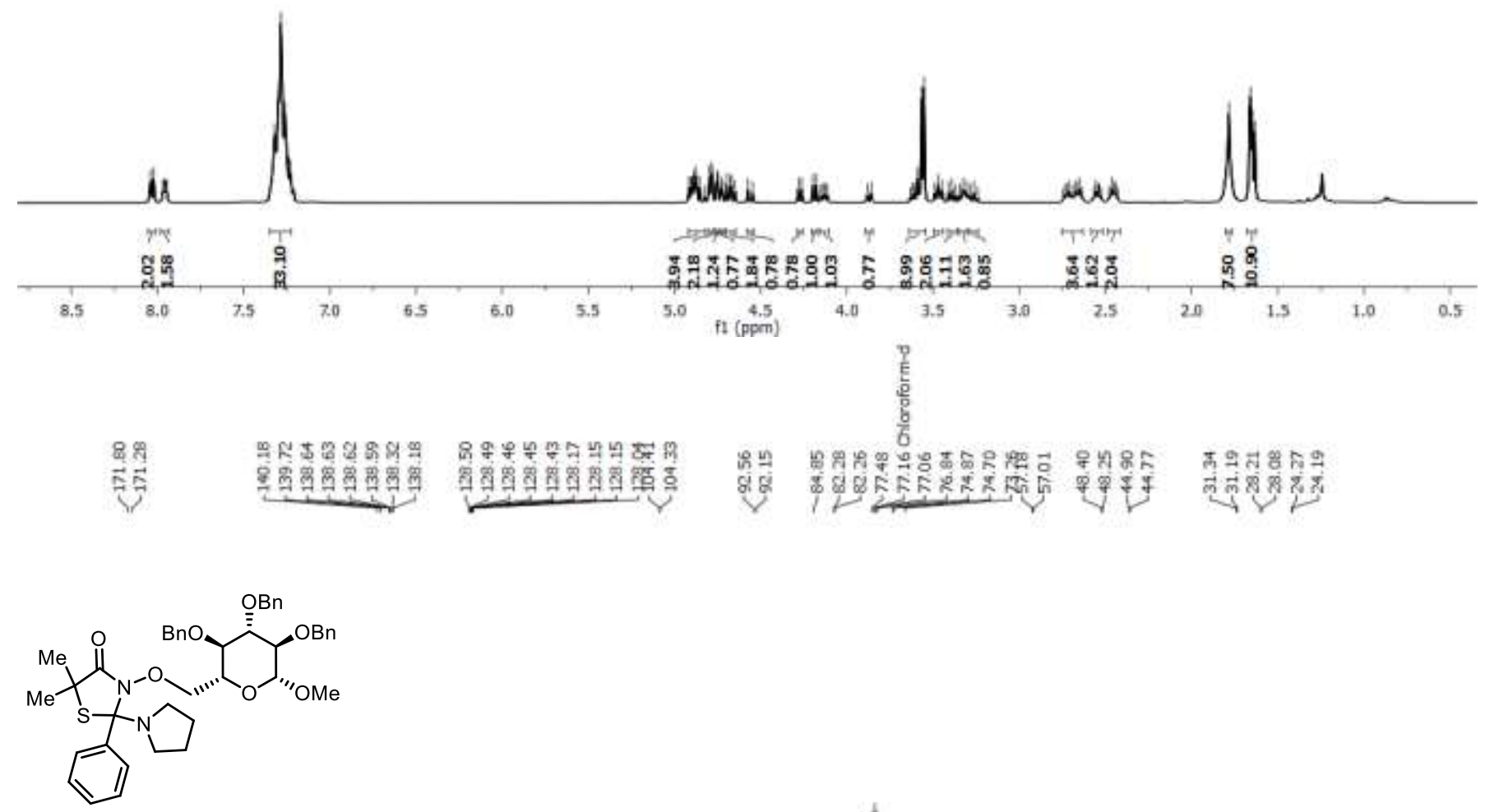

$1 \mathrm{~g} "\left(100 \mathrm{MHz}, \mathrm{CDCl}_{3}\right)$

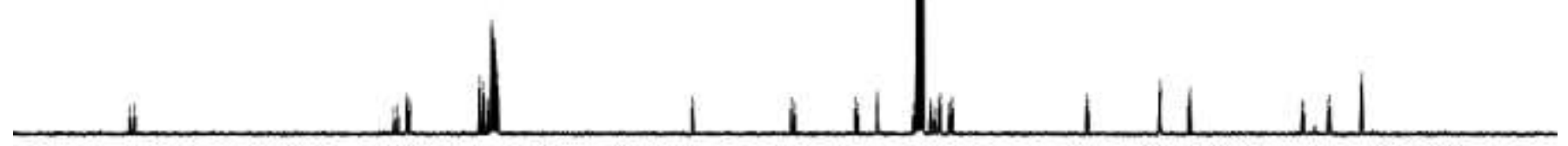

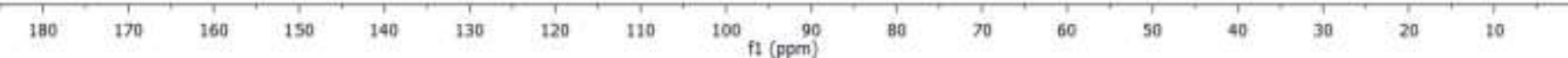


$\frac{1}{\frac{1}{5}}$

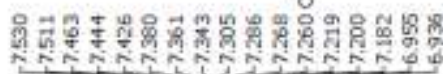

i

햄

Me

2a $\left(400 \mathrm{MHz}, \mathrm{CDCl}_{3}\right)$

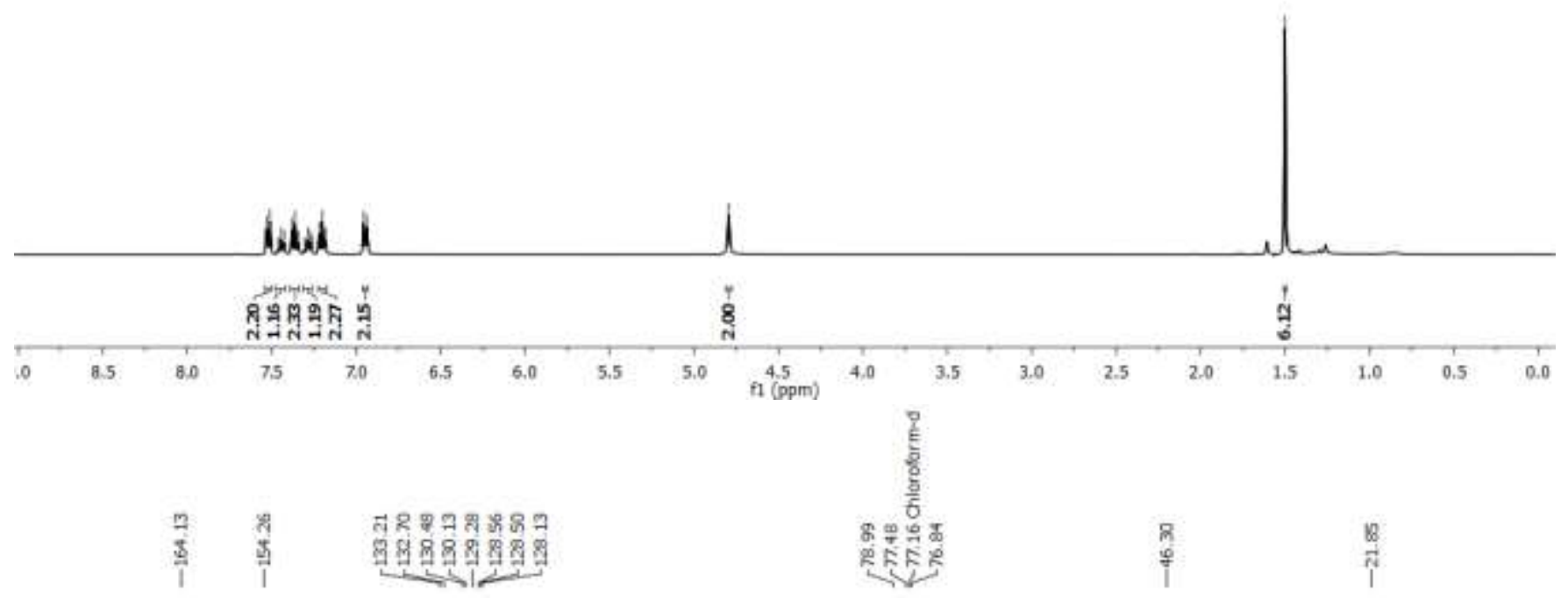

Me

2a $\left(100 \mathrm{MHz}, \mathrm{CDCl}_{3}\right)$

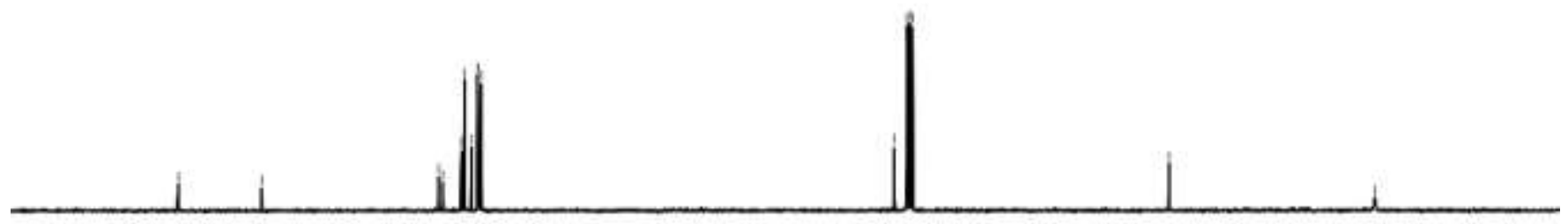

$\begin{array}{llllllllll}180 & 170 & 160 & 150 & 140 & 130 & 120 & 110 & 100 & 90 \\ 61(\mathrm{ppm})\end{array}$

$\begin{array}{lllllll}70 & 60 & 50 & 40 & 30 & 20 & 10\end{array}$ 


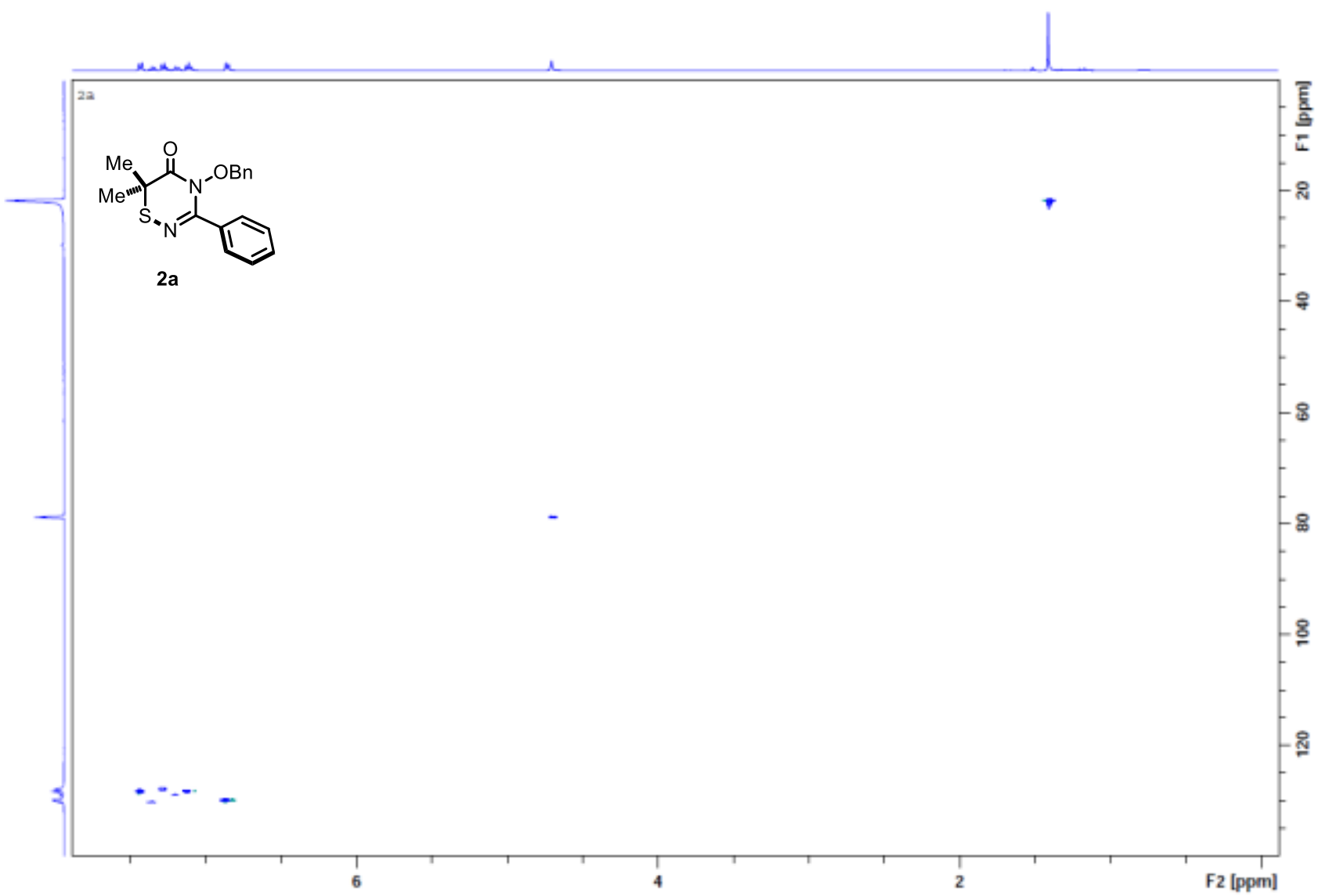

S69 


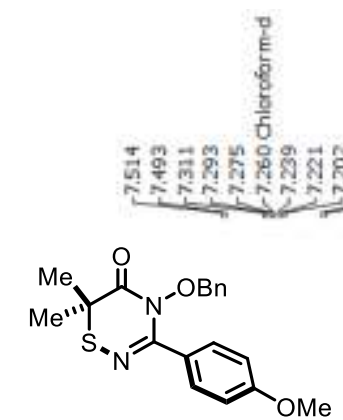

2b (400 $\mathrm{MHZ}, \mathrm{CDCl}_{3}$ )
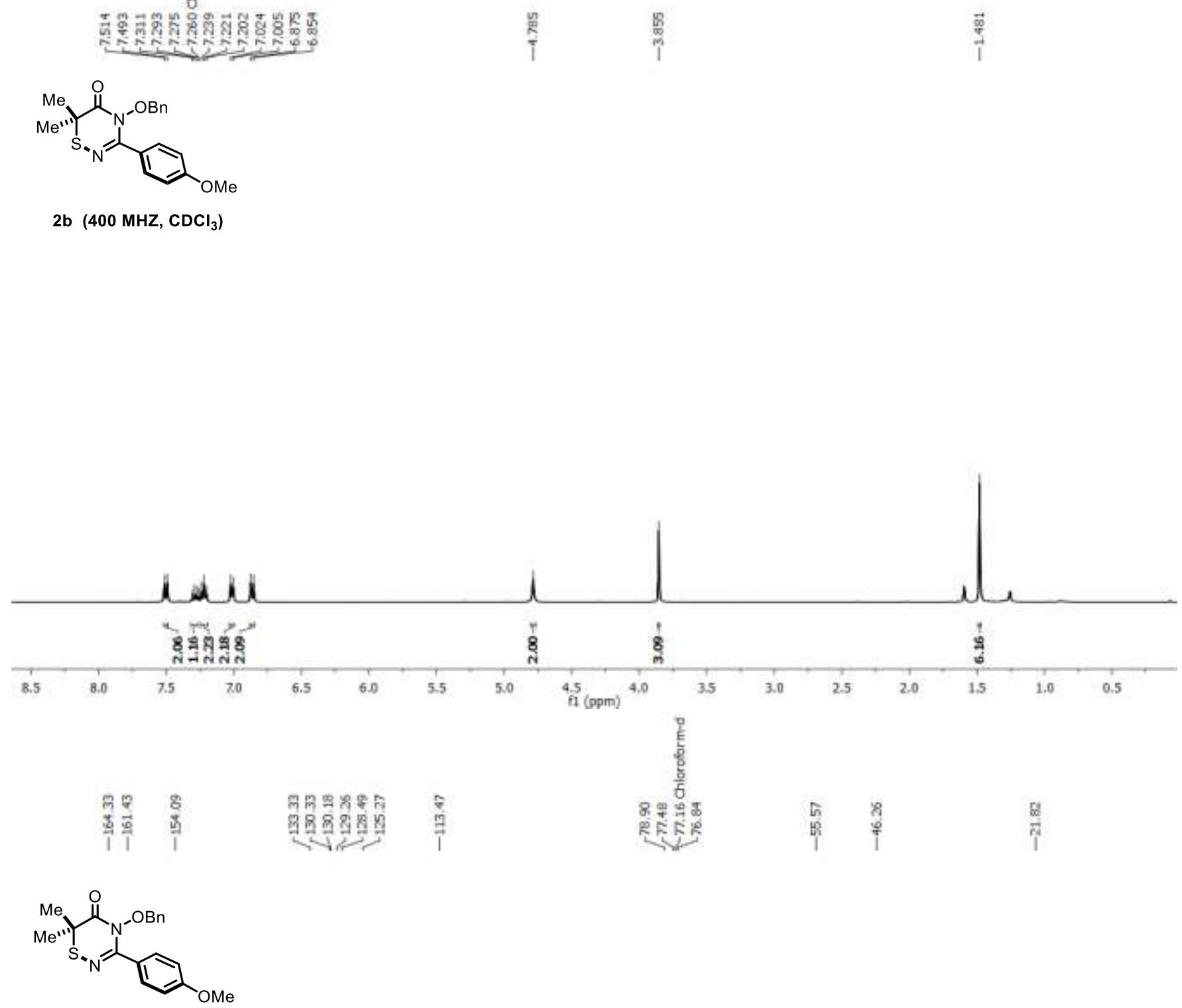

2b (100 MHZ, $\left.\mathrm{CDCl}_{3}\right)$

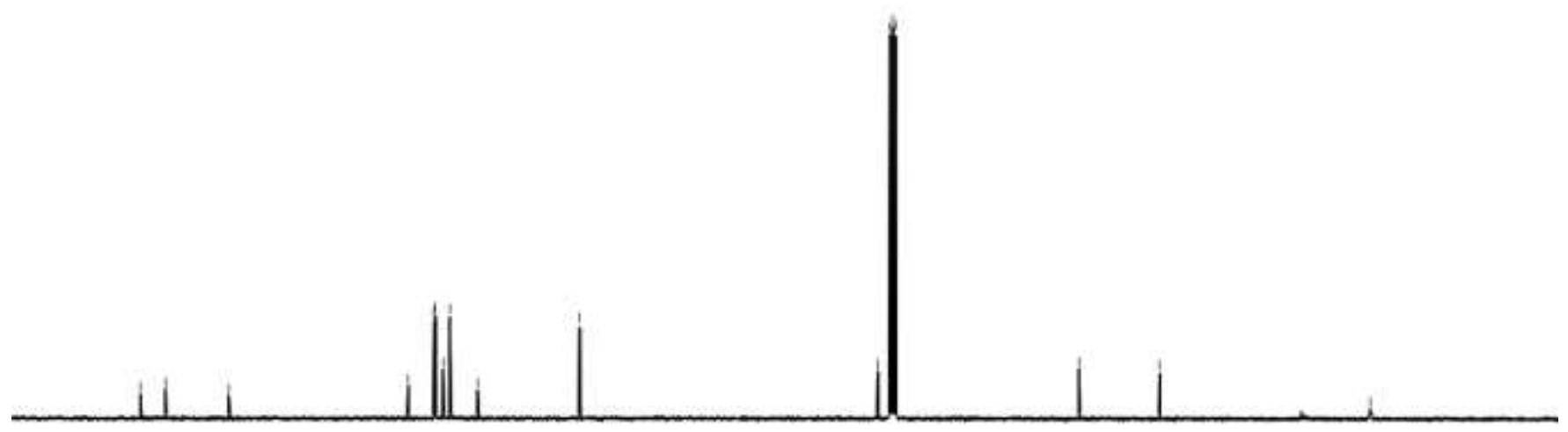

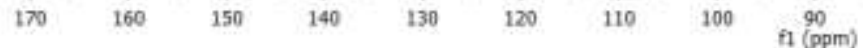

$\begin{array}{llllllll}80 & 70 & 60 & 50 & 40 & 30 & 20 & 10\end{array}$ 

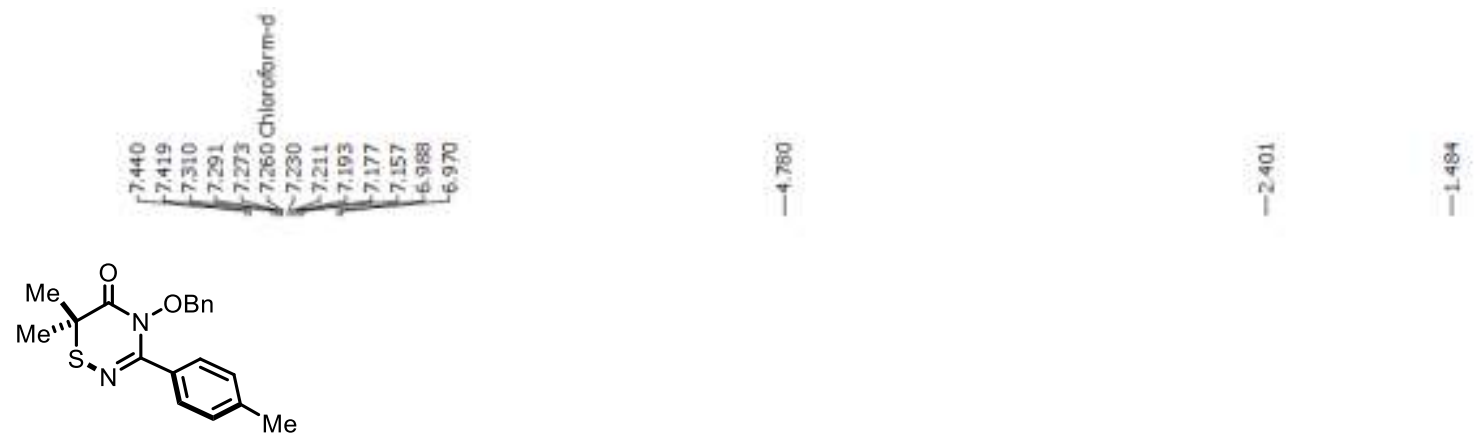

2c $\left(400 \mathrm{MHz}, \mathrm{CDCl}_{3}\right)$
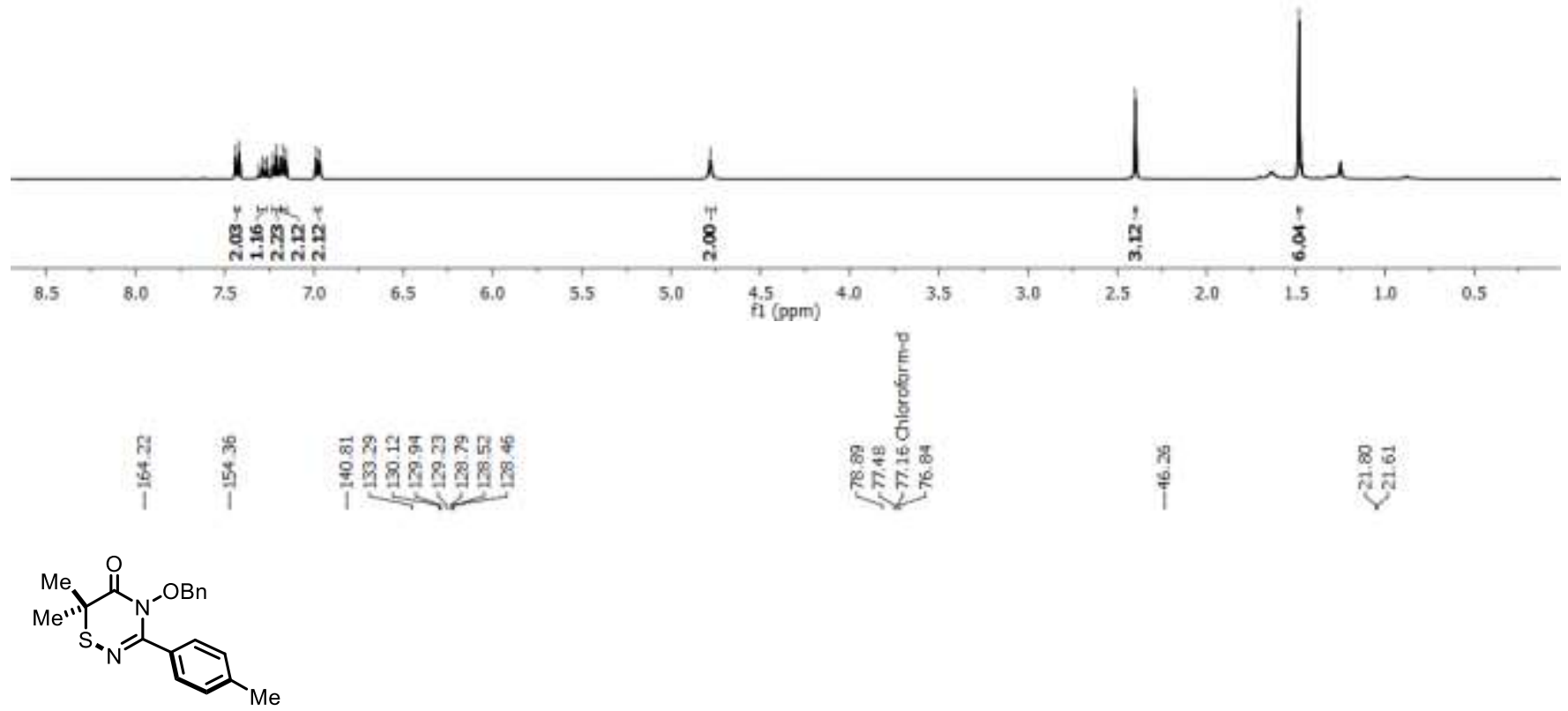

2c $\left(100 \mathrm{MHz}, \mathrm{CDCl}_{3}\right)$
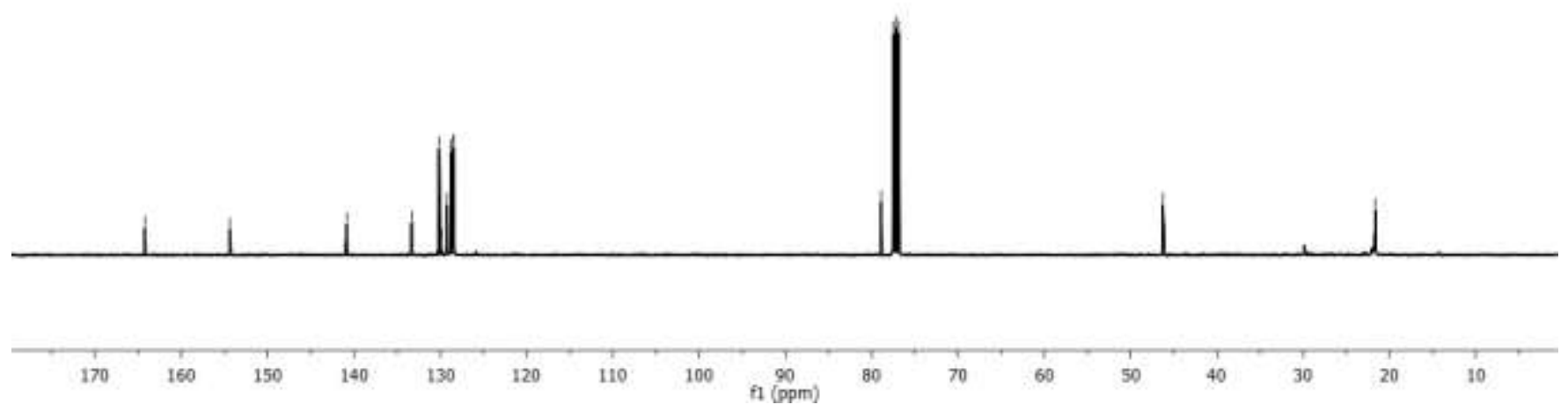


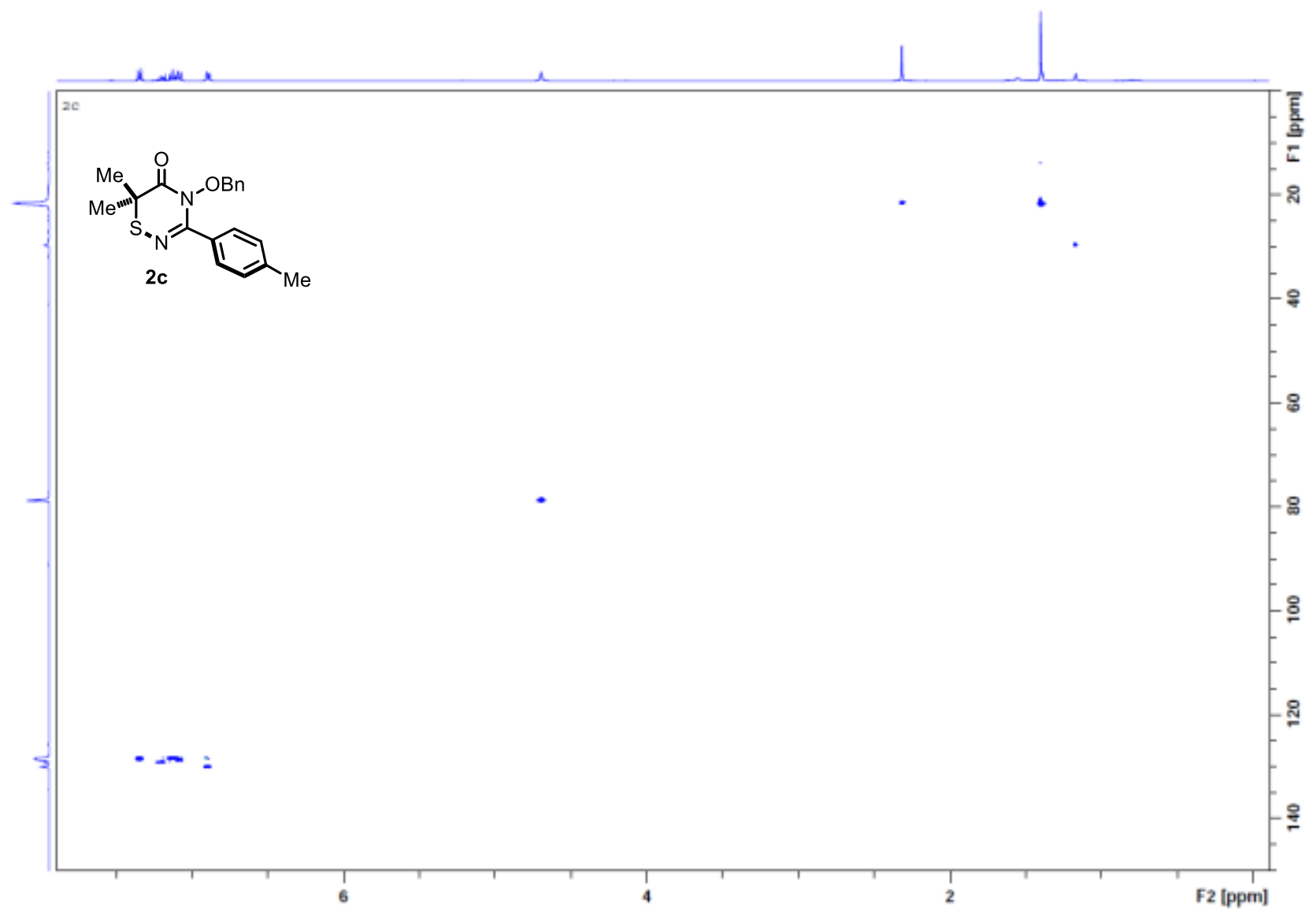



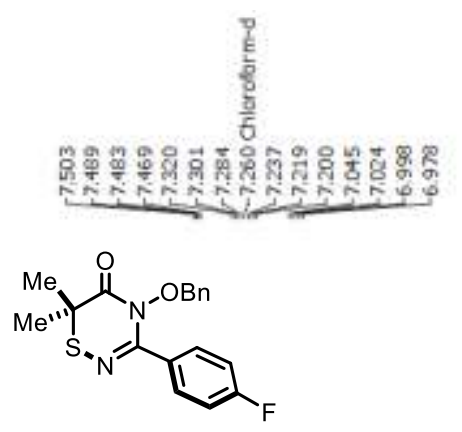

2d $\left(400 \mathrm{MHz}, \mathrm{CDCl}_{3}\right)$

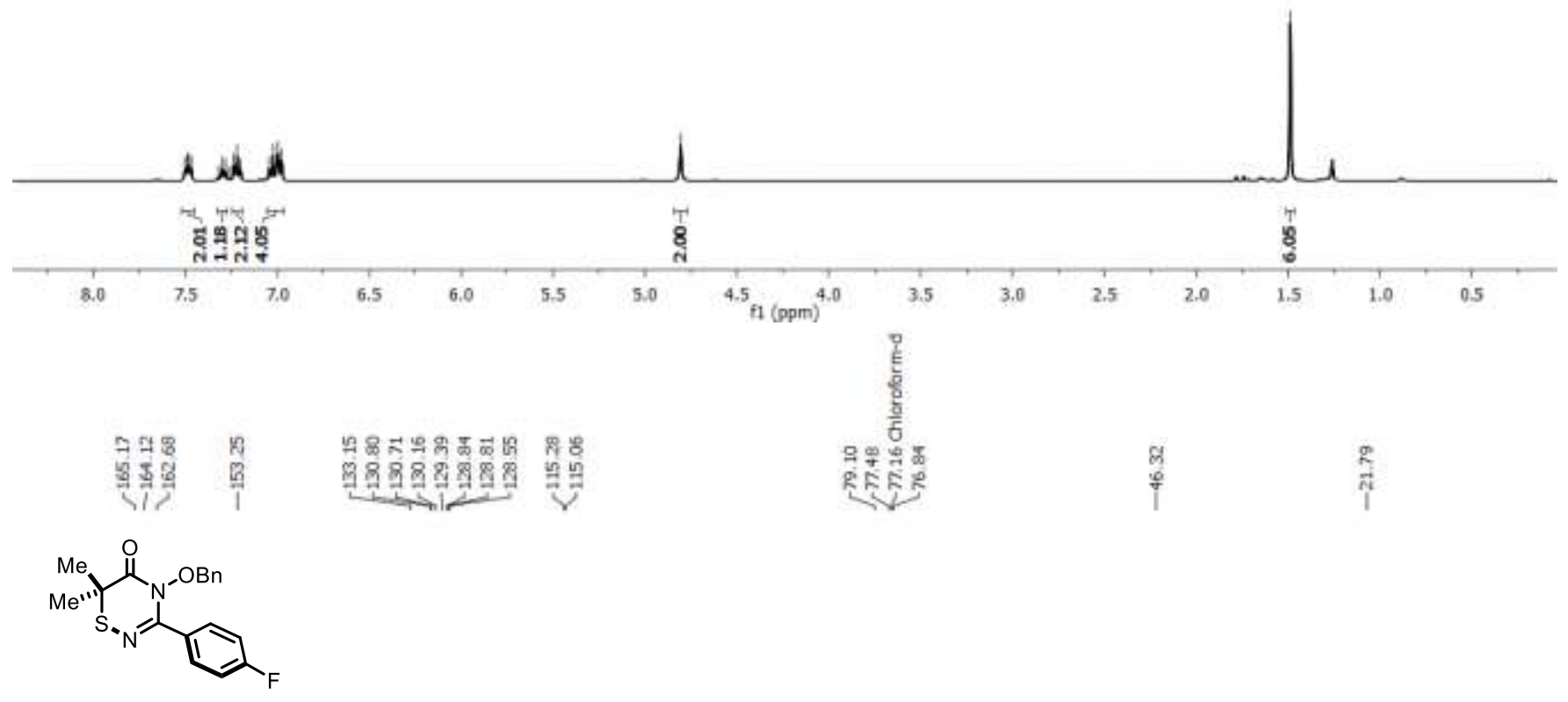

2d $\left(100 \mathrm{MHz}, \mathrm{CDCl}_{3}\right)$

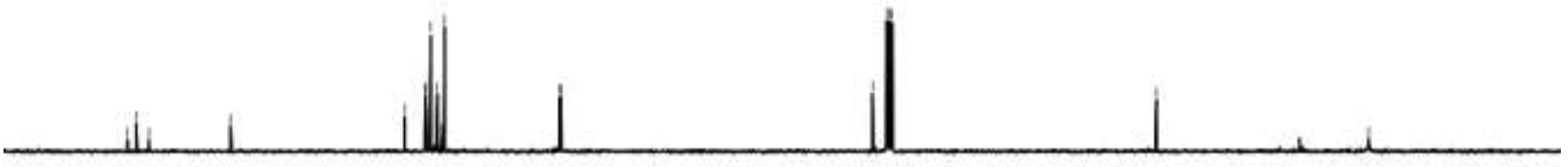

$\begin{array}{lllllllll}170 & 160 & 150 & 140 & 130 & 120 & 110 & 100 & 90 \\ \mathrm{f1} & (\mathrm{ppm})\end{array}$ 


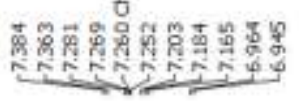

$\stackrel{1}{i}$

$\frac{\$}{1}$
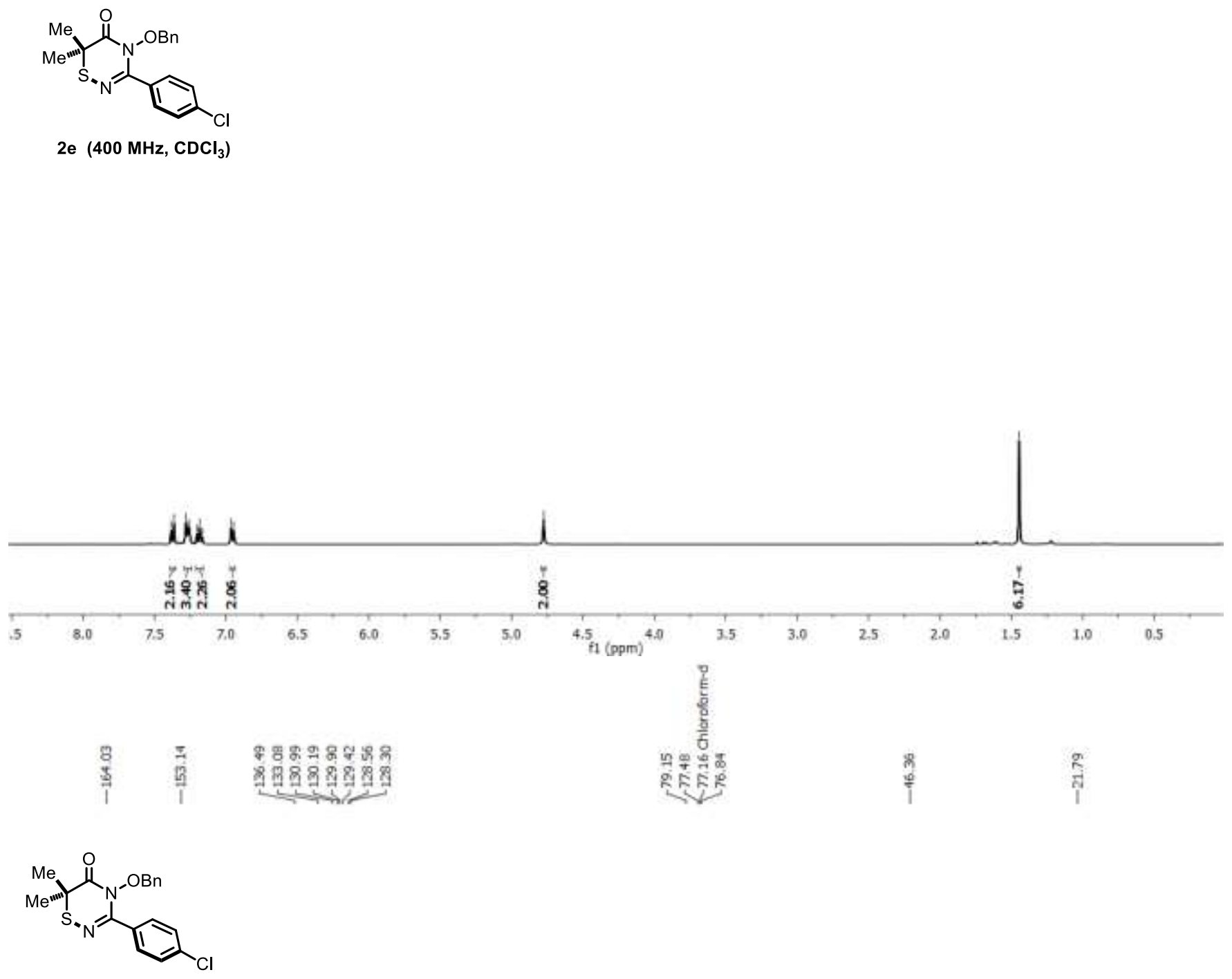

2e $\left(100 \mathrm{MHz}, \mathrm{CDCl}_{3}\right)$
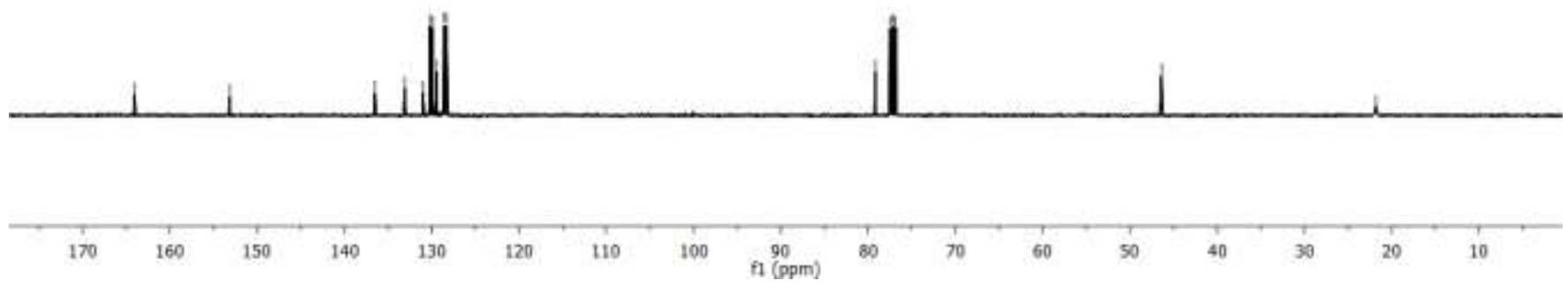

S74 

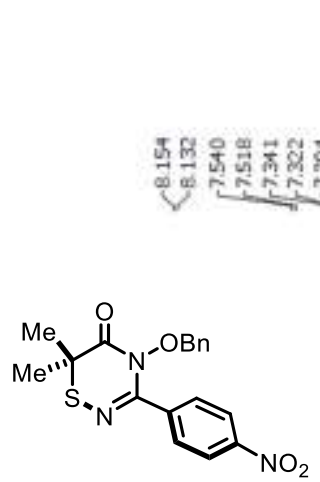

2f $\left(400 \mathrm{MHz}, \mathrm{CDCl}_{3}\right)$
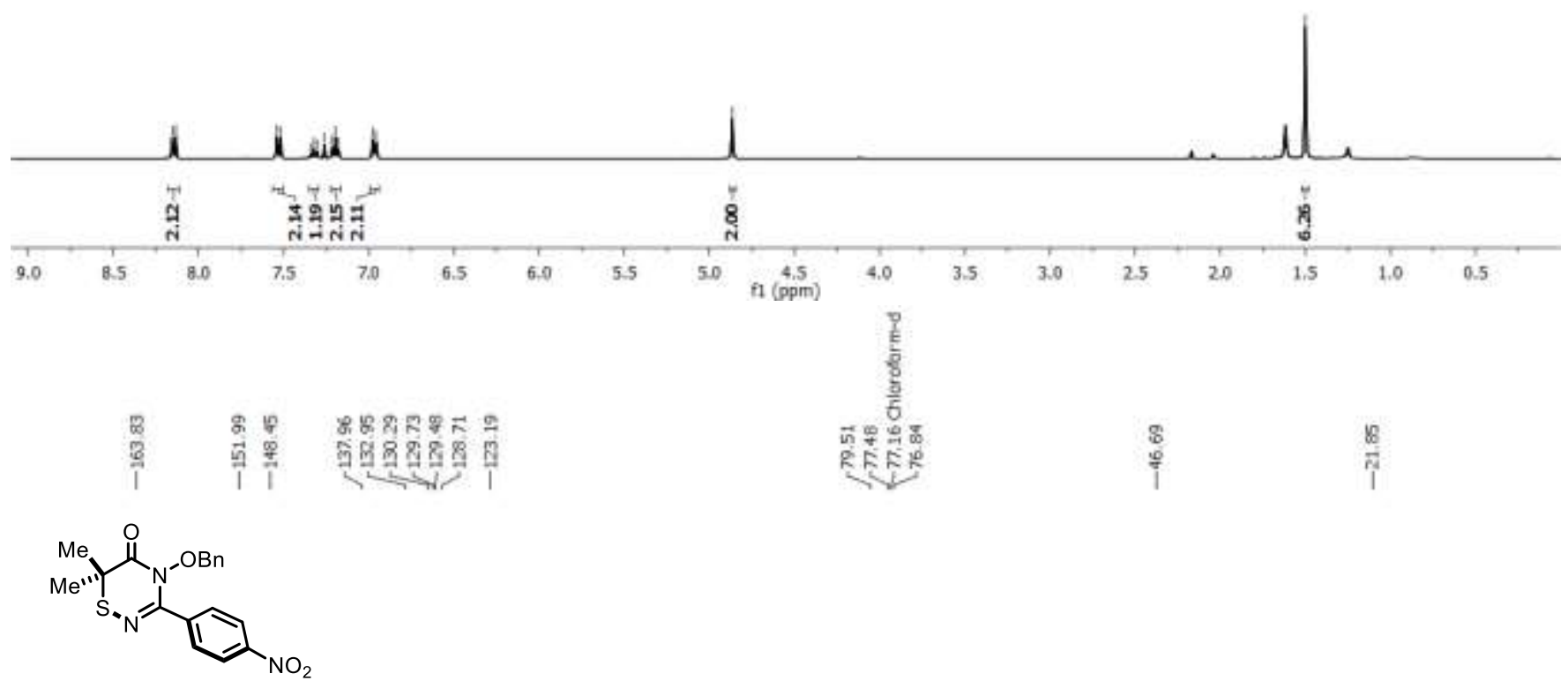

2f $\left(100 \mathrm{MHz}^{-\mathrm{CDCl}_{3}}\right)$
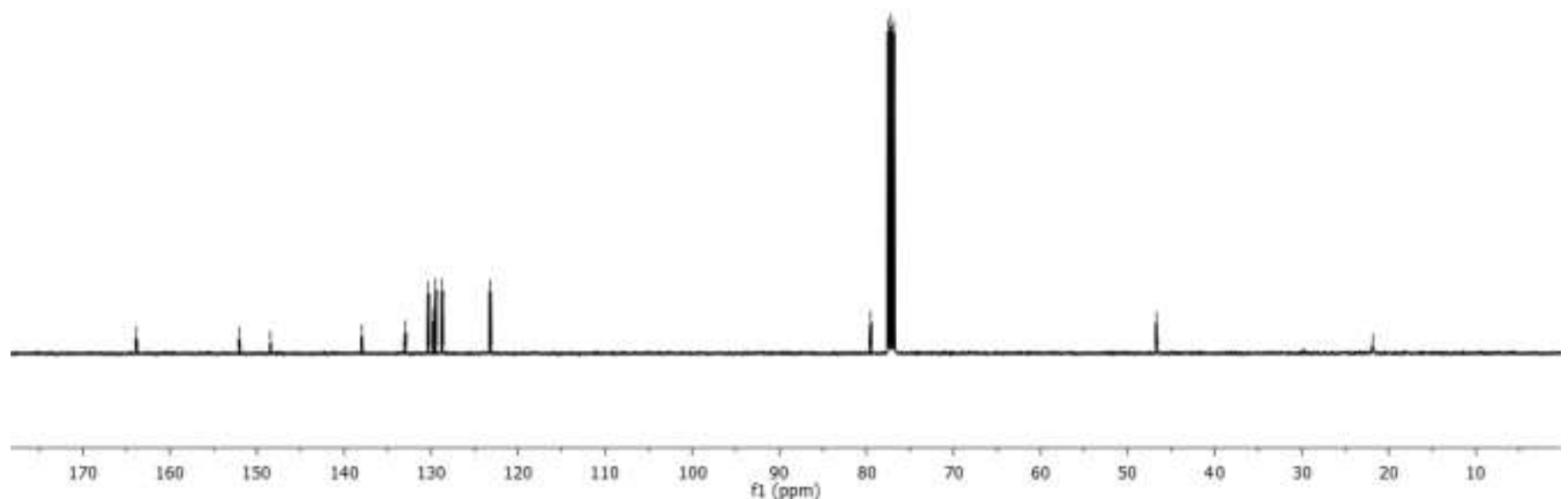

2f $\left(400 \mathrm{MHz}, \mathrm{CDCl}_{3}\right)$ 


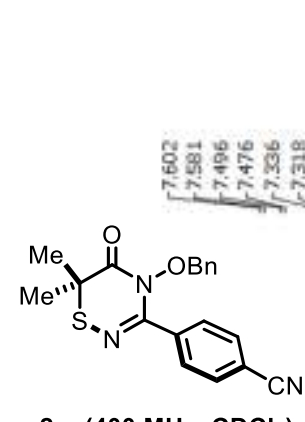

2g $\left(400 \mathrm{MHz}, \mathrm{CDCl}_{3}\right)$
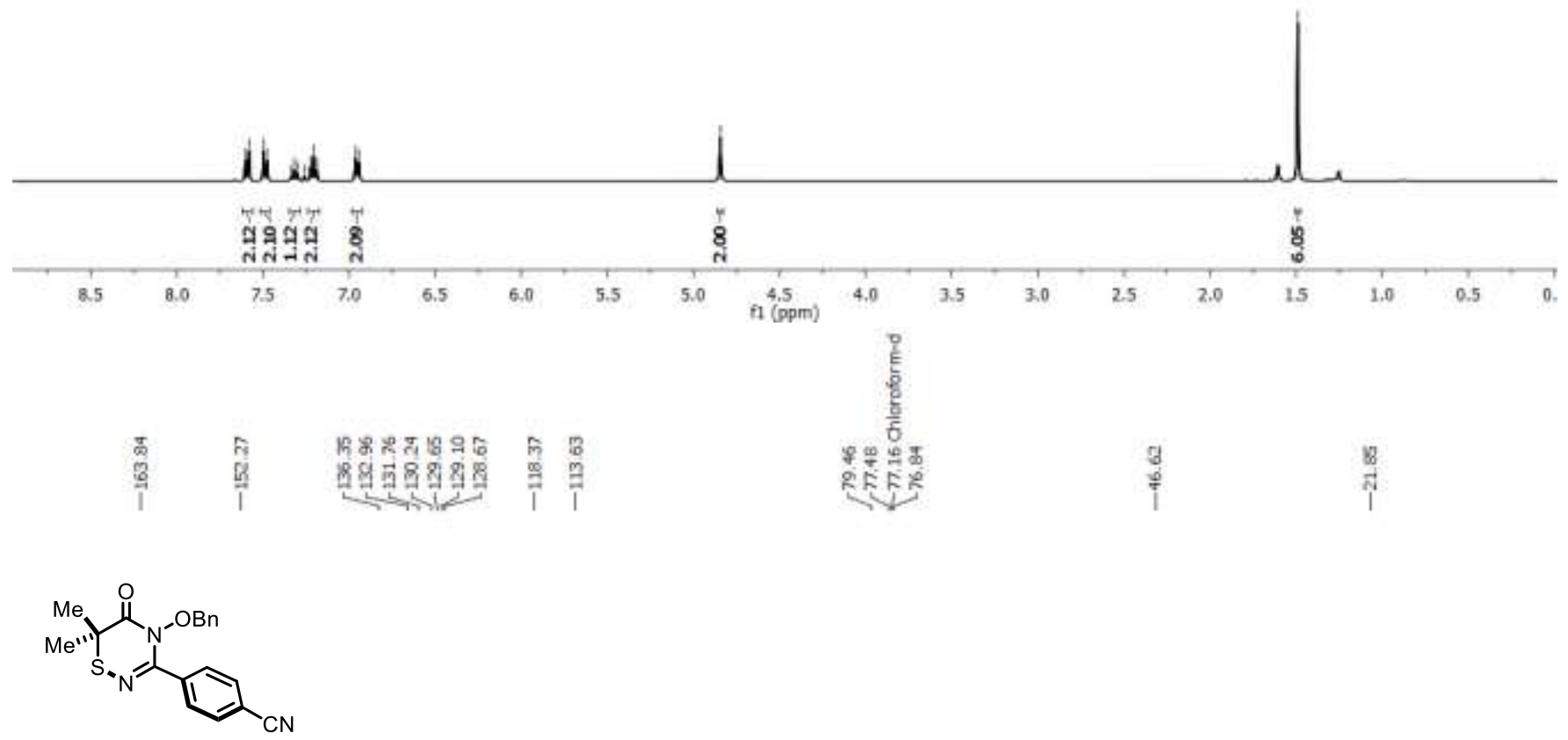

2g (100 MHz, $\left.\mathrm{CDCl}_{3}\right)$
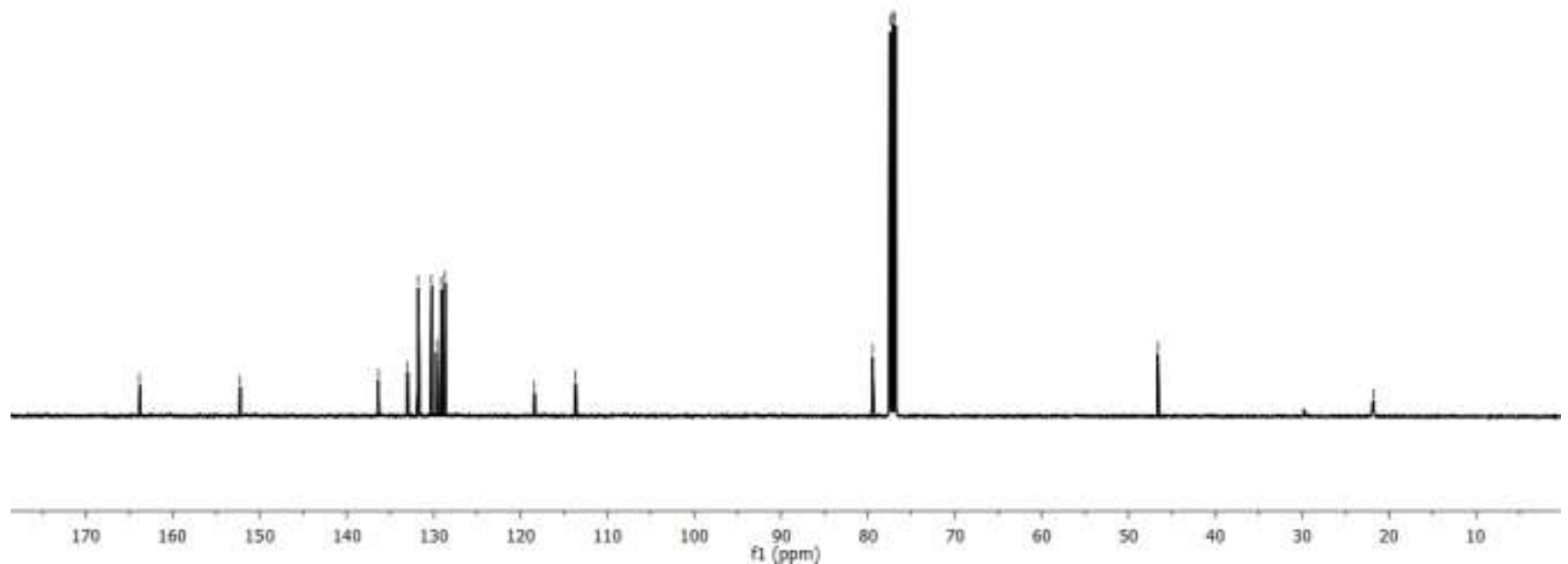

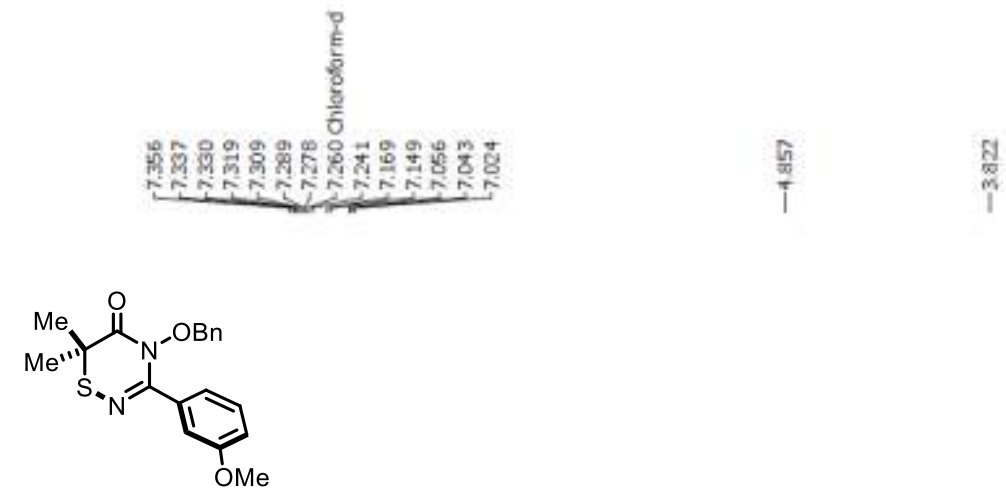

2h (400 $\mathrm{MHz}, \mathrm{CDCl}_{3}$ )

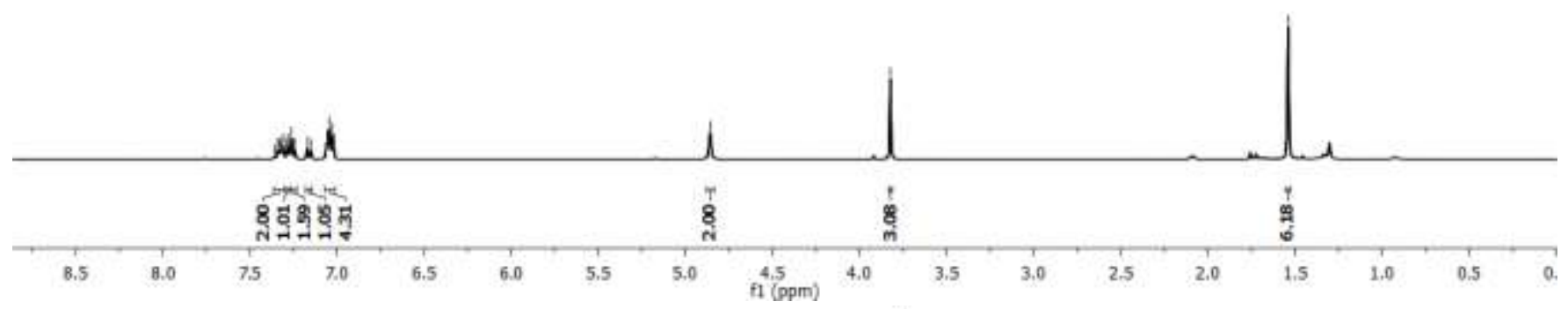

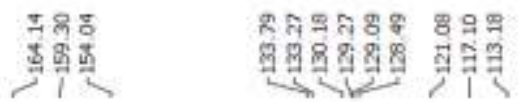
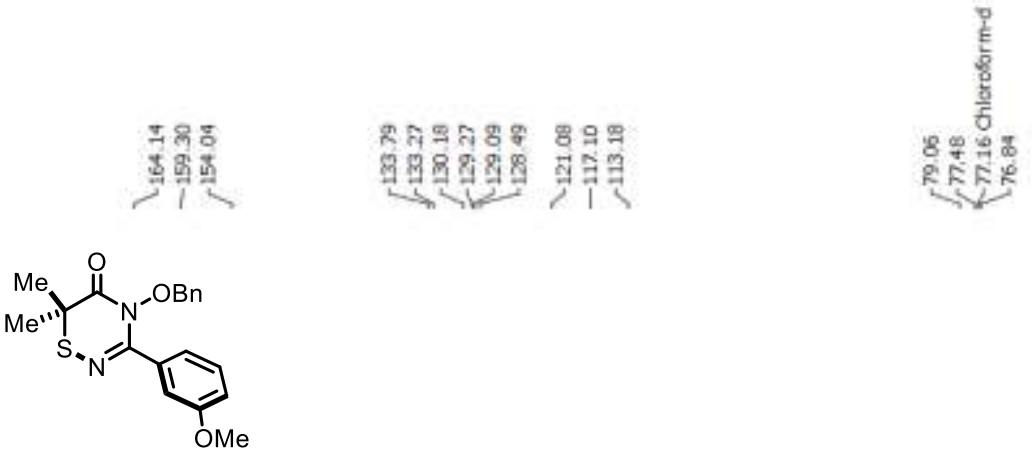

2h (100 MHz, $\left.\mathrm{CDCl}_{3}\right)$
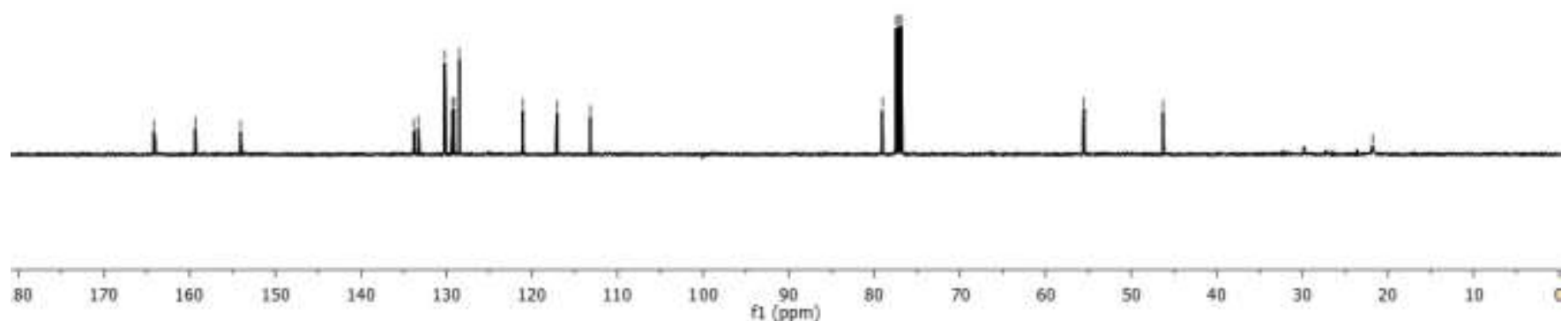


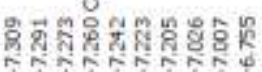

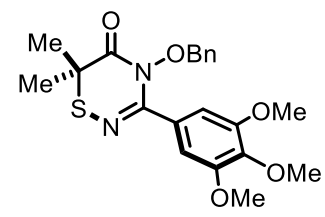

2i $\left(400 \mathrm{MHz}, \mathrm{CDCl}_{3}\right)$
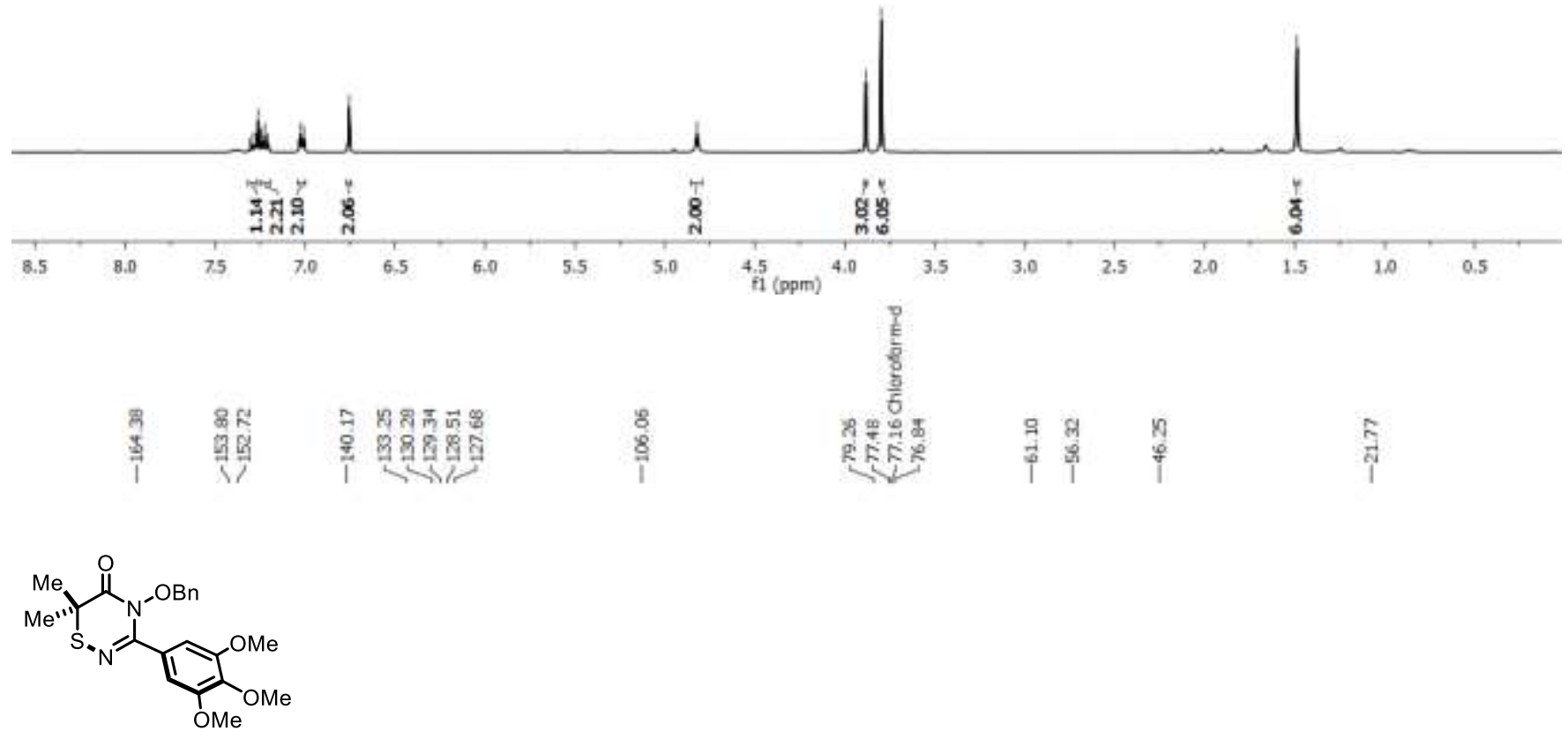

2i $\left(100 \mathrm{MHz}, \mathrm{CDCl}_{3}\right)$

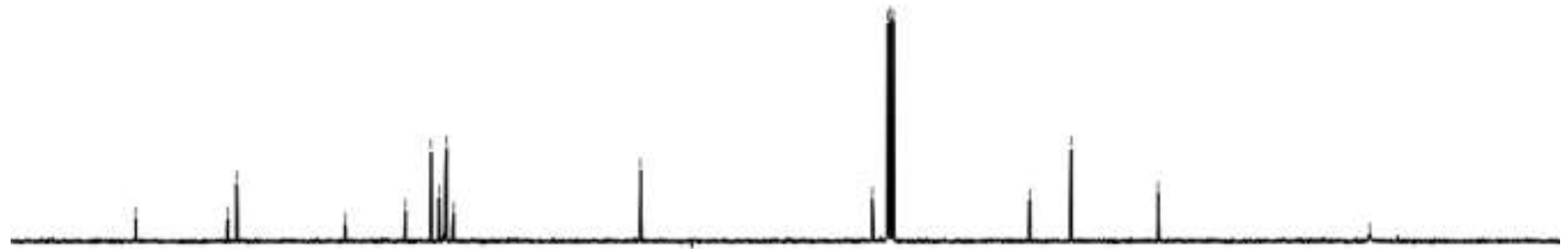

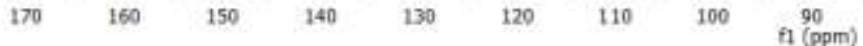

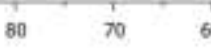




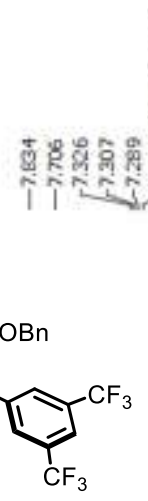

2j $\left.\left(400 \mathrm{MHz}^{\mathrm{CDCl}}\right)_{3}\right)$
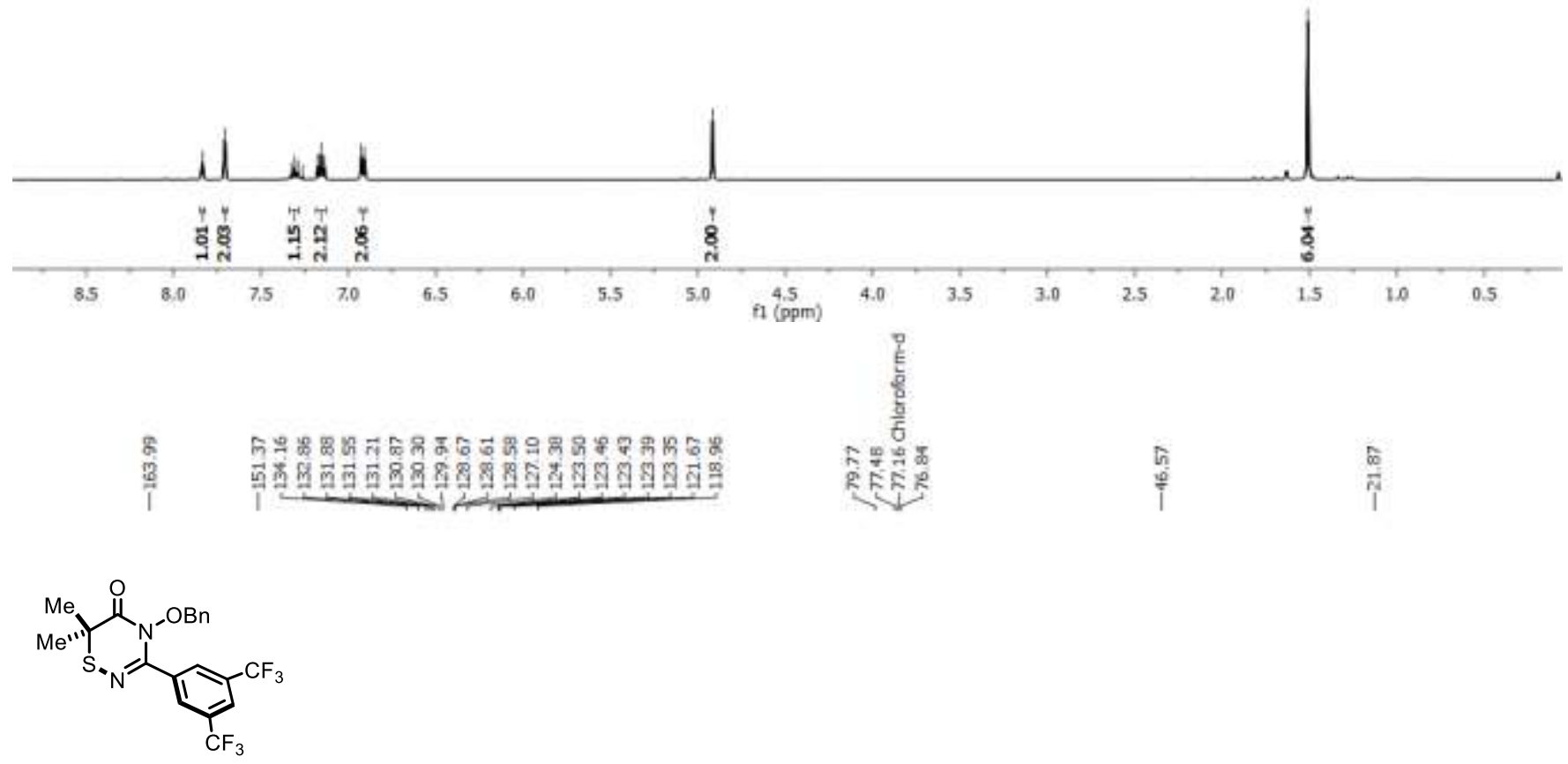

2j $\left(100 \mathrm{MHz}, \mathrm{CDCl}_{3}\right)$

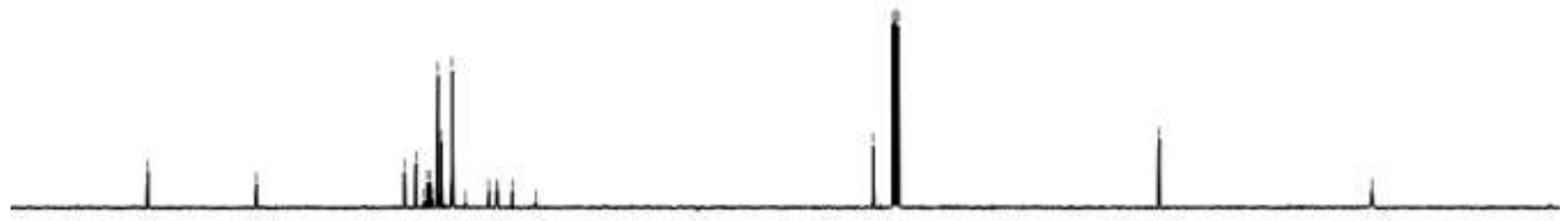

$\begin{array}{lllllllll}170 & 160 & 150 & 140 & 130 & 120 & 110 & 100 & 90\end{array}$ 


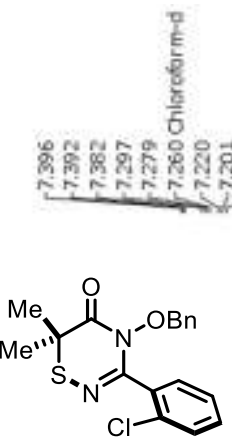

2k (400 $\mathrm{MHz}, \mathrm{CDCl}_{3}$ )
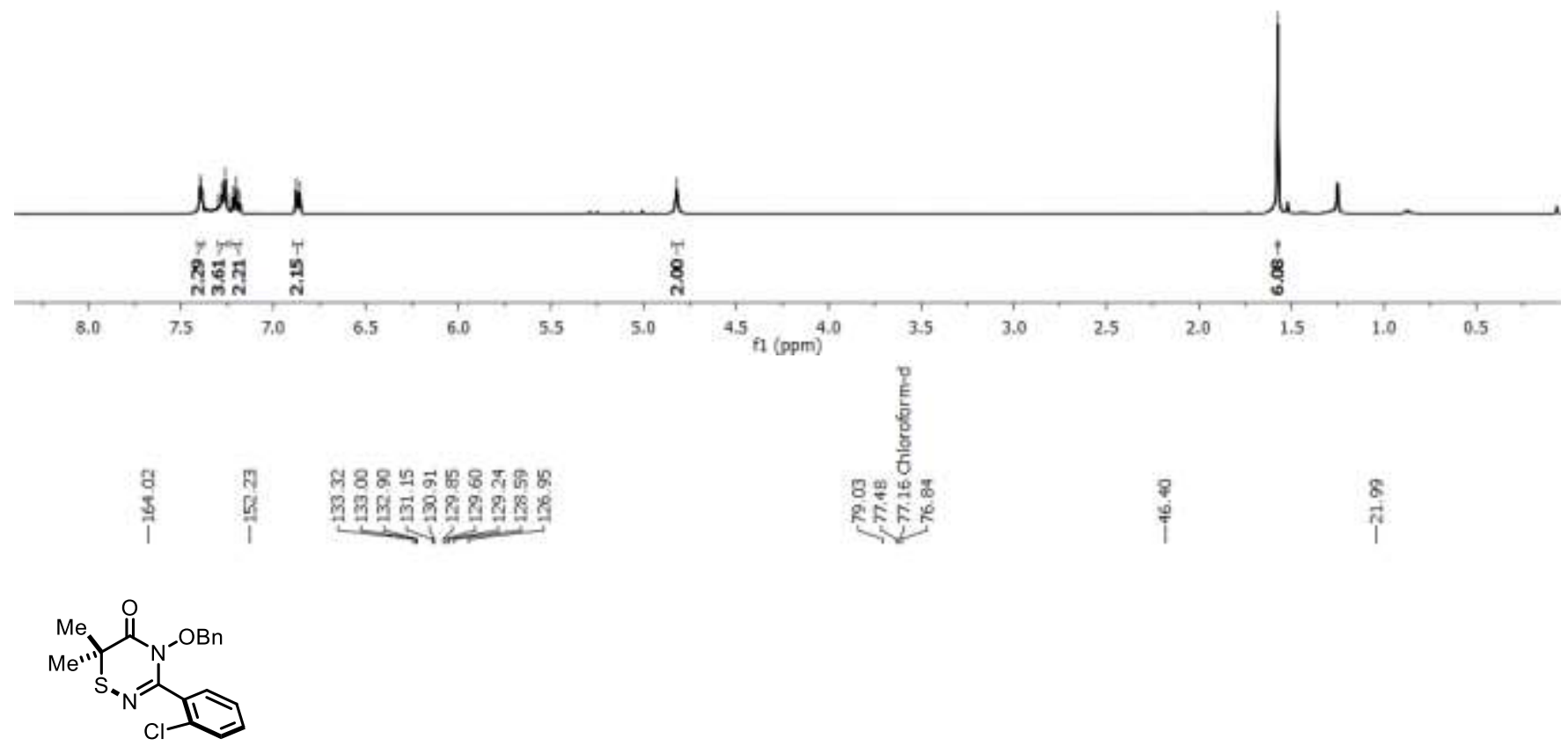

2k (100 $\left.\mathrm{MHz}, \mathrm{CDCl}_{3}\right)$
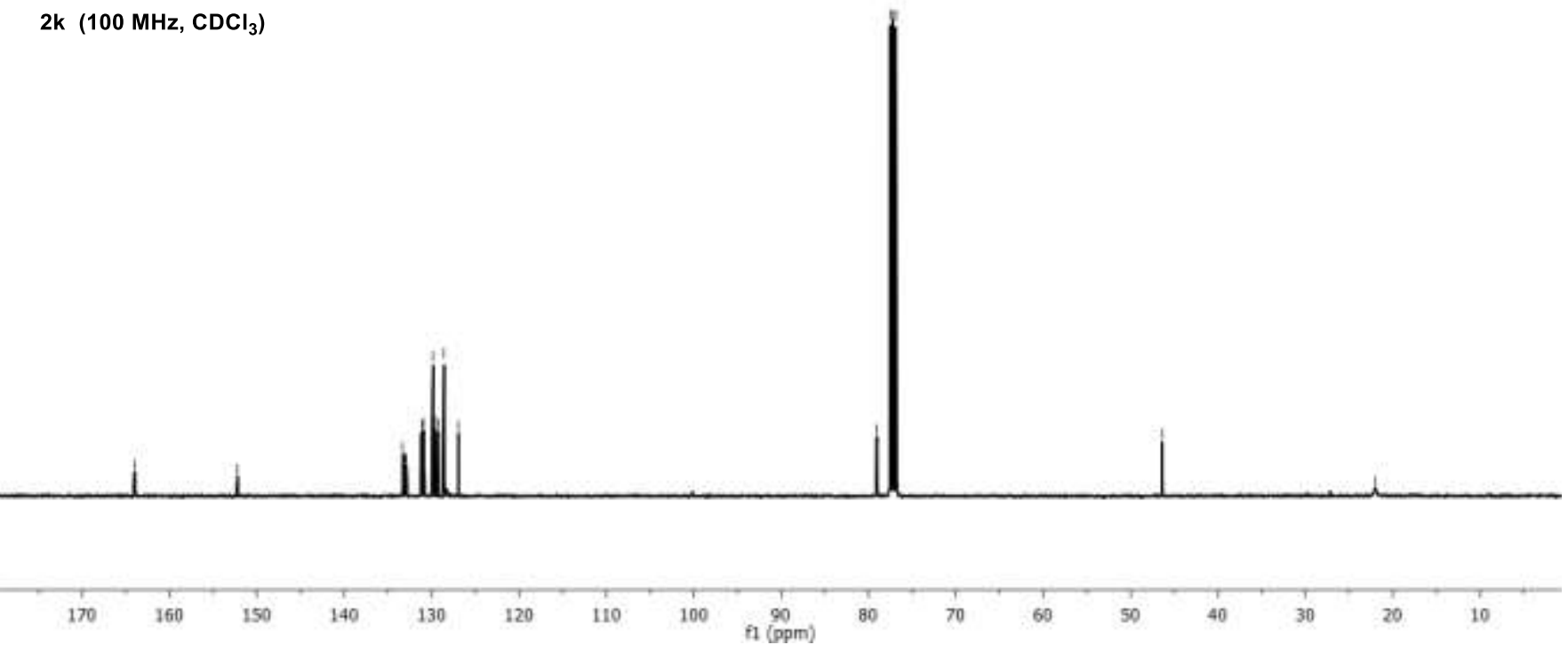


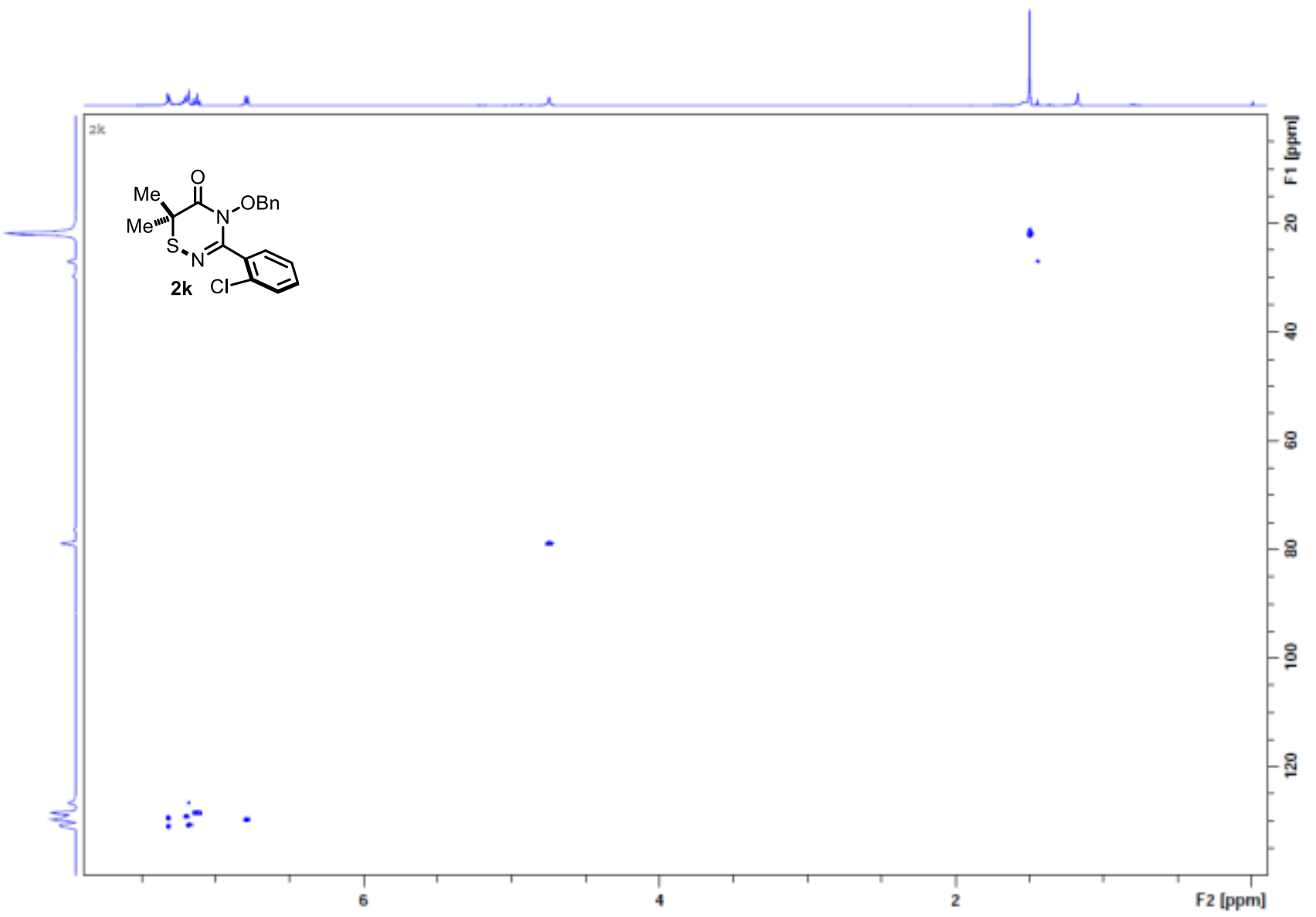

S81 
告)

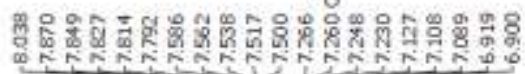

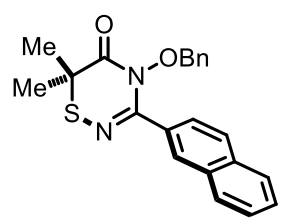

$2 \mathrm{~m}\left(400 \mathrm{MHz}, \mathrm{CDCl}_{3}\right)$
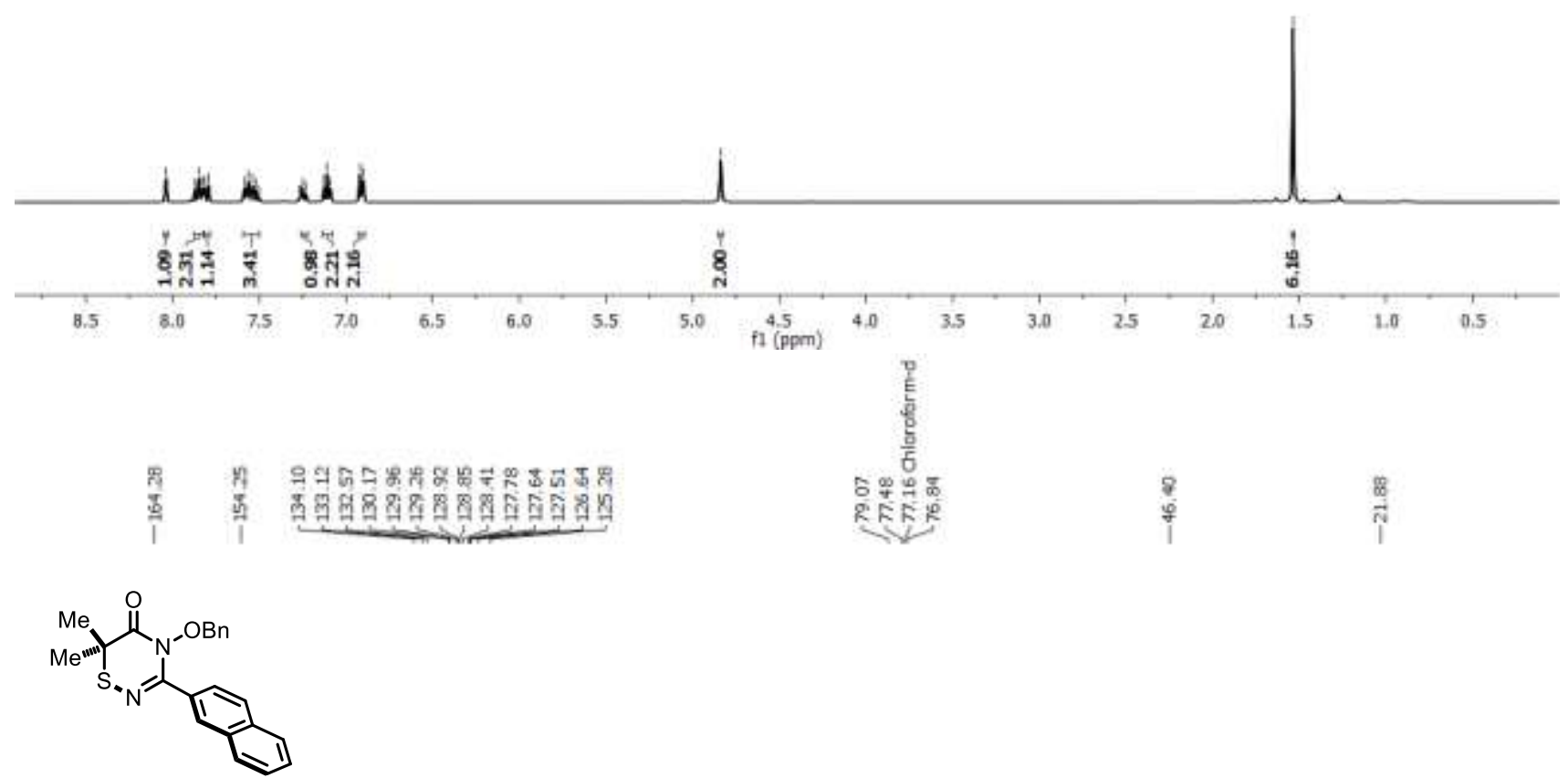

2m $\left(100 \mathrm{MHz}, \mathrm{CDCl}_{3}\right)$

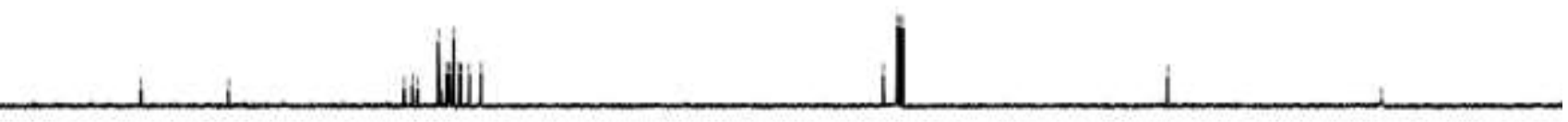

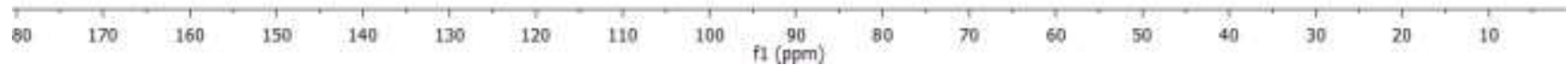




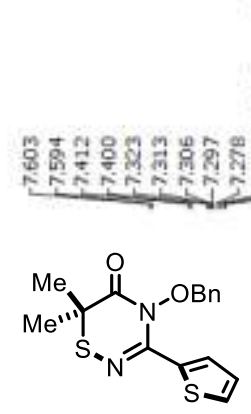

2n $\left(400 \mathrm{MHz}, \mathrm{CDCl}_{3}\right)$
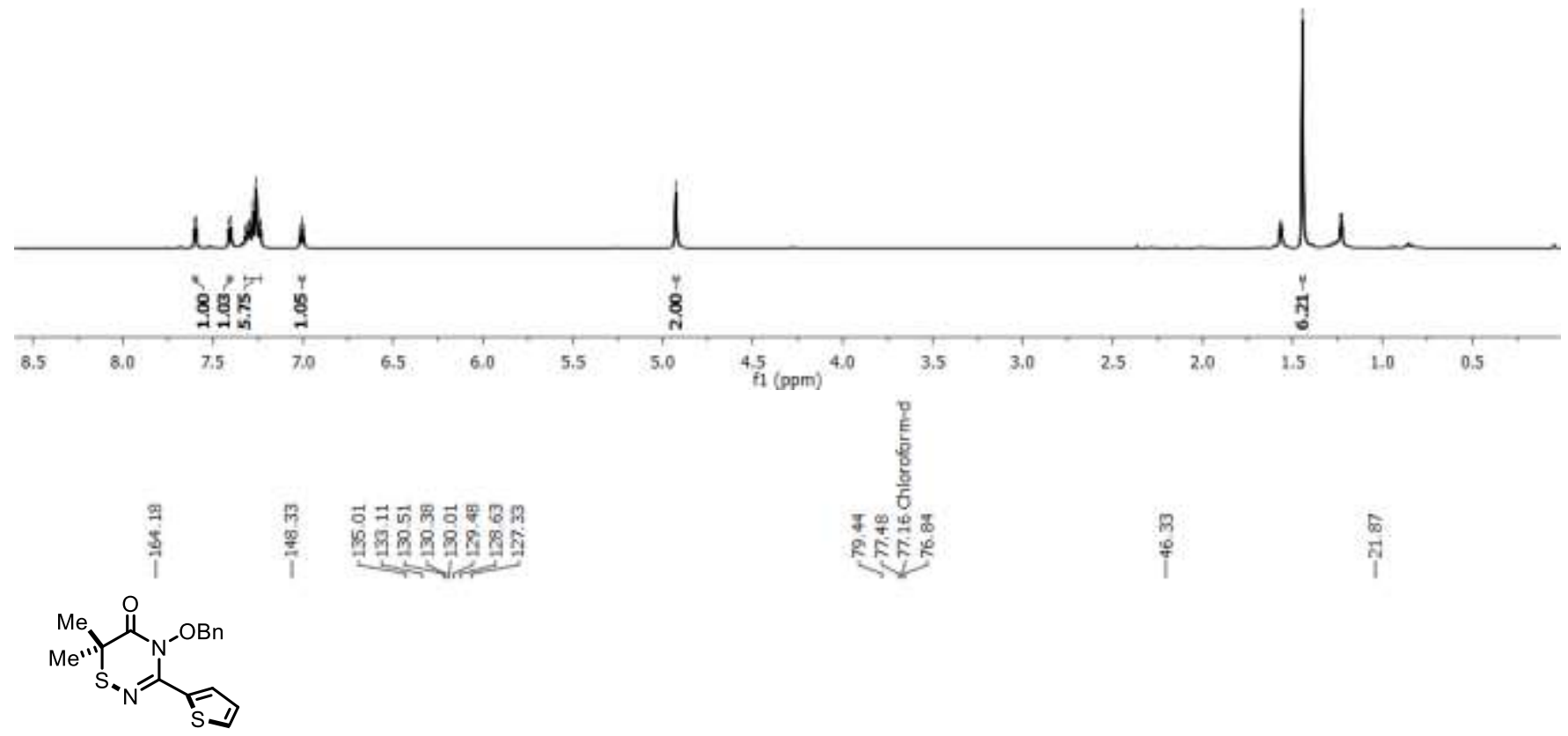

2n (100 $\left.\mathrm{MHz}, \mathrm{CDCl}_{3}\right)$
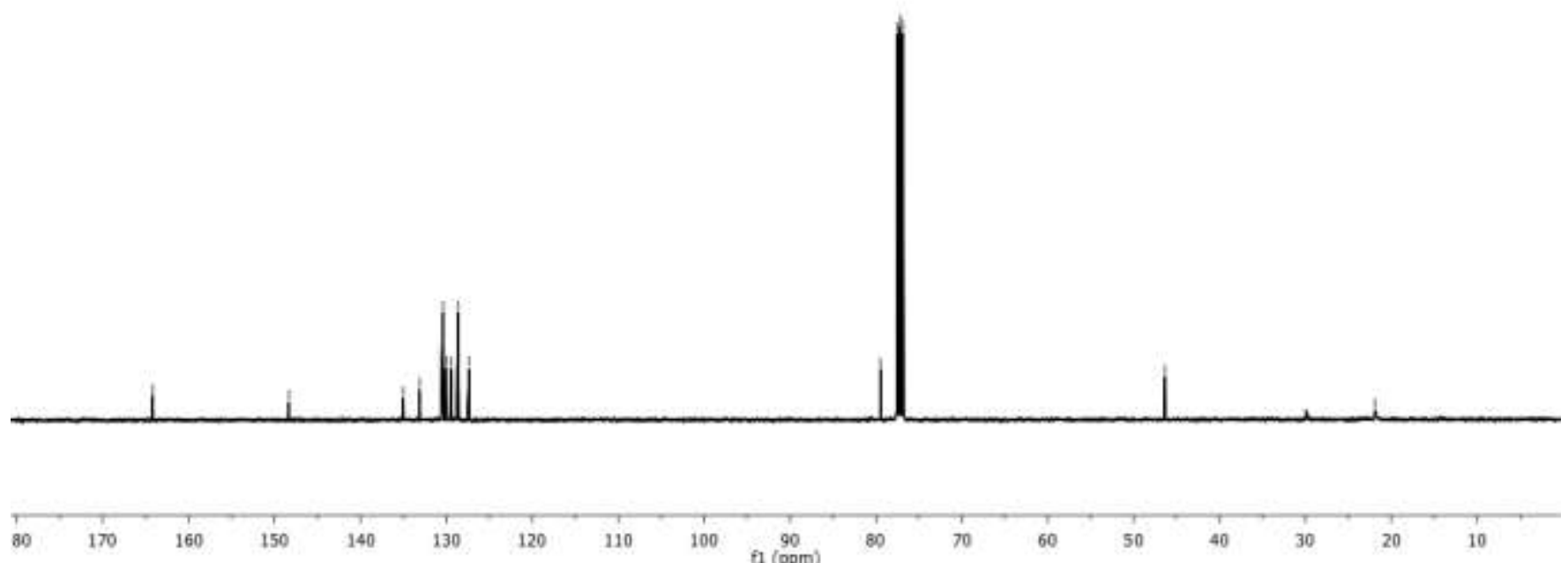

$\frac{8}{4}$

है 


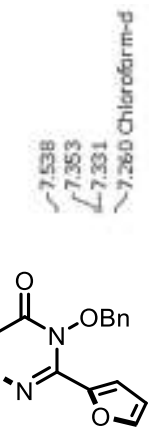

$20\left(400 \mathrm{MHz} \mathrm{CDCl}_{3}\right.$ )
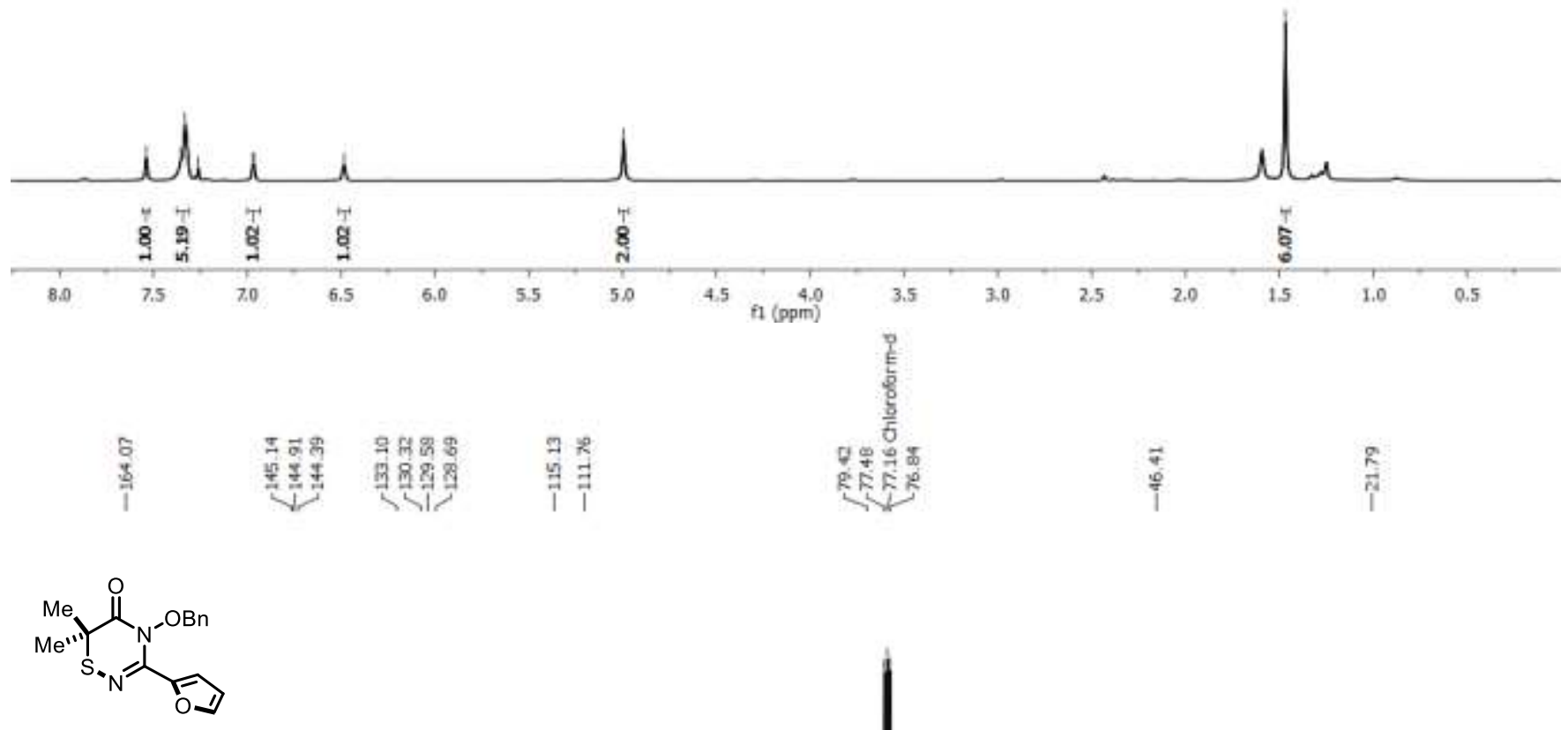

2o $\left(100 \mathrm{MHz}, \mathrm{CDCl}_{3}\right)$
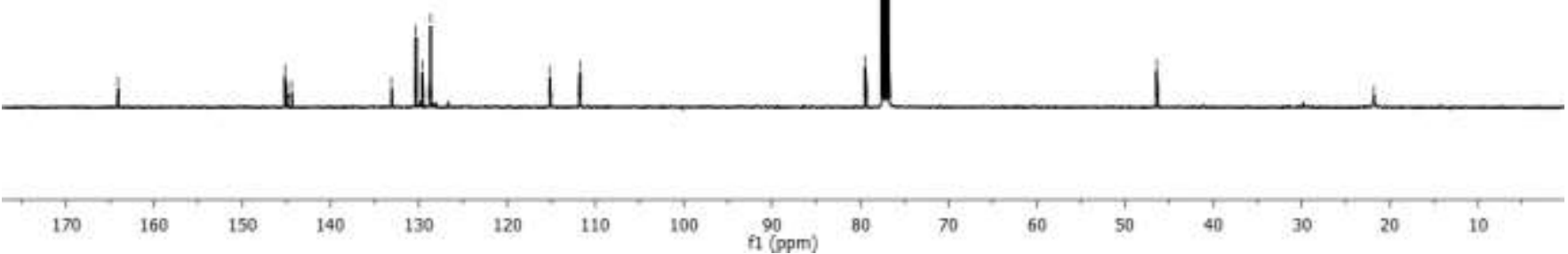


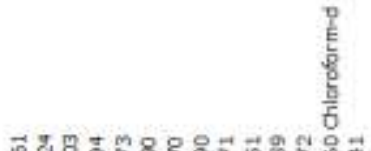

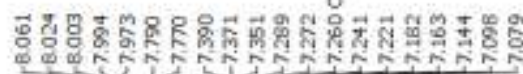

$\stackrel{\text { gू }}{\text { † }}$

$\stackrel{8}{8}$

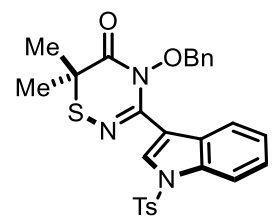

2p $\left(400 \mathrm{MHz}, \mathrm{CDCl}_{3}\right)$

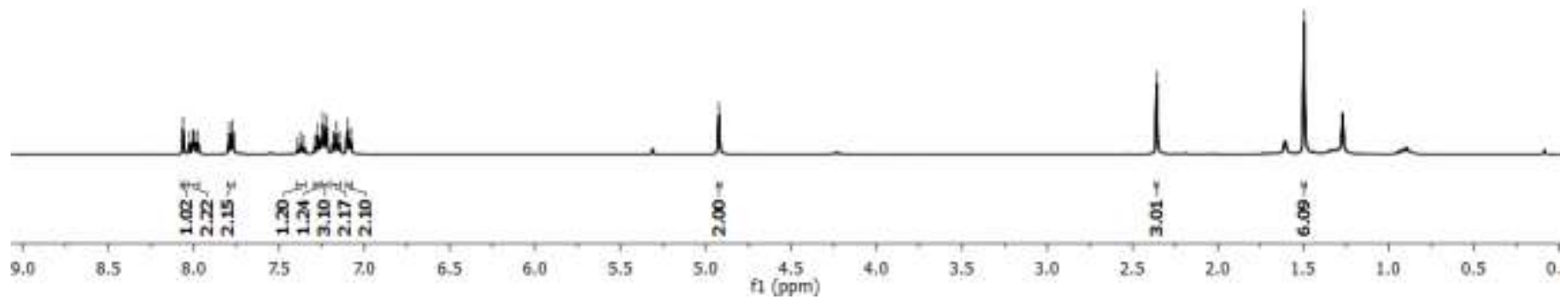

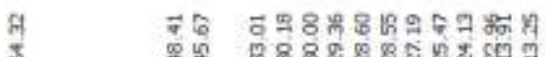

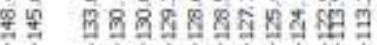
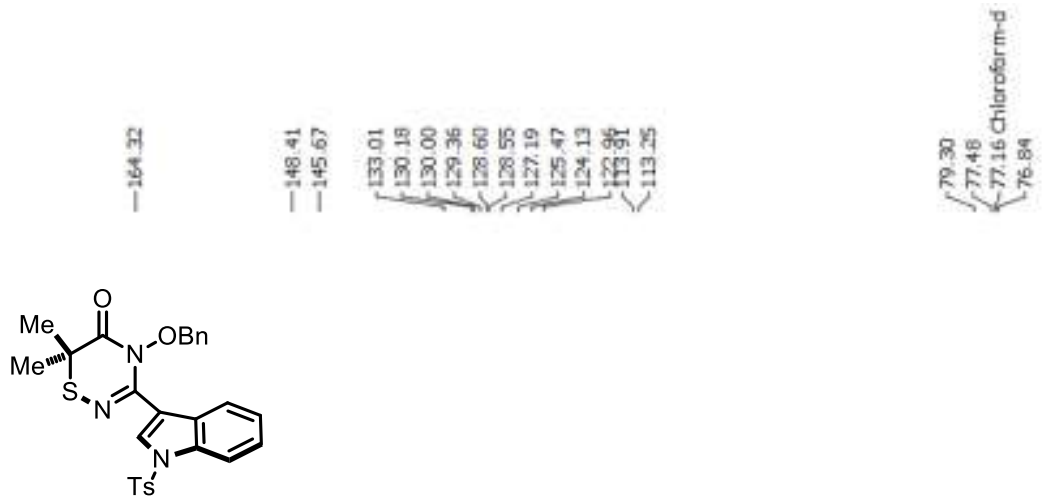

2p $\left(100 \mathrm{MHz}, \mathrm{CDCl}_{3}\right)$

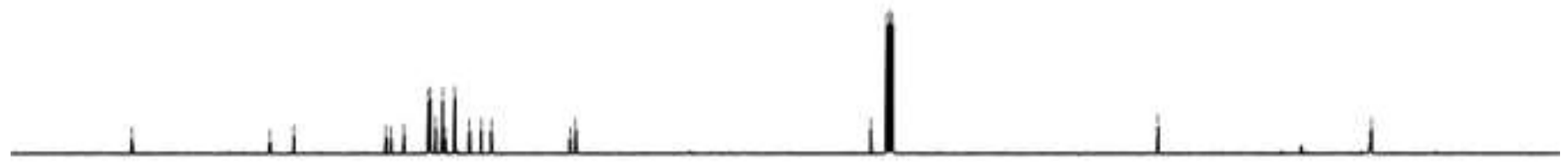

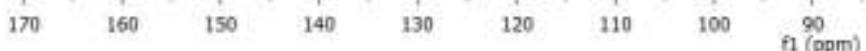

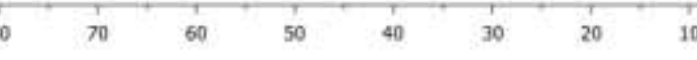



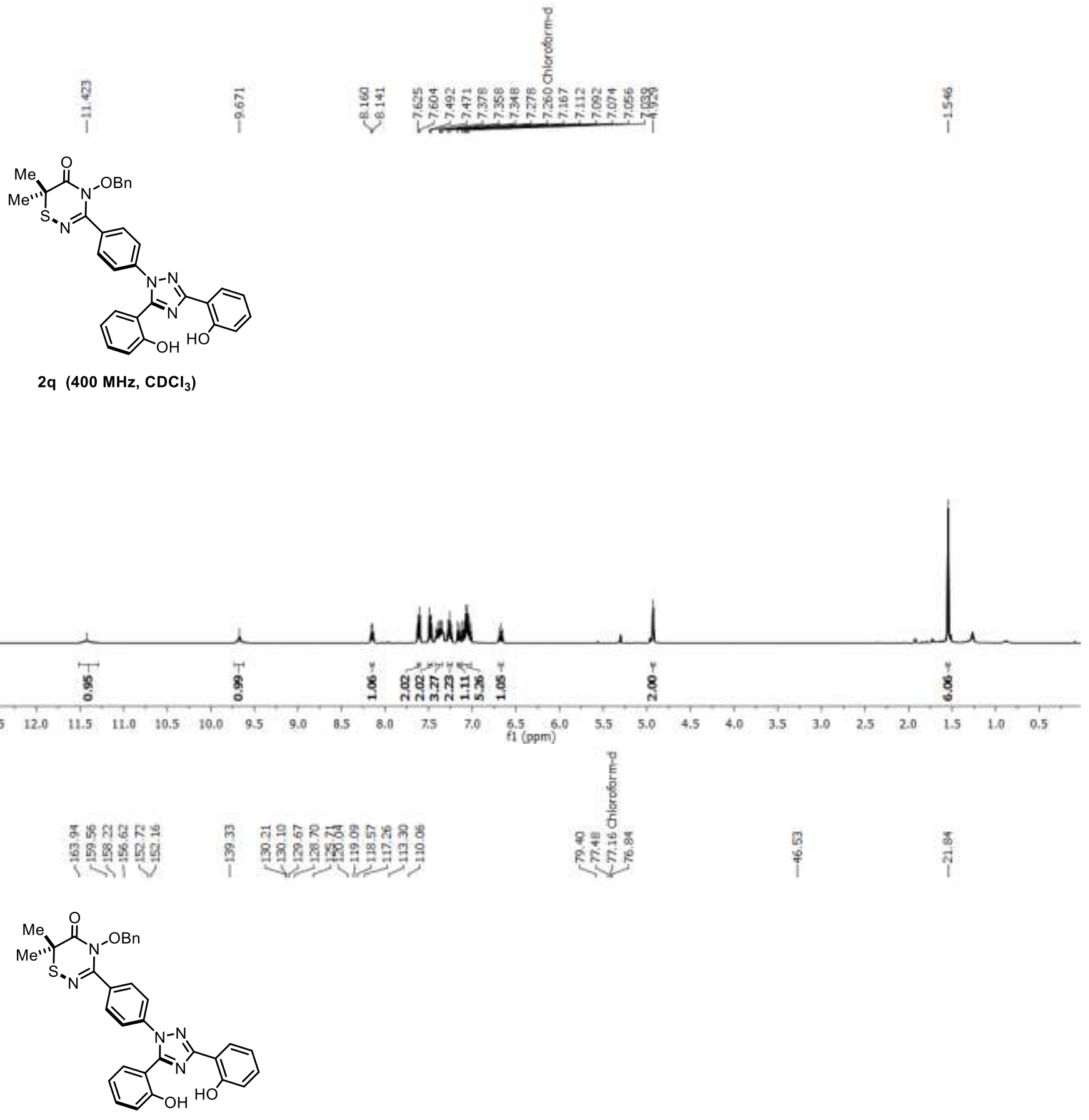

2q $\left(100 \mathrm{MHz}, \mathrm{CDCl}_{3}\right)$

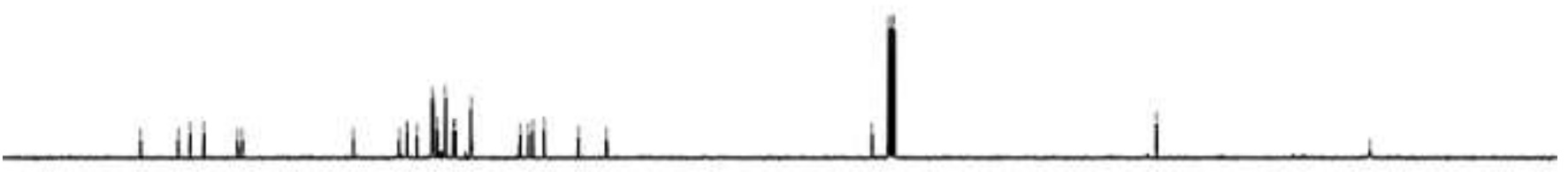

$\begin{array}{lllllllll}170 & 160 & 150 & 140 & 130 & 120 & 110 & 100 & 90 \\ f 1(\mathrm{ppm})\end{array}$

$\begin{array}{llllllll}80 & 70 & 60 & 50 & 40 & 30 & 20 & 10\end{array}$ 


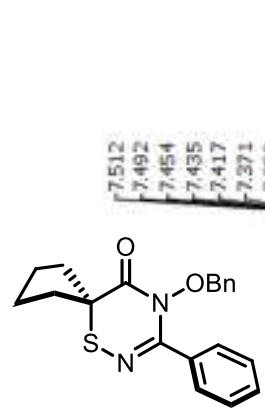

3a $\left(400 \mathrm{MHz}, \mathrm{CDCl}_{3}\right)$

3a $\left(400 \mathrm{MHz}, \mathrm{CDCl}_{3}\right)$
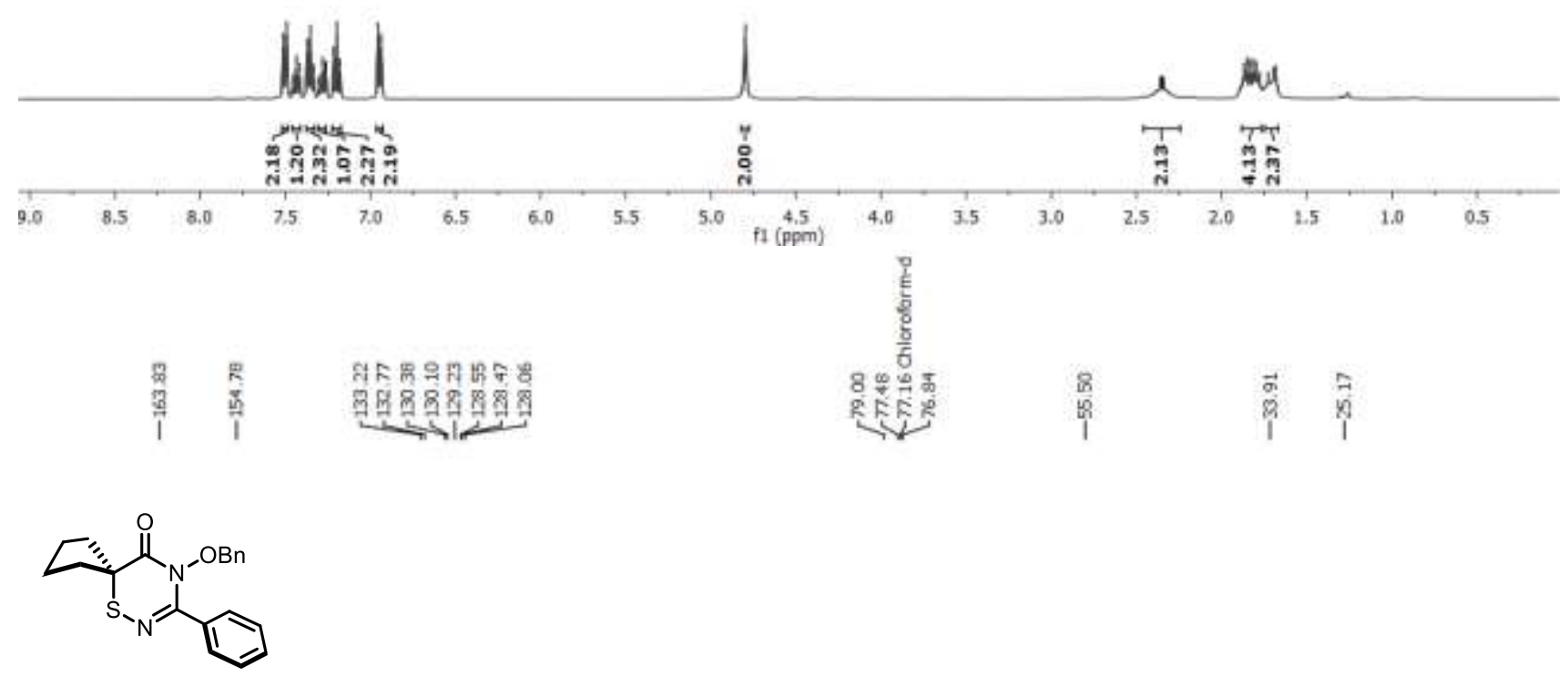

3a $\left(100 \mathrm{MHz}, \mathrm{CDCl}_{3}\right)$

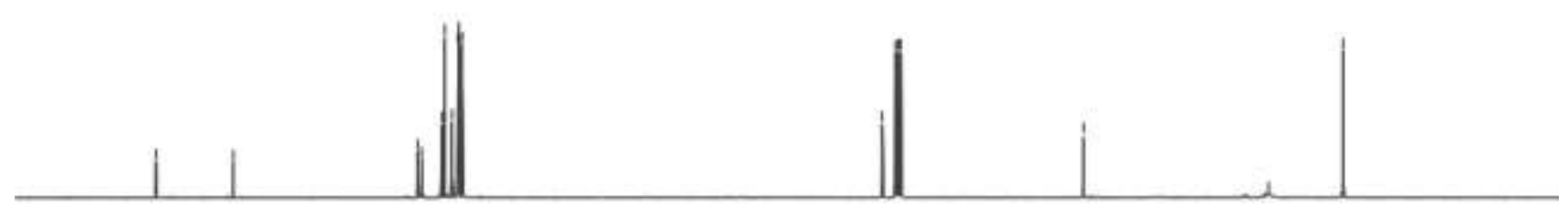

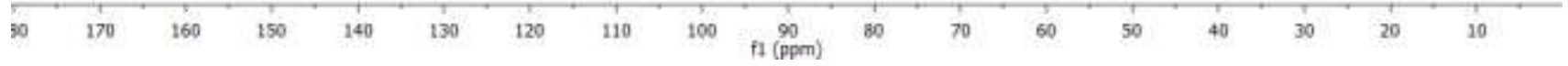




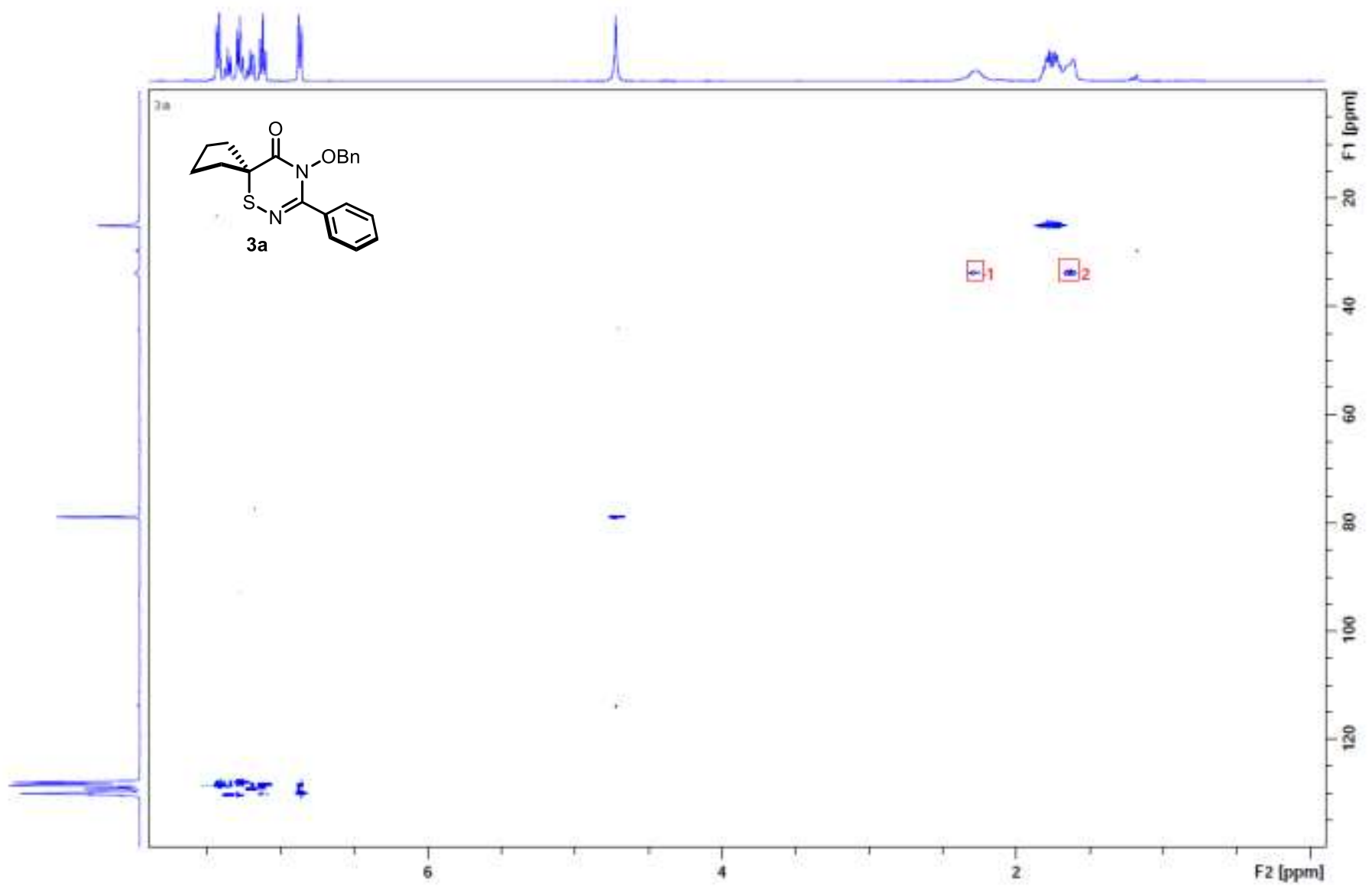

S88 
$\frac{1}{\frac{5}{5}}$

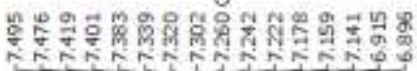

$\stackrel{\text { ng }}{\Upsilon}$

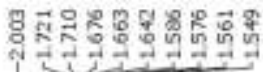<smiles>CCCC1SN=C(c2ccccc2)N(OCc2ccccc2)C1=O</smiles>

3b $\left(400 \mathrm{MHz}, \mathrm{CDCl}_{3}\right)$

Wilid
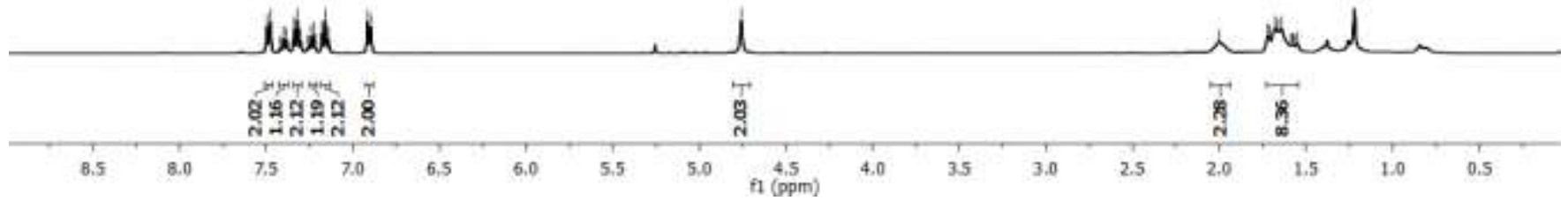

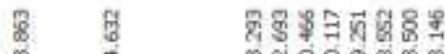

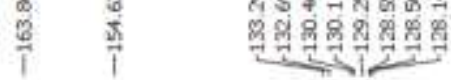

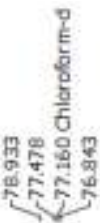

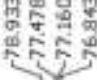

ตุ.

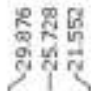

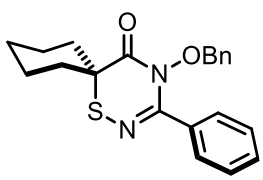

3b $\left(100 \mathrm{MHz}, \mathrm{CDCl}_{3}\right)$

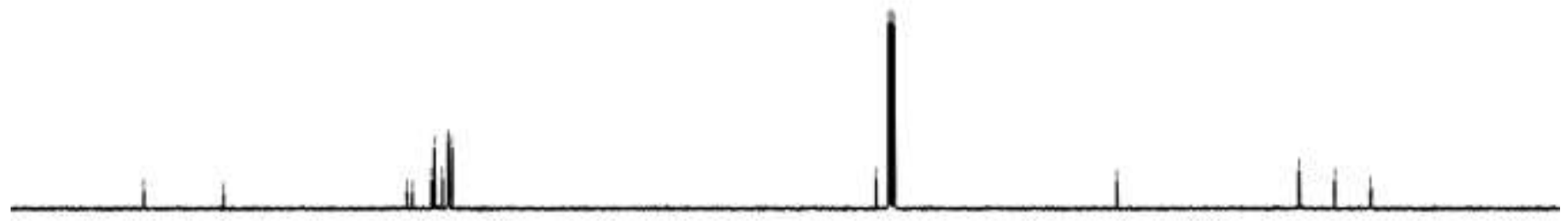

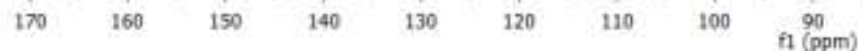

$\begin{array}{lllllllll}80 & 70 & 60 & 50 & 40 & 30 & 20 & 10 & 0\end{array}$



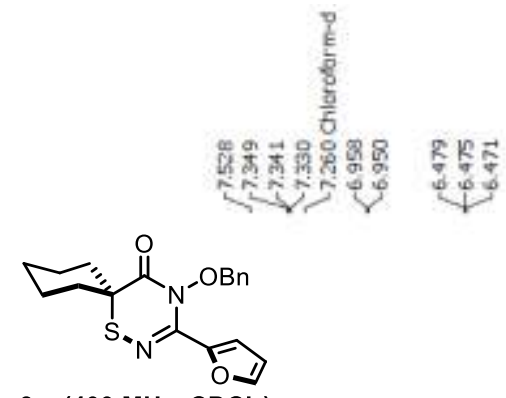

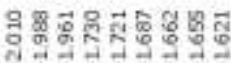

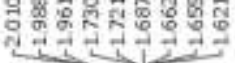

3c $\left(400 \mathrm{MHz}, \mathrm{CDCl}_{3}\right)$

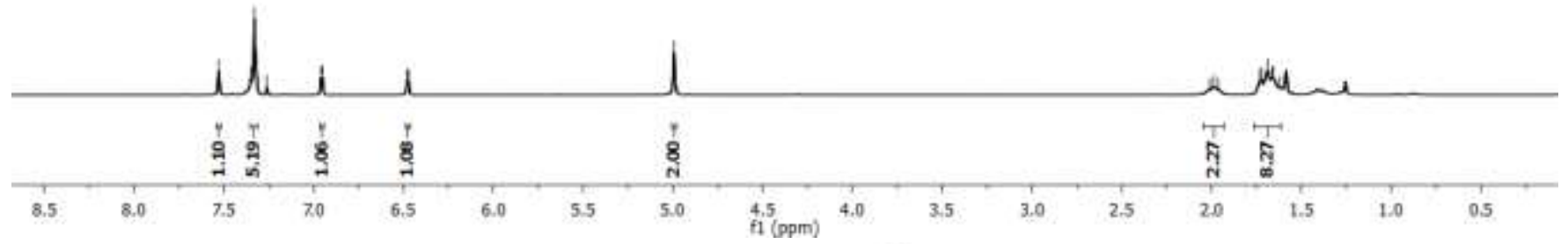

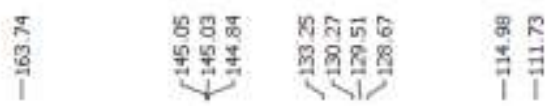

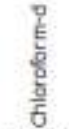

尊

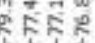

i

$2 \sin$

ชูํํㄹ

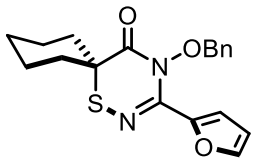

3c $\left(100 \mathrm{MHz}, \mathrm{CDCl}_{3}\right)$

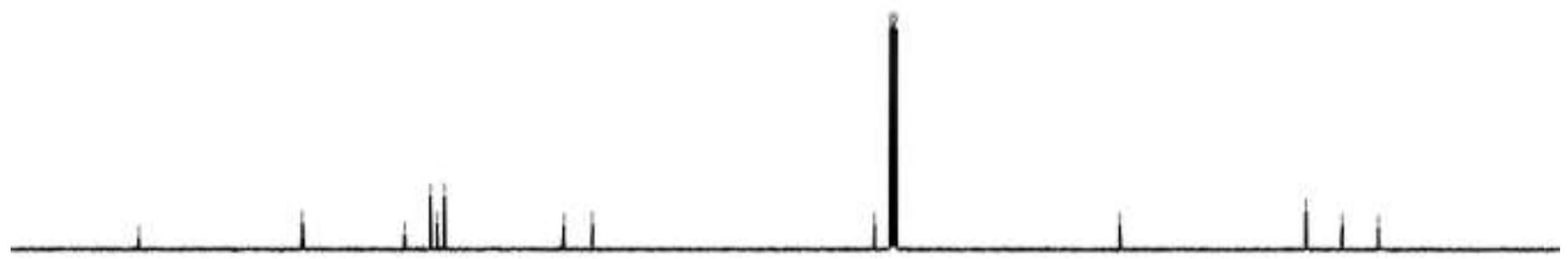

$\begin{array}{lllllllll}170 & 160 & 150 & 140 & 130 & 120 & 110 & 100 & 90\end{array}$

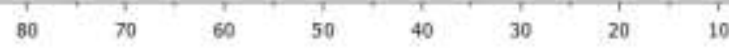

10 

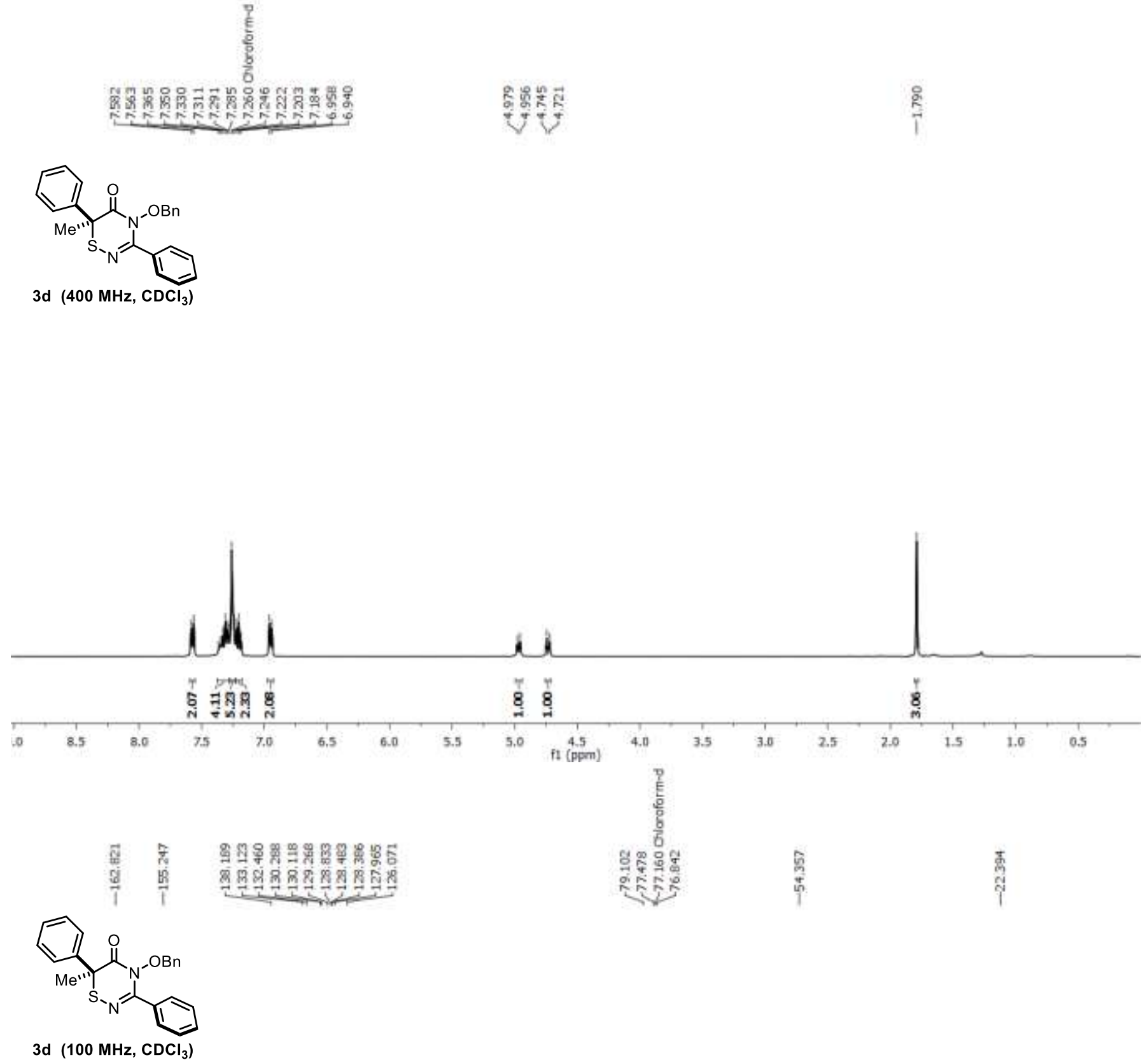

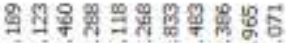

ติ

d $\left(100 \mathrm{MHz}, \mathrm{CDCl}_{3}\right)$
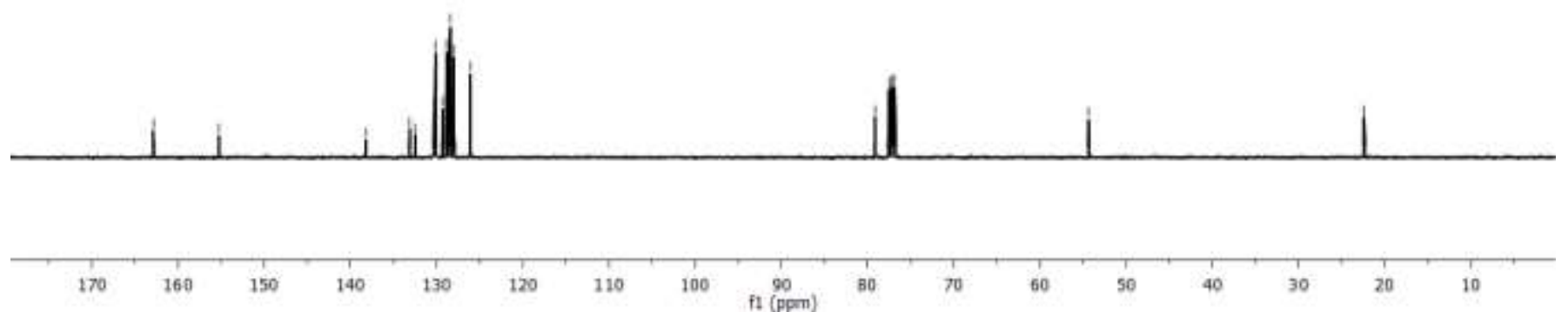


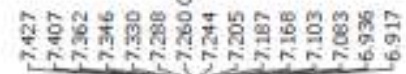

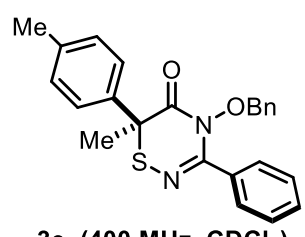

3e $\left(400 \mathrm{MHz}, \mathrm{CDCl}_{3}\right)$
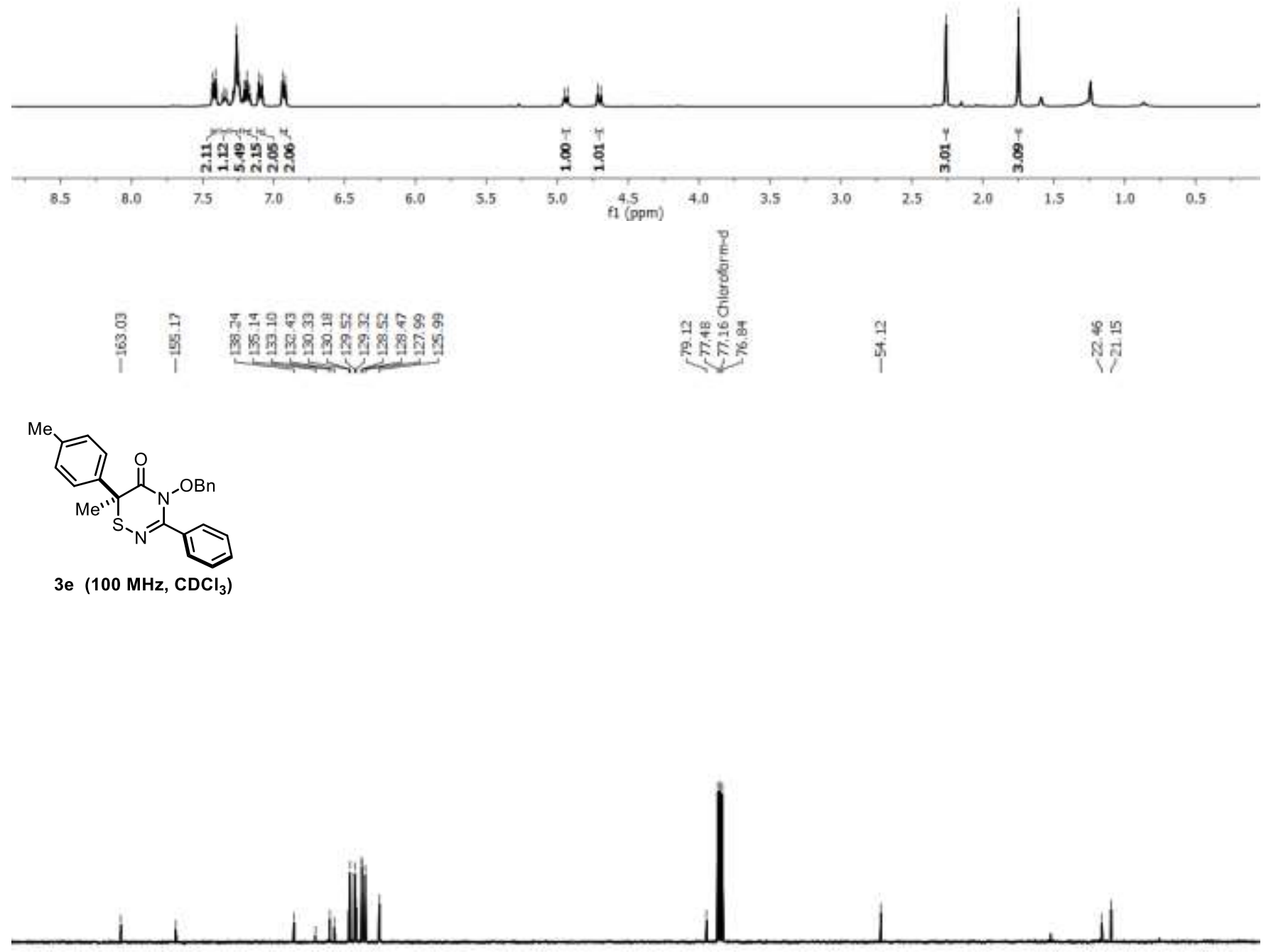

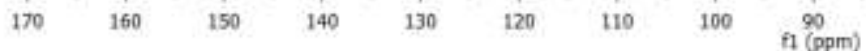

$\begin{array}{llllllll}70 & 60 & 50 & 40 & 30 & 20 & 10 & 6\end{array}$




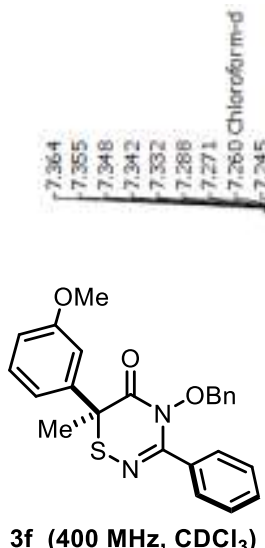

3f (400 $\mathrm{MHz}, \mathrm{CDCl}_{3}$ )
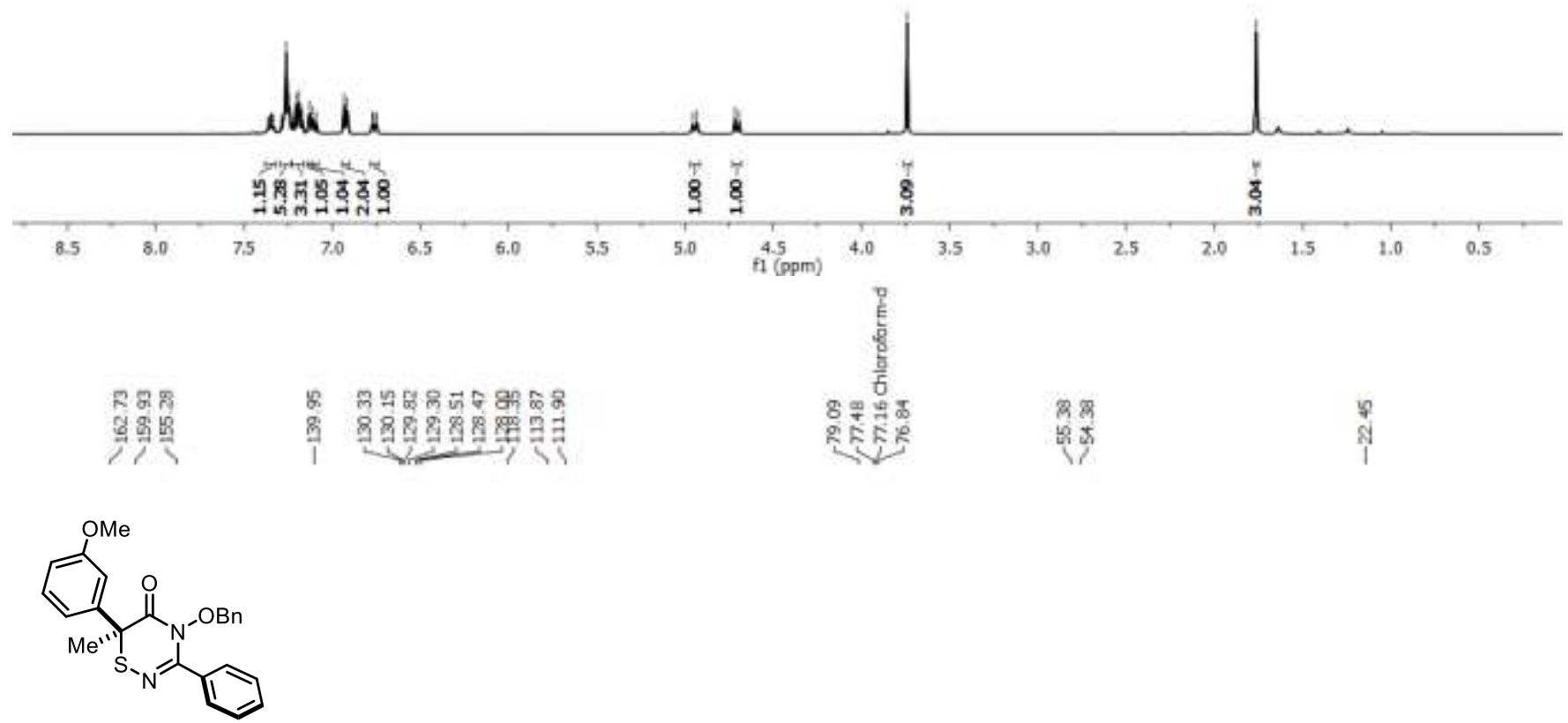

3f $\left(100 \mathrm{MHz}, \mathrm{CDCl}_{3}\right)$ 


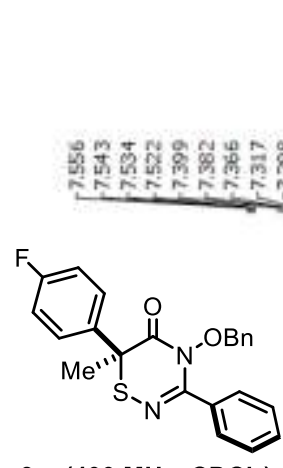

$3 g\left(400 \mathrm{MHz}, \mathrm{CDCl}_{3}\right)$

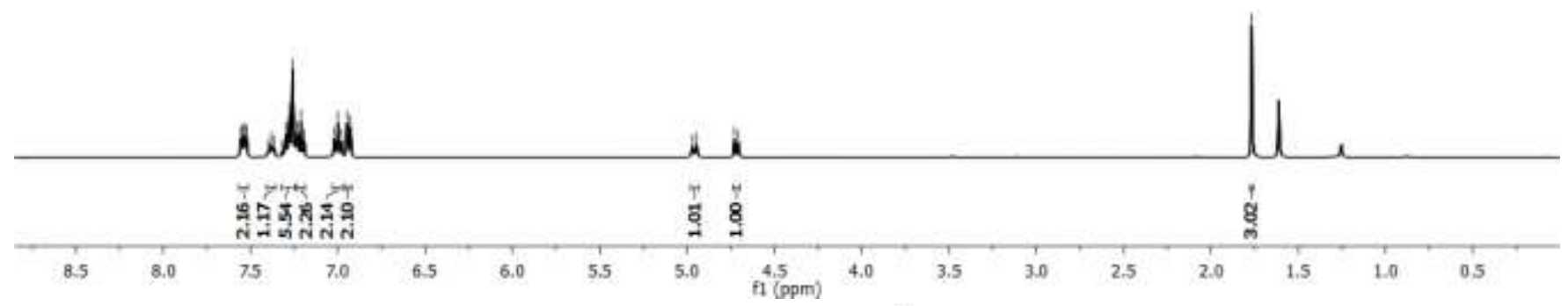

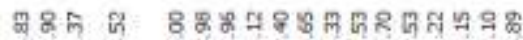

운웅
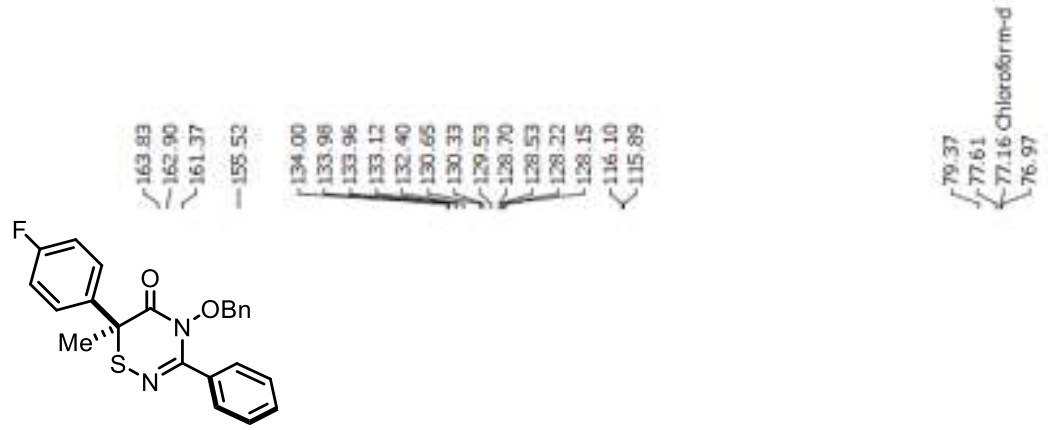

$3 g\left(100 \mathrm{MHz}, \mathrm{CDCl}_{3}\right)$
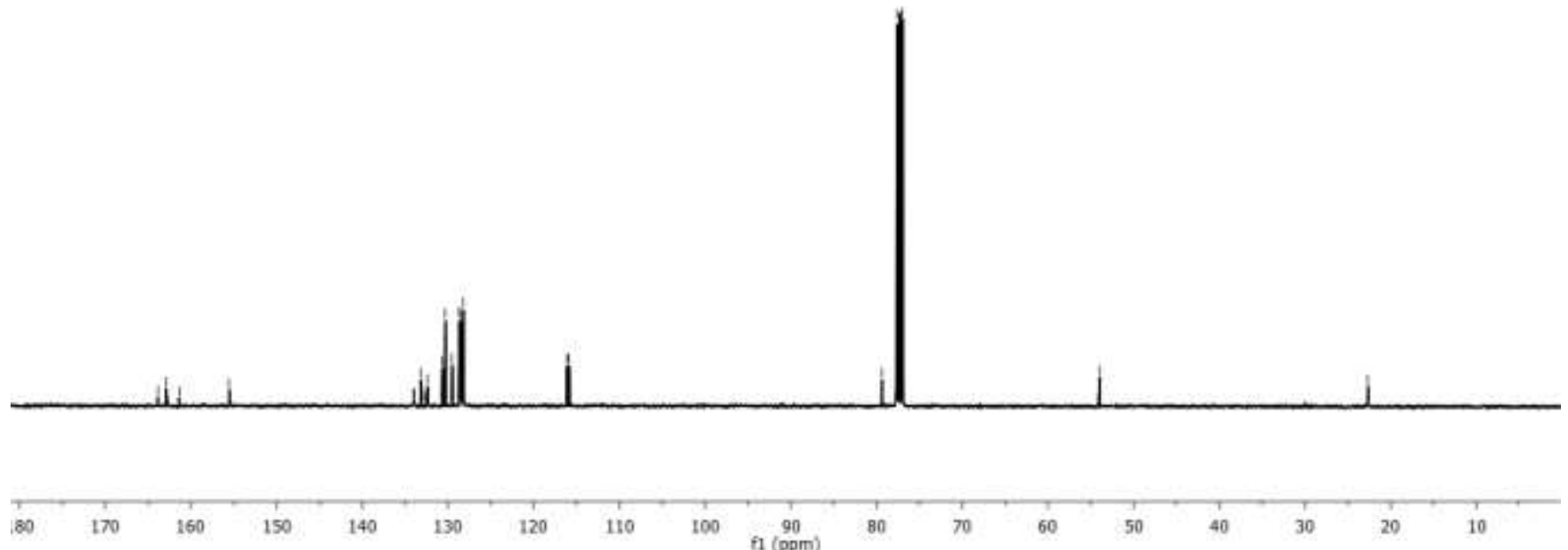

\$

8 


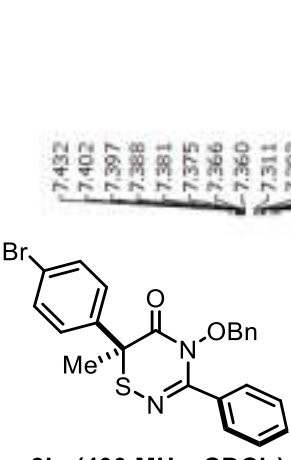

3h (400 $\mathrm{MHz}, \mathrm{CDCl}_{3}$ )
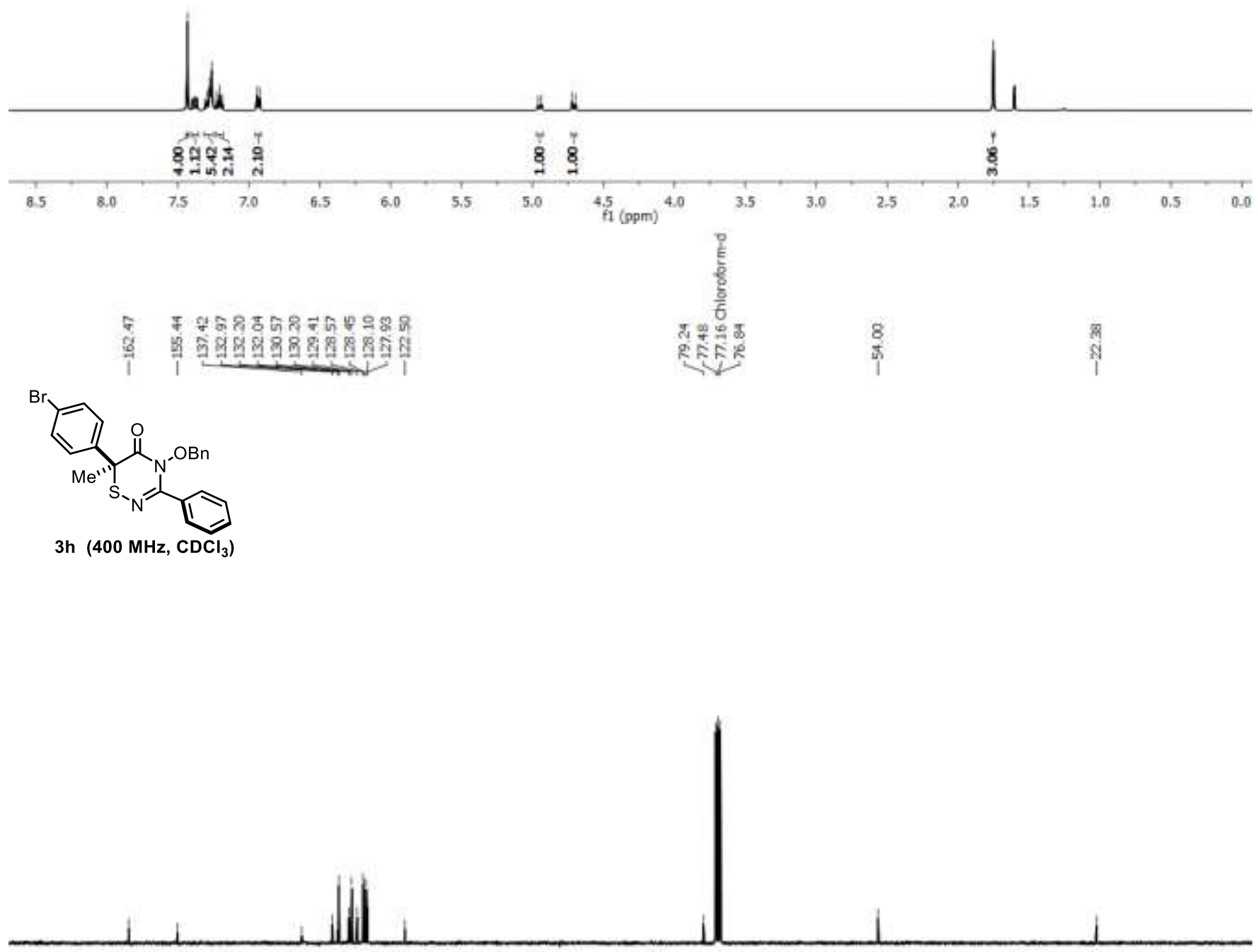

$\begin{array}{lllllllll}170 & 160 & 150 & 140 & 130 & 120 & 110 & 100 & 90 \\ 61(\mathrm{pm})\end{array}$

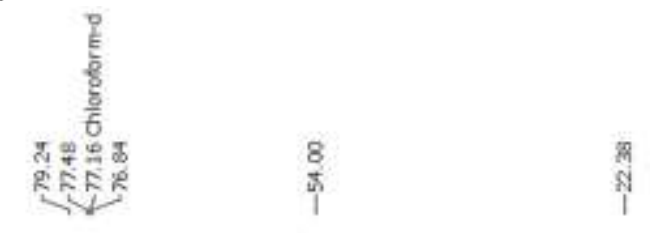

$3 \mathrm{~h}\left(400 \mathrm{MHz}, \mathrm{CDCl}_{3}\right)$ 


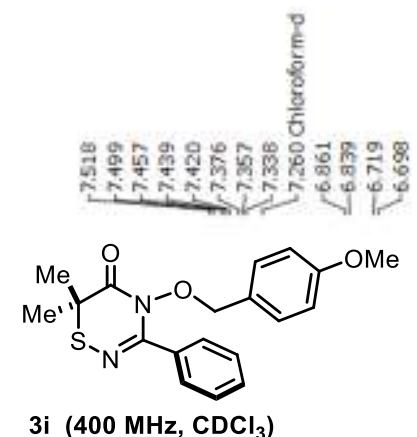

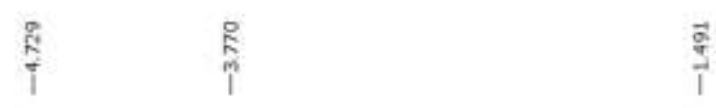

3i $\left(400 \mathrm{MHz}, \mathrm{CDCl}_{3}\right)$

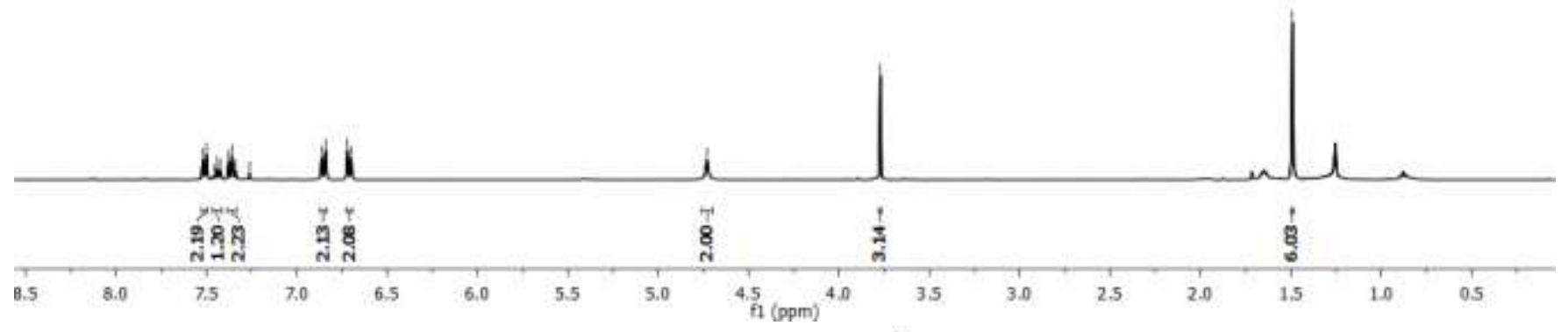

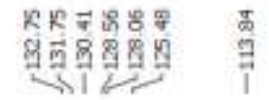

$\frac{1}{\frac{1}{8}}$

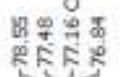

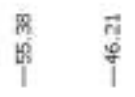

$\frac{8}{7}$<smiles>COc1ccc(CON2C(=O)C(N)(N)SN=C2c2ccccc2)cc1</smiles>

3i $\left(100 \mathrm{MHz}, \mathrm{CDCl}_{3}\right)$

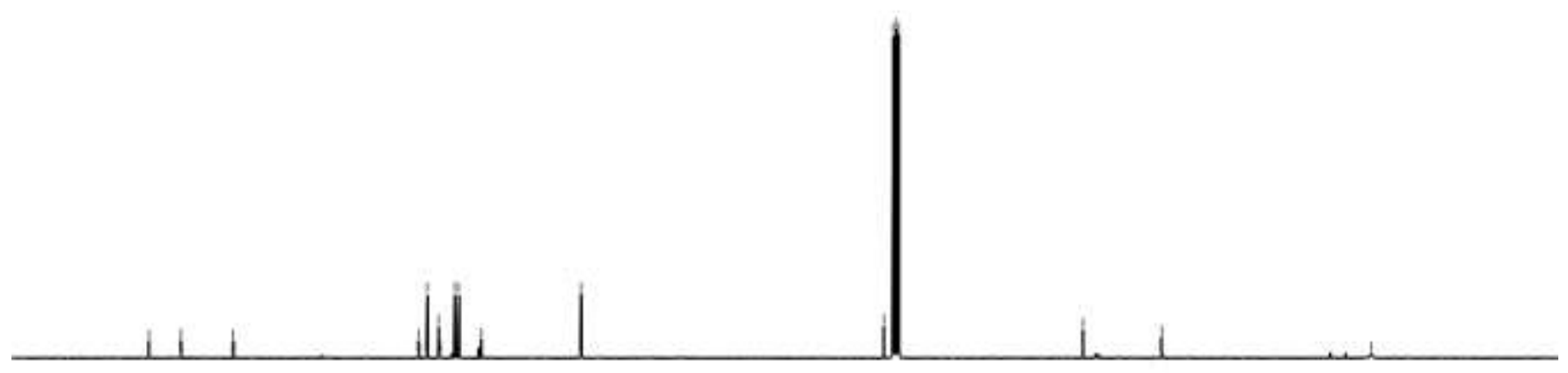

$\begin{array}{lllllllll}170 & 160 & 150 & 140 & 130 & 120 & 110 & 100 & 90 \\ 11(\mathrm{ppm})\end{array}$

80

70

$60 \quad 50$

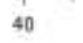




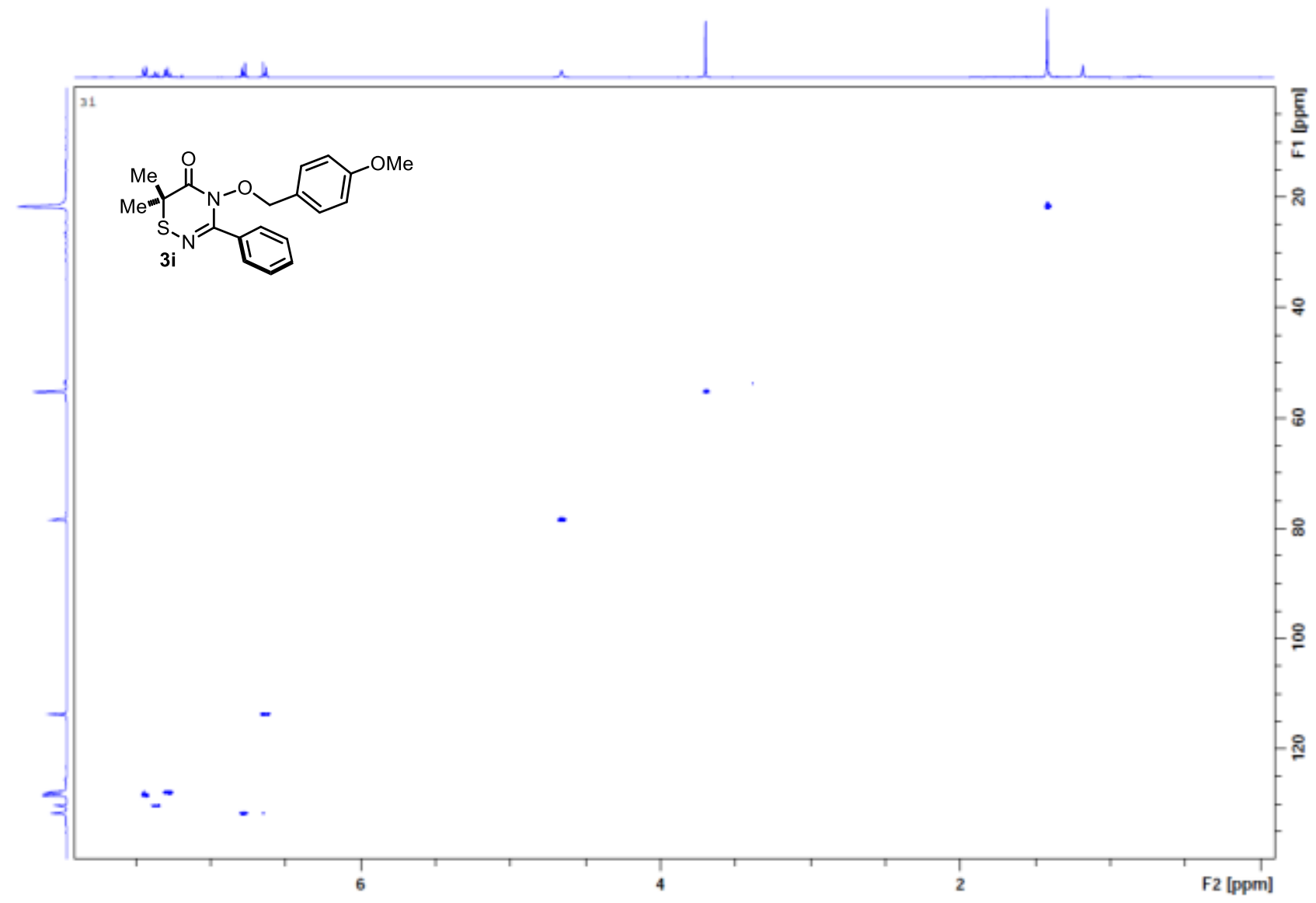



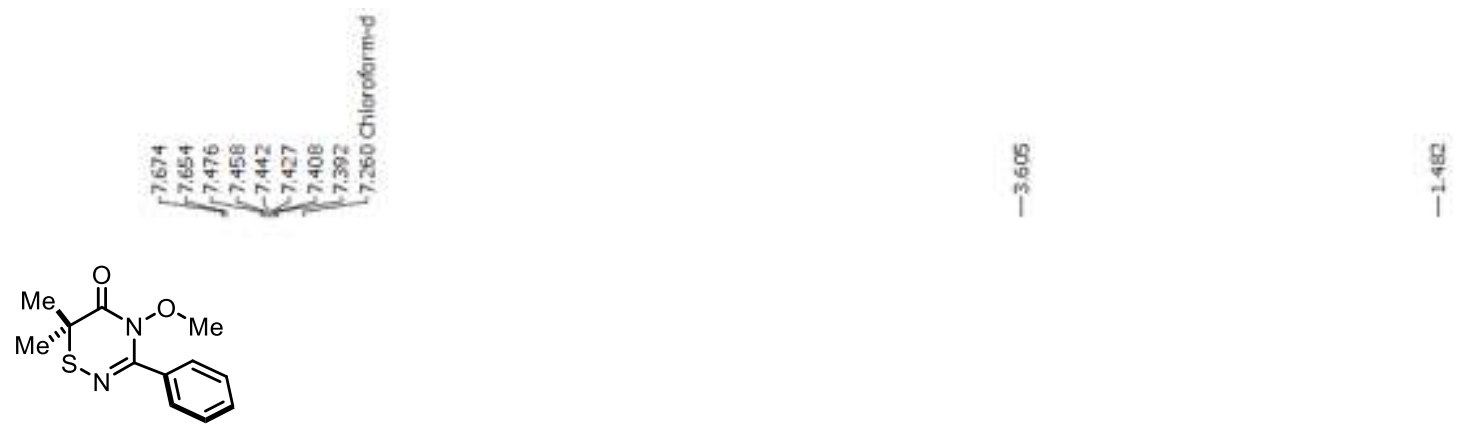

3j (400 MHz, $\mathrm{CDCl}_{3}$ )
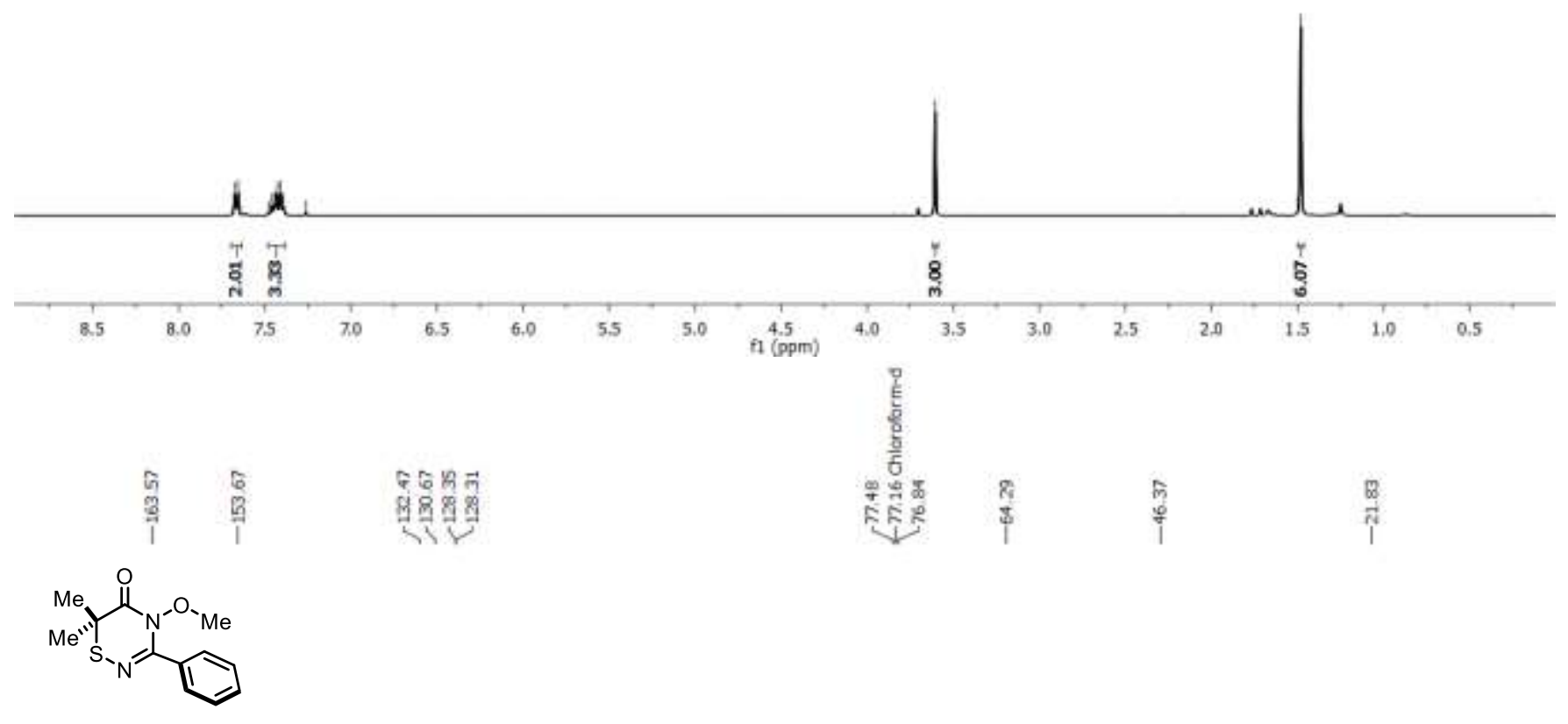

3j $\left(100 \mathrm{MHz}, \mathrm{CDCl}_{3}\right)$
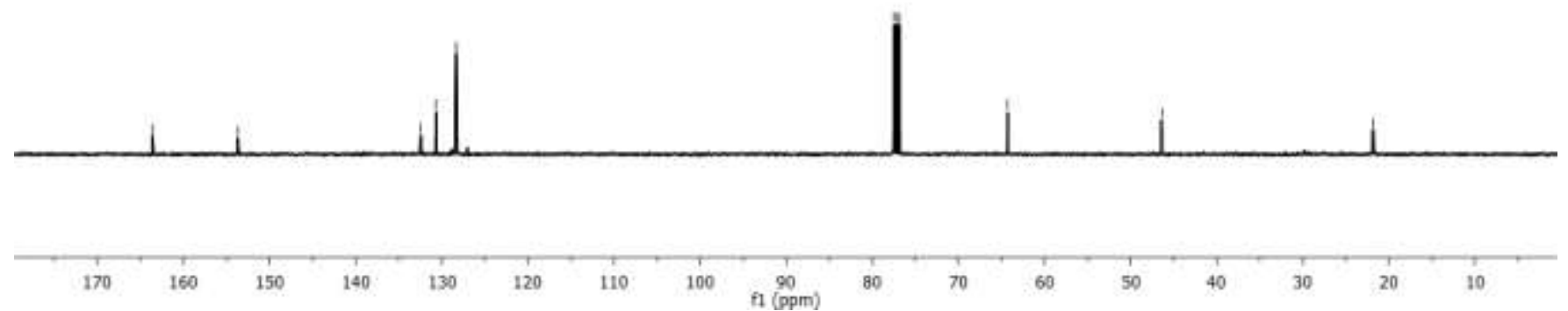


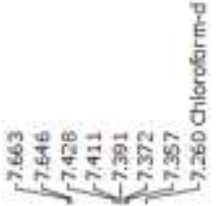

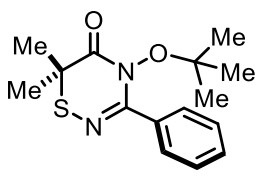

3k $\left(400 \mathrm{MHz}, \mathrm{CDCl}_{3}\right)$

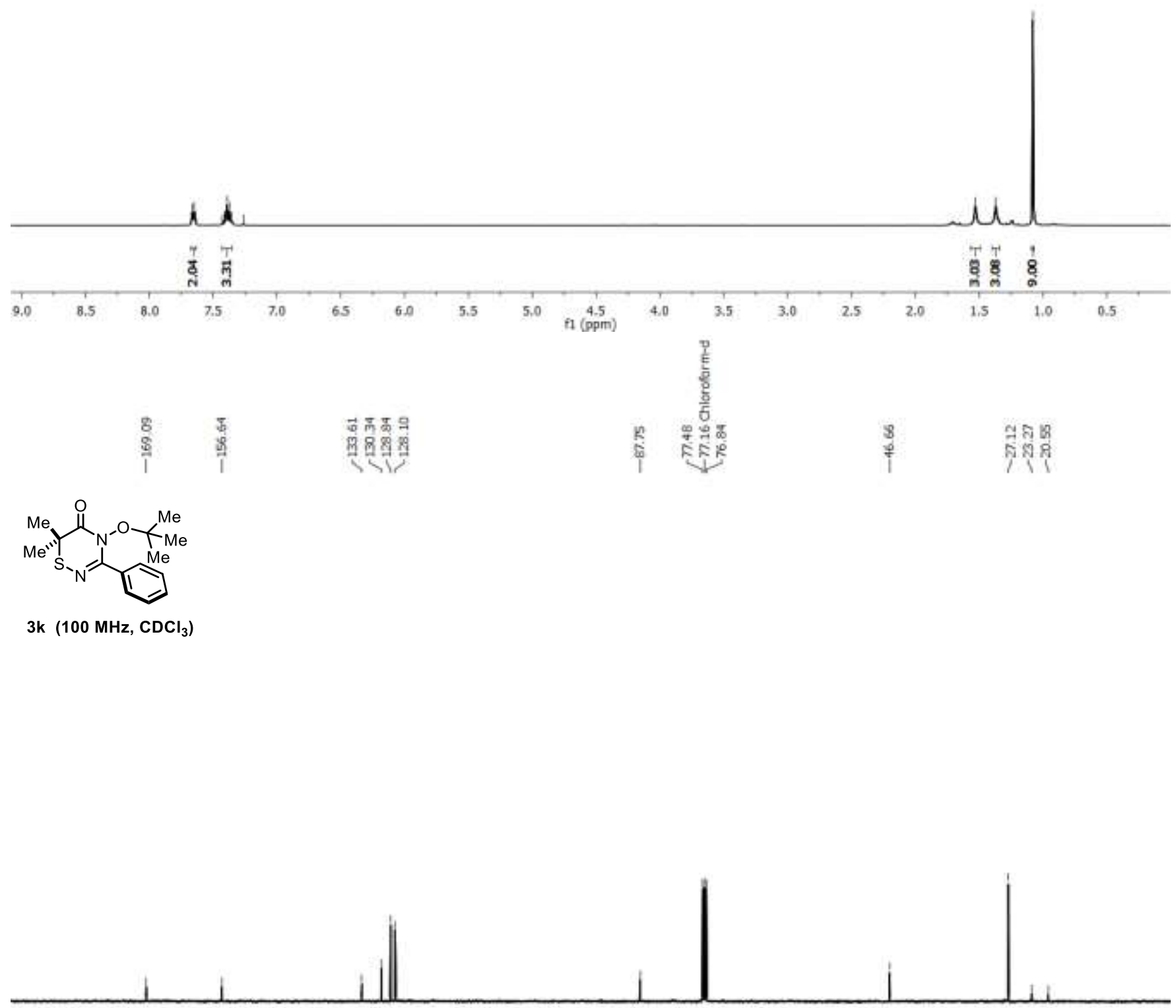

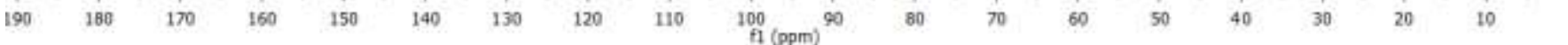




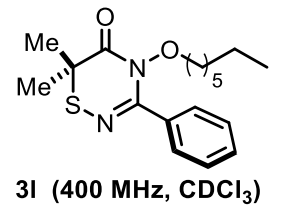

3) $\left(400 \mathrm{MHz}, \mathrm{CDCl}_{3}\right)$

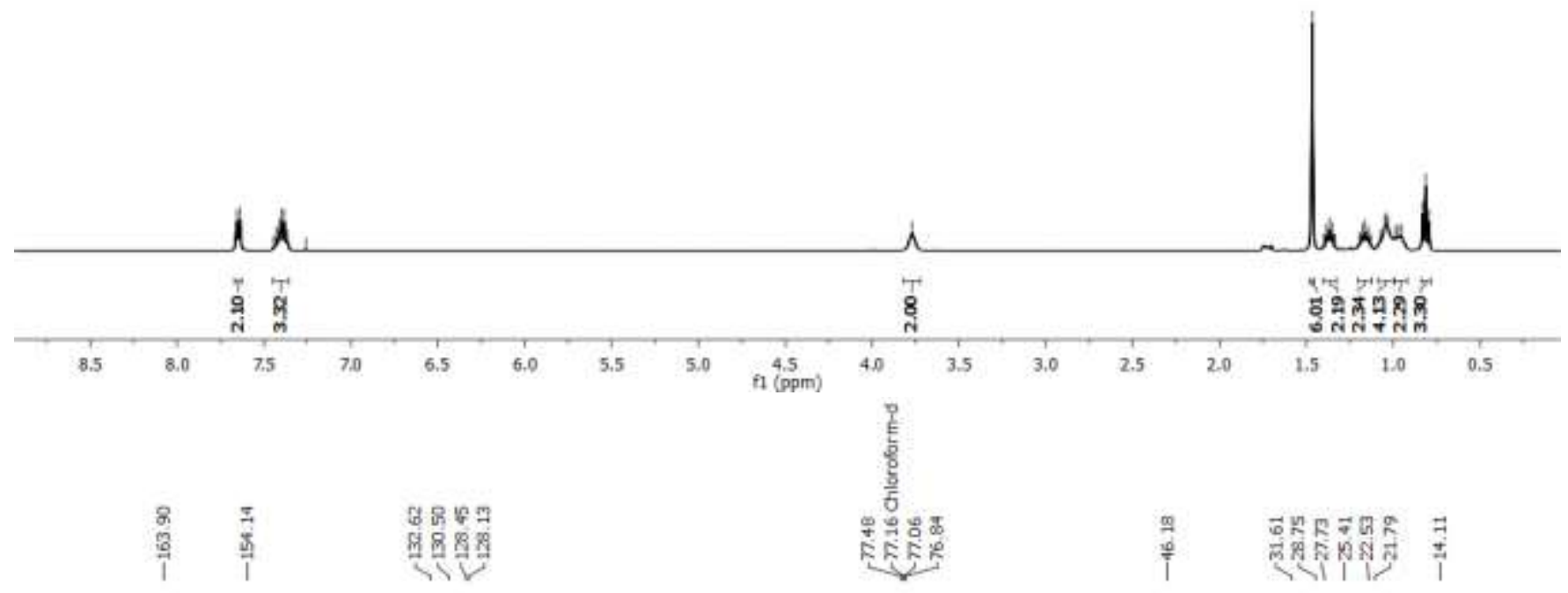<smiles>CCCON1C(=O)C(C)(C)SN=C1c1ccccc1</smiles>

31 (100 $\left.\mathrm{MHz}, \mathrm{CDCl}_{3}\right)$
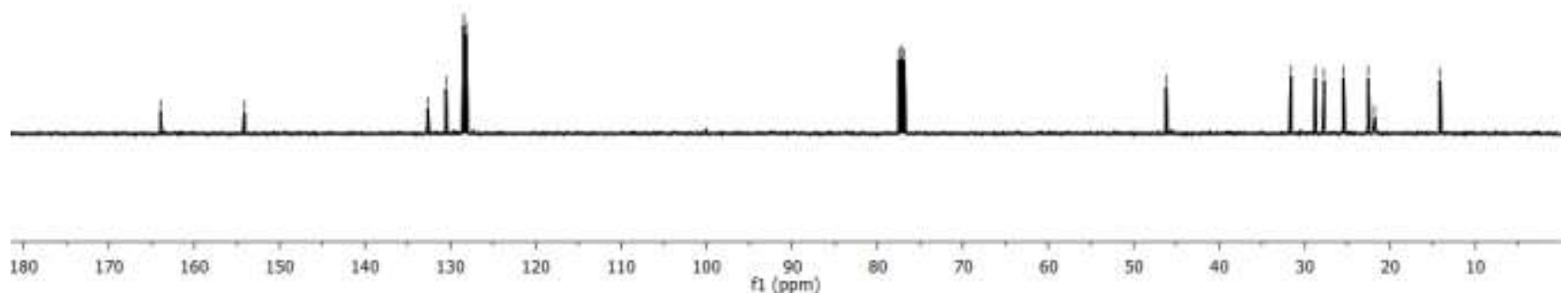

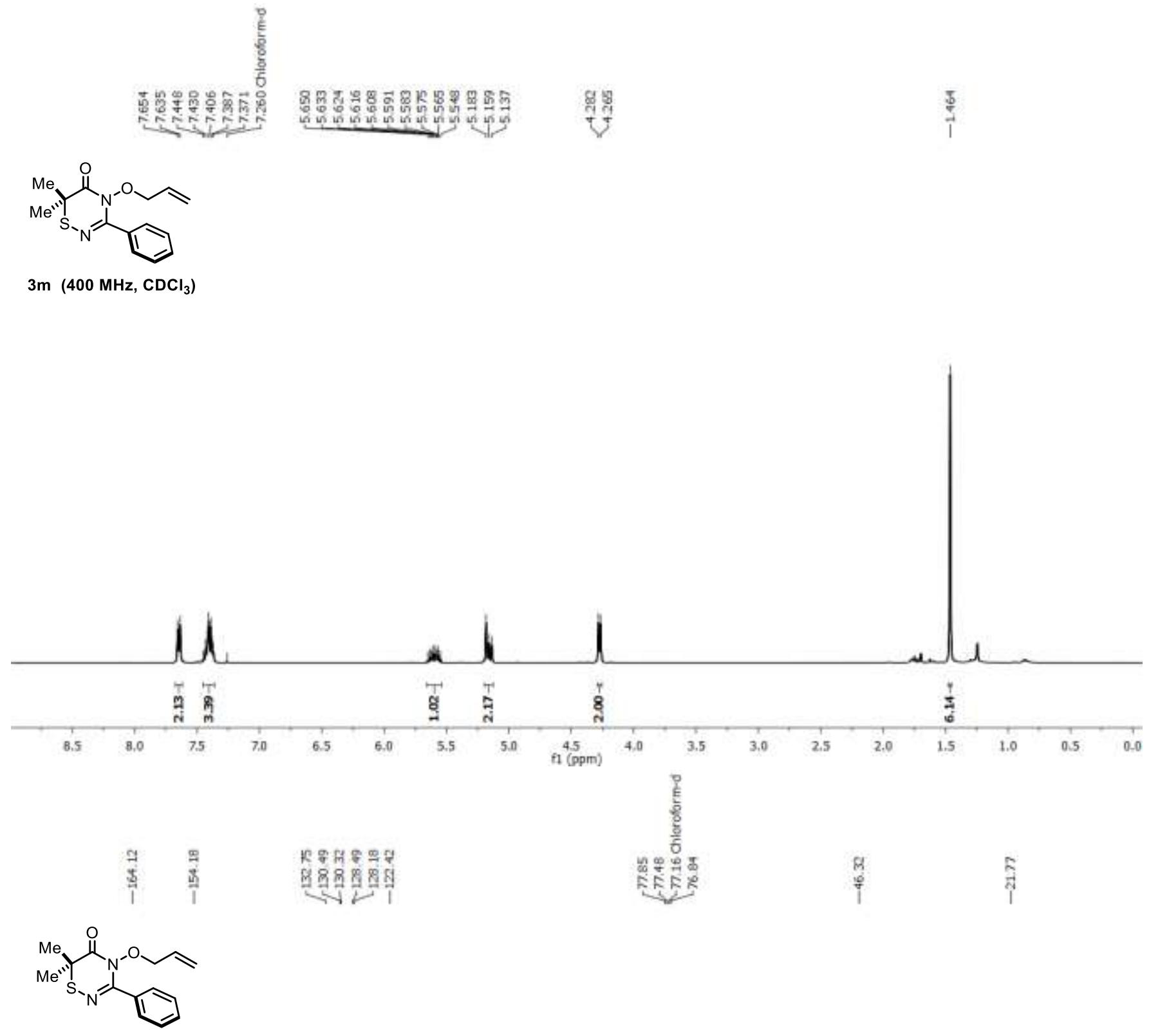

3m $\left(100 \mathrm{MHz}, \mathrm{CDCl}_{3}\right)$

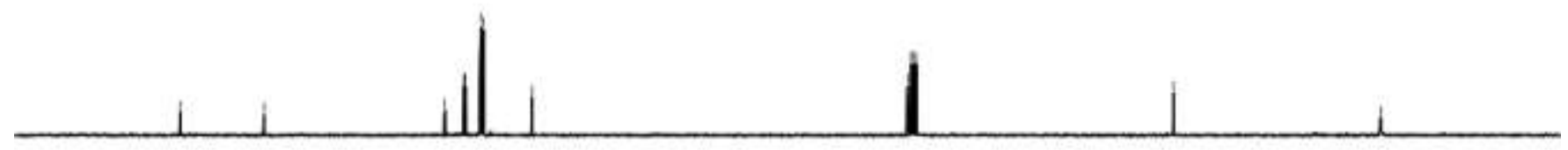

$\begin{array}{lllllllll}180 & 170 & 160 & 150 & 140 & 130 & 120 & 110 & 100\end{array}$ 

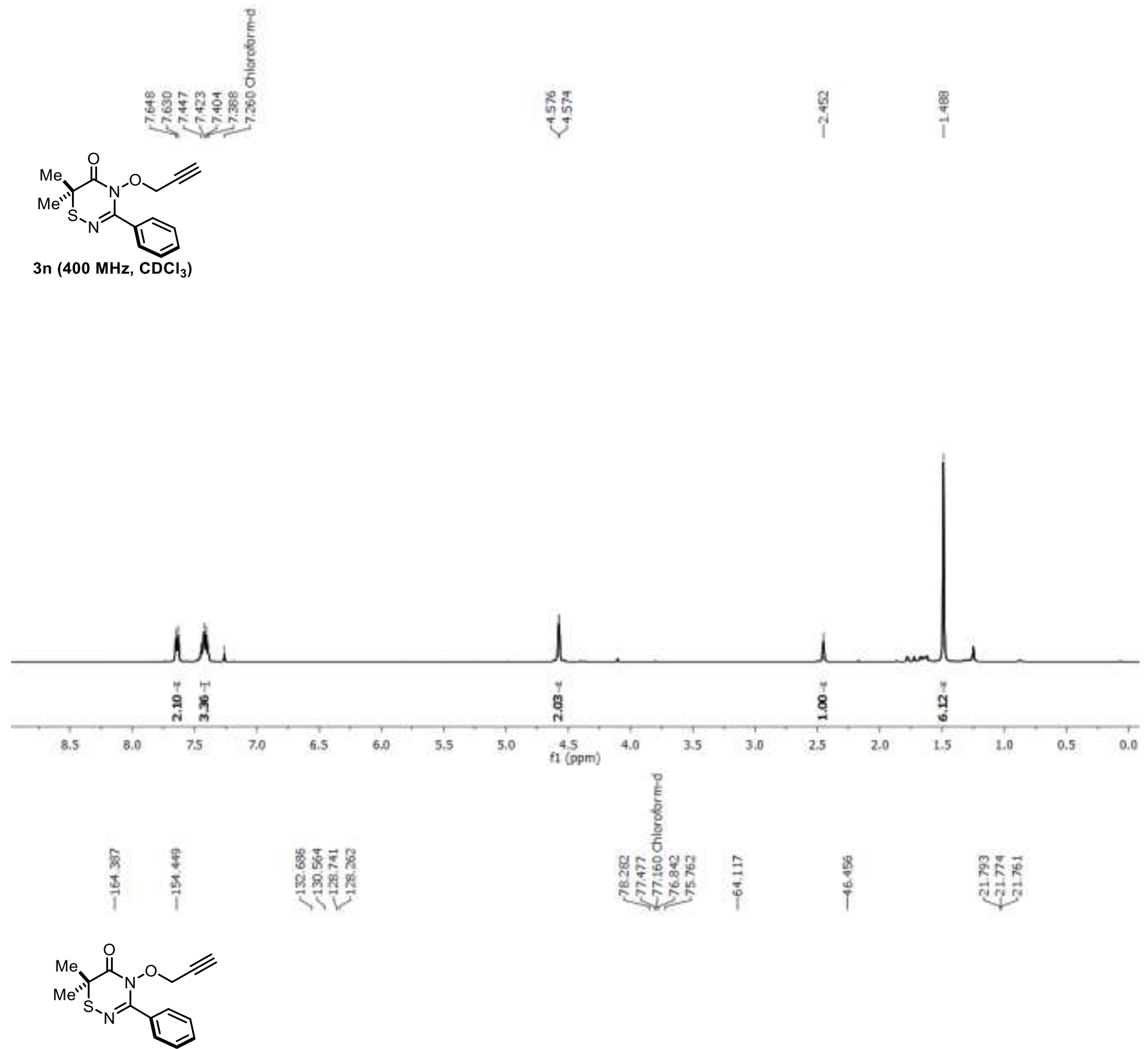

3n $\left(100 \mathrm{MHz}, \mathrm{CDCl}_{3}\right)$
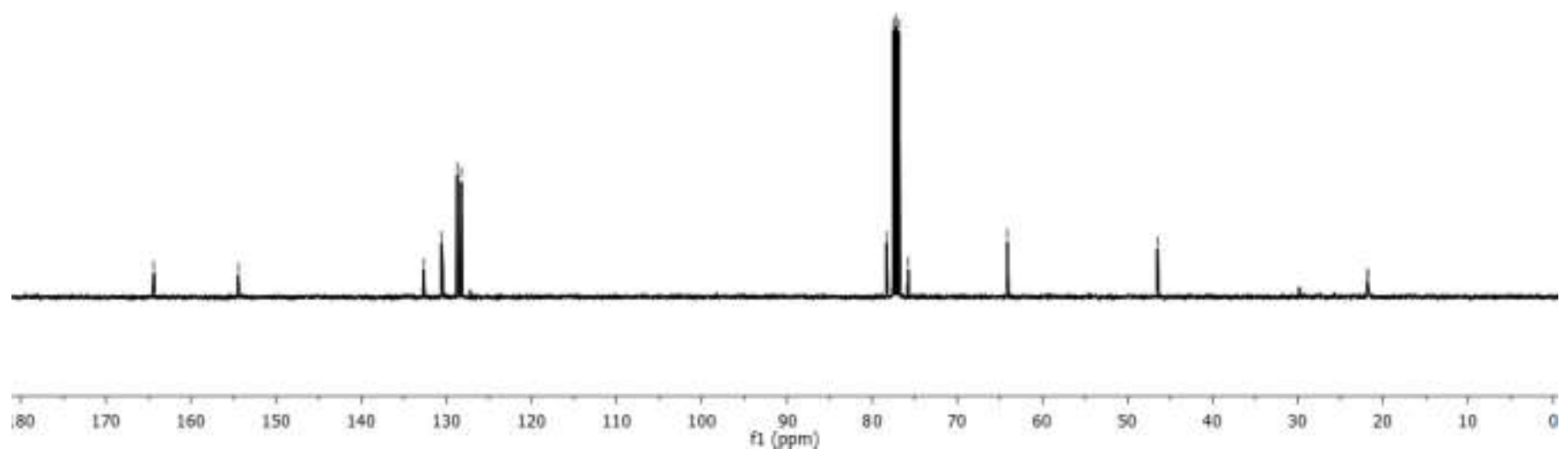


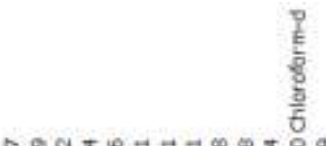

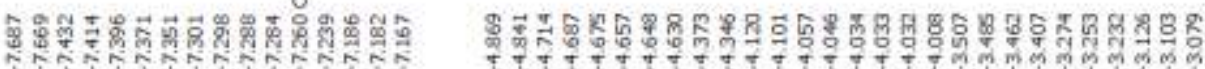

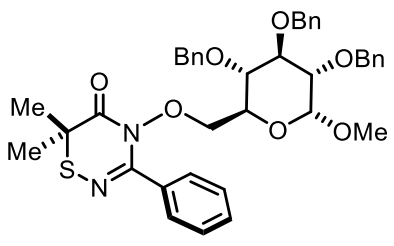

3o $\left(400 \mathrm{MHz}, \mathrm{CDCl}_{3}\right)$

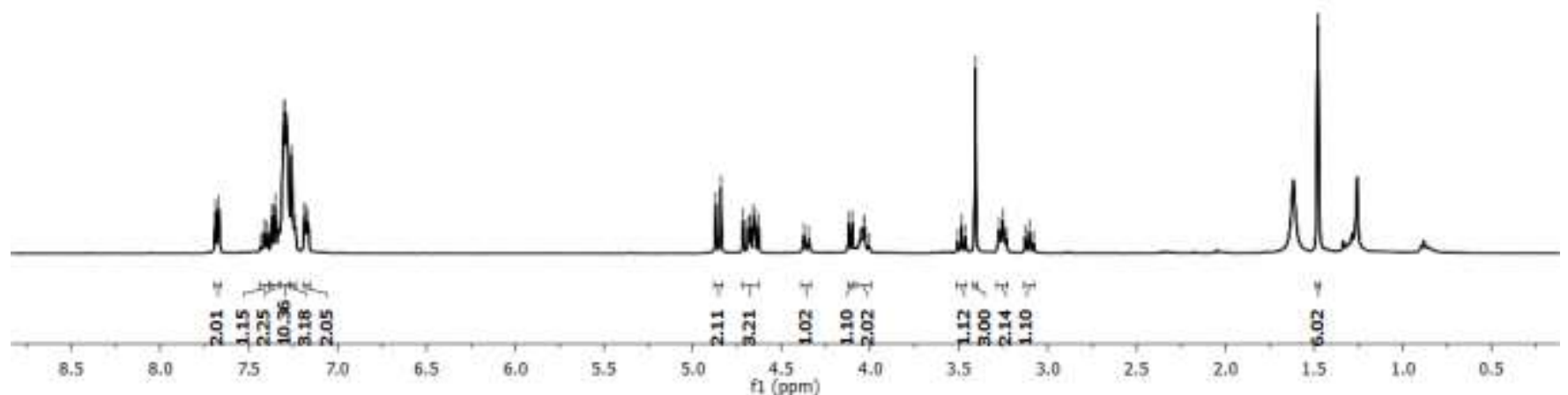<smiles>CO[C@H]1OC(CON2C(=O)C(C)(C)SN=C2c2ccccc2)[C@H](OCc2ccccc2)[C@H](Cc2ccccc2)[C@@H]1O</smiles>

3o $\left(100 \mathrm{MHz}, \mathrm{CDCl}_{3}\right)$

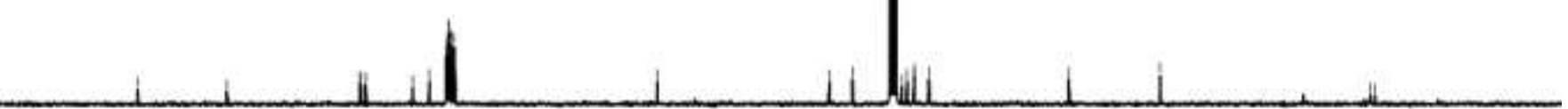

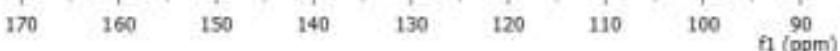

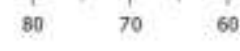

50

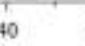

$20 \quad 10$ 

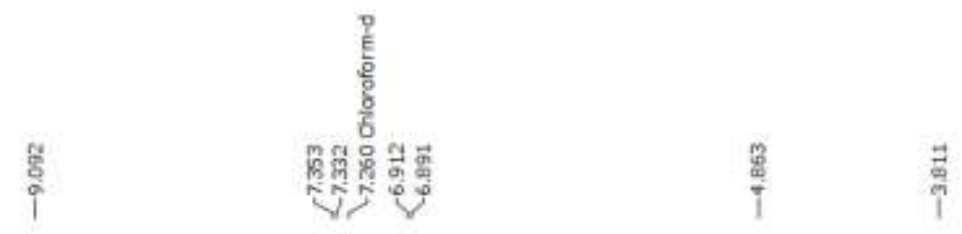

i

${ }_{\mathrm{Br}}^{\mathrm{O}} \mathrm{N}_{\mathrm{H}}^{-O P M B}$

4d $\left(400 \mathrm{MHz}, \mathrm{CDCl}_{3}\right)$

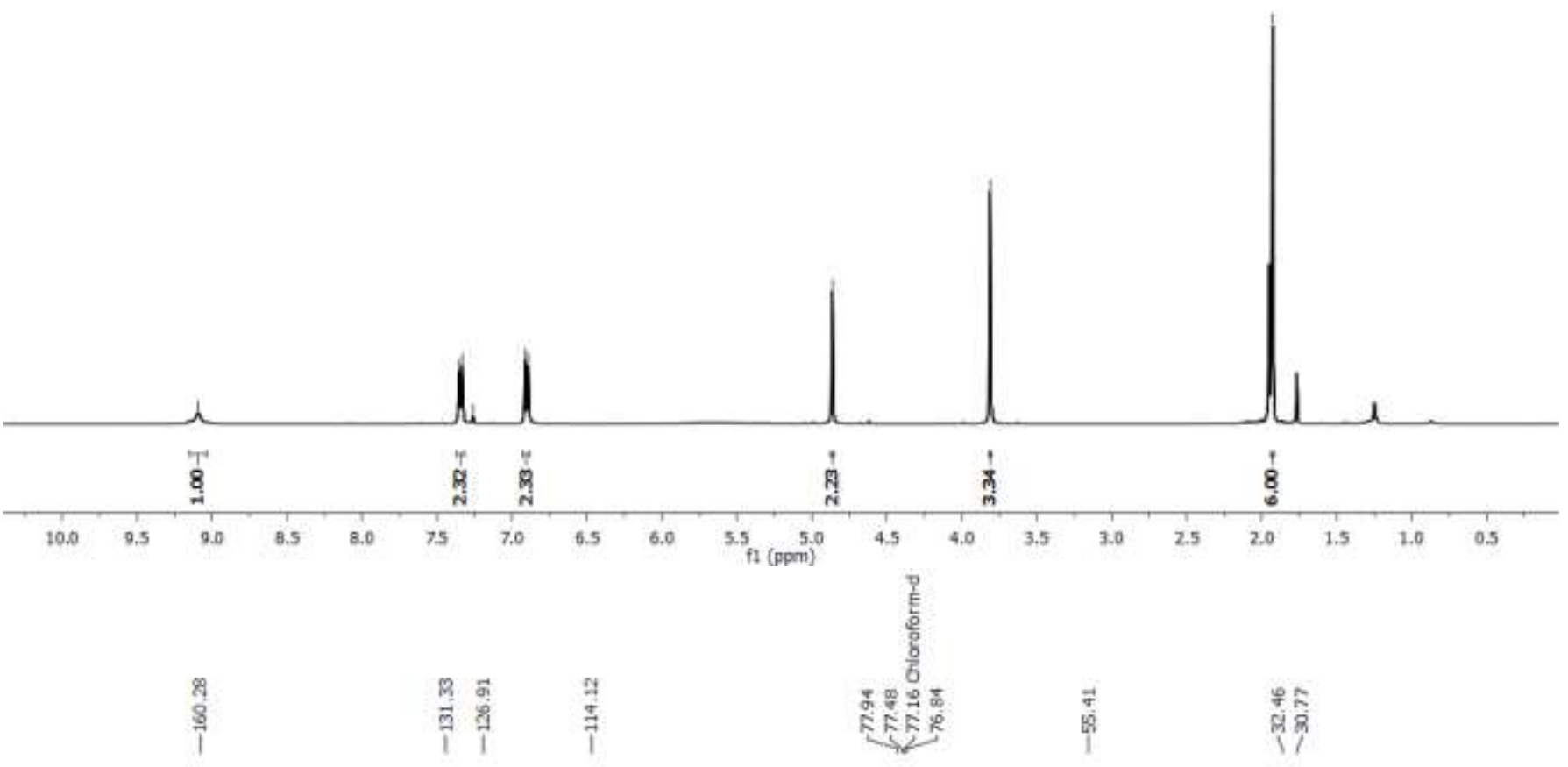

${ }_{\mathrm{Br}}^{\mathrm{O}} \mathrm{N}_{\mathrm{H}}^{-O P M B}$

4d $\left(100 \mathrm{MHz}, \mathrm{CDCl}_{3}\right)$
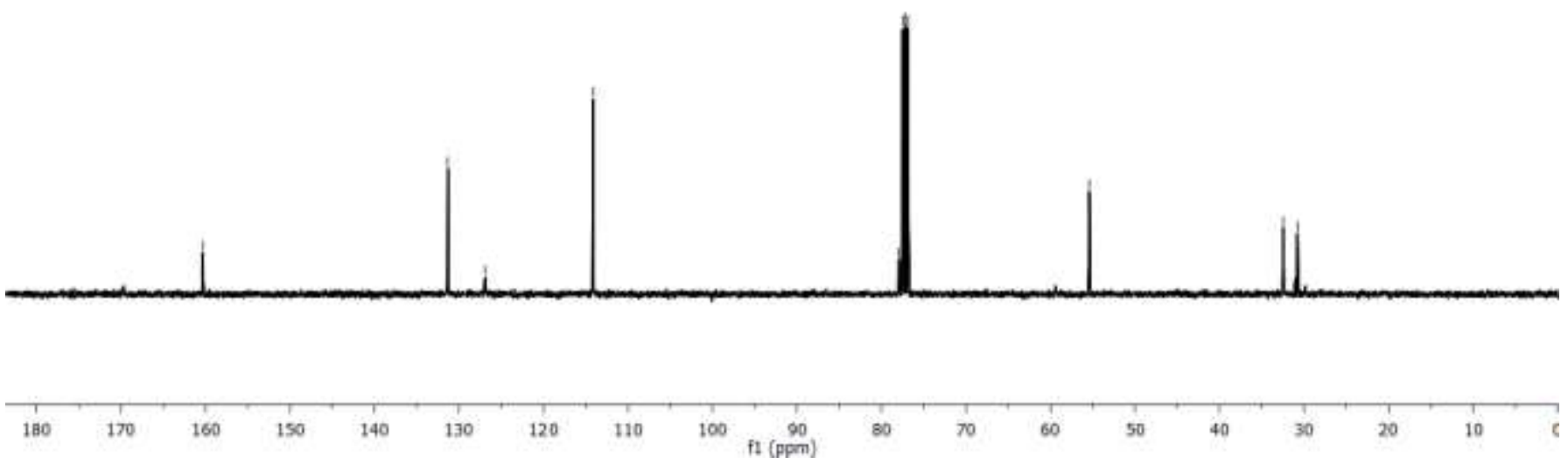

S104 

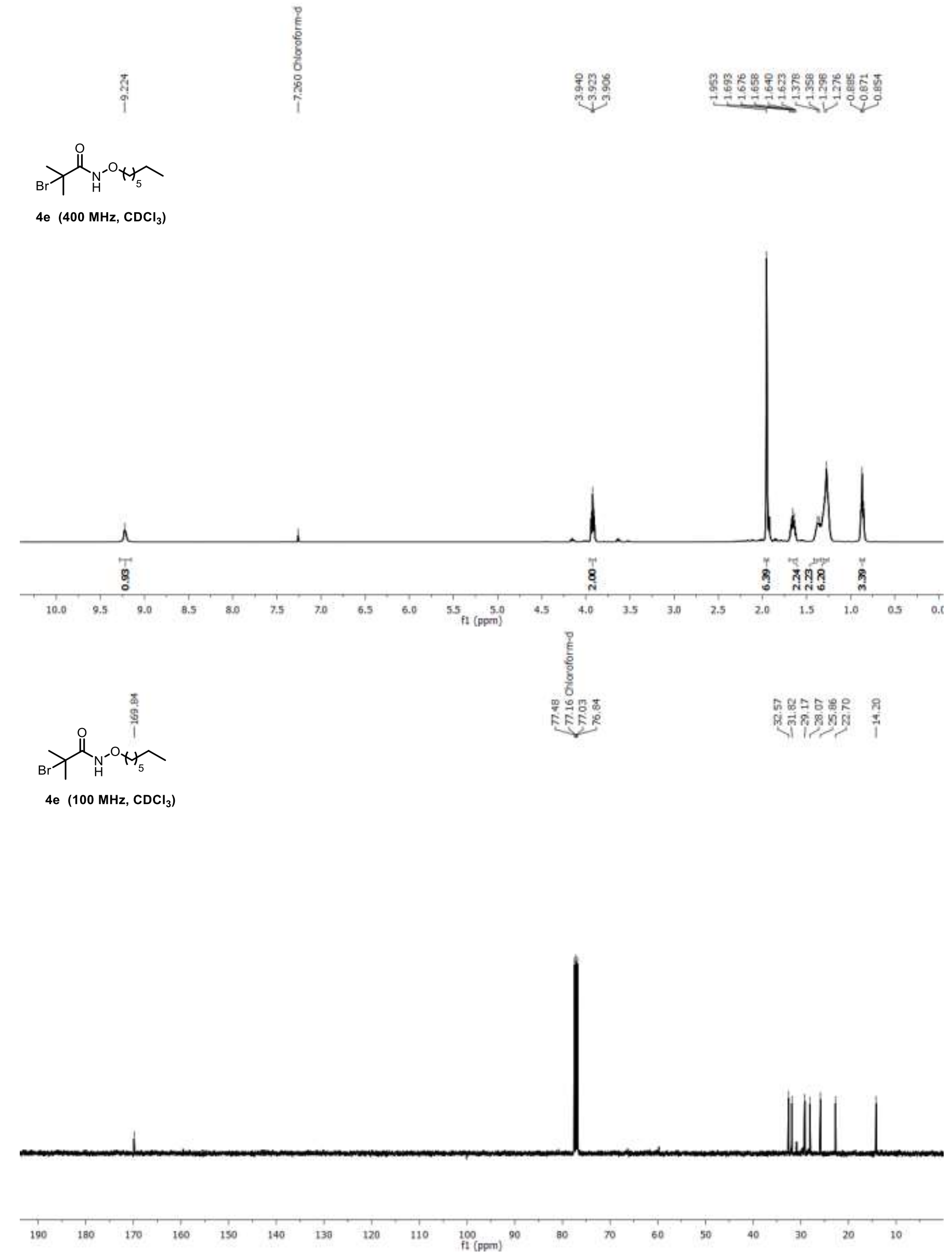


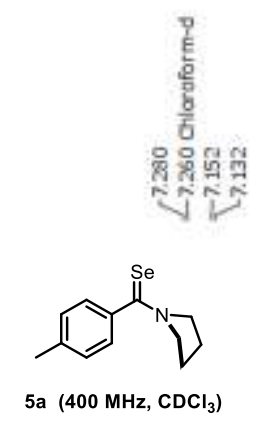

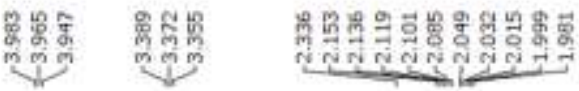

5a $\left(400 \mathrm{MHz}, \mathrm{CDCl}_{3}\right)$
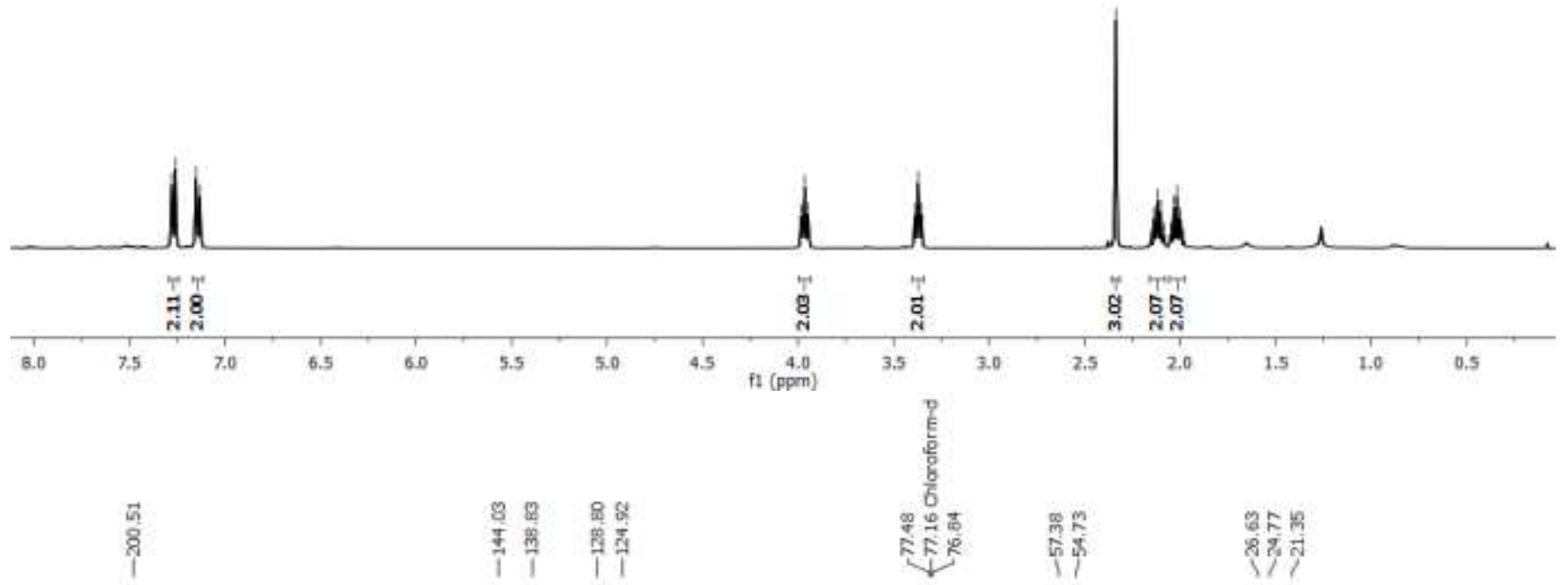

grog

สำ

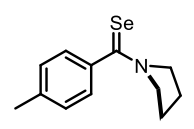

5a $\left(100 \mathrm{MHz}, \mathrm{CDCl}_{3}\right)$

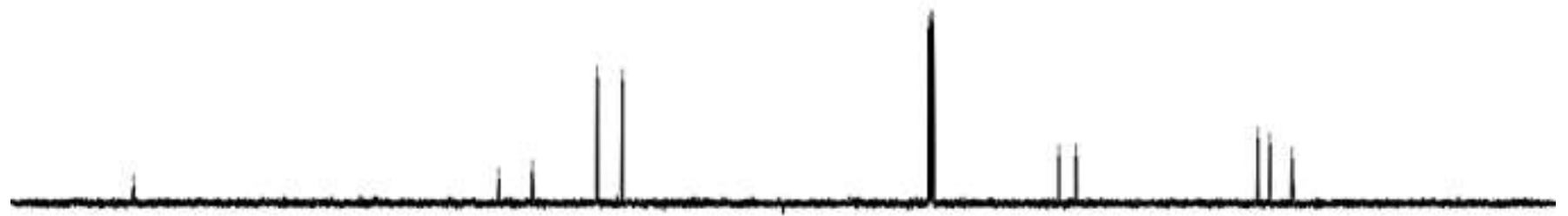

$\begin{array}{lllllllllllllllllllllllllll}210 & 200 & 190 & 180 & 170 & 160 & 150 & 140 & 130 & 120 & 110 & 100 & 90 & 80 & 70 & 60 & 50 & 40 & 30 & 20 & 10 & 0 & -10 & 100\end{array}$ 


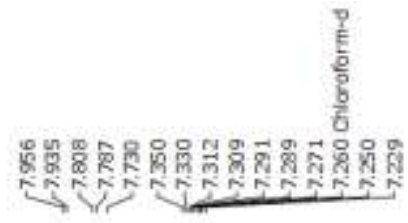

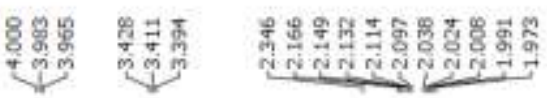

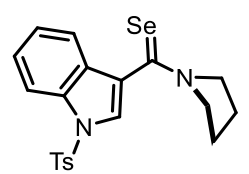

5c $\left(400 \mathrm{MHz}, \mathrm{CDCl}_{3}\right)$
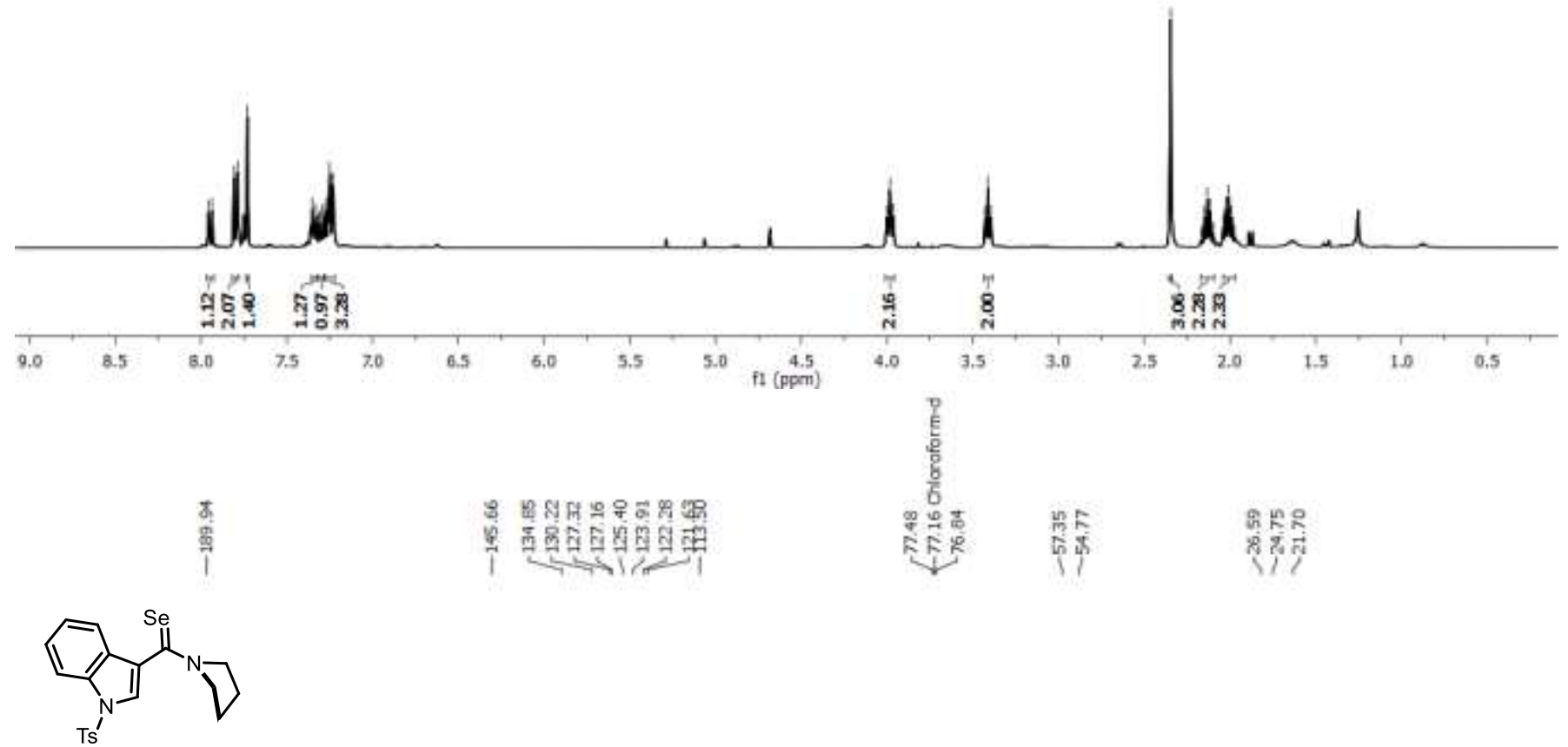

5c $\left(100 \mathrm{MHz}, \mathrm{CDCl}_{3}\right)$

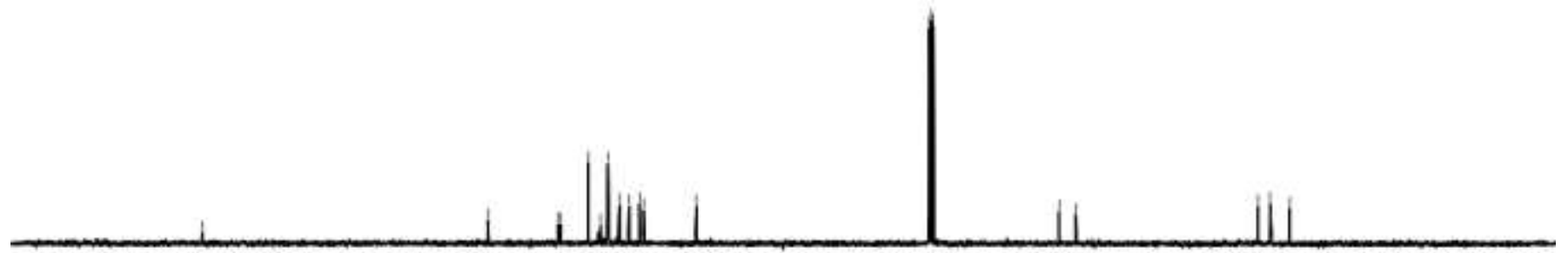

$\begin{array}{lllllllllllllllllllllllllll}210 & 200 & 190 & 180 & 170 & 160 & 150 & 140 & 130 & 120 & 110 & 100 & 90 & 80 & 70 & 60 & 50 & 40 & 30 & 20 & 10 & 0 & -10 & 0\end{array}$ 

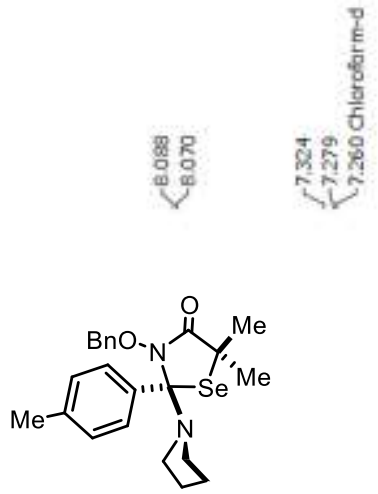

6a $\left(400 \mathrm{MHz}, \mathrm{CDCl}_{3}\right.$ )
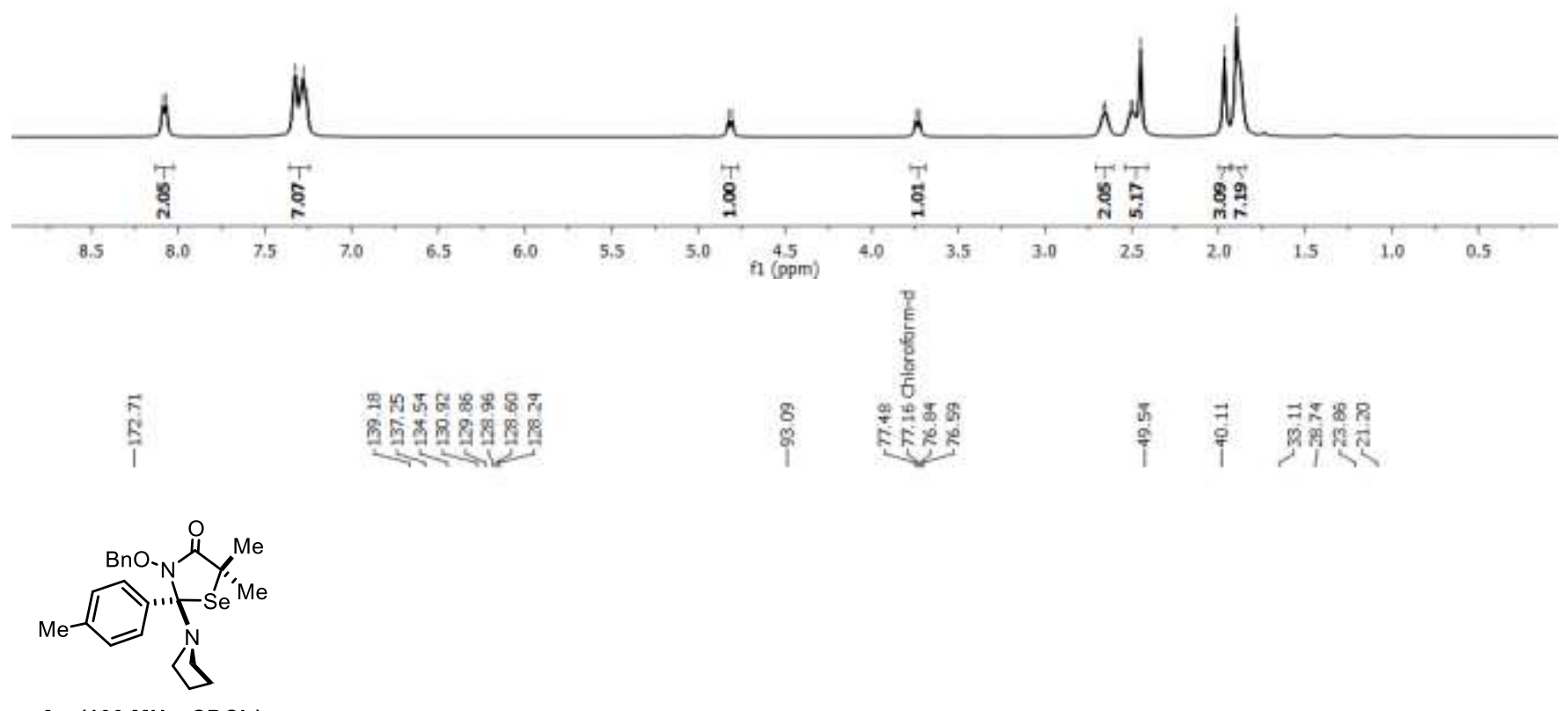

6a (100 MHz, $\mathrm{CDCl}_{3}$ )
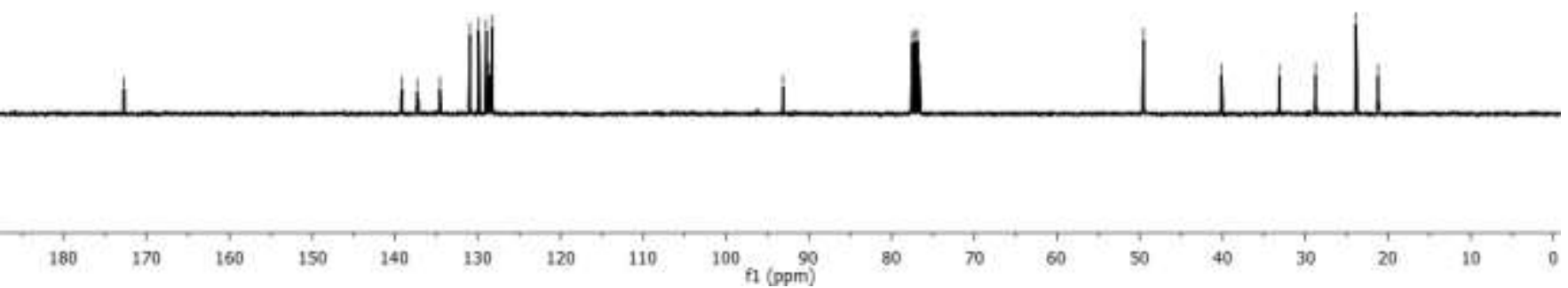


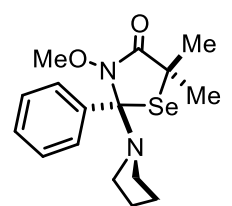

6b $\left(400 \mathrm{MHz}, \mathrm{CDCl}_{3}\right)$
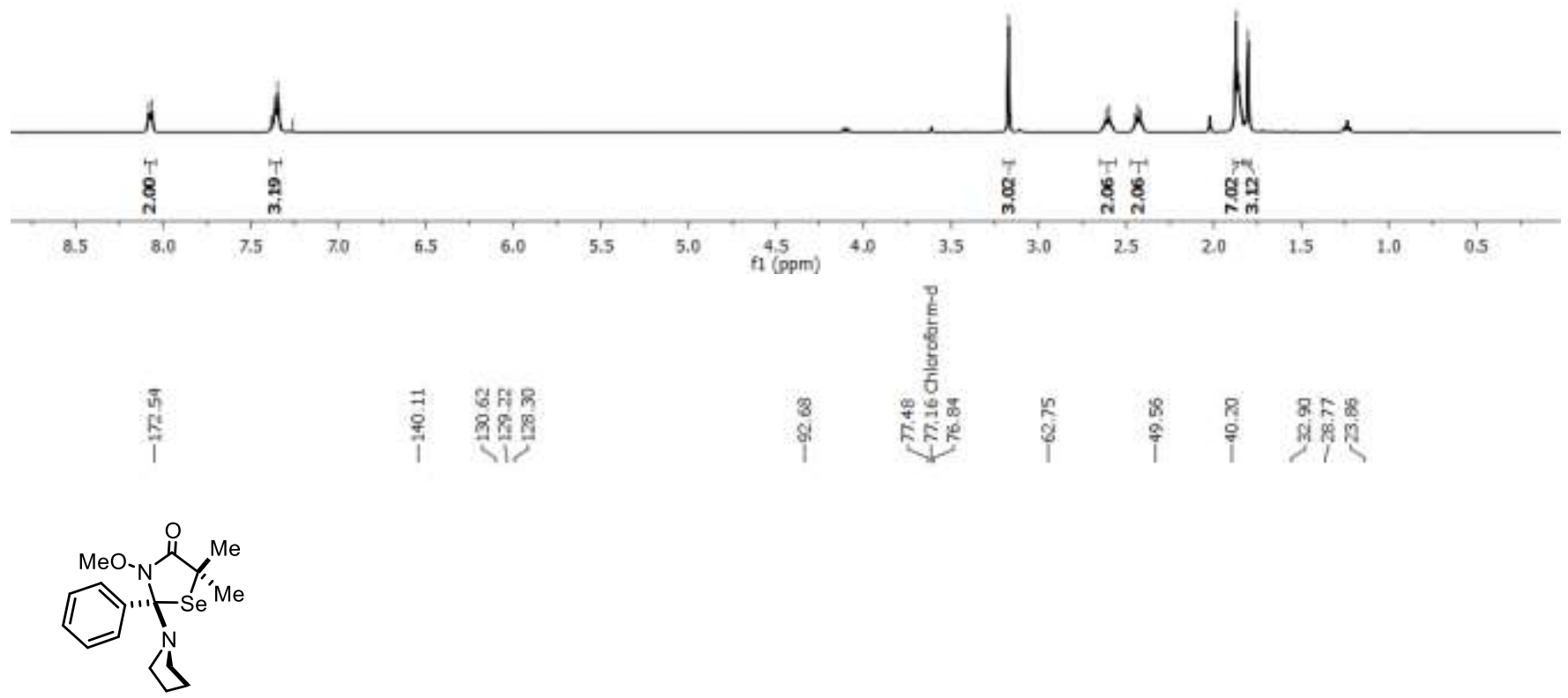

6b $\left(100 \mathrm{MHz}, \mathrm{CDCl}_{3}\right)$
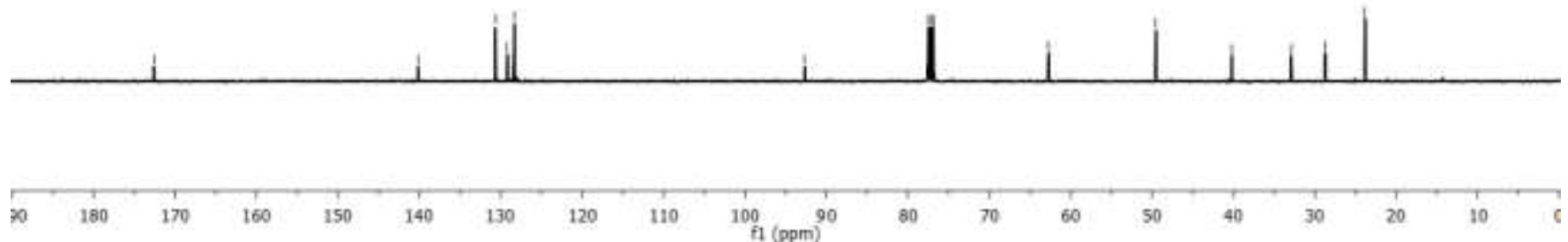


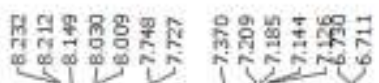

踢

新

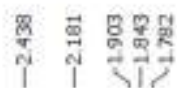

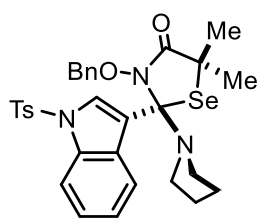

6c $\left(400 \mathrm{MHz} \mathrm{CDCl}_{3}\right)$
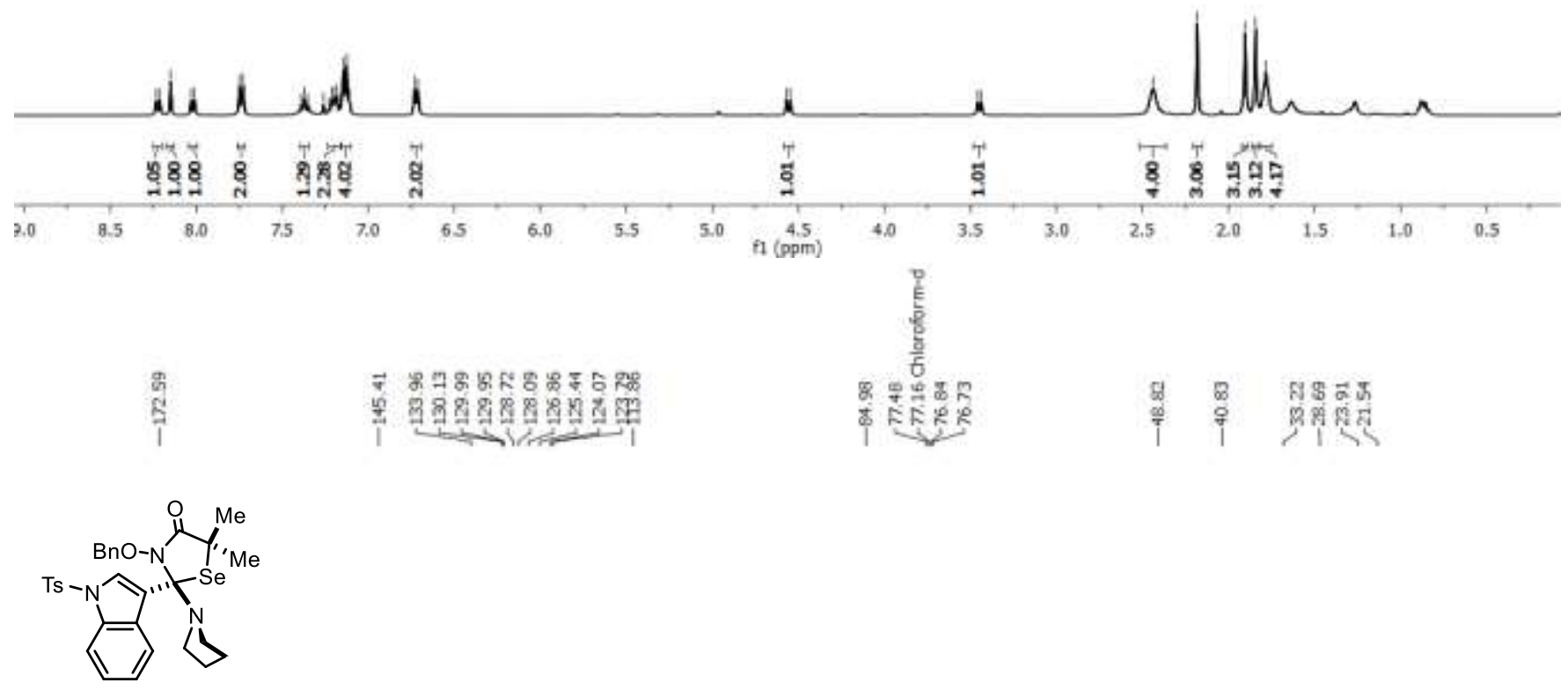

6c $\left(100 \mathrm{MHz}, \mathrm{CDCl}_{3}\right)$

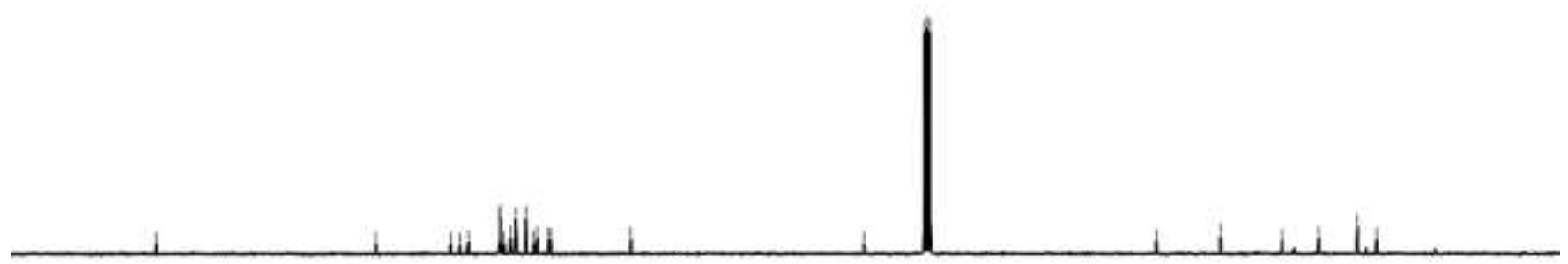

$\begin{array}{llllllllllllllllllllllll}90 & 180 & 170 & 160 & 150 & 140 & 130 & 120 & 110 & 100 & 90 & 80 & 70 & 60 & 50 & 40 & 30 & 20 & 10 & 0\end{array}$ 


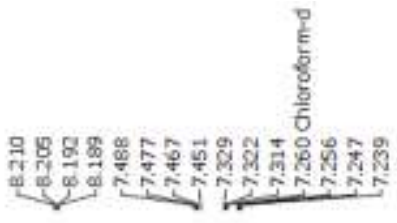

\section{侸}

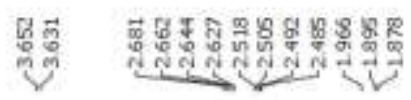

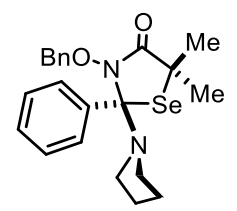

6d $\left(400 \mathrm{MHz}, \mathrm{CDCl}_{3}\right)$
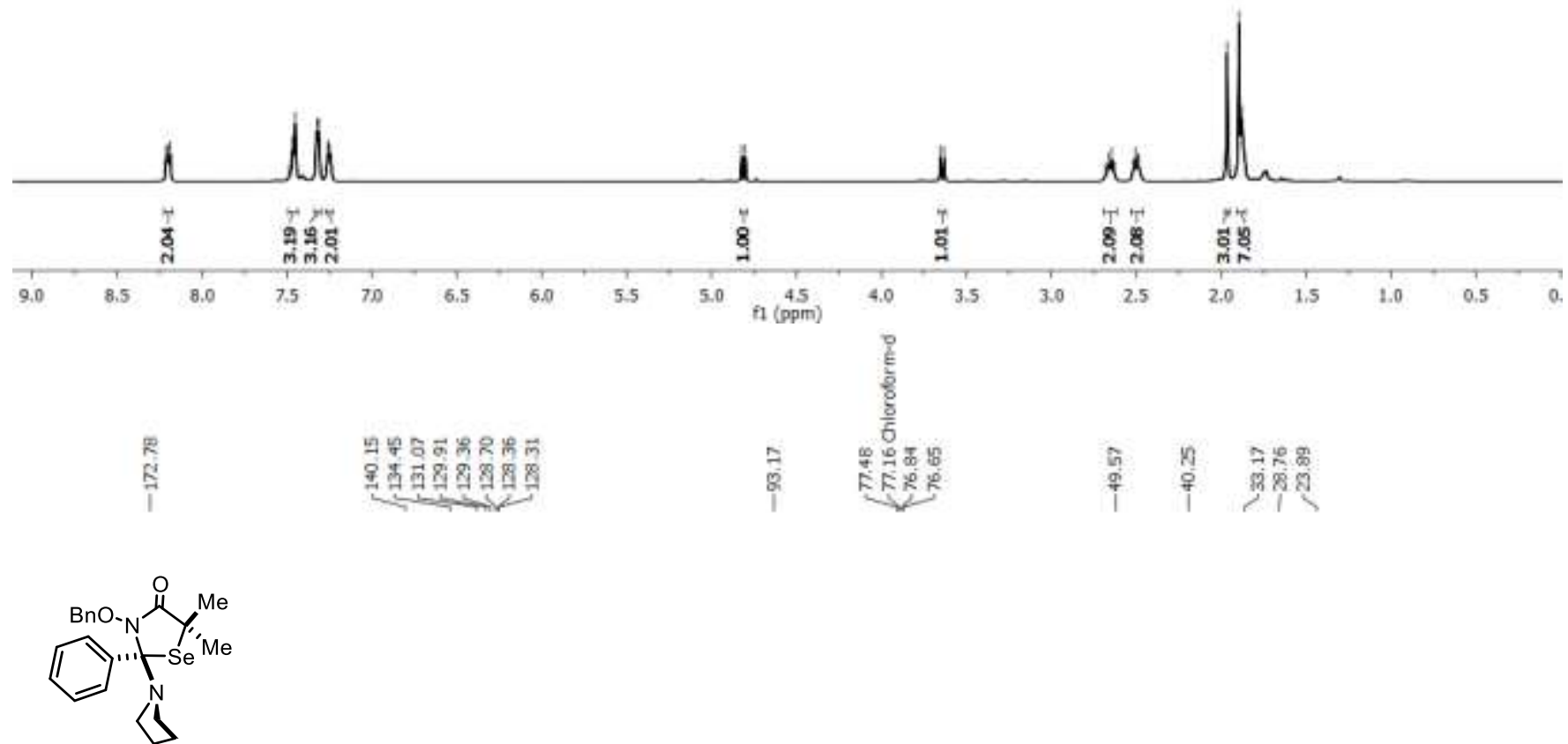

6d (400 $\mathrm{MHz}, \mathrm{CDCl}_{3}$ )

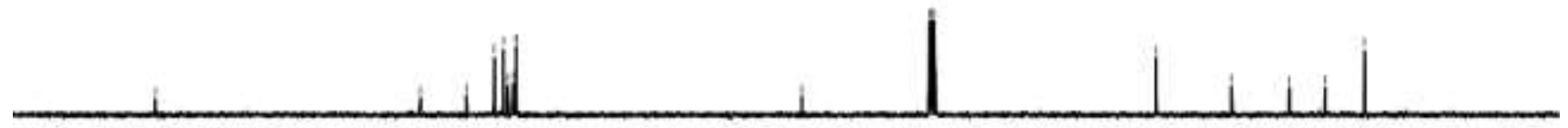




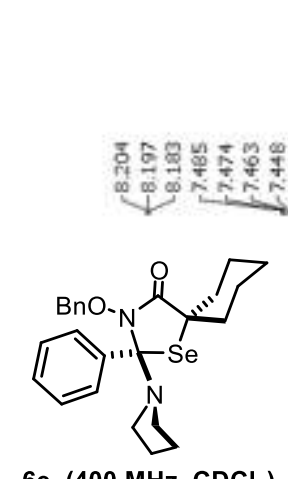

6e $\left(400 \mathrm{MHz}, \mathrm{CDCl}_{3}\right)$
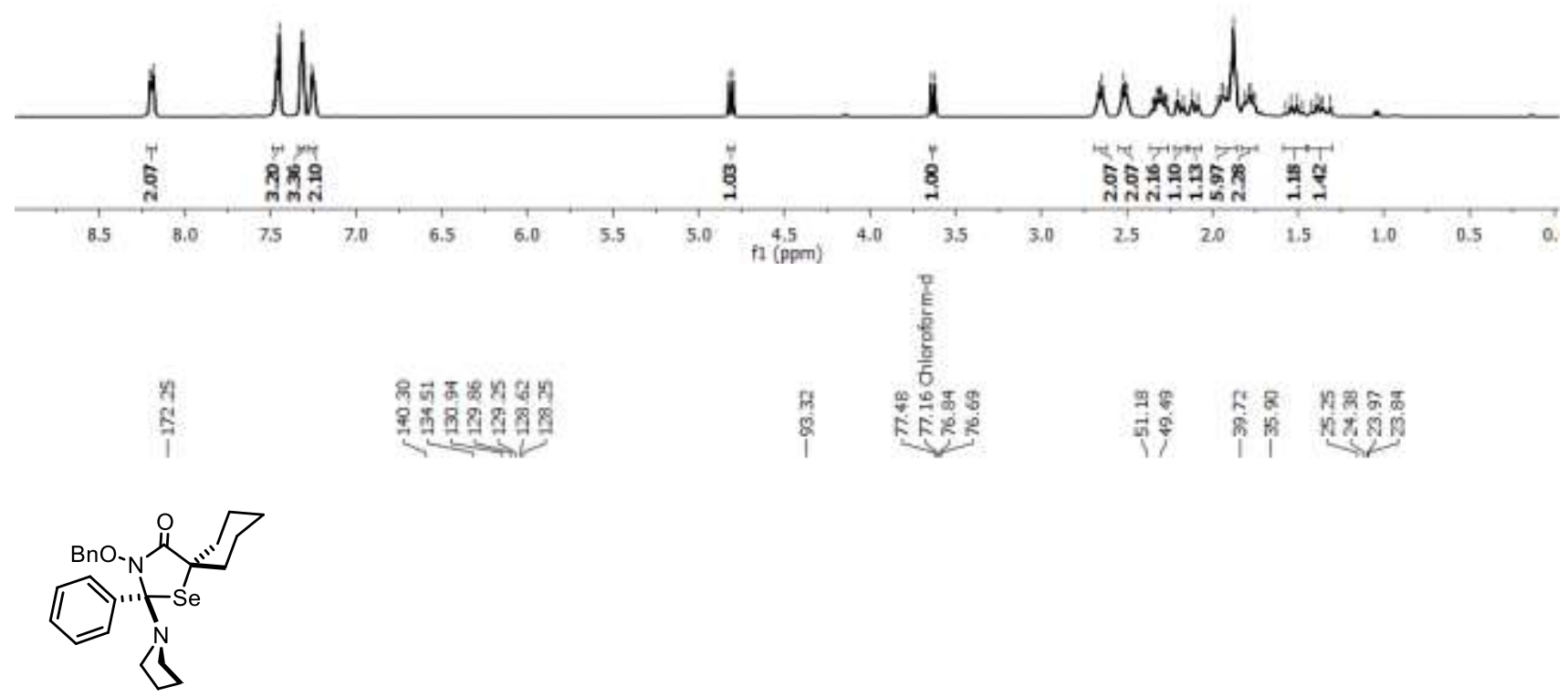

6e $\left(100 \mathrm{MHz}, \mathrm{CDCl}_{3}\right)$

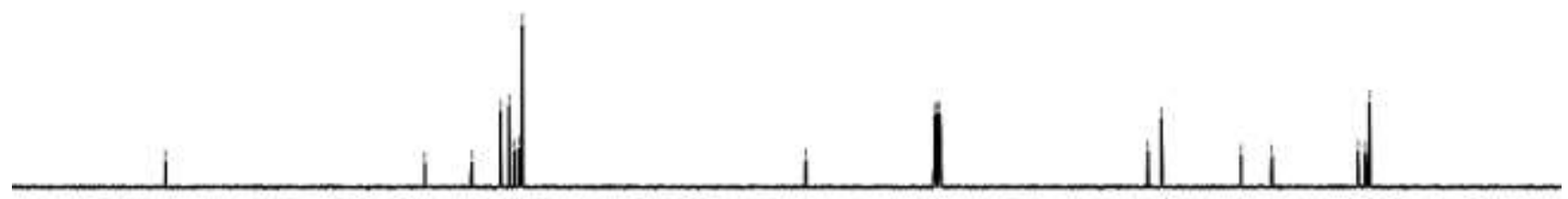

$\begin{array}{llllllllllllllllllllllll}90 & 180 & 170 & 160 & 150 & 140 & 130 & 120 & 110 & 100 & 90 & 80 & 70 & 60 & 50 & 40 & 30 & 20 & 10 & \end{array}$




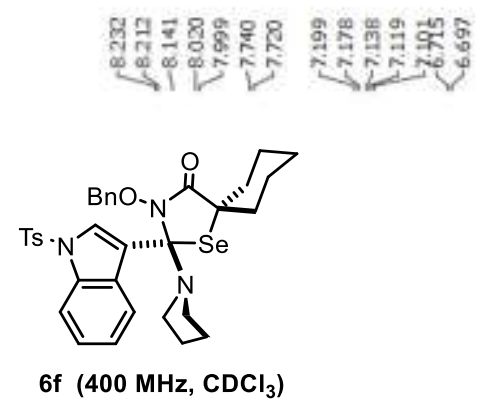

6f $\left(400 \mathrm{MHz}, \mathrm{CDCl}_{3}\right)$

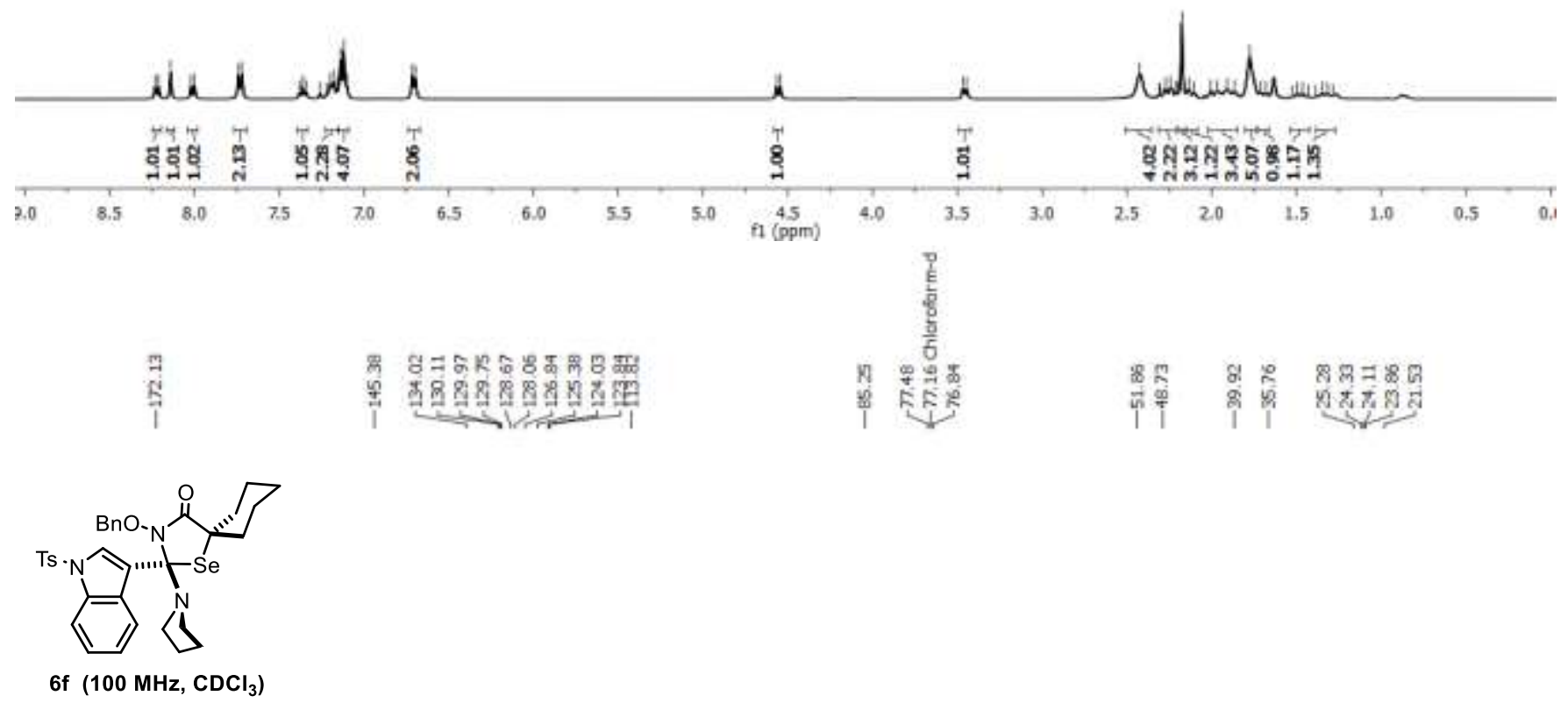

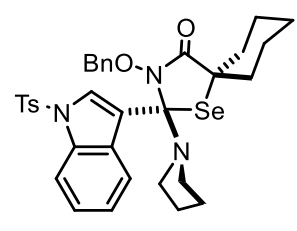

6f (100 $\left.\mathrm{MHz}, \mathrm{CDCl}_{3}\right)$

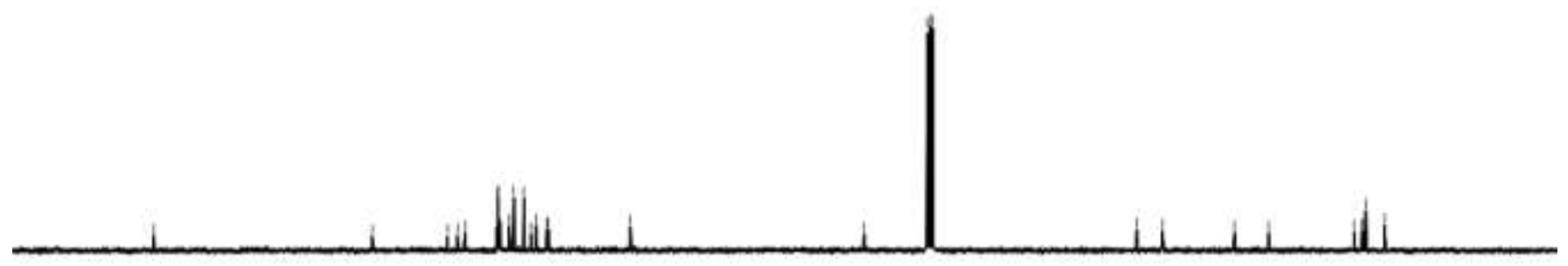

$\begin{array}{lllllllll}180 & 170 & 160 & 150 & 140 & 130 & 120 & 110 & 100\end{array}$

$80 \quad 70 \quad 60$ 


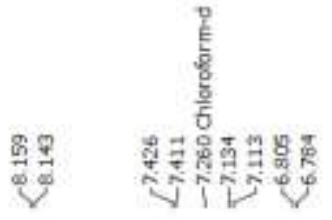

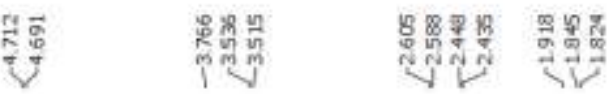

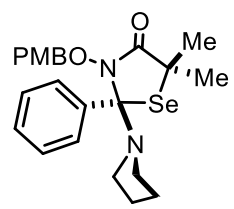

$6 \mathrm{~g}\left(400 \mathrm{MHz}, \mathrm{CDCl}_{3}\right)$
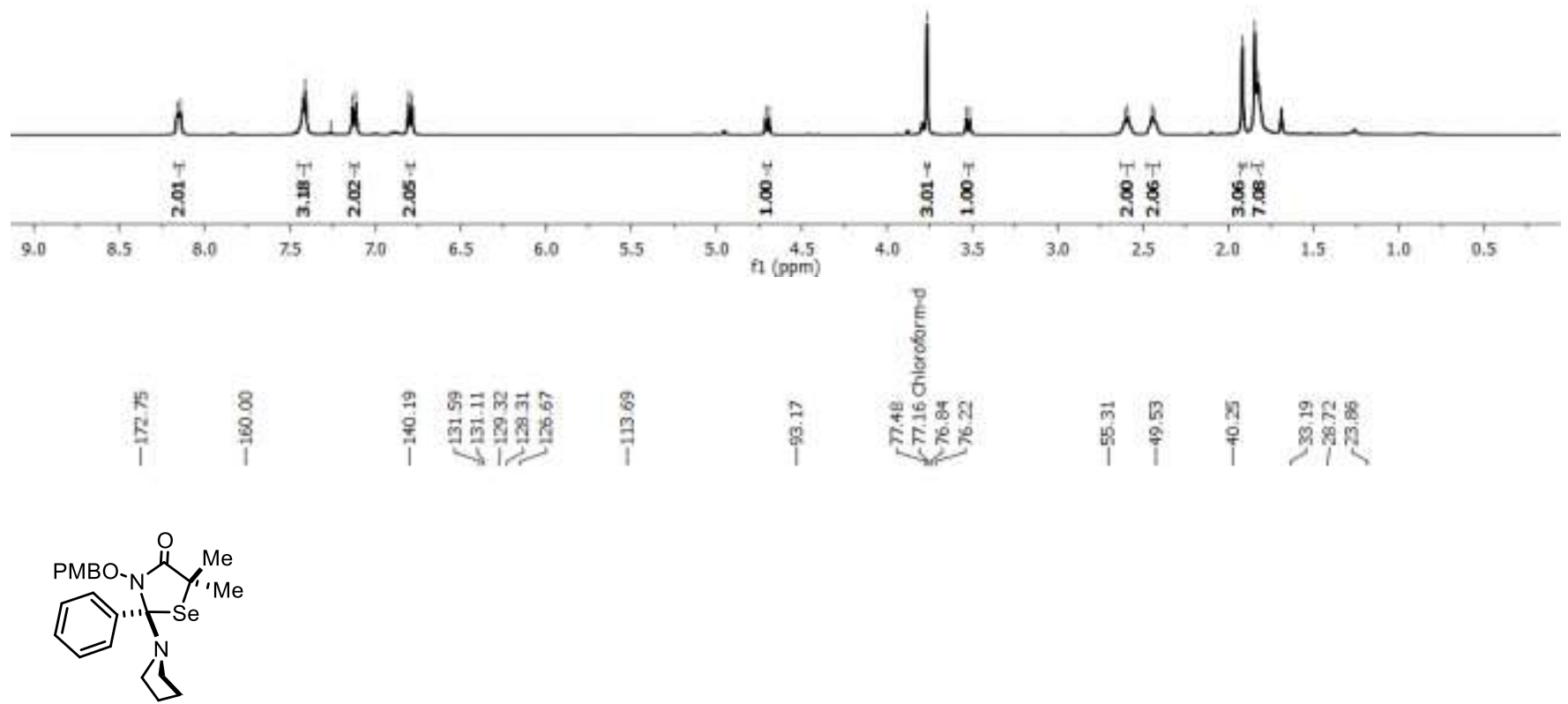

6g (100 $\left.\mathrm{MHz}, \mathrm{CDCl}_{3}\right)$

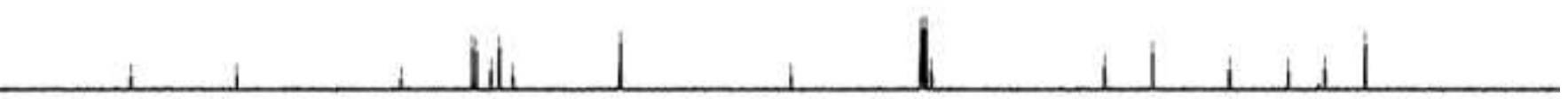

$\begin{array}{lllllllll}180 & 170 & 160 & 150 & 140 & 130 & 120 & 110 & 100\end{array}$ 


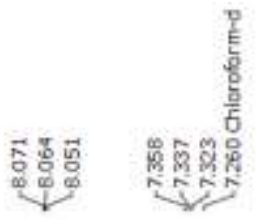

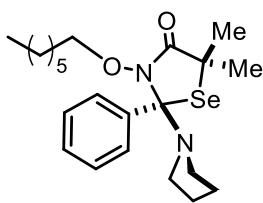

6h $\left(400 \mathrm{MHz}, \mathrm{CDCl}_{3}\right)$
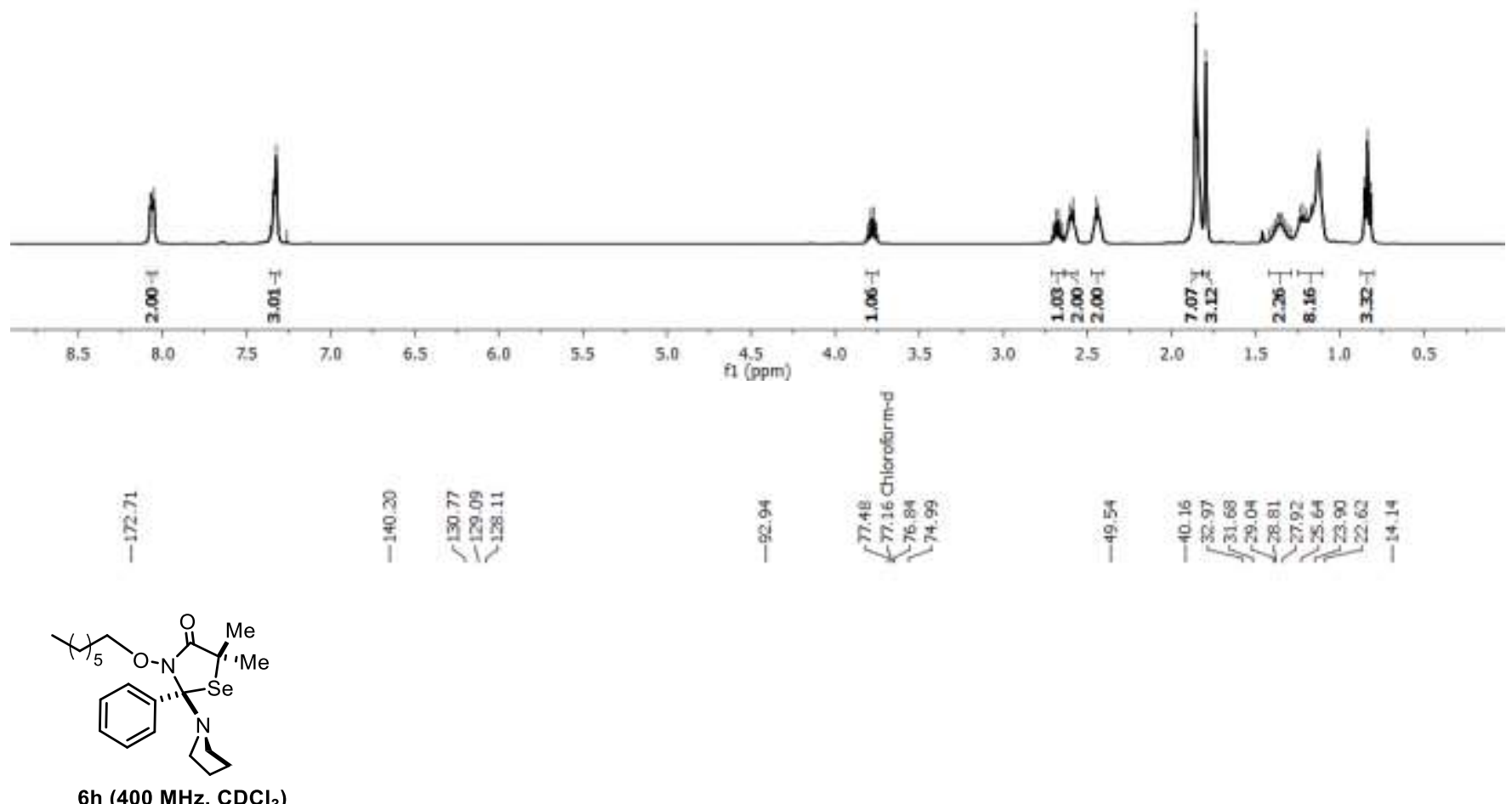

6h $\left(400 \mathrm{MHz}, \mathrm{CDCl}_{3}\right)$

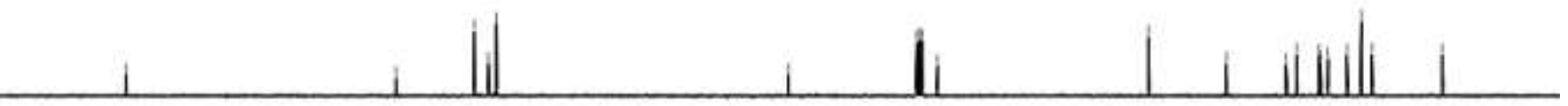

$\begin{array}{lllllllll}180 & 170 & 160 & 150 & 140 & 130 & 120 & 110 & 100\end{array}$ 

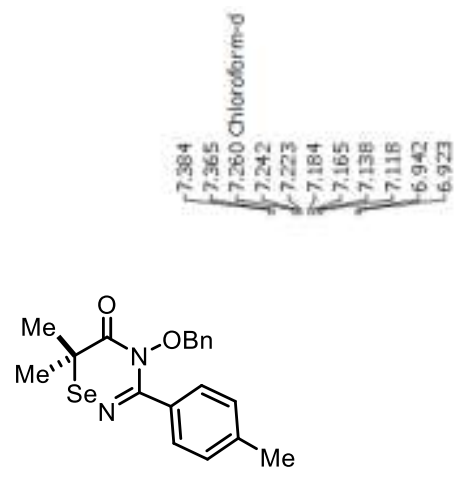

7a $\left(400 \mathrm{MHz}, \mathrm{CDCl}_{3}\right)$
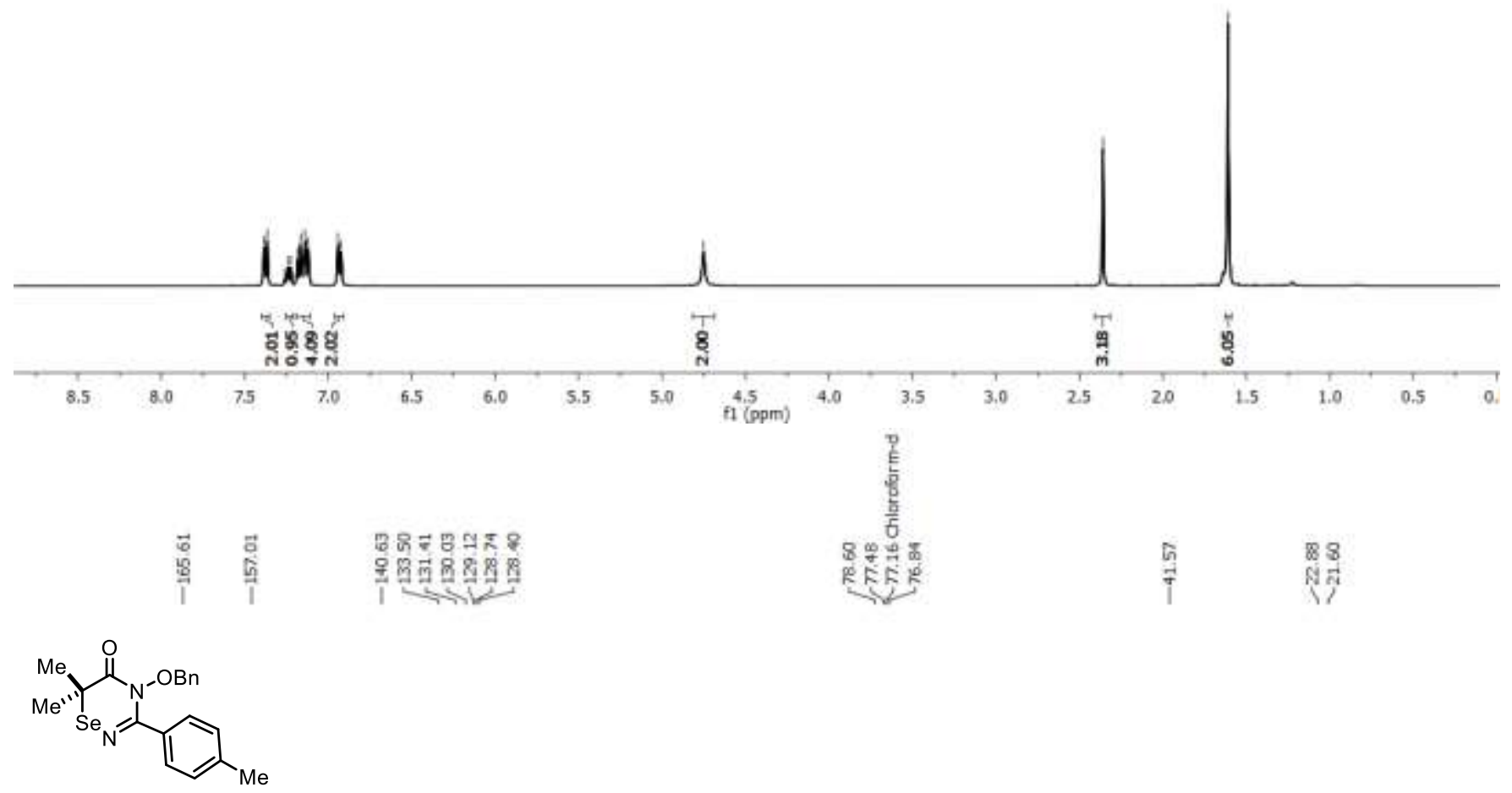

7a $\left(400 \mathrm{MHz}, \mathrm{CDCl}_{3}\right)$
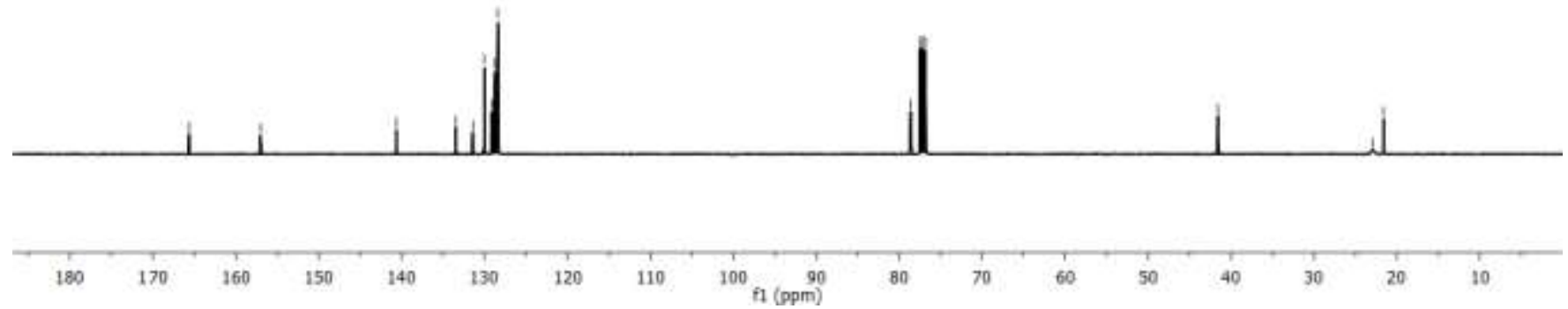

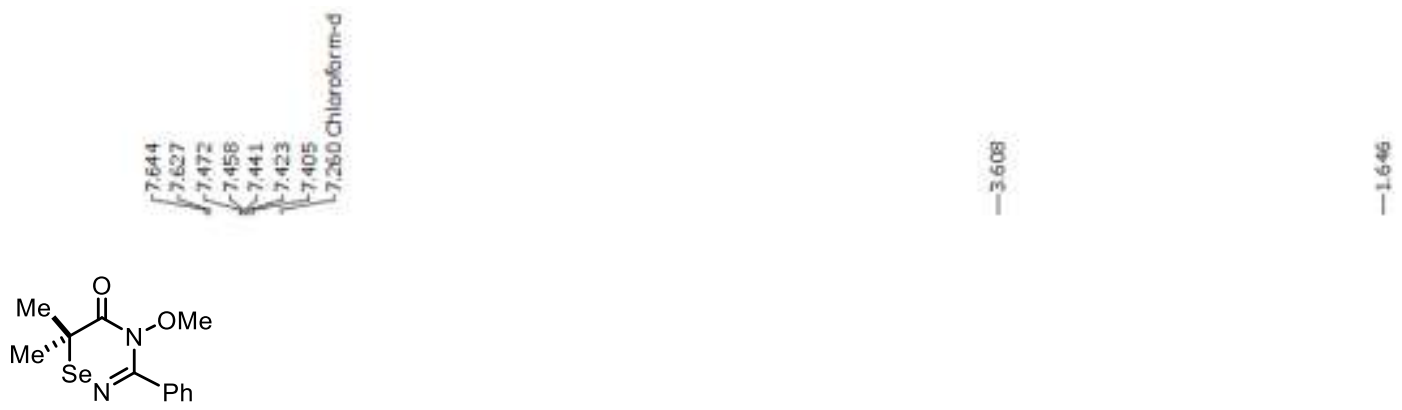

7b $\left(400 \mathrm{MHz}^{\mathrm{CDCl}}\right)_{3}$
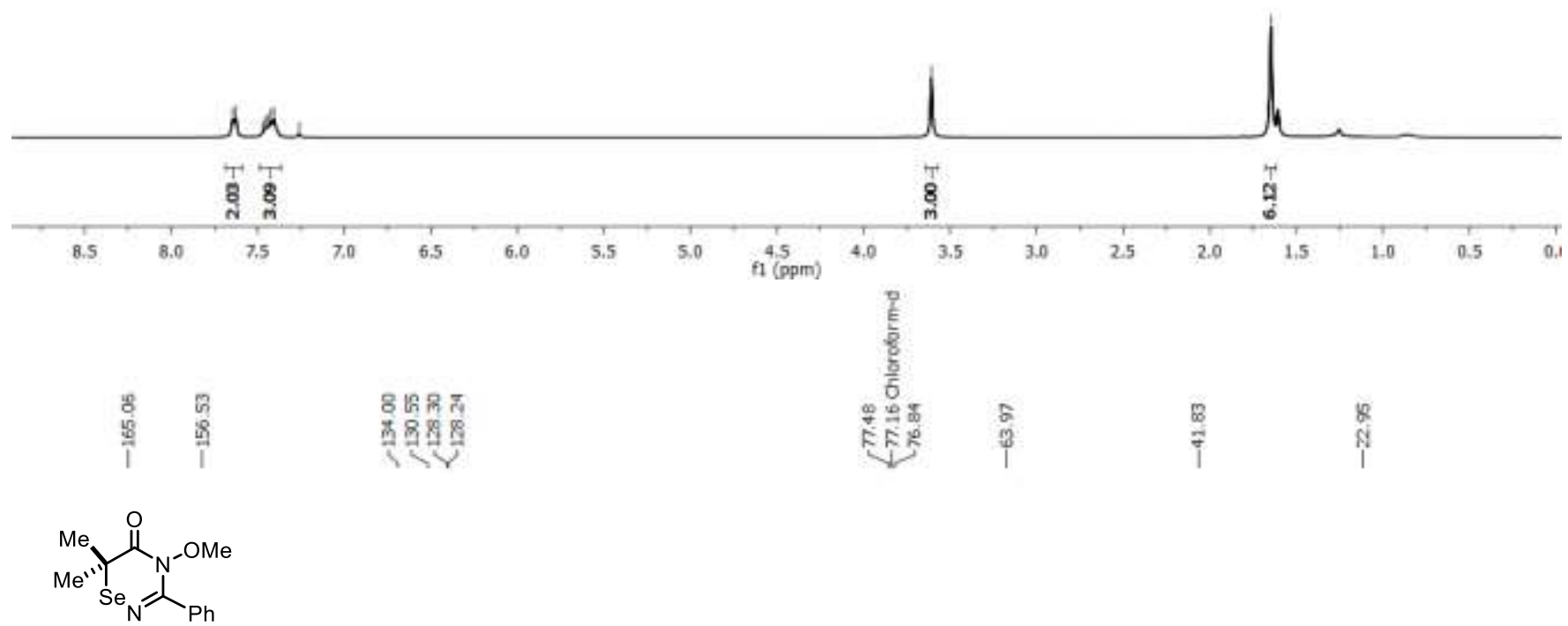

7b $\left(100 \mathrm{MHz}, \mathrm{CDCl}_{3}\right)$

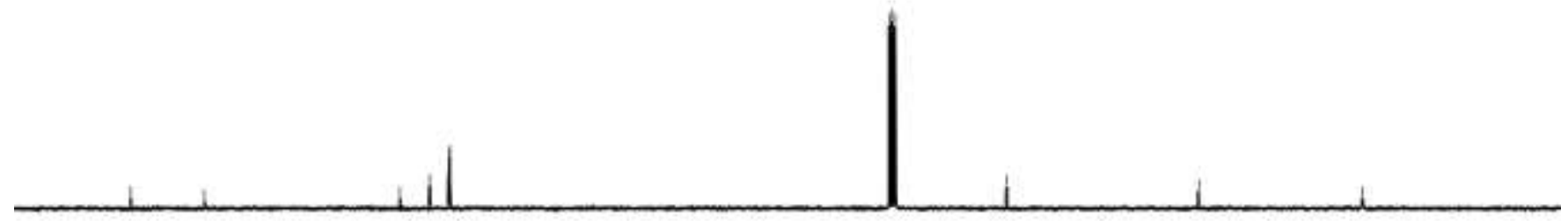

$\begin{array}{llllllll}170 & 160 & 150 & 140 & 130 & 120 & 110 & 100\end{array}$

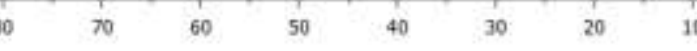




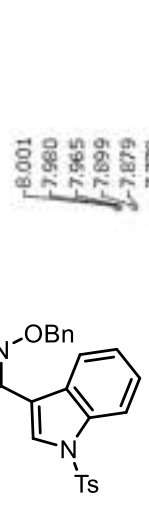

7c $\left(400 \mathrm{MHz}, \mathrm{CDCl}_{3}\right)$
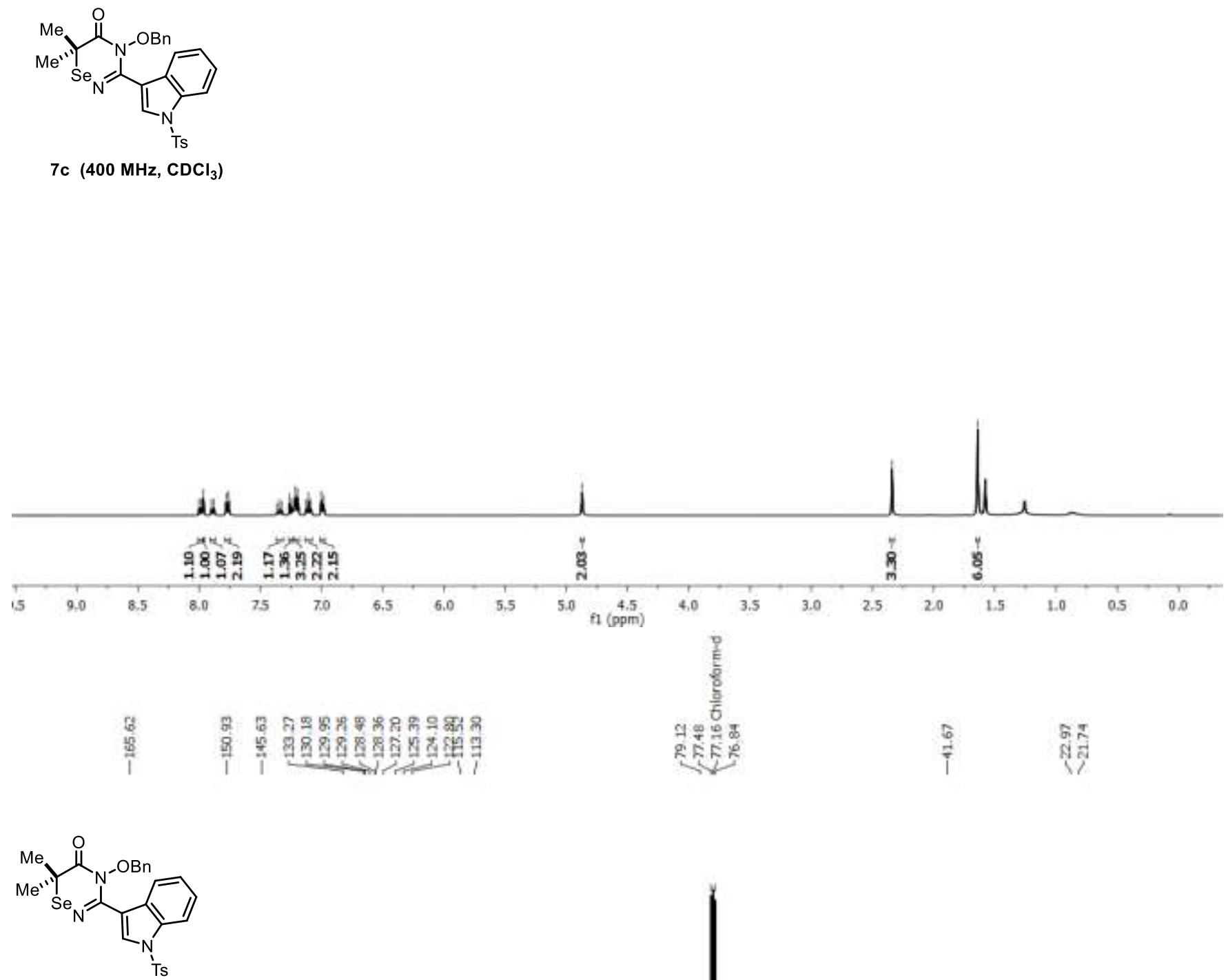

7c $\left(100 \mathrm{MHz}, \mathrm{CDCl}_{3}\right)$
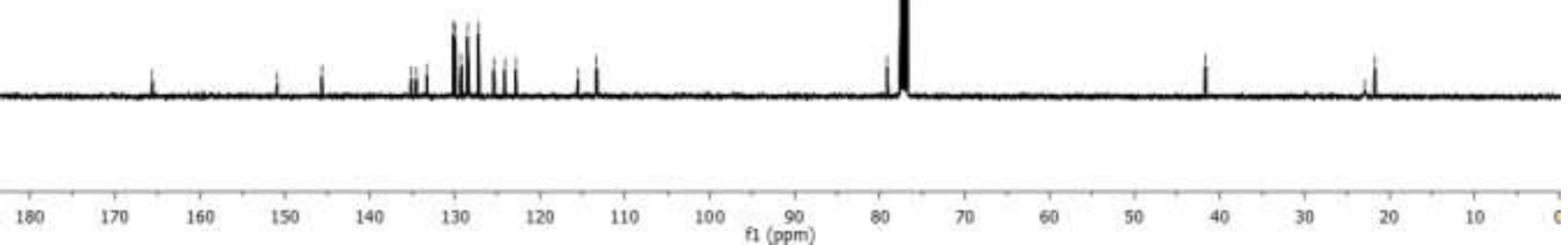


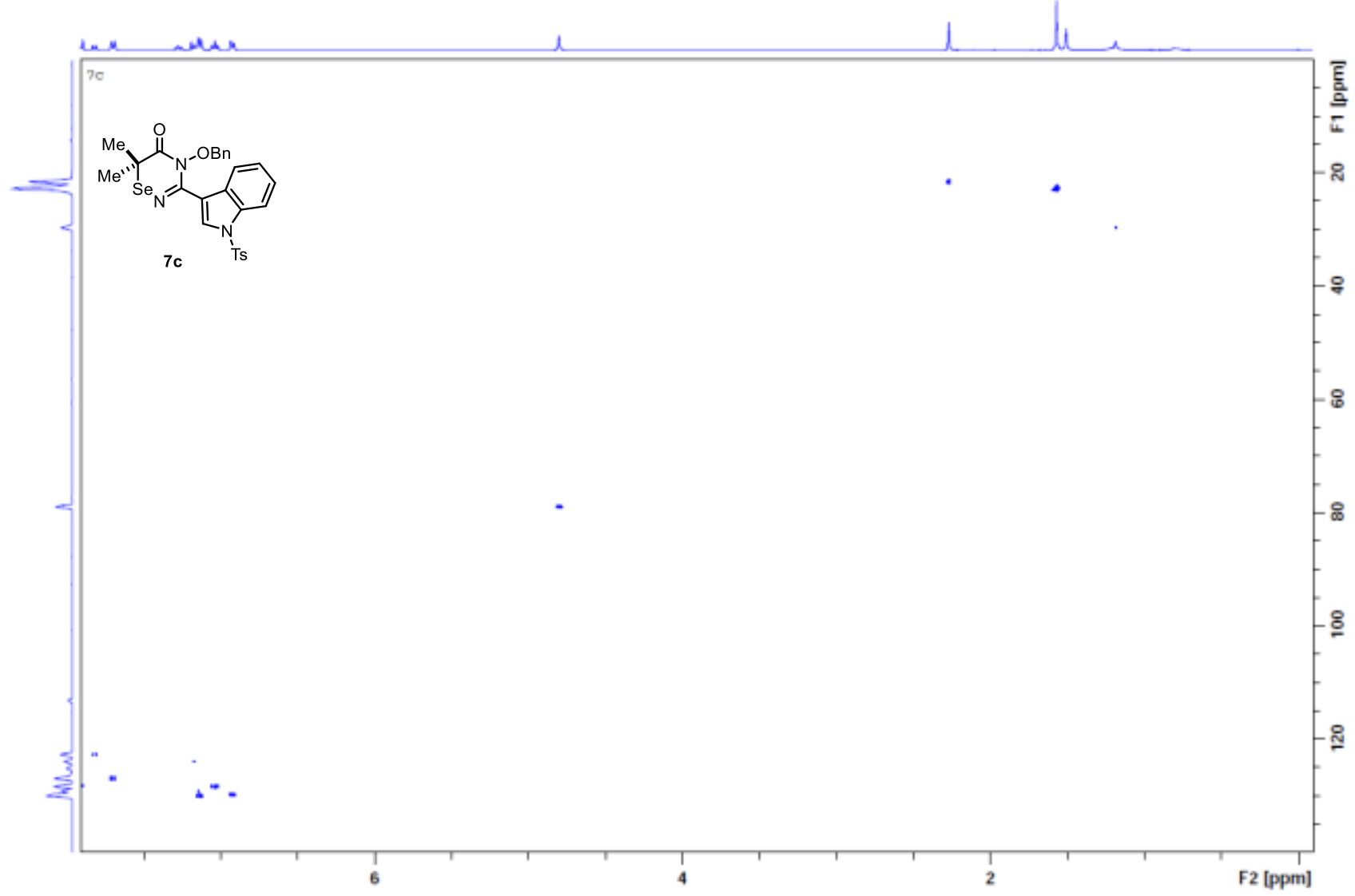



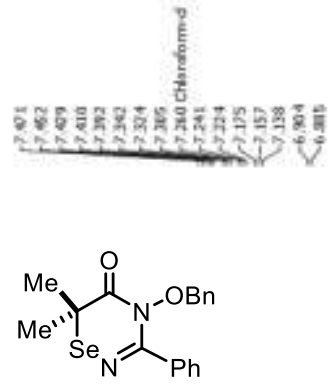

7d $\left(400 \mathrm{MHz}, \mathrm{CDCl}_{3}\right)$
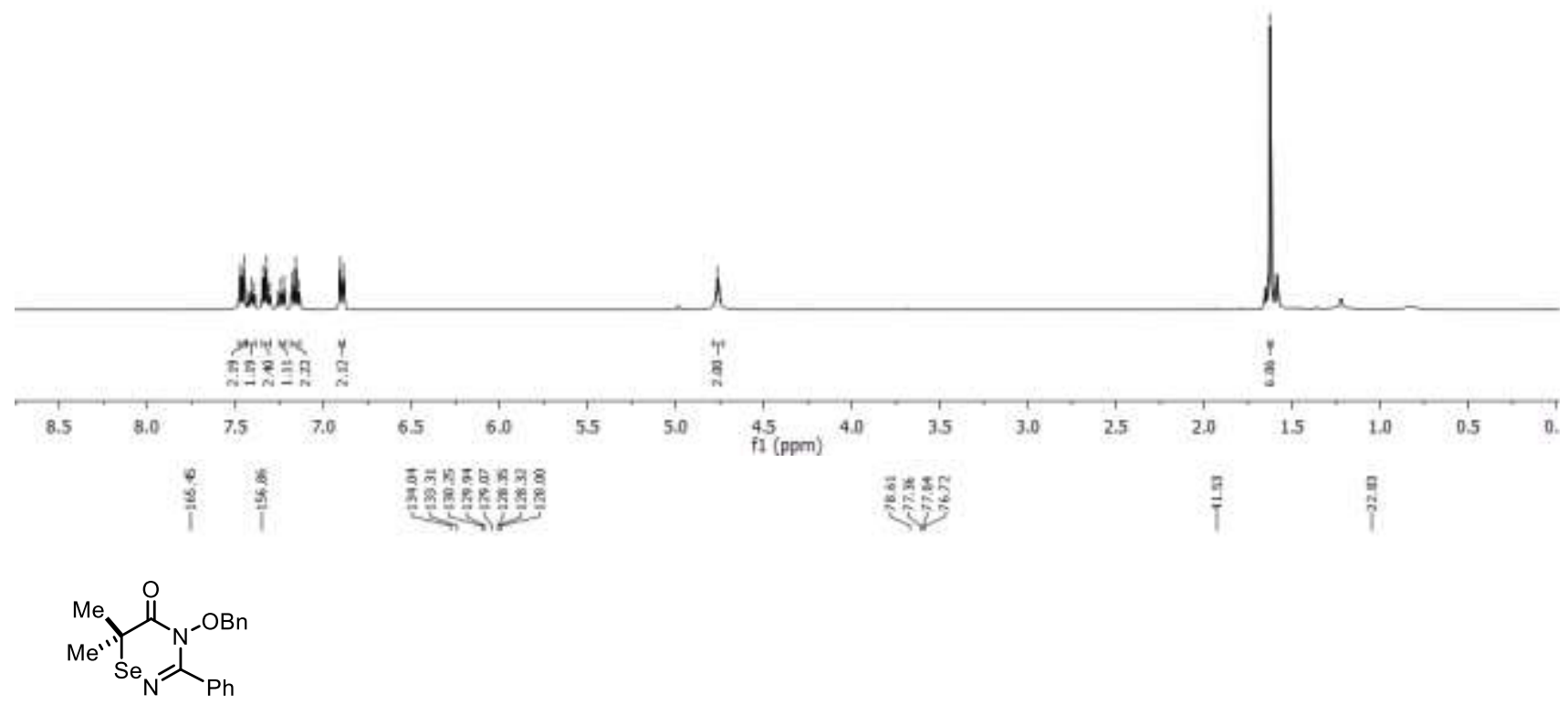

7d $\left(100 \mathrm{MHz}, \mathrm{CDCl}_{3}\right)$

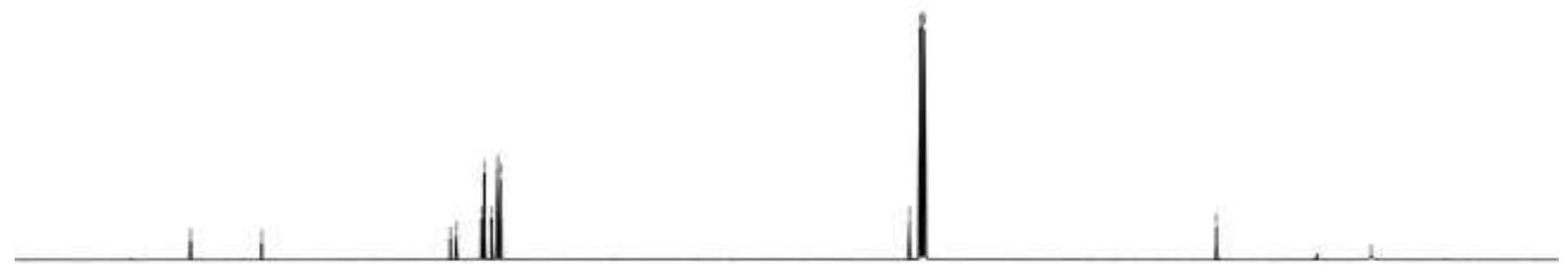

$180 \quad 170 \quad 160$

$150 \quad 140$

130

120

110

${ }^{100} \mathrm{fl}(\mathrm{ppm})$

80

$70 \quad 60$

50

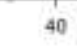

30

$20 \quad 10$ 

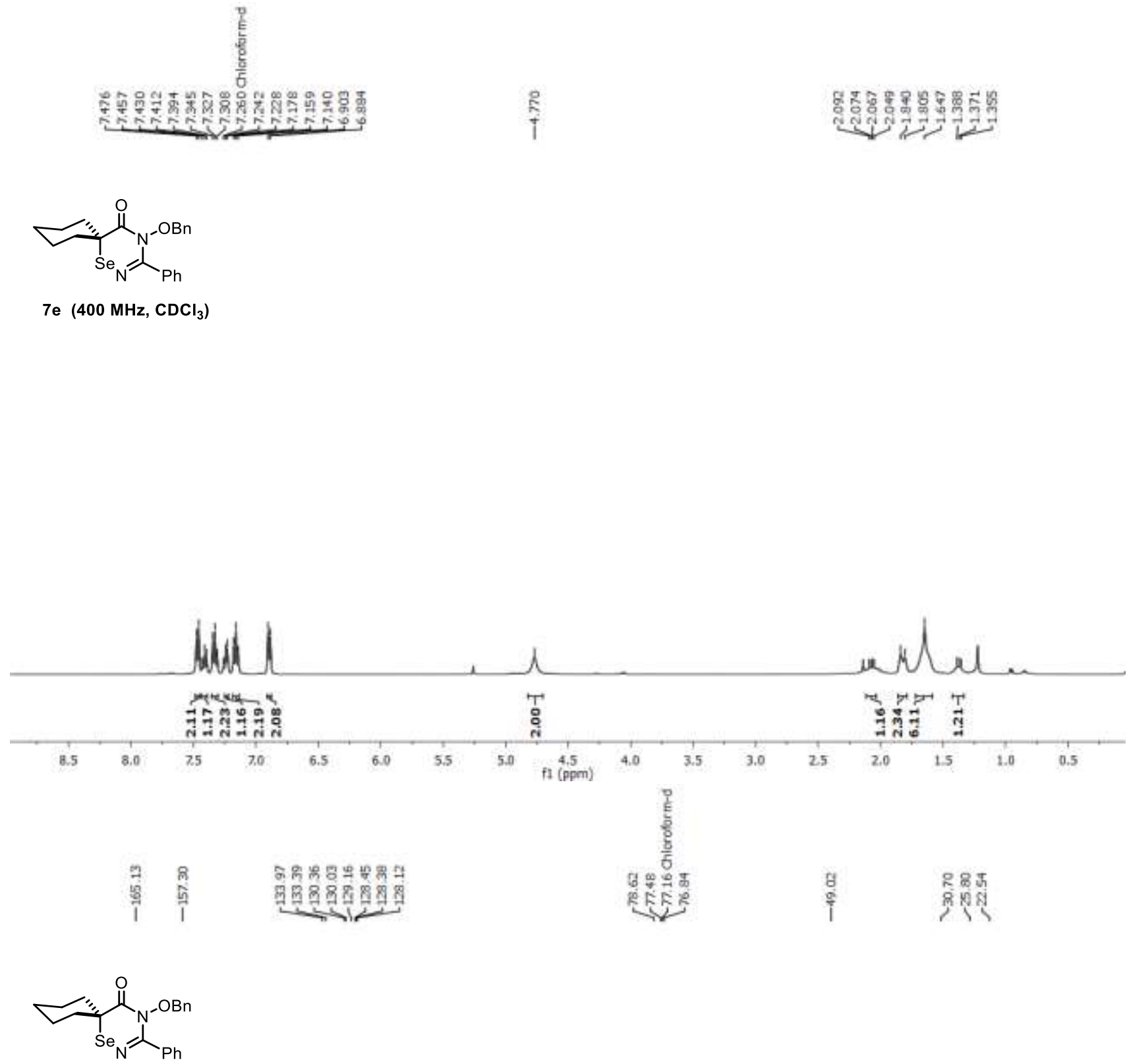

7e $\left(100 \mathrm{MHz}, \mathrm{CDCl}_{3}\right)$

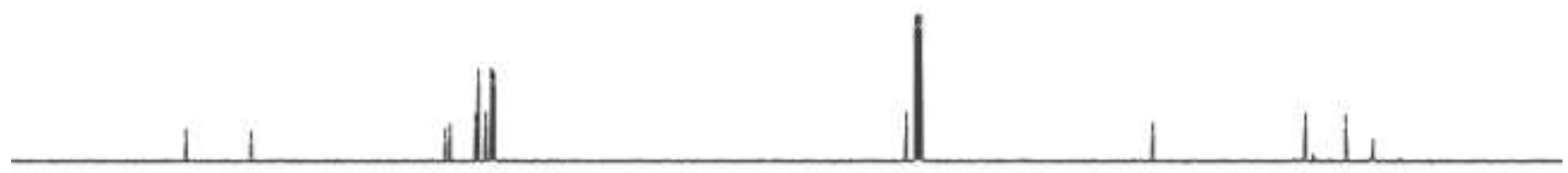

$\begin{array}{lllllllllllllllllllllllll}180 & 170 & 160 & 150 & 140 & 130 & 120 & 110 & 100 & 90 & 80 & 70 & 60 & 50 & 40 & 30 & 20 & 10 & \end{array}$


$\frac{\sqrt{2}}{\frac{1}{2}}$

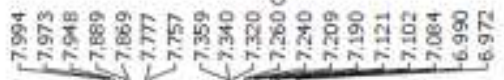

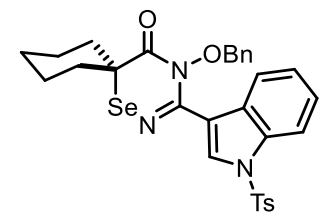

7f $\left(400 \mathrm{MHz}, \mathrm{CDCl}_{3}\right)$
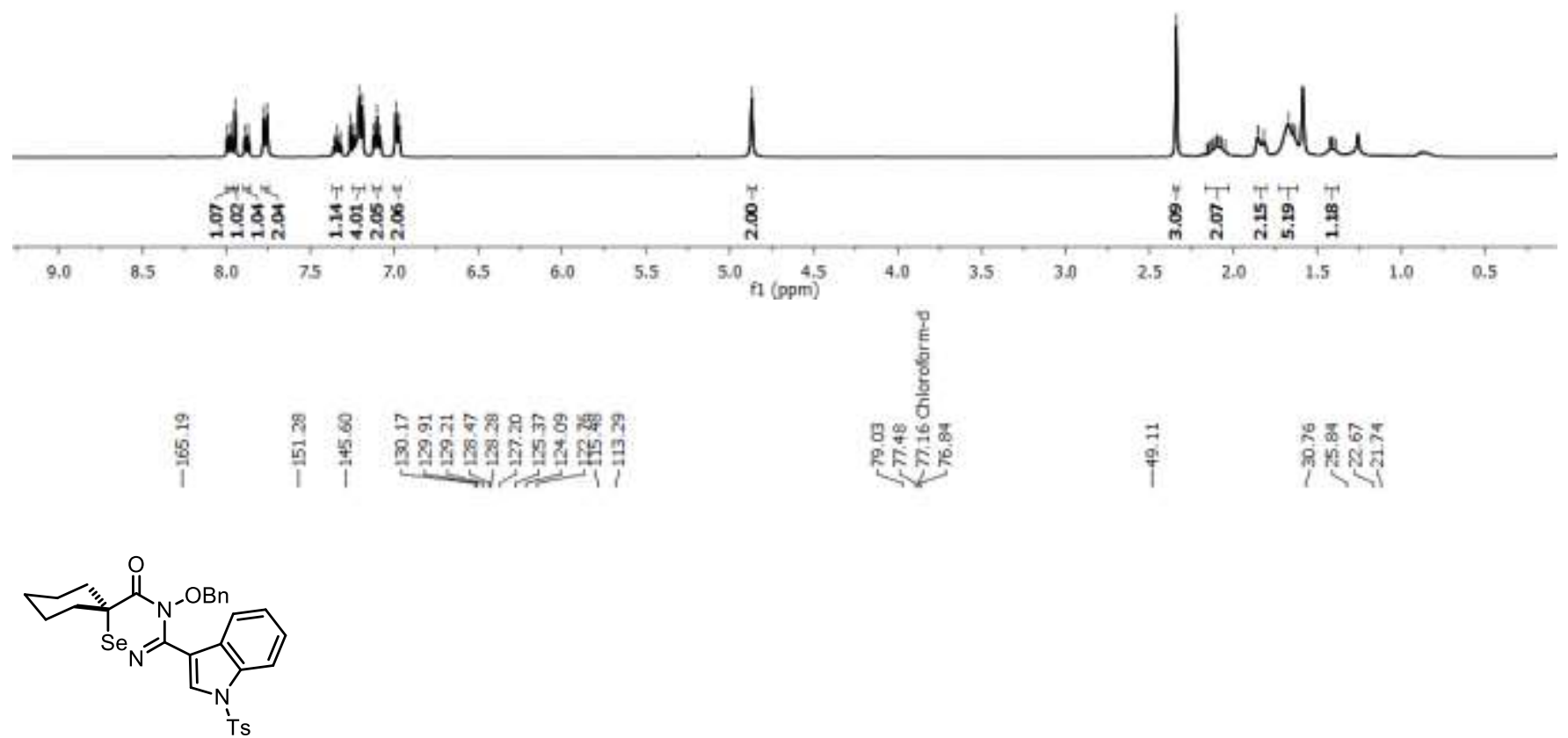

7f $\left(100 \mathrm{MHz}, \mathrm{CDCl}_{3}\right)$

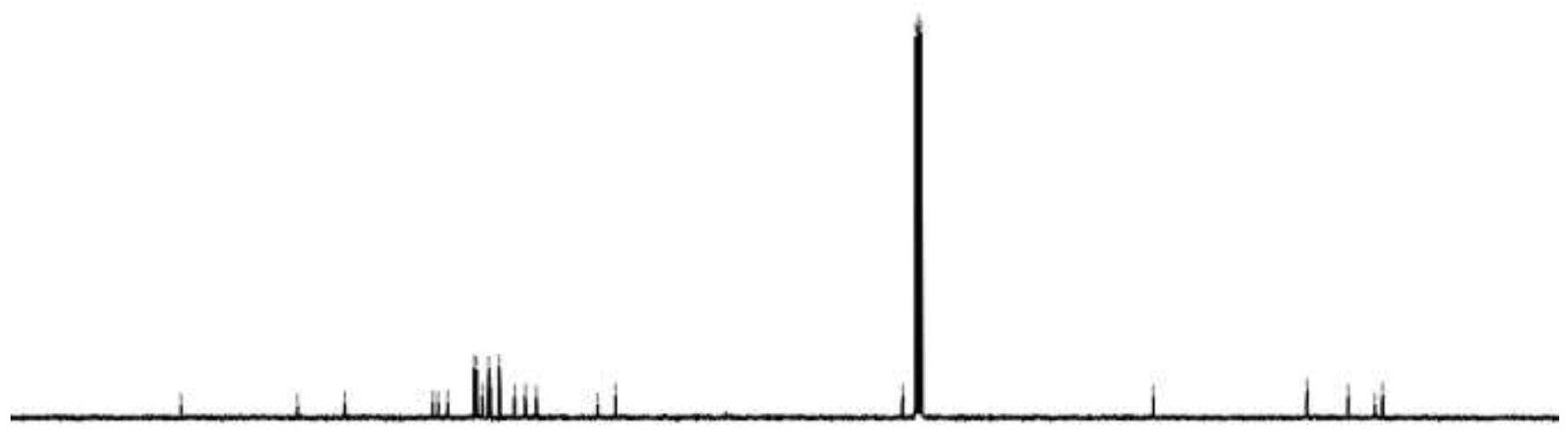

$\begin{array}{llllllllll}180 & 170 & 160 & 150 & 140 & 130 & 120 & 110 & 100 & 90\end{array}$

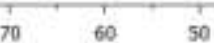

40

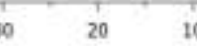




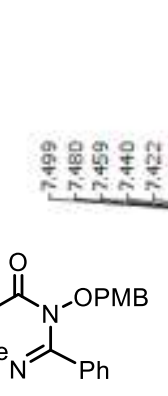

$7 \mathrm{~g}\left(400 \mathrm{MHz}, \mathrm{CDCl}_{3}\right)$
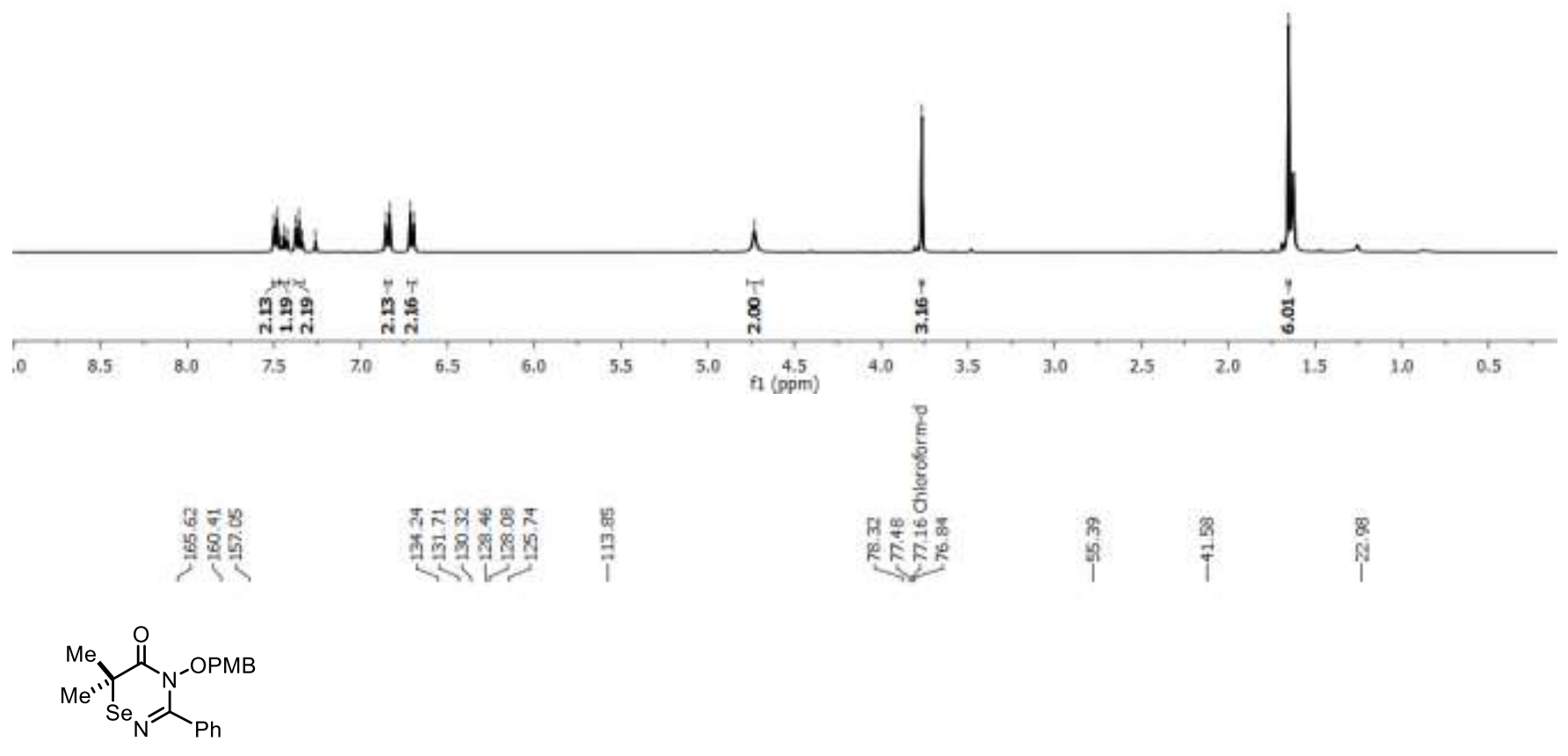

$7 g\left(100 \mathrm{MHz} \mathrm{CDCl}_{3}\right)$
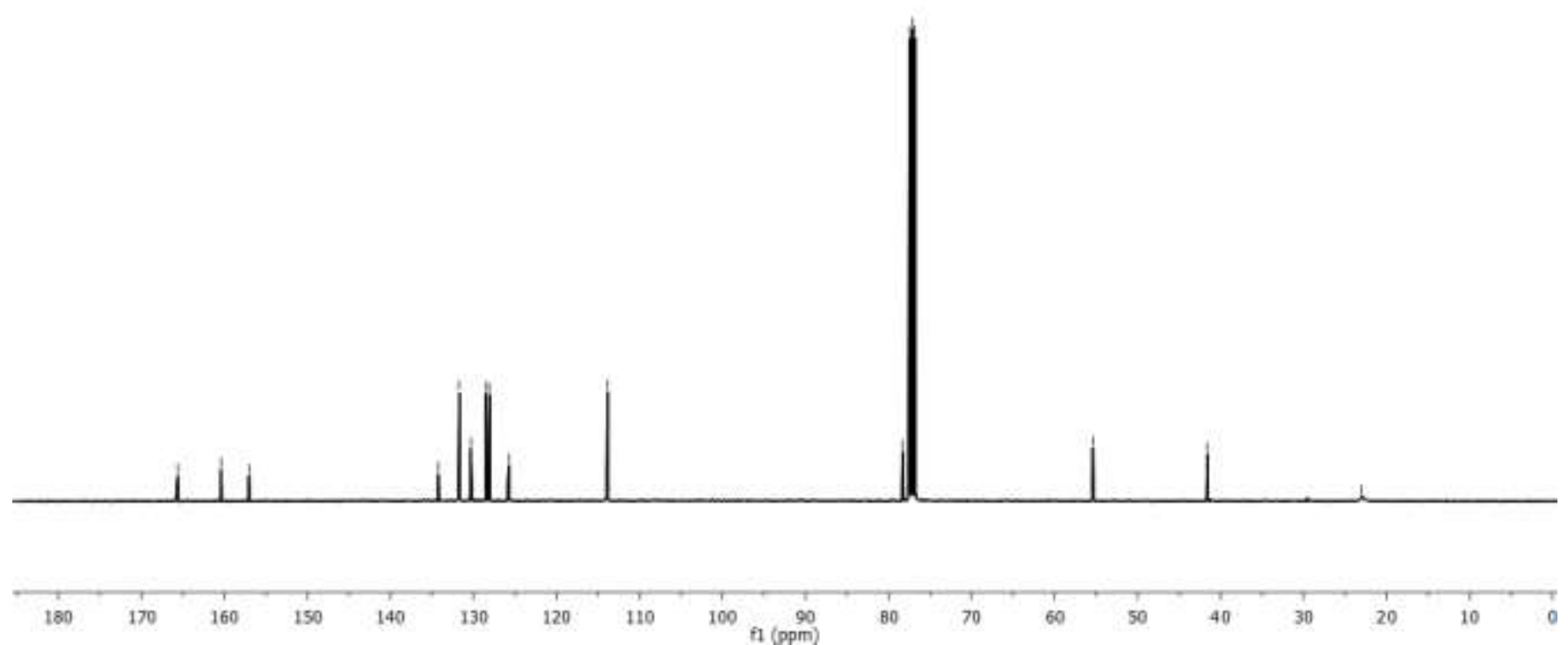


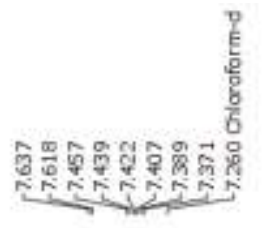

$\mathrm{Me}_{\mathrm{Se}^{2}}^{\mathrm{Me}} \mathrm{Cl}_{\mathrm{Ph}}^{\mathrm{O}}$

7h $\left(400 \mathrm{MHz} \mathrm{CDCl}_{3}\right)$
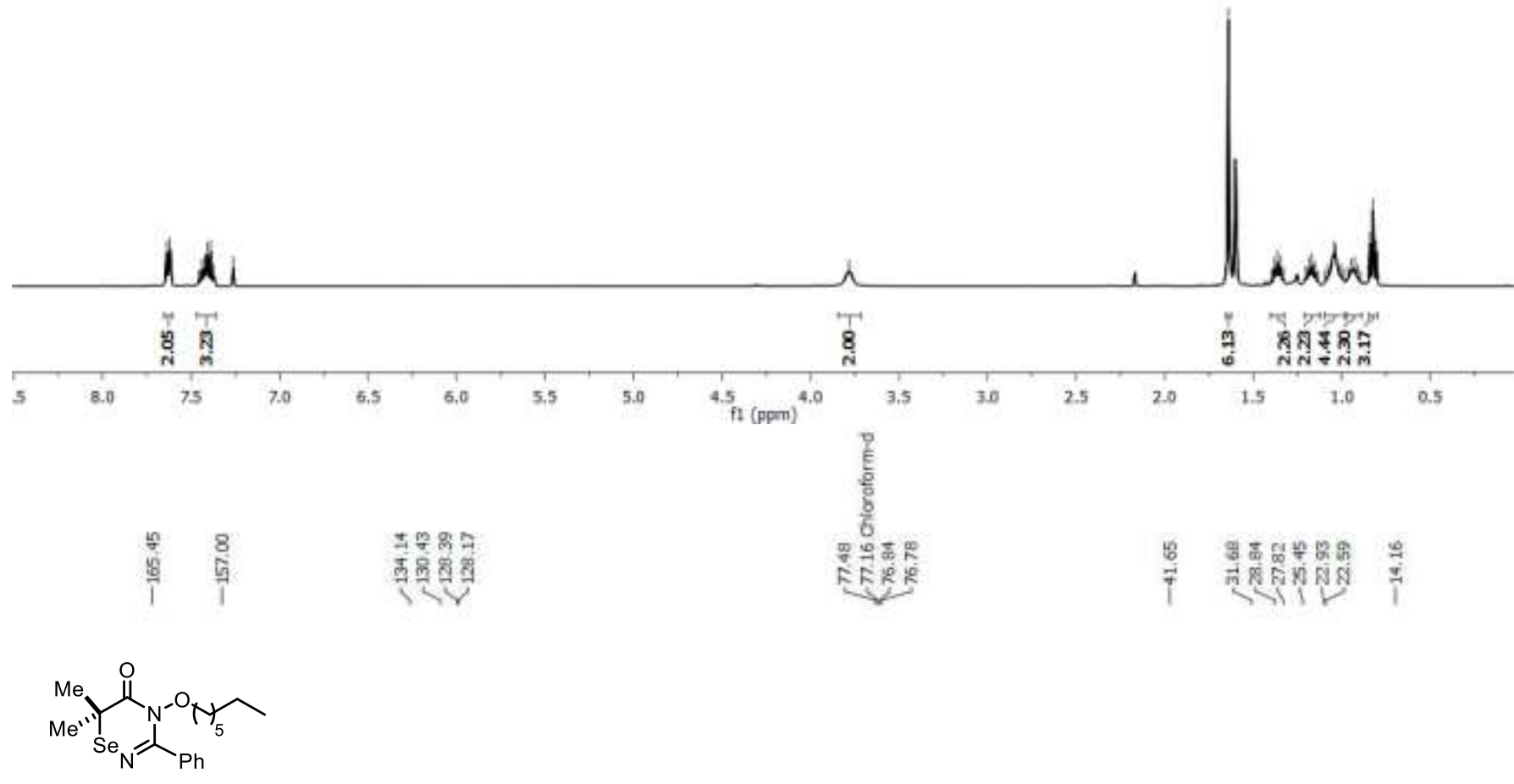

7h $\left(100 \mathrm{MHz}, \mathrm{CDCl}_{3}\right)$
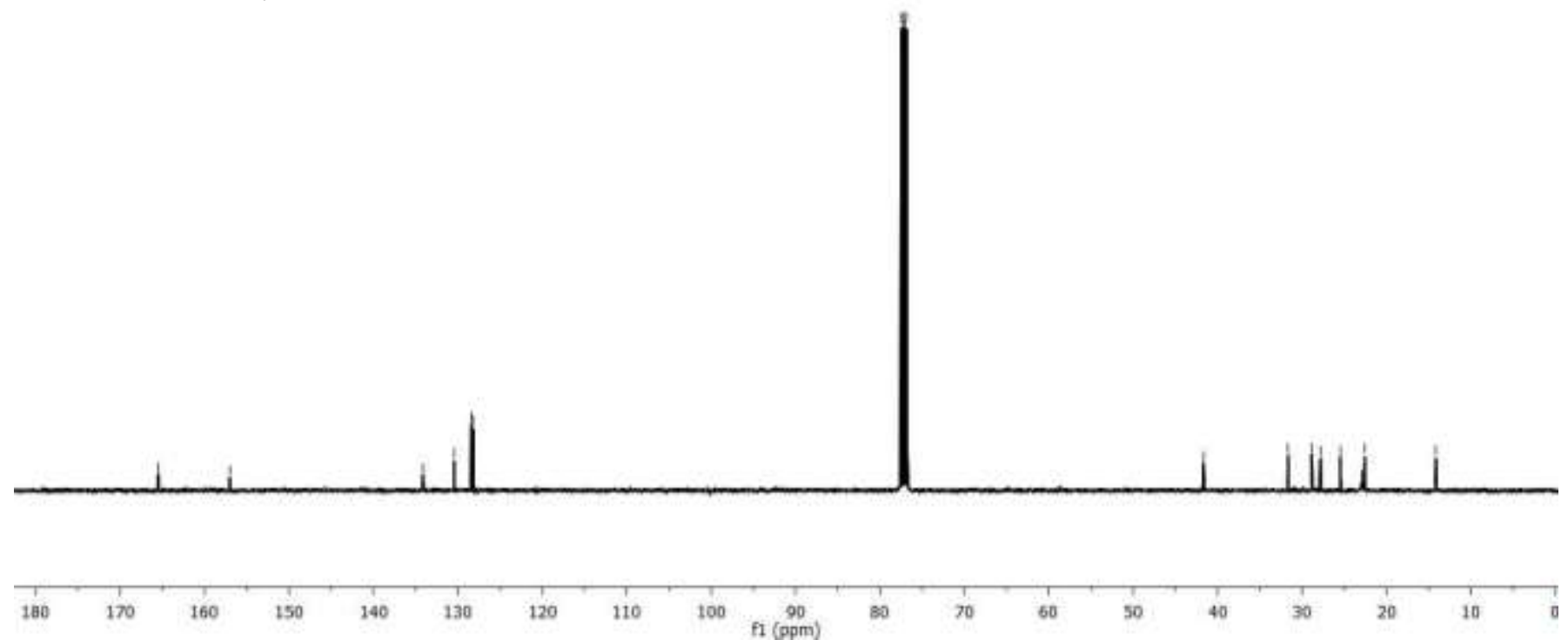


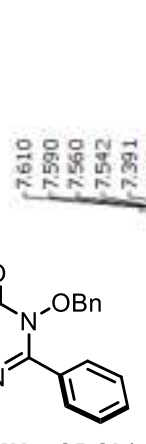

$8\left(400 \mathrm{MHz}, \mathrm{CDCl}_{3}\right)$
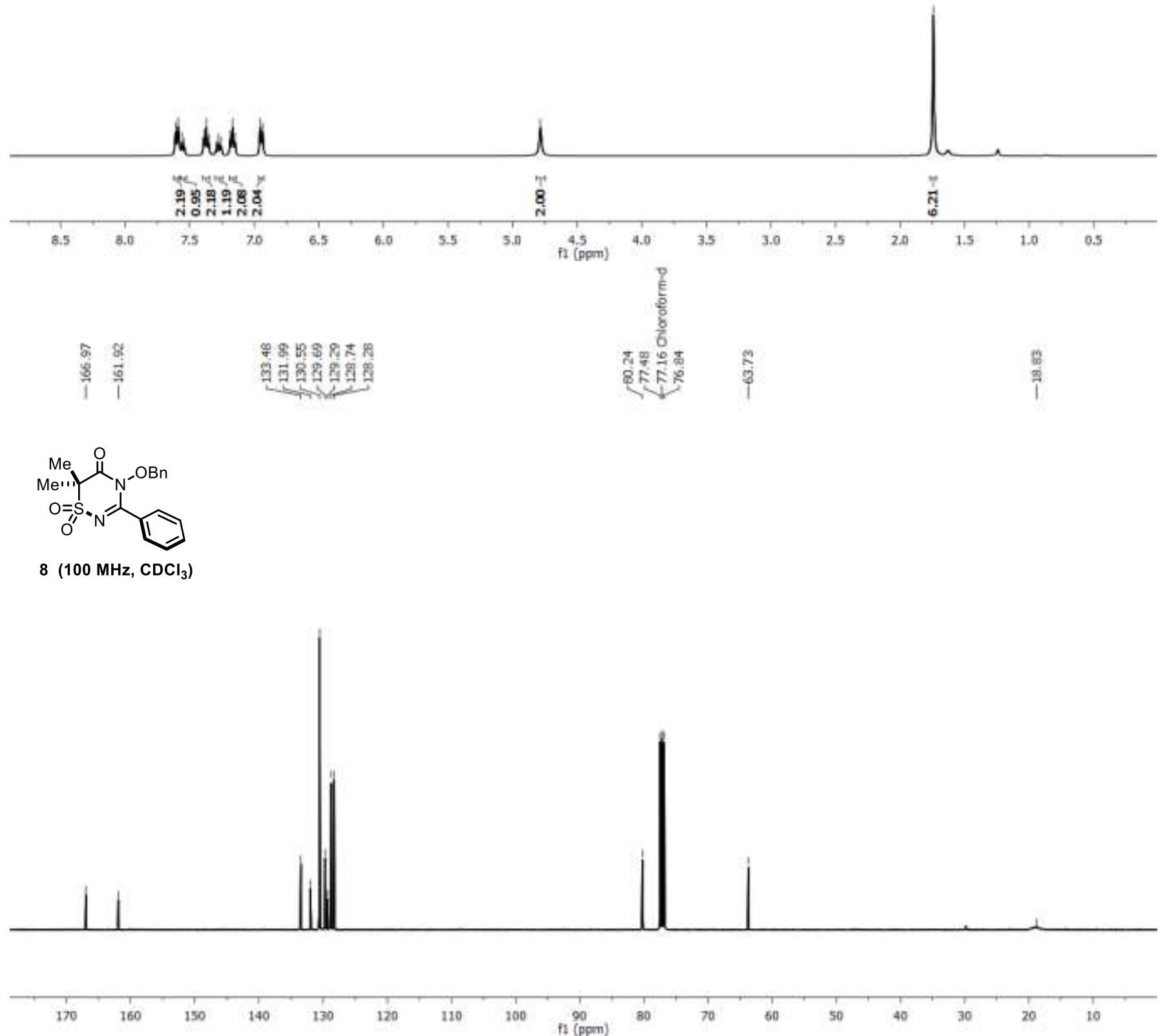

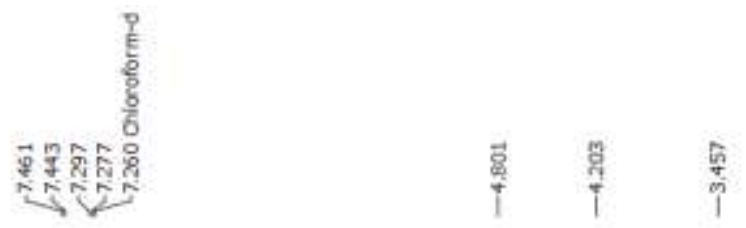

$\underset{+}{\stackrel{5}{+}}$
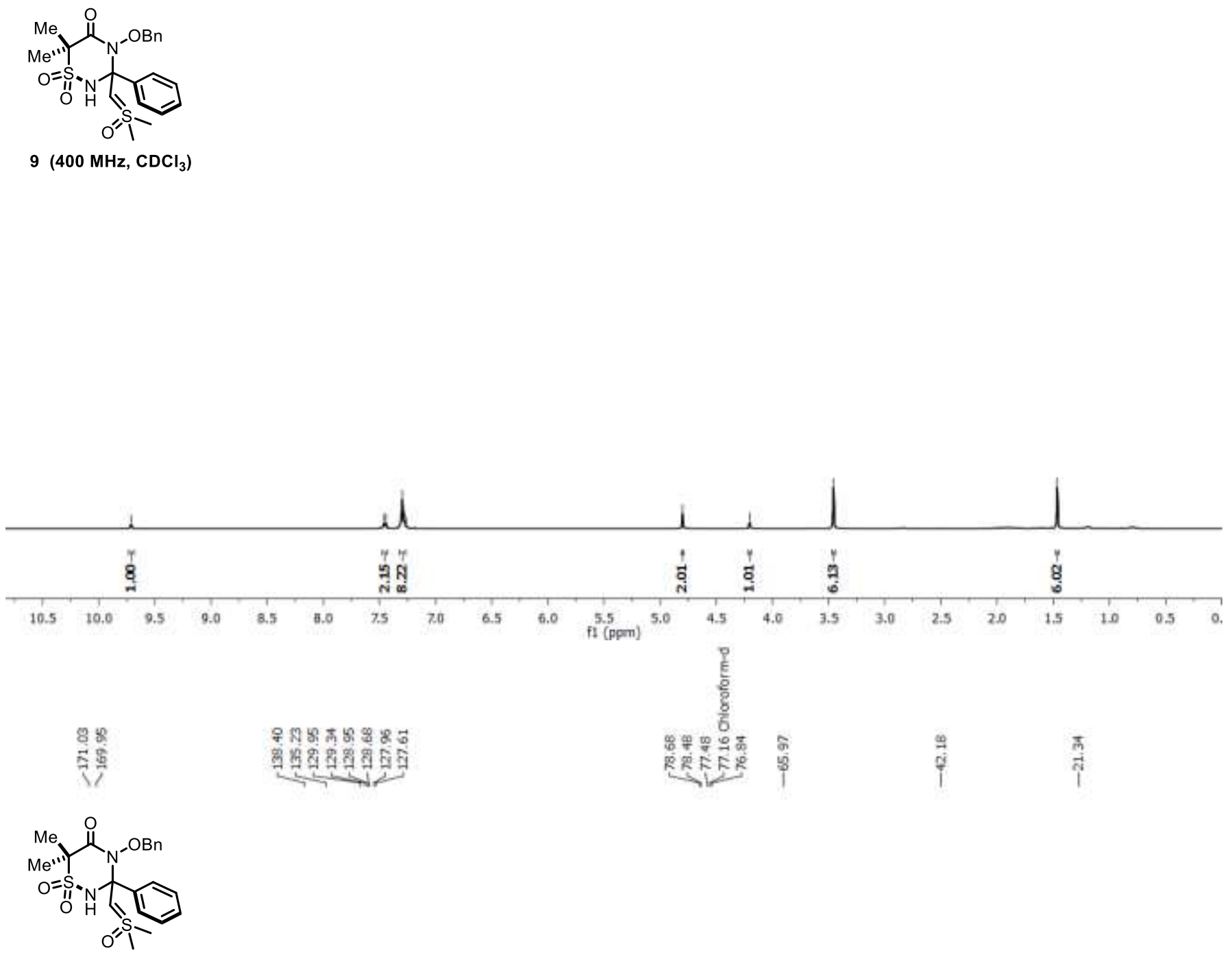

$9\left(100 \mathrm{MHz}, \mathrm{CDCl}_{3}\right)$
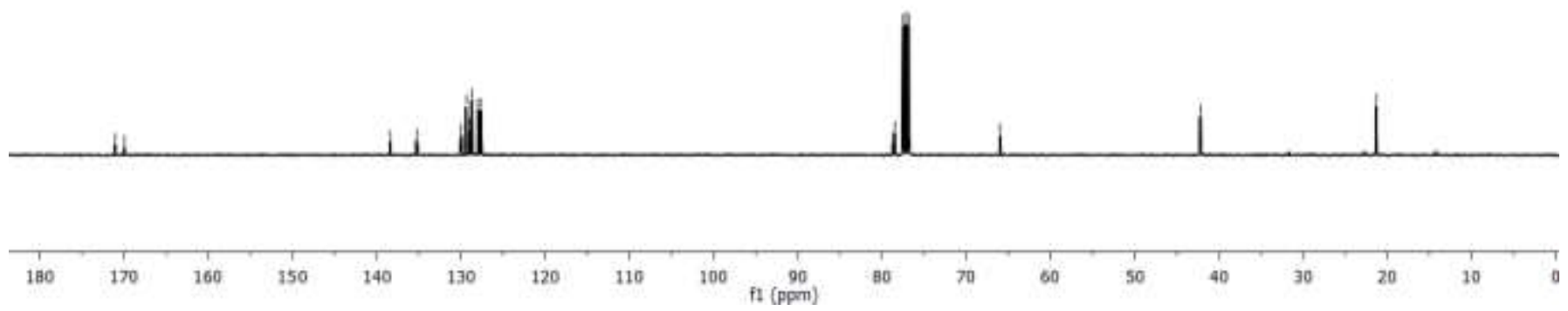

S126 

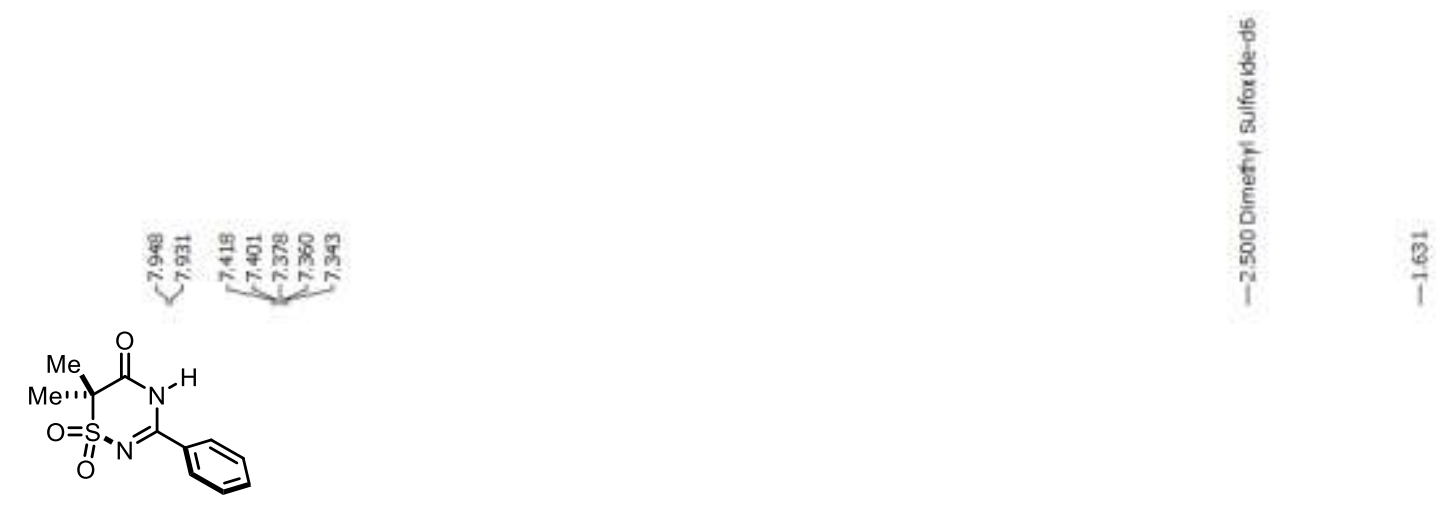

10 (400 MHz, DMSD-d6)
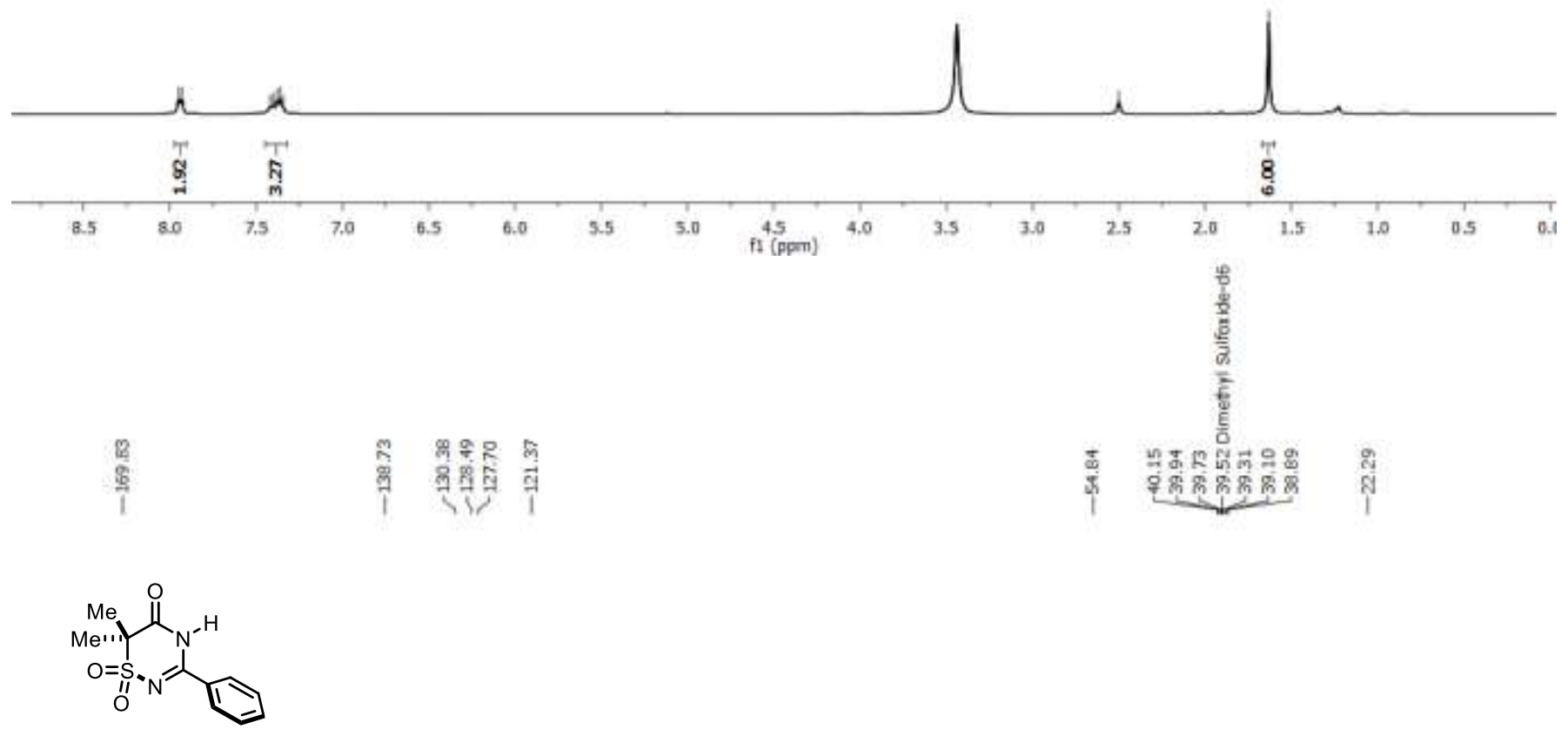

10 (400 MHz, DMSD-d6)
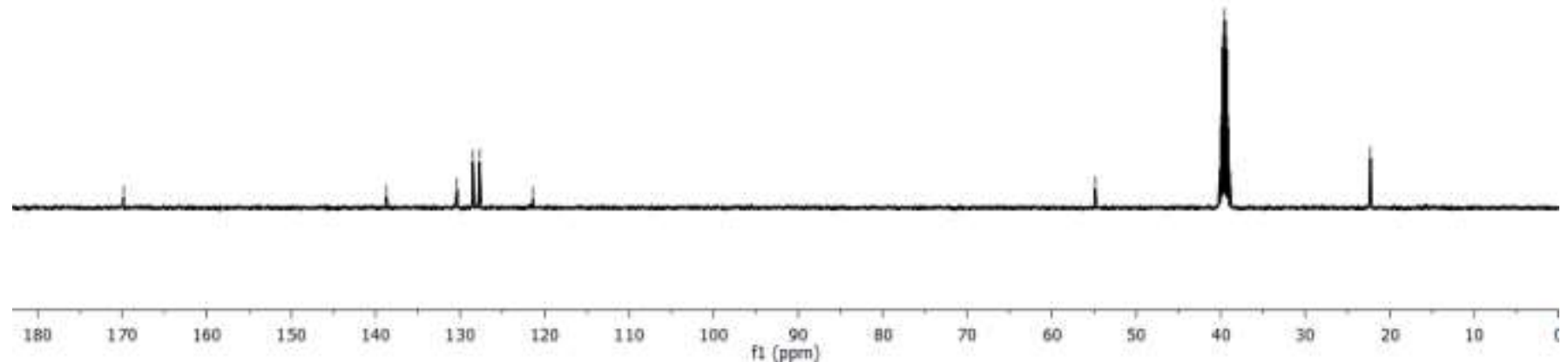

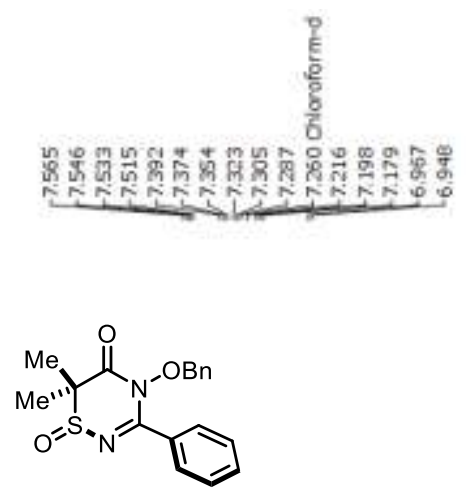

$11\left(400 \mathrm{MHz}, \mathrm{CDCl}_{3}\right)$
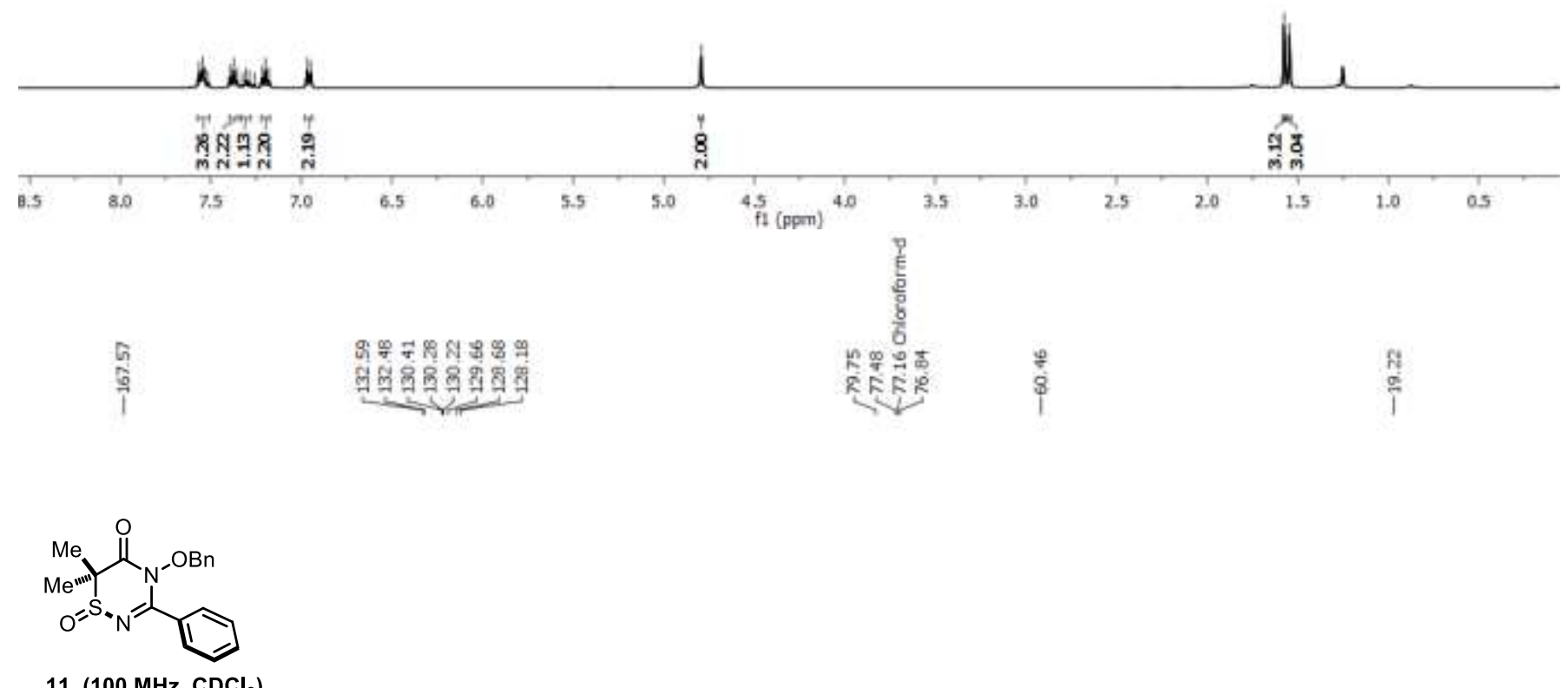

$11\left(100 \mathrm{MHz}, \mathrm{CDCl}_{3}\right)$
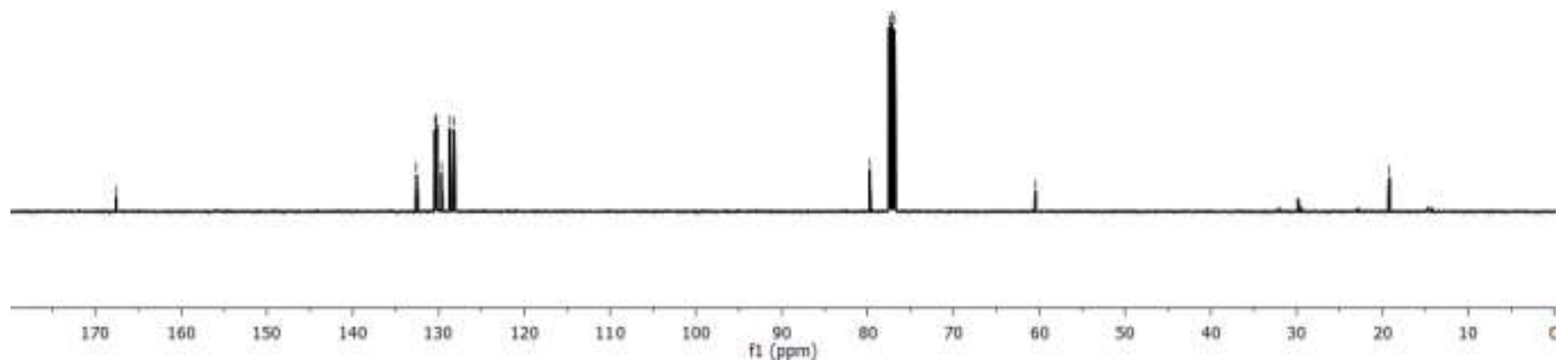


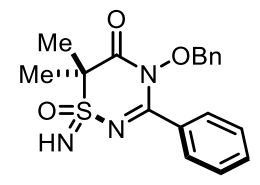

12 (400 MHz, DMSO-d6)
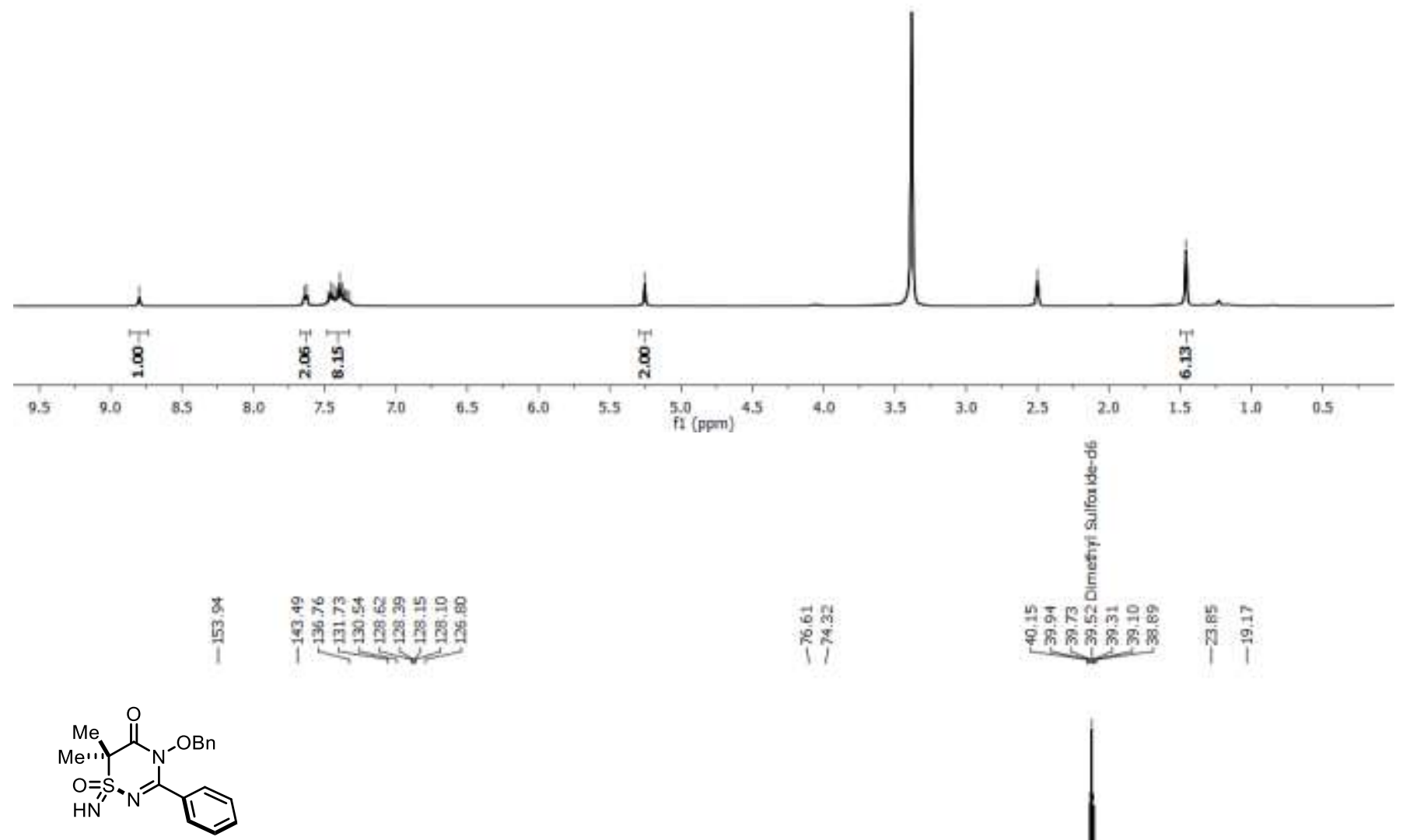

12 (100 MHz, DMSO-d6)
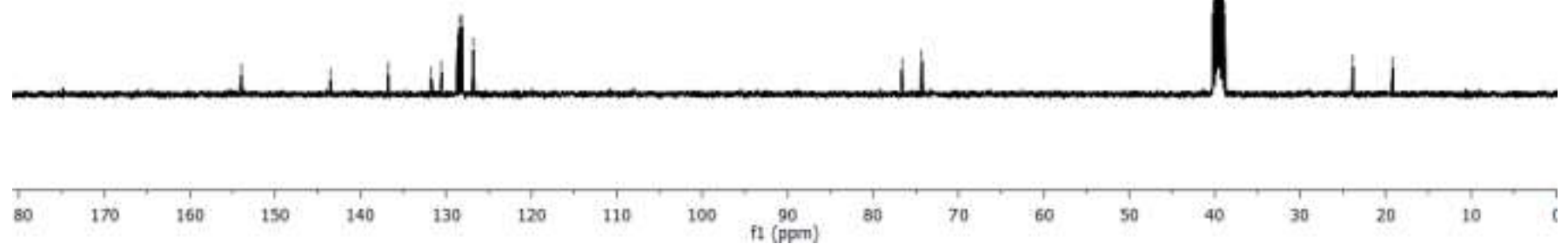


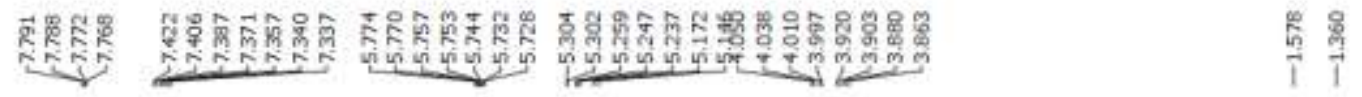
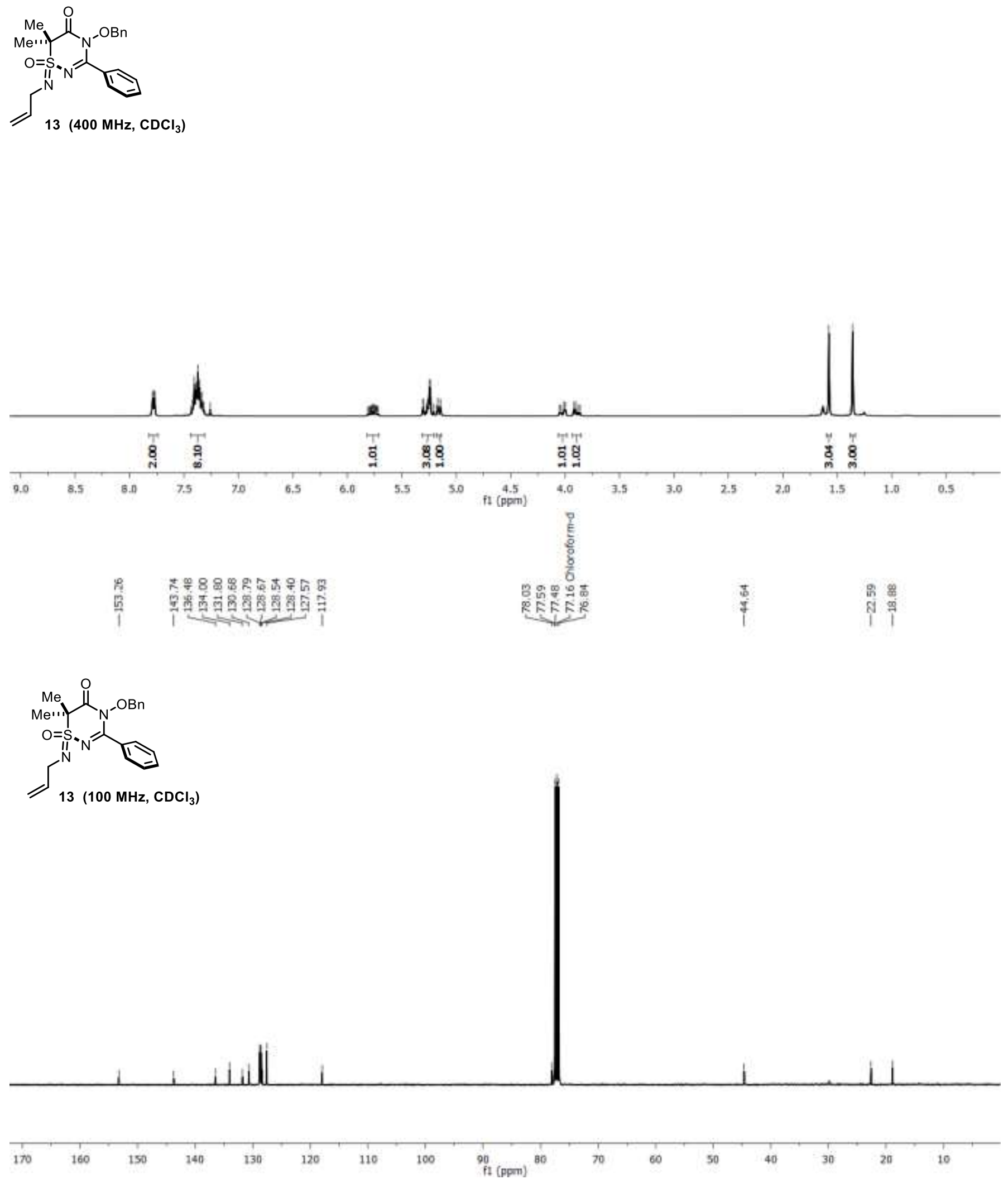


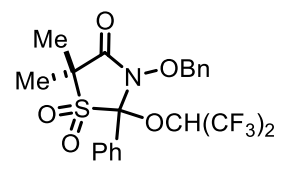

$14\left(400 \mathrm{MHz}, \mathrm{CDCl}_{3}\right)$
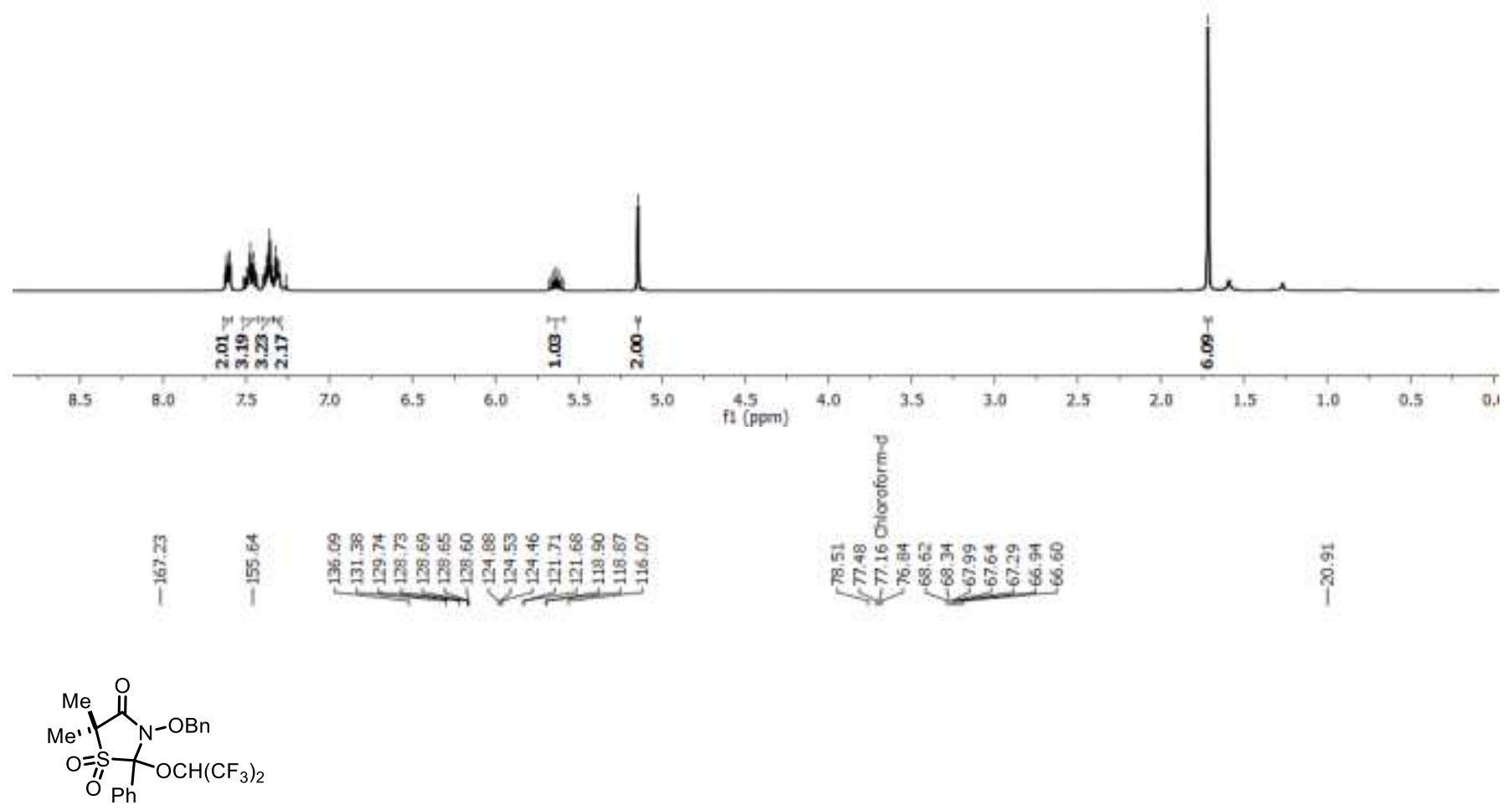

$14\left(100 \mathrm{MHz}, \mathrm{CDCl}_{3}\right)$
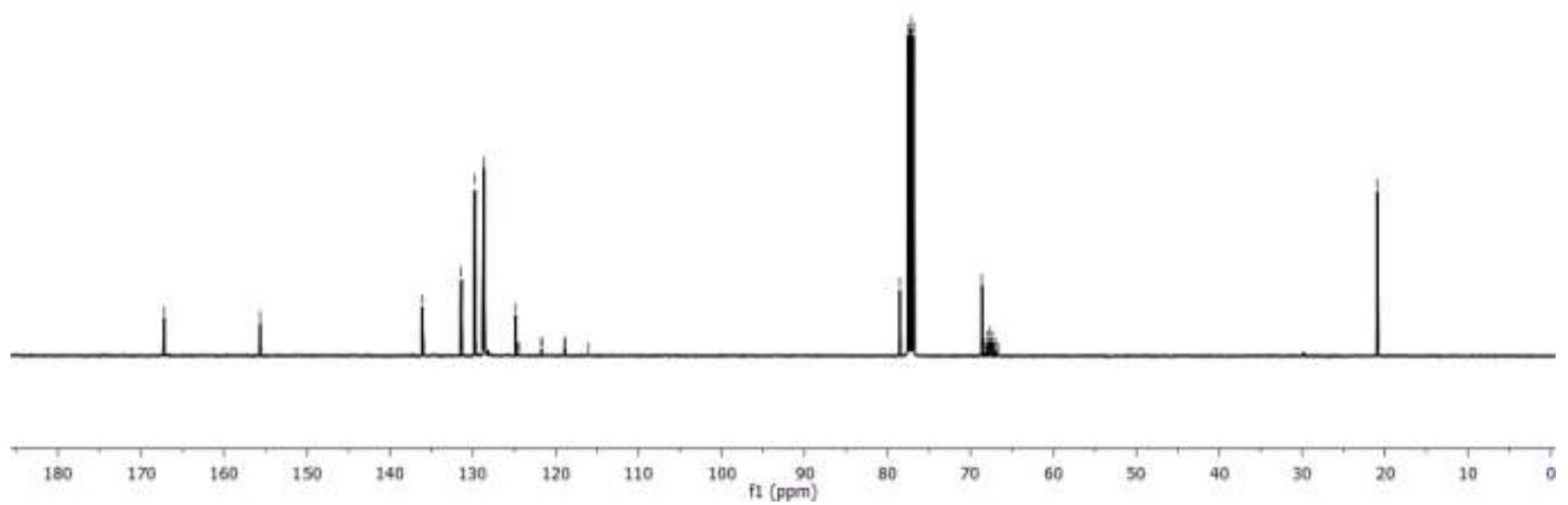


\section{Computational Studies}

The computations were performed with the Gaussian 16 software package. ${ }^{7}$ The geometries of all compounds and transition states were optimized with the BP86 functional ${ }^{8}$ and the 6$31 \mathrm{G}(\mathrm{d}, \mathrm{p})$ basis set at $298.15 \mathrm{~K}$. Other combination of functionals (B3LYP, B3P86, M06-2X, M06, M06L, MN15, MN15L, PBE, $\omega B$ 97-XD) and basis set (6-311+G(d,p), def2-TZVPP) were tested. While significant differences in energies were obtained, the relative position of the intermediates and transition states remained the same. Empirical dispersion correction also did not change the conclusions reached in this study. Frequency calculations furnished the thermal correction to the Gibbs free Energy $\mathrm{G}_{\text {corr. }}$. Using the gas phase geometry, single point calculations at the BP86/6-31G(d,p) level were carried out to obtain the energies in solution ESCRF. The SMD model was used to take the solvent effect into account, using the parameters of 2-propanol, except $\varepsilon$ set at the HFIP value of $16.7 .^{9}$ The free energy in solution at $298.15 \mathrm{~K}$ was obtained by adding the single point energy to the thermal correction to the Gibbs free energy $\left(\mathrm{G}=\mathrm{E}_{\mathrm{SCRF}}+\mathrm{G}_{\mathrm{corr}}\right)$. The discussed energies are $\Delta G_{298}$ in $\mathrm{kcal} / \mathrm{mol}$.

Results and discussion. Azide A was used as model compound for this study (Figure S2. Also see Scheme 6a from the main text). In $\mathbf{A}$, the $\mathrm{N}_{3}$ group occupies the axial position of the heterocycle and is oriented towards it. Conformational analysis showed that this particular isomer is the most stable. The stabilizing endo orientation of the $\mathrm{N}_{3}$ group can be attributed to a HOMO/LUMO interaction (hyperconjugation). Indeed, the HOMO is centered on the sulfur atom and the LUMO on the neighboring central and external nitrogen atoms of the $\mathrm{N}_{3}$ moiety $\left(\mathrm{S} \cdots \mathrm{N}^{2} 3.05 \AA\right.$ ). However, since the next steps are of $\mathrm{S}_{\mathrm{N}} 2$ type, $\mathbf{A}$ is not the reactive isomer. The reactive one is actually $\mathbf{A}^{\prime}, 1.3 \mathrm{kcal} / \mathrm{mol}$ less stable than $\mathbf{A}$, in which the $\mathrm{N}_{3}$ group adopts an exo orientation relatively to the heterocycle. 


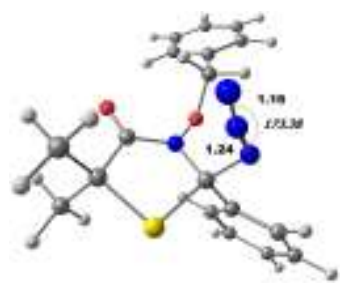

$\mathbf{A}(0.0)$

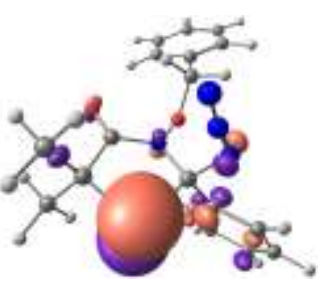

HOMO of A

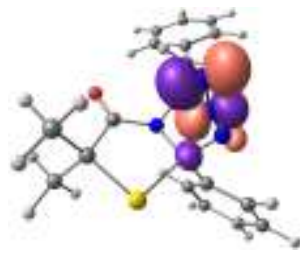

LUMO of $\mathbf{A}$

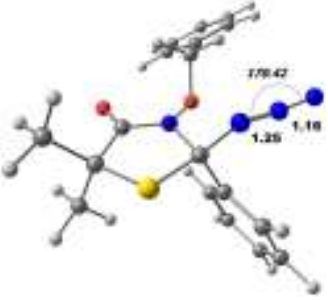

$\mathbf{A}^{\prime}(1.3)$

Figure S2. Endo and exo isomers $\mathbf{A}$ and $\mathbf{A}^{\prime}$ (relative free energies in $\mathrm{kcal} / \mathrm{mol}$ ). Frontier molecular orbitals of A. Selected bond length $(\AA)$ and angles $\left(^{\circ}\right)$

The pathways leading to the three possible heterocycles $\mathbf{G}, \mathbf{2 a}$ and $\mathbf{D}$ from azide $\mathbf{A}$ ' were next studied (Scheme 1). N-N, S-N and C-N attacks to the internal azide nitrogen atom were modeled through $\mathbf{T} \mathbf{S}_{\mathbf{A}^{\prime} \mathbf{E}}, \mathbf{T S}_{\mathbf{A}^{\prime} \mathbf{H}}$ and $\mathbf{T} \mathbf{S}_{\mathbf{A}^{\prime} \mathbf{B}}$, respectively. In contrast with $\mathbf{T S}_{\mathbf{A}^{\prime} \mathbf{E}}$ and $\mathbf{T} \mathbf{S}_{\mathbf{A}^{\prime} \mathbf{H}}$, the highest-lying transition state $\mathbf{T S}_{\mathbf{A}^{\prime} \mathbf{B}}$ does not correspond to the direct elimination of $\mathrm{N}_{2}$ but produces the 1,2,3-triazole $\mathbf{B}$ after [3+2] addition of the $\mathrm{N}_{3}$ dipole to a phenyl double bond. Elimination of $\mathrm{N}_{2}$ could then be computed from $\mathbf{B}$ to give the 5-membered ring heterocycle, first as an adduct with $\mathrm{N}_{2}(\mathbf{C})$ and then free (D). Although $\mathbf{D}$ is formed in a strongly exergonic manner and is the most stable of the three possible heterocycles $(-61.9$ vs -49.5 or -43.6 $\mathrm{kcal} / \mathrm{mol}$ ), $\mathbf{T} \mathbf{S}_{\mathbf{A}} \mathbf{B}, \mathbf{B}$ and $\mathbf{T S}_{\mathbf{B C}}$ lie above $40 \mathrm{kcal} / \mathrm{mol}$ and are clearly inaccessible at room temperature. Another unlikely option is the formation of $\mathbf{G}$ since the N-N attack transition state $\mathbf{T S}_{\mathbf{A}^{\prime} \mathbf{E}}$ has a free energy of $37.6 \mathrm{kcal} / \mathrm{mol}$. It leads to the 3 -membered ring diazo zwitterion $\mathbf{E}$ as an adduct with $\mathrm{N}_{2}$, located at $9.1 \mathrm{kcal} / \mathrm{mol}$ on the energy surface. After removal of $\mathrm{N}_{2}$ the free energy goes down to $4.8 \mathrm{kcal} / \mathrm{mol}$. finally, the $\mathrm{C}-\mathrm{N}$ bond cleavage transition state $\mathbf{T S} \mathbf{S}_{\mathbf{F G}}$, lying at $7.3 \mathrm{kcal} / \mathrm{mol}$, furnishes heterocycle $\mathbf{G}$ at $-43.6 \mathrm{kcal} / \mathrm{mol}$. The most accessible route, marked in green in Scheme 5a, is eventually that corresponding to the S-N attack through $\mathbf{T S}_{\mathbf{A}^{\prime} \mathbf{H}}$ located at $27.6 \mathrm{kcal} / \mathrm{mol}$. It connects $\mathbf{A}^{\boldsymbol{\prime}}$ to the 3 -membered ring zwitterion $\mathbf{H}$ as an adduct with $\mathrm{N}_{2}$, which is formed in an exergonic manner $(-6.6 \mathrm{kcal} / \mathrm{mol})$. Removal of $\mathrm{N}_{2}$ further releases $\mathrm{kcal} / \mathrm{mol}$

to give 
$-10.9 \mathrm{kcal} / \mathrm{mol}$. Cleavage of the $\mathrm{C}-\mathrm{S}$ bond to give the experimentally observed heterocycle $\mathbf{2 a}$ requires only $0.9 \mathrm{kcal} / \mathrm{mol}$ to reach $\mathbf{T S} \mathbf{I}(\mathbf{2 a})$ (at $-10.0 \mathrm{kcal} / \mathrm{mol}$ ). Overall, $\mathbf{2 a}$ is more stable than A by $49.5 \mathrm{kcal} / \mathrm{mol}$.

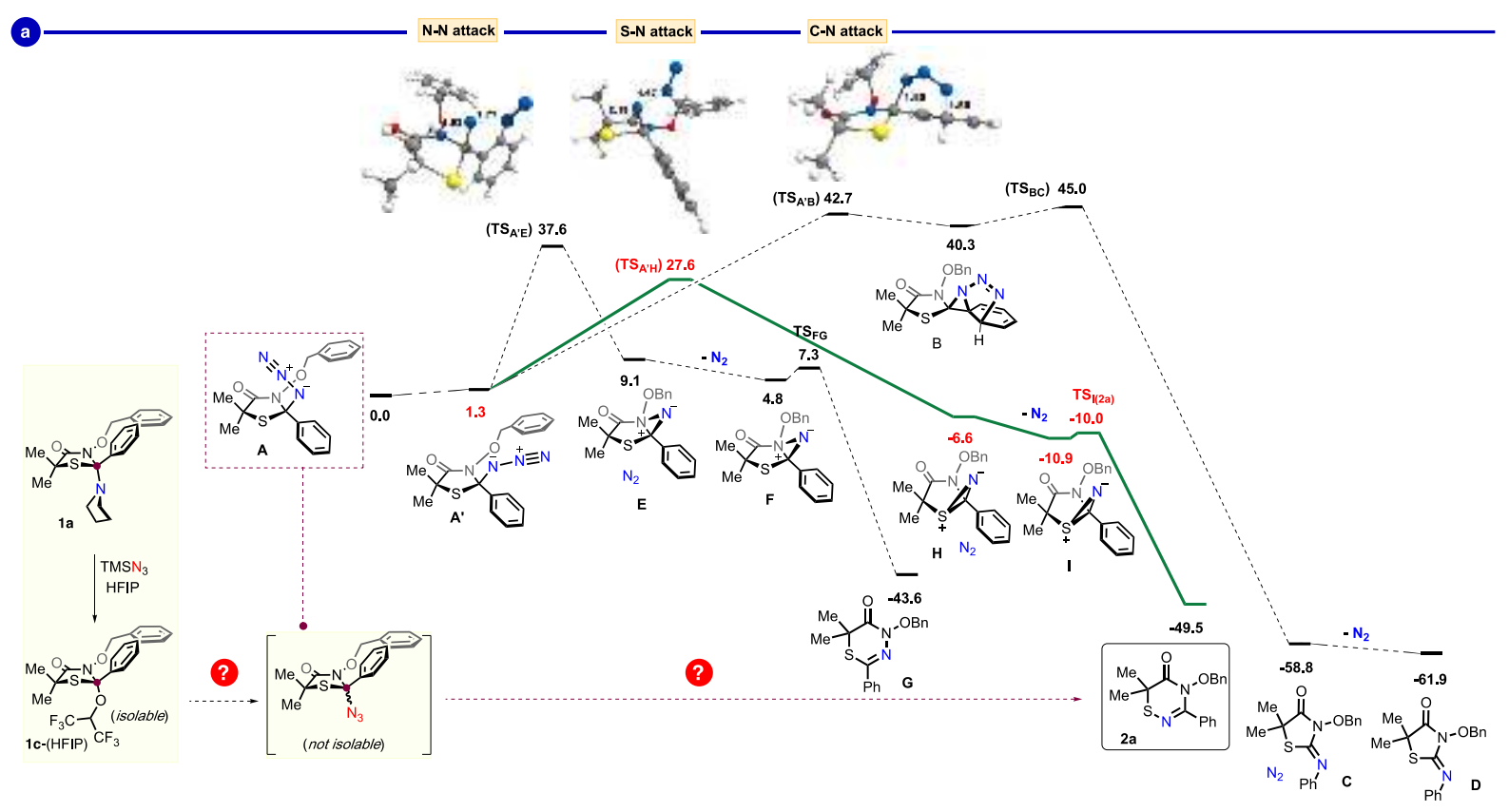

Scheme 6a (from main text). Computed pathways to possible products $\mathbf{G}, \mathbf{2 a}$ or $\mathbf{D}$

While the computations support the formation of the experimentally observed isomer $\mathbf{2 a}$ as the best option, the first barrier of the pathway to $\mathbf{2 a}$ remains quite high for a reaction taking place at $\mathrm{rt}(27.6 \mathrm{kcal} / \mathrm{mol})$. We reinvestigated all these options with the azide occupying the equatorial position of the 5-membered ring instead of the axial one but found virtually the same energies. The possible formation of a nitrenium was also envisaged, but these attempts systematically led to compound I. Although the role of HFIP is implicitly taken into account in these calculations, we decided to explicitly introduce a molecule of solvant in the optimized structures. We have shown on various occasions that the optimization of alcohol adducts instead of naked molecules can lead to a significant decrease of the reaction barriers. ${ }^{10}$ Various isomers of the azide and HFIP were analyzed, leading to $\mathbf{K}$ as the most stable one (Figure S3). 
The striking feature of this adduct is the endo orientation of the benzyl group relatively to the heterocycle when HFIP is present. Analysis of the electron density indicated that the strongest noncovalent interaction is the hydrogen bond between the $\mathrm{OH}$ group of HFIP and the $\mathrm{S}$ atom $\left(\rho_{\max }=0.0233 \mathrm{e} / \AA^{3}\right)$. There are then three weaker hydrogen bonds $\left(\rho_{\max }=0.0054 \mathrm{e} / \AA^{3}\right)$, one between the $\mathrm{CH}$ hydrogen of HFIP and the internal $\mathrm{N}$ atom of the $\mathrm{N}_{3}$ moiety and two between a fluorine atom of $\mathrm{CF}_{3}$ group and two phenyl hydrogens, enforcing this specific conformation. We then studied the C-N attack and obtained $\mathbf{T S} \mathbf{S K C}$, in which the benzyl group retrieves its exo orientation. This time, the migration of the phenyl group takes place in a concerted fashion to give C'. This direct process could not be modeled in the absence of HFIP ( $c f$. Scheme 5a). While the barrier in the explicit presence of HFIP is much lower ( $26.7 \mathrm{vs} 42.7 / 45.0 \mathrm{kcal} / \mathrm{mol})$, it remains relatively high for a reaction performed at $\mathrm{rt}$. The $\mathrm{N}-\mathrm{N}$ attack pathway was found stepwise as before, yet at a still very high free energy cost $(39.3 \mathrm{kcal} / \mathrm{mol})$. The $\mathrm{S}-\mathrm{N}$ attack on the other hand was found markedly facilitated from the HFIP adduct $\mathbf{K}$ compared to $\mathbf{A}^{\prime}$. The barrier went down to $19.9 \mathrm{kcal} / \mathrm{mol}$ via $\mathbf{T S} \mathbf{K}\left(\mathbf{2} \mathbf{a}^{\mathbf{}}\right)$. It is also noteworthy that the reaction is now concerted.

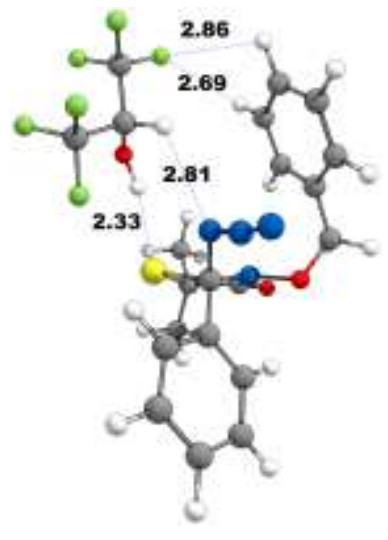

$\mathbf{K}(0.0)$

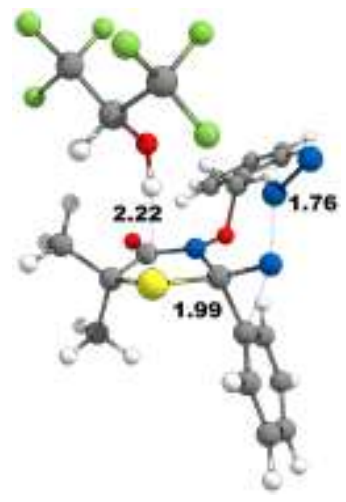

TSKC' $^{\prime}$ (26.7)

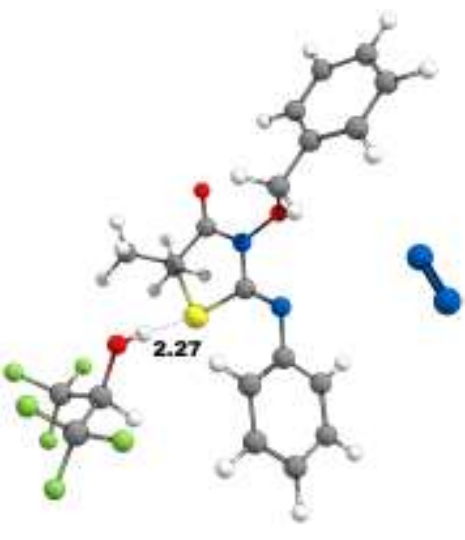

C' (-62.4) 


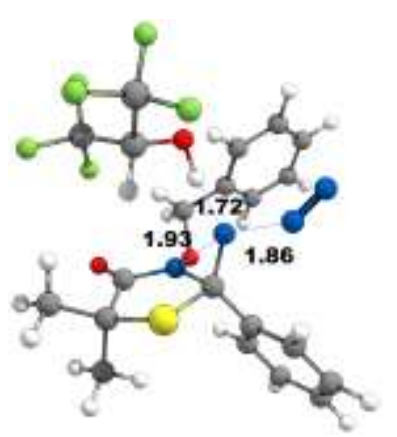

TSKG' $^{\prime}(39.3)$

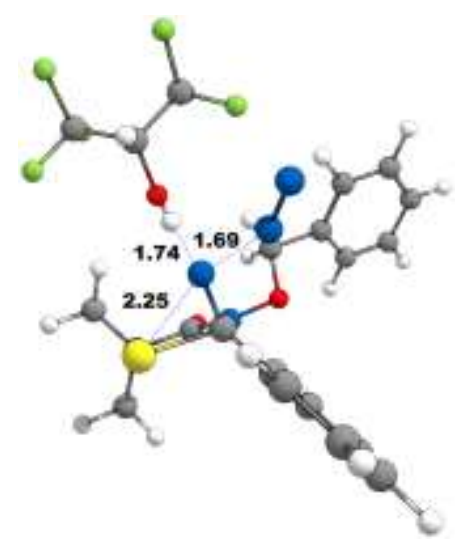

TSк2а' (19.9)

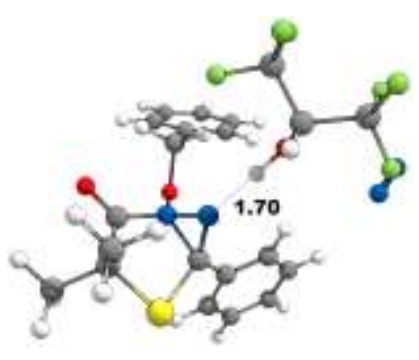

$\mathbf{G}^{\prime}(6.4)$

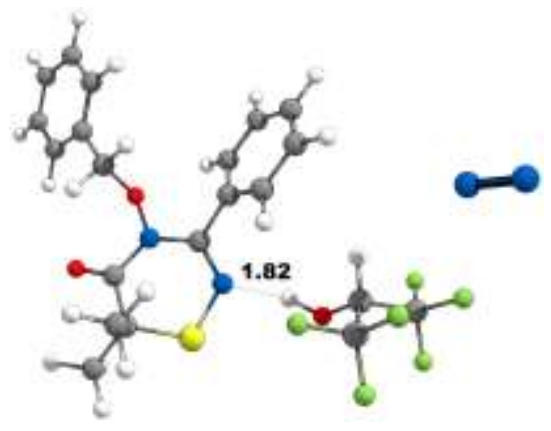

$\mathbf{2} \mathbf{a}^{\prime}(-50.8)$

Figure S3. Endo and exo isomers A and A' $\left(\Delta G_{298}, \mathrm{kcal} / \mathrm{mol}\right)$. Selected bond length $(\AA)$ and angles $\left({ }^{\circ}\right)$

A frontier molecular orbitals analysis was performed to rationalize the role of HFIP. Since A' and $\mathbf{K}$ have different conformations, the less stable isomer K', showing a single hydrogen bond as in $\mathbf{T S}_{\mathbf{K} 2 \mathbf{a}}$, was used for the comparison (Figure S4). Clearly, the hydrogen bond between HFIP and the internal azide nitrogen has a strong stabilizing influence on the LUMO level and reduces the HOMO/LUMO gap (from 3.33 to $2.95 \mathrm{eV}$ ). 

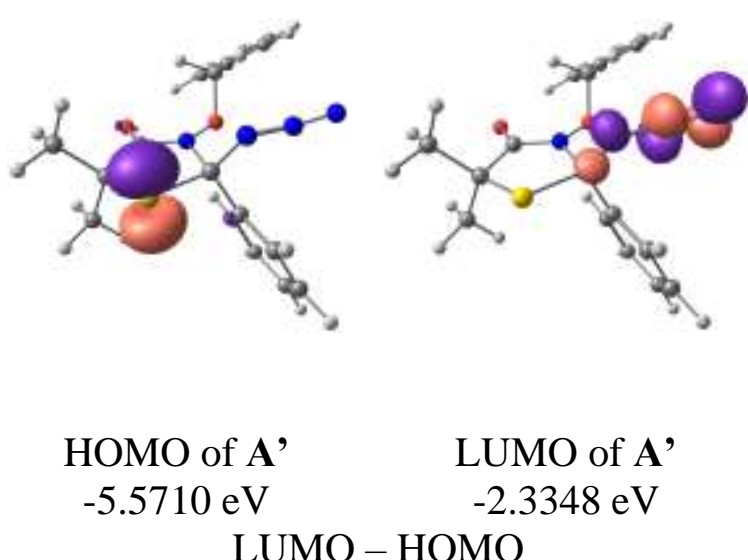

$3.3261 \mathrm{Ev}$



HOMO of $\mathbf{K}^{\prime}$ $-5.6525 \mathrm{eV}$

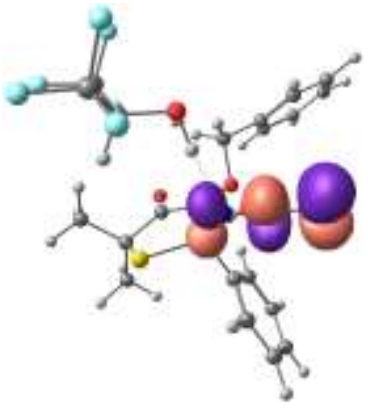

LUMO of $\mathbf{K}^{\prime}$ $-2.6979 \mathrm{eV}$

$$
\text { LUMO - HOMO }
$$

$2.9545 \mathrm{eV}$

Figure S4. Frontier orbitals of A' and K'.

The same comparison was made between $\mathbf{T S}_{\mathbf{A}^{\prime} \mathbf{H}}$ and $\mathbf{T} \mathbf{S}_{\mathbf{K} 2 \mathbf{a}}$ ' since the conformations are the same. The 2.6385 eV HOMO/LUMO gap in $\mathbf{T S}_{\mathbf{A}^{\prime} \mathbf{H}}$ goes down to $2.2971 \mathrm{eV}$ in $\mathbf{T} \mathbf{S}_{\mathbf{k} 2 \mathrm{a}}$. Thus, HFIP plays the role of a Lewis acid in this reaction.

In another study, ${ }^{10}$ it was shown that the use of several H-bonded molecules of HFIP could further lower the energy barriers, but this was not the case here (Figure S5). With one or two additional HFIP H-bonded to the one activating the azide functionality, the barriers increased to 22.1 and to $24.3 \mathrm{kcal} / \mathrm{mol}$ respectively. With two independent HFIP molecules on the azide, there was virtually no effect on the barrier height $(20.4 \mathrm{kcal} / \mathrm{mol})$. In fact, HFIP can form many types of clusters, the acidity of which depending on subtle factors such as conformation and aggregation, ${ }^{11}$ so it is very difficult to know which precise cluster actually catalyzes best the reaction. However, this computational study shows the dramatic impact a single explicit molecule of HFIP can have in this reaction. Besides, this study clearly predicts the experimentally observed product. 


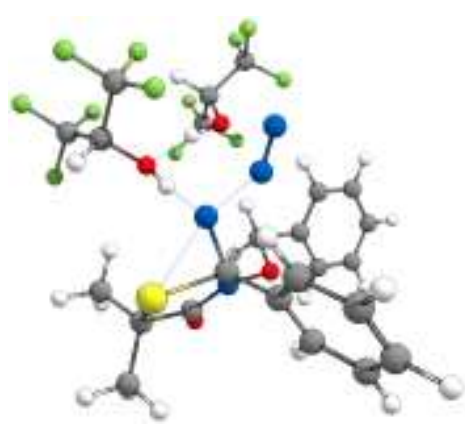

[TSK2a']' (22.1)

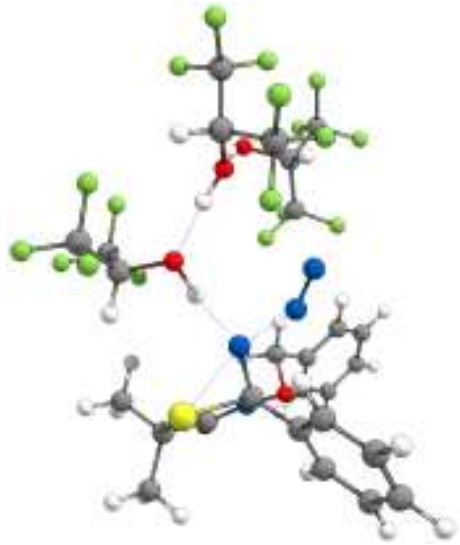

[TSK2a']" (24.3)

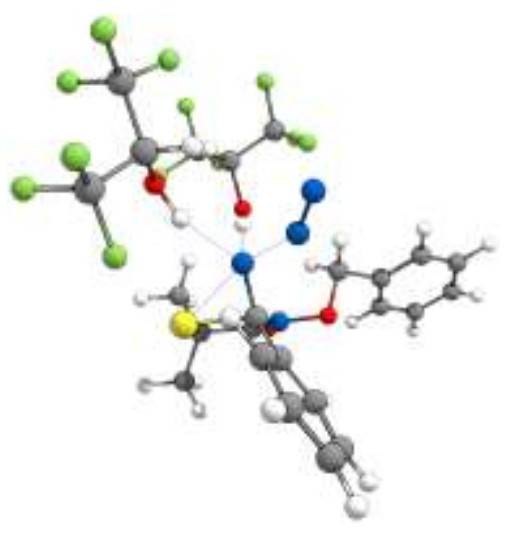

$\left[\right.$ TSK2a'] $^{\prime}, "(20.4)$

Figure S5. S-N attack transitions states with 2 or 3 HFIP molecules $\left(\Delta G_{298}, \mathrm{kcal} / \mathrm{mol}\right)$. 
Table S2. Coordinates (x,y,z), thermal correction to Gibbs free energy (Hartree), SCRF energies (Hartree) and imaginary frequencies $\left(\mathrm{cm}^{-1}\right)$ of the computed species.

\begin{tabular}{|c|c|c|c|c|c|c|c|}
\hline \multicolumn{4}{|c|}{$\begin{array}{c}\mathbf{N}_{\mathbf{2}} \\
\text { Thermal correction to Gibbs Free Energy= } \\
0.013118 \\
\mathrm{E}(\mathrm{RB}-\mathrm{P} 86)=-109.521755494\end{array}$} & \multicolumn{4}{|c|}{$\begin{array}{c}\text { HFIP } \\
\text { Thermal correction to Gibbs Free Energy= } \\
0.024665 \\
\mathrm{E}(\mathrm{RB}-\mathrm{P} 86)=-789.799106869\end{array}$} \\
\hline $\mathrm{N}$ & 0 & 0 & 0.558769 & 0 & -0.039976 & 1.877454 & -0.024897 \\
\hline \multirow[t]{11}{*}{$\mathrm{N}$} & 0 & 0 & -0.558769 & $\mathrm{C}$ & -0.005183 & 0.550911 & -0.50345 \\
\hline & & & & C & -1.302441 & -0.132579 & -0.031454 \\
\hline & & & & $\mathrm{F}$ & -2.372852 & 0.473773 & -0.601709 \\
\hline & & & & $\mathrm{F}$ & -1.450035 & -0.078024 & 1.309929 \\
\hline & & & & $\mathrm{F}$ & -1.304291 & -1.438631 & -0.412454 \\
\hline & & & & $\mathrm{C}$ & 1.274646 & -0.169332 & -0.025333 \\
\hline & & & & $\mathrm{F}$ & 1.445236 & -1.359937 & -0.64676 \\
\hline & & & & $\mathrm{F}$ & 1.305367 & -0.36947 & 1.308872 \\
\hline & & & & $\mathrm{F}$ & 2.344969 & 0.629085 & -0.35092 \\
\hline & & & & $\mathrm{H}$ & -0.00163 & 0.483308 & -1.611712 \\
\hline & & & & $\mathrm{H}$ & 0.803762 & 2.291889 & -0.290315 \\
\hline \multicolumn{4}{|c|}{$\begin{array}{c}\mathbf{A} \\
\text { Thermal correction to Gibbs Free Energy= } \\
0.268771 \\
\mathrm{E}(\mathrm{RB}-\mathrm{P} 86)=-1464.36830860\end{array}$} & \multicolumn{4}{|c|}{$\begin{array}{c}\mathbf{A}^{\prime} \\
\text { Thermal correction to Gibbs Free Energy= } \\
0.267178 \\
\mathrm{E}(\mathrm{RB}-\mathrm{P} 86)=-1464.36468943\end{array}$} \\
\hline $\mathrm{N}$ & 1.212444 & 0.264681 & 1.951628 & $\mathrm{C}$ & 1.414289 & -0.08929 & -0.71292 \\
\hline$S$ & 2.891634 & -0.905328 & -0.004469 & $\mathrm{~N}$ & 1.139718 & -0.57072 & -2.099042 \\
\hline C & 1.429581 & 0.190915 & 0.467029 & $\mathrm{~N}$ & 0.490033 & -1.63598 & -2.188216 \\
\hline $\mathrm{N}$ & 0.316003 & -0.467095 & -0.1951 & $S$ & 2.769093 & 1.194721 & -0.912477 \\
\hline C & 1.659244 & 1.620292 & -0.010139 & $\mathrm{~N}$ & 0.297607 & 0.706315 & -0.230558 \\
\hline $\mathrm{N}$ & 1.242909 & -0.811776 & 2.571417 & C & 1.825532 & -1.219789 & 0.231729 \\
\hline $\mathrm{N}$ & 1.245364 & -1.742248 & 3.258087 & $\mathrm{C}$ & 1.903379 & 2.473935 & 0.14095 \\
\hline C & 1.850222 & -2.254421 & -0.774256 & C & 0.460886 & 1.965563 & 0.311457 \\
\hline C & 0.409129 & -1.71482 & -0.785049 & C & 1.888926 & 3.830925 & -0.584871 \\
\hline 0 & -0.912856 & 0.192962 & -0.213905 & C & 2.55619 & 2.589599 & 1.534462 \\
\hline C & 2.250635 & 2.570287 & 0.841839 & $\mathrm{O}$ & -0.437619 & 2.609755 & 0.853083 \\
\hline $\mathrm{C}$ & 1.334798 & 1.980766 & -1.332089 & $\mathrm{H}$ & 1.300593 & 4.548233 & 0.011449 \\
\hline C & 1.906225 & -3.562457 & 0.03866 & $\mathrm{H}$ & 2.914094 & 4.221141 & -0.696263 \\
\hline C & 2.299929 & -2.492206 & -2.230304 & $\mathrm{H}$ & 1.436875 & 3.745497 & -1.585042 \\
\hline 0 & -0.545058 & -2.326396 & -1.263935 & $\mathrm{H}$ & 1.974208 & 3.294892 & 2.152015 \\
\hline $\mathrm{H}$ & 1.219611 & -4.296669 & -0.415927 & $\mathrm{H}$ & 2.588371 & 1.614139 & 2.044502 \\
\hline $\mathrm{H}$ & 2.927264 & -3.977203 & 0.02592 & $\mathrm{H}$ & 3.587915 & 2.965712 & 1.441812 \\
\hline $\mathrm{H}$ & 1.610382 & -3.404803 & 1.087158 & 0 & -0.907691 & 0.025116 & -0.016523 \\
\hline $\mathrm{H}$ & 1.620214 & -3.223314 & -2.699056 & C & 2.803019 & -2.145955 & -0.188125 \\
\hline $\mathrm{H}$ & 2.277184 & -1.559971 & -2.8154 & C & 1.274602 & -1.335217 & 1.521811 \\
\hline $\mathrm{H}$ & 3.325991 & -2.894406 & -2.254922 & C & -1.94676 & 0.474156 & -0.969724 \\
\hline C & -1.809945 & -0.270744 & 0.86635 & $\mathrm{C}$ & -3.27462 & 0.142802 & -0.34391 \\
\hline C & 2.517803 & 3.866541 & 0.374771 & $\mathrm{H}$ & -1.803896 & -0.040107 & -1.93658 \\
\hline $\mathrm{H}$ & 2.492572 & 2.292961 & 1.871381 & $\mathrm{H}$ & -1.827272 & 1.561083 & -1.105573 \\
\hline $\mathrm{C}$ & 1.595755 & 3.277577 & -1.791406 & $\mathrm{C}$ & -4.106324 & -0.853316 & -0.8863 \\
\hline
\end{tabular}




\begin{tabular}{|c|c|c|c|c|c|c|c|}
\hline $\mathrm{H}$ & 0.86563 & 1.248015 & -1.994637 & $C$ & -3.685977 & 0.838751 & 0.81213 \\
\hline C & -3.218835 & -0.004015 & 0.407525 & $\mathrm{C}$ & -5.345776 & -1.141642 & -0.29325 \\
\hline $\mathrm{H}$ & -1.566945 & 0.272424 & 1.79645 & $\mathrm{H}$ & -3.780768 & -1.40372 & -1.776327 \\
\hline $\mathrm{H}$ & -1.633888 & -1.350673 & 1.008381 & $\mathrm{C}$ & -4.919268 & 0.544891 & 1.407569 \\
\hline C & -4.04407 & 0.906984 & 1.090953 & $\mathrm{H}$ & -3.021722 & 1.601037 & 1.233244 \\
\hline C & -3.718173 & -0.679035 & -0.726096 & $\mathrm{C}$ & -5.752603 & -0.443077 & 0.85372 \\
\hline C & -5.361453 & 1.131615 & 0.660543 & $\mathrm{H}$ & -5.990093 & -1.914829 & -0.724135 \\
\hline $\mathrm{H}$ & -3.65303 & 1.440279 & 1.965095 & $\mathrm{H}$ & -6.71845 & -0.668242 & 1.318058 \\
\hline C & -5.029823 & -0.447597 & -1.159642 & $\mathrm{H}$ & -5.235409 & 1.088908 & 2.303714 \\
\hline $\mathrm{H}$ & -3.059815 & -1.374571 & -1.257422 & $\mathrm{C}$ & 3.226239 & -3.16685 & 0.671571 \\
\hline C & -5.854746 & 0.455112 & -0.465403 & $\mathrm{H}$ & 3.239614 & -2.056743 & -1.188493 \\
\hline $\mathrm{H}$ & -5.999119 & 1.838767 & 1.200995 & $\mathrm{C}$ & 1.702175 & -2.361521 & 2.379403 \\
\hline $\mathrm{H}$ & -5.413473 & -0.974325 & -2.039658 & $\mathrm{H}$ & 0.495777 & -0.641753 & 1.849436 \\
\hline $\mathrm{H}$ & -6.881141 & 0.631343 & -0.804046 & $C$ & 2.677477 & -3.27752 & 1.960267 \\
\hline C & 2.191296 & 4.224308 & -0.94125 & $\mathrm{H}$ & 3.987829 & -3.877178 & 0.334402 \\
\hline $\mathrm{H}$ & 2.979015 & 4.597783 & 1.046311 & $\mathrm{H}$ & 3.007357 & -4.076712 & 2.631571 \\
\hline $\mathrm{H}$ & 1.328989 & 3.550489 & -2.817367 & $\mathrm{H}$ & 1.26102 & -2.445888 & 3.377718 \\
\hline $\mathrm{H}$ & 2.395268 & 5.237056 & -1.303475 & $\mathrm{~N}$ & -0.094126 & -2.595134 & -2.461353 \\
\hline \multicolumn{4}{|c|}{$\begin{array}{c}\text { TS }_{\mathbf{A}^{\prime} \mathbf{B}} \\
\text { Thermal correction to Gibbs Free Energy= } \\
0.270115 \\
\mathrm{E}(\mathrm{RB}-\mathrm{P} 86)=-1464.30148670 \\
\text { Frequency }-351.2016\end{array}$} & \multicolumn{4}{|c|}{$\begin{array}{c}\text { B } \\
\text { Thermal correction to Gibbs Free Energy }= \\
0.270709 \\
E(\text { RB-P86) }=-1464.30591188\end{array}$} \\
\hline$C$ & -1.64038 & -1.251903 & -0.352588 & $C$ & -1.619719 & -1.266684 & -0.226514 \\
\hline $\mathrm{N}$ & -1.136481 & -0.673467 & 1.337419 & $\mathrm{~N}$ & -1.145729 & -0.814741 & 1.18711 \\
\hline C & -1.319631 & 0.136239 & 0.03812 & $C$ & -1.293644 & 0.173529 & 0.047407 \\
\hline $\mathrm{N}$ & -2.291856 & -0.844465 & 2.009856 & $N$ & -2.323097 & -0.883876 & 2.027681 \\
\hline$S$ & -2.635543 & 1.409989 & -0.114974 & $S$ & -2.609092 & 1.433511 & -0.068794 \\
\hline C & -1.418628 & 2.826012 & -0.177389 & $C$ & -1.396469 & 2.856117 & -0.169922 \\
\hline $\mathrm{N}$ & -0.108138 & 0.797303 & -0.35196 & $\mathrm{~N}$ & -0.08331 & 0.831757 & -0.345465 \\
\hline C & -0.043681 & 2.181081 & -0.411495 & $C$ & -0.019963 & 2.213423 & -0.411637 \\
\hline O & 1.085023 & 0.077203 & -0.418219 & 0 & 1.103172 & 0.104867 & -0.429059 \\
\hline C & -3.055313 & -1.662068 & -0.031634 & $\mathrm{C}$ & -3.099851 & -1.633518 & -0.013092 \\
\hline C & -0.757272 & -2.16978 & -1.036741 & $C$ & -0.76414 & -2.198063 & -0.991019 \\
\hline $\mathrm{N}$ & -3.317568 & -1.337821 & 1.50163 & $\mathrm{~N}$ & -3.336941 & -1.355461 & 1.482139 \\
\hline C & -1.385203 & 3.588059 & 1.163564 & $\mathrm{C}$ & -1.35246 & 3.641045 & 1.156766 \\
\hline C & -1.763718 & 3.760042 & -1.350027 & $C$ & -1.760864 & 3.76772 & -1.354322 \\
\hline O & 0.990223 & 2.819145 & -0.598659 & 0 & 1.01038 & 2.855019 & -0.608756 \\
\hline C & 1.868759 & 0.17093 & 0.842295 & $C$ & 1.882313 & 0.174345 & 0.834785 \\
\hline C & -3.408495 & -3.066633 & -0.371365 & $C$ & -3.471241 & -3.032452 & -0.399224 \\
\hline $\mathrm{H}$ & -3.797655 & -0.963751 & -0.477843 & $\mathrm{H}$ & -3.796484 & -0.918663 & -0.504196 \\
\hline C & -1.191135 & -3.43384 & -1.340907 & $C$ & -1.239858 & -3.41724 & -1.357499 \\
\hline $\mathrm{H}$ & 0.245527 & -1.831143 & -1.299458 & $\mathrm{H}$ & 0.253604 & -1.879601 & -1.220811 \\
\hline $\mathrm{H}$ & -0.606181 & 4.367822 & 1.116755 & $\mathrm{H}$ & -0.576055 & 4.421723 & 1.087669 \\
\hline $\mathrm{H}$ & -2.358956 & 4.067069 & 1.354901 & $\mathrm{H}$ & -2.325298 & 4.121279 & 1.349798 \\
\hline $\mathrm{H}$ & -1.161166 & 2.913023 & 2.004498 & $\mathrm{H}$ & -1.117747 & 2.982282 & 2.007591 \\
\hline $\mathrm{H}$ & -0.981107 & 4.533068 & -1.425809 & $\mathrm{H}$ & -0.983824 & 4.543872 & -1.452257 \\
\hline $\mathrm{H}$ & -1.809778 & 3.210085 & -2.302563 & $\mathrm{H}$ & -1.81428 & 3.201089 & -2.296631 \\
\hline $\mathrm{H}$ & -2.734224 & 4.254654 & -1.180666 & $\mathrm{H}$ & -2.732358 & 4.259635 & -1.18266 \\
\hline
\end{tabular}




\begin{tabular}{|c|c|c|c|c|c|c|c|}
\hline C & 3.278621 & -0.211708 & 0.485278 & $C$ & 3.287725 & -0.228638 & 0.482874 \\
\hline $\mathrm{H}$ & 1.413999 & -0.497726 & 1.590272 & $\mathrm{H}$ & 1.412067 & -0.494237 & 1.573842 \\
\hline $\mathrm{H}$ & 1.813367 & 1.216819 & 1.186522 & $\mathrm{H}$ & 1.842546 & 1.218555 & 1.187198 \\
\hline C & -2.517246 & -3.892844 & -0.983101 & $\mathrm{C}$ & -2.591988 & -3.851332 & -1.027079 \\
\hline $\mathrm{H}$ & -4.417282 & -3.400636 & -0.110567 & $\mathrm{H}$ & -4.492389 & -3.351457 & -0.169719 \\
\hline $\mathrm{H}$ & -0.521793 & -4.11623 & -1.874294 & $\mathrm{H}$ & -0.591476 & -4.103662 & -1.9117 \\
\hline C & 3.863757 & -1.38526 & 0.995079 & $C$ & 3.851946 & -1.414447 & 0.987772 \\
\hline C & 4.02544 & 0.617207 & -0.378174 & $\mathrm{C}$ & 4.051115 & 0.593767 & -0.372224 \\
\hline C & 5.185572 & -1.721142 & 0.661835 & $\mathrm{C}$ & 5.169762 & -1.768944 & 0.657962 \\
\hline $\mathrm{H}$ & 3.281652 & -2.034033 & 1.659382 & $\mathrm{H}$ & 3.256833 & -2.058176 & 1.645398 \\
\hline C & 5.341122 & 0.276787 & -0.717445 & C & 5.362663 & 0.234742 & -0.708247 \\
\hline $\mathrm{H}$ & 3.555225 & 1.521756 & -0.778255 & $\mathrm{H}$ & 3.597019 & 1.507965 & -0.769081 \\
\hline C & 5.924387 & -0.891281 & -0.195368 & $\mathrm{C}$ & 5.92519 & -0.945547 & -0.190949 \\
\hline $\mathrm{H}$ & 5.636052 & -2.632609 & 1.068148 & $\mathrm{H}$ & 5.604095 & -2.689897 & 1.060417 \\
\hline $\mathrm{H}$ & 5.916579 & 0.924334 & -1.387153 & $\mathrm{H}$ & 5.950986 & 0.877156 & -1.371662 \\
\hline $\mathrm{H}$ & 6.954731 & -1.153501 & -0.457699 & $\mathrm{H}$ & 6.952373 & -1.222373 & -0.450617 \\
\hline $\mathrm{H}$ & -2.805711 & -4.919846 & -1.229795 & $\mathrm{H}$ & -2.902129 & -4.861447 & -1.314652 \\
\hline \multicolumn{4}{|c|}{$\begin{array}{c}\text { TS }_{\mathrm{BC}} \\
\text { Thermal correction to Gibbs Free Energy }= \\
0.267521 \\
\mathrm{E}(\mathrm{RB}-\mathrm{P} 86)=-1464.29533494 \\
\text { Frequency } \quad-392.7833 \\
\end{array}$} & \multicolumn{4}{|c|}{$\begin{array}{c}\text { C } \\
\text { Thermal correction to Gibbs Free Energy= } \\
0.253954 \\
E(R B-P 86)=-1464.44734009\end{array}$} \\
\hline C & -1.154635 & 0.166639 & 0.500541 & $C$ & 2.912443 & 1.457701 & 0.105589 \\
\hline C & -1.64255 & -1.25381 & -0.064771 & $\mathrm{~N}$ & 1.616495 & 1.02722 & -0.245798 \\
\hline $\mathrm{N}$ & -1.026981 & -1.00812 & 1.24889 & $\mathrm{C}$ & 1.315254 & -0.208952 & -0.37957 \\
\hline C & -3.147852 & -1.464064 & 0.00692 & $\mathrm{~N}$ & -2.774895 & -1.69337 & 3.464576 \\
\hline C & -0.878249 & -2.098147 & -0.994377 & $S$ & 2.345595 & -1.68512 & -0.252558 \\
\hline $\mathrm{N}$ & -2.467242 & -1.44941 & 2.358081 & $\mathrm{C}$ & 0.945262 & -2.829369 & -0.753701 \\
\hline$S$ & -2.397219 & 1.490297 & 0.778655 & $\mathrm{~N}$ & 0.000008 & -0.605311 & -0.681368 \\
\hline $\mathrm{N}$ & 0.041213 & 0.8756 & 0.112406 & $\mathrm{C}$ & -0.308556 & -1.945386 & -0.882192 \\
\hline $\mathrm{N}$ & -3.359773 & -1.783894 & 1.657596 & O & -0.966034 & 0.348375 & -0.955857 \\
\hline C & -1.462942 & 2.721917 & -0.28199 & $C$ & 3.607258 & 0.957539 & 1.231676 \\
\hline C & -0.031171 & 2.15915 & -0.400157 & $\mathrm{C}$ & 3.495225 & 2.495418 & -0.656314 \\
\hline C & -1.434539 & 4.08657 & 0.42469 & $\mathrm{~N}$ & -3.546375 & -2.17132 & 4.116418 \\
\hline C & -2.068536 & 2.826741 & -1.696597 & C & 0.715638 & -3.896379 & 0.331377 \\
\hline $\mathrm{O}$ & 1.188324 & 0.131027 & -0.156286 & C & 1.240176 & -3.47238 & -2.124235 \\
\hline $\mathrm{O}$ & 0.908008 & 2.776628 & -0.89663 & O & -1.429182 & -2.372756 & -1.141959 \\
\hline C & 2.067488 & 0.085963 & 1.025451 & C & -1.613855 & 0.822108 & 0.279835 \\
\hline C & -3.719794 & -2.472566 & -0.893677 & $\mathrm{C}$ & 4.869676 & 1.470055 & 1.564698 \\
\hline $\mathrm{H}$ & -3.733552 & -0.524035 & 0.01599 & $\mathrm{H}$ & 3.140891 & 0.188963 & 1.855815 \\
\hline C & -1.496111 & -3.020579 & -1.788611 & C & 4.761672 & 2.990115 & -0.323082 \\
\hline $\mathrm{H}$ & 0.205202 & -1.959229 & -0.997393 & $\mathrm{H}$ & 2.93868 & 2.892196 & -1.51055 \\
\hline $\mathrm{H}$ & -0.78934 & 4.767635 & -0.154494 & $\mathrm{H}$ & -0.163766 & -4.501412 & 0.054518 \\
\hline $\mathrm{H}$ & -2.447197 & 4.51897 & 0.480392 & $\mathrm{H}$ & 1.592115 & -4.559561 & 0.413353 \\
\hline $\mathrm{H}$ & -1.030728 & 4.00301 & 1.445534 & $\mathrm{H}$ & 0.531895 & -3.437493 & 1.315367 \\
\hline $\mathrm{H}$ & -1.444336 & 3.500102 & -2.30825 & $\mathrm{H}$ & 0.372105 & -4.080727 & -2.429039 \\
\hline $\mathrm{H}$ & -2.109812 & 1.843409 & -2.191366 & $\mathrm{H}$ & 1.423851 & -2.708357 & -2.895685 \\
\hline $\mathrm{H}$ & -3.090733 & 3.235572 & -1.646745 & $\mathrm{H}$ & 2.127089 & -4.123454 & -2.059791 \\
\hline C & 3.443132 & -0.271685 & 0.525849 & $\mathrm{C}$ & -2.965082 & 1.359951 & -0.113938 \\
\hline
\end{tabular}




\begin{tabular}{|c|c|c|c|c|c|c|c|}
\hline $\mathrm{H}$ & 1.667778 & -0.652549 & 1.741578 & $\mathrm{H}$ & -0.974464 & 1.59186 & 0.745714 \\
\hline $\mathrm{H}$ & 2.052159 & 1.088796 & 1.488184 & $\mathrm{H}$ & -1.707145 & -0.039859 & 0.964767 \\
\hline C & -2.929372 & -3.205307 & -1.733956 & $C$ & 5.456588 & 2.480722 & 0.787448 \\
\hline $\mathrm{H}$ & -4.807161 & -2.594633 & -0.89661 & $\mathrm{H}$ & 5.394357 & 1.077338 & 2.44214 \\
\hline $\mathrm{H}$ & -0.905712 & -3.636023 & -2.474505 & $\mathrm{H}$ & 5.206362 & 3.785405 & -0.930533 \\
\hline C & 4.122389 & -1.400575 & 1.018116 & $\mathrm{C}$ & -3.334991 & 2.681118 & 0.19475 \\
\hline C & 4.063314 & 0.543909 & -0.443972 & $\mathrm{C}$ & -3.877942 & 0.522665 & -0.788812 \\
\hline $\mathrm{H}$ & -3.393921 & -3.92981 & -2.411631 & $\mathrm{C}$ & -4.61002 & 3.159083 & -0.147469 \\
\hline C & 5.41566 & -1.704283 & 0.56318 & $\mathrm{H}$ & -2.620941 & 3.337066 & 0.705688 \\
\hline $\mathrm{H}$ & 3.636638 & -2.04153 & 1.762694 & $\mathrm{C}$ & -5.145574 & 1.004546 & -1.139862 \\
\hline C & 5.348924 & 0.233117 & -0.90541 & $\mathrm{H}$ & -3.571966 & -0.4975 & -1.044243 \\
\hline $\mathrm{H}$ & 3.517474 & 1.409147 & -0.83524 & $\mathrm{C}$ & -5.515958 & 2.321611 & -0.815688 \\
\hline C & 6.029325 & -0.888588 & -0.399511 & $\mathrm{H}$ & -4.891173 & 4.187916 & 0.100092 \\
\hline $\mathrm{H}$ & 5.939654 & -2.581935 & 0.955607 & $\mathrm{H}$ & -5.849254 & 0.351502 & -1.666538 \\
\hline $\mathrm{H}$ & 5.824913 & 0.868348 & -1.659698 & $\mathrm{H}$ & -6.508762 & 2.694775 & -1.088042 \\
\hline $\mathrm{H}$ & 7.036125 & -1.126886 & -0.758317 & $\mathrm{H}$ & 6.442399 & 2.876786 & 1.05055 \\
\hline \multicolumn{4}{|c|}{$\begin{array}{c}\text { D } \\
\text { Thermal correction to Gibbs Free Energy= } \\
0.262101 \\
E(\text { RB-P86) }=-1354.92550861\end{array}$} & \multicolumn{4}{|c|}{$\begin{array}{c}\text { TS }_{\mathrm{A}^{\prime} \mathrm{E}} \\
\text { Thermal correction to Gibbs Free Energy= } \\
0.264569 \\
\mathrm{E}(\mathrm{RB}-\mathrm{P} 86)=-1464.30416690 \\
\text { Frequency }-551.1879\end{array}$} \\
\hline C & -2.560292 & -1.525908 & -0.123247 & $\mathrm{~N}$ & 0.591792 & -0.760063 & -0.228279 \\
\hline $\mathrm{N}$ & -1.278349 & -0.952076 & -0.24398 & $\mathrm{~N}$ & 1.140907 & -0.025979 & 1.470514 \\
\hline C & -1.072839 & 0.305938 & -0.135663 & $\mathrm{C}$ & 1.345171 & 0.46905 & 0.175212 \\
\hline$S$ & -2.233876 & 1.665852 & 0.10899 & $\mathrm{~N}$ & 1.913783 & 1.02488 & 2.669032 \\
\hline C & -0.891428 & 2.970665 & -0.020891 & $S$ & 3.088706 & 0.177521 & -0.576784 \\
\hline $\mathrm{N}$ & 0.227877 & 0.836156 & -0.210271 & $\mathrm{C}$ & 2.856663 & -1.67476 & -0.497282 \\
\hline C & 0.439915 & 2.208285 & -0.145885 & $\mathrm{C}$ & 0.748516 & 1.787848 & -0.288968 \\
\hline O & 1.296874 & 0.010994 & -0.518716 & $C$ & 1.335192 & -1.929839 & -0.516658 \\
\hline C & -3.413563 & -1.273091 & 0.97629 & 0 & -0.761855 & -0.705519 & -0.478007 \\
\hline C & -2.962776 & -2.459791 & -1.104674 & $\mathrm{C}$ & -0.068772 & 2.515412 & 0.597092 \\
\hline C & -0.870408 & 3.840363 & 1.249136 & C & 0.974037 & 2.286776 & -1.587168 \\
\hline C & -1.092965 & 3.826738 & -1.28782 & $\mathrm{~N}$ & 2.159526 & 1.047017 & 3.772468 \\
\hline $\mathrm{O}$ & 1.541235 & 2.74733 & -0.194128 & $\mathrm{C}$ & 3.470493 & -2.285547 & 0.781372 \\
\hline C & 1.853786 & -0.614156 & 0.693871 & $\mathrm{C}$ & 3.463814 & -2.314923 & -1.761105 \\
\hline C & -4.653211 & -1.922588 & 1.069483 & 0 & 0.799844 & -2.999028 & -0.772863 \\
\hline $\mathrm{H}$ & -3.08792 & -0.589159 & 1.766169 & C & -1.534272 & -1.392876 & 0.585373 \\
\hline C & -4.208198 & -3.091965 & -1.009041 & C & -0.639793 & 3.732726 & 0.197763 \\
\hline $\mathrm{H}$ & -2.284652 & -2.666546 & -1.937938 & $\mathrm{H}$ & -0.248963 & 2.115611 & 1.598705 \\
\hline $\mathrm{H}$ & -0.021411 & 4.541223 & 1.186281 & C & 0.394211 & 3.499228 & -1.986685 \\
\hline $\mathrm{H}$ & -1.804082 & 4.419453 & 1.336911 & $\mathrm{H}$ & 1.606321 & 1.720329 & -2.277044 \\
\hline $\mathrm{H}$ & -0.754167 & 3.226819 & 2.156214 & $\mathrm{H}$ & 3.264544 & -3.370041 & 0.813034 \\
\hline $\mathrm{H}$ & -0.252183 & 4.534702 & -1.379163 & $\mathrm{H}$ & 4.562192 & -2.133911 & 0.776855 \\
\hline $\mathrm{H}$ & -1.128309 & 3.2021 & -2.193978 & $\mathrm{H}$ & 3.045673 & -1.810514 & 1.677323 \\
\hline $\mathrm{H}$ & -2.033703 & 4.396921 & -1.220098 & $\mathrm{H}$ & 3.254189 & -3.397457 & -1.757004 \\
\hline C & 3.275224 & -0.997178 & 0.374562 & $\mathrm{H}$ & 3.041475 & -1.877112 & -2.678737 \\
\hline $\mathrm{H}$ & 1.2334 & -1.4862 & 0.963705 & $\mathrm{H}$ & 4.555566 & -2.164404 & -1.771918 \\
\hline $\mathrm{H}$ & 1.810491 & 0.132616 & 1.50752 & C & -2.988337 & -1.116519 & 0.319931 \\
\hline C & -5.060617 & -2.827191 & 0.076811 & $\mathrm{H}$ & -1.180396 & -0.967694 & 1.542387 \\
\hline
\end{tabular}




\begin{tabular}{|c|c|c|c|c|c|c|c|}
\hline $\mathrm{H}$ & -5.301658 & -1.72152 & 1.928871 & $\mathrm{H}$ & -1.302179 & -2.468967 & 0.543433 \\
\hline $\mathrm{H}$ & -4.5122 & -3.803526 & -1.783971 & $\mathrm{C}$ & -0.409963 & 4.227621 & -1.095405 \\
\hline C & 3.709533 & -2.329259 & 0.495434 & $\mathrm{H}$ & -1.264371 & 4.295411 & 0.89938 \\
\hline C & 4.186816 & -0.003927 & -0.040408 & $\mathrm{H}$ & 0.574928 & 3.877323 & -2.998058 \\
\hline C & 5.045224 & -2.666856 & 0.224702 & C & -3.488378 & 0.200627 & 0.386518 \\
\hline $\mathrm{H}$ & 2.998221 & -3.104013 & 0.803497 & C & -3.868131 & -2.169232 & 0.004742 \\
\hline C & 5.516366 & -0.344181 & -0.320944 & $C$ & -4.845169 & 0.455572 & 0.147978 \\
\hline $\mathrm{H}$ & 3.832969 & 1.02653 & -0.152684 & $\mathrm{H}$ & -2.803152 & 1.024521 & 0.611995 \\
\hline C & 5.949301 & -1.674921 & -0.184306 & $\mathrm{C}$ & -5.229714 & -1.916136 & -0.222203 \\
\hline $\mathrm{H}$ & 5.376229 & -3.70564 & 0.325259 & $\mathrm{H}$ & -3.481366 & -3.192318 & -0.060784 \\
\hline $\mathrm{H}$ & 6.219401 & 0.430064 & -0.64537 & C & -5.719604 & -0.603123 & -0.151875 \\
\hline $\mathrm{H}$ & 6.99014 & -1.937652 & -0.400533 & $\mathrm{H}$ & -5.223982 & 1.481602 & 0.198925 \\
\hline \multirow[t]{3}{*}{$\mathrm{H}$} & -6.029328 & -3.33081 & 0.153502 & $\mathrm{H}$ & -5.905698 & -2.74363 & -0.460629 \\
\hline & & & & $\mathrm{H}$ & -6.780899 & -0.403116 & -0.332405 \\
\hline & & & & $\mathrm{H}$ & -0.854913 & 5.17798 & -1.407877 \\
\hline \multicolumn{4}{|c|}{$\begin{array}{c}\mathbf{E} \\
\text { Thermal correction to Gibbs Free Energy= } \\
0.255548 \\
\mathrm{E}(\mathrm{RB}-\mathrm{P} 86)=-1464.34054568\end{array}$} & \multicolumn{4}{|c|}{$\begin{array}{c}\mathbf{F} \\
\text { Thermal correction to Gibbs Free Energy= } \\
0.261579 \\
E(\text { RB-P86) }=-1354.81857051\end{array}$} \\
\hline $\mathrm{N}$ & 0.827701 & -0.674806 & -0.193848 & $\mathrm{~N}$ & -0.848445 & -0.688691 & -0.204766 \\
\hline $\mathrm{N}$ & 1.134835 & -0.251934 & 1.152486 & $\mathrm{~N}$ & -1.125793 & -0.240481 & -1.549088 \\
\hline C & 1.441746 & 0.652687 & 0.107582 & $\mathrm{C}$ & -1.458386 & 0.642692 & -0.493584 \\
\hline $\mathrm{N}$ & -0.018113 & 0.201614 & 4.356424 & $S$ & -3.226539 & 0.63517 & 0.133744 \\
\hline S & 3.195464 & 0.660855 & -0.558414 & C & -3.266616 & -1.234101 & 0.149492 \\
\hline C & 3.238373 & -1.207595 & -0.614024 & $\mathrm{C}$ & -0.687439 & 1.893024 & -0.183326 \\
\hline C & 0.661184 & 1.90635 & -0.162497 & $\mathrm{C}$ & -1.811523 & -1.740674 & 0.183847 \\
\hline C & 1.783952 & -1.716877 & -0.624238 & 0 & 0.508803 & -0.823571 & 0.336045 \\
\hline 0 & -0.539592 & -0.801103 & -0.707537 & C & 0.201349 & 2.387142 & -1.158526 \\
\hline C & -0.206244 & 2.381963 & 0.840912 & C & -0.858068 & 2.594222 & 1.026655 \\
\hline C & 0.802252 & 2.62708 & -1.364883 & $\mathrm{C}$ & -3.971223 & -1.791848 & -1.106834 \\
\hline $\mathrm{N}$ & 0.657195 & -0.542879 & 4.844381 & $\mathrm{C}$ & -3.964123 & -1.714918 & 1.440019 \\
\hline C & 3.974377 & -1.790155 & 0.612665 & 0 & -1.447394 & -2.867955 & 0.442682 \\
\hline C & 3.90527 & -1.659484 & -1.931008 & $\mathrm{C}$ & 1.345865 & -1.536053 & -0.627104 \\
\hline $\mathrm{O}$ & 1.416003 & -2.839731 & -0.896524 & C & 0.916791 & 3.569882 & -0.92299 \\
\hline C & -1.357186 & -1.531329 & 0.259513 & $\mathrm{H}$ & 0.311572 & 1.832404 & -2.095214 \\
\hline C & -0.929442 & 3.566522 & 0.640079 & $\mathrm{C}$ & -0.134282 & 3.771713 & 1.261143 \\
\hline $\mathrm{H}$ & -0.294231 & 1.814393 & 1.772182 & $\mathrm{H}$ & -1.554511 & 2.210242 & 1.778311 \\
\hline C & 0.070511 & 3.806095 & -1.564079 & $\mathrm{H}$ & -3.950237 & -2.895678 & -1.09016 \\
\hline $\mathrm{H}$ & 1.482137 & 2.256974 & -2.138341 & $\mathrm{H}$ & -5.021608 & -1.457154 & -1.118003 \\
\hline $\mathrm{H}$ & 3.954993 & -2.893416 & 0.57316 & $\mathrm{H}$ & -3.466728 & -1.43336 & -2.015715 \\
\hline $\mathrm{H}$ & 5.024114 & -1.45336 & 0.605436 & $\mathrm{H}$ & -3.92121 & -2.815766 & 1.485719 \\
\hline $\mathrm{H}$ & 3.491778 & -1.452259 & 1.541072 & $\mathrm{H}$ & -3.480604 & -1.303986 & 2.339703 \\
\hline $\mathrm{H}$ & 3.86356 & -2.759234 & -1.998869 & $\mathrm{H}$ & -5.020235 & -1.401537 & 1.436614 \\
\hline $\mathrm{H}$ & 3.399088 & -1.230871 & -2.809735 & $\mathrm{C}$ & 2.781778 & -1.288965 & -0.236295 \\
\hline $\mathrm{H}$ & 4.960484 & -1.34346 & -1.946661 & $\mathrm{H}$ & 1.11574 & -1.132322 & -1.631858 \\
\hline C & -2.799793 & -1.286368 & -0.106604 & $\mathrm{H}$ & 1.100298 & -2.612472 & -0.603503 \\
\hline $\mathrm{H}$ & -1.113774 & -1.139421 & 1.265517 & $\mathrm{C}$ & 0.752034 & 4.262166 & 0.28807 \\
\hline $\mathrm{H}$ & -1.106253 & -2.605918 & 0.21702 & $\mathrm{H}$ & 1.598509 & 3.955299 & -1.688168 \\
\hline C & -0.794453 & 4.278419 & -0.563227 & $\mathrm{H}$ & -0.262709 & 4.30805 & 2.206762 \\
\hline
\end{tabular}




\begin{tabular}{|c|c|c|c|c|c|c|c|}
\hline $\mathrm{H}$ & -1.59422 & 3.937797 & 1.426844 & $C$ & 3.308126 & 0.019369 & -0.228867 \\
\hline $\mathrm{H}$ & 0.175929 & 4.357665 & -2.50377 & $\mathrm{C}$ & 3.616724 & -2.365051 & 0.117712 \\
\hline C & -3.336433 & 0.017342 & -0.07203 & C & 4.647393 & 0.241177 & 0.117566 \\
\hline C & -3.629904 & -2.35921 & -0.481245 & $\mathrm{H}$ & 2.656805 & 0.861611 & -0.484555 \\
\hline C & -4.681106 & 0.237617 & -0.397853 & $\mathrm{C}$ & 4.961587 & -2.144386 & 0.454296 \\
\hline $\mathrm{H}$ & -2.689142 & 0.857453 & 0.200441 & $\mathrm{H}$ & 3.208852 & -3.382174 & 0.126864 \\
\hline C & -4.980003 & -2.140377 & -0.797347 & $C$ & 5.479012 & -0.840402 & 0.45572 \\
\hline $\mathrm{H}$ & -3.214036 & -3.372279 & -0.522764 & $\mathrm{H}$ & 5.046246 & 1.261018 & 0.121793 \\
\hline C & -5.507617 & -0.841081 & -0.757113 & $\mathrm{H}$ & 5.601927 & -2.991076 & 0.722464 \\
\hline $\mathrm{H}$ & -5.08818 & 1.253792 & -0.369266 & $\mathrm{H}$ & 6.526518 & -0.665225 & 0.72225 \\
\hline $\mathrm{H}$ & -5.616612 & -2.984519 & -1.081908 & $\mathrm{H}$ & 1.310401 & 5.185898 & 0.471981 \\
\hline $\mathrm{H}$ & -6.559348 & -0.66726 & -1.007418 & & & & \\
\hline $\mathrm{H}$ & -1.359231 & 5.203359 & -0.719539 & & & & \\
\hline \multicolumn{4}{|c|}{$\begin{array}{c}\text { TS }_{\mathrm{FG}} \\
\text { Thermal correction to Gibbs Free Energy= } \\
0.260246 \\
\mathrm{E}(\mathrm{RB}-\mathrm{P} 86)=-1354.81320848 \\
\text { Frequency }-163.9661 \\
\end{array}$} & \multicolumn{4}{|c|}{$\begin{array}{c}\mathbf{G} \\
\text { Thermal correction to Gibbs Free Energy= } \\
0.263179 \\
\mathrm{E}(\mathrm{RB}-\mathrm{P} 86)=-1354.89739294\end{array}$} \\
\hline $\mathrm{N}$ & -0.554417 & -0.730215 & -0.189707 & C & -1.043977 & 2.568179 & 0.130467 \\
\hline C & -1.744318 & 0.532599 & -0.664916 & $S$ & -2.265302 & 1.548844 & -0.84342 \\
\hline $\mathrm{N}$ & -1.00407 & -0.307571 & -1.482981 & C & -1.937149 & -0.027084 & -0.071985 \\
\hline C & -1.290844 & -1.793772 & 0.41652 & $\mathrm{~N}$ & -0.757974 & -0.359982 & 0.385769 \\
\hline $\mathrm{O}$ & 0.79922 & -0.56879 & 0.234882 & $\mathrm{~N}$ & 0.296088 & 0.483593 & 0.205407 \\
\hline$S$ & -3.415625 & -0.049908 & -0.270351 & $C$ & 0.329938 & 1.877323 & 0.055721 \\
\hline C & -1.321235 & 1.86248 & -0.187554 & 0 & 1.388254 & 2.493688 & -0.069264 \\
\hline C & -2.801171 & -1.81244 & 0.043503 & $\mathrm{C}$ & -0.974543 & 3.942334 & -0.551698 \\
\hline C & -3.002181 & -2.693629 & -1.206343 & $\mathrm{C}$ & -1.474241 & 2.685103 & 1.606573 \\
\hline C & -3.589042 & -2.352754 & 1.250103 & 0 & 1.519349 & -0.107107 & 0.537831 \\
\hline C & -0.188924 & 2.458313 & -0.793444 & $\mathrm{C}$ & 3.602533 & -0.883403 & -0.33825 \\
\hline C & -2.031733 & 2.577023 & 0.805892 & C & 4.387881 & 0.2331 & 0.017095 \\
\hline $\mathrm{O}$ & -0.766704 & -2.623679 & 1.145337 & C & 5.750079 & 0.072178 & 0.301538 \\
\hline C & 1.690461 & -1.158676 & -0.770331 & C & 6.34202 & -1.201087 & 0.228317 \\
\hline C & 0.230054 & 3.733981 & -0.40143 & $\mathrm{C}$ & 5.56445 & -2.315121 & -0.122146 \\
\hline $\mathrm{H}$ & 0.330794 & 1.901762 & -1.577855 & $\mathrm{C}$ & 4.196613 & -2.156501 & -0.397015 \\
\hline C & -1.618579 & 3.860463 & 1.178937 & C & 2.142332 & -0.687693 & -0.656374 \\
\hline $\mathrm{H}$ & -2.895845 & 2.108529 & 1.286975 & C & -3.032137 & -1.019538 & 0.004861 \\
\hline $\mathrm{H}$ & -2.641113 & -3.716805 & -0.999877 & $\mathrm{C}$ & -4.382131 & -0.645779 & -0.179699 \\
\hline $\mathrm{H}$ & -4.074209 & -2.744242 & -1.459431 & C & -5.400317 & -1.608465 & -0.129397 \\
\hline $\mathrm{H}$ & -2.447092 & -2.272635 & -2.057803 & $\mathrm{C}$ & -5.090169 & -2.953479 & 0.11962 \\
\hline $\mathrm{H}$ & -3.19983 & -3.350957 & 1.508215 & $\mathrm{C}$ & -3.749552 & -3.333056 & 0.314776 \\
\hline $\mathrm{H}$ & -3.480279 & -1.701891 & 2.131257 & C & -2.728634 & -2.380631 & 0.253551 \\
\hline $\mathrm{H}$ & -4.659553 & -2.437599 & 1.002927 & $\mathrm{H}$ & -0.696952 & 3.850785 & -1.613473 \\
\hline C & 3.100335 & -0.855303 & -0.335533 & $\mathrm{H}$ & -1.94724 & 4.455178 & -0.477405 \\
\hline $\mathrm{H}$ & 1.453199 & -0.721944 & -1.757109 & $\mathrm{H}$ & -0.206637 & 4.553022 & -0.053472 \\
\hline $\mathrm{H}$ & 1.500476 & -2.247696 & -0.801278 & $\mathrm{H}$ & -2.452511 & 3.188185 & 1.676863 \\
\hline C & -0.485153 & 4.439624 & 0.581285 & $\mathrm{H}$ & -1.552889 & 1.698425 & 2.090206 \\
\hline $\mathrm{H}$ & 1.108571 & 4.187204 & -0.871606 & $\mathrm{H}$ & -0.728527 & 3.283331 & 2.158648 \\
\hline $\mathrm{H}$ & -2.172239 & 4.407375 & 1.948747 & $\mathrm{H}$ & 3.91033 & 1.216785 & 0.075773 \\
\hline C & 3.906928 & 0.039246 & -1.063353 & $\mathrm{H}$ & 6.354772 & 0.942064 & 0.578595 \\
\hline
\end{tabular}




\begin{tabular}{|c|c|c|c|c|c|c|c|}
\hline C & 3.624613 & -1.466664 & 0.821937 & $\mathrm{H}$ & 7.408018 & -1.323701 & 0.44701 \\
\hline $\mathrm{H}$ & -0.161786 & 5.442164 & 0.879999 & $\mathrm{H}$ & 6.019773 & -3.309522 & -0.174766 \\
\hline C & 5.220737 & 0.31117 & -0.651244 & $\mathrm{H}$ & 3.584961 & -3.027004 & -0.66089 \\
\hline $\mathrm{H}$ & 3.503106 & 0.519395 & -1.961955 & $\mathrm{H}$ & 2.006334 & 0.023357 & -1.491146 \\
\hline C & 4.933209 & -1.189534 & 1.239521 & $\mathrm{H}$ & 1.642978 & -1.641351 & -0.90988 \\
\hline $\mathrm{H}$ & 2.994328 & -2.155404 & 1.394523 & $\mathrm{H}$ & -4.632868 & 0.406502 & -0.345628 \\
\hline C & 5.734928 & -0.301941 & 0.501668 & $\mathrm{H}$ & -6.440984 & -1.301501 & -0.27494 \\
\hline $\mathrm{H}$ & 5.84176 & 1.003177 & -1.229491 & $\mathrm{H}$ & -5.886464 & -3.703449 & 0.163386 \\
\hline $\mathrm{H}$ & 5.331144 & -1.668777 & 2.139964 & $\mathrm{H}$ & -3.499557 & -4.381351 & 0.508154 \\
\hline $\mathrm{H}$ & 6.75934 & -0.089983 & 0.825167 & $\mathrm{H}$ & -1.683294 & -2.667457 & 0.395194 \\
\hline \multicolumn{4}{|c|}{$\begin{array}{c}\text { TS }_{\mathrm{A}^{\prime} \mathrm{H}} \\
\text { Thermal correction to Gibbs Free Energy= } \\
0.265952 \\
\mathrm{E}(\mathrm{RB}-\mathrm{P} 86)=-1464.32146061 \\
\text { Frequency }-606.7124\end{array}$} & \multicolumn{4}{|c|}{$\begin{array}{c}\mathbf{H} \\
\text { Thermal correction to Gibbs Free Energy= } \\
0.255533 \\
\mathrm{E}(\mathrm{RB}-\mathrm{P} 86)=-1464.36554319\end{array}$} \\
\hline $\mathrm{N}$ & 1.853037 & -0.191423 & 1.619486 & $\mathrm{~N}$ & 2.032606 & -0.182662 & 1.038131 \\
\hline$S$ & 2.881319 & -1.211933 & -0.031562 & $S$ & 2.951445 & -0.531534 & -0.404114 \\
\hline C & 1.519844 & 0.063982 & 0.267371 & C & 1.348978 & 0.429825 & -0.005923 \\
\hline $\mathrm{N}$ & 0.32133 & -0.614318 & -0.292563 & $\mathrm{~N}$ & 0.298006 & -0.338485 & -0.714926 \\
\hline C & 1.755484 & 1.427762 & -0.347819 & $\mathrm{C}$ & 1.254027 & 1.909614 & -0.186308 \\
\hline $\mathrm{N}$ & 0.970841 & 0.793112 & 2.645448 & $\mathrm{~N}$ & 2.203367 & -2.158617 & 3.757652 \\
\hline $\mathrm{N}$ & 0.850973 & 1.005354 & 3.756354 & $\mathrm{~N}$ & 2.353881 & -1.27544 & 4.425302 \\
\hline C & 1.690984 & -2.619227 & -0.347892 & C & 2.02591 & -1.998003 & -1.183644 \\
\hline C & 0.29476 & -1.973454 & -0.496097 & $\mathrm{C}$ & 0.520121 & -1.651049 & -1.092261 \\
\hline O & -0.88172 & 0.093436 & -0.362785 & 0 & -1.0287 & 0.09774 & -0.628223 \\
\hline C & 2.580538 & 2.345569 & 0.331794 & C & 1.846334 & 2.73128 & 0.795473 \\
\hline C & 1.21331 & 1.781341 & -1.600811 & $C$ & 0.60708 & 2.498731 & -1.294312 \\
\hline C & 1.665904 & -3.592226 & 0.845257 & $\mathrm{C}$ & 2.299935 & -3.271015 & -0.371279 \\
\hline C & 2.079453 & -3.322569 & -1.660721 & C & 2.458647 & -2.107687 & -2.653533 \\
\hline $\mathrm{O}$ & -0.713762 & -2.636708 & -0.749968 & O & -0.36462 & -2.466731 & -1.35528 \\
\hline $\mathrm{H}$ & 0.878607 & -4.344898 & 0.672785 & $\mathrm{H}$ & 1.624458 & -4.070014 & -0.718666 \\
\hline $\mathrm{H}$ & 2.635556 & -4.106276 & 0.947559 & $\mathrm{H}$ & 3.343771 & -3.601961 & -0.499475 \\
\hline $\mathrm{H}$ & 1.4505 & -3.052102 & 1.780144 & $\mathrm{H}$ & 2.107656 & -3.091862 & 0.699393 \\
\hline $\mathrm{H}$ & 1.313325 & -4.082906 & -1.886208 & $\mathrm{H}$ & 1.878964 & -2.910646 & -3.138819 \\
\hline $\mathrm{H}$ & 2.127017 & -2.614867 & -2.503465 & $\mathrm{H}$ & 2.276601 & -1.170163 & -3.204798 \\
\hline $\mathrm{H}$ & 3.056629 & -3.823882 & -1.56318 & $\mathrm{H}$ & 3.53033 & -2.359524 & -2.729805 \\
\hline C & -1.744587 & -0.132589 & 0.820308 & $C$ & -1.631254 & -0.293149 & 0.663413 \\
\hline C & 2.849363 & 3.603711 & -0.225615 & $C$ & 1.790755 & 4.125451 & 0.672322 \\
\hline $\mathrm{H}$ & 3.011256 & 2.055411 & 1.29419 & $\mathrm{H}$ & 2.345523 & 2.247301 & 1.640443 \\
\hline C & 1.483559 & 3.040238 & -2.153144 & C & 0.559517 & 3.892987 & -1.414451 \\
\hline $\mathrm{H}$ & 0.56867 & 1.077547 & -2.133184 & $\mathrm{H}$ & 0.140524 & 1.863155 & -2.051283 \\
\hline C & -3.163045 & 0.095362 & 0.371253 & $\mathrm{C}$ & -3.12349 & -0.276777 & 0.468407 \\
\hline $\mathrm{H}$ & -1.451857 & 0.564533 & 1.62426 & $\mathrm{H}$ & -1.306406 & 0.414724 & 1.448083 \\
\hline $\mathrm{H}$ & -1.592058 & -1.171598 & 1.154691 & $\mathrm{H}$ & -1.266434 & -1.304427 & 0.910464 \\
\hline C & -3.741506 & -0.781928 & -0.570154 & C & -3.709333 & -1.18405 & -0.439343 \\
\hline C & -3.919763 & 1.170281 & 0.871667 & $\mathrm{C}$ & -3.942244 & 0.626124 & 1.17023 \\
\hline C & -5.062129 & -0.586086 & -0.993962 & $C$ & -5.09688 & -1.189338 & -0.630957 \\
\hline $\mathrm{H}$ & -3.136065 & -1.606952 & -0.960246 & $\mathrm{H}$ & -3.058384 & -1.871465 & -0.990085 \\
\hline C & -5.245974 & 1.361101 & 0.452144 & C & -5.333805 & 0.614213 & 0.983949 \\
\hline
\end{tabular}




\begin{tabular}{|c|c|c|c|c|c|c|c|}
\hline $\mathrm{H}$ & -3.468225 & 1.858471 & 1.595598 & $\mathrm{H}$ & -3.487084 & 1.338552 & 1.867862 \\
\hline C & -5.817658 & 0.483549 & -0.481651 & $\mathrm{C}$ & -5.911841 & -0.29282 & 0.082735 \\
\hline $\mathrm{H}$ & -5.507273 & -1.270689 & -1.723587 & $\mathrm{H}$ & -5.546856 & -1.896132 & -1.335976 \\
\hline $\mathrm{H}$ & -5.829634 & 2.197418 & 0.850593 & $\mathrm{H}$ & -5.964733 & 1.316547 & 1.53851 \\
\hline $\mathrm{H}$ & -6.851178 & 0.632349 & -0.811777 & $\mathrm{H}$ & -6.996847 & -0.30146 & -0.065413 \\
\hline C & 2.300604 & 3.955222 & -1.468488 & $C$ & 1.149482 & 4.708514 & -0.433149 \\
\hline $\mathrm{H}$ & 3.490547 & 4.309087 & 0.312782 & $\mathrm{H}$ & 2.24815 & 4.758469 & 1.439483 \\
\hline $\mathrm{H}$ & 1.050333 & 3.308963 & -3.121966 & $\mathrm{H}$ & 0.059597 & 4.346332 & -2.276368 \\
\hline $\mathrm{H}$ & 2.508499 & 4.93817 & -1.903676 & $\mathrm{H}$ & 1.107944 & 5.798368 & -0.531024 \\
\hline \multicolumn{4}{|c|}{$\begin{array}{l}\text { I } \\
\text { to Gibbs Free Energy= } \\
61587 \\
-1354.84366218\end{array}$} & \multicolumn{4}{|c|}{$\begin{array}{c}\mathbf{T S}_{\mathbf{1 2 a}} \\
\text { Thermal correction to Gibbs Free Energy= } \\
0.262232 \\
\mathrm{E}(\mathrm{RB}-\mathrm{P} 86)=-1354.84279603 \\
\text { Frequency }-26.5530\end{array}$} \\
\hline $\mathrm{N}$ & -2.012869 & -0.213518 & -1.673336 & $S$ & -3.276815 & -0.589252 & -0.014588 \\
\hline$S$ & -3.056201 & -0.870865 & -0.437676 & $C$ & -1.573352 & 0.35508 & -0.508411 \\
\hline C & -1.507628 & 0.245754 & -0.462702 & $N$ & -2.496931 & -0.008425 & -1.466791 \\
\hline $\mathrm{N}$ & -0.481747 & -0.567901 & 0.234893 & $C$ & -2.356696 & -2.210974 & 0.242494 \\
\hline C & -1.559054 & 1.672997 & -0.024086 & $\mathrm{~N}$ & -0.518236 & -0.599712 & -0.229774 \\
\hline C & -2.103553 & -2.391854 & 0.189791 & $C$ & -1.364592 & 1.758371 & -0.045057 \\
\hline C & -0.632551 & -1.93797 & 0.35282 & $\mathrm{C}$ & -0.834848 & -1.92297 & 0.102171 \\
\hline O & 0.804912 & -0.040031 & 0.389625 & 0 & 0.784739 & -0.190116 & 0.119266 \\
\hline C & -2.104375 & 2.616542 & -0.919699 & $C$ & -1.968404 & 2.77674 & -0.814751 \\
\hline C & -1.092689 & 2.096042 & 1.239513 & $\mathrm{C}$ & -0.636033 & 2.10217 & 1.116567 \\
\hline C & -2.170729 & -3.50831 & -0.861315 & $\mathrm{C}$ & -2.771395 & -3.205985 & -0.855995 \\
\hline C & -2.69088 & -2.797376 & 1.549771 & $C$ & -2.636794 & -2.73566 & 1.660441 \\
\hline O & 0.282614 & -2.729719 & 0.583626 & 0 & 0.011738 & -2.788624 & 0.303257 \\
\hline $\mathrm{H}$ & -1.473084 & -4.31275 & -0.575975 & $C$ & 1.654654 & -0.224615 & -1.065309 \\
\hline $\mathrm{H}$ & -3.189833 & -3.923228 & -0.930345 & $C$ & -1.83653 & 4.119086 & -0.437835 \\
\hline $\mathrm{H}$ & -1.871627 & -3.119946 & -1.849029 & $\mathrm{H}$ & -2.541405 & 2.479431 & -1.698281 \\
\hline $\mathrm{H}$ & -2.102336 & -3.635266 & 1.959479 & $\mathrm{C}$ & -0.517451 & 3.445201 & 1.493564 \\
\hline $\mathrm{H}$ & -2.65689 & -1.966363 & 2.273837 & $\mathrm{H}$ & -0.168175 & 1.320485 & 1.718973 \\
\hline $\mathrm{H}$ & -3.737862 & -3.129138 & 1.442438 & $\mathrm{H}$ & -2.108611 & -4.087007 & -0.818318 \\
\hline C & 1.579174 & -0.15529 & -0.863866 & $\mathrm{H}$ & -3.812445 & -3.536969 & -0.709157 \\
\hline C & -2.180692 & 3.967436 & -0.55742 & $\mathrm{H}$ & -2.678192 & -2.730261 & -1.846299 \\
\hline $\mathrm{H}$ & -2.462639 & 2.259038 & -1.889907 & $\mathrm{H}$ & -2.045343 & -3.649338 & 1.83267 \\
\hline C & -1.176518 & 3.447082 & 1.597878 & $\mathrm{H}$ & -2.36384 & -1.996262 & 2.431745 \\
\hline $\mathrm{H}$ & -0.662236 & 1.367089 & 1.930874 & $\mathrm{H}$ & -3.705661 & -2.982939 & 1.774829 \\
\hline C & 3.033364 & -0.079615 & -0.483254 & $C$ & 3.073554 & -0.261285 & -0.5601 \\
\hline $\mathrm{H}$ & 1.28575 & 0.659072 & -1.551569 & $\mathrm{H}$ & 1.461061 & 0.664856 & -1.693464 \\
\hline $\mathrm{H}$ & 1.330785 & -1.127266 & -1.322563 & $\mathrm{H}$ & 1.405225 & -1.133916 & -1.638838 \\
\hline C & 3.586139 & -1.092105 & 0.328962 & $\mathrm{C}$ & -1.112052 & 4.455577 & 0.717304 \\
\hline C & 3.848439 & 0.980749 & -0.918443 & $\mathrm{H}$ & -2.301409 & 4.903399 & -1.043777 \\
\hline C & 4.938688 & -1.043514 & 0.689929 & $\mathrm{H}$ & 0.039619 & 3.706219 & 2.399095 \\
\hline $\mathrm{H}$ & 2.936412 & -1.904064 & 0.672687 & $C$ & 3.500226 & -1.354817 & 0.222434 \\
\hline C & 5.206172 & 1.023871 & -0.563471 & $\mathrm{C}$ & 3.979207 & 0.773284 & -0.855666 \\
\hline $\mathrm{H}$ & 3.417474 & 1.773507 & -1.540704 & $\mathrm{C}$ & 4.818337 & -1.410602 & 0.69318 \\
\hline C & 5.751872 & 0.012437 & 0.241657 & $\mathrm{H}$ & 2.782025 & -2.146873 & 0.459523 \\
\hline $\mathrm{H}$ & 5.363125 & -1.832193 & 1.319964 & $\mathrm{C}$ & 5.303067 & 0.711476 & -0.391849 \\
\hline
\end{tabular}




\begin{tabular}{|c|c|c|c|c|c|c|c|}
\hline $\mathrm{H}$ & 5.835241 & 1.849892 & -0.91111 & $\mathrm{H}$ & 3.64613 & 1.62894 & -1.454224 \\
\hline $\mathrm{H}$ & 6.810047 & 0.046349 & 0.521633 & $\mathrm{C}$ & 5.723188 & -0.379764 & 0.383648 \\
\hline C & -1.719027 & 4.384681 & 0.701968 & $\mathrm{H}$ & 5.14428 & -2.261615 & 1.300248 \\
\hline $\mathrm{H}$ & -2.600714 & 4.696239 & -1.258082 & $\mathrm{H}$ & 6.003328 & 1.518345 & -0.631452 \\
\hline $\mathrm{H}$ & -0.81656 & 3.771145 & 2.579597 & $\mathrm{H}$ & 6.754516 & -0.427786 & 0.748622 \\
\hline $\mathrm{H}$ & -1.78066 & 5.440312 & 0.986479 & $\mathrm{H}$ & -1.012232 & 5.50432 & 1.016295 \\
\hline \multicolumn{4}{|c|}{$\begin{array}{c}\mathbf{2 a} \\
\text { Thermal correction to Gibbs Free Energy= } \\
0.264230 \\
\mathrm{E}(\mathrm{RB}-\mathrm{P} 86)=-1354.90782422\end{array}$} & \multicolumn{4}{|c|}{$\begin{array}{c}\mathbf{K} \\
\text { Thermal correction to Gibbs Free Energy= } \\
0.313229 \\
\mathrm{E}(\mathrm{RB}-\mathrm{P} 86)=-2254.16638243\end{array}$} \\
\hline$S$ & 3.339287 & -0.82389 & 1.133493 & $S$ & -0.960344 & -0.973539 & 1.597767 \\
\hline C & 1.458366 & 0.732875 & 0.19823 & $\mathrm{~N}$ & -0.433789 & -0.27353 & -0.934805 \\
\hline $\mathrm{N}$ & 2.603278 & 0.683691 & 0.825515 & $\mathrm{C}$ & -1.616024 & -0.307351 & -0.035863 \\
\hline $\mathrm{N}$ & 0.748703 & -0.419184 & -0.232585 & $\mathrm{~N}$ & -0.685701 & -0.246962 & -2.160012 \\
\hline C & 0.866073 & 2.072256 & -0.067861 & $\mathrm{C}$ & -1.805996 & 0.33645 & 2.627545 \\
\hline C & 2.75324 & -1.870347 & -0.298316 & $\mathrm{~N}$ & -2.022567 & 1.045164 & 0.321397 \\
\hline C & 1.224754 & -1.746147 & -0.288913 & $\mathrm{C}$ & -2.749418 & -1.181353 & -0.574745 \\
\hline O & -0.639635 & -0.292041 & -0.416961 & $C$ & -2.301045 & 1.398167 & 1.626495 \\
\hline C & 0.089073 & 2.342917 & -1.216736 & 0 & -2.524275 & 1.807779 & -0.745546 \\
\hline C & 1.155268 & 3.123611 & 0.831201 & $C$ & -2.446724 & -2.480748 & -1.034005 \\
\hline C & 3.172459 & -3.317855 & -0.010718 & $\mathrm{C}$ & -4.082979 & -0.732373 & -0.590631 \\
\hline C & 3.346666 & -1.350727 & -1.621566 & $\mathrm{~N}$ & -0.738636 & -0.232907 & -3.312423 \\
\hline O & 0.457304 & -2.70401 & -0.371186 & $\mathrm{C}$ & -0.800219 & 0.97257 & 3.603604 \\
\hline C & -1.354534 & -0.45953 & 0.860368 & $\mathrm{C}$ & -3.022339 & -0.24934 & 3.373528 \\
\hline C & -0.38942 & 3.639049 & -1.45201 & 0 & -2.845944 & 2.449328 & 1.964761 \\
\hline $\mathrm{H}$ & -0.13942 & 1.540373 & -1.921056 & $\mathrm{C}$ & -1.883115 & 3.147272 & -0.826302 \\
\hline C & 0.667979 & 4.413561 & 0.592925 & $\mathrm{C}$ & -3.466562 & -3.318497 & -1.500459 \\
\hline $\mathrm{H}$ & 1.77147 & 2.908163 & 1.708493 & $\mathrm{H}$ & -1.410948 & -2.836175 & -1.009678 \\
\hline $\mathrm{H}$ & 2.812992 & -3.972343 & -0.820228 & $C$ & -5.101057 & -1.578154 & -1.060418 \\
\hline $\mathrm{H}$ & 4.271279 & -3.39192 & 0.041223 & $\mathrm{H}$ & -4.324261 & 0.281888 & -0.261896 \\
\hline $\mathrm{H}$ & 2.740347 & -3.683424 & 0.933518 & $\mathrm{H}$ & -1.296046 & 1.803551 & 4.132392 \\
\hline $\mathrm{H}$ & 2.930615 & -1.924559 & -2.468799 & $\mathrm{H}$ & -0.461069 & 0.233462 & 4.347977 \\
\hline $\mathrm{H}$ & 3.123117 & -0.284584 & -1.783172 & $\mathrm{H}$ & 0.08275 & 1.363256 & 3.074027 \\
\hline $\mathrm{H}$ & 4.441773 & -1.475797 & -1.615667 & $\mathrm{H}$ & -3.547008 & 0.565553 & 3.900035 \\
\hline C & -2.794403 & -0.731566 & 0.515282 & $\mathrm{H}$ & -3.726712 & -0.732693 & 2.678571 \\
\hline $\mathrm{H}$ & -1.239258 & 0.45873 & 1.46574 & $\mathrm{H}$ & -2.693865 & -0.997694 & 4.112561 \\
\hline $\mathrm{H}$ & -0.899934 & -1.313885 & 1.390411 & $\mathrm{C}$ & -0.397506 & 3.096977 & -1.051241 \\
\hline C & -0.107094 & 4.676466 & -0.549384 & $\mathrm{H}$ & -2.150467 & 3.697756 & 0.0889 \\
\hline $\mathrm{H}$ & -0.986183 & 3.837651 & -2.348049 & $\mathrm{H}$ & -2.420739 & 3.575789 & -1.687193 \\
\hline $\mathrm{H}$ & 0.893311 & 5.217041 & 1.301607 & $\mathrm{C}$ & -4.798219 & -2.869605 & -1.514432 \\
\hline C & -3.812404 & 0.172493 & 0.868411 & $\mathrm{H}$ & -3.221023 & -4.325807 & -1.851099 \\
\hline C & -3.1258 & -1.914178 & -0.178712 & $\mathrm{H}$ & -6.134678 & -1.218113 & -1.075071 \\
\hline C & -5.150824 & -0.106864 & 0.549513 & $C$ & 0.483348 & 3.165924 & 0.047178 \\
\hline $\mathrm{H}$ & -3.55471 & 1.097082 & 1.397478 & $\mathrm{C}$ & 0.135222 & 2.945425 & -2.347774 \\
\hline C & -4.460439 & -2.187322 & -0.504891 & $\mathrm{C}$ & 1.86818 & 3.05103 & -0.139818 \\
\hline $\mathrm{H}$ & -2.321907 & -2.602215 & -0.460404 & $\mathrm{H}$ & 0.07376 & 3.3139 & 1.052773 \\
\hline C & -5.475551 & -1.286256 & -0.138035 & $\mathrm{C}$ & 1.520565 & 2.842621 & -2.539168 \\
\hline $\mathrm{H}$ & -5.938168 & 0.599082 & 0.833325 & $\mathrm{H}$ & -0.541339 & 2.901122 & -3.208548 \\
\hline $\mathrm{H}$ & -4.71254 & -3.106804 & -1.043424 & $\mathrm{C}$ & 2.387838 & 2.883942 & -1.434203 \\
\hline
\end{tabular}




\begin{tabular}{|c|c|c|c|c|c|c|c|}
\hline $\mathrm{H}$ & -6.518876 & -1.504001 & -0.389557 & $\mathrm{H}$ & 2.54278 & 3.081424 & 0.720065 \\
\hline \multirow[t]{15}{*}{$\mathrm{H}$} & -0.488176 & 5.685887 & -0.735388 & $\mathrm{H}$ & 1.924391 & 2.7275 & -3.55025 \\
\hline & & & & $\mathrm{H}$ & 3.468305 & 2.788423 & -1.577967 \\
\hline & & & & $\mathrm{H}$ & -5.594866 & -3.525321 & -1.879738 \\
\hline & & & & $\mathrm{O}$ & 2.307504 & -0.527012 & 1.568059 \\
\hline & & & & C & 2.856949 & -0.750602 & 0.292958 \\
\hline & & & & $\mathrm{H}$ & 1.322738 & -0.542029 & 1.474797 \\
\hline & & & & C & 2.755684 & -2.243704 & -0.104542 \\
\hline & & & & C & 4.314677 & -0.254391 & 0.334658 \\
\hline & & & & $\mathrm{H}$ & 2.347026 & -0.178928 & -0.507195 \\
\hline & & & & $\mathrm{F}$ & 1.457654 & -2.657493 & 0.023435 \\
\hline & & & & $\mathrm{F}$ & 3.515681 & -3.047306 & 0.674894 \\
\hline & & & & $\mathrm{F}$ & 3.118461 & -2.440951 & -1.399988 \\
\hline & & & & $\mathrm{F}$ & 4.941811 & -0.498881 & -0.84835 \\
\hline & & & & $\mathrm{F}$ & 5.029083 & -0.839239 & 1.321984 \\
\hline & & & & $\mathrm{F}$ & 4.346842 & 1.091769 & 0.544779 \\
\hline \multicolumn{4}{|c|}{$\begin{array}{c}\text { TS }_{\mathrm{Kc}} \\
\text { Thermal correction to Gibbs Free Energy }= \\
0.306001 \\
\mathrm{E}(\text { RB-P86) }=-2254.11661257 \\
\text { Frequency }-539.1369\end{array}$} & \multicolumn{4}{|c|}{$\begin{array}{c}\text { C' }^{\prime} \\
\text { Thermal correction to Gibbs Free Energy= } \\
0.297847 \\
E(\text { RB-P86 })=-2254.25058150\end{array}$} \\
\hline$N$ & 0.942054 & 1.392197 & -1.697902 & $\mathrm{~N}$ & -1.069764 & -1.034926 & 1.283345 \\
\hline$N$ & -0.367049 & 0.375618 & -2.279816 & $\mathrm{~N}$ & -3.479935 & -4.475641 & -1.383589 \\
\hline C & 1.000405 & 1.43394 & -0.346195 & C & -0.838251 & 0.214417 & 1.176733 \\
\hline$S$ & -0.450631 & 2.163713 & 0.683846 & $S$ & 0.706127 & 1.126008 & 1.469674 \\
\hline C & 0.161658 & 1.390901 & 2.276999 & C & -0.068892 & 2.786559 & 1.044971 \\
\hline$N$ & 1.300676 & 0.235871 & 0.465328 & $\mathrm{~N}$ & -1.830295 & 1.133745 & 0.809357 \\
\hline C & 2.192654 & 2.456978 & -0.569092 & C & -0.045816 & -1.957347 & 1.599111 \\
\hline C & 1.066698 & 0.224557 & 1.833631 & C & -1.542263 & 2.492312 & 0.706511 \\
\hline 0 & 2.278676 & -0.644187 & -0.004577 & $\mathrm{O}$ & -3.143546 & 0.715245 & 0.675573 \\
\hline C & 1.918537 & 3.836878 & -0.709728 & C & 1.05889 & -2.164926 & 0.740887 \\
\hline C & 3.527421 & 1.991885 & -0.592355 & C & -0.185812 & -2.756818 & 2.754436 \\
\hline$N$ & -0.808688 & 0.020824 & -3.256533 & $\mathrm{~N}$ & -3.014168 & -5.479947 & -1.23228 \\
\hline C & -1.019318 & 0.851244 & 3.100113 & C & 0.627085 & 3.405291 & -0.181967 \\
\hline C & 1.003884 & 2.39677 & 3.0891 & C & -0.006442 & 3.722689 & 2.266891 \\
\hline 0 & 1.501929 & -0.622999 & 2.610264 & $\mathrm{O}$ & -2.354182 & 3.350874 & 0.381461 \\
\hline C & 1.676641 & -1.855077 & -0.589633 & C & -3.417519 & 0.255183 & -0.701713 \\
\hline C & 2.973486 & 4.747851 & -0.814731 & C & 2.017117 & -3.140977 & 1.055851 \\
\hline $\mathrm{H}$ & 0.880032 & 4.177422 & -0.720294 & $\mathrm{H}$ & 1.152966 & -1.58275 & -0.181513 \\
\hline C & 4.576516 & 2.915478 & -0.682466 & C & 0.784848 & -3.717876 & 3.064872 \\
\hline $\mathrm{H}$ & 3.72239 & 0.919392 & -0.528461 & $\mathrm{H}$ & -1.057484 & -2.605163 & 3.397866 \\
\hline $\mathrm{H}$ & -0.62067 & 0.326203 & 3.983554 & $\mathrm{H}$ & 0.119208 & 4.349835 & -0.437413 \\
\hline $\mathrm{H}$ & -1.660835 & 1.680165 & 3.443119 & $\mathrm{H}$ & 1.685748 & 3.612633 & 0.037658 \\
\hline $\mathrm{H}$ & -1.627625 & 0.140626 & 2.520125 & $\mathrm{H}$ & 0.582804 & 2.736879 & -1.056394 \\
\hline $\mathrm{H}$ & 1.429719 & 1.883089 & 3.967625 & $\mathrm{H}$ & -0.544466 & 4.654307 & 2.025739 \\
\hline $\mathrm{H}$ & 1.831011 & 2.812513 & 2.491988 & $\mathrm{H}$ & -0.474816 & 3.264714 & 3.152013 \\
\hline $\mathrm{H}$ & 0.371724 & 3.23017 & 3.435598 & $\mathrm{H}$ & 1.039832 & 3.968275 & 2.510058 \\
\hline C & 2.671721 & -2.974018 & -0.417692 & C & -4.908302 & 0.332668 & -0.895415 \\
\hline $\mathrm{H}$ & 1.44734 & -1.669415 & -1.653553 & $\mathrm{H}$ & -3.031525 & -0.771875 & -0.817616 \\
\hline
\end{tabular}




\begin{tabular}{|c|c|c|c|c|c|c|c|}
\hline $\mathrm{H}$ & 0.734858 & -2.051139 & -0.048831 & $\mathrm{H}$ & -2.883764 & 0.932003 & -1.392885 \\
\hline C & 4.303686 & 4.289247 & -0.79503 & $\mathrm{C}$ & 1.89001 & -3.915165 & 2.219603 \\
\hline $\mathrm{H}$ & 2.76083 & 5.816358 & -0.916923 & $\mathrm{H}$ & 2.858733 & -3.296678 & 0.373972 \\
\hline $\mathrm{H}$ & 5.611015 & 2.558743 & -0.68164 & $\mathrm{H}$ & 0.671576 & -4.323382 & 3.970155 \\
\hline C & 3.127919 & -3.708349 & -1.527218 & $C$ & -5.657386 & -0.817482 & -1.20261 \\
\hline C & 3.140802 & -3.299605 & 0.872494 & $C$ & -5.561187 & 1.577228 & -0.774775 \\
\hline C & 4.028302 & -4.771542 & -1.353081 & $\mathrm{C}$ & -7.043373 & -0.724874 & -1.40681 \\
\hline $\mathrm{H}$ & 2.772999 & -3.448627 & -2.531317 & $\mathrm{H}$ & -5.152298 & -1.786409 & -1.284041 \\
\hline C & 4.048027 & -4.353504 & 1.042461 & C & -6.945639 & 1.665595 & -0.967973 \\
\hline $\mathrm{H}$ & 2.794085 & -2.707284 & 1.726277 & $\mathrm{H}$ & -4.971734 & 2.463225 & -0.515711 \\
\hline C & 4.489896 & -5.094241 & -0.068046 & $C$ & -7.688319 & 0.515632 & -1.288837 \\
\hline $\mathrm{H}$ & 4.373782 & -5.341565 & -2.22171 & $\mathrm{H}$ & -7.619451 & -1.623359 & -1.650755 \\
\hline $\mathrm{H}$ & 4.410294 & -4.602194 & 2.045476 & $\mathrm{H}$ & -7.448352 & 2.633463 & -0.871349 \\
\hline $\mathrm{H}$ & 5.195479 & -5.920376 & 0.069298 & $\mathrm{H}$ & -8.769897 & 0.587734 & -1.443952 \\
\hline $\mathrm{H}$ & 5.128169 & 5.004805 & -0.878031 & $\mathrm{H}$ & 2.639033 & -4.675943 & 2.46022 \\
\hline O & -2.289366 & -0.389209 & 0.048764 & 0 & 2.455137 & 0.422662 & -1.183024 \\
\hline C & -3.660498 & -0.068396 & 0.074595 & $C$ & 3.739083 & 0.096062 & -0.7012 \\
\hline $\mathrm{H}$ & -1.773506 & 0.457174 & 0.14302 & $\mathrm{H}$ & 1.905265 & 0.668405 & -0.396707 \\
\hline C & -4.384593 & -1.183547 & 0.854097 & C & 4.57604 & 1.375578 & -0.473953 \\
\hline C & -4.196019 & 0.119135 & -1.363446 & C & 4.373392 & -0.872427 & -1.717147 \\
\hline $\mathrm{H}$ & -3.877442 & 0.876592 & 0.609967 & $\mathrm{H}$ & 3.715258 & -0.439685 & 0.268541 \\
\hline $\mathrm{F}$ & -3.949461 & -1.19282 & 2.144374 & $\mathrm{~F}$ & 3.896744 & 2.195771 & 0.389947 \\
\hline $\mathrm{F}$ & -4.152403 & -2.406 & 0.326225 & $\mathrm{~F}$ & 4.789223 & 2.067054 & -1.614807 \\
\hline $\mathrm{F}$ & -5.728317 & -0.97453 & 0.873994 & $\mathrm{~F}$ & 5.777433 & 1.098213 & 0.092436 \\
\hline$F$ & -5.493821 & 0.524398 & -1.366518 & $\mathrm{~F}$ & 5.643378 & -1.192173 & -1.354184 \\
\hline $\mathrm{F}$ & -4.10202 & -1.011468 & -2.099927 & $\mathrm{~F}$ & 4.403167 & -0.355754 & -2.964848 \\
\hline $\mathrm{F}$ & -3.460574 & 1.086869 & -1.982441 & $\mathrm{~F}$ & 3.650233 & -2.026796 & -1.759365 \\
\hline \multicolumn{4}{|c|}{$\begin{array}{c}\text { TS }_{\mathrm{KG}} \\
\text { Thermal correction to Gibbs Free Energy }= \\
0.307429 \\
\mathrm{E}(\mathrm{RB}-\mathrm{P} 86)=-2254.09784522 \\
\text { Frequency }-440.6032\end{array}$} & \multicolumn{4}{|c|}{$\begin{array}{c}\text { G' } \\
\text { Thermal correction to Gibbs Free Energy= } \\
0.309326 \\
\mathrm{E}(\mathrm{RB}-\mathrm{P} 86)=-2254.15222850\end{array}$} \\
\hline $\mathrm{N}$ & 1.035224 & -0.666622 & -1.026461 & $\mathrm{~N}$ & 1.814075 & -0.578141 & -0.522437 \\
\hline $\mathrm{N}$ & 0.605212 & 0.283212 & 0.599359 & $\mathrm{~N}$ & 0.6963 & -1.231035 & 0.145105 \\
\hline $\mathrm{N}$ & 0.872608 & 0.966218 & 2.313776 & $\mathrm{~N}$ & -2.754329 & 1.38129 & 3.196943 \\
\hline C & 1.301029 & -0.904124 & 0.413734 & $C$ & 1.8381 & -1.003843 & 0.940632 \\
\hline C & 0.32711 & -1.652717 & -1.81738 & $C$ & 2.530849 & -1.508564 & -1.42664 \\
\hline 0 & 1.834332 & 0.199684 & -1.748867 & 0 & 1.81138 & 0.80807 & -0.867528 \\
\hline $\mathrm{N}$ & 0.809368 & 1.958856 & 2.843354 & $\mathrm{~N}$ & -3.568819 & 2.142304 & 3.122871 \\
\hline$S$ & 0.237375 & -2.548392 & 0.745225 & $S$ & 3.033917 & -2.424453 & 1.122563 \\
\hline C & 2.720205 & -1.121443 & 0.897517 & $\mathrm{C}$ & 1.967084 & 0.054443 & 1.999453 \\
\hline C & 0.058233 & -2.950333 & -1.057689 & $C$ & 2.97497 & -2.796559 & -0.70946 \\
\hline C & 2.951765 & -1.291499 & 2.278476 & $C$ & 0.81126 & 0.691698 & 2.505387 \\
\hline C & 3.808204 & -1.178012 & 0.005296 & $\mathrm{C}$ & 3.233828 & 0.394386 & 2.522079 \\
\hline C & -1.346364 & -3.490408 & -1.376648 & C & 1.984545 & -3.940204 & -1.029937 \\
\hline C & 1.146414 & -3.962151 & -1.503145 & $\mathrm{C}$ & 4.397293 & -3.144816 & -1.195996 \\
\hline O & 0.11862 & -1.460957 & -2.999623 & O & 2.70699 & -1.238077 & -2.595041 \\
\hline $\mathrm{H}$ & -1.417841 & -3.698592 & -2.457198 & $\mathrm{H}$ & 1.948959 & -4.112173 & -2.119895 \\
\hline $\mathrm{H}$ & -1.519553 & -4.429553 & -0.826928 & $\mathrm{H}$ & 2.326661 & -4.864964 & -0.537216 \\
\hline
\end{tabular}




\begin{tabular}{|c|c|c|c|c|c|c|c|}
\hline $\mathrm{H}$ & -2.136128 & -2.774139 & -1.111288 & $\mathrm{H}$ & 0.977874 & -3.687786 & -0.668648 \\
\hline $\mathrm{H}$ & 1.069967 & -4.147879 & -2.588462 & $\mathrm{H}$ & 4.380583 & -3.294876 & -2.287971 \\
\hline $\mathrm{H}$ & 2.15874 & -3.595155 & -1.271604 & $\mathrm{H}$ & 5.116156 & -2.345058 & -0.960729 \\
\hline $\mathrm{H}$ & 0.996257 & -4.911553 & -0.964748 & $\mathrm{H}$ & 4.739163 & -4.075612 & -0.71642 \\
\hline C & 1.064492 & 1.370756 & -2.279724 & $C$ & 0.746449 & 1.105767 & -1.856937 \\
\hline C & 4.251241 & -1.494732 & 2.759692 & $\mathrm{C}$ & 0.93305 & 1.652063 & 3.518645 \\
\hline $\mathrm{H}$ & 2.110297 & -1.286364 & 2.978372 & $\mathrm{H}$ & -0.177271 & 0.437271 & 2.106462 \\
\hline C & 5.107579 & -1.391084 & 0.493531 & $C$ & 3.345198 & 1.36166 & 3.528033 \\
\hline $\mathrm{H}$ & 3.64673 & -1.037957 & -1.065877 & $\mathrm{H}$ & 4.127365 & -0.100573 & 2.130937 \\
\hline C & 1.401648 & 2.640156 & -1.545566 & $\mathrm{C}$ & 0.65889 & 2.605531 & -1.924963 \\
\hline $\mathrm{H}$ & -0.005076 & 1.126739 & -2.226065 & $\mathrm{H}$ & -0.195437 & 0.660491 & -1.49886 \\
\hline $\mathrm{H}$ & 1.375651 & 1.404772 & -3.336272 & $\mathrm{H}$ & 1.037636 & 0.660727 & -2.821769 \\
\hline C & 5.335503 & -1.545129 & 1.868119 & $C$ & 2.195253 & 1.989961 & 4.030951 \\
\hline $\mathrm{H}$ & 4.4147 & -1.625059 & 3.83414 & $\mathrm{H}$ & 0.032419 & 2.131546 & 3.913259 \\
\hline $\mathrm{H}$ & 5.944872 & -1.431682 & -0.210639 & $\mathrm{H}$ & 4.332646 & 1.626331 & 3.919367 \\
\hline C & 0.448417 & 3.260988 & -0.71265 & $\mathrm{C}$ & -0.165803 & 3.30926 & -1.025027 \\
\hline C & 2.667261 & 3.239684 & -1.710135 & $\mathrm{C}$ & 1.416006 & 3.323193 & -2.871162 \\
\hline $\mathrm{H}$ & 6.350259 & -1.708567 & 2.244307 & $\mathrm{H}$ & 2.283258 & 2.740675 & 4.823075 \\
\hline C & 0.75893 & 4.462203 & -0.056196 & $C$ & -0.237789 & 4.708166 & -1.075933 \\
\hline $\mathrm{H}$ & -0.536562 & 2.800466 & -0.577837 & $\mathrm{H}$ & -0.757401 & 2.750435 & -0.292326 \\
\hline C & 2.980133 & 4.432931 & -1.045548 & $C$ & 1.344572 & 4.722821 & -2.92354 \\
\hline $\mathrm{H}$ & 3.409204 & 2.766597 & -2.363554 & $\mathrm{H}$ & 2.060339 & 2.777936 & -3.569946 \\
\hline C & 2.024025 & 5.047875 & -0.219317 & $\mathrm{C}$ & 0.517426 & 5.416464 & -2.02537 \\
\hline $\mathrm{H}$ & 0.009981 & 4.939183 & 0.583871 & $\mathrm{H}$ & -0.887356 & 5.246838 & -0.378453 \\
\hline $\mathrm{H}$ & 3.965741 & 4.890536 & -1.179699 & $\mathrm{H}$ & 1.931836 & 5.2726 & -3.666122 \\
\hline $\mathrm{H}$ & 2.264993 & 5.985068 & 0.29309 & $\mathrm{H}$ & 0.457905 & 6.508992 & -2.068038 \\
\hline $\mathrm{H}$ & -1.062157 & 0.704889 & 0.628869 & $\mathrm{H}$ & -0.862121 & -0.55503 & 0.201943 \\
\hline O & -1.979923 & 1.116956 & 0.658071 & 0 & -1.812695 & -0.204341 & 0.204715 \\
\hline C & -2.910644 & 0.072802 & 0.567072 & $\mathrm{C}$ & -2.660279 & -1.270252 & -0.14055 \\
\hline C & -3.456398 & -0.028545 & -0.874017 & $C$ & -3.011546 & -1.218214 & -1.645832 \\
\hline C & -4.020132 & 0.311328 & 1.610332 & $C$ & -3.907065 & -1.21247 & 0.764129 \\
\hline $\mathrm{H}$ & -2.466908 & -0.91624 & 0.80209 & $\mathrm{H}$ & -2.195729 & -2.260976 & 0.030893 \\
\hline$F$ & -2.403632 & -0.12132 & -1.746216 & $\mathrm{~F}$ & -1.857449 & -1.179568 & -2.371403 \\
\hline $\mathrm{F}$ & -4.1961 & 1.041624 & -1.238987 & $\mathrm{~F}$ & -3.740134 & -0.123731 & -1.971326 \\
\hline $\mathrm{F}$ & -4.214732 & -1.148998 & -1.048078 & $\mathrm{~F}$ & -3.703657 & -2.321338 & -2.038388 \\
\hline $\mathrm{F}$ & -4.99891 & -0.63223 & 1.511611 & $\mathrm{~F}$ & -4.803579 & -2.182049 & 0.434314 \\
\hline$F$ & -4.595696 & 1.529075 & 1.475537 & $\mathrm{~F}$ & -4.535373 & -0.015096 & 0.689094 \\
\hline $\mathrm{F}$ & -3.493617 & 0.233001 & 2.860629 & $\mathrm{~F}$ & -3.543126 & -1.410956 & 2.059611 \\
\hline \multicolumn{4}{|c|}{$\begin{array}{c}\text { TS }_{\text {K2a' }} \\
\text { Thermal correction to Gibbs Free Energy= } \\
0.310401 \\
\mathrm{E}(\text { RB-P86) }=-2254.13175203 \\
\text { Frequency }-574.6450\end{array}$} & \multicolumn{4}{|c|}{$\begin{array}{c}\mathbf{2 a}^{\prime} \\
\text { Thermal correction to Gibbs Free Energy= } \\
0.302274 \\
\mathrm{E}(\mathrm{RB}-\mathrm{P} 86)=-2254.23638353\end{array}$} \\
\hline $\mathrm{N}$ & 0.237761 & -1.17969 & 1.185242 & $N$ & -0.202579 & 1.224421 & -0.588253 \\
\hline $\mathrm{N}$ & 0.439017 & 0.194036 & 2.15211 & $\mathrm{~N}$ & -4.779863 & -3.218966 & 1.56156 \\
\hline C & 1.399655 & -1.31763 & 0.372712 & $\mathrm{C}$ & 0.842121 & 0.461262 & -0.390711 \\
\hline $\mathrm{N}$ & 0.03113 & 0.716181 & 3.071137 & $\mathrm{~N}$ & -5.723097 & -3.668296 & 1.957437 \\
\hline$S$ & 0.714024 & -3.033749 & 0.004117 & $S$ & -0.035292 & 2.930314 & -0.662034 \\
\hline $\mathrm{N}$ & 1.374629 & -0.656761 & -0.93985 & $\mathrm{~N}$ & 2.145786 & 0.973781 & -0.233086 \\
\hline
\end{tabular}




\begin{tabular}{|c|c|c|c|c|c|c|c|}
\hline C & 2.763623 & -1.290494 & 1.03569 & $\mathrm{C}$ & 0.609344 & -1.012839 & -0.310069 \\
\hline C & 0.477887 & -2.727486 & -1.833096 & C & 1.401797 & 3.241029 & 0.475627 \\
\hline C & 0.947835 & -1.276231 & -2.090599 & $\mathrm{C}$ & 2.533945 & 2.311859 & 0.018231 \\
\hline C & -0.997276 & -2.877037 & -2.24129 & $\mathrm{C}$ & 1.018761 & 2.937564 & 1.938055 \\
\hline C & 1.388494 & -3.702749 & -2.605696 & $\mathrm{C}$ & 1.813938 & 4.70845 & 0.286961 \\
\hline O & 1.75293 & 0.693039 & -0.934727 & 0 & 3.207165 & 0.090184 & -0.483551 \\
\hline C & 2.882672 & -1.702096 & 2.379386 & $\mathrm{C}$ & -0.428188 & -1.464093 & 0.537323 \\
\hline C & 3.918706 & -0.908609 & 0.325184 & $\mathrm{C}$ & 1.321634 & -1.951009 & -1.09008 \\
\hline $\mathrm{O}$ & 0.905008 & -0.754916 & -3.204896 & O & 3.714162 & 2.648295 & -0.0277 \\
\hline $\mathrm{H}$ & -1.090092 & -2.602426 & -3.305251 & $\mathrm{H}$ & 1.898136 & 3.071436 & 2.592718 \\
\hline $\mathrm{H}$ & -1.326626 & -3.92185 & -2.116362 & $\mathrm{H}$ & 0.226716 & 3.629792 & 2.266394 \\
\hline $\mathrm{H}$ & -1.650481 & -2.218891 & -1.648671 & $\mathrm{H}$ & 0.645282 & 1.908652 & 2.06133 \\
\hline $\mathrm{H}$ & 1.324007 & -3.451242 & -3.677853 & $\mathrm{H}$ & 2.694139 & 4.923806 & 0.912207 \\
\hline $\mathrm{H}$ & 2.440564 & -3.621138 & -2.289802 & $\mathrm{H}$ & 2.081831 & 4.920805 & -0.759701 \\
\hline $\mathrm{H}$ & 1.056032 & -4.744523 & -2.467614 & $\mathrm{H}$ & 0.991154 & 5.376004 & 0.59098 \\
\hline C & 0.613363 & 1.581078 & -1.297316 & $\mathrm{C}$ & 3.845061 & -0.343706 & 0.772966 \\
\hline C & 4.136475 & -1.724638 & 3.003954 & $\mathrm{C}$ & -0.740069 & -2.828923 & 0.612637 \\
\hline $\mathrm{H}$ & 1.986697 & -2.012908 & 2.925134 & $\mathrm{H}$ & -0.985177 & -0.734456 & 1.132215 \\
\hline C & 5.170446 & -0.927274 & 0.957024 & C & 0.998585 & -3.313198 & -1.012621 \\
\hline $\mathrm{H}$ & 3.834711 & -0.573494 & -0.711518 & $\mathrm{H}$ & 2.114938 & -1.612002 & -1.758137 \\
\hline C & 1.09789 & 2.988669 & -1.086825 & $\mathrm{C}$ & 5.163659 & -0.958756 & 0.388327 \\
\hline $\mathrm{H}$ & -0.253447 & 1.336334 & -0.662941 & $\mathrm{H}$ & 3.182112 & -1.063675 & 1.285682 \\
\hline $\mathrm{H}$ & 0.363843 & 1.376456 & -2.35144 & $\mathrm{H}$ & 3.988756 & 0.549625 & 1.404581 \\
\hline C & 5.284535 & -1.33563 & 2.294947 & $\mathrm{C}$ & -0.026615 & -3.756963 & -0.162081 \\
\hline $\mathrm{H}$ & 4.216242 & -2.047698 & 4.046797 & $\mathrm{H}$ & -1.54315 & -3.164148 & 1.276236 \\
\hline $\mathrm{H}$ & 6.059739 & -0.616316 & 0.399655 & $\mathrm{H}$ & 1.548691 & -4.030518 & -1.629872 \\
\hline C & 0.520484 & 3.803559 & -0.094215 & C & 5.420244 & -2.324366 & 0.60753 \\
\hline C & 2.136299 & 3.509041 & -1.886769 & $\mathrm{C}$ & 6.156177 & -0.151019 & -0.205204 \\
\hline $\mathrm{H}$ & 6.263871 & -1.349914 & 2.783876 & $\mathrm{H}$ & -0.271398 & -4.822488 & -0.106493 \\
\hline C & 0.967022 & 5.120956 & 0.09123 & C & 6.661594 & -2.877156 & 0.255062 \\
\hline $\mathrm{H}$ & -0.292521 & 3.40447 & 0.52148 & $\mathrm{H}$ & 4.645479 & -2.954671 & 1.058686 \\
\hline C & 2.59196 & 4.819842 & -1.691924 & $\mathrm{C}$ & 7.390541 & -0.706299 & -0.566145 \\
\hline $\mathrm{H}$ & 2.584899 & 2.87754 & -2.660943 & $\mathrm{H}$ & 5.939797 & 0.907364 & -0.383932 \\
\hline C & 2.006107 & 5.629161 & -0.703686 & C & 7.646628 & -2.068911 & -0.33293 \\
\hline $\mathrm{H}$ & 0.504527 & 5.749456 & 0.859236 & $\mathrm{H}$ & 6.856793 & -3.939291 & 0.43495 \\
\hline $\mathrm{H}$ & 3.399721 & 5.215173 & -2.316615 & $\mathrm{H}$ & 8.157583 & -0.075672 & -1.02725 \\
\hline $\mathrm{H}$ & 2.357104 & 6.65599 & -0.557109 & $\mathrm{H}$ & 8.614073 & -2.499774 & -0.611009 \\
\hline $\mathrm{H}$ & -1.18413 & -0.666322 & 0.323741 & $\mathrm{H}$ & -1.922614 & 0.837602 & -1.023773 \\
\hline $\mathrm{O}$ & -1.990849 & -0.335019 & -0.189375 & O & -2.87418 & 0.876366 & -1.326265 \\
\hline C & -3.074551 & -0.23417 & 0.697508 & C & -3.650758 & 0.053047 & -0.505072 \\
\hline C & -4.287518 & -0.967536 & 0.086959 & $\mathrm{C}$ & -4.934525 & -0.292816 & -1.284834 \\
\hline C & -3.364465 & 1.254319 & 0.997524 & C & -3.942623 & 0.734694 & 0.852808 \\
\hline $\mathrm{H}$ & -2.883257 & -0.717061 & 1.675359 & $\mathrm{H}$ & -3.171215 & -0.917855 & -0.26211 \\
\hline$F$ & -3.981064 & -2.28301 & -0.082837 & $\mathrm{~F}$ & -4.619742 & -1.004909 & -2.396053 \\
\hline$F$ & -4.635686 & -0.462762 & -1.118961 & $\mathrm{~F}$ & -5.613119 & 0.811777 & -1.67118 \\
\hline$F$ & -5.370709 & -0.897332 & 0.907335 & $\mathrm{~F}$ & -5.773295 & -1.058119 & -0.527183 \\
\hline$F$ & -4.345255 & 1.400496 & 1.925603 & $\mathrm{~F}$ & -4.61159 & -0.088487 & 1.707602 \\
\hline $\mathrm{F}$ & -3.71677 & 1.946372 & -0.108431 & $\mathrm{~F}$ & -4.652459 & 1.876954 & 0.726187 \\
\hline
\end{tabular}




\begin{tabular}{|c|c|c|c|c|c|c|c|}
\hline \multirow{2}{*}{\multicolumn{4}{|c|}{ 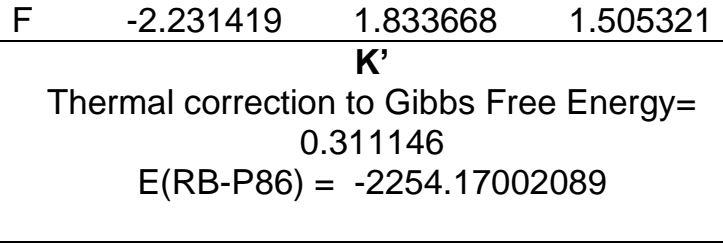 }} & \multirow{2}{*}{\multicolumn{4}{|c|}{$\begin{array}{c}F \quad-2.751312 \quad 1.054117 \quad 1.454035 \\
{\left[\text { TS }_{\text {K2a'] }}\right.} \\
\text { Thermal correction to Gibbs Free Energy }= \\
0.353516 \\
\text { E(RB-P86) }=-3043.94237458 \\
\text { Frequency } \quad-566.2355\end{array}$}} \\
\hline & & & & & & & \\
\hline$S$ & -0.242035 & 2.628164 & 0.367281 & $\mathrm{~N}$ & 1.411827 & 1.380833 & -0.939366 \\
\hline $\mathrm{N}$ & 0.235648 & 0.773707 & -1.516097 & $\mathrm{~N}$ & 0.911367 & 0.178915 & -2.040067 \\
\hline C & 0.944379 & 1.352434 & -0.330887 & $C$ & 2.606246 & 0.923396 & -0.319304 \\
\hline $\mathrm{N}$ & 0.974584 & 0.267426 & -2.397609 & $S$ & 2.693311 & 2.672203 & 0.39553 \\
\hline C & 0.17563 & 2.172938 & 2.138771 & $\mathrm{C}$ & 2.570995 & 2.118415 & 2.185546 \\
\hline$N$ & 0.982177 & 0.370861 & 0.739649 & $N$ & 2.462881 & 0.081819 & 0.87592 \\
\hline C & 2.304976 & 1.942801 & -0.703 & $\mathrm{C}$ & 3.77485 & 0.529796 & -1.202142 \\
\hline C & 0.808274 & 0.76923 & 2.056343 & $N$ & 0.170812 & -0.045986 & -2.864098 \\
\hline O & 1.685658 & -0.811219 & 0.45742 & $C$ & 2.539258 & 0.57231 & 2.156947 \\
\hline C & 2.375895 & 2.935947 & -1.703021 & $\mathrm{C}$ & 1.288637 & 2.637621 & 2.857246 \\
\hline C & 3.48429 & 1.521495 & -0.059704 & $\mathrm{C}$ & 3.837483 & 2.595467 & 2.922006 \\
\hline $\mathrm{N}$ & 1.511546 & -0.208677 & -3.297914 & 0 & 2.38223 & -1.297138 & 0.636878 \\
\hline C & -1.109118 & 2.113561 & 2.981849 & $\mathrm{C}$ & 3.865415 & 1.064978 & -2.503711 \\
\hline C & 1.200167 & 3.154922 & 2.743444 & $C$ & 4.797422 & -0.317254 & -0.729755 \\
\hline O & 1.097299 & 0.08227 & 3.032563 & 0 & 2.515128 & -0.107015 & 3.183299 \\
\hline C & 0.79114 & -1.979538 & 0.667204 & $\mathrm{C}$ & 1.08466 & -1.854156 & 1.106954 \\
\hline C & 3.608339 & 3.499509 & -2.052684 & $\mathrm{C}$ & 4.957809 & 0.750313 & -3.322846 \\
\hline $\mathrm{H}$ & 1.458747 & 3.27794 & -2.194691 & $\mathrm{H}$ & 3.079159 & 1.735716 & -2.862125 \\
\hline C & 4.717467 & 2.094873 & -0.412132 & $\mathrm{C}$ & 5.885691 & -0.632025 & -1.555363 \\
\hline $\mathrm{H}$ & 3.44478 & 0.734298 & 0.697686 & $\mathrm{H}$ & 4.728748 & -0.751598 & 0.270645 \\
\hline $\mathrm{H}$ & -0.841511 & 1.814496 & 4.00886 & $\mathrm{H}$ & 1.245443 & 2.233792 & 3.882492 \\
\hline $\mathrm{H}$ & -1.595823 & 3.102199 & 3.020025 & $\mathrm{H}$ & 1.297129 & 3.738659 & 2.913841 \\
\hline $\mathrm{H}$ & -1.820654 & 1.37598 & 2.583292 & $\mathrm{H}$ & 0.386831 & 2.312659 & 2.317081 \\
\hline $\mathrm{H}$ & 1.488314 & 2.800378 & 3.747738 & $\mathrm{H}$ & 3.818753 & 2.169176 & 3.939135 \\
\hline $\mathrm{H}$ & 2.106526 & 3.22988 & 2.122649 & $\mathrm{H}$ & 4.756967 & 2.257545 & 2.418728 \\
\hline $\mathrm{H}$ & 0.756489 & 4.159341 & 2.835189 & $\mathrm{H}$ & 3.858475 & 3.694737 & 2.999518 \\
\hline C & 1.615106 & -3.196646 & 0.352475 & $\mathrm{C}$ & 1.160357 & -3.335009 & 0.853391 \\
\hline $\mathrm{H}$ & -0.091023 & -1.874613 & 0.013669 & $\mathrm{H}$ & 0.254934 & -1.386212 & 0.552625 \\
\hline $\mathrm{H}$ & 0.472159 & -1.961311 & 1.722066 & $\mathrm{H}$ & 1.001597 & -1.624306 & 2.182146 \\
\hline C & 4.784093 & 3.081404 & -1.405636 & $\mathrm{C}$ & 5.970685 & -0.100161 & -2.8517 \\
\hline $\mathrm{H}$ & 3.650542 & 4.271789 & -2.827109 & $\mathrm{H}$ & 5.017086 & 1.171622 & -4.331371 \\
\hline $\mathrm{H}$ & 5.628961 & 1.759257 & 0.092346 & $\mathrm{H}$ & 6.667754 & -1.301529 & -1.183657 \\
\hline C & 1.410027 & -3.922805 & -0.835875 & $\mathrm{C}$ & 0.252958 & -3.957561 & -0.024408 \\
\hline C & 2.619946 & -3.613373 & 1.249871 & $\mathrm{C}$ & 2.140949 & -4.112151 & 1.504342 \\
\hline C & 2.188821 & -5.05493 & -1.120103 & $\mathrm{H}$ & 6.82239 & -0.348704 & -3.492883 \\
\hline $\mathrm{H}$ & 0.630042 & -3.602774 & -1.535569 & $C$ & 0.321779 & -5.342446 & -0.242959 \\
\hline C & 3.40452 & -4.738286 & 0.962579 & $\mathrm{H}$ & -0.519908 & -3.359099 & -0.516132 \\
\hline $\mathrm{H}$ & 2.779788 & -3.046034 & 2.173128 & $\mathrm{C}$ & 2.216801 & -5.491925 & 1.273262 \\
\hline C & 3.188053 & -5.462152 & -0.22239 & $\mathrm{H}$ & 2.842797 & -3.624429 & 2.188995 \\
\hline $\mathrm{H}$ & 2.016034 & -5.617934 & -2.042956 & $C$ & 1.305506 & -6.109804 & 0.399529 \\
\hline $\mathrm{H}$ & 4.18148 & -5.056174 & 1.665476 & $\mathrm{H}$ & -0.394209 & -5.820898 & -0.919021 \\
\hline $\mathrm{H}$ & 3.796428 & -6.345277 & -0.443668 & $\mathrm{H}$ & 2.981912 & -6.089048 & 1.780438 \\
\hline $\mathrm{H}$ & 5.74722 & 3.524807 & -1.677275 & $\mathrm{H}$ & 1.360461 & -7.189457 & 0.224555 \\
\hline
\end{tabular}




\begin{tabular}{|c|c|c|c|c|c|c|c|}
\hline 0 & -2.115906 & -0.758484 & -1.131649 & $\mathrm{H}$ & -0.01422 & 1.574324 & -0.17037 \\
\hline C & -3.100181 & -0.021346 & -0.442127 & $\mathrm{O}$ & -0.959455 & 1.637973 & 0.243248 \\
\hline $\mathrm{H}$ & -1.358234 & -0.145429 & -1.309913 & C & -1.557877 & 2.874354 & -0.066515 \\
\hline C & -3.489771 & -0.798186 & 0.82964 & C & -2.623582 & 3.151467 & 1.013717 \\
\hline C & -4.295521 & 0.247745 & -1.381887 & C & -2.141578 & 2.880475 & -1.499579 \\
\hline $\mathrm{H}$ & -2.733934 & 0.967924 & -0.105868 & $\mathrm{H}$ & -0.83191 & 3.708683 & -0.02333 \\
\hline $\mathrm{F}$ & -2.393964 & -0.917292 & 1.639482 & $\mathrm{~F}$ & -2.027634 & 3.290265 & 2.225987 \\
\hline$F$ & -3.937982 & -2.043831 & 0.559894 & $\mathrm{~F}$ & -3.519048 & 2.135038 & 1.104874 \\
\hline$F$ & -4.447142 & -0.141552 & 1.538271 & $\mathrm{~F}$ & -3.304453 & 4.295004 & 0.750489 \\
\hline $\mathrm{F}$ & -5.240216 & 1.017976 & -0.77555 & $\mathrm{~F}$ & -2.603803 & 4.10827 & -1.84197 \\
\hline$F$ & -4.888697 & -0.895097 & -1.800255 & $\mathrm{~F}$ & -3.157371 & 1.989989 & -1.64278 \\
\hline \multirow[t]{13}{*}{$\mathrm{F}$} & -3.855005 & 0.919718 & -2.479712 & $\mathrm{~F}$ & -1.163584 & 2.542554 & -2.382926 \\
\hline & & & & $\mathrm{H}$ & -1.69836 & 0.132822 & -0.191138 \\
\hline & & & & $\mathrm{O}$ & -1.898465 & -0.776244 & -0.545697 \\
\hline & & & & C & -3.243187 & -1.076582 & -0.264625 \\
\hline & & & & C & -3.3817 & -1.69794 & 1.144191 \\
\hline & & & & C & -3.754461 & -2.000751 & -1.385552 \\
\hline & & & & $\mathrm{H}$ & -3.892058 & -0.181159 & -0.275246 \\
\hline & & & & $\mathrm{F}$ & -2.799557 & -0.86674 & 2.052369 \\
\hline & & & & $\mathrm{F}$ & -2.773574 & -2.906233 & 1.238842 \\
\hline & & & & $\mathrm{F}$ & -4.685228 & -1.851767 & 1.498205 \\
\hline & & & & $\mathrm{F}$ & -5.027045 & -2.411562 & -1.138225 \\
\hline & & & & $\mathrm{F}$ & -2.978419 & -3.106207 & -1.529169 \\
\hline & & & & $\mathrm{F}$ & -3.75219 & -1.336741 & -2.56836 \\
\hline \multicolumn{4}{|c|}{$\begin{array}{c}{\left[\text { TS }_{\text {K2a' }}\right]^{\prime \prime}} \\
\text { Thermal correction to Gibbs Free Energy }= \\
0.396535 \\
\mathrm{E}(\mathrm{RB}-\mathrm{P} 86)=-3833.74810922 \\
\text { Frequency }-570.7333\end{array}$} & \multicolumn{4}{|c|}{$\begin{array}{c}{\left[\text { TS }_{\left.\mathrm{K} 2 \mathbf{a}^{\prime}\right]^{\prime \prime \prime}}\right.} \\
\text { Thermal correction to Gibbs Free Energy= } \\
0.353755 \\
\mathrm{E}(\mathrm{RB}-\mathrm{P} 86)=-3043.93794941 \\
\text { Frequency }-582.2989\end{array}$} \\
\hline $\mathrm{N}$ & 1.502703 & -1.261526 & 0.780491 & $\mathrm{~N}$ & 0.131837 & -0.915747 & -0.02055 \\
\hline $\mathrm{N}$ & 0.857043 & 0.136424 & 1.566046 & $\mathrm{~N}$ & -0.071823 & -0.769031 & -1.680916 \\
\hline C & 2.908319 & -1.177266 & 0.883103 & C & -0.921238 & -1.739454 & 0.485642 \\
\hline$S$ & 2.860177 & -2.949094 & 0.216329 & $S$ & 0.115469 & -1.809925 & 2.060659 \\
\hline C & 3.842794 & -2.562634 & -1.338034 & C & -1.141339 & -0.921698 & 3.133169 \\
\hline $\mathrm{N}$ & 3.624291 & -0.446424 & -0.177662 & $\mathrm{~N}$ & -2.129839 & -1.030241 & 0.914459 \\
\hline C & 3.537028 & -1.008298 & 2.252639 & C & -1.193987 & -3.07099 & -0.184343 \\
\hline$N$ & -0.104584 & 0.633304 & 1.891011 & $\mathrm{~N}$ & 0.448926 & -0.416845 & -2.623849 \\
\hline C & 4.147918 & -1.048019 & -1.29291 & C & -2.318344 & -0.565962 & 2.197751 \\
\hline C & 3.053014 & -2.880822 & -2.617378 & C & -0.549728 & 0.355447 & 3.750545 \\
\hline C & 5.162727 & -3.357438 & -1.272143 & C & -1.62985 & -1.910812 & 4.212123 \\
\hline O & 3.79507 & 0.928426 & 0.018546 & O & -3.082706 & -0.749532 & -0.070571 \\
\hline C & 2.823628 & -1.432578 & 3.392875 & C & -0.130756 & -3.76181 & -0.803173 \\
\hline C & 4.835997 & -0.482414 & 2.404433 & C & -2.477337 & -3.652591 & -0.160209 \\
\hline 0 & 4.756361 & -0.472296 & -2.196477 & O & -3.297753 & 0.072864 & 2.578977 \\
\hline C & 2.96519 & 1.723347 & -0.927246 & C & -3.078157 & 0.689345 & -0.463165 \\
\hline C & 3.398007 & -1.324488 & 4.666128 & C & -0.352031 & -5.011244 & -1.396808 \\
\hline $\mathrm{H}$ & 1.821587 & -1.853536 & 3.269649 & $\mathrm{H}$ & 0.872796 & -3.327218 & -0.798013 \\
\hline C & 5.403568 & -0.372302 & 3.681562 & C & -2.693372 & -4.900092 & -0.762972 \\
\hline $\mathrm{H}$ & 5.391622 & -0.134929 & 1.529969 & $\mathrm{H}$ & -3.309795 & -3.120009 & 0.305584 \\
\hline
\end{tabular}




\begin{tabular}{|c|c|c|c|c|c|c|c|}
\hline $\mathrm{H}$ & 3.670481 & -2.597584 & -3.485715 & $\mathrm{H}$ & -1.352091 & 0.871334 & 4.303656 \\
\hline $\mathrm{H}$ & 2.828378 & -3.958352 & -2.684121 & $\mathrm{H}$ & 0.25903 & 0.102623 & 4.455629 \\
\hline $\mathrm{H}$ & 2.11483 & -2.308463 & -2.67013 & $\mathrm{H}$ & -0.161099 & 1.037427 & 2.979702 \\
\hline $\mathrm{H}$ & 5.790935 & -3.047445 & -2.12424 & $\mathrm{H}$ & -2.438363 & -1.420385 & 4.780096 \\
\hline $\mathrm{H}$ & 5.713922 & -3.15745 & -0.340005 & $\mathrm{H}$ & -2.024818 & -2.839991 & 3.77176 \\
\hline $\mathrm{H}$ & 4.975397 & -4.44105 & -1.347268 & $\mathrm{H}$ & -0.814812 & -2.165371 & 4.908811 \\
\hline C & 3.549746 & 3.109 & -0.921089 & $\mathrm{C}$ & -4.441332 & 0.96516 & -1.033204 \\
\hline $\mathrm{H}$ & 1.913418 & 1.707753 & -0.599483 & $\mathrm{H}$ & -2.273877 & 0.868816 & -1.195184 \\
\hline $\mathrm{H}$ & 3.059061 & 1.255991 & -1.920683 & $\mathrm{H}$ & -2.884043 & 1.285522 & 0.441001 \\
\hline C & 4.689182 & -0.792856 & 4.814428 & $\mathrm{C}$ & -1.634685 & -5.582744 & -1.381738 \\
\hline $\mathrm{H}$ & 2.834865 & -1.65679 & 5.543878 & $\mathrm{H}$ & 0.481431 & -5.540329 & -1.869406 \\
\hline $\mathrm{H}$ & 6.407421 & 0.050099 & 3.790231 & $\mathrm{H}$ & -3.696871 & -5.33687 & -0.750766 \\
\hline C & 2.830177 & 4.200762 & -0.401508 & $\mathrm{C}$ & -4.615165 & 1.239757 & -2.401957 \\
\hline C & 4.84417 & 3.313779 & -1.443451 & $\mathrm{C}$ & -5.566024 & 0.945005 & -0.181616 \\
\hline $\mathrm{H}$ & 5.136492 & -0.705184 & 5.809654 & $\mathrm{H}$ & -1.807554 & -6.557302 & -1.849213 \\
\hline C & 3.39285 & 5.48696 & -0.415221 & $\mathrm{C}$ & -5.894112 & 1.506322 & -2.915496 \\
\hline $\mathrm{H}$ & 1.822358 & 4.046895 & -0.002214 & $\mathrm{H}$ & -3.74309 & 1.252443 & -3.065627 \\
\hline C & 5.407941 & 4.595807 & -1.446221 & $\mathrm{C}$ & -6.842991 & 1.202568 & -0.696106 \\
\hline $\mathrm{H}$ & 5.396095 & 2.456047 & -1.84224 & $\mathrm{H}$ & -5.419145 & 0.722962 & 0.880605 \\
\hline C & 4.681765 & 5.685073 & -0.933299 & $\mathrm{C}$ & -7.008797 & 1.486162 & -2.063293 \\
\hline $\mathrm{H}$ & 2.823452 & 6.333569 & -0.018389 & $\mathrm{H}$ & -6.019651 & 1.726709 & -3.980534 \\
\hline $\mathrm{H}$ & 6.412337 & 4.749584 & -1.854295 & $\mathrm{H}$ & -7.71211 & 1.188655 & -0.030232 \\
\hline $\mathrm{H}$ & 5.120661 & 6.688286 & -0.94137 & $\mathrm{H}$ & -8.007229 & 1.69274 & -2.462771 \\
\hline $\mathrm{H}$ & 0.106291 & -1.695293 & -0.092856 & $\mathrm{H}$ & -0.031909 & 0.860556 & 0.431253 \\
\hline O & -0.821756 & -1.994804 & -0.361554 & 0 & -0.176312 & 1.804742 & 0.722868 \\
\hline C & -0.753416 & -2.974541 & -1.371731 & $\mathrm{C}$ & 0.890844 & 2.591736 & 0.238578 \\
\hline C & -1.042394 & -2.333329 & -2.747349 & $\mathrm{C}$ & 1.309071 & 3.566745 & 1.355582 \\
\hline C & -1.724112 & -4.116513 & -1.006992 & $\mathrm{C}$ & 0.453386 & 3.316066 & -1.052782 \\
\hline $\mathrm{H}$ & 0.253508 & -3.425747 & -1.428377 & $\mathrm{H}$ & 1.787033 & 1.992256 & -0.011648 \\
\hline $\mathrm{F}$ & -0.12631 & -1.348406 & -2.974501 & $\mathrm{~F}$ & 1.764158 & 2.863934 & 2.428601 \\
\hline $\mathrm{F}$ & -2.273094 & -1.772197 & -2.791466 & $\mathrm{~F}$ & 0.277668 & 4.338076 & 1.775094 \\
\hline$F$ & -0.94243 & -3.237234 & -3.752815 & $\mathrm{~F}$ & 2.310731 & 4.386116 & 0.939513 \\
\hline$F$ & -1.709484 & -5.092226 & -1.947221 & $\mathrm{~F}$ & 1.479331 & 3.995218 & -1.625199 \\
\hline $\mathrm{F}$ & -3.005967 & -3.666878 & -0.888163 & $\mathrm{~F}$ & -0.566922 & 4.180453 & -0.847701 \\
\hline$F$ & -1.363812 & -4.66154 & 0.178968 & $\mathrm{~F}$ & 0.022379 & 2.382639 & -1.960556 \\
\hline $\mathrm{H}$ & -2.123261 & -0.944494 & -0.130241 & $\mathrm{H}$ & 1.984426 & -0.65738 & 0.166463 \\
\hline $\mathrm{O}$ & -2.858953 & -0.320856 & 0.133005 & 0 & 2.834936 & -0.14828 & 0.191016 \\
\hline C & -3.773961 & -1.008437 & 0.959823 & $\mathrm{C}$ & 3.691419 & -0.648094 & -0.806796 \\
\hline C & -5.186201 & -0.46282 & 0.668858 & $\mathrm{C}$ & 4.292533 & -2.007198 & -0.383113 \\
\hline C & -3.355483 & -0.848646 & 2.438024 & $\mathrm{C}$ & 4.759213 & 0.432376 & -1.06671 \\
\hline $\mathrm{H}$ & -3.798801 & -2.091227 & 0.745393 & $\mathrm{H}$ & 3.187622 & -0.822983 & -1.778687 \\
\hline $\mathrm{F}$ & -5.477208 & -0.634297 & -0.64465 & $\mathrm{~F}$ & 3.263485 & -2.857569 & -0.063059 \\
\hline$F$ & -5.282525 & 0.860206 & 0.951709 & $\mathrm{~F}$ & 5.093825 & -1.91381 & 0.698292 \\
\hline $\mathrm{F}$ & -6.119886 & -1.123717 & 1.399019 & $\mathrm{~F}$ & 4.994643 & -2.580244 & -1.393213 \\
\hline $\mathrm{F}$ & -4.214799 & -1.480294 & 3.276479 & $\mathrm{~F}$ & 5.649303 & 0.009684 & -2.002606 \\
\hline $\mathrm{F}$ & -3.278019 & 0.453433 & 2.807674 & $\mathrm{~F}$ & 5.438637 & 0.759502 & 0.053666 \\
\hline $\mathrm{F}$ & -2.121829 & -1.404314 & 2.611595 & $\mathrm{~F}$ & 4.156568 & 1.556589 & -1.538922 \\
\hline $\mathrm{H}$ & -3.263498 & 1.030249 & -1.011218 & & & & \\
\hline
\end{tabular}




\begin{tabular}{|lrrr|r|}
\hline O & -3.316929 & 1.953824 & -1.357044 & \\
C & -2.77997 & 2.765029 & -0.344735 & \\
C & -3.599095 & 4.068498 & -0.285052 & \\
C & -1.27871 & 3.017117 & -0.611672 & \\
H & -2.844402 & 2.30012 & 0.656441 & \\
F & -4.865211 & 3.798089 & 0.119362 & \\
F & -3.665364 & 4.684968 & -1.488004 & \\
F & -3.053217 & 4.943037 & 0.608388 & \\
F & -0.666935 & 3.629452 & 0.446095 & \\
F & -1.050785 & 3.762891 & -1.714168 & \\
F & -0.649411 & 1.811897 & -0.791603 & \\
\hline
\end{tabular}




\section{Crystallographic Data}

\section{Compound 2a:}

Crystal structures of 2a was obtained using a Bruker D8 Quest equipped with a microfocus source for generating Mo Ka radiation $(\lambda=0.71073 \AA)$ and a PHOTON II CMOS detector. Data were recorded at $298 \mathrm{~K}$. Integration and scaling of the recorded data were performed by SAINT ${ }^{12}$ and SADABS program ${ }^{13}$ respectively. Molecular structures were solved by direct methods using SHELXT-2018 ${ }^{3}$ and refined by fullmatrix least-squares on $\mathrm{F}^{2}$ using SHELXL-2018/3 version. ${ }^{14}$ All non-hydrogen atoms in the compounds were refined anisotropically and hydrogen atoms were placed at calculated positions using riding models.

Table S3. Crystal data and refinement parameters

\begin{tabular}{|c|c|}
\hline Compound code & $2 a$ \\
\hline Empirical formula & $\mathrm{C}_{18} \mathrm{H}_{18} \mathrm{~N}_{2} \mathrm{O}_{2} \mathrm{~S}_{1}$ \\
\hline Formula weight & 326.40 \\
\hline Wavelength/ $\AA$ & 0.71073 \\
\hline Crystal system & Monoclinic \\
\hline Space group & $P 2_{1} / n$ \\
\hline$a / \AA$ & $8.8520(9)$ \\
\hline$b / \AA$ & $18.4314(16)$ \\
\hline$c / \AA$ & $10.4241(10)$ \\
\hline$\alpha /\left(^{\circ}\right)$ & 90 \\
\hline$\beta /\left(^{\circ}\right)$ & $101.034(3)$ \\
\hline$\gamma /\left({ }^{\circ}\right)$ & 90 \\
\hline $\mathrm{V} / \AA^{3}$ & 1669.3(3) \\
\hline Z & 4 \\
\hline$D_{\text {cal }} / \mathrm{g} \mathrm{cm}^{-3}$ & 1.299 \\
\hline $\mathrm{T} / \mathrm{K}$ & $298(2)$ \\
\hline$\mu / \mathrm{mm}^{-1}$ & 0.205 \\
\hline$F_{000}$ & 688 \\
\hline Theta ranges for data collection $\left(^{\circ}\right)$ & 2.277 to 33.174 \\
\hline Reflections measured & 62944 \\
\hline Unique reflections & 6346 \\
\hline Observed reflections & 4107 \\
\hline
\end{tabular}




\begin{tabular}{|l|l|}
\hline Parameters & 210 \\
\hline Data completeness & 0.995 \\
\hline$R$ int & 0.0418 \\
\hline final $R(I>2 \sigma(I))$ & 0.0452 \\
\hline final $R$ (all data) & 0.0819 \\
\hline final $w R_{2}(I>2 \sigma(I))$ & 0.1109 \\
\hline final $w R_{2}$ (all data) & 0.1387 \\
\hline GOF on $\mathrm{F}^{2}$ & 1.047 \\
\hline CCDC no & $\mathbf{2 0 4 8 6 7 6}$ \\
\hline
\end{tabular}

Crystallization information: Solvent system: Chloroform-methanol mixture Method: Slow diffusion technique 
ORTEP diagram of 2a (CCDC No 2048676): Atoms are shown with $50 \%$ probability of thermal ellipsoids

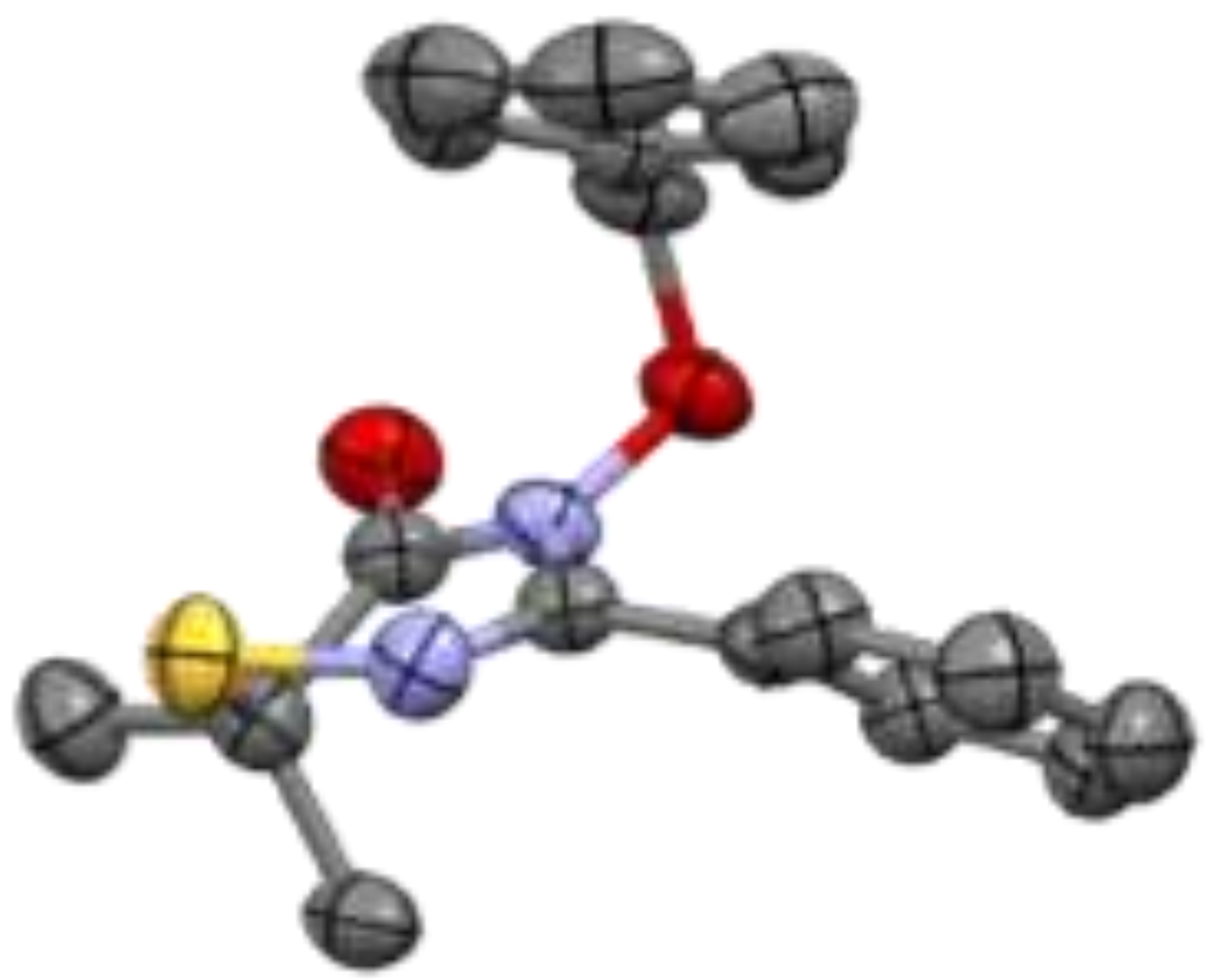




\section{Compound 3d:}

Crystal structures of $\mathbf{3} \mathbf{d}$ was determined using similar method as was used for compound 2a, described above.

Table S4. Crystal data and refinement parameters

\begin{tabular}{|c|c|}
\hline Compound code & 3d \\
\hline Empirical formula & $\mathrm{C}_{23} \mathrm{H}_{20} \mathrm{~N}_{2} \mathrm{O}_{2} \mathrm{~S}_{1}$ \\
\hline Formula weight & 388.47 \\
\hline Wavelength/ $\AA$ & 0.71073 \\
\hline Crystal system & Monoclinic \\
\hline Space group & $P 21 / c$ \\
\hline$a / \AA$ & $10.1166(12)$ \\
\hline$b / \AA$ & $23.387(3)$ \\
\hline$c / \AA$ & $8.8600(10)$ \\
\hline$\alpha /\left(^{\circ}\right)$ & 90 \\
\hline$\beta /\left(^{\circ}\right)$ & $104.733(4)$ \\
\hline$Y /\left(^{\circ}\right)$ & 90 \\
\hline $\mathrm{V} / \AA^{3}$ & 2027.3(4) \\
\hline Z & 4 \\
\hline$D_{\text {cal }} / \mathrm{g} \mathrm{cm}^{-3}$ & 1.273 \\
\hline $\mathrm{T} / \mathrm{K}$ & $298(2)$ \\
\hline$\mu / \mathrm{mm}^{-1}$ & 0.180 \\
\hline$F_{000}$ & 816 \\
\hline Theta ranges for data collection $\left(^{\circ}\right)$ & 2.256 to 28.300 \\
\hline Reflections measured & 41861 \\
\hline Unique reflections & 5015 \\
\hline Observed reflections & 3864 \\
\hline Parameters & 254 \\
\hline Data completeness & 0.995 \\
\hline$R_{\text {int }}$ & 0.0376 \\
\hline final $R(I>2 \sigma(I))$ & 0.0414 \\
\hline final $R$ (all data) & 0.0575 \\
\hline final $w R_{2}(I>2 \sigma(I))$ & 0.1047 \\
\hline
\end{tabular}




\begin{tabular}{|l|l|}
\hline final $w R_{2}$ (all data) & 0.1186 \\
\hline GOF on $F^{2}$ & 1.025 \\
\hline CCDC no & $\mathbf{2 0 4 8 6 7 7}$ \\
\hline
\end{tabular}

Crystallization information: Solvent system: Chloroform-methanol mixture Method: Slow diffusion technique 
ORTEP diagram of 3d (CCDC No 2048677): Atoms are shown with $50 \%$ probability of thermal ellipsoids

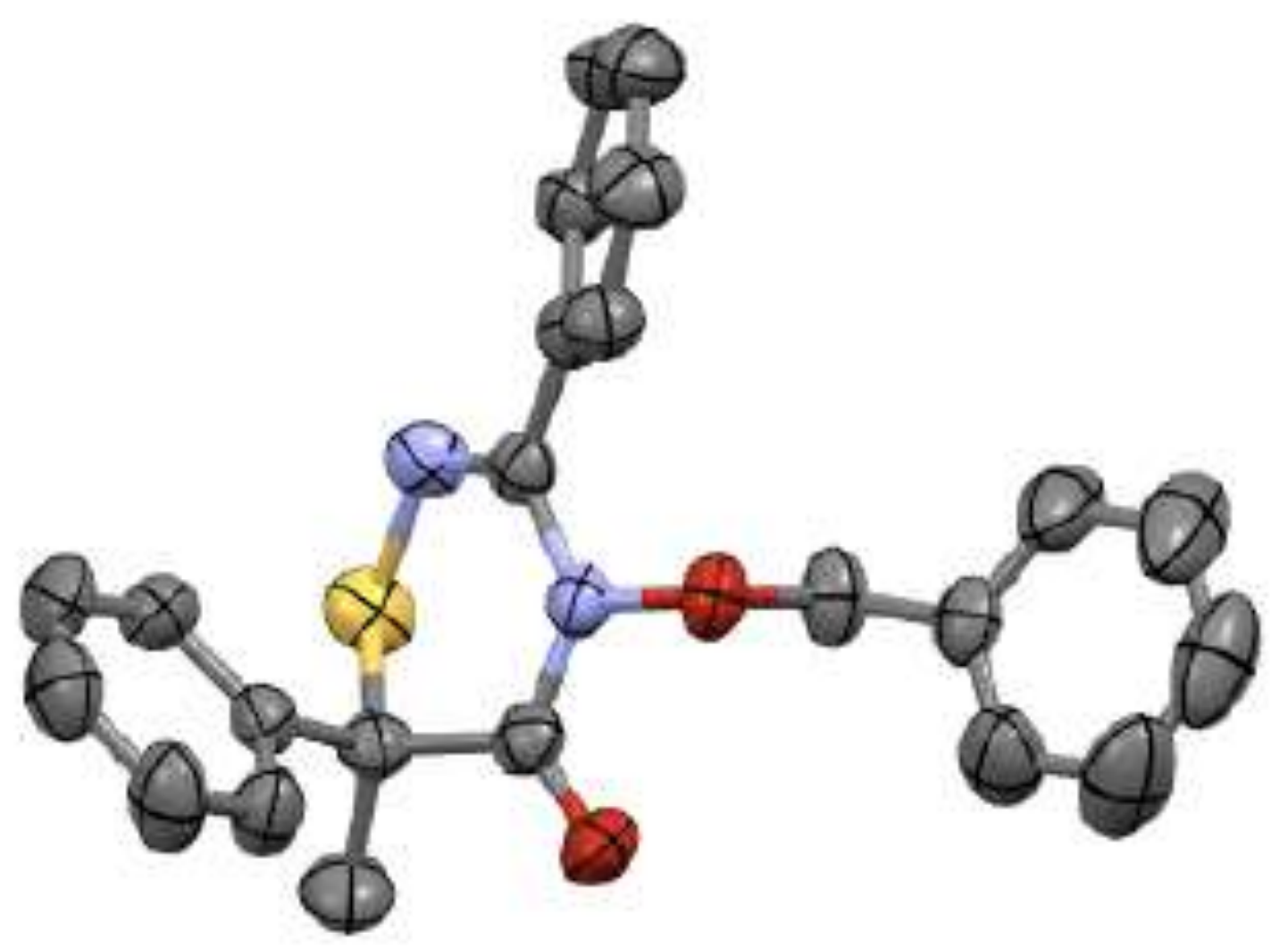




\section{Compound 7a:}

Crystal structures of $\mathbf{7 a}$ was determined using similar method as was used for compound 2a, described above.

Table S5. Crystal data and refinement parameters

\begin{tabular}{|c|c|}
\hline Compound code & $7 a$ \\
\hline Empirical formula & $\mathrm{C}_{19} \mathrm{H}_{20} \mathrm{~N}_{2} \mathrm{O}_{2} \mathrm{Se}_{1}$ \\
\hline Formula weight & 387.33 \\
\hline Wavelength/ $\AA$ & 0.71073 \\
\hline Crystal system & Monoclinic \\
\hline Space group & $C 2 / c$ \\
\hline$a / \AA$ & $28.028(4)$ \\
\hline$b / \AA$ & $7.8188(9)$ \\
\hline$c / \AA$ & $19.487(3)$ \\
\hline$\alpha /\left(^{\circ}\right)$ & 90 \\
\hline$\beta /\left(^{\circ}\right)$ & $120.458(4)$ \\
\hline$y /\left(^{\circ}\right)$ & 90 \\
\hline $\mathrm{V} / \AA^{3}$ & $3681.1(8)$ \\
\hline Z & 8 \\
\hline$D_{\text {cal }} / \mathrm{g} \mathrm{cm}^{-3}$ & 1.398 \\
\hline $\mathrm{T} / \mathrm{K}$ & $298(2)$ \\
\hline$\mu / \mathrm{mm}^{-1}$ & 2.053 \\
\hline$F_{000}$ & 1584 \\
\hline Theta ranges for data collection $\left(^{\circ}\right)$ & 2.139 to 28.404 \\
\hline Reflections measured & 82461 \\
\hline Unique reflections & 4607 \\
\hline Observed reflections & 3979 \\
\hline Parameters & 220 \\
\hline Data completeness & 0.996 \\
\hline$R_{\text {int }}$ & 0.0267 \\
\hline final $R(I>2 \sigma(I))$ & 0.0327 \\
\hline final $R$ (all data) & 0.0375 \\
\hline
\end{tabular}




\begin{tabular}{|l|l|}
\hline final $w R_{2}(\mathrm{I}>2 \sigma(\mathrm{l}))$ & 0.0963 \\
\hline final $w R_{2}($ all data) & 0.0997 \\
\hline GOF on $\mathrm{F}^{2}$ & 1.054 \\
\hline CCDC no & $\mathbf{2 0 4 8 6 7 9}$ \\
\hline
\end{tabular}

Crystallization information: Solvent system: Chloroform-methanol mixture Method: Slow diffusion technique 
ORTEP diagram of 7 a (CCDC No 2048679): Atoms are shown with $50 \%$ probability of thermal ellipsoids

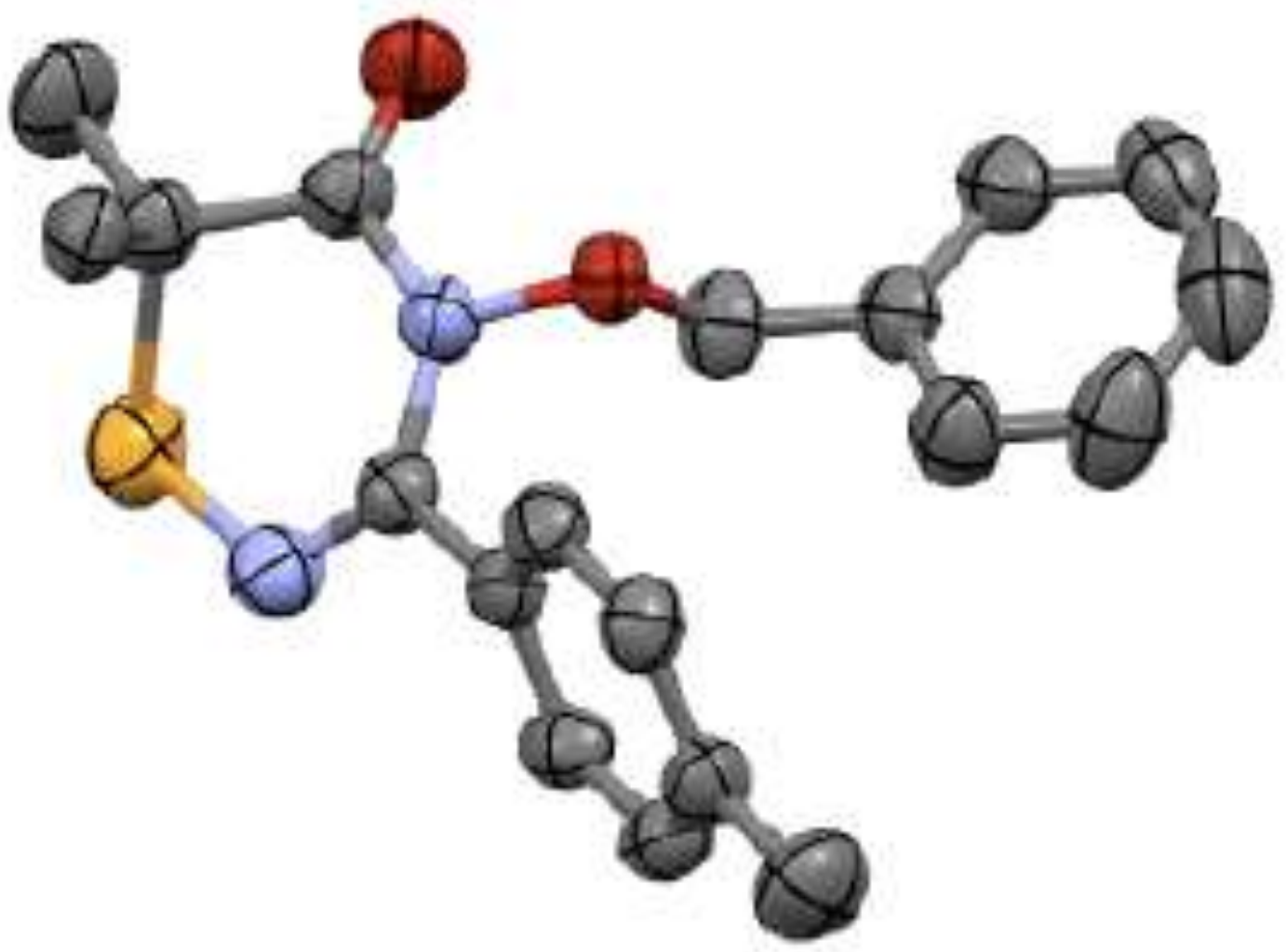




\section{Compound 11:}

Crystal structures of $\mathbf{1 1}$ was determined using similar method as was used for compound $\mathbf{2 a}$, described above.

Table S6. Crystal data and refinement parameters

\begin{tabular}{|c|c|}
\hline Compound code & 11 \\
\hline Empirical formula & $\mathrm{C}_{18} \mathrm{H}_{18} \mathrm{~N}_{2} \mathrm{O}_{3} \mathrm{~S}_{1}$ \\
\hline Formula weight & 342.40 \\
\hline Wavelength/ $\AA$ & 0.71073 \\
\hline Crystal system & Monoclinic \\
\hline Space group & $P 2_{1} / n$ \\
\hline$a / \AA$ & $9.5138(13)$ \\
\hline$b / \AA$ & $10.6279(16)$ \\
\hline$c / \AA$ & 17.402(3) \\
\hline$\alpha /\left(^{\circ}\right)$ & 90 \\
\hline$\beta /\left(^{\circ}\right)$ & 101.083(4) \\
\hline$\gamma /\left(^{\circ}\right)$ & 90 \\
\hline $\mathrm{V} / \AA^{3}$ & 1726.7(4) \\
\hline Z & 4 \\
\hline$D_{\text {cal }} / \mathrm{g} \mathrm{cm}^{-3}$ & 1.317 \\
\hline$T / K$ & $298(2)$ \\
\hline$\mu / \mathrm{mm}^{-1}$ & 0.205 \\
\hline$F_{000}$ & 720 \\
\hline Theta ranges for data collection $\left(^{\circ}\right)$ & 2.257 to 28.405 \\
\hline Reflections measured & 69477 \\
\hline Unique reflections & 4322 \\
\hline Observed reflections & 2391 \\
\hline Parameters & 219 \\
\hline Data completeness & 0.995 \\
\hline$R_{\text {int }}$ & 0.1295 \\
\hline final $R(I>2 \sigma(I))$ & 0.0602 \\
\hline
\end{tabular}




\begin{tabular}{|l|l|}
\hline final $R$ (all data) & 0.1284 \\
\hline final $w R_{2}(\mathrm{I}>2 \sigma(\mathrm{I}))$ & 0.1389 \\
\hline final $w R_{2}$ (all data) & 0.1745 \\
\hline GOF on $\mathrm{F}^{2}$ & 1.022 \\
\hline CCDC no & $\mathbf{2 0 4 8 6 7 8}$ \\
\hline
\end{tabular}

Crystallization information: Solvent system: Chloroform-methanol mixture Method: Slow diffusion technique 
ORTEP diagram of 11 (CCDC No 2048678): Atoms are shown with $50 \%$ probability of thermal ellipsoids

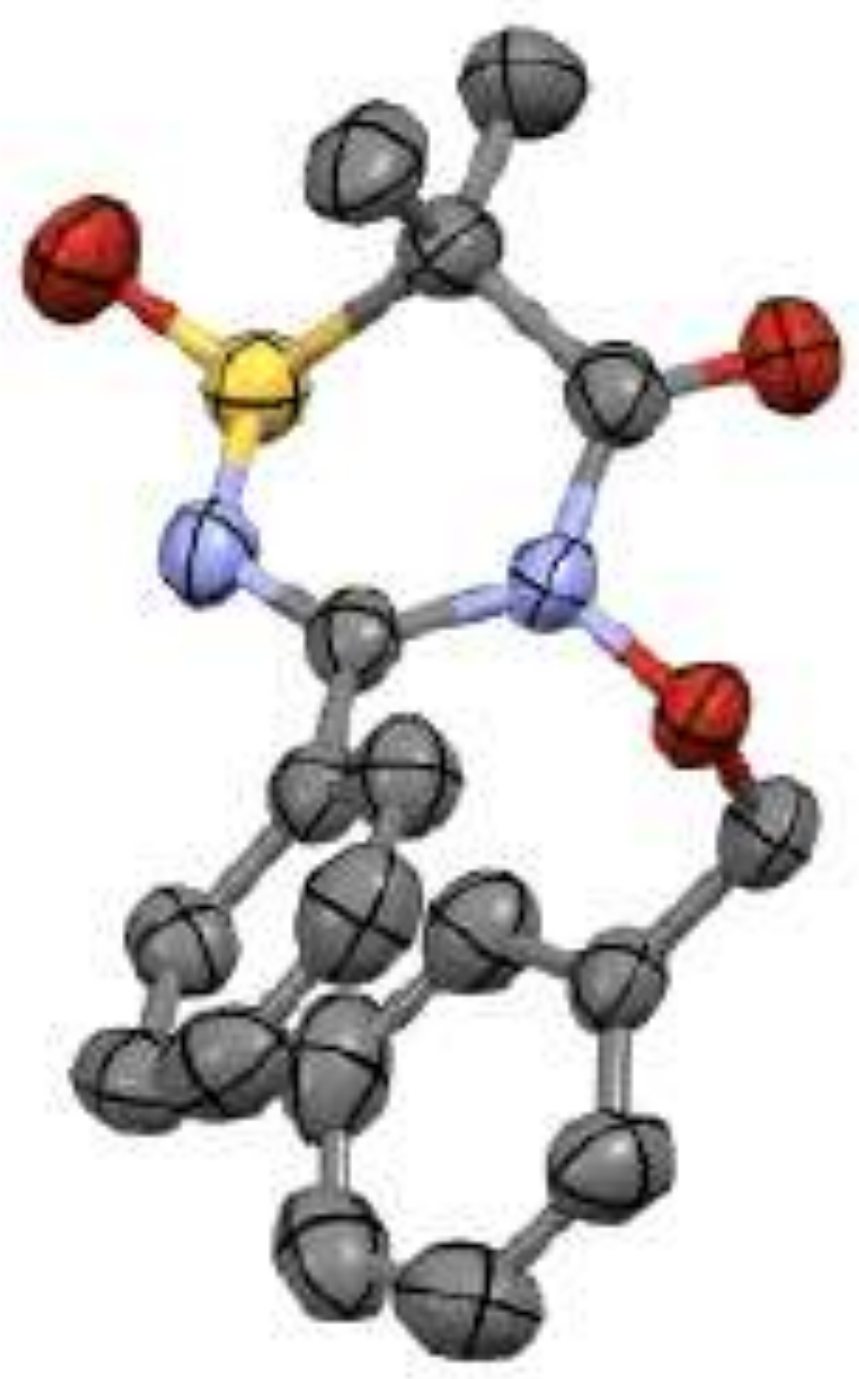




\section{References.}

(1) See ref. 10 from the main text.

(2) C. Tselepis, J. S. Fossey, R. Byravan, Iron Complexing agents and uses thereof in the treatment and prevention of colorectal cancer. US2016/0296629 A1, 2016.

(3) G. Malik, X. Guinchard, D. Crich Asymmetric Synthesis of Polyhydroxylated NAlkoxypiperidines by Ring-Closing Double Reductive Amination: Facile Preparation of Isofagomine and Analogues: Org. Lett. 2012, 14, 596-599.

(4) C. S. Jeffrey, K. L. Barnes, , J. A. Eickhoff, C. R. Carson, J. Am. Chem. Soc., 2011, $133,7688$.

(5) S.Thurow, E. J. Lenardão, X. J. Baringo, D. J. Procter, Org. Lett. 2017, 19, 1, 50-53.

(6) See ref. 18a from the main text.

(7) Gaussian 16, Revision B.01, M. J. Frisch, G. W. Trucks, H. B. Schlegel, G. E. Scuseria, M. A. Robb, J. R. Cheeseman, G. Scalmani, V. Barone, G. A. Petersson, H. Nakatsuji, X. Li, M. Caricato, A. V. Marenich, J. Bloino, B. G. Janesko, R. Gomperts, B. Mennucci, H. P. Hratchian, J. V. Ortiz, A. F. Izmaylov, J. L. Sonnenberg, D. Williams-Young, F. Ding, F. Lipparini, F. Egidi, J. Goings, B. Peng, A. Petrone, T. Henderson, D. Ranasinghe, V. G. Zakrzewski, J. Gao, N. Rega, G. Zheng, W. Liang, M. Hada, M. Ehara, K. Toyota, R. Fukuda, J. Hasegawa, M. Ishida, T. Nakajima, Y. Honda, O. Kitao, H. Nakai, T. Vreven, K. Throssell, J. A. Montgomery, Jr., J. E. Peralta, F. Ogliaro, M. J. Bearpark, J. J. Heyd, E. N. Brothers, K. N. Kudin, V. N. Staroverov, T. A. Keith, R. Kobayashi, J. Normand, K. Raghavachari, A. P. Rendell, J. C. Burant, S. S. lyengar, J. Tomasi, M. Cossi, J. M. Millam, M. Klene, C. Adamo, R. Cammi, J. W. Ochterski, R. L. Martin, K. Morokuma, O. Farkas, J. B. Foresman, and D. J. Fox, Gaussian, Inc., Wallingford CT, 2016.

(8) Becke, A. D. Phys. Rev. A. 1988, 38, 3098-3100.

(9) G.-X. Li, C. A. Morales-Rivera, F. Gao, Y. Wang, G. He, P. Liu, G. Chen, Chem. Sci. 2017, 8, 7180-7185.

(10) See ref. 23 from the main text.

(11) See ref. 17 from the main text.

(12) SAINT, Version 6.45; Bruker AXS Inc.: Madison, WI, 2003;

(13) SADABS, Version 2.05; Bruker AXS Inc.: Madison, WI, 2002.

(14) G. M. Sheldrick, Acta Cryst. Sect. A. 2015, 71, 3-8 